Supporting Information

\title{
Development of Immobilized SPINOL-Derived Chiral Phosphoric Acids for Catalytic Continuous Flow Processes. Use in the Catalytic Desymmetrization of 3,3-Disubstituted Oxetanes
}

\author{
Junshan Lai, $\dagger, \S$ Mauro Fianchini, ${ }^{\dagger}$ and Miquel A. Pericàs $s^{\dagger,} \hbar, *$ \\ $\dagger$ Institute of Chemical Research of Catalonia (ICIQ), The Barcelona Institute of Science and Technology, Av. Països \\ Catalans, 16, 43007 Tarragona (Spain) \\ $\S$ Universitat Rovira i Virgili, Departament de Química Analítica i Química Orgànica, c/Marcel·lí Domingo, 1, 43007 \\ Tarragona (Spain) \\ ¥ Departament de Química Inorgànica i Orgànica, Universitat de Barcelona (UB), 08028 Barcelona (Spain) \\ mapericas@iciq.es
}

\section{Table of Contents}

1. General information

2. Preparation of the immobilized SPINOL-derived chiral phosphoric acids

2.1. Preparation of Cat $\mathbf{b}$

2.2. Preparation of Cat $\mathrm{c}$

2.3. Preparation of Cat $\mathbf{d}$ S4

2.4. Preparation of Cat $\mathrm{e}$ S6

2.5. Preparation of Cat $f$ S9

2.6. Preparation of Cat $\mathbf{g}$

3. Experimental procedures

3.1. Optimization of catalyst and solvent for the desymmetrization reaction

3.2. Recycling of Cat $\mathbf{f}$ in the desymmetrization of 22a with 23a in batch

3.3. Optimization of the desymmetrization of 22a with 23a mediated by Cat $\mathbf{f}$ in flow

3.4. Continuous flow desymmetrization of oxetanes 22 with thiols 23 mediated by Cat $\mathbf{f}$

3.5. Desymmetrization of oxetanes $\mathbf{2 2}$ with thiols $\mathbf{2 3}$ mediated by Cat $\mathbf{g}$ in batch

4. Compound characterization data

5. Computational section

6. X-ray diffraction data

7. References

8. NMR spectra

9. HPLC chromatograms 


\section{General information}

Unless otherwise noted, all reactions were conducted under air. All commercial reagents were used as received; 3,3'-Diaryl-SPINOLs $\mathbf{S 1}$ and $\mathbf{1 2}$ were synthesized according to the reported procedures. ${ }^{1 \mathrm{a}} \mathbf{2 2 \mathrm { g }}$ is commercially available, 22n was prepared according to a reported procedure. ${ }^{1 b}$ All other oxetanes were prepared from the coupling of 3-oxetanone with the corresponding Grignard reagents. Flash chromatography was carried out using 60 mesh silicagel and dry-packed columns. For the continuous flow system, the packed bed reactor was an adjustable volume, jacketed Omnifit glass column with $10 \mathrm{~mm} \varnothing$, and a dual syringe pump system was used for the circulation of reactants and solvent. Thin layer chromatography was carried out using Merck TLC Silicagel 60 F254 aluminum sheets. Components were visualized by UV light $(\lambda=254 \mathrm{~nm})$ and stained with phosphomolybdic dip. NMR spectra were recorded at $298 \mathrm{~K}$ on a Bruker Avance 400 Ultrashield apparatus. ${ }^{1} \mathrm{H}$ NMR spectroscopy chemical shifts are quoted in ppm relative to tetramethylsilane (TMS). $\mathrm{CDCl}_{3}$ was used as internal standard for ${ }^{13} \mathrm{C}$ NMR spectra. Chemical shifts are given in ppm and coupling constants in Hz. IR spectra were recorded on a Bruker Tensor 27 FTIR spectrometer and are reported in wavenumbers $\left(\mathrm{cm}^{-1}\right)$. Elemental analyses were performed by MEDAC Ltd. (Surrey, UK) on a LECO CHNS 932 micro-analyzer. High performance liquid chromatography (HPLC) was performed on Agilent Technologies chromatographs (1100 and 1200 Series), using Chiralpak AD-H columns and guard columns. FAB mass spectra were obtained on a Fisons V6-Quattro instrument, ESI mass spectra were obtained on a Waters LCT Premier Instrument and Cl and El spectra were obtained on a Waters GCT spectrometer. Specific optical rotation measurements were carried out on a Jasco P-1030 polarimeter. 


\section{Preparation of the immobilized SPINOL-derived chiral phosphoric acids}

\subsection{Preparation of Cat $b$}

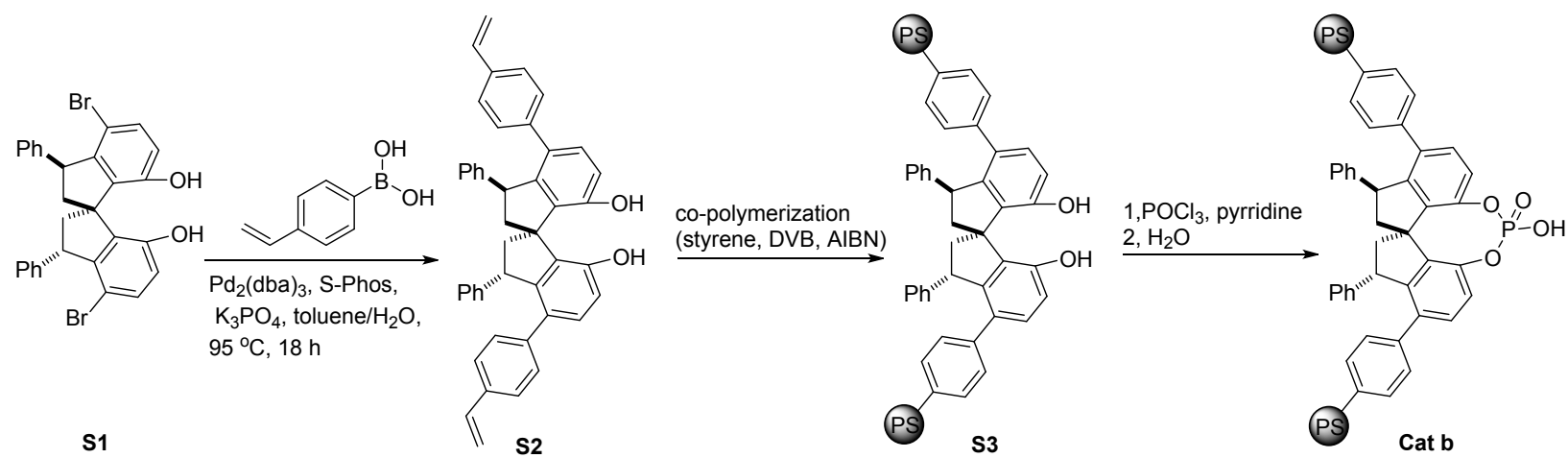

Scheme S1. The procedure of preparation of Cat $\mathbf{b}$

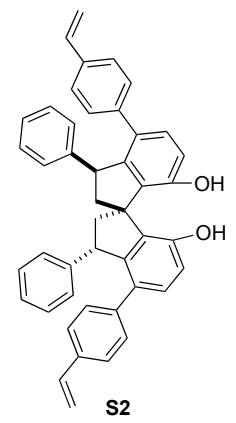

S2 was synthesized following a typical Suzuki coupling procedure. Under $\mathrm{N}_{2}$, to a Schlenk tube containing S1 (1.12 g, 2 mmol), 4-vinylphenylboronic acid (1.18 g, 8 mmol, 4.0 equiv), $\operatorname{Pd}_{2}\left(d_{b a}\right)_{3}$ (36.8 mg, $2 \mathrm{~mol} \%$ ), S-Phos (66 mg, $8 \mathrm{~mol} \%$ ) and $\mathrm{K}_{3} \mathrm{PO}_{4}(1.7 \mathrm{~g}, 8 \mathrm{mmol}, 4.0$ equiv) was added degased toluene $(15 \mathrm{~mL})$ and water $(5 \mathrm{~mL})$. The reaction mixture was stirred and heated to $95{ }^{\circ} \mathrm{C}$ for $18 \mathrm{~h}$. After being cooled to room temperature, $\mathrm{HCl}(2 \mathrm{~N})$ was added to the reaction mixture. The mixture was then extracted with $\mathrm{CH}_{2} \mathrm{Cl}_{2}(40 \mathrm{~mL} \times 3)$. The combined organic layers were dried over $\mathrm{MgSO}_{4}$ and concentrated under reduced pressure. The crude product was purified by flash column chromatography on silicagel using cyclohexane/ $\mathrm{CH}_{2} \mathrm{Cl}_{2}(5: 1)$ as the eluent to give compound $\mathbf{S 2}$ as a slightly yellow solid. (791 $\mathrm{mg}, 65 \%$ yield).

Yellow solid. Carbonization or polymerization starts from $280^{\circ} \mathrm{C}$; Melting point could not be detected.

${ }^{1} \mathrm{H}$ NMR (400 MHz, Chloroform-d) $\delta 7.12$ (d, J = 8.2 Hz, 2H), 7.06 (d, J = 8.2 Hz, 4H), 6.99-6.79 (m, 16H), 6.58 (dd, $J=$ 17.6, $10.9 \mathrm{~Hz}, 2 \mathrm{H}), 5.61$ (dd, $J=17.6,1.0 \mathrm{~Hz}, 2 \mathrm{H}), 5.15$ (dd, $J=10.8,1.0 \mathrm{~Hz}, 2 \mathrm{H}), 4.99(\mathrm{~s}, 2 \mathrm{H}), 4.73$ (dd, $J=10.1,7.7$ $\mathrm{Hz}, 2 \mathrm{H}), 2.94$ (dd, $J=13.3,7.7 \mathrm{~Hz}, 2 \mathrm{H}), 2.37$ (dd, $J=13.2,10.3 \mathrm{~Hz}, 2 \mathrm{H})$.

${ }^{13} \mathrm{C}$ NMR (101 MHz, $\left.\mathrm{CDCl}_{3}\right) \delta 151.9(x 2), 145.3$ (x2), $143.9(x 2), 139.7(x 2), 136.7(x 2), 135.3$ (x2), $133.1(x 2), 131.8$ (x2), $131.7(x 2), 128.8(x 4), 128.0(x 4), 127.7(x 4), 125.6(x 2), 125.3(x 4), 115.2(x 2), 113.0(x 2), 55.6(x 2), 50.0(x 3)$. IR (neat): 3527, 3020, 2927, 2867, 1595, 1480, 1261, 1201, 988, 906, 822, 762, $698 \mathrm{~cm}^{-1}$.

HRMS (ESI): m/z: [M-H] ${ }^{+}\left(\mathrm{C}_{45} \mathrm{H}_{35} \mathrm{O}_{2}\right)$, calcd.: 607.2643; found: 607.2627.

$[\alpha]_{D}^{25}=+288\left(c=0.1, \mathrm{CH}_{2} \mathrm{Cl}_{2}\right)$.

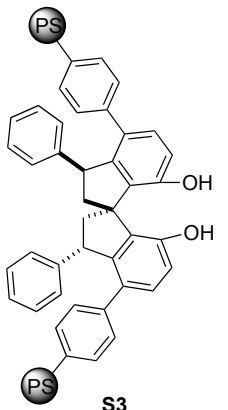

S3 was synthesized following a modification of a literature procedure. ${ }^{2}$ A $100 \mathrm{~mL}$ reactor was charged with a suspension of polyvinyl alcohol (PV-OH) (100 mg, $0.96 \mu \mathrm{mol}, 0.001$ equiv.) in $72 \mathrm{~mL}$ of degassed MiliQ water. The solution was heated at $100{ }^{\circ} \mathrm{C}$ until PV-OH was dissolved. Then, it was cooled to RT and a solution of boric acid (449 mg, $7.26 \mathrm{mmol}$ ) in $18 \mathrm{~mL}$ of degassed MiliQ 
water was transferred to the reactor. Later, a degassed solution containing divinylbenzene (DVB), filtered on a short pad of silica immediately before use, $(80 \%, 119 \mu \mathrm{L}, 0.68 \mathrm{mmol}, 0.65$ equiv.), BINOL derivative $\mathbf{S 2}$ (645 mg, $1.06 \mathrm{mmol})$, styrene ( $2.9 \mathrm{~mL}, 25.5 \mathrm{mmol}, 23.66$ equiv.) and AIBN (31 mg, $0.19 \mathrm{mmol}, 0.18$ equiv.) in toluene (2.4 mL) was transferred to the reactor. After that, the system was heated at $90{ }^{\circ} \mathrm{C}$ and magnetically stirred at $440 \mathrm{rpm}$ overnight, the aqueous solution was decanted off and the resin was washed with water $\left(50^{\circ} \mathrm{C}\right)$ several times, followed by $\mathrm{MeOH}$ and $\mathrm{CH}_{2} \mathrm{Cl}_{2}$. Finally, it was dried overnight in a $40{ }^{\circ} \mathrm{C}$ vacuum oven to furnish $2.7 \mathrm{~g}$ of light-yellow beads.

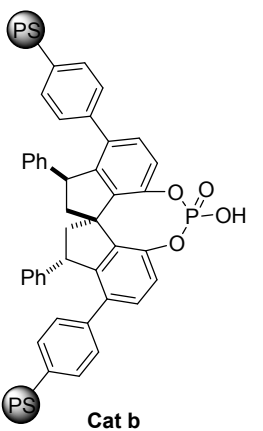

Cat $\mathbf{b}$ was synthesized following a modification of a literature procedure. ${ }^{2}$ In an oven-dried Schlenk tube, resin S3 (2.7 g, ca. $1.06 \mathrm{mmol})$ was suspended in pyridine $(20 \mathrm{~mL})$ under Ar. Then, $\mathrm{POCl}_{3}(495 \mu \mathrm{L}, 5.3 \mathrm{mmol}, 5$ eq.) was added and the reaction mixture was heated in the sealed Schlenk tube at $120^{\circ} \mathrm{C}$. After 2 days, it was cooled to RT and $5 \mathrm{~mL}$ of water were added. Then the system was sealed again and heated at $100{ }^{\circ} \mathrm{C}$ overnight. The resin was filtered and washed with water, THF/water, THF, $2 \mathrm{M} \mathrm{HCl}$ /EtOAC, EtOAC/DCM, and DCM and dried overnight in a 40 ${ }^{\circ} \mathrm{C}$ vacuum oven to give $2.7 \mathrm{~g}$ of brown beads.

P elemental analysis (\%): 0.34

$f_{(P)}: 0.11 \mathrm{mmol} / \mathrm{g}$ resin

\subsection{Preparation of Cat $c$}

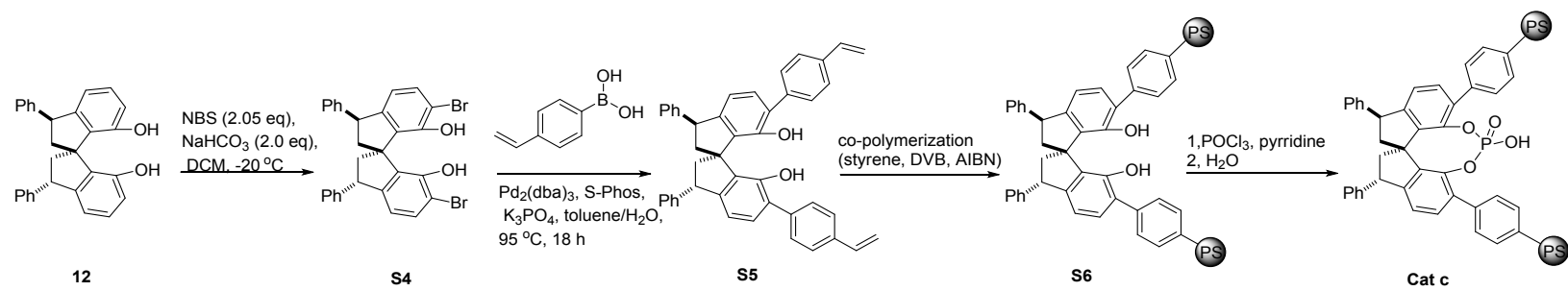

Scheme S2. The procedure of preparation of Cat c

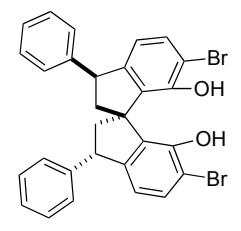

S4

S4 was synthesized according to the reported procedure. ${ }^{3}$ In a Schlenk tube, SPINOL 12 (3.232 g, $8 \mathrm{mmol}$ ) and $\mathrm{NaHCO}_{3}\left(1.344 \mathrm{~g}, 16 \mathrm{mmol}, 2.0\right.$ equiv) were mixed with $\mathrm{CH}_{2} \mathrm{Cl}_{2}(80 \mathrm{~mL})$, and the mixture was stirred and cooled to $-20^{\circ} \mathrm{C}$. Then, NBS (2.92 g, $16.4 \mathrm{mmol}, 2.05$ equiv) was added slowly. After being stirred at $-20^{\circ} \mathrm{C}$ for $2 \mathrm{~h}$, the reaction mixture was poured into $\mathrm{HCl}(2 \mathrm{~N})$. The reaction mixture was then extracted with $\mathrm{CH}_{2} \mathrm{Cl}_{2}(40 \mathrm{~mL} \times 3)$. After removal of solvent by rotaevaporation, the residue was purified by column chromatography (silicagel, cyclohexane/ethyl acetate $(v / v=20 / 1)$ as eluent), affording the expected compound $\mathbf{S 4}$ as a Light yellow solid ( $3.6 \mathrm{~g}, 80 \%$ yield).

Light yellow solid. m.p. $241^{\circ} \mathrm{C}$.

${ }^{1} \mathrm{H}$ NMR (500 MHz, Chloroform-d) 8 7.33-7.24 (m, 12H), 6.36 (dd, J = 8.1, 1.2 Hz, 2H), $5.43(\mathrm{~d}, J=1.4 \mathrm{~Hz}, 2 \mathrm{H}), 4.39$ (dd, $J=10.6,7.7 \mathrm{~Hz}, 2 \mathrm{H}$ ), 2.76 (dd, $J=12.8,7.6 \mathrm{~Hz}, 2 \mathrm{H}$ ), 2.49 (dd, $J=12.9,10.8 \mathrm{~Hz}, 2 \mathrm{H}$ ). 
${ }^{13} \mathrm{C}$ NMR (126 MHz, $\mathrm{CDCl}_{3}$ ) $\delta 148.4$ (x2), 144.0 (x2), 134.5 (x2), $131.2(x 2), 128.6(x 2), 128.4(x 2), 128.3(x 4), 126.7$ (x2), $118.5(x 2), 108.5(x 2), 99.9(x 2), 57.4,49.9(x 2), 48.0(x 2)$.

IR (neat): 3492, 3061, 3023, 2935, 2863, 1579, 1493, 1445, 1317, 1237, 1160, 907, 806, 759, $698 \mathrm{~cm}^{-1}$.

HRMS (ESI): m/z: [M-H] ${ }^{+}\left(\mathrm{C}_{29} \mathrm{H}_{21} \mathrm{Br}_{2} \mathrm{O}_{2}\right)$, calcd.: 558.9914; found: 558.9903.

$[\alpha]_{D}^{25}=+65\left(c=0.1, \mathrm{CH}_{2} \mathrm{Cl}_{2}\right)$.

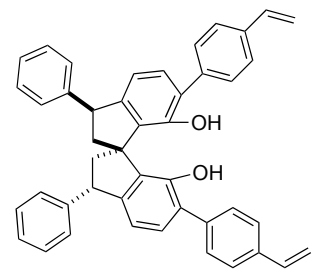

S5

S5 was synthesized following a modification of a literature procedure. ${ }^{3}$ Under $\mathrm{N}_{2}$, to a Schlenk tube containing S4 (1.12 g, 2 mmol), 4-vinylphenylboronic acid ( $1.18 \mathrm{~g}, 8 \mathrm{mmol}, 4.0$ equiv), $\mathrm{Pd}_{2}(\mathrm{dba})_{3}(36.8 \mathrm{mg}, 2 \mathrm{~mol} \%)$, S-Phos (66 mg, $8 \mathrm{~mol} \%$ ) and $\mathrm{K}_{3} \mathrm{PO}_{4}(34 \mathrm{mmol})$ was added toluene $(20 \mathrm{~mL})$ and water $(20 \mathrm{~mL})$. The reaction mixture was stirred and heated to $95^{\circ} \mathrm{C}$ for $18 \mathrm{~h}$. After being cooled to room temperature, $\mathrm{HCl}(2 \mathrm{~N})$ was added to the reaction mixture. The mixture was then extracted with $\mathrm{CH}_{2} \mathrm{Cl}_{2}(40 \mathrm{~mL} \times 3)$. After removal of solvent by rota-evaporation, the residue was purified by column chromatography [silicagel, cyclohexane/ dichloromethane ( $/ \mathrm{v}=5 / 2)$ as eluent], affording the expected product as a white solid. (780 mg, 64\% yield).

Light yellow solid. Carbonization or polymerization, Melting point cannot be detected.

${ }^{1} \mathrm{H}$ NMR (400 MHz, Chloroform-d) $\delta 7.46(\mathrm{t}, J=2.1 \mathrm{~Hz}, 8 \mathrm{H}), 7.33$ (dd, J = 4.1, 2.1 Hz, 8H), 7.29-7.20 (m, 2H), 7.20-7.12 $(\mathrm{m}, 2 \mathrm{H}), 6.72$ (ddd, $J=17.7,10.9,1.8 \mathrm{~Hz}, 2 \mathrm{H}), 6.58(\mathrm{dd}, J=7.6,2.2 \mathrm{~Hz}, 2 \mathrm{H}), 5.76(\mathrm{~d}, J=17.6 \mathrm{~Hz}, 2 \mathrm{H}), 5.31-5.18(\mathrm{~m}$, $4 \mathrm{H}), 4.50(\mathrm{dd}, J=10.8,7.4 \mathrm{~Hz}, 2 \mathrm{H}), 2.87(\mathrm{dd}, J=13.0,7.5 \mathrm{~Hz}, 2 \mathrm{H}), 2.55(\mathrm{t}, J=11.9 \mathrm{~Hz}, 2 \mathrm{H})$.

${ }^{13} \mathrm{C}$ NMR (101 MHz, $\left.\mathrm{CDCl}_{3}\right) \delta 149.3$ (x2), 148.3 (x2), 143.9 (x2), 136.7 (x2), $136.6(x 2), 136.4$ (x2), 132.2 (x2), 130.6 (x2), $129.4(x 4), 128.6(x 4), 128.4(x 4), 126.9(x 2), 126.7(x 2), 126.5(x 4), 117.9(x 2), 114.1(x 2), 56.3,50.0(x 2), 48.0$ $(x 2)$.

IR (neat): 3497, 3024, 2958, 2927, 2864, 1601, 1576, 1446, 1400, 1228, 1117, 990, 909, 848, 818, $759,698 \mathrm{~cm}^{-1}$. HRMS (ESI): m/z: [M-H] ${ }^{+}\left(\mathrm{C}_{45} \mathrm{H}_{35} \mathrm{O}_{2}\right)$, calcd.: 607.2643; found: 607.2657.

$[\alpha]_{D}^{25}=+232\left(c=0.1, \mathrm{CH}_{2} \mathrm{Cl}_{2}\right)$.

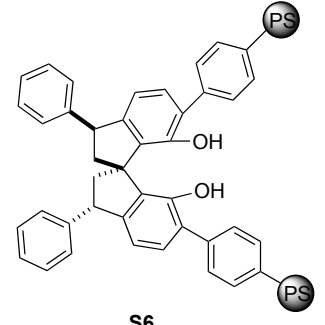

S6

S6 was synthesized following a modification of a literature procedure. ${ }^{2}$ A $100 \mathrm{~mL}$ reactor was charged with a suspension of polyvinyl alcohol (PV-OH) (100 mg, $0.96 \mu \mathrm{mol}, 0.001$ equiv.) in $72 \mathrm{~mL}$ of degassed MiliQ water. The solution was heated at $100{ }^{\circ} \mathrm{C}$ until PV-OH was dissolved. Then, it was cooled to RT and a solution of boric acid (449 mg, $7.26 \mathrm{mmol}$ ) in 18 $\mathrm{mL}$ of degassed MiliQ water was transferred to the reactor. Later, a degassed solution containing divinylbenzene (DVB), filtered on a short pad of silica immediately before use,

(80\%, $119 \mu \mathrm{L}, 0.68 \mathrm{mmol}, 0.65$ equiv.), BINOL derivative S5 (645 mg, $1.06 \mathrm{mmol}$ ), styrene (2.9 ml, $25.5 \mathrm{mmol}, 23.66$ equiv.) and AIBN (31 mg, $0.19 \mathrm{mmol}, 0.18$ equiv.) in toluene $(2.4 \mathrm{~mL})$ was transferred to the reactor. After that, the system was heated at $90{ }^{\circ} \mathrm{C}$ and magnetically stirred at $440 \mathrm{rpm}$ overnight, the aqueous solution was decanted off 
and the resin was washed with water $\left(50^{\circ} \mathrm{C}\right)$ several times, followed by $\mathrm{MeOH}$ and $\mathrm{CH}_{2} \mathrm{Cl}_{2}$. Finally, it was dried overnight in a $40^{\circ} \mathrm{C}$ vacuum oven to furnish $3.5 \mathrm{~g}$ of light-yellow beads.

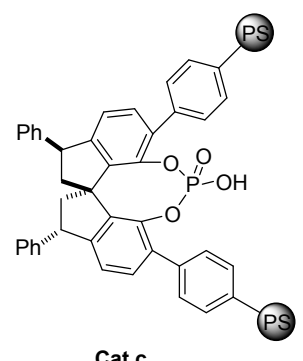

Cat c

Cat c was synthesized following a modification of a literature procedure. ${ }^{2}$ In an oven-dried Schlenk tube, resin S6 (3.5 g, ca. $1.06 \mathrm{mmol})$ was suspended in pyridine $(20 \mathrm{~mL})$ under Ar. Then, $\mathrm{POCl}_{3}$ (495 $\mu \mathrm{L}, 5.3 \mathrm{mmol}, 5$ eq.) was added and the reaction mixture was heated in the sealed Schlenk tube at $120^{\circ} \mathrm{C}$. After 2 days, it was cooled to RT and $5 \mathrm{~mL}$ of water were added. Then the system was sealed again and heated at $100{ }^{\circ} \mathrm{C}$ overnight. The resin was filtered and washed with water, THF/water, THF, $2 \mathrm{M} \mathrm{HCl} / \mathrm{EtOAC}$, EtOAC/DCM, and DCM and dried overnight in a $40^{\circ} \mathrm{C}$ vacuum oven to give $3.5 \mathrm{~g}$ of brown beads.

P elemental analysis (\%): 0.93

$\mathrm{f}_{(\mathrm{P})}: 0.3 \mathrm{mmol} / \mathrm{g}$ resin

\subsection{Preparation of Cat $d$}<smiles>Oc1c(F)ccc2c1C1(CC(c3ccccc3)CC1c1ccccc1)C(c1ccccc1)C2c1ccccc1</smiles>

S4

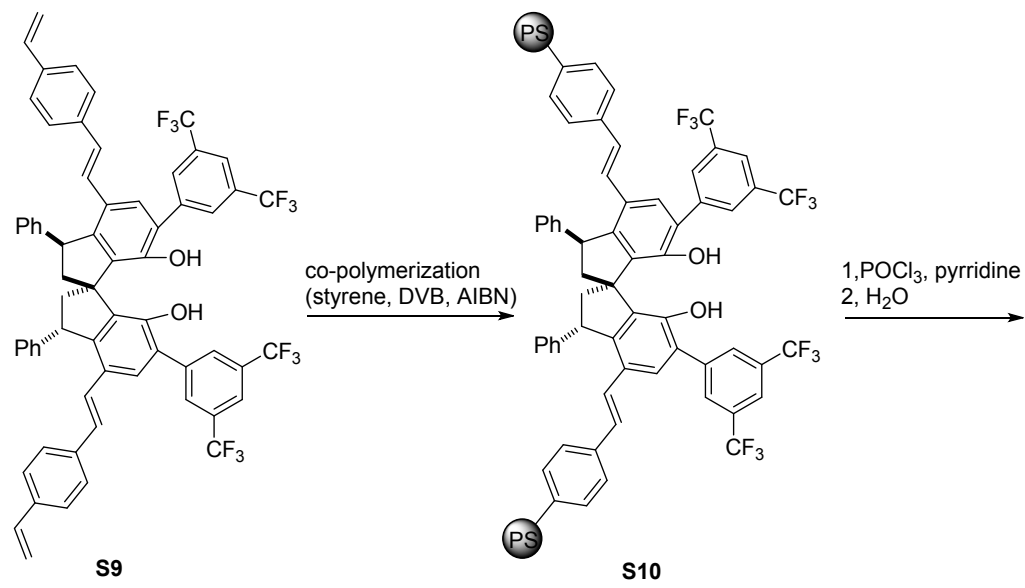

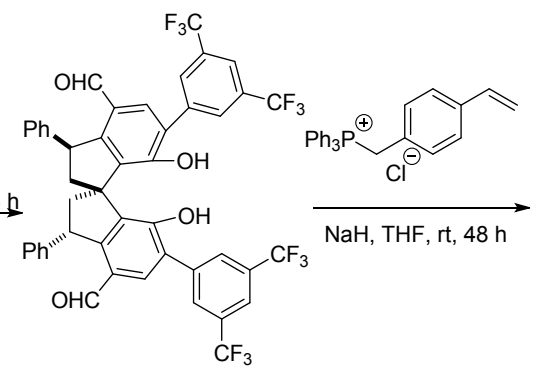

S8

Scheme S3. The procedure of preparation of Cat d

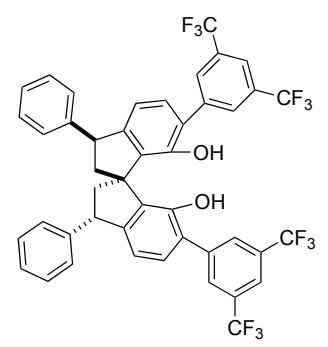

S7

S7 was synthesized following a modification of a literature procedure. ${ }^{3}$ Under $\mathrm{N}_{2}$, to a Schlenk tube containing S4 (1.124 g, 2 mmol), (3,5-bis(trifluoromethyl)phenyl)boronic acid (2.064 g, $8 \mathrm{mmol}, 4.0$ equiv), $\mathrm{Pd}_{2}(\mathrm{dba})_{3}(36.8 \mathrm{mg}, 2 \mathrm{~mol} \%)$, S-Phos (66 mg, $8 \mathrm{~mol} \%$ ) and 
$\mathrm{K}_{3} \mathrm{PO}_{4}(34 \mathrm{mmol})$ was added toluene $(20 \mathrm{~mL})$ and water $(20 \mathrm{~mL})$. The reaction mixture was stirred and heated to $95^{\circ} \mathrm{C}$ for $18 \mathrm{~h}$. After being cooled to room temperature, $\mathrm{HCl}(2 \mathrm{~N})$ was added to the reaction mixture. The mixture was then extracted with $\mathrm{CH}_{2} \mathrm{Cl}_{2}(30 \mathrm{~mL} \times 3)$. After removal of solvent by rota-evaporation, the residue was purified by column chromatography [silicagel, cyclohexane/ dichloromethane $(v / v=5 / 2)$ as eluent], affording the expected product as a white solid. (1.34 $\mathrm{g}, 81 \%$ yield).

White solid. m.p. $140^{\circ} \mathrm{C}$.

${ }^{1} \mathrm{H}$ NMR $(400 \mathrm{MHz}$, Chloroform-d) $\delta 8.01(\mathrm{~s}, 4 \mathrm{H}), 7.84(\mathrm{~s}, 2 \mathrm{H}), 7.44-7.23(\mathrm{~m}, 12 \mathrm{H}), 6.73(\mathrm{dq}, J=7.8,1.3 \mathrm{~Hz}, 2 \mathrm{H}), 5.17$ $(\mathrm{t}, J=1.8 \mathrm{~Hz}, 2 \mathrm{H}), 4.57(\mathrm{dd}, J=11.0,7.5 \mathrm{~Hz}, 2 \mathrm{H}), 3.05-2.85(\mathrm{~m}, 2 \mathrm{H}), 2.51(\mathrm{t}, J=12.1 \mathrm{~Hz}, 2 \mathrm{H})$.

${ }^{13} \mathrm{C}$ NMR (101 MHz, CDCl $) \delta 150.0$ (x2), 149.6 (x2), 142.6 (x2), 139.5 (x2), 131.9 (x2), 131.7 (x4, q, $\left.{ }^{2} \mathrm{~J}_{(\mathrm{C}-\mathrm{F})}=33 \mathrm{~Hz}\right)$, 130.9 (x2), 129.5 (x4), 128.9 (x4), 128.3 (x4), 127.2 (x2), 125.1 (x2), 123.4 (x4, q, $\left.{ }^{1} \mathrm{~J}_{(\mathrm{C}-\mathrm{F})}=275 \mathrm{~Hz}\right), 121.0$ (x2), 119.1 ( $\mathrm{x} 2), 55.8,50.0(\mathrm{x} 2), 48.0(\mathrm{x} 2)$.

${ }^{19} \mathrm{~F} \mathrm{NMR}\left(376 \mathrm{MHz}, \mathrm{CDCl}_{3}\right) \delta-62.90$.

IR (neat): 3525, 3030, 2955, 2869, 1617, 1579, 1495, 1457, 1418, 1381, 1275, 1169, 1125, 1000, 897, 823, 762, 699, $682 \mathrm{~cm}^{-1}$.

HRMS (ESI): $\mathrm{m} / \mathrm{z}:[\mathrm{M}-\mathrm{H}]^{+}\left(\mathrm{C}_{45} \mathrm{H}_{27} \mathrm{~F}_{12} \mathrm{O}_{2}\right)$, calcd.: 827.1825; found: 829.1813 .

$[\alpha]_{D}^{25}=+53\left(c=0.1, \mathrm{CH}_{2} \mathrm{Cl}_{2}\right)$.

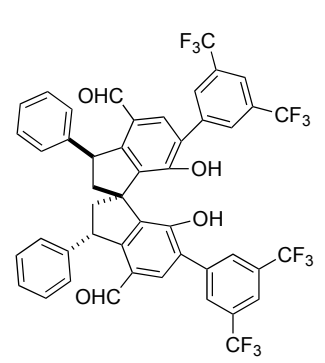

S8

For the preparation of $\mathbf{S 8}$, to a Schlenk tube containing $\mathbf{S 7}(829 \mathrm{mg}, 1 \mathrm{mmol})$ under Ar, hexamethylenetetramine (HMTA) $(561 \mathrm{mg}, 4 \mathrm{mmol}, 4.0$ equiv), and trifluoroacetic acid (4 $\mathrm{mL}$ ) were added. The reaction mixture was stirred and heated to $70^{\circ} \mathrm{C}$ for $6 \mathrm{~h}$. After being cooled to room temperature, $4 \mathrm{~mL} \mathrm{HCl}(6 \mathrm{~N})$ was added to the reaction mixture and heated at $50{ }^{\circ} \mathrm{C}$ for two more hours. The mixture was then extracted with dichloromethane. After removal of solvent by rota-evaporation, the residue was purified by column chromatography [silicagel, cyclohexane/ ethyl acetate $(v / v=5 / 1)$ as eluent], affording the expected product as a light yellow solid. (575 mg, 65\% yield).

Light yellow solid. m.p. $215^{\circ} \mathrm{C}$.

${ }^{1} \mathrm{H}$ NMR $(400 \mathrm{MHz}$, Chloroform-d) $\delta 9.59(\mathrm{~s}, 2 \mathrm{H}), 8.04-7.79(\mathrm{~m}, 8 \mathrm{H}), 7.38-7.22(\mathrm{~m}, 11 \mathrm{H}), 5.94(\mathrm{~d}, J=4.4 \mathrm{~Hz}, 2 \mathrm{H})$, 4.98 (dd, $J=10.1,7.8 \mathrm{~Hz}, 2 \mathrm{H}$ ), 3.09 (dd, $J=13.4,7.9 \mathrm{~Hz}, 2 \mathrm{H}), 2.51$ (dd, $J=13.3,10.2 \mathrm{~Hz}, 2 \mathrm{H}$ ).

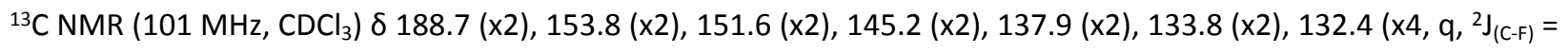
$33 \mathrm{~Hz}), 131.9$ (x2), $129.5(\mathrm{x} 2), 129.4(\mathrm{x} 6), 127.3(\mathrm{x} 6), 127.2(\mathrm{x} 2), 126.2(\mathrm{x} 2), 123.1\left(\mathrm{x} 4, \mathrm{q},{ }^{1} \mathrm{~J}_{(\mathrm{C}-\mathrm{F})}=274 \mathrm{~Hz}\right), 122.0(\mathrm{x} 2)$, , $56.4,50.1(x 2), 49.2(x 2)$.

${ }^{19} \mathrm{~F}$ NMR $\left(376 \mathrm{MHz}, \mathrm{CDCl}_{3}\right) \delta-62.99$.

IR (neat): 3502, 3061, 2929, 2870, 1671, 1600, 1566, 1479, 1453, 1377, 1275, 1171, 1126, 1032, 896, 845, 767, 701, $682 \mathrm{~cm}^{-1}$.

HRMS (ESI): m/z: [M-H] $]^{+}\left(\mathrm{C}_{47} \mathrm{H}_{27} \mathrm{~F}_{12} \mathrm{O}_{4}\right)$, calcd.: 883.1723; found: 883.1726 . 
$[\alpha]_{D}^{25}=+52\left(c=0.1, \mathrm{CH}_{2} \mathrm{Cl}_{2}\right)$.

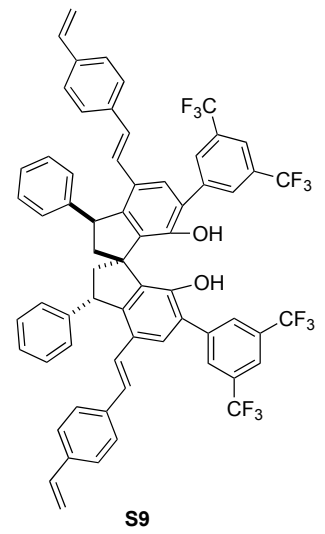

White solid. Decomposed by carbonization or polymerization upon heating. A melting point could not be detected.

S9: To a Schlenk tube, S8 $(500 \mathrm{mg}, 0.56 \mathrm{mmol})$, (4-vinylbenzyl) triphenylphosphonium chloride (930 mg, $2.24 \mathrm{mmol}, 4.0$ equiv) and $15 \mathrm{~mL}$ THF were added under Ar. The mixture was cooled to $0{ }^{\circ} \mathrm{C}$ with an ice bath, then $\mathrm{NaH}$ (a mixture of $60 \%$ sodium hydride $(\mathrm{w} / \mathrm{w}$ ) in mineral oil, $134 \mathrm{mg}, 3.36 \mathrm{mmol}, 6 \mathrm{eq}$ ) suspended in $5 \mathrm{~mL}$ THF was added dropwise. The reaction mixture was stirred at rt for $48 \mathrm{~h}$, then cooled to $0{ }^{\circ} \mathrm{C}$ with an ice bath, and $1 \mathrm{~mL}$ water was added dropwise to quench the reaction. The mixture was then extracted with dichloromethane $(3 \times 10 \mathrm{~mL})$. After removal of solvent by rota-evaporation, the residue was purified by column chromatography [silicagel, cyclohexane/ dichloromethane $(v / v=5 / 2)$ as eluent], affording the expected product as a white solid. (377 mg, 62\% yield).

${ }^{1} \mathrm{H}$ NMR (400 MHz, Chloroform-d) $\delta 8.08$ (d, J = 4.2 Hz, 4H), 7.90 (d, J = 3.7 Hz, 2H), $7.64-7.56$ (m, $\left.2 \mathrm{H}\right), 7.44-7.34$ (m, 8H), $7.33-7.28(\mathrm{~m}, 2 \mathrm{H}), 7.24(\mathrm{dd}, J=8.3,2.5 \mathrm{~Hz}, 4 \mathrm{H}), 6.92(\mathrm{dd}, J=8.1,3.2 \mathrm{~Hz}, 4 \mathrm{H}), 6.78(\mathrm{dd}, J=16.2,3.5 \mathrm{~Hz}, 2 \mathrm{H})$, $6.63(\mathrm{ddd}, J=16.2,7.5,3.1 \mathrm{~Hz}, 4 \mathrm{H}), 5.70$ (dd, $J=17.7,2.3 \mathrm{~Hz}, 2 \mathrm{H}), 5.37$ (q, J = 3.2, $2.7 \mathrm{~Hz}, 2 \mathrm{H}$ ), 5.19 (dd, J = $11.1,2.0$ $\mathrm{Hz}, 2 \mathrm{H}), 4.73(\mathrm{td}, J=8.9,7.5,3.2 \mathrm{~Hz}, 2 \mathrm{H}), 3.13-2.92(\mathrm{~m}, 2 \mathrm{H}), 2.50(\mathrm{td}, J=10.5,9.9,5.4 \mathrm{~Hz}, 2 \mathrm{H})$.

${ }^{13} \mathrm{C}$ NMR (101 MHz, $\mathrm{CDCl}_{3}$ ) $\delta 149.0$ (x2), 146.0 (x2), 144.3 (x2), 139.3 (x2), 136.8 (x2), 136.7 (x2), 136.4 (x4), 132.0 (x2), $131.8\left(x 4, q,{ }^{2} J_{(c-F)}=33 \mathrm{~Hz}\right), 129.6(x 6), 129.2(x 6), 129.1(x 2), 128.3(x 2), 128.0(x 6), 127.9(x 2), 127.1(x 2), 126.5$ (x4), $126.3(x 4), 124.7(x 2), 123.5\left(x 4, q^{1}{ }^{1} J_{(C-F)}=274 \mathrm{~Hz}\right), 121.3(x 2), 113.6(x 2), 55.7,50.0(x 2), 49.6(x 2)$.

${ }^{19} \mathrm{~F} \mathrm{NMR}\left(376 \mathrm{MHz} \mathrm{CDCl}_{3}\right) \delta-62.73,-62.74$.

IR (neat): 3501, 3028, 2937, 2865, 1600, 1510, 1479, 1463, 1407, 1380, 1333, 1275, 1171, 1127, 1031, 989, 961, 893, $845,822,767,700,681 \mathrm{~cm}^{-1}$. HRMS (ESI): m/z: [M-H] ${ }^{+}\left(\mathrm{C}_{65} \mathrm{H}_{43} \mathrm{~F}_{12} \mathrm{O}_{2}\right)$, calcd.: 1083.3064 ; found: 1083.3077. $[\alpha]_{D}^{25}=-84\left(c=0.1, \mathrm{CH}_{2} \mathrm{Cl}_{2}\right)$.

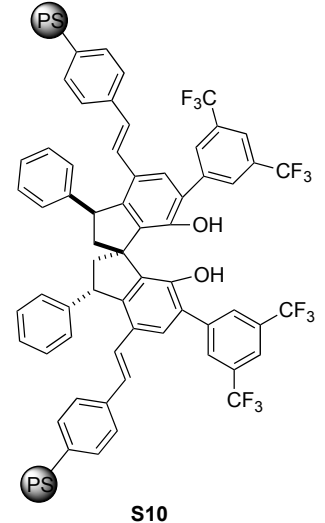

S10
S10 was synthesized following a modification of a literature procedure. ${ }^{2}$ A $100 \mathrm{~mL}$ reactor was charged with a suspension of polyvinyl alcohol (PV-OH) (50 mg, $0.58 \mu \mathrm{mol}, 0.002$ equiv.) in $36 \mathrm{~mL}$ of degassed MiliQ water. The solution was heated at $100{ }^{\circ} \mathrm{C}$ until PV-OH was dissolved. Then, it was cooled to RT and a solution of boric acid (225 mg, $3.63 \mathrm{mmol})$ in $9 \mathrm{~mL}$ of degassed MiliQ water was transferred to the reactor. Later, a degassed solution containing divinylbenzene (DVB), filtered on a short pad of silica immediately before use, (80\%, $60 \mu \mathrm{L}, 0.34 \mathrm{mmol}, 1.3$ equiv.), SPINOL derivative $\mathbf{S 9}$ (293 mg, $0.27 \mathrm{mmol}$ ), styrene (1.45 $\mathrm{ml}, 12.75 \mathrm{mmol}, 47.75$ equiv.) and AIBN (15.5 mg, $0.1 \mathrm{mmol}, 0.35$ equiv.) in toluene (1.2 mL) were added to the reactor. After that, the system was heated at $80{ }^{\circ} \mathrm{C}$ and magnetically stirred at $440 \mathrm{rpm}$. After two days, the aqueous solution was decanted off and the resin was washed with water (50 
${ }^{\circ} \mathrm{C}$ ) several times, followed by $\mathrm{MeOH}$ and $\mathrm{CH}_{2} \mathrm{Cl}_{2}$. Finally, it was dried overnight in a $40{ }^{\circ} \mathrm{C}$ vacuum oven to furnish 1.5 $\mathrm{g}$ of light-yellow beads.

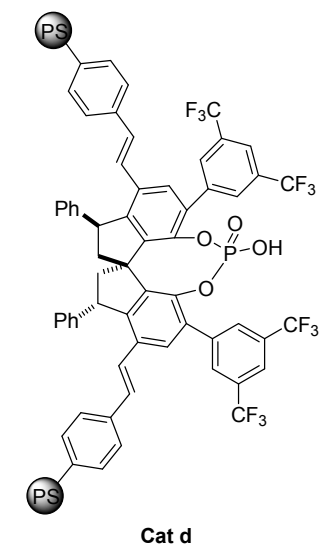

$f_{(P)}: 0.18 \mathrm{mmol} / \mathrm{g}$ resin
Cat $\mathbf{d}$ was synthesized following a modification of a literature procedure. ${ }^{2}$ In an oven-dried Schlenk tube, resin $\mathbf{S 1 0}$ (1.5 g, ca. $0.27 \mathrm{mmol}$ ) was suspended in pyridine $(5 \mathrm{~mL})$ under Ar. Then, $\mathrm{POCl}_{3}(126 \mu \mathrm{L}, 1.35 \mathrm{mmol}, 5$ eq.) was added, and the reaction mixture was heated in the sealed Schlenk tube at $120^{\circ} \mathrm{C}$. After 2 days, it was cooled to room temperature and 1.25 $\mathrm{mL}$ of water were added. Then the system was sealed again and heated at $100^{\circ} \mathrm{C}$ overnight. The resin was then filtered and sequentially washed with water, THF/water, THF, $2 \mathrm{M}$ $\mathrm{HCl} / \mathrm{EtOAC}, \mathrm{EtOAC} / \mathrm{DCM}$, and DCM, and finally dried overnight in a vacuum oven at $40{ }^{\circ} \mathrm{C}$ to afford $1.5 \mathrm{~g}$ of brown beads.

P elemental analysis (\%): 0.56 


\subsection{Preparation of Cat $\mathrm{e}$}
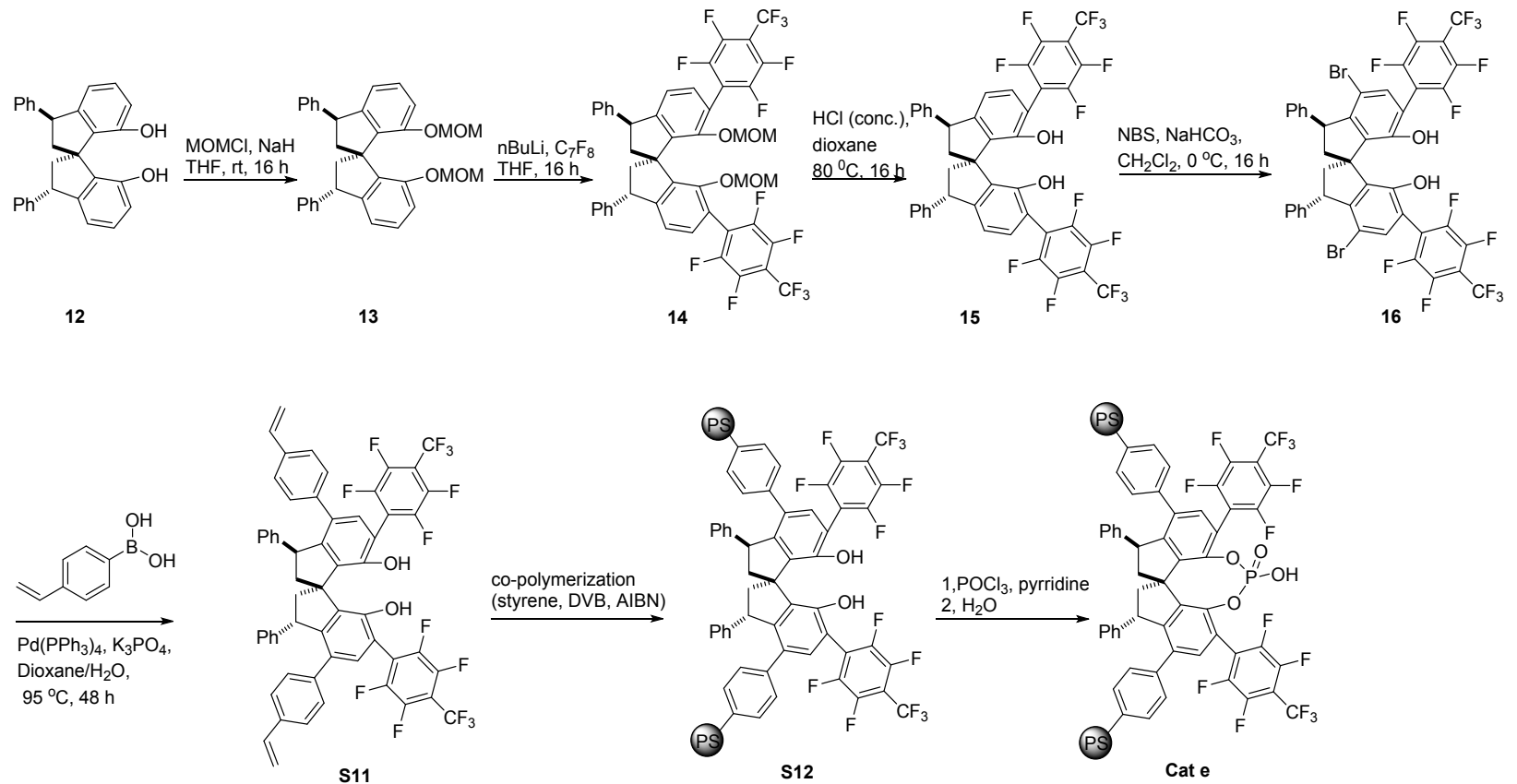

Scheme S4. The procedure of preparation of Cat $\mathbf{e}$

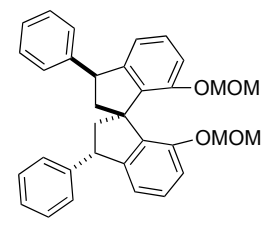

13

13: In an oven-dried Schlenk tube under Argon, $\mathrm{NaH}$ (a mixture of $60 \%$ sodium hydride $(\mathrm{w} / \mathrm{w})$ in mineral oil, $2.4 \mathrm{~g}, 60 \mathrm{mmol}, 3 \mathrm{eq}$ ) was suspended in $200 \mathrm{~mL}$ anhydrous THF, and the mixture was stirred and cooled to $0{ }^{\circ} \mathrm{C}$ in an ice bath. SPINOL $12(8.09 \mathrm{~g}, 20 \mathrm{mmol})$ was dissolved in $40 \mathrm{~mL}$ anhydrous THF and added to the mixture dropwise with syringe. The mixture was stirred at this temperature for 1 hour, then removed the ice bath and stirred at $\mathrm{rt}$ for $15 \mathrm{~min}$. The mixture was cooled to $0{ }^{\circ} \mathrm{C}$ again and Chloromethyl methyl ether $(4.83 \mathrm{~g}, 4.56 \mathrm{~mL}, 60 \mathrm{mmol}, 3 \mathrm{eq})$ was added dropwise with syringe. After being stirred at $\mathrm{rt}$ for $16 \mathrm{~h}$, the reaction mixture was quenched with $8 \mathrm{~mL}$ water. The reaction mixture was then extracted with $\mathrm{CH}_{2} \mathrm{Cl}_{2}(100 \mathrm{~mL} \times 3)$. After removal of solvent by rota-evaporation, the residue was purified by column chromatography (silicagel, cyclohexane/ethyl acetate $(v / v=20 / 1)$ as eluent), affording the expected compound 13 as a white solid ( $8.79 \mathrm{~g}, 90 \%$ yield).

White solid. m.p. $142.6^{\circ} \mathrm{C}$.

${ }^{1} \mathrm{H}$ NMR (400 MHz, Chloroform-d) $\delta 7.37-7.28(\mathrm{~m}, 8 \mathrm{H}), 7.26-7.21(\mathrm{~m}, 2 \mathrm{H}), 7.10-7.03(\mathrm{~m}, 2 \mathrm{H}), 6.81(\mathrm{~d}, J=8.1 \mathrm{~Hz}$, $2 \mathrm{H}), 6.53(\mathrm{~d}, J=7.5 \mathrm{~Hz}, 2 \mathrm{H}), 5.00(\mathrm{~d}, J=6.3 \mathrm{~Hz}, 2 \mathrm{H}), 4.95(\mathrm{~d}, J=6.3 \mathrm{~Hz}, 2 \mathrm{H}), 4.47(\mathrm{t}, J=9.2 \mathrm{~Hz}, 2 \mathrm{H}), 3.17(\mathrm{~s}, 6 \mathrm{H}), 2.77$ (dd, $J=12.7,7.9 \mathrm{~Hz}, 2 \mathrm{H}), 2.50(\mathrm{t}, J=11.8 \mathrm{~Hz}, 2 \mathrm{H})$.

${ }^{13} \mathrm{C}$ NMR (101 MHz, CDCl $)^{2}$ ) 153.3 (x2), 148.1 (x2), 145.5 (x2), 137.4, 128.5 (x6), 128.4 (x2), 128.3, 128.0 (x2), 126.3 (x2), $118.2(x 2), 111.1(x 2), 93.7(x 2), 57.3(x 2), 55.8,50.3(x 2), 48.5(x 2)$.

IR (neat): 3064, 3026, 2954, 2926, 2853, 2823, 1587, 1473, 1406, 1251, 1150, 1080, 1022, 918, 794, 764, 746, 698 $\mathrm{cm}^{-1}$. 
HRMS (ESI): m/z: [M+Na] ${ }^{+}\left(\mathrm{C}_{33} \mathrm{H}_{32} \mathrm{NaO}_{4}\right)$, calcd.: 515.2193; found: 515.2195.

$[\alpha]_{D}^{25}=+55\left(c=0.1, \mathrm{CH}_{2} \mathrm{Cl}_{2}\right)$.

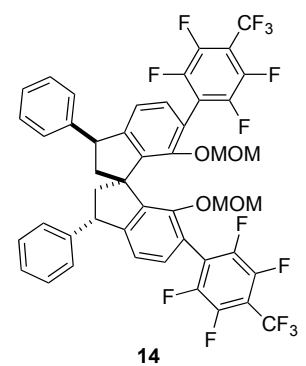

14 was synthesized following a modification of a literature procedure. ${ }^{4}$ In a Schlenk tube under Argon, 13 (7.88 g, $16 \mathrm{mmol}$ ) was dissolved in $160 \mathrm{~mL}$ anhydrous THF, and the mixture was stirred and cooled to $0^{\circ} \mathrm{C}$ with ice bath. $n$-BuLi (2.5 M in hexanes, $19.2 \mathrm{~mL}, 48 \mathrm{mmol}$ ) was added to the mixture dropwise with syringe. The mixture was stirred at $r$ for 3 hours, then cooled to $-78{ }^{\circ} \mathrm{C}$. Perfluorotoluene $(26.43 \mathrm{~g}, 7.93 \mathrm{~mL}, 112 \mathrm{mmol}, 7 \mathrm{eq})$ was added dropwise with syringe. The mixture was stirred at this temperature for one more hour and allowed to warm to rt slowly. After being stirred at $\mathrm{rt}$ for $16 \mathrm{~h}$, the reaction mixture was quenched with $20 \mathrm{~mL}$ water by dropwise at $0^{\circ} \mathrm{C}$ (ice bath). The reaction mixture was then extracted with $\mathrm{CH}_{2} \mathrm{Cl}_{2}(80 \mathrm{~mL} \times 3)$. After removal of solvent by rotaevaporation, the residue was purified by column chromatography (silica gel, cyclohexane/ dichloromethane ( $\mathrm{v} / \mathrm{v}=$ 5/2) as eluent), affording the expected compound 14 as a white solid (13.77 g, 93\% yield).

White solid. m.p. $184.1^{\circ} \mathrm{C}$.

${ }^{1} \mathrm{H}$ NMR (400 MHz, Chloroform-d) $\delta 7.37$ (dd, $\left.J=4.3,1.3 \mathrm{~Hz}, 8 \mathrm{H}\right), 7.32-7.26(\mathrm{~m}, 2 \mathrm{H}), 7.06(\mathrm{dd}, J=7.7,1.4 \mathrm{~Hz}, 2 \mathrm{H})$, $6.85-6.78(\mathrm{~m}, 2 \mathrm{H}), 4.60(\mathrm{dd}, J=6.0,1.7 \mathrm{~Hz}, 2 \mathrm{H}), 4.53(\mathrm{dd}, J=11.1,7.4 \mathrm{~Hz}, 2 \mathrm{H}), 4.38(\mathrm{dd}, J=6.0,1.6 \mathrm{~Hz}, 2 \mathrm{H}), 2.94(\mathrm{~d}$, $J=1.6 \mathrm{~Hz}, 6 \mathrm{H}), 2.92-2.84(\mathrm{~m}, 2 \mathrm{H}), 2.55(\mathrm{t}, J=11.9 \mathrm{~Hz}, 2 \mathrm{H})$.

${ }^{13} \mathrm{C}$ NMR (101 MHz, $\mathrm{CDCl}_{3}$ ) $\delta 153.3$ (x2), 150.8 (x2), 143.1 (x2), $141.6(x 2), 130.9(x 4), 128.8$ (x8), 128.4 (x8), 126.9 (x4), $120.9(x 2), 117.6(x 4), 99.3(x 2), 57.6,56.1(x 2), 50.0(x 2), 48.3(x 2)$.

${ }^{19} \mathrm{~F} \mathrm{NMR}\left(376 \mathrm{MHz}, \mathrm{CDCl}_{3}\right) \delta-56.18(\mathrm{t}, \mathrm{J}=21.7 \mathrm{~Hz}, 6 \mathrm{~F}),-137.85(\mathrm{dd}, \mathrm{J}=22.6,12.5 \mathrm{~Hz}, 2 \mathrm{~F}),-138.13$ (dd, J = 21.7, 12.1 $H z, 2 F),-141.13--141.52(m, 2 F),-141.56--141.89(m, 2 F)$.

IR (neat): 3064, 3030, 2954, 2934, 2838, 1657, 1601, 1478, 1430, 1392, 1338, 1258, 1187, 1136, 1086, 1013, 984 , $944,901,827,765,714,700 \mathrm{~cm}^{-1}$.

HRMS (ESI): m/z: [M+Na] ${ }^{+}\left(\mathrm{C}_{47} \mathrm{H}_{30} \mathrm{~F}_{14} \mathrm{NaO}_{4}\right)$, calcd.: 947.1813; found: 947.1802 .

$[\alpha]_{D}^{25}=+85\left(c=0.1, \mathrm{CH}_{2} \mathrm{Cl}_{2}\right)$.

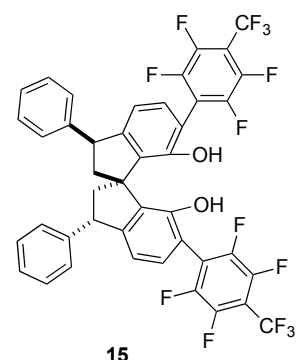

15
15: Compound 14 (6.50 g, $7 \mathrm{mmol}$ ) was dissolved in $35 \mathrm{~mL}$ 1,4-dioxane, $7 \mathrm{~mL}$ con. $\mathrm{HCl}$ was added and heated at $80{ }^{\circ} \mathrm{C}$ for $16 \mathrm{~h}$. The reaction mixture was then extracted with dichloromethane. After removal of solvent by rota-evaporation, the residue was purified by column chromatography (silicagel, cyclohexane/ dichloromethane ( $/ \mathrm{v}=5 / 2$ ) as eluent), affording the expected compound 15 as a white solid ( $4.685 \mathrm{~g}, 80 \%$ yield).

Note: the polarity of 14 and 15 are almost the same. The consumption of 14 couldn't be

checked by TLC. It should be detected by $1 H N M R$.

white solid. m.p. $169.7^{\circ} \mathrm{C}$. 
${ }^{1} \mathrm{H}$ NMR (400 MHz, Chloroform-d) $\delta 7.37(\mathrm{t}, J=7.4 \mathrm{~Hz}, 4 \mathrm{H}), 7.33-7.26(\mathrm{~m}, 6 \mathrm{H}), 7.21(\mathrm{~d}, J=7.8 \mathrm{~Hz}, 2 \mathrm{H}), 6.74(\mathrm{~d}, J=$ $7.8 \mathrm{~Hz}, 2 \mathrm{H}), 5.08(\mathrm{~s}, 2 \mathrm{H}), 4.58$ (dd, $J=11.0,7.4 \mathrm{~Hz}, 2 \mathrm{H}), 2.98$ (dd, $J=13.3,7.4 \mathrm{~Hz}, 2 \mathrm{H}), 2.50(\mathrm{dd}, J=13.1,11.1 \mathrm{~Hz}, 2 \mathrm{H})$. ${ }^{13} \mathrm{C}$ NMR (101 MHz, $\mathrm{CDCl}_{3}$ ) $\delta 151.5$ (x2), 150.4 (x2), 142.2 (x2), 132.6 (x4), 130.6 (x2), 128.9 (x8), 128.2 (x8), 127.3 (x4), $118.8(x 4), 113.0(x 2), 55.6,50.3(x 2), 47.7(x 2)$.

${ }^{19} \mathrm{~F} \mathrm{NMR}\left(376 \mathrm{MHz}, \mathrm{CDCl}_{3}\right) \delta-56.27(\mathrm{t}, \mathrm{J}=21.7 \mathrm{~Hz}, 6 \mathrm{~F}),-136.93(\mathrm{p}, \mathrm{J}=16.3 \mathrm{~Hz}, 2 \mathrm{~F}),-138.44(\mathrm{dd}, \mathrm{J}=22.2,12.4 \mathrm{~Hz}, 2 \mathrm{~F})$, $-140.61--141.52(m, 4 F)$.

IR (neat): 3530, 3029, 2954, 1659, 1602, 1479, 1454, 1428, 1338, 1257, 1186, 1145, 982, 901, 827, 763, 714,699 $\mathrm{cm}^{-1}$.

HRMS (ESI): m/z: [M+Na] ${ }^{+}\left(\mathrm{C}_{43} \mathrm{H}_{22} \mathrm{~F}_{14} \mathrm{NaO}_{2}\right)$, calcd.: 859.1294; found: 859,1290.

$[\alpha]_{D}^{25}=+85\left(c=0.1, \mathrm{CH}_{2} \mathrm{Cl}_{2}\right)$.

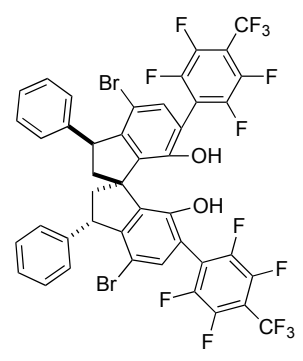

16

a) Preparation of 16 from 15: In a Schlenk tube, 15 (4.6 g, $5.5 \mathrm{mmol}$ ) and $\mathrm{NaHCO}_{3}(924 \mathrm{mg}, 11$ mmol, 2.0 equiv) were mixed with dichloromethane $(55 \mathrm{~mL})$, and the mixture was stirred and cooled to $0^{\circ} \mathrm{C}$. Then, NBS (2.007 g, $11.275 \mathrm{mmol}, 2.05$ equiv) was added slowly. After being stirred at $0{ }^{\circ} \mathrm{C}$ for $16 \mathrm{~h}$, the reaction mixture was poured into $\mathrm{HCl}(2 \mathrm{~N})$. The reaction mixture was then extracted with dichloromethane $(50 \mathrm{~mL} \times 3)$. After removal of solvent by rotaevaporation, the residue was purified by column chromatography (silicagel, cyclohexane/ethyl acetate $(v / v=20 / 1)$ as eluent), affording the expected compound 16 as a white solid (4.375 g, $80 \%$ yield).

b) Preparation of 16 from 14: Compound 14 (13.0 g, $14 \mathrm{mmol}$ ) was dissolved in $70 \mathrm{~mL}$ 1,4-dioxane, $14 \mathrm{~mL}$ con. $\mathrm{HCl}$ was added and heated at $80^{\circ} \mathrm{C}$ for $16 \mathrm{~h}$. The reaction mixture was then extracted with dichloromethane. After removal of solvent by rota-evaporation, we obtained $11.5 \mathrm{~g}$ yellow solid. The obtained yellow solid was used in the next step directly without purification.

In a $250 \mathrm{~mL}$ round bottle, the obtained yellow solid was dissolved in $100 \mathrm{~mL} \mathrm{MeCN}$, and cooled with ice bath. One drop of $\mathrm{Br}_{2}$ was added to the mixture. Then, NBS $(5.11 \mathrm{~g}, 28.7 \mathrm{mmol}, 2.05$ equiv) was added slowly. After being stirred at $0^{\circ} \mathrm{C}$ for $4 \mathrm{~h}$ and TLC showed SM consumed completely, $20 \mathrm{~mL}$ saturated $\mathrm{Na}_{2} \mathrm{~S}_{2} \mathrm{O}_{4}$ was added to the mixture to quench the reaction. The double phase mixture was separated and the aqueous phase was extracted with dichloromethane $(20 \mathrm{~mL} \times 3)$. After removal of solvent by rota-evaporation, the residue was purified by column chromatography (silicagel, cyclohexane/ethyl acetate ( $/ \mathrm{v}=20 / 1$ ) as eluent), affording the expected compound 16 as a white solid $(9.71 \mathrm{~g}, 70 \%$ yield).

Light yellow solid. m.p. $175^{\circ} \mathrm{C}$.

${ }^{1} \mathrm{H}$ NMR (400 MHz, Chloroform-d) $\delta 7.37-7.18(\mathrm{~m}, 12 \mathrm{H}), 5.11(\mathrm{~s}, 2 \mathrm{H}), 4.59(\mathrm{dd}, J=10.1,7.9 \mathrm{~Hz}, 2 \mathrm{H}), 2.99(\mathrm{dd}, J=$ 13.6, $7.9 \mathrm{~Hz}, 2 \mathrm{H}), 2.37$ (dd, $J=13.5,10.3 \mathrm{~Hz}, 2 \mathrm{H})$.

${ }^{13} \mathrm{C}$ NMR (126 MHz, CDCl3) $\delta 149.6$ (x2), $148.2(x 2), 142.7$ (x2), $136.0(x 4), 134.1(x 2), 128.8(x 8), 128.1(x 8), 126.9$ $(x 4), 115.2(x 4), 112.5(x 2), 56.5,51.6(x 2), 49.6(x 2)$. 
${ }^{19} \mathrm{~F} \mathrm{NMR}\left(376 \mathrm{MHz}, \mathrm{CDCl}_{3}\right) \delta-56.31(\mathrm{t}, \mathrm{J}=21.7 \mathrm{~Hz}, 6 \mathrm{~F}),-136.21--137.16(\mathrm{p}, \mathrm{J}=16.0 \mathrm{~Hz}, 2 \mathrm{~F}),-137.90(\mathrm{dd}, \mathrm{J}=21.8$, $12.4 \mathrm{~Hz}, 2 \mathrm{~F}),-139.56--140.78(\mathrm{~m}, 4 \mathrm{~F})$.

IR (neat): 3538, 3067, 3028, 2956, 2937, 2871, 1660, 1602, 1480, 1454, 1337, 1261, 1232, 1143, 987, 843, 763, 715, $699,676 \mathrm{~cm}^{-1}$.

HRMS (ESI): m/z: [M-H] ${ }^{+}\left(\mathrm{C}_{43} \mathrm{H}_{19} \mathrm{Br}_{2} \mathrm{~F}_{14} \mathrm{O}_{2}\right)$, calcd.: 990.9534 ; found: 990.9505.

$[\alpha]_{D}^{25}=+87\left(c=0.1, \mathrm{CH}_{2} \mathrm{Cl}_{2}\right)$.

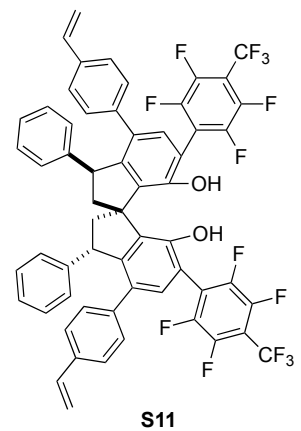

S11: Under $\mathrm{N}_{2}$, to a Schlenk tube containing 16 (2.0 g, 2 mmol), 4-vinylphenylboronic acid (0.9 g, $6 \mathrm{mmol}, 3.0$ equiv), $\mathrm{Pd}(\mathrm{PPh} 3) 4(116 \mathrm{mg}, 0.1 \mathrm{mmol}, 5 \mathrm{~mol} \%)$ and $\mathrm{K}_{3} \mathrm{PO}_{4}(1.272 \mathrm{~g}, 6 \mathrm{mmol}$, $3.0 \mathrm{eq})$, degassed 1,4-dioxane $(15 \mathrm{~mL})$ and water $(5 \mathrm{~mL})$ were added. The reaction mixture was stirred and heated to $95{ }^{\circ} \mathrm{C}$ for $48 \mathrm{~h}$., cooled to room temperature and extracted with $\mathrm{CH}_{2} \mathrm{Cl}_{2}(20 \mathrm{~mL} \times 3)$. The combined organic layers were dried over $\mathrm{MgSO}_{4}$ and concentrated under reduced pressure. The crude product was purified by flash column chromatography on silicagel using cyclohexane/ $\mathrm{CH}_{2} \mathrm{Cl}_{2}(5: 1)$ as the eluent to give compound $\mathbf{S 1 1}$ as a slightly

yellow solid. (1.33 g, 64\% yield).

Light yellow solid. Decomposed upon heating by carbonization or polymerization; a melting point could not be detected.

${ }^{1} \mathrm{H}$ NMR (400 MHz, Chloroform-d) $\delta 7.91(\mathrm{~d}, J=1.3 \mathrm{~Hz}, 2 \mathrm{H}), 7.26-6.88(\mathrm{~m}, 2 \mathrm{H}), 6.64(\mathrm{dd}, J=17.6,10.9 \mathrm{~Hz}, 2 \mathrm{H}), 5.69$ $(\mathrm{d}, J=17.6 \mathrm{~Hz}, 2 \mathrm{H}), 5.22(\mathrm{~d}, J=10.9 \mathrm{~Hz}, 2 \mathrm{H}), 5.00(\mathrm{t}, J=8.6 \mathrm{~Hz}, 2 \mathrm{H}), 3.25$ (ddd, J = 13.7, 8.6, $1.4 \mathrm{~Hz}, 2 \mathrm{H}), 2.77(\mathrm{ddd}, J$ $=13.7,8.9,1.5 \mathrm{~Hz}, 2 \mathrm{H})$.

${ }^{13} \mathrm{C}$ NMR (101 MHz, $\left.\mathrm{CDCl}_{3}\right) \delta 152.4(x 2), 147.8$ (x2), 144.5 (x2), $139.2(x 2), 137.4(x 2), 136.5$ (x4), 136.1 (x2), 132.8 (x2), $128.9(x 6), 128.0(x 12), 125.9(x 4), 125.5(x 6), 124.3(x 2), 120.7(x 2), 113.7(x 4), 56.8,52.0(x 2), 51.2(x 2)$.

${ }^{19} \mathrm{~F} \mathrm{NMR}\left(376 \mathrm{MHz}, \mathrm{CDCl}_{3}\right) \delta-55.36(\mathrm{t}, \mathrm{J}=21.8 \mathrm{~Hz}, 6 \mathrm{~F}),-138.61--139.77(\mathrm{pd}, \mathrm{J}=21.1,5.8 \mathrm{~Hz}, 2 \mathrm{~F}),-144.06(\mathrm{pd}, \mathrm{J}=$ 21.1, $5.8 \mathrm{~Hz}, 2 \mathrm{~F}),-145.79(\mathrm{t}, \mathrm{J}=19.6 \mathrm{~Hz}, 4 \mathrm{~F})$.

IR (neat): 3538, 3027, 2939, 2871, 1663, 1629, 1603, 1516, 1464, 1427, 1321, 1234, 1184, 1136, 986, 906, 841, 756, $714,697 \mathrm{~cm}^{-1}$.

HRMS (ESI): m/z: [M-H] ${ }^{+}\left(\mathrm{C}_{59} \mathrm{H}_{33} \mathrm{~F}_{14} \mathrm{O}_{2}\right)$, calcd.: 1039.2257; found: 1039.2268.

$[\alpha]_{D}^{25}=+24\left(c=0.1, \mathrm{CH}_{2} \mathrm{Cl}_{2}\right)$.

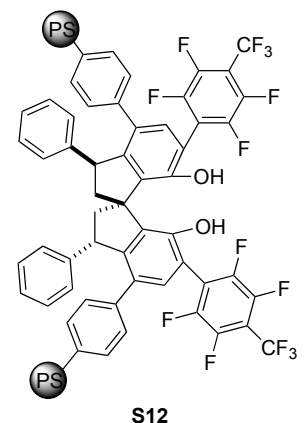

S12

S12 was synthesized following a modification of a literature procedure. ${ }^{2}$ A $100 \mathrm{~mL}$ reactor was charged with a suspension of polyvinyl alcohol (PV-OH) (100 mg, $0.96 \mu \mathrm{mol}, 0.001$ equiv.) in $72 \mathrm{~mL}$ of degassed MiliQ water. The solution was heated at $100{ }^{\circ} \mathrm{C}$ until PV-OH was dissolved. Then, it was cooled to RT and a solution of boric acid ( $449 \mathrm{mg}, 7.26 \mathrm{mmol}$ ) in $18 \mathrm{~mL}$ of degassed MiliQ water was transferred to the reactor. Later, a degassed solution containing divinylbenzene (DVB), filtered on a short pad of silica immediately before use, $(80 \%, 119 \mu \mathrm{L}$, 
$0.68 \mathrm{mmol}, 0.65$ equiv.), BINOL derivative S11 (1.1 g, $1.06 \mathrm{mmol})$, styrene $(2.9 \mathrm{ml}, 25.5 \mathrm{mmol}, 23.66$ equiv.) and AIBN (31 mg, $0.19 \mathrm{mmol}, 0.18$ equiv.) in toluene $(2.4 \mathrm{~mL})$ was transferred to the reactor. After that, the system was heated at $90{ }^{\circ} \mathrm{C}$ and magnetically stirred at $440 \mathrm{rpm}$ overnight, the aqueous solution was decanted off and the resin was washed with water $\left(50^{\circ} \mathrm{C}\right)$ several times, followed by $\mathrm{MeOH}$ and $\mathrm{CH}_{2} \mathrm{Cl}_{2}$. Finally, it was dried overnight in a vacuum oven at $40{ }^{\circ} \mathrm{C}$ to furnish $3.0 \mathrm{~g}$ of light-yellow beads.

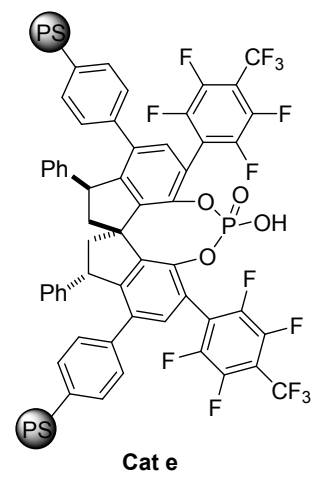

$f_{(P)}: 0.13 \mathrm{mmol} / \mathrm{g}$ resin

Cat e was synthesized following a modification of a literature procedure. ${ }^{2}$ In an oven-dried Schlenk tube, resin S12 (3.0 g, ca. $1.06 \mathrm{mmol}$ ) was suspended in pyridine (20 mL) under Ar. Then, $\mathrm{POCl}_{3}(495 \mu \mathrm{L}, 5.3 \mathrm{mmol}, 5$ eq.) was added, and the reaction mixture was heated in the sealed Schlenk tube at $120^{\circ} \mathrm{C}$. After 2 days, it was cooled to RT and $5 \mathrm{~mL}$ of water were added. Then the system was sealed again and heated at $100{ }^{\circ} \mathrm{C}$ overnight. The resin was filtered and washed with water, THF/water, THF, $2 \mathrm{M} \mathrm{HCl} /$ EtOAC, EtOAC/DCM, and DCM and dried overnight in a $40{ }^{\circ} \mathrm{C}$ vacuum oven to give $3.0 \mathrm{~g}$ of brown beads.

P elemental analysis (\%): 0.40 


\subsection{Preparation of Cat $f$}

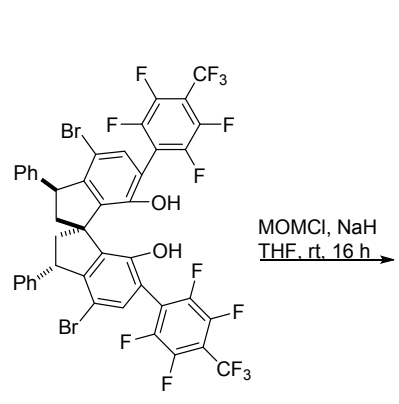

16

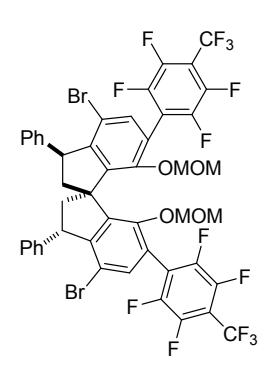

17
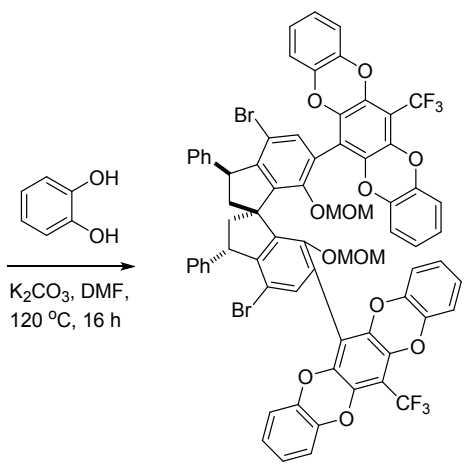

18

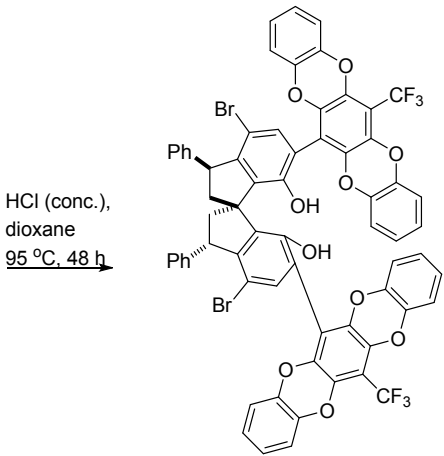

19

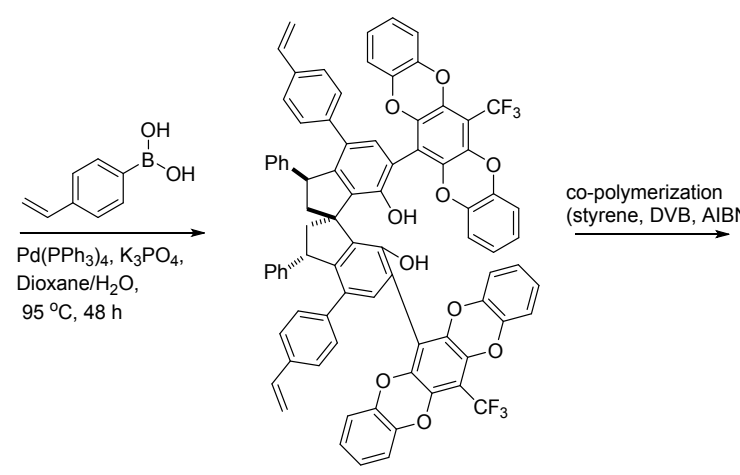

20

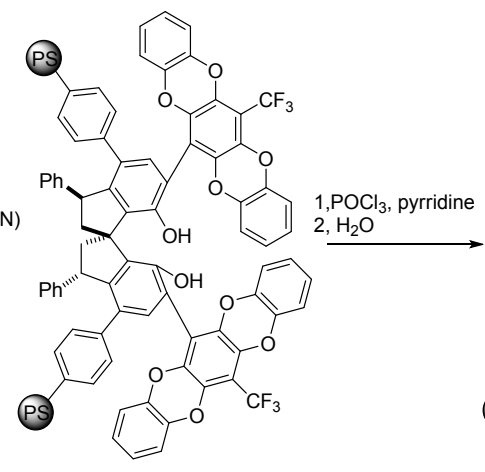

21

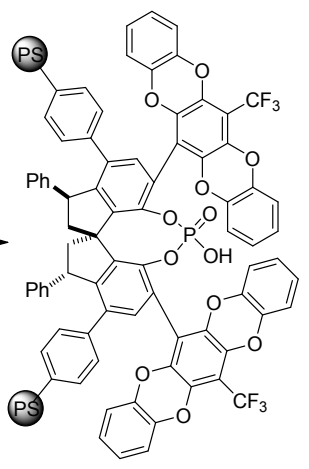

Cat $f$

Scheme S5. The procedure of preparation of Cat $\mathbf{f}$

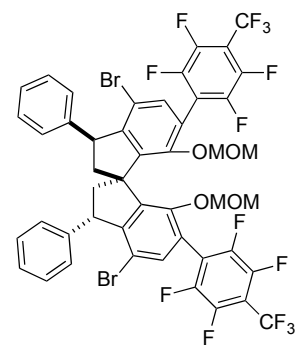

17

Preparation of 17: In a Schlenk tube under $\mathrm{Ar}, \mathrm{NaH}$ (a mixture of $60 \%$ sodium hydride $(\mathrm{w} / \mathrm{w}$ ) in mineral oil, $0.6 \mathrm{~g}, 15 \mathrm{mmol}, 3 \mathrm{eq}$ ) was dissolved in $50 \mathrm{~mL}$ anhydrous THF, and the mixture was stirred and cooled to $0^{\circ} \mathrm{C}$ with ice bath. SPINOL 16 (5.0 g, $\left.5 \mathrm{mmol}\right)$ was dissolved in 10 $\mathrm{mL}$ anhydrous THF and added to the mixture dropwise with syringe. The mixture was stirred at this temperature for 1 hour, then removed the ice bath and stirred at rt for $15 \mathrm{~min}$. The mixture was cooled to $0{ }^{\circ} \mathrm{C}$ again and Chloromethyl methyl ether $(1.21 \mathrm{~g}, 1.14 \mathrm{~mL}, 15 \mathrm{mmol}$, $3 \mathrm{eq}$ ) was added dropwise with syringe. After being stirred at $\mathrm{rt}$ for $16 \mathrm{~h}$, the reaction mixture was quenched with $2 \mathrm{~mL}$ water. The reaction mixture was then extracted with dichloromethane. After removal of solvent by rota-evaporation, the residue was purified by column chromatography (silicagel, cyclohexane $/ \mathrm{CH}_{2} \mathrm{Cl}_{2}(\mathrm{v} / \mathrm{v}$ $=5 / 2$ ) as eluent), affording the expected compound 17 as a white solid ( $4.44 \mathrm{~g}, 82 \%$ yield).

White solid. m. p. $177.2^{\circ} \mathrm{C}$.

${ }^{1} \mathrm{H}$ NMR (400 MHz, Chloroform-d) $\delta 7.32-7.25(\mathrm{~m}, 12 \mathrm{H}), 4.68$ (dd, J = 6.1, $\left.1.9 \mathrm{~Hz}, 2 \mathrm{H}\right), 4.55$ (dd, J=10.2, 7.9 Hz, 2H), $4.44(\mathrm{dd}, J=6.2,1.9 \mathrm{~Hz}, 2 \mathrm{H}), 3.06(\mathrm{~s}, 6 \mathrm{H}), 2.95-2.89(\mathrm{~m}, 2 \mathrm{H}), 2.34(\mathrm{dd}, J=13.0,10.3 \mathrm{~Hz}, 2 \mathrm{H})$.

${ }^{13} \mathrm{C}$ NMR (101 MHz, CDCl $) \delta 152.5$ (x2), 147.5 (x2), 144.5 (x2), 143.5 (x2), 135.0 (x4), 128.6 (x8), 128.2 (x8), 126.7 (x4), $121.9(x 2), 119.5(x 2), 115.3(x 2), 99.3(x 2), 58.4,56.3(x 2), 51.4(x 2), 50.5(x 2)$. 
${ }^{19} \mathrm{~F} \mathrm{NMR}\left(376 \mathrm{MHz}, \mathrm{CDCl}_{3}\right) \delta-56.27(\mathrm{t}, J=21.7 \mathrm{~Hz}, 6 \mathrm{~F}),-137.37(\mathrm{dd}, J=20.3,12.3 \mathrm{~Hz}, 2 \mathrm{~F}),-138.03--138.40(\mathrm{~m}, 2 \mathrm{~F}),-$ $140.71--141.33(\mathrm{~m}, 2 \mathrm{~F})$.

IR (neat): 3063, 3027, 2943, 1660, 1603, 1550, 1480, 1452, 1415, 1386, 1336, 1262, 1143, 1082, 1031, 986, 907, 845, $763,733,714,699,675 \mathrm{~cm}^{-1}$.

HRMS (ESI): m/z: [M+Na] ${ }^{+}\left(\mathrm{C}_{47} \mathrm{H}_{28} \mathrm{Br}_{2} \mathrm{~F}_{14} \mathrm{NaO}_{4}\right)$, calcd.: 1103.0023; found: 1103.0036 .

$[\alpha]_{D}^{25}=+96\left(c=0.1, \mathrm{CH}_{2} \mathrm{Cl}_{2}\right)$.

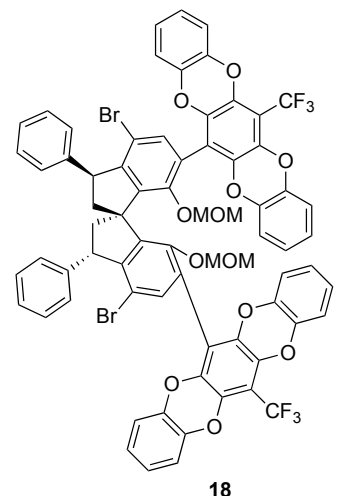

Preparation of 18: In a Schlenk tube was added 17 (4.33 g, 4 mmol), pyrocatechol (1.98 g, $18 \mathrm{mmol}, 4.5$ equiv) and $\mathrm{K}_{2} \mathrm{CO}_{3}(11.04 \mathrm{~g}, 80 \mathrm{mmol}, 20$ equiv). The flask was reflashed with Ar three times. $50 \mathrm{~mL}$ degassed DMF was added and stirred at $120{ }^{\circ} \mathrm{C}$ overnight. The reaction mixture was cooled to $\mathrm{rt}$ and quenched with $100 \mathrm{~mL}$ water and $100 \mathrm{~mL}$ ethyl acetate. The biphasic solution was separated and the organic solution was washed with saturated $\mathrm{NaHCO}_{3}$, water and brine, dried with $\mathrm{MgSO}_{4}$. After removal of solvent by rotaevaporation, the residue was purified by column chromatography (silicagel, cyclohexane/ethyl acetate ( $/ / v=20 / 1)$ as eluent), affording the expected compound 18 as a white solid (4.91 g, 90\% yield).

White solid. m. p. $227.9^{\circ} \mathrm{C}$.

${ }^{1} \mathrm{H}$ NMR (400 MHz, Chloroform-d) $\delta 7.42(\mathrm{dt}, J=5.8,3.4 \mathrm{~Hz}, 4 \mathrm{H}), 7.30-7.25(\mathrm{~m}, 8 \mathrm{H}), 6.89(\mathrm{~d}, J=4.3 \mathrm{~Hz}, 4 \mathrm{H}), 6.86(\mathrm{~d}$, $J=4.3 \mathrm{~Hz}, 4 \mathrm{H}), 6.81-6.77(\mathrm{~m}, 2 \mathrm{H}), 6.69-6.65(\mathrm{~m}, 2 \mathrm{H}), 6.59(\mathrm{~d}, J=7.9 \mathrm{~Hz}, 2 \mathrm{H}), 6.29(\mathrm{~d}, J=7.9 \mathrm{~Hz}, 2 \mathrm{H}), 4.77-4.71$ $(\mathrm{m}, 2 \mathrm{H}), 4.64-4.56(\mathrm{~m}, 4 \mathrm{H}), 3.02(\mathrm{~d}, J=3.0 \mathrm{~Hz}, 8 \mathrm{H}), 2.68-2.59(\mathrm{~m}, 2 \mathrm{H})$.

${ }^{13} \mathrm{C}$ NMR (101 MHz, $\left.\mathrm{CDCl}_{3}\right) \delta 152.7$ (x2), 145.8 (x2), 144.5 (x2), $144.2(x 2), 141.3(x 2), 141.0(x 2), 140.8(x 2), 136.9$ (x2), $136.6(x 2), 136.4(x 2), 135.8(x 2), 135.4(x 2), 128.7(x 6), 128.5(x 6), 126.3(x 2), 124.8(x 2), 124.6(x 2), 124.5(x 2)$, $124.2(x 2), 123.3(x 2, q, J=277 \mathrm{~Hz}), 118.8(x 2), 116.6(x 2), 116.5(x 2), 116.3(x 2), 115.9(x 2), 114.2(x 2), 106.4(x 2, q$, $\left.{ }^{1} \mathrm{~J}_{(\mathrm{C}-\mathrm{F})}=32 \mathrm{~Hz}\right), 98.9(\mathrm{x} 2), 58.5,56.5(\mathrm{x} 2), 51.8(\mathrm{x} 2), 50.6(\mathrm{x} 2)$.

${ }^{19} \mathrm{~F} \mathrm{NMR}\left(376 \mathrm{MHz} \mathrm{CDCl}_{3}\right) \delta-55.00$.

IR (neat): 3063, 3026, 2940, 1645, 1602, 1495, 1438, 1311, 1253, 1158, 1121, 1100, 1028, 986, 875, 744, 729,697 $\mathrm{cm}^{-1}$.

HRMS (ESI): m/z: [M+Na] ${ }^{+}\left(\mathrm{C}_{71} \mathrm{H}_{44} \mathrm{Br}_{2} \mathrm{~F}_{6} \mathrm{NaO}_{12}\right)$, calcd.: 1383.0996; found: 1383.0974 .

$[\alpha]_{D}^{25}=+185\left(c=0.1, \mathrm{CH}_{2} \mathrm{Cl}_{2}\right)$.

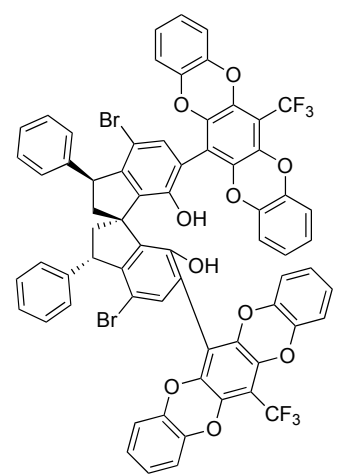

19 a) Preparation of 19 from 18: In a round-bottom flask equipped with a condenser, compound 18 (4.85 g, $3.56 \mathrm{mmol}$ ) was dissolved in $30 \mathrm{~mL}$ 1,4-dioxane, $7 \mathrm{~mL}$ con. $\mathrm{HCl}$ was added, and the mixture was heated at $95{ }^{\circ} \mathrm{C}$ for $48 \mathrm{~h}$. The reaction mixture was then extracted with dichloromethane. After removal of solvent by rota-evaporation, the residue 
was purified by column chromatography (silicagel, cyclohexane/ dichloromethane ( $\mathrm{v} / \mathrm{v}=5 / 2$ ) as eluent), affording the expected compound 19 as a white solid (1.91 g, 42\% yield).

Note: the polarity of 18 and 19 are almost the same. The consumption of 18 couldn't be checked by TLC; it should be detected by ${ }^{1} H$ NMR or HPLC.

b) Preparation of 19 from 16: In a Schlenk tube under Argon, NaH (a mixture of 60\% sodium hydride (w/w) in mineral oil, $1.08 \mathrm{~g}, 27 \mathrm{mmol}, 3 \mathrm{eq}$ ) was suspended in $100 \mathrm{~mL}$ anhydrous THF, and the mixture was stirred and cooled to $0{ }^{\circ} \mathrm{C}$ with an ice bath. SPINOL $16(8.93 \mathrm{~g}, 9 \mathrm{mmol})$ was dissolved in $30 \mathrm{~mL}$ anhydrous THF and added to the mixture dropwise via syringe. The mixture was stirred at this temperature for 1 hour, the ice bath was then removed and stirring was continued at rt for $15 \mathrm{~min}$. The mixture was cooled again to $0^{\circ} \mathrm{C}$ and chloromethyl methyl ether $(2.17 \mathrm{~g}$, $2.05 \mathrm{~mL}, 27 \mathrm{mmol}, 3 \mathrm{eq}$ ) was added dropwise via syringe. After being stirred at $\mathrm{rt}$ for $16 \mathrm{~h}$, the reaction mixture was quenched with $4 \mathrm{~mL}$ water and extracted with dichloromethane (3x $100 \mathrm{~mL}$ ). After removal of solvent by rotaevaporation, the residue was passed through a short pad of silicagel eluting with cyclohexane/EA $(v / v=2 / 1)$ and the clear eluate was evaporated to dryness. The solid residue $(9.23 \mathrm{~g})$ was placed in a a Shlenck tube, catechol (4.46 g, 40.5 mmol, 4.5 equiv) and $\mathrm{K}_{2} \mathrm{CO}_{3}(24.84 \mathrm{~g}, 180 \mathrm{mmol}, 20$ equiv) were added, and an $\mathrm{Ar}$ atmosphere was established ( $3 \mathrm{X}$ vacuum/refill). Degassed DMF $\left(120 \mathrm{~mL}\right.$ ) was added, and the misture stirred at $120{ }^{\circ} \mathrm{C}$ for $48 \mathrm{~h}$. The reaction mixture was cooled to $\mathrm{rt}$ and quenched with $400 \mathrm{~mL}$ water and $400 \mathrm{~mL}$ ethyl acetate. The biphasic solution was separated and the organic solution was washed with saturated $\mathrm{NaHCO}_{3}$, water and brine, dried with $\mathrm{MgSO}_{4}$. After removal of solvent by rota-evaporation, the residue was used in the next step without purification. The obtained yellow semi-solid was dissolved in $90 \mathrm{~mL}$ 1,4-dioxane, $9 \mathrm{~mL}$ con. $\mathrm{HCl}$ was added and the solution was heated at 95 ${ }^{\circ} \mathrm{C}$ for $48 \mathrm{~h}$. The reaction mixture was then extracted with dichloromethane (3x $\left.100 \mathrm{~mL}\right)$. After removal of solvent by rota-evaporation, the residue was purified by column chromatography (silicagel, cyclohexane/ dichloromethane ( $\mathrm{v} / \mathrm{v}$ $=5 / 2$ ) as eluent), affording the expected compound 19 as a white solid (6.75 g, 59\% yield).

Light yellow solid. Decomposed by carbonization or polymerization upon heating, or m. p. $>300{ }^{\circ} \mathrm{C}$. A melting point could not be detected.

${ }^{1} \mathrm{H}$ NMR (400 MHz, Chloroform-d) $\delta 7.34$ - $7.22(\mathrm{~m}, 12 \mathrm{H}), 6.93-6.88(\mathrm{~m}, 4 \mathrm{H}), 6.85$ - $6.76(\mathrm{~m}, 4 \mathrm{H}), 6.72(\mathrm{td}, \mathrm{J}=7.7$, $1.7 \mathrm{~Hz}, 2 \mathrm{H}), 6.66-6.57(\mathrm{~m}, 6 \mathrm{H}), 5.16(\mathrm{~s}, 2 \mathrm{H}), 4.62(\mathrm{dd}, J=10.2,7.5 \mathrm{~Hz}, 2 \mathrm{H}), 3.08-2.97(\mathrm{~m}, 2 \mathrm{H}), 2.43(\mathrm{dd}, J=13.2$, $10.4 \mathrm{~Hz}, 2 \mathrm{H})$.

${ }^{13} \mathrm{C}$ NMR (101 MHz, $\mathrm{CDCl}_{3}$ ) $\delta 149.6$ (x2), 146.4 (x2), 143.5 (x2), 141.1 (x2), 140.8 (x2), 140.3 (x2), 139.9 (x2), 136.7 (x2), 136.3 (x2), 135.8 (x2), 135.8 (x2), 135.4 (x2), 134.3 (x2), 128.7 (x4), 128.3 (x4), 126.6 (x2), 124.6 (x2), 124.5 (x2), $124.5(x 2), 124.3(x 2), 122.3(x 2, q, J=274 \mathrm{~Hz}), 118.9(x 2), 116.5(x 4), 116.4(x 2), 116.1(x 2), 115.8(x 2), 111.9(x 2)$, $106.4\left(x 2, q^{1}{ }^{1} \mathrm{~J}_{(\mathrm{C}-\mathrm{F})}=32 \mathrm{~Hz}\right), 56.6,51.5(\mathrm{x} 2), 49.9(\mathrm{x} 2)$.

${ }^{19} \mathrm{~F} \mathrm{NMR}\left(376 \mathrm{MHz} \mathrm{CDCl}_{3}\right) \delta-55.11$.

IR (neat): 3507, 3059, 3028, 2930, 2866, 1648, 1602, 1495, 1439, 1311, 1255, 1124, 1099, 1030, 988, 905, 876, 743, $728,697 \mathrm{~cm}^{-1}$.

HRMS (ESI): m/z: $[M+N a]^{+}\left(\mathrm{C}_{67} \mathrm{H}_{36} \mathrm{Br}_{2} \mathrm{~F}_{6} \mathrm{NaO}_{10}\right)$, calcd.: 1295.0472; found: 1295.0464 . 
$[\alpha]_{D}^{25}=-32\left(c=0.1, \mathrm{CH}_{2} \mathrm{Cl}_{2}\right)$.

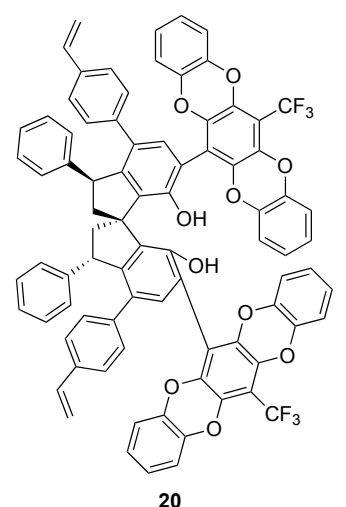

20: Under $\mathrm{N}_{2}$, to a Schlenk tube containing 19 (1.8 g, $\left.1.41 \mathrm{mmol}\right)$, 4-vinylphenylboronic acid (626 mg, $4.23 \mathrm{mmol}, 3.0$ equiv), $\mathrm{Pd}\left(\mathrm{PPh}_{3}\right)_{4}(130 \mathrm{mg}, 0.07 \mathrm{mmol}, 5 \mathrm{~mol} \%)$ and $\mathrm{K}_{3} \mathrm{PO}_{4}(0.9 \mathrm{~g}$, $4.23 \mathrm{mmol}, 3.0 \mathrm{eq})$ was added degassed 1,4-dioxane $(9 \mathrm{~mL})$ and water $(3 \mathrm{~mL})$. The reaction mixture was stirred and heated to $95{ }^{\circ} \mathrm{C}$ for $48 \mathrm{~h}$. and then it was cooled to room temperature and extracted with $\mathrm{CH}_{2} \mathrm{Cl}_{2}(10 \mathrm{~mL} \times 3)$. The combined organic layers were dried over $\mathrm{MgSO}_{4}$ and concentrated under reduced pressure. The crude product was purified by flash column chromatography on silicagel using cyclohexane $/ \mathrm{CH}_{2} \mathrm{Cl}_{2}(5: 2)$ as the eluent to give compound 20 ( $838 \mathrm{mg}, 45 \%$ yield) as a slightly yellow solid. The reaction can also be performed with $\mathrm{Pd}_{2}(\mathrm{dba})_{3}(2 \mathrm{~mol} \%)$ and S-Phos $(8 \mathrm{~mol} \%)$ in 3:1 toluene/water $\left(95^{\circ} \mathrm{C}, 18 \mathrm{~h}\right), 20$ being obtained in almost identical yield (44\%).

Light yellow solid. Decomposed by carbonization or polymerization upon heating, or m. p. $>300^{\circ} \mathrm{C}$. A melting point could not be detected.

${ }^{1} \mathrm{H}$ NMR $(500 \mathrm{MHz}$, Chloroform-d) $\delta 7.10(\mathrm{~s}, 2 \mathrm{H}), 7.06(\mathrm{~d}, J=8.4 \mathrm{~Hz}, 4 \mathrm{H}), 7.02(\mathrm{~d}, J=8.3 \mathrm{~Hz}, 4 \mathrm{H}), 7.00-6.89(\mathrm{~m}, 10 \mathrm{H})$, $6.88(\mathrm{~d}, J=2.1 \mathrm{~Hz}, 1 \mathrm{H}), 6.86(\mathrm{dd}, J=2.9,1.7 \mathrm{~Hz}, 2 \mathrm{H}), 6.85(\mathrm{~d}, J=1.4 \mathrm{~Hz}, 1 \mathrm{H}), 6.84-6.80(\mathrm{~m}, 2 \mathrm{H}), 6.76-6.72(\mathrm{~m}, 6 \mathrm{H})$, $6.66-6.62(\mathrm{~m}, 2 \mathrm{H}), 6.57(\mathrm{~d}, J=10.9 \mathrm{~Hz}, 1 \mathrm{H}), 6.56-6.52(\mathrm{~m}, 3 \mathrm{H}), 5.60(\mathrm{dd}, J=17.5,1.0 \mathrm{~Hz}, 2 \mathrm{H}), 5.37(\mathrm{~s}, 2 \mathrm{H}), 5.14$ (dd, $J=10.8,1.0 \mathrm{~Hz}, 2 \mathrm{H}$ ), 4.88 (dd, $J=10.3,7.5 \mathrm{~Hz}, 2 \mathrm{H}$ ), 3.14 (dd, $J=13.3,7.5 \mathrm{~Hz}, 2 \mathrm{H}$ ), 2.51 (dd, $J=13.1,10.5 \mathrm{~Hz}, 2 \mathrm{H}$ ).

${ }^{13} \mathrm{C} \mathrm{NMR} \mathrm{(126} \mathrm{MHz,} \mathrm{CDCl} 3$ ) $\delta 149.6$ (x2), 146.0 (x2), 143.3 (x2), 141.3 (x2), 140.9 (x2), 140.5 (x2), 140.0 (x2), 139.0 (x2), $136.6(x 4), 136.2(x 2), 135.9(x 2), 135.6(x 2), 135.5(x 2), 133.5(x 2), 133.0(x 2), 131.9(x 2), 128.8(x 4), 128.0(x 4)$, 127.9 (x4), 125.8 (x2), $125.3(x 4), 124.4$ (x2), 124.3 (x2), 124.2 (x2), 124.1 (x2), 122.4(x2, q, J = $222 \mathrm{~Hz}), 117.9$ (x2), $117.2(x 2), 116.5(x 4), 116.4(x 2), 116.1(x 2), 113.2(x 2), 105.8\left(x 2, q^{1}{ }^{1}{ }_{(C-F)}=32 \mathrm{~Hz}\right), 55.9,50.2(x 2), 50.1(x 2)$.

${ }^{19} \mathrm{~F}$ NMR $\left(376 \mathrm{MHz}, \mathrm{CDCl}_{3}\right) \delta-55.00$.

IR (neat): 3511, 3059, 3027, 2932, 2862, 1647, 1603, 1495, 1439, 1310, 1256, 1165, 1124, 1098, 1031, 988, 906, 885, $842,743,730,698 \mathrm{~cm}^{-1}$.

HRMS (ESI): $\mathrm{m} / \mathrm{z}:[\mathrm{M}+\mathrm{Na}]^{+}\left(\mathrm{C}_{83} \mathrm{H}_{50} \mathrm{~F}_{6} \mathrm{NaO}_{10}\right)$, calcd.: 1343.3200 ; found: 1343.3176 .

$[\alpha]_{D}{ }^{25}=+50\left(c=0.1, \mathrm{CH}_{2} \mathrm{Cl}_{2}\right)$.

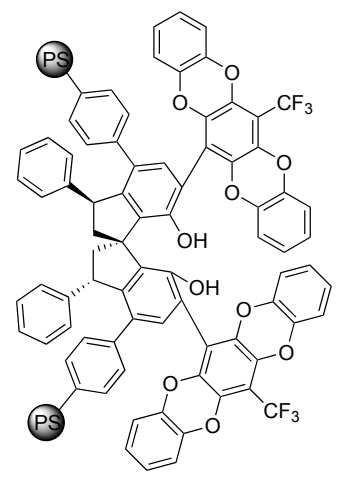

21
21 was synthesized following a modification of a literature procedure. ${ }^{2}$ A $100 \mathrm{~mL}$ reactor was charged with a suspension of polyvinyl alcohol (PV-OH) $(100 \mathrm{mg}, 0.96 \mu \mathrm{mol}, 0.002$ equiv.) in $72 \mathrm{~mL}$ of degassed MiliQ water. The solution was heated at $100{ }^{\circ} \mathrm{C}$ until PV-OH was dissolved. Then, it was cooled to rt and a solution of boric acid (449 $\mathrm{mg}, 7.26 \mathrm{mmol}$ ) in $18 \mathrm{~mL}$ of degassed MiliQ water was transferred to the reactor. Later, a degassed solution containing divinylbenzene (DVB), filtered on a short pad of silica immediately before use, (80\%, $119 \mu \mathrm{L}, 0.68 \mathrm{mmol}, 1.3$ equiv.), BINOL derivative 20 (700 mg, $0.53 \mathrm{mmol}$ ), styrene 
( $2.9 \mathrm{ml}, 25.5 \mathrm{mmol}, 47.75$ equiv.) and AIBN (31 mg, $0.19 \mathrm{mmol}, 0.35$ equiv.) in toluene ( $2.4 \mathrm{~mL})$ was transferred to the reactor. After that, the system was heated at $90{ }^{\circ} \mathrm{C}$ and magnetically stirred at $440 \mathrm{rpm}$. After two days, the aqueous solution was decanted off and the resin was washed with water $\left(50^{\circ} \mathrm{C}\right)$ several times, followed by $\mathrm{MeOH}$ and $\mathrm{CH}_{2} \mathrm{Cl}_{2}$. Finally, it was dried overnight in a $40{ }^{\circ} \mathrm{C}$ vacuum oven to furnish $3.0 \mathrm{~g}$ of yellow beads.

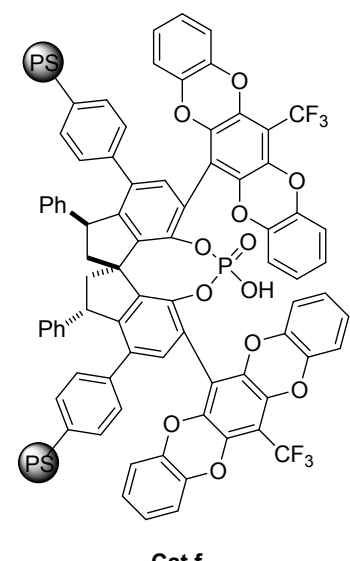

Cat $f$
Cat $f$ was synthesized following a modification of a literature procedure. ${ }^{2}$ In an oven-dried Schlenk tube, resin 21 ( $3.0 \mathrm{~g}$, ca. $0.53 \mathrm{mmol})$ was suspended in pyridine $(20 \mathrm{~mL})$ under Ar. Then, $\mathrm{POCl}_{3}(248 \mu \mathrm{L}, 2.65 \mathrm{mmol}, 5$ eq.) was added and the reaction mixture was heated in the sealed Schlenk tube at $120^{\circ} \mathrm{C}$. After 2 days, it was cooled to RT and $5 \mathrm{~mL}$ of water were added. Then the system was sealed again and heated at $100^{\circ} \mathrm{C}$ overnight. The resin was filtered and washed with water, THF/water, THF, $2 \mathrm{M} \mathrm{HCl} / E t O A c, E t O A C / D C M$, and DCM and dried overnight in a $40^{\circ} \mathrm{C}$ vacuum oven to give $3.0 \mathrm{~g}$ of brown beads.

P elemental analysis (\%): 0.53

$f_{(P)}: 0.17 \mathrm{mmol} / \mathrm{g}$ resin 


\subsection{Preparation of Cat $\mathrm{g}$}

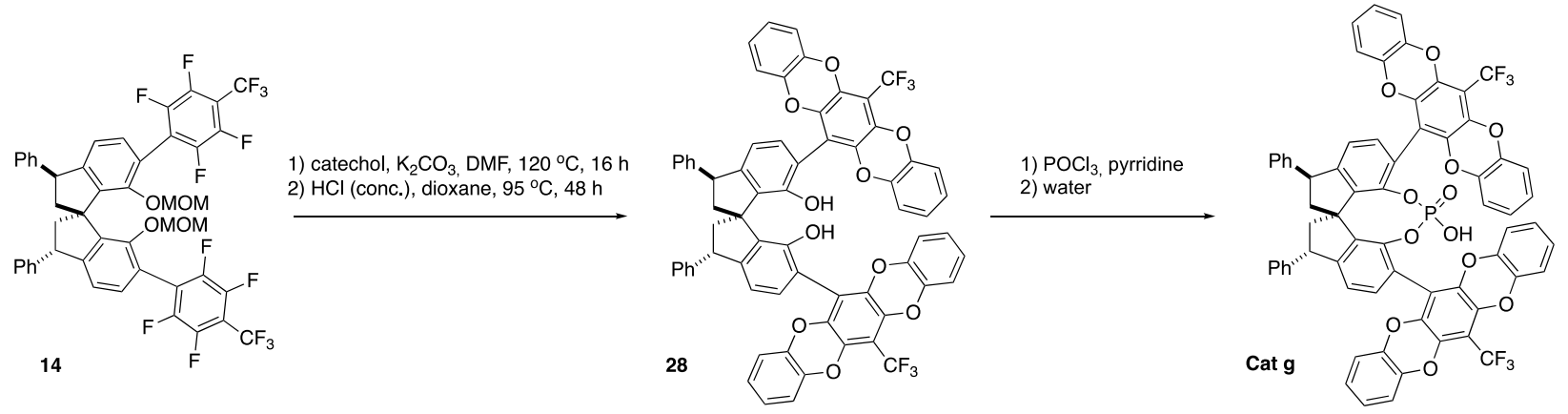

Scheme S6. The procedure of preparation of Cat $\mathbf{g}$

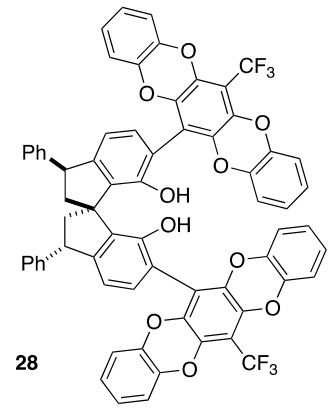

28: The protected diol 14 (924 mg, 1 mmol) was placed In a Schlenk tube, pyrocatechol (495 $\mathrm{mg}, 4.5 \mathrm{mmol}, 4.5$ equiv) and $\mathrm{K}_{2} \mathrm{CO}_{3}(2.75 \mathrm{~g}, 20 \mathrm{mmol}, 20$ equiv) were added, and an $\mathrm{Ar}$ atmosphere was established (3X vacuum/refill). Degassed anhydrous DMF (12 mL) was added, and the mixture was stirred at $120^{\circ} \mathrm{C}$ overnight. The reaction mixture was cooled to $\mathrm{rt}$ and quenched with $30 \mathrm{~mL}$ water and $30 \mathrm{~mL}$ ethyl acetate. Phases were separated, and the organic one was washed with saturated $\mathrm{NaHCO}_{3}$, water and brine, and dried with $\mathrm{MgSO}_{4}$. Removal of solvent by rota-evaporation afforded $1.2 \mathrm{~g}$ of a yellow semi-solid, which was dissolved in 1,4-dioxane $(10 \mathrm{~mL})$. Concentrated aqueous $\mathrm{HCl}(1.0 \mathrm{~mL})$ was added, and the solution was heated at $95{ }^{\circ} \mathrm{C}$ for $48 \mathrm{~h}$. The reaction mixture was then extracted with dichloromethane ( $\left.3 \times 15 \mathrm{~mL}\right)$. After solvent removal by rota-evaporation, the residue was purified by column chromatography [silicagel, cyclohexane/ dichloromethane $(v / v=5 / 2)$ as eluent], affording the expected compound 28 as a white solid (647 $\mathrm{mg}, 58 \%$ yield).

Light yellow solid. Decomposed by carbonization or polymerization upon heating, or m. p. $>300^{\circ} \mathrm{C}$. A melting point could not be detected.

${ }^{1} \mathrm{H}$ NMR $\left(400 \mathrm{MHz}, \mathrm{CDCl}_{3}\right) \delta$ 7.32-7.15 (m, 10H), $7.00(\mathrm{~d}, J=7.7 \mathrm{~Hz}, 2 \mathrm{H}), 6.79(\mathrm{dd}, J=5.9,1.8 \mathrm{~Hz}, 4 \mathrm{H}), 6.74-6.63(\mathrm{~m}$, $4 \mathrm{H}), 6.61-6.45(\mathrm{~m}, 10 \mathrm{H}), 5.08(\mathrm{~s}, 2 \mathrm{H}), 4.52(\mathrm{dd}, J=11.0,7.1 \mathrm{~Hz}, 2 \mathrm{H}), 2.92(\mathrm{dd}, J=13.0,7.1 \mathrm{~Hz}, 2 \mathrm{H}), 2.44(\mathrm{t}, J=12.0 \mathrm{~Hz}$, $2 \mathrm{H})$.

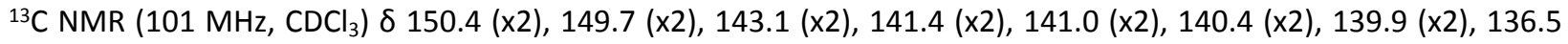
(x2), 136.1 (x2), 135.8 (x2), 135.6 (x2), 132.3 (x2), $130.7(x 2), 128.8(x 4), 128.4(x 4), 126.9(x 2), 124.4(x 2), 124.2(x 2)$, $124.2(x 2), 124.1(x 2), 122.4(x 2, q, 1 J=276 \mathrm{~Hz}), 118.3(x 2), 118.1(x 2), 116.6(x 2), 116.5(x 2), 116.3(x 2), 115.9(x 2)$, $106.1(x 2), 105.5\left(x 2, q,{ }^{1} J_{(C-F)}=33 \mathrm{~Hz}\right), 55.8,50.2(x 2), 48.0(x 2)$.

${ }^{19} \mathrm{~F} \mathrm{NMR}\left(376 \mathrm{MHz}^{\mathrm{CDCl}}{ }_{3}\right) \delta-54.96$.

IR (neat): 3510, 3060, 3028, 2930, 2860, 1648, 1600, 1496, 1440, 1311, 1257, 1166, 1120, 1030, 985, 900, 887, 843 $\mathrm{cm}^{-1}$.

HRMS (ESI): $\mathrm{m} / \mathrm{z}:[\mathrm{M}+\mathrm{Na}]^{+}\left(\mathrm{C}_{67} \mathrm{H}_{38} \mathrm{~F}_{6} \mathrm{NaO}_{10}\right)$, calcd.: 1139.2261; found: 1139.2242 .

$[\alpha]_{D}^{25}=-48\left(c=0.1, \mathrm{CH}_{2} \mathrm{Cl}_{2}\right)$. 


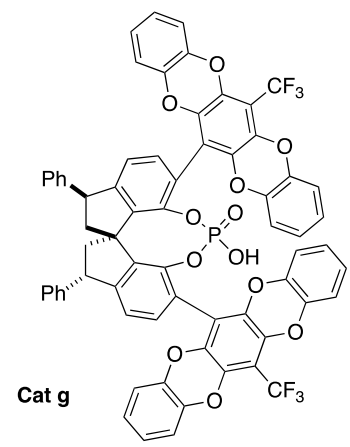

Cat g: To an oven-dried Schlenk tube, 28 (500 $\mathrm{mg}, 0.448 \mathrm{mmol}$ ) and pyridine $(5 \mathrm{~mL})$ were added under $\mathrm{Ar}$. Then, $\mathrm{POCl}_{3}(126 \mu \mathrm{L}, 1.35 \mathrm{mmol}, 3$ eq.) was added, and the reaction mixture was heated at $100{ }^{\circ} \mathrm{C}$ in the sealed Schlenk tube. After 18 hours, the reaction mixture was cooled to room temperature and water $(5 \mathrm{~mL})$ was added. The system was sealed again and heated at $100{ }^{\circ} \mathrm{C}$ overnight. Afterwards, the solution was cooled to rt, diluted with $\mathrm{DCM}(20 \mathrm{~mL})$, washed with $2 \mathrm{~N} \mathrm{HCl}(3 \times 10 \mathrm{~mL})$. Volatiles were removed under vacuum and the residue was purified by column chromatography on silicagel (ethyl acetate/hexane=1:1). The collected product was redissolved in DCM $(5 \mathrm{~mL}), 6 \mathrm{~N} \mathrm{HCl}(5 \mathrm{~mL})$ was added, and the mixture stirred for 1 hour at room temperature. By evaporation of the organic phase, compound Cat g (413 mg, 78\% yield) was obtained as a slightly yellow solid.

Light yellow solid. Decomposed by carbonization or polymerization upon heating, or m. p. $>300^{\circ} \mathrm{C}$. A melting point could not be detected.

${ }^{1} \mathrm{H} \mathrm{NMR}\left(500 \mathrm{MHz}, \mathrm{CDCl}_{3}\right) \delta 7.40(\mathrm{t}, J=7.4 \mathrm{~Hz}, 4 \mathrm{H}), 7.33(\mathrm{dd}, J=12.4,7.3 \mathrm{~Hz}, 6 \mathrm{H}), 7.23(\mathrm{~d}, J=7.7 \mathrm{~Hz}, 2 \mathrm{H}), 6.90-6.77$ (m, 14H), $6.69(\mathrm{~d}, J=7.7 \mathrm{~Hz}, 2 \mathrm{H}), 6.45(\mathrm{~d}, J=8.0 \mathrm{~Hz}, 2 \mathrm{H}), 4.63(\mathrm{dd}, J=10.8,6.3 \mathrm{~Hz}, 2 \mathrm{H}), 2.98(\mathrm{dd}, J=12.3,6.3 \mathrm{~Hz}, 2 \mathrm{H})$, $2.43(\mathrm{t}, J=11.6 \mathrm{~Hz}, 2 \mathrm{H})$.

${ }^{13} \mathrm{C}$ NMR (126 MHz, CDCl $)$ ठ 149.6 (x2), 143.6 (x2), 143.2 (x2), 141.6 (x2), 141.4 (x2), 141.3 (x2), 141.1 (x2), 139.7 (x2), $137.1(x 2), 136.5(x 2), 136.2(x 2), 135.7(x 2), 131.4(x 2), 128.8(x 4), 128.6(x 4), 128.5(x 2), 127.1(x 2), 125.6(x 2)$, $124.3(x 2), 124.2$ (x2), $124.1(x 4), 122.8(x 2), 122.4(x 2), 122.3(x 2, q, 1 J=275 \mathrm{~Hz}), 117.9(x 2), 116.6(x 2), 116.5(x 2)$, $116.3(x 2), 116.0(x 2), 106.1\left(x 2, q,{ }^{1} J_{(C-F)}=33 \mathrm{~Hz}\right), 57.7,50.1(x 2), 49.2(x 2)$.

${ }^{19} \mathrm{~F} \mathrm{NMR}\left(471 \mathrm{MHz}, \mathrm{CDCl}_{3}\right) \delta-54.91$.

31P NMR (162 MHz, $\left.\mathrm{CDCl}_{3}\right) \delta-7.09$.

IR (neat): 3648, 3358, 3081, 3062, 3025, 2960, 2926, 2858, 1640, 1600, 1493, 1434, 1308, 1256, 1165, 1120, 1090, $1020,985,906,834,747,730,698 \mathrm{~cm}^{-1}$.

HRMS (ESI): $\mathrm{m} / \mathrm{z}:[\mathrm{M}-\mathrm{H}]^{+}\left(\mathrm{C}_{67} \mathrm{H}_{36} \mathrm{~F}_{6} \mathrm{O}_{12} \mathrm{P}\right)$, calcd.: 1177.1854; found: 1177.1843.

$[\alpha]_{D}^{25}=240\left(c=0.1, \mathrm{CH}_{2} \mathrm{Cl}_{2}\right)$. 


\section{Experimental procedures}

\subsection{Optimization of catalyst and solvent for the desymmetrization reaction}

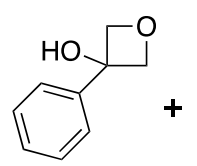

$22 a$
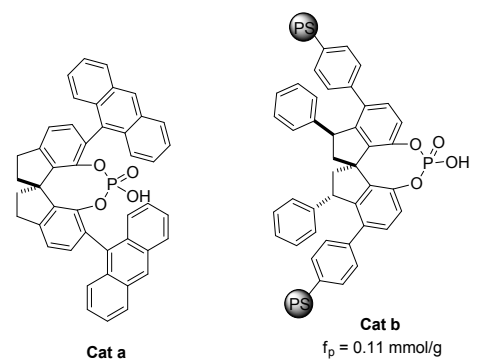<smiles>Sc1nc2ccccc2s1</smiles>

$23 a$

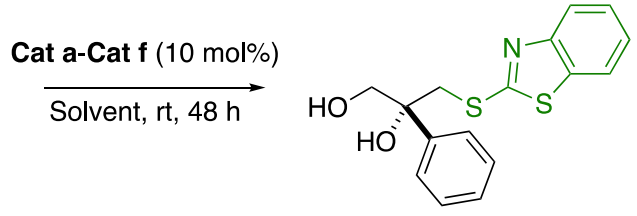

(R)-24aa
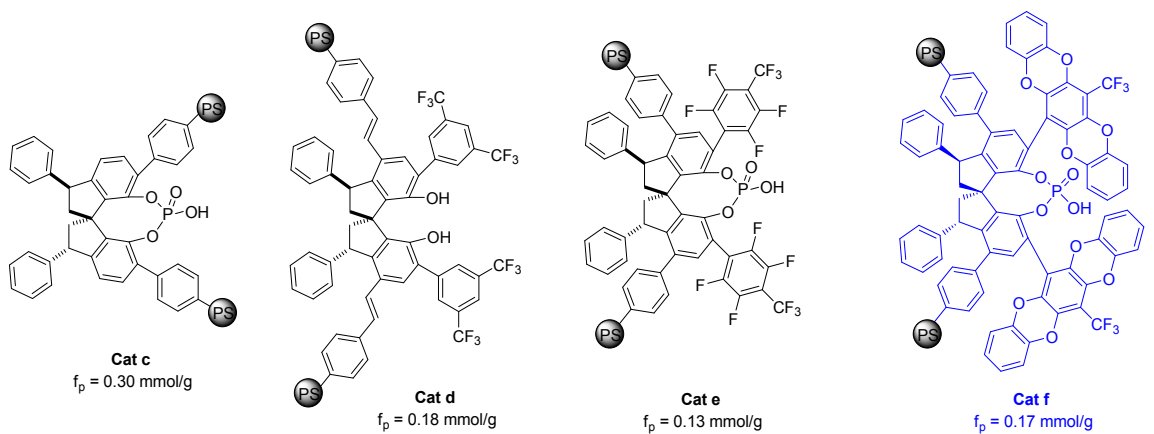

Scheme S7. Optimization of reaction conditions

These experiments refer to Table 1 in the main text. To a $10 \mathrm{~mL}$ glass vial were sequentially added Cat $\mathbf{b}$-Cat $\mathbf{f}$ (10 $\mathrm{mol} \%$ loading) and the indicated solvent $(1 \mathrm{~mL})$, followed by oxetane $22 \mathrm{a}(30 \mathrm{mg}, 0.2 \mathrm{mmol})$ and $23 \mathrm{a}(42 \mathrm{mg}, 0.25$ $\mathrm{mmol}$ ). The reaction mixture was shaken at room temperature for $48 \mathrm{~h}$, or at $60^{\circ} \mathrm{C}$ for $16 \mathrm{~h}$ in specified cases. Then, it was filtered and the resin beads were washed with DCM $(3 \times 0.25 \mathrm{~mL})$. The solvent was concentrated under reduced pressure and the product was isolated after purification by column chromatography on silicagel with cyclohexane/ethyl acetate $(\mathrm{EtOAc} / \mathrm{c}-\mathrm{Hex}=1: 5)$ to yield 24aa.

\subsection{Recycling of Cat $f$ in the desymmetrization of 22a with 23a in batch}<smiles>OC1(c2ccccc2)COC1</smiles>

22a

$$
\underset{\mathrm{CHCl}_{3}, 60^{\circ} \mathrm{C}, 16 \mathrm{~h}}{\text { Cat } \mathrm{f}(10 \mathrm{~mol} \%)}
$$<smiles>OC[C@@](O)(CSc1nc2ccccc2s1)c1ccccc1</smiles>

$(R)-24 a a$

Scheme S8. Recycling experiments of Cat $\mathrm{f}$ in batch

To a $10 \mathrm{~mL}$ glass vial were sequentially Cat $\mathrm{f}\left(59 \mathrm{mg}, \mathrm{f}_{\mathrm{p}}=0.17 \mathrm{mmol} / \mathrm{g}, 5 \mathrm{~mol} \%\right.$ loading) and $4 \mathrm{~mL} \mathrm{CHCl}$, followed by oxetane 22a (30 mg, $0.2 \mathrm{mmol}$ ) and 23a (42 mg, $0.25 \mathrm{mmol})$. The reaction mixture was shaken at $60{ }^{\circ} \mathrm{C}$ for $16 \mathrm{~h}$. Then, it was filtered and the resin beads were washed with $\mathrm{CHCl}_{3}(3 \times 0.25 \mathrm{~mL})$. The solvent was removed under reduced pressure and the residue was purified by column chromatography on silicagel with cyclohexane/ethyl acetate (EtOAc/c-Hex $=1: 5)$ to yield 24aa. 
Table S1. Recycling experiments in batch

\begin{tabular}{cccc}
\hline Run & Conversion (\%) & Yield (\%) & ee (\%) \\
\hline 1 & 92 & 90 & 95 \\
2 & 89 & 90 & 96 \\
3 & 92 & 85 & 97 \\
4 & 90 & 89 & 97 \\
5 & 93 & 90 & 97 \\
6 & 90 & 90 & 97 \\
7 & 90 & 85 & 97 \\
8 & 90 & 87 & 95 \\
9 & 93 & 90 & 96 \\
10 & 92 & 90 & 95 \\
11 & 91 & 90 & 95 \\
12 & 89 & 85 & 95 \\
13 & 92 & 89 & 95 \\
14 & 91 & 82 & 94 \\
15 & 91 & 85 & 95 \\
16 & 90 & 84 & 94
\end{tabular}

Isolated yield.

\subsection{Optimization of the desymmetrization of $22 \mathrm{a}$ with $23 a$ mediated by Cat $\mathrm{f}$ in flow}

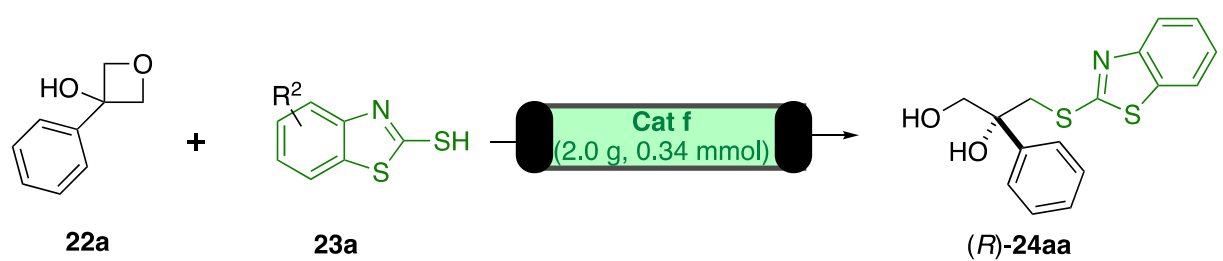

Scheme S9. Parameter optimization for the flow experiment

Cat f $\left(2.0 \mathrm{~g}, \mathrm{f}_{\mathrm{p}}=0.17 \mathrm{mmol} / \mathrm{g}, 0.34 \mathrm{mmol}\right)$ was placed in a size-adjustable, jacketed glass Omnifit column (10 $\left.\mathrm{mm} \varnothing\right)$, and temperature was controlled at $60{ }^{\circ} \mathrm{C}$ by an external circulating pump. A stream of $\mathrm{CHCl}_{3}$ was passed for $1 \mathrm{~h}$ at $0.1 \mathrm{~mL} \mathrm{~min}{ }^{-1}$ to swell the polymer, and size of the packed bed was adjusted. The reagents were then introduced in the system using the two-pump system, represented in Figure 4 of the main text: One of the pumps was used to circulate a solution of 22a (300 mg, 2 mmol, 1.0 eq) and 23a (418 mg, 1.25 eq.) in $\mathrm{CHCl}_{3}$ (40 mL for $0.05 \mathrm{M} ; 20 \mathrm{~mL}$ for $0.1 \mathrm{M}$ ), while the other was used to circulate $\mathrm{CHCl}_{3}$ at $0.5 \mathrm{~mL} \mathrm{~min}{ }^{-1}$ for $1 \mathrm{~h}$ between the eight individual experiments in Table S2. The collected outstream was concentrated under reduced pressure and purified by column chromatography on silicagel, eluting with cyclohexane/ethyl acetate (EtOAc/c-Hex $=1: 5$ ) to yield 24aa. 
Table S2. Parameter optimization for the flow experiment

\begin{tabular}{cccccc}
\hline Flow rate & concentration & Temperature & conversion & yield (\%) & ee (\%) \\
\hline $0.5 \mathrm{ml} / \mathrm{min}$ & $0.05 \mathrm{M}$ & $\mathrm{rt}$ & 84 & 80 & 95 \\
$0.3 \mathrm{ml} / \mathrm{min}$ & $0.05 \mathrm{M}$ & $\mathrm{rt}$ & 89 & 85 & 96 \\
$1 \mathrm{ml} / \mathrm{min}$ & $\mathbf{0 . 0 5 \mathrm { M }}$ & $60^{\circ} \mathrm{C}$ & 92 & 90 & 97 \\
$2 \mathrm{ml} / \mathrm{min}$ & $0.05 \mathrm{M}$ & $60^{\circ} \mathrm{C}$ & 78 & 72 & 95 \\
$2 \mathrm{ml} / \mathrm{min}$ & $0.1 \mathrm{M}$ & $60^{\circ} \mathrm{C}$ & 81 & 80 & 95 \\
$1.0 \mathrm{ml} / \mathrm{min}$ & $0.1 \mathrm{M}$ & $60^{\circ} \mathrm{C}$ & 87 & 80 & 94 \\
$0.75 \mathrm{ml} / \mathrm{min}$ & $0.1 \mathrm{M}$ & $60^{\circ} \mathrm{C}$ & 87 & 82 & 95 \\
$0.5 \mathrm{ml} / \mathrm{min}$ & $\mathbf{0 . 1 \mathrm { M }}$ & $60^{\circ} \mathrm{C}$ & 98 & 90 & 95 \\
\hline
\end{tabular}

\subsection{Continuous flow desymmetrization of oxetanes 22 with thiols 23 mediated by Cat $f$}

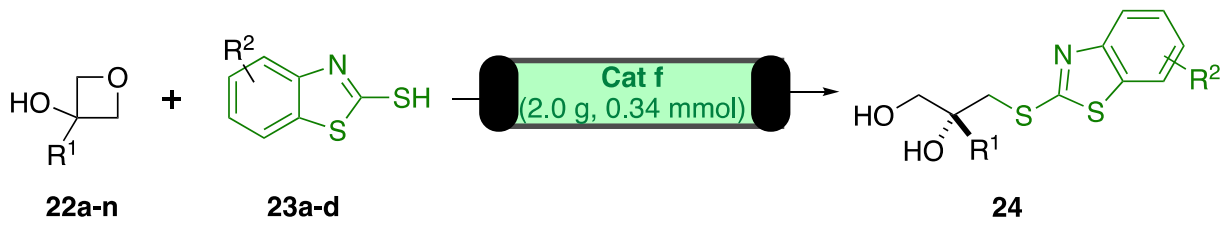

Scheme S10. Continuous flow process

a) Processes at $\mathbf{2} \mathbf{~ m m o l ~ s c a l e : ~ U s i n g ~ t h e ~ s a m e ~ s e t - u p ~ d e p i c t e d ~ a b o v e , ~ f i l l e d ~ w i t h ~ t h e ~ s a m e ~ s a m p l e ~ o f ~ C a t ~} \mathbf{f}\left(2.0 \mathrm{~g}\right.$, $f_{p}=$ $0.17 \mathrm{mmol} / \mathrm{g}, 0.34 \mathrm{mmol})$, previously swollen with $\mathrm{CHCl}_{3}$ and operated at the same temperature $\left(60{ }^{\circ} \mathrm{C}\right)$, the reactants were circulated through the reactor using the two-pump system. One of the syringes contained the oxetane (22a-n, $2.00 \mathrm{mmol}, 1.00 \mathrm{eq})$ and the thiol (23a-d, $2.50 \mathrm{mmol}, 1.25 \mathrm{eq})$ to be reacted, dissolved in $\mathrm{CHCl}_{3}(20$ $\mathrm{mL}$ ), while the other syringe was filled with chloroform for rinsing the resin between individual experiments. A threeway valve was intercalated between the syringes and the reactor, to act as a flow selector, and a back pressure regulator was placed at the outlet of the reactor to prevent the formation of bubbles near the boiling point of chloroform. Flow rate in the preparative experiments was $0.5 \mathrm{~mL} / \mathrm{min}$. In each case, when the circulation of the solution containing the reactants was complete, the channel used for the addition of the reactants and the packed bed reactor was rinsed with $\mathrm{CHCl}_{3}$ at $0.5 \mathrm{~mL} \mathrm{~min}^{-1}$ for $1 \mathrm{~h}$. The collected outstream was concentrated under reduced pressure and purified by column chromatography on silicagel with cyclohexane/ethyl acetate $($ EtOAc/c-Hex $=1: 5)$ to yield the corresponding products 24 .

b) Processes at $\mathbf{2 0} \mathbf{~ m m o l ~ s c a l e : ~ W i t h ~ t h e ~ s a m e ~ s e t - u p ~ a n d ~ c a t a l y s t ~ s a m p l e , ~ o p e r a t e d ~ u n d e r ~ t h e ~ s a m e ~ e x p e r i m e n t a l ~}$ conditions, the large-scale preparation of 24 aa (one run at $20 \mathrm{mmol}$ scale and one run at $18 \mathrm{mmol}$ scale) and $24 \mathrm{na}$ (20 mmol scale) was performed with the results shown in Scheme 3 of the main text. Results for the two runs in the 
preparation of 24aa, performed in a non-consecutive manner, indicate tha Cat $\mathbf{f}$ can be stored for 15 months without losses in its catalytic performance.

\subsection{Desymmetrization of oxetanes 22 with thiols 23 mediated by Cat $\mathbf{g}$ in batch}

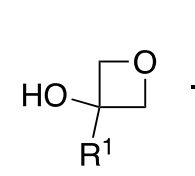

22<smiles>Sc1nc2[R]cccc2s1</smiles>

23

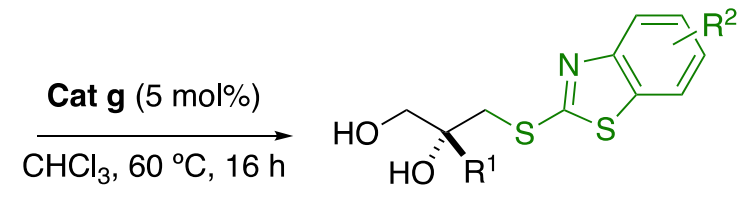

$(R)-24$

Scheme S11. Desymmetrization of oxetanes $\mathbf{2 2}$ with thiols $\mathbf{2 3}$ mediated by Cat $\mathrm{g}$ in batch

In a tall reaction vial with screw-on cap, 22 (0.2 mmol), 23 (0.25 mmol), Cat g (12 mg, $5 \mathrm{~mol} \%$ ) and chloroform (4 mL) were placed. The system was stirred at $60{ }^{\circ} \mathrm{C}$ (bath temperature) for $16 \mathrm{~h}$. Then, the products were purified as in the flow experiments mediated by Cat $\mathbf{f}$. The results (yield and enantiomeric excess) for the fifteen examples studied are summarized in Table S3. For comparison purposes, the results achieved with Cat $\mathbf{f}$ in the preparation of the same products in flow (Figure 5 in the main text) are also included in the table.

Table S3. Desymmetrization of 22 with 23 leading to 24 mediated by the homogeneous catalyst Cat g.

\begin{tabular}{cccc}
\hline Entry & Product & $\begin{array}{c}\text { with Cat g } \\
\text { Yield [\%]; ee [\%] }\end{array}$ & $\begin{array}{c}\text { with Cat f } \\
\text { Yield [\%]; ee [\%] }\end{array}$ \\
\hline $\mathbf{1}$ & 24aa & $87 ; 95$ & $95 ; 95$ \\
2 & 24ba & 78; 98 & $80 ; 95$ \\
3 & 24ca & $83 ;>99$ & $90 ; 99$ \\
4 & 24da & $86 ; 95$ & $65 ; 79$ \\
5 & 24ea & $90 ; 73$ & $85 ; 70$ \\
6 & 24ga & $84 ; 7$ & $75 ; 0$ \\
7 & 24ha & $87 ; 13$ & $80 ; 37$ \\
8 & 24ia & $90 ; 90$ & $85 ; 90$ \\
9 & 24ja & $85 ; 92$ & $92 ; 91$ \\
10 & 24ka & $88 ; 92$ & $84 ; 90$ \\
11 & 24ma & $87 ; 97$ & $95 ; 93$ \\
12 & 24na & $95 ; 78$ & $95 ; 72$ \\
13 & 24ab & $96 ; 95$ & $90 ;>99$ \\
14 & 24ac & $85 ; 85$ & $88 ; 87$ \\
15 & 24ad & $80 ; 74$ & $78 ; 92$ \\
\hline
\end{tabular}




\section{Compound characterization data}

(Data on prepared amounts and yields refer to the flow processes summarized in Figure 5 in the main text)<smiles>OCC(O)(CSc1nc2ccccc2s1)c1ccccc1</smiles>

24aa
(R)-3-(benzo[d]thiazol-2-ylthio)-2-phenylpropane-1,2-diol

Colorless solid. $603 \mathrm{mg}, 95 \%$ yield. Reported compound. ${ }^{5}$

${ }^{1} \mathrm{H}$ NMR $(400 \mathrm{MHz}$, Chloroform-d) $\delta 7.86(\mathrm{dt}, J=8.1,0.9 \mathrm{~Hz}, 1 \mathrm{H}), 7.73(\mathrm{dt}, J=7.9$, $0.9 \mathrm{~Hz}, 1 \mathrm{H}), 7.56-7.49(\mathrm{~m}, 2 \mathrm{H}), 7.47-7.26(\mathrm{~m}, 5 \mathrm{H}), 5.37(\mathrm{~s}, 1 \mathrm{H}), 4.00(\mathrm{dd}, J=11.2$, $7.3 \mathrm{~Hz}, 1 \mathrm{H}), 3.93-3.83(\mathrm{~m}, 2 \mathrm{H}), 3.82-3.74(\mathrm{~m}, 2 \mathrm{H})$.

${ }^{13} \mathrm{C}$ NMR $\left(101 \mathrm{MHz}, \mathrm{CDCl}_{3}\right) \delta 169.2,151.8,142.5,135.2,128.4(\times 2), 127.6,126.4$, 125.5 (x2), 124.8, 121.1, 121.0, 76.4, 68.3, 42.6.

$[\alpha]_{D}^{25}=+112.2\left(c=0.1, \mathrm{CH}_{2} \mathrm{Cl}_{2}\right)$.

HPLC (Daicel Chiralpak AD-H, hexane $/ i-P r O H=80: 20$, flow rate $1.0 \mathrm{~mL} / \mathrm{min}, \lambda=210 \mathrm{~nm}$ ): minor isomer: $t_{R}=12.6 \mathrm{~min}$; major isomer: $t_{R}=15.4$ min.<smiles>COc1ccc(C(O)(CO)CSc2nc3ccccc3s2)cc1</smiles>

24ba
(R)-3-(benzo[d] thiazol-2-ylthio)-2-(4-methoxyphenyl)propane-1,2-diol Colorless solid. M.p. $167.8^{\circ} \mathrm{C} .592 \mathrm{mg}, 80 \%$ yield.

${ }^{1} \mathrm{H}$ NMR $(400 \mathrm{MHz}$, Chloroform-d) $\delta 7.88-7.82(\mathrm{~m}, 1 \mathrm{H}), 7.73$ (dt, $J=8.0,0.8$ $\mathrm{Hz}, 1 \mathrm{H}), 7.48-7.40(\mathrm{~m}, 3 \mathrm{H}), 7.33(\mathrm{~d}, J=1.0 \mathrm{~Hz}, 1 \mathrm{H}), 6.91(\mathrm{~d}, J=8.8 \mathrm{~Hz}, 2 \mathrm{H})$, $5.18(\mathrm{~s}, 1 \mathrm{H}), 4.02-3.94(\mathrm{~m}, 1 \mathrm{H}), 3.90(\mathrm{dd}, J=12.3,5.3 \mathrm{~Hz}, 1 \mathrm{H}), 3.85-3.71$ $(\mathrm{m}, 6 \mathrm{H})$.

${ }^{13} \mathrm{C}$ NMR $\left(101 \mathrm{MHz}, \mathrm{CDCl}_{3}\right) \delta 169.2,159.0,151.8,135.2,134.5,126.8(x 2)$, $126.4,124.8,121.1,121.0,113.7$ (x2), 76.1, 68.2, 55.2, 42.7.

IR (neat): 3368, 2957, 1609, 1579, 1512, 1452, 1419, 1307, 1246, 1209, 1180, 1080, 1011, 968, 815, 751, $565 \mathrm{~cm}^{-1}$. HRMS (ESI): $\mathrm{m} / \mathrm{z}:[\mathrm{M}+\mathrm{Na}]^{+}\left(\mathrm{C}_{17} \mathrm{H}_{17} \mathrm{NNaO}_{3} \mathrm{~S}_{2}\right)$, calcd.: 370.0542 ; found: 370.0538 .

$[\alpha]_{D}^{25}=+132.0\left(c=0.1, \mathrm{CH}_{2} \mathrm{Cl}_{2}\right)$.

HPLC (Daicel Chiralpak AD-H, hexane $/ i-\mathrm{PrOH}=80: 20$, flow rate $1.0 \mathrm{~mL} / \mathrm{min}, \lambda=210 \mathrm{~nm}$ ): minor isomer: $t_{\mathrm{R}}=37.6 \mathrm{~min}$; major isomer: $t_{R}=39.3 \mathrm{~min}$.<smiles>OCC(O)(CSc1nc2ccccc2s1)c1ccc2c(c1)OCO2</smiles>

24ca

(R)-2-(benzo[d][1,3]dioxol-5-yl)-3-(benzo[d] thiazol-2-ylthio)propane-1,2diol Colorless solid. M.p. $120.4{ }^{\circ} \mathrm{C} .650 \mathrm{mg}, 90 \%$ yield.

${ }^{1} \mathrm{H}$ NMR (400 MHz, Chloroform-d) $\delta 7.82$ (ddd, $\left.J=8.2,1.2,0.6 \mathrm{~Hz}, 1 \mathrm{H}\right), 7.70$ (ddd, $J=8.0,1.3,0.6 \mathrm{~Hz}, 1 \mathrm{H}$ ), 7.41 (ddd, $J=8.3,7.3,1.3 \mathrm{~Hz}, 1 \mathrm{H}$ ), 7.30 (ddd, $J=$ 8.0, 7.3, 1.2 Hz, 1H), $7.05(\mathrm{~d}, J=1.8 \mathrm{~Hz}, 1 \mathrm{H}), 6.94(\mathrm{dd}, J=8.1,1.8 \mathrm{~Hz}, 1 \mathrm{H}), 6.78$ $(\mathrm{d}, J=8.1 \mathrm{~Hz}, 1 \mathrm{H}), 5.92(\mathrm{~s}, 2 \mathrm{H}), 5.42(\mathrm{~s}, 1 \mathrm{H}), 4.01(\mathrm{~d}, J=6.9 \mathrm{~Hz}, 1 \mathrm{H}), 3.94-3.81$ $(\mathrm{m}, 2 \mathrm{H}), 3.77-3.66(\mathrm{~m}, 2 \mathrm{H})$.

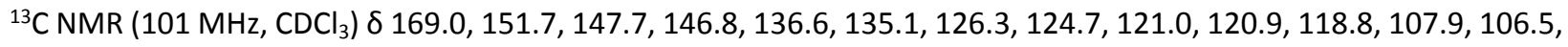
101.0, 76.3, 68.4, 42.7 .

IR (neat): 3366, 3200, 2888, 1501, 1419, 1396, 1244, 1079, 1039, 1011, 933, 858, 824, $749 \mathrm{~cm}^{-1}$. HRMS (ESI): $\mathrm{m} / \mathrm{z}:[\mathrm{M}+\mathrm{Na}]^{+}\left(\mathrm{C}_{17} \mathrm{H}_{15} \mathrm{NNaO}_{4} \mathrm{~S}_{2}\right)$, calcd.: 384.0335 ; found: 384.0319 .

$[\alpha]_{D}^{25}=+169.5\left(c=0.1, \mathrm{CH}_{2} \mathrm{Cl}_{2}\right)$.

HPLC (Daicel Chiralpak AD-H, hexane $/ \mathrm{i}-\mathrm{PrOH}=80: 20$, flow rate $1.0 \mathrm{~mL} / \mathrm{min}, \lambda=210 \mathrm{~nm}$ ): minor isomer: $\mathrm{t}_{\mathrm{R}}=22.5 \mathrm{~min}$; major isomer: $t_{R}=24.3 \mathrm{~min}$.<smiles>Cc1ccccc1C(O)(CO)CSc1nc2ccccc2s1</smiles>

24da
(R)-3-(benzo[d]thiazol-2-ylthio)-2-(o-tolyl)propane-1,2-diol Colorless solid. M.p. $106.7^{\circ} \mathrm{C} .457 \mathrm{mg}, 65 \%$ yield.

${ }^{1} \mathrm{H}$ NMR $(400 \mathrm{MHz}$, Chloroform-d) $\delta 7.89$ (dd, $J=8.1,1.1 \mathrm{~Hz}, 1 \mathrm{H}), 7.74(\mathrm{~d}, J=8.0 \mathrm{~Hz}$, $1 \mathrm{H}), 7.53-7.43(\mathrm{~m}, 2 \mathrm{H}), 7.39-7.31(\mathrm{~m}, 1 \mathrm{H}), 7.25-7.13(\mathrm{~m}, 3 \mathrm{H}), 5.38(\mathrm{q}, \mathrm{J}=2.4$, $1.5 \mathrm{~Hz}, 1 \mathrm{H}), 4.21(\mathrm{~d}, J=8.0 \mathrm{~Hz}, 2 \mathrm{H}), 4.00(\mathrm{~s}, 2 \mathrm{H}), 3.88(\mathrm{~d}, J=5.8 \mathrm{~Hz}, 1 \mathrm{H}), 2.62(\mathrm{~s}, 3 \mathrm{H})$. 
${ }^{13} \mathrm{C} \mathrm{NMR}\left(101 \mathrm{MHz}, \mathrm{CDCl}_{3}\right) \delta 169.2,151.7,139.4,136.0,135.1,132.7,127.7,126.9,126.4,125.6,124.7,121.1,120.9$, 77.7, 67.0, 41.0, 22.4.

IR (neat): 3398, 3054, 2928, 2856, 1492, 1451, 1417, 1390, 1344, 1309, 1235, 1208, 1075, 1053, 1022, 1005, 966, $882,752,722,568 \mathrm{~cm}^{-1}$.

HRMS (ESI): $\mathrm{m} / \mathrm{z}:[\mathrm{M}+\mathrm{Na}]^{+}\left(\mathrm{C}_{17} \mathrm{H}_{17} \mathrm{NNaO}_{2} \mathrm{~S}_{2}\right)$, calcd.: 354.0593; found: 354.0583 .

$[\alpha]_{D}^{25}=+81.9\left(c=0.1, \mathrm{CH}_{2} \mathrm{Cl}_{2}\right)$.

HPLC (Daicel Chiralpak AD-H, hexane/i-PrOH = 80:20, flow rate $1.0 \mathrm{~mL} / \mathrm{min}, \lambda=210 \mathrm{~nm}$ ): $\operatorname{minor}$ isomer: $t_{R}=13.1 \mathrm{~min}$; major isomer: $t_{R}=16.5 \mathrm{~min}$.<smiles>C#C[C@](O)(CO)CSc1nc2ccccc2s1</smiles>

24ea

(R)-2-((benzo[d]thiazol-2-ylthio)methyl)but-3-yne-1,2-diol

Colorless solid. $411 \mathrm{mg}, 85 \%$ yield. Reported compound. 5

${ }^{1} \mathrm{H}$ NMR $(400 \mathrm{MHz}$, Chloroform-d) $\delta 7.85-7.78(\mathrm{~m}, 1 \mathrm{H}), 7.76-7.70(\mathrm{~m}, 1 \mathrm{H}), 7.42$ (ddd, $J=8.3,7.2,1.3 \mathrm{~Hz}, 1 \mathrm{H}), 7.32(\mathrm{td}, J=7.6,1.2 \mathrm{~Hz}, 1 \mathrm{H}), 5.15(\mathrm{~s}, 1 \mathrm{H}), 4.51(\mathrm{t}, J=8.1 \mathrm{~Hz}$, $1 \mathrm{H}), 3.84(\mathrm{dd}, J=11.8,6.5 \mathrm{~Hz}, 1 \mathrm{H}), 3.80-3.71(\mathrm{~m}, 2 \mathrm{H}), 3.65(\mathrm{~d}, J=14.6 \mathrm{~Hz}, 1 \mathrm{H}), 2.58$ (s, $1 \mathrm{H})$.

${ }^{13} \mathrm{C} \mathrm{NMR}\left(101 \mathrm{MHz}, \mathrm{CDCl}_{3}\right) \delta 168.9,151.6,135.0,126.5,124.9,121.1,120.9,83.2,74.6$, $71.6,66.8,40.6$.

$[\alpha]_{D}^{25}=-28.9\left(c=0.1, \mathrm{CH}_{2} \mathrm{Cl}_{2}\right)$.

HPLC (Daicel Chiralpak AD-H, hexane/i-PrOH $=80: 20$, flow rate $1.0 \mathrm{~mL} / \mathrm{min}, \lambda=210 \mathrm{~nm}$ ): minor isomer: $\mathrm{t}_{\mathrm{R}}=12.9 \mathrm{~min}$; major isomer: $t_{R}=15.3 \mathrm{~min}$.

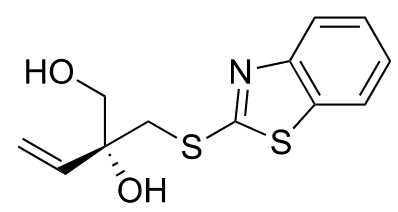

$24 \mathrm{fa}$

(R)-2-((benzo[d] thiazol-2-ylthio)methyl)but-3-ene-1,2-diol

Colorless solid. $455 \mathrm{mg}, 85 \%$ yield.

${ }^{1} \mathrm{H}$ NMR (400 MHz, Chloroform-d) $\delta 7.83$ (dd, $\left.J=8.3,1.1 \mathrm{~Hz}, 1 \mathrm{H}\right), 7.74(\mathrm{~d}, J=8.0 \mathrm{~Hz}$, $1 \mathrm{H}), 7.50-7.38(\mathrm{~m}, 1 \mathrm{H}), 7.36-7.25(\mathrm{~m}, 1 \mathrm{H}), 6.04(\mathrm{dd}, J=17.3,10.9 \mathrm{~Hz}, 1 \mathrm{H}), 5.55$ (dd, $J=17.3,1.4 \mathrm{~Hz}, 1 \mathrm{H}), 5.32(\mathrm{dd}, J=10.8,1.4 \mathrm{~Hz}, 1 \mathrm{H}), 4.65-4.50(\mathrm{~m}, 1 \mathrm{H}), 4.42(\mathrm{~d}, J=14.4$ $\mathrm{Hz}, 1 \mathrm{H}), 3.79-3.51(\mathrm{~m}, 4 \mathrm{H})$.

${ }^{13} \mathrm{C}$ NMR $\left(101 \mathrm{MHz}, \mathrm{CDCl}_{3}\right) \delta 169.1,151.7,138.4,134.9,126.3,124.7,121.0,120.9$,

$116.2,75.3,66.6,40.2$.

IR (neat): 3333, 2926, 2871, 2243, 1642, 1561, 1456, 1425, 1310, 1240, 1128, 1076, 1020, 993, 926, 754, 724, 670 $\mathrm{cm}^{-1}$.

HRMS (ESI): $\mathrm{m} / \mathrm{z}:[\mathrm{M}+\mathrm{Na}]^{+}\left(\mathrm{C}_{12} \mathrm{H}_{13} \mathrm{NNaO}_{2} \mathrm{~S}_{2}\right)$, calcd.: 290.0280; found: 290.0281 .

$[\alpha]_{D}{ }^{25}=+7.71\left(c=0.1, \mathrm{CH}_{2} \mathrm{Cl}_{2}\right)$.

HPLC (Daicel Chiralpak AD-H, hexane $/ i-\mathrm{PrOH}=80: 20$, flow rate $1.0 \mathrm{~mL} / \mathrm{min}, \lambda=210 \mathrm{~nm}$ ): minor isomer: $\mathrm{t}_{\mathrm{R}}=22.6 \mathrm{~min}$; major isomer: $t_{R}=24.1 \mathrm{~min}$.<smiles>OCC(O)CSc1nc2ccccc2s1</smiles>

24ga

3-(benzo[d]thiazol-2-ylthio)propane-1,2-diol

Colorless oil. $394 \mathrm{mg}, 75 \%$ yield.

${ }^{1} \mathrm{H}$ NMR $(400 \mathrm{MHz}$, Chloroform-d) $\delta 7.80$ (dd, $J=8.3,1.3 \mathrm{~Hz}, 1 \mathrm{H}), 7.69$ (dd, $J=8.1$, $1.4 \mathrm{~Hz}, 1 \mathrm{H}), 7.42-7.33(\mathrm{~m}, 1 \mathrm{H}), 7.33-7.18(\mathrm{~m}, 1 \mathrm{H}), 4.67(\mathrm{~d}, J=5.4 \mathrm{~Hz}, 1 \mathrm{H}), 4.26-$ $4.01(\mathrm{~m}, 2 \mathrm{H}), 3.73(\mathrm{~d}, \mathrm{~J}=4.8 \mathrm{~Hz}, 2 \mathrm{H}), 3.60-3.39(\mathrm{~m}, 2 \mathrm{H})$.

${ }^{13} \mathrm{CNMR}\left(101 \mathrm{MHz}, \mathrm{CDCl}_{3}\right)$ $\delta 168.3,152.0,135.0,126.3,124.6,121.0$ (x2), 71.4, 64.1, 36.1.

IR (neat): 3320, 2926, 2872, 1644, 1455, 1424, 1310, 1238, 1074, 1020, 996, 905,

$752,724 \mathrm{~cm}^{-1}$.

HRMS (ESI): $\mathrm{m} / \mathrm{z}:[\mathrm{M}+\mathrm{Na}]^{+}\left(\mathrm{C}_{10} \mathrm{H}_{11} \mathrm{NNaO}_{2} \mathrm{~S}_{2}\right)$, calcd.: 264,0129; found: 264,0125.

HPLC (Daicel Chiralpak AD-H, hexane $/ \mathrm{i}-\mathrm{PrOH}=80: 20$, flow rate $1.0 \mathrm{~mL} / \mathrm{min}, \lambda=210 \mathrm{~nm}$ ): $\mathrm{t}_{\mathrm{R} 1}=12.6 \mathrm{~min} ; \mathrm{t}_{\mathrm{R} 2}=15.4$ $\min$.<smiles>C[C@](O)(CO)CSc1nc2ccccc2s1</smiles>

24ha
(R)-3-(benzo $[d]$ thiazol-2-ylthio)-2-methylpropane-1,2-diol

Colorless oil. $445 \mathrm{mg}, 80 \%$ yield. 
${ }^{1} \mathrm{H}$ NMR (400 MHz, Chloroform-d) $\delta 7.82(\mathrm{~d}, J=8.1 \mathrm{~Hz}, 1 \mathrm{H}), 7.74(\mathrm{dd}, J=8.1,1.3 \mathrm{~Hz}, 1 \mathrm{H}), 7.46-7.39(\mathrm{~m}, 1 \mathrm{H}), 7.36-$ $7.30(\mathrm{~m}, 1 \mathrm{H}), 4.90(\mathrm{t}, J=7.6 \mathrm{~Hz}, 1 \mathrm{H}), 3.93(\mathrm{t}, J=5.1 \mathrm{~Hz}, 1 \mathrm{H}), 3.69-3.56(\mathrm{~m}, 2 \mathrm{H}), 3.49-3.34(\mathrm{~m}, 2 \mathrm{H}), 1.33(\mathrm{~s}, 3 \mathrm{H})$.

${ }^{13} \mathrm{C}$ NMR $\left(101 \mathrm{MHz}, \mathrm{CDCl}_{3}\right) \delta$ 169.2, 151.7, 134.8, 126.4, 124.7, 121.0, 120.8, 73.18, 67.07, 40.7, 22.9.

IR (neat): 3347, 2971, 2930, 2871, 1456, 1426, 1375, 1310, 1238, 1167, 1127, 1077, 1046, 1003, 904, 754, 724 cm-1. HRMS (ESI): $\mathrm{m} / \mathrm{z}:[\mathrm{M}+\mathrm{Na}]^{+}\left(\mathrm{C}_{11} \mathrm{H}_{13} \mathrm{NNaO}_{2} \mathrm{~S}_{2}\right)$, calcd.: 278.0280 ; found: 278.0275 .

$[\alpha]_{D}{ }^{25}=-10.9\left(c=0.1, \mathrm{CH}_{2} \mathrm{Cl}_{2}\right)$.

HPLC (Daicel Chiralpak AD-H, hexane/i-PrOH = 90:10, flow rate $1.0 \mathrm{~mL} / \mathrm{min}, \lambda=210 \mathrm{~nm}$ ): $\operatorname{minor}$ isomer: $t_{R}=19.1 \mathrm{~min}$; major isomer: $t_{R}=20.6 \mathrm{~min}$.<smiles>CC(C)(C)[C@](O)(CO)CSc1nc2ccccc2s1</smiles>

24ia

(R)-2-((benzo[d]thiazol-2-ylthio)methyl)hexane-1,2-diol

Colorless oil. $505 \mathrm{mg}, 85 \%$ yield.

${ }^{1} \mathrm{H}$ NMR (400 MHz, Chloroform-d) $\delta 7.83-7.76(\mathrm{~m}, 1 \mathrm{H}), 7.71(\mathrm{dd}, J=8.0,1.3 \mathrm{~Hz}, 1 \mathrm{H})$, $7.40(\mathrm{td}, J=8.2,7.8,1.4 \mathrm{~Hz}, 1 \mathrm{H}), 7.34-7.25(\mathrm{~m}, 1 \mathrm{H}), 4.89(\mathrm{ddt}, J=6.4,4.3,2.3 \mathrm{~Hz}, 1 \mathrm{H})$, $3.72(\mathrm{q}, J=4.6 \mathrm{~Hz}, 1 \mathrm{H}), 3.66-3.57(\mathrm{~m}, 2 \mathrm{H}), 3.49-3.32(\mathrm{~m}, 2 \mathrm{H}), 1.66-1.55(\mathrm{~m}, 2 \mathrm{H})$, $1.50-1.29(\mathrm{~m}, 4 \mathrm{H}), 0.93(\mathrm{t}, J=7.1 \mathrm{~Hz}, 3 \mathrm{H})$.

${ }^{13} \mathrm{C}$ NMR $\left(101 \mathrm{MHz} \mathrm{CDCl}_{3}\right) \delta 169.3,151.7,134.8,126.3,124.7,121.0,120.8,74.7,65.6$,

$38.8,35.2,25.0,23.2,14.0$.

IR (neat): 3059, 3063, 2954, 2930, 2869, 1456, 1426, 1310, 1240, 1053, 1019, 1002, 906, 754, $724 \mathrm{~cm}^{-1}$.

HRMS (ESI): $\mathrm{m} / \mathrm{z}:[\mathrm{M}+\mathrm{Na}]^{+}\left(\mathrm{C}_{14} \mathrm{H}_{19} \mathrm{NNaO}_{2} \mathrm{~S}_{2}\right)$, calcd.: 320.0749 ; found: 320.0746 .

$[\alpha]_{D}^{25}=-46.8\left(c=0.1, \mathrm{CH}_{2} \mathrm{Cl}_{2}\right)$.

HPLC (Daicel Chiralpak AD-H, hexane/ $i-\mathrm{PrOH}=80: 20$, flow rate $1.0 \mathrm{~mL} / \mathrm{min}, \lambda=210 \mathrm{~nm}$ ): minor isomer: $t_{R}=10.4 \mathrm{~min}$; major isomer: $t_{R}=12.8 \mathrm{~min}$.

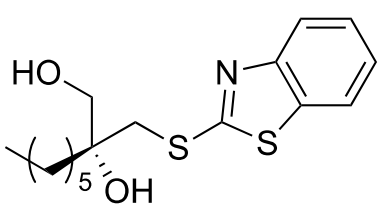

24ja

(R)-2-((benzo[d]thiazol-2-ylthio)methyl)octane-1,2-diol

Colorless oil. $585 \mathrm{mg}, 92 \%$ yield.

${ }^{1} \mathrm{H}$ NMR (400 MHz, Chloroform-d) $\delta 7.80(\mathrm{~d}, J=8.1 \mathrm{~Hz}, 1 \mathrm{H}), 7.72(\mathrm{~d}, J=7.9 \mathrm{~Hz}, 1 \mathrm{H})$, $7.41(\mathrm{t}, J=7.7 \mathrm{~Hz}, 1 \mathrm{H}), 7.34-7.23(\mathrm{~m}, 1 \mathrm{H}), 4.92-4.80(\mathrm{~m}, 1 \mathrm{H}), 3.73-3.57(\mathrm{~m}, 3 \mathrm{H})$, $3.45(\mathrm{dd}, J=11.9,8.0 \mathrm{~Hz}, 1 \mathrm{H}), 3.36(\mathrm{~d}, J=14.7 \mathrm{~Hz}, 1 \mathrm{H}), 1.61(\mathrm{dd}, J=10.1,7.4 \mathrm{~Hz}, 2 \mathrm{H})$, $1.51-1.19(\mathrm{~m}, 8 \mathrm{H}), 0.93-0.83(\mathrm{~m}, 3 \mathrm{H})$.

${ }^{13} \mathrm{C}$ NMR $\left(101 \mathrm{MHz}^{\mathrm{CDCl}}\right)_{3} \delta 169.3,151.8,134.8,126.3,124.7,121.0,120.8,74.8$, $65.62,38.8,35.5,31.7,29.8,22.9,22.5,14.0$.

IR (neat): 3355, 2926, 2855, 1561, 1456, 1426, 1310,1277, 1240, 1060, 1003, 909, 753, $724 \mathrm{~cm}^{-1}$. HRMS (ESI): $\mathrm{m} / \mathrm{z}:[\mathrm{M}+\mathrm{Na}]^{+}\left(\mathrm{C}_{16} \mathrm{H}_{23} \mathrm{NNaO}_{2} \mathrm{~S}_{2}\right)$, calcd.: 348,1068; found: 348,1058.

$[\alpha]_{D}^{25}=-46.1\left(c=0.1, \mathrm{CH}_{2} \mathrm{Cl}_{2}\right)$.

HPLC (Daicel Chiralpak AD-H, hexane/ $-\mathrm{PrOH}=80: 20$, flow rate $1.0 \mathrm{~mL} / \mathrm{min}, \lambda=210 \mathrm{~nm}$ ): major isomer: $t_{\mathrm{R}}=14.2 \mathrm{~min}$; minor isomer: $t_{R}=16.1 \mathrm{~min}$.

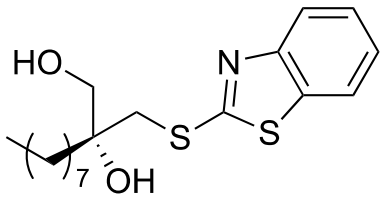

$24 \mathrm{ka}$

(R)-2-((benzo[d]thiazol-2-ylthio)methyl)decane-1,2-diol

Colorless oil. $593 \mathrm{mg}, 84 \%$ yield.

${ }^{1} \mathrm{H} \mathrm{NMR}(400 \mathrm{MHz}$, Chloroform-d) $87.80(\mathrm{~d}, J=8.1 \mathrm{~Hz}, 1 \mathrm{H}), 7.71(\mathrm{~d}, J=7.9 \mathrm{~Hz}, 1 \mathrm{H}), 7.40$ (t, $J=7.8 \mathrm{~Hz}, 1 \mathrm{H}), 7.29(\mathrm{dd}, J=14.9,7.3 \mathrm{~Hz}, 1 \mathrm{H}), 4.94-4.81(\mathrm{~m}, 1 \mathrm{H}), 3.74-3.55(\mathrm{~m}$, $3 \mathrm{H}), 3.48-3.31(\mathrm{~m}, 2 \mathrm{H}), 1.60(\mathrm{dd}, J=8.7,6.1 \mathrm{~Hz}, 2 \mathrm{H}), 1.43-1.22(\mathrm{~m}, 12 \mathrm{H}), 0.88(\mathrm{t}, J=$ $6.7 \mathrm{~Hz}, 3 \mathrm{H})$.

${ }^{13} \mathrm{C}$ NMR $\left(101 \mathrm{MHz}, \mathrm{CDCl}_{3}\right) \delta$ 169.3, 151.7, 134.8, 126.3, 124.7, 121.0, 120.8, 74.8, 65.6, $38.8,35.5,31.8,30.1,29.5,29.2,22.9,22.6,14.1$.

IR (neat): 3355, 2922, 2852, 1457, 1427, 1310, 1240, 1046, 1003, 753, $724 \mathrm{~cm}^{-1}$.

HRMS (ESI): $\mathrm{m} / \mathrm{z}:\left[\mathrm{M}+\mathrm{Na}^{+}\left(\mathrm{C}_{18} \mathrm{H}_{27} \mathrm{NNaO}_{2} \mathrm{~S}_{2}\right)\right.$, calcd.: 376.1375 ; found: 376.1371 .

$[\alpha]_{D}{ }^{25}=41.2\left(c=0.1, \mathrm{CH}_{2} \mathrm{Cl}_{2}\right)$.

HPLC (Daicel Chiralpak AD-H, hexane $/ i-\operatorname{PrOH}=80: 20$, flow rate $1.0 \mathrm{~mL} / \mathrm{min}, \lambda=210 \mathrm{~nm}$ ): minor isomer: $t_{R}=8.4 \mathrm{~min}$; major isomer: $t_{R}=10.6 \mathrm{~min}$.<smiles>CCC(C)(CO)CSc1nc2ccccc2s1</smiles>

(R)-2-((benzo[d]thiazol-2-ylthio)methyl)tetradecane-1,2-diol

Colorless oil. $708 \mathrm{mg}, 82 \%$ yield. 
${ }^{1} \mathrm{H}$ NMR $(400 \mathrm{MHz}$, Chloroform-d) $\delta 7.82(\mathrm{dd}, J=8.2,1.1 \mathrm{~Hz}, 1 \mathrm{H}), 7.74(\mathrm{dd}, J=8.1,1.3 \mathrm{~Hz}, 1 \mathrm{H}), 7.46-7.40(\mathrm{~m}, 1 \mathrm{H})$, $7.35-7.29(\mathrm{~m}, 1 \mathrm{H}), 4.91(\mathrm{t}, J=7.2 \mathrm{~Hz}, 1 \mathrm{H}), 3.74-3.59(\mathrm{~m}, 3 \mathrm{H}), 3.52-3.34(\mathrm{~m}, 2 \mathrm{H}), 1.63(\mathrm{dd}, J=8.8,6.0 \mathrm{~Hz}, 2 \mathrm{H}), 1.30$ (d, $J=17.4 \mathrm{~Hz}, 20 \mathrm{H}), 0.94-0.84(\mathrm{~m}, 3 \mathrm{H})$.

${ }^{13} \mathrm{C} \mathrm{NMR}\left(101 \mathrm{MHz}, \mathrm{CDCl}_{3}\right) \delta 169.3,151.7,134.8,126.3,124.7,121.0,120.8,74.8,65.6,38.8,35.5,31.7,30.1,29.6$, 29.6, 29.5, 29.5, 29.3, 22.9, 22.6, 14.1 .

IR (neat): 3355, 2921, 2851, 1457, 1427, 1310, 1241, 1128, 1020, 1003, 908, 753, $724 \mathrm{~cm}^{-1}$.

HRMS (ESI): $\mathrm{m} / \mathrm{z}:[\mathrm{M}+\mathrm{Na}]^{+}\left(\mathrm{C}_{22} \mathrm{H}_{35} \mathrm{NNaO}_{2} \mathrm{~S}_{2}\right)$, calcd.: 432.2001; found: 432.1997.

$[\alpha]_{D}^{25}=-50.0\left(c=0.1, \mathrm{CH}_{2} \mathrm{Cl}_{2}\right)$.

HPLC (Daicel Chiralpak AD-H, hexane $/ \mathrm{i}-\mathrm{PrOH}=80: 20$, flow rate $1.0 \mathrm{~mL} / \mathrm{min}, \lambda=210 \mathrm{~nm}$ ): $\mathrm{minor}$ isomer: $t_{R}=6.7 \mathrm{~min}$; major isomer: $t_{R}=7.6 \mathrm{~min}$.<smiles>OC[C@@](O)(CSc1nc2ccccc2s1)c1ccc(Cl)s1</smiles>

24ma

(R)-3-(benzo[d] thiazol-2-ylthio)-2-(5-chlorothiophen-2-yl)propane-1,2-

diol

Colorless solid. M.p. $96.8^{\circ} \mathrm{C} .675 \mathrm{mg}, 95 \%$ yield.

${ }^{1} \mathrm{H}$ NMR (400 MHz, Chloroform-d) $\delta 7.82(\mathrm{~d}, J=8.2 \mathrm{~Hz}, 1 \mathrm{H}), 7.75-7.69(\mathrm{~m}$, $1 \mathrm{H}), 7.45-7.39(\mathrm{~m}, 1 \mathrm{H}), 7.36-7.29(\mathrm{~m}, 1 \mathrm{H}), 6.80(\mathrm{~d}, J=1.9 \mathrm{~Hz}, 2 \mathrm{H}), 5.69(\mathrm{t}, J$ $=4.7 \mathrm{~Hz}, 1 \mathrm{H}), 4.42(\mathrm{~s}, 1 \mathrm{H}), 3.91-3.70(\mathrm{~m}, 4 \mathrm{H})$.

${ }^{13} \mathrm{C}$ NMR $\left(101 \mathrm{MHz}, \mathrm{CDCl}_{3}\right) \delta 169.0,151.5,146.0,135.0,129.5,126.5,126.0$, 125.0, 122.6, 121.1, 121.0, 76.1, 67.8, 42.4 .

IR (neat): 3212, 1453, 1423, 1399, 1238, 1215, 1081, 997, 924, 791, $755 \mathrm{~cm}^{-1}$.

HRMS (ESI): $\mathrm{m} / \mathrm{z}:[\mathrm{M}+\mathrm{Na}]^{+}\left(\mathrm{C}_{14} \mathrm{H}_{12} \mathrm{ClNNaO}_{2} \mathrm{~S}_{3}\right)$, calcd.: 379.9611; found: 379.9611 .

$[\alpha]_{D}{ }^{25}=+130.5\left(c=0.1, \mathrm{CH}_{2} \mathrm{Cl}_{2}\right)$.

HPLC (Daicel Chiralpak AD-H, hexane $/ \mathrm{i}-\mathrm{PrOH}=80: 20$, flow rate $1.0 \mathrm{~mL} / \mathrm{min}, \lambda=210 \mathrm{~nm}$ ): minor isomer: $\mathrm{t}_{\mathrm{R}}=12.7 \mathrm{~min}$; major isomer: $t_{R}=15.6 \mathrm{~min}$.<smiles>C[C@](CO)(COc1ccccc1)CSc1nc2ccccc2s1</smiles>

24na

(R)-3-(benzo $[d]$ thiazol-2-ylthio)-2-((benzyloxy)methyl)-2-methylpropan-1-ol

Colorless oil. $682 \mathrm{mg}, 95 \%$ yield.

${ }^{1} \mathrm{H}$ NMR (400 MHz, Chloroform-d) $\delta 7.84$ (dd, $J=8.1,1.1 \mathrm{~Hz}, 1 \mathrm{H}$ ), 7.74 (dd, $J=8.0$, $1.2 \mathrm{~Hz}, 1 \mathrm{H}), 7.44-7.27(\mathrm{~m}, 7 \mathrm{H}), 5.00(\mathrm{t}, J=7.2 \mathrm{~Hz}, 1 \mathrm{H}), 4.57(\mathrm{~d}, J=1.6 \mathrm{~Hz}, 2 \mathrm{H}), 3.63$ (d, $J=14.3 \mathrm{~Hz}, 1 \mathrm{H}), 3.56-3.45(\mathrm{~m}, 5 \mathrm{H}), 1.13(\mathrm{~s}, 3 \mathrm{H})$.

${ }^{13} \mathrm{C} \mathrm{NMR}\left(101 \mathrm{MHz}, \mathrm{CDCl}_{3}\right) \delta 169.6,152.0,138.2,134.8,128.3,127.5,127.4,126.2$, $124.5,120.9,120.8,74.9,73.4,65.4,41.7,37.8,19.5$.

IR (neat): 3354, 3061, 3029, 2858, 1602, 1496, 1454, 1426, 1361, 1309, 1277, 1205, 1095, 1046, 994, 895, 842, 753, 725, 696, $608 \mathrm{~cm}^{-1}$.

HRMS (ESI): $\mathrm{m} / \mathrm{z}:[\mathrm{M}+\mathrm{Na}]^{+}\left(\mathrm{C}_{19} \mathrm{H}_{21} \mathrm{NNaO}_{2} \mathrm{~S}_{2}\right)$, calcd.: 382.0906; found: 382.0895 .

$[\alpha]_{D}^{25}=-25.2\left(c=0.1, \mathrm{CH}_{2} \mathrm{Cl}_{2}\right)$.

HPLC (Daicel Chiralpak AD-H, hexane $/ i-P r O H=80: 20$, flow rate $1.0 \mathrm{~mL} / \mathrm{min}, \lambda=210 \mathrm{~nm}$ ): minor isomer: $t_{R}=9.0 \mathrm{~min}$; major isomer: $\mathrm{t}_{\mathrm{R}}=13.9 \mathrm{~min}$.

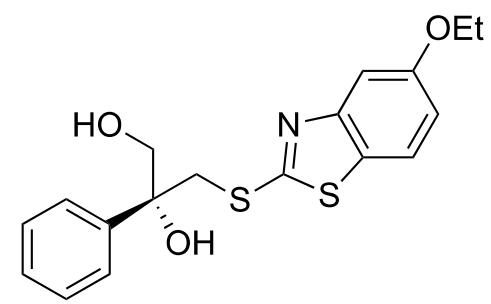

24ab

(R)-3-((5-ethoxybenzo[d]thiazol-2-yl)thio)-2-phenylpropane-1,2-diol Colorless solid. $650 \mathrm{mg}, 90 \%$ yield. Reported compound. ${ }^{5}$

${ }^{1} \mathrm{H}$ NMR $(400 \mathrm{MHz}$, Chloroform-d) $87.75(\mathrm{~d}, J=8.8 \mathrm{~Hz}, 1 \mathrm{H}), 7.59-7.50(\mathrm{~m}, 2 \mathrm{H})$, $7.44-7.27(\mathrm{~m}, 3 \mathrm{H}), 7.20(\mathrm{~d}, J=2.4 \mathrm{~Hz}, 1 \mathrm{H}), 7.04(\mathrm{dd}, J=8.8,2.4 \mathrm{~Hz}, 1 \mathrm{H}), 5.47(\mathrm{~d}$, $J=2.3 \mathrm{~Hz}, 1 \mathrm{H}), 4.16-3.94(\mathrm{~m}, 4 \mathrm{H}), 3.92-3.69(\mathrm{~m}, 3 \mathrm{H}), 1.47(\mathrm{t}, J=7.0 \mathrm{~Hz}, 3 \mathrm{H})$.

${ }^{13} \mathrm{C}$ NMR $\left(101 \mathrm{MHz}, \mathrm{CDCl}_{3}\right) \delta 165.7,156.7,146.2,142.6,136.5,128.3,127.5$, $125.5,121.5,115.6,104.8,76.4,68.3,64.1,42.8,14.7$.

$[\alpha]_{D}{ }^{25}=+115.6\left(c=0.1, \mathrm{CH}_{2} \mathrm{Cl}_{2}\right)$.

HPLC (Daicel Chiralpak AD-H, hexane $/ i-\mathrm{PrOH}=80: 20$, flow rate $1.0 \mathrm{~mL} / \mathrm{min}, \lambda=$ $210 \mathrm{~nm}$ ): minor isomer: $t_{R}=19.7 \mathrm{~min}$; major isomer: $t_{R}=28.5 \mathrm{~min}$.<smiles>OCC(O)(CSc1nc2ccc(Cl)cc2s1)c1ccccc1</smiles>

(R)-3-((6-chlorobenzo[d]thiazol-2-yl)thio)-2-phenylpropane-1,2-diol Colorless solid. $620 \mathrm{mg}, 88 \%$ yield. Reported compound. ${ }^{5}$ 
${ }^{1} \mathrm{H}$ NMR (400 MHz, Chloroform- $\left.d\right) \delta 7.88(\mathrm{~d}, J=2.1 \mathrm{~Hz}, 1 \mathrm{H}), 7.64(\mathrm{~d}, J=8.5 \mathrm{~Hz}, 1 \mathrm{H}), 7.58-7.51(\mathrm{~m}, 2 \mathrm{H}), 7.43-7.38$ $(\mathrm{m}, 2 \mathrm{H}), 7.33(\mathrm{td}, J=8.5,1.7 \mathrm{~Hz}, 2 \mathrm{H}), 5.20(\mathrm{~d}, J=2.5 \mathrm{~Hz}, 1 \mathrm{H}), 4.03-3.88(\mathrm{~m}, 2 \mathrm{H}), 3.86-3.77(\mathrm{~m}, 2 \mathrm{H}), 3.69(\mathrm{t}, J=6.7$ $\mathrm{Hz}, 1 \mathrm{H})$.

${ }^{13} \mathrm{C}$ NMR $\left(101 \mathrm{MHz}, \mathrm{CDCl}_{3}\right) \delta 171.3,152.6,142.3,133.4,132.6,128.4,128.3,127.7,125.5,125.5,125.2,121.7,121.0$, $76.4,68.5,42.7$.

$[\alpha]_{D}^{25}=+168.8\left(c=0.1, \mathrm{CH}_{2} \mathrm{Cl}_{2}\right)$.

HPLC (Daicel Chiralpak AD-H, hexane $/ \mathrm{i}-\mathrm{PrOH}=80: 20$, flow rate $1.0 \mathrm{~mL} / \mathrm{min}, \lambda=210 \mathrm{~nm}$ ): $\operatorname{minor}$ isomer: $\mathrm{t}_{\mathrm{R}}=7.5 \mathrm{~min}$; major isomer: $t_{R}=8.3 \mathrm{~min}$.

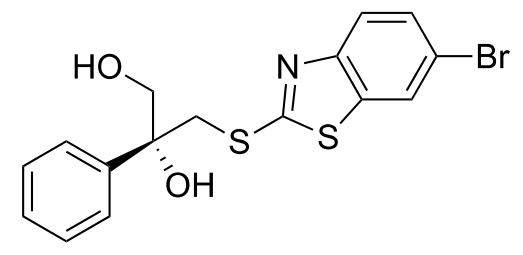

24ad
(R)-3-((6-bromobenzo[d] thiazol-2-yl)thio)-2-phenylpropane-1,2-diol Light-yellow solid. M.p. $150.6^{\circ} \mathrm{C} .616 \mathrm{mg}, 78 \%$ yield.

${ }^{1} \mathrm{H}$ NMR (400 MHz, Chloroform-d) $\delta 8.05(\mathrm{~d}, J=1.9 \mathrm{~Hz}, 1 \mathrm{H}), 7.65-7.51(\mathrm{~m}$, $3 \mathrm{H}), 7.48-7.30(\mathrm{~m}, 4 \mathrm{H}), 5.17(\mathrm{~d}, J=2.6 \mathrm{~Hz}, 1 \mathrm{H}), 3.98(\mathrm{dd}, J=20.0,13.8 \mathrm{~Hz}$, $2 \mathrm{H}), 3.86-3.75(\mathrm{~m}, 2 \mathrm{H}), 3.64(\mathrm{~d}, J=6.9 \mathrm{~Hz}, 1 \mathrm{H})$.

${ }^{13} \mathrm{C}$ NMR $\left(101 \mathrm{MHz}, \mathrm{CDCl}_{3}\right) \delta 171.2,152.9,142.3,134.0,128.4,127.8,127.7$, $125.5,125.2,124.0,122.0,121.7,121.0,120.1,76.4,68.5,42.7$.

IR (neat): 3529, 3089, 2911, 1497, 1446, 1409, 1246, 1202, 1071, 1050, 1024,

$959,858,696,569 \mathrm{~cm}^{-1}$.

HRMS (ESI): $\mathrm{m} / \mathrm{z}:[\mathrm{M}+\mathrm{Na}]^{+}\left(\mathrm{C}_{16} \mathrm{H}_{14} \mathrm{BrNNaO}_{2} \mathrm{~S}_{2}\right)$, calcd.: 417.9542 ; found: 417.9548.

$[\alpha]_{D}^{25}=+139.3\left(c=0.1, \mathrm{CH}_{2} \mathrm{Cl}_{2}\right)$.

HPLC (Daicel Chiralpak AD-H, hexane $/ \mathrm{i}-\mathrm{PrOH}=80: 20$, flow rate $1.0 \mathrm{~mL} / \mathrm{min}, \lambda=210 \mathrm{~nm}$ ): $\operatorname{minor}$ isomer: $t_{\mathrm{R}}=7.8 \mathrm{~min}$; major isomer: $t_{R}=8.8 \mathrm{~min}$. 


\section{Computational section}

The calculations were carried out using G09 D.01 program. ${ }^{6}$ M06-2X was selected for this investigation given the excellent performances of this functional reported for kinetics and thermodynamics of the main group and the noncovalent interactions (particularly $\pi-\pi$ stacking of arenes). ${ }^{7}$ Our recent study pointed out that M06-2X-D3 is particularly suitable to produce kinetically relevant model for the chemistry of phosphine oxides vis-à-vis with experiments. ${ }^{8}$ Furthermore, M06-2X-D3 delivers better performances than M06-2X in describing weak interactions. ${ }^{9}$ Preliminary stationary points used as good guesses for higher level calculations have been located using multi-layer $\mathrm{ONIOM}^{10-16}$ (DFT:PM6) calculations. Guesses structures were firstly optimized at M06-2X-D3/DGDZVP17 level in gas phase and, secondly, the solvent correction was introduced during the single-point energy refinement at M06-2XD3/DGTZVP/SMD ${ }^{18}$ (chloroform, $\varepsilon=4$ 4113). Single-point energy corrections have been calculated using an "ultrafine" (99 radial shells by 590 angular points) grid to avoid potential problems deriving from quadrature issues of the Minnesota functionals. ${ }^{19}$ Vibrational analysis was carried out to identify the nature of stationary points and to obtain ZPE, enthalpic $(H)$ and free energy corrections $(G)$ to the potential energy via statistical thermodynamics. $\Delta H,-T \Delta S$ and $\Delta G$ have been calculated at $333 \mathrm{~K}$ and $1 \mathrm{M}$. GoodVibes v. $2.0 .3^{20}$ has been used to introduce the quasi-harmonic correction to the entropic term derived from low-frequency vibrations ( $v<100 \mathrm{~cm}^{-1}$ cut off) via Grimme's RRHO corrections. Basis set superimposition error (BSSE) have been estimated at M06-2X-D3/DGTZVP level in gas phase for each stationary point using the counterpoise scheme implemented in $\mathrm{G} 09$ based on Boys and Bernardi method ${ }^{21-}$ ${ }^{22}$ (the partition schemes for reactants, transition states and products are displayed in Fig. S1, S2 and S3) and added as a correction to the total single-point potential energy. For simplicity and clarity in the computational profiles, Van der Waals complexes found to be endergonic with respect to the reactants or the products separated at infinite distance have been removed from the profiles. CHIMERA v.1.13.123 and VMD v.1.9.3 $3^{24}$ have been used to visualize, render and generate all the graphical content included in the computational work via POV-Ray ${ }^{25}$ and Tachyon ${ }^{26}$ libraries, respectively. 


\section{Cartesian coordinates, vibrations and non-corrected values for energies}

\section{Benzo[d]thiazole-2-thiol}

\begin{tabular}{|c|c|c|c|c|c|}
\hline \multirow{2}{*}{$\begin{array}{l}\text { Center } \\
\text { Number }\end{array}$} & \multicolumn{2}{|c|}{ Atomic } & Atomic & \multicolumn{2}{|c|}{ Coordinates (Angstroms) } \\
\hline & & nber & Type & $X \quad Y$ & Z \\
\hline 1 & 6 & 0 & -5.197433 & -5.732430 & -0.074661 \\
\hline 2 & 6 & 0 & -3.806657 & -5.925135 & 0.032468 \\
\hline 3 & 6 & 0 & -2.949692 & -4.818007 & 0.017625 \\
\hline 4 & 6 & 0 & -3.498127 & -3.548786 & -0.103458 \\
\hline 5 & 6 & 0 & -4.887310 & -3.368910 & -0.210385 \\
\hline 6 & 6 & 0 & -5.752396 & -4.457232 & -0.197110 \\
\hline 7 & 6 & 0 & -4.420597 & -8.034746 & 0.132690 \\
\hline 8 & 1 & 0 & -1.879067 & -4.973913 & 0.100985 \\
\hline 9 & 1 & 0 & -2.845872 & -2.681660 & -0.115837 \\
\hline 10 & 1 & 0 & -5.294044 & -2.367387 & -0.305097 \\
\hline 11 & 1 & 0 & -6.825224 & -4.316067 & -0.279644 \\
\hline 12 & 16 & 0 & -4.332215 & $5 \quad-9.784615$ & 0.257634 \\
\hline 13 & 1 & 0 & -2.991580 & -9.788737 & 0.307133 \\
\hline 14 & 16 & 0 & -6.002580 & $\begin{array}{ll}0 & -7.279050\end{array}$ & -0.027200 \\
\hline 15 & 7 & 0 & -3.398468 & -7.249414 & 0.148947 \\
\hline
\end{tabular}

$\begin{array}{lccc}\text { Frequencies -- } & 104.1360 & 189.6270 & 191.9167 \\ \text { Frequencies -- } & 257.6143 & 292.9460 & 374.3202 \\ \text { Frequencies -- } & 387.7570 & 432.4147 & 511.7332 \\ \text { Frequencies -- } & 531.1037 & 599.6660 & 601.4862 \\ \text { Frequencies -- } & 659.7282 & 719.7802 & 741.3648 \\ \text { Frequencies -- } & 777.7204 & 876.0156 & 876.4382 \\ \text { Frequencies -- } & 917.5204 & 964.2259 & 1003.3537 \\ \text { Frequencies -- } & 1051.3082 & 1060.8864 & 1114.9294 \\ \text { Frequencies -- } & 1153.9121 & 1186.5066 & 1280.0095 \\ \text { Frequencies -- } & 1314.7471 & 1360.6119 & 1486.8536 \\ \text { Frequencies -- } & 1507.5366 & 1575.1762 & 1648.6841 \\ \text { Frequencies -- } & 1685.1011 & 2764.3400 & 3227.9745 \\ \text { Frequencies -- } & 3235.8498 & 3243.8381 & 3252.5495 \\ \text { SCF Done: E(RM062X/DGDZVP) } & -1120.65058655 \\ \text { Sum of electronic and zero-point Energies= } & -1120.547701 \\ \text { Sum of electronic and thermal Energies= } & -1120.537731 \\ \text { Sum of electronic and thermal Free Energies= } & -1120.586309 \\ \text { SCF Done: E(RM062X/DGTZVP/SMD) = -1120.81574335 }\end{array}$

\section{TS1Ha}

Center Atomic Atomic Coordinates (Angstroms)

\begin{tabular}{cccccc} 
Number & Number & Type & $X$ & $Y$ & $Z$ \\
\hline 1 & 16 & 0 & -1.473481 & -3.787416 & 0.669706 \\
2 & 6 & 0 & -1.977063 & -3.449680 & -0.951264 \\
3 & 16 & 0 & -2.491276 & -4.744429 & -2.012884 \\
4 & 7 & 0 & -2.018660 & -2.262290 & -1.497572 \\
5 & 6 & 0 & -2.791159 & -3.576982 & -3.275803 \\
6 & 6 & 0 & -2.464242 & -2.291791 & -2.814314 \\
7 & 6 & 0 & -3.269671 & -3.779701 & -4.571262 \\
8 & 6 & 0 & -2.607366 & -1.179170 & -3.649944 \\
9 & 6 & 0 & -3.411917 & -2.668770 & -5.395015 \\
10 & 1 & 0 & -3.522388 & -4.773110 & -4.927289
\end{tabular}

$\begin{array}{cccccc}11 & 6 & 0 & -3.082940 & -1.382141 & -4.938229 \\ 12 & 1 & 0 & -2.345041 & -0.189776 & -3.287181 \\ 13 & 1 & 0 & -3.781682 & -2.800622 & -6.406438 \\ 14 & 1 & 0 & -3.201416 & -0.533714 & -5.603998 \\ 15 & 1 & 0 & -0.733582 & -2.533570 & 0.795868 \\ 16 & 1 & 0 & -1.956285 & -1.022280 & -0.773234 \\ 17 & 16 & 0 & -1.895690 & 0.338794 & -0.061592 \\ 18 & 6 & 0 & -0.376256 & -0.005234 & 0.689085 \\ 19 & 16 & 0 & 0.640687 & 1.305961 & 1.267513 \\ 20 & 7 & 0 & 0.120822 & -1.198791 & 0.886286 \\ 21 & 6 & 0 & 1.809871 & 0.137629 & 1.825022 \\ 22 & 6 & 0 & 1.358788 & -1.157850 & 1.516560 \\ 23 & 6 & 0 & 3.026980 & 0.345441 & 2.475586 \\ 24 & 6 & 0 & 2.136098 & -2.271812 & 1.854706 \\ 25 & 6 & 0 & 3.789672 & -0.768462 & 2.810632 \\ 26 & 1 & 0 & 3.371503 & 1.346882 & 2.712485 \\ 27 & 6 & 0 & 3.347195 & -2.064609 & 2.501508 \\ 28 & 1 & 0 & 1.788526 & -3.270414 & 1.606990 \\ 29 & 1 & 0 & 4.740681 & -0.630899 & 3.314542 \\ 30 & 1 & 0 & 3.961783 & -2.917171 & 2.771174\end{array}$

$\begin{array}{lccc}\text { Frequencies -- } & -877.5730 & 12.5050 & 22.4898 \\ \text { Frequencies -- } & 30.6378 & 49.6159 & 65.9400 \\ \text { Frequencies -- } & 103.7174 & 106.6099 & 186.7690 \\ \text { Frequencies -- } & 192.5403 & 196.3899 & 216.4836 \\ \text { Frequencies -- } & 285.8666 & 297.6936 & 354.3899 \\ \text { Frequencies -- } & 383.4696 & 389.6339 & 398.3790 \\ \text { Frequencies -- } & 410.0559 & 429.8172 & 430.5120 \\ \text { Frequencies -- } & 511.9237 & 512.9308 & 532.3886 \\ \text { Frequencies -- } & 537.3675 & 599.1356 & 604.1531 \\ \text { Frequencies -- } & 611.9578 & 615.9727 & 696.6839 \\ \text { Frequencies -- } & 707.8178 & 725.1419 & 733.0035 \\ \text { Frequencies -- } & 739.9246 & 742.1845 & 769.4200 \\ \text { Frequencies -- } & 773.9651 & 842.5311 & 868.8566 \\ \text { Frequencies -- } & 869.1053 & 881.7151 & 889.0184 \\ \text { Frequencies -- } & 956.7807 & 957.9128 & 980.0724 \\ \text { Frequencies -- } & 996.8577 & 1001.5645 & 1046.2482 \\ \text { Frequencies -- } & 1049.1485 & 1059.2169 & 1060.3222 \\ \text { Frequencies -- } & 1111.4573 & 1115.8374 & 1149.8279 \\ \text { Frequencies -- } & 1157.7927 & 1167.4540 & 1184.9782 \\ \text { Frequencies -- } & 1186.1485 & 1252.8510 & 1285.2786 \\ \text { Frequencies -- } & 1290.2932 & 1297.5739 & 1341.8349 \\ \text { Frequencies -- } & 1347.5923 & 1365.1360 & 1370.0329 \\ \text { Frequencies -- } & 1489.9900 & 1493.6516 & 1510.1384 \\ \text { Frequencies -- } & 1510.6733 & 1547.2346 & 1557.5746 \\ \text { Frequencies -- } & 1654.1143 & 1658.0178 & 1684.4211 \\ \text { Frequencies -- } & 1686.5783 & 3220.9730 & 3222.6524 \\ \text { Frequencies -- } & 3226.9273 & 3227.7955 & 3235.7067 \\ \text { Frequencies -- } & 3239.7196 & 3245.2103 & 3253.2800\end{array}$

SCF Done: $E(R M 062 X / D G D Z V P)=-2241.31134437$

Sum of electronic and zero-point Energies= $\quad-2241.109213$ Sum of electronic and thermal Energies= $\quad-2241.088358$ Sum of electronic and thermal Free Energies $=\quad-2241.166226$ SCF Done: $E(R M 062 X / D G T Z V P / S M D)=-2241.64215807$

\section{Benzo[d]thiazole-2(3H)-thione}

Center Atomic Atomic Coordinates (Angstroms) Number Number Type $X \quad$ Y $\quad Z$

\footnotetext{
$\begin{array}{llllll}1 & 16 & 0 & -0.000084 & 0.000000 & -0.003844\end{array}$
} 


$\begin{array}{cccccc}2 & 6 & 0 & 0.002414 & 0.000000 & 1.639210 \\ 3 & 16 & 0 & 1.441255 & 0.000000 & 2.662505 \\ 4 & 7 & 0 & -1.097081 & 0.000000 & 2.449341 \\ 5 & 6 & 0 & 0.481924 & 0.000000 & 4.131178 \\ 6 & 6 & 0 & -0.884822 & 0.000000 & 3.822530 \\ 7 & 6 & 0 & 0.906254 & 0.000000 & 5.457015 \\ 8 & 6 & 0 & -1.851089 & 0.000000 & 4.826276 \\ 9 & 6 & 0 & -0.056759 & 0.000000 & 6.464996 \\ 10 & 1 & 0 & 1.963937 & 0.000000 & 5.699337 \\ 11 & 6 & 0 & -1.420521 & 0.000000 & 6.150531 \\ 12 & 1 & 0 & -2.908322 & 0.000000 & 4.579951 \\ 13 & 1 & 0 & 0.257290 & 0.000000 & 7.503078 \\ 14 & 1 & 0 & -2.155788 & 0.000000 & 6.948005 \\ 15 & 1 & 0 & -2.016575 & 0.000000 & 2.019252\end{array}$

$\begin{array}{cccc}\text { Frequencies -- } & 90.2679 & 188.0419 & 209.0542 \\ \text { Frequencies -- } & 281.7423 & 384.9194 & 403.5443 \\ \text { Frequencies -- } & 430.1006 & 504.7645 & 523.1969 \\ \text { Frequencies -- } & 555.3698 & 576.3708 & 621.9335 \\ \text { Frequencies -- } & 668.5946 & 723.5974 & 734.7883 \\ \text { Frequencies -- } & 761.3246 & 870.6349 & 883.2073 \\ \text { Frequencies -- } & 951.5913 & 992.0682 & 1055.2400 \\ \text { Frequencies -- } 1074.2844 & 1115.1751 & 1161.3382 \\ \text { Frequencies -- } 1180.2769 & 1254.2635 & 1295.5396 \\ \text { Frequencies -- } 1315.8380 & 1368.1783 & 1460.3870 \\ \text { Frequencies -- } 1517.8067 & 1536.7507 & 1673.0820 \\ \text { Frequencies -- } 1688.7806 & 3228.2050 & 3240.9820 \\ \text { Frequencies -- } 3244.5452 & 3257.8298 & 3597.0997\end{array}$

SCF Done: $E(R M 062 X / D G D Z V P)=-1120.66395890$

Sum of electronic and zero-point Energies $=\quad-1120.557749$ Sum of electronic and thermal Energies $=\quad-1120.548147$ Sum of electronic and thermal Free Energies $=\quad-1120.596096$ SCF Done: $E(R M 062 X / D G T Z V P / S M D)=-1120.83289725$

\section{TS1Hb}

\begin{tabular}{ccccccc} 
Center & \multicolumn{2}{c}{ Atomic } & \multicolumn{2}{c}{ Atomic } & \multicolumn{3}{c}{ Coordinates (Angstroms) } \\
Number & Number & Type & $X$ & $Y$ & $Z$ \\
\hline 1 & 16 & 0 & -1.972467 & -2.043090 & 1.390312 \\
2 & 6 & 0 & -1.628666 & -2.088370 & -0.285707 \\
3 & 16 & 0 & -1.300976 & -3.313174 & -1.460024 \\
4 & 7 & 0 & -1.623344 & -0.854746 & -0.767183 \\
5 & 6 & 0 & -1.144772 & -2.064266 & -2.690865 \\
6 & 6 & 0 & -1.353520 & -0.789697 & -2.126458 \\
7 & 6 & 0 & -0.860768 & -2.216943 & -4.046712 \\
8 & 6 & 0 & -1.278171 & 0.354235 & -2.925505 \\
9 & 6 & 0 & -0.787985 & -1.071807 & -4.834566 \\
10 & 1 & 0 & -0.701215 & -3.200105 & -4.477920 \\
11 & 6 & 0 & -0.995035 & 0.198847 & -4.276682 \\
12 & 1 & 0 & -1.439805 & 1.332868 & -2.486040 \\
13 & 1 & 0 & -0.568456 & -1.166096 & -5.892714 \\
14 & 1 & 0 & -0.934834 & 1.076291 & -4.912100 \\
15 & 1 & 0 & -1.911863 & -0.565860 & 0.541642
\end{tabular}

Frequencies -- -1791.7319

Frequencies -- 231.5125

Frequencies -- 422.2681

Frequencies -- 513.4839

Frequencies -- 707.0575

Frequencies -- 768.8068

Frequencies -- 904.9025
96.6327

274.3485

427.5883

576.2388

719.4309

863.4105

960.5877
192.3924

392.1086

504.0108

643.0212

736.6681

877.2180

998.4210

$\begin{array}{llll}\text { Frequencies -- } & 1056.3680 & 1080.8570 & 1143.8096 \\ \text { Frequencies -- } & 1159.0840 & 1182.7738 & 1290.7266 \\ \text { Frequencies -- } 1316.7638 & 1364.6377 & 1466.7644 \\ \text { Frequencies -- } 1502.6788 & 1513.3681 & 1653.8101 \\ \text { Frequencies -- } 1682.0835 & 1747.0970 & 3228.4493 \\ \text { Frequencies -- } 3241.8355 & 3249.2991 & 3257.4515\end{array}$

SCF Done: $E(R M 062 X / D G D Z V P)=-1120.59695302$

Sum of electronic and zero-point Energies $=\quad-1120.496832$ Sum of electronic and thermal Energies $=\quad-1120.487519$ Sum of electronic and thermal Free Energies $=\quad-1120.534950$ SCF Done: $E($ RM062X/DGTZVP/SMD) $=-1120.76598765$

\section{(R)-3-(benzo[d]thiazol-2-ylthio)-2- phenylpropane-1,2-diol ((R)-Product)}

\begin{tabular}{|c|c|c|c|c|c|}
\hline \multirow{2}{*}{$\begin{array}{l}\text { Center } \\
\text { Number }\end{array}$} & \multicolumn{2}{|c|}{ Atomic } & Atomic & \multicolumn{2}{|c|}{ Coordinates (Angstroms } \\
\hline & & ber & Type & $X \quad Y$ & Z \\
\hline 1 & 8 & 0 & -0.137702 & 0.169497 & 0.049577 \\
\hline 2 & 6 & 0 & -0.051436 & 0.047498 & 1.454043 \\
\hline 3 & 6 & 0 & 2.448661 & 0.021304 & 1.235620 \\
\hline 4 & 1 & 0 & -0.048046 & 1.058219 & 1.867595 \\
\hline 5 & 1 & 0 & -0.910328 & -0.498300 & 1.864997 \\
\hline 6 & 6 & 0 & 1.222407 & -0.714184 & 1.857707 \\
\hline 7 & 8 & 0 & $1.061215-$ & -1.974578 & 1.228583 \\
\hline 8 & 1 & 0 & -0.067433 & -0.734033 & -0.300271 \\
\hline 9 & 6 & 0 & 1.283306 & -0.826834 & 3.372721 \\
\hline 10 & 6 & 0 & 1.179643 & -2.067850 & 4.001951 \\
\hline 11 & 6 & 0 & 1.426868 & 0.322316 & 4.159580 \\
\hline 12 & 6 & 0 & 1.231518 & -2.161106 & 5.393803 \\
\hline 13 & 1 & 0 & 1.053756 & -2.960535 & 3.397579 \\
\hline 14 & 6 & 0 & 1.470627 & 0.232231 & 5.548139 \\
\hline 15 & 1 & 0 & 1.521287 & 1.298347 & 3.687566 \\
\hline 16 & 6 & 0 & 1.376833 & -1.013816 & 6.170506 \\
\hline 17 & 1 & 0 & 1.154747 & -3.134168 & 5.869435 \\
\hline 18 & 1 & 0 & 1.585081 & 1.132780 & 6.143676 \\
\hline 19 & 1 & 0 & 1.416123 & -1.087072 & 7.252951 \\
\hline 20 & 1 & 0 & 2.235750 & 1.087695 & 1.115465 \\
\hline 21 & 1 & 0 & 2.627584 & -0.383504 & 0.237871 \\
\hline 22 & 16 & 0 & 4.027536 & 0.046623 & 2.147091 \\
\hline 23 & 6 & 0 & 4.408637 & -1.648305 & 2.277567 \\
\hline 24 & 16 & 0 & 5.849297 & -2.094559 & 3.180661 \\
\hline 25 & 7 & 0 & 3.725491 & -2.630411 & 1.777491 \\
\hline 26 & 6 & 0 & 5.491104 & -3.769117 & 2.848139 \\
\hline 27 & 6 & 0 & 4.311402 & -3.853851 & 2.087382 \\
\hline 28 & 6 & 0 & 6.191920 & -4.912455 & 3.234661 \\
\hline 29 & 6 & 0 & 3.813126 & -5.104955 & 1.706976 \\
\hline 30 & 6 & 0 & 5.686851 & -6.149181 & 2.848309 \\
\hline 31 & 1 & 0 & 7.102489 & -4.842458 & 3.820733 \\
\hline 32 & 6 & 0 & 4.506997 & -6.243901 & 2.092353 \\
\hline 33 & 1 & 0 & 2.901945 & -5.164285 & 1.119420 \\
\hline 34 & 1 & 0 & 6.214055 & -7.052529 & 3.136986 \\
\hline 35 & 1 & 0 & 4.134579 & -7.221376 & 1.803956 \\
\hline 36 & 1 & 0 & 1.936227 & -2.418282 & 1.252864 \\
\hline
\end{tabular}

$\begin{array}{cccc}\text { Frequencies -- } & 11.1939 & 29.0324 & 49.2269 \\ \text { Frequencies -- } & 68.6393 & 80.4601 & 112.4797 \\ \text { Frequencies -- } & 116.4288 & 141.3818 & 148.6469 \\ \text { Frequencies -- } & 190.5215 & 199.1509 & 255.5933 \\ \text { Frequencies -- } & 284.5231 & 301.4874 & 334.1762 \\ \text { Frequencies -- } & 342.1714 & 366.6704 & 383.0561 \\ \text { Frequencies -- } & 412.4321 & 414.6108 & 431.1679 \\ \text { Frequencies -- } & 456.2651 & 491.7236 & 512.7939\end{array}$




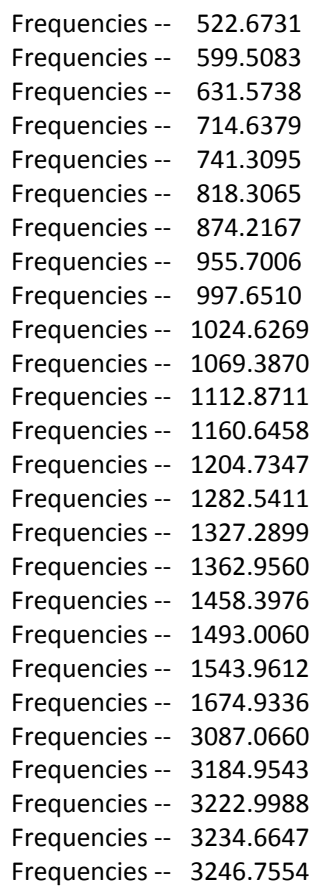

$\begin{array}{llllll}27 & 6 & 0 & -2.145414 & -3.719390 & 3.926971 \\ 28 & 6 & 0 & -3.350859 & -4.792721 & 5.763560 \\ 29 & 6 & 0 & -2.594976 & -4.698023 & 3.033159 \\ 30 & 6 & 0 & -3.791218 & -5.757490 & 4.864476 \\ 31 & 1 & 0 & -3.641797 & -4.831206 & 6.808248 \\ 32 & 6 & 0 & -3.416121 & -5.709450 & 3.511522 \\ 33 & 1 & 0 & -2.295100 & -4.650930 & 1.991108 \\ 34 & 1 & 0 & -4.434373 & -6.557920 & 5.215140 \\ 35 & 1 & 0 & -3.773960 & -6.475257 & 2.831243 \\ 36 & 1 & 0 & -0.031946 & -2.389801 & 2.270611\end{array}$

Frequencies -- 7.3793

Frequencies -- 58.4343

Frequencies -- 127.1108

Frequencies -- 190.7003

Frequencies -- 289.3714

Frequencies -- 349.3119

Frequencies -- 410.7551

Frequencies -- 457.2284

Frequencies -- 515.5604

Frequencies -- 595.8564

Frequencies -- 629.1138

Frequencies -- 714.8316

Frequencies -- 741.8447

Frequencies -- 808.3213

Frequencies -- 877.5223

Frequencies -- 954.1881

Frequencies -- 1000.3282

Frequencies -- 1026.8347

Frequencies -- 1070.9474

Frequencies -- 1120.8695

Frequencies -- 1169.6011

Frequencies -- 1207.4729

Frequencies -- 1282.9883

Frequencies -- 1325.8941

Frequencies -- 1364.7489

Frequencies -- 1458.8594

Frequencies -- 1492.2632

Frequencies -- 1546.8266

Frequencies -- 1675.2617

Frequencies -- 3079.9400

Frequencies -- 3189.4115

Frequencies -- 3224.8059

Frequencies -- 3238.1836

Frequencies -- 3250.4507

$\begin{array}{lc}26.6969 & 44.4741 \\ 79.5700 & 104.0162 \\ 142.1247 & 184.0885 \\ 207.1183 & 242.6401 \\ 297.5904 & 332.8236 \\ 364.9423 & 380.4041 \\ 414.0055 & 434.0302 \\ 490.3359 & 512.6722 \\ 531.2132 & 580.9667 \\ 612.2785 & 624.9165 \\ 659.2603 & 700.3386 \\ 719.7310 & 724.1803 \\ 775.7372 & 778.9930 \\ 871.0917 & 875.8713 \\ 887.4267 & 940.6716 \\ 964.5050 & 999.9646 \\ 1007.2379 & 1016.1054 \\ 1038.2963 & 1056.8233 \\ 1107.0287 & 1110.7151 \\ 1131.5375 & 1158.7500 \\ 1177.0084 & 1184.7394 \\ 1212.5091 & 1253.1698 \\ 1306.8909 & 1318.1476 \\ 1342.7872 & 1363.7224 \\ 1384.1251 & 1439.8099 \\ 1462.8623 & 1489.1134 \\ 1510.1182 & 1513.2463 \\ 1560.0568 & 1652.0152 \\ 1686.7621 & 1699.6260 \\ 3114.3386 & 3171.2100 \\ 3202.3302 & 3215.9925 \\ 3228.5957 & 3236.5053 \\ 3246.0368 & 3250.2697 \\ 3546.0167 & 3790.1303 \\ & \end{array}$

SCF Done: $E(R M 062 X / D G D Z V P)=-1619.92907204$

Sum of electronic and zero-point Energies $=\quad-1619.646255$ Sum of electronic and thermal Energies $=\quad-1619.623754$ Sum of electronic and thermal Free Energies $=\quad-1619.703735$ SCF Done: $E(R M 062 X / D G T Z V P / S M D)=-1620.21547081$

\section{3-Phenyloxetan-3-ol}

\begin{tabular}{|c|c|c|c|c|c|}
\hline \multirow{2}{*}{$\begin{array}{l}\text { Center } \\
\text { Number }\end{array}$} & \multicolumn{2}{|c|}{ Atomic } & Atomic & \multicolumn{2}{|c|}{ Coordinates (Angstroms) } \\
\hline & & mber & Type & $\begin{array}{ll}X & Y\end{array}$ & z \\
\hline 1 & 8 & 0 & 0.137658 & -1.198681 & -0.829289 \\
\hline 2 & 6 & 0 & 0.643784 & -2.316164 & -0.076728 \\
\hline 3 & 6 & 0 & 0.627681 & -1.756377 & -2.062601 \\
\hline 4 & 1 & 0 & 1.565488 & -2.057008 & 0.460875 \\
\hline 5 & 1 & 0 & -0.098332 & -2.716187 & 0.618987 \\
\hline 6 & 6 & 0 & 0.908929 & -3.116080 & -1.375785 \\
\hline 7 & 8 & 0 & 2.191843 & -3.665920 & -1.540846 \\
\hline
\end{tabular}




$\begin{array}{cccccc}8 & 1 & 0 & 2.850772 & -2.983051 & -1.346468 \\ 9 & 1 & 0 & 1.542429 & -1.250796 & -2.399142 \\ 10 & 1 & 0 & -0.127271 & -1.737493 & -2.853028 \\ 11 & 6 & 0 & -0.121405 & -4.180875 & -1.668324 \\ 12 & 6 & 0 & -1.482843 & -3.869581 & -1.560073 \\ 13 & 6 & 0 & 0.258507 & -5.469243 & -2.045596 \\ 14 & 6 & 0 & -2.449224 & -4.835522 & -1.825640 \\ 15 & 1 & 0 & -1.787414 & -2.865784 & -1.267276 \\ 16 & 6 & 0 & -0.713272 & -6.436024 & -2.311607 \\ 17 & 1 & 0 & 1.312669 & -5.708626 & -2.128060 \\ 18 & 6 & 0 & -2.066496 & -6.124414 & -2.202785 \\ 19 & 1 & 0 & -3.501660 & -4.583345 & -1.738465 \\ 20 & 1 & 0 & -0.408402 & -7.436311 & -2.604027 \\ 21 & 1 & 0 & -2.819737 & -6.878855 & -2.407977\end{array}$

$\begin{array}{llcc}\text { Frequencies -- } & 53.1801 & 117.8919 & 140.2864 \\ \text { Frequencies -- } & 232.9607 & 309.8066 & 324.8309 \\ \text { Frequencies -- } & 342.7273 & 410.6848 & 413.7259 \\ \text { Frequencies -- } & 416.4638 & 522.9715 & 559.2501 \\ \text { Frequencies -- } & 628.6483 & 700.1318 & 718.0760 \\ \text { Frequencies -- } & 777.9285 & 803.7395 & 875.2143 \\ \text { Frequencies -- } & 921.3744 & 948.5266 & 965.0766 \\ \text { Frequencies -- } & 1005.3082 & 1015.2464 & 1019.6501 \\ \text { Frequencies -- } 1043.8363 & 1064.9114 & 1087.4103 \\ \text { Frequencies -- } & 1090.0886 & 1098.6886 & 1117.9587 \\ \text { Frequencies -- } & 1154.2205 & 1178.9953 & 1182.4369 \\ \text { Frequencies -- } & 1210.6908 & 1217.0152 & 1263.9193 \\ \text { Frequencies -- } & 1298.0914 & 1342.7895 & 1368.1090 \\ \text { Frequencies -- } & 1389.4218 & 1422.9881 & 1499.0289 \\ \text { Frequencies -- } & 1526.5169 & 1548.2840 & 1555.3147 \\ \text { Frequencies -- } & 1678.5990 & 1700.9109 & 3062.7541 \\ \text { Frequencies -- } & 3072.4638 & 3158.3777 & 3164.8192 \\ \text { Frequencies -- } & 3194.7523 & 3216.8129 & 3224.3511 \\ \text { Frequencies -- } & 3240.2954 & 3250.9207 & 3833.2432 \\ \text { SCF Done: E(RM062X/DGDZVP) } & -499.212120525 \\ \text { Sum of electronic and zero-point Energies= } & -499.037424 \\ \text { Sum of electronic and thermal Energies= } & -499.026165 \\ \text { Sum of electronic and thermal Free Energies= } & -499.077392 \\ \text { SCF Done: E(RM062X/DGTZVP/SMD) } & -499.335004990 \\ & & & \end{array}$

\section{Cat a}

Center Atomic Atomic Coordinates (Angstroms) Number Number Type $X \quad$ Y $\quad Z$

$\begin{array}{cccccc}1 & 15 & 0 & -0.266770 & -0.212339 & 0.299723 \\ 2 & 8 & 0 & -0.205384 & -0.083627 & 1.908538 \\ 3 & 8 & 0 & 1.276424 & -0.130695 & -0.147704 \\ 4 & 8 & 0 & -0.715947 & 1.211554 & -0.243746 \\ 5 & 8 & 0 & -1.046064 & -1.383866 & -0.099364 \\ 6 & 6 & 0 & 0.408371 & 1.036608 & 2.461450 \\ 7 & 6 & 0 & 2.048358 & -1.224112 & 0.254419 \\ 8 & 6 & 0 & 1.781666 & 1.039539 & 2.652848 \\ 9 & 6 & 0 & -0.379547 & 2.143945 & 2.803408 \\ 10 & 6 & 0 & 2.656003 & -1.197117 & 1.500289 \\ 11 & 6 & 0 & 2.154724 & -2.333480 & -0.591821 \\ 12 & 6 & 0 & 2.803792 & -0.070968 & 2.503877 \\ 13 & 6 & 0 & 2.404543 & 2.210316 & 3.098257 \\ 14 & 6 & 0 & 0.264016 & 3.277777 & 3.311032 \\ 15 & 6 & 0 & 3.297667 & -2.347818 & 1.968791 \\ 16 & 6 & 0 & 2.861625 & -3.446473 & -0.122211 \\ 17 & 6 & 0 & 2.964324 & -0.888247 & 3.817322 \\ 18 & 6 & 0 & 4.087826 & 0.766411 & 2.240402\end{array}$

\begin{tabular}{|c|c|c|c|c|c|}
\hline 19 & 6 & 0 & 3.906690 & 2.030816 & 3.101398 \\
\hline 20 & 6 & 0 & 1.653280 & 3.329658 & 3.436390 \\
\hline 21 & 6 & 0 & 3.756340 & -2.141370 & 3.395960 \\
\hline 22 & 6 & 0 & 3.409108 & -3.473666 & 1.161402 \\
\hline 23 & 1 & 0 & 2.949880 & -4.311450 & -0.774069 \\
\hline 24 & 1 & 0 & 3.448304 & -0.310452 & 4.609948 \\
\hline 25 & 1 & 0 & 1.969287 & -1.184265 & 4.166655 \\
\hline 26 & 1 & 0 & 5.000053 & 0.207662 & 2.467485 \\
\hline 27 & 1 & 0 & 4.110430 & 1.044262 & 1.181183 \\
\hline 28 & 1 & 0 & 4.278058 & 1.878871 & 4.121166 \\
\hline 29 & 1 & 0 & 4.837201 & -1.965376 & 3.436349 \\
\hline 30 & 1 & 0 & 3.908136 & -4.368863 & 1.522232 \\
\hline 31 & 1 & 0 & 2.135750 & 4.237257 & 3.788477 \\
\hline 32 & 1 & 0 & 3.542489 & -3.007171 & 4.027472 \\
\hline 33 & 1 & 0 & 4.426954 & 2.899800 & 2.690985 \\
\hline 34 & 6 & 0 & 1.568892 & -2.325261 & -1.962687 \\
\hline 35 & 6 & 0 & 0.499732 & -3.183239 & -2.282229 \\
\hline 36 & 6 & 0 & 2.127497 & -1.480784 & -2.943562 \\
\hline 37 & 6 & 0 & -0.140522 & -4.012716 & -1.302599 \\
\hline 38 & 6 & 0 & -0.000854 & -3.216869 & -3.627595 \\
\hline 39 & 6 & 0 & 1.606485 & -1.509560 & -4.280870 \\
\hline 40 & 6 & 0 & 3.221080 & -0.594021 & -2.668839 \\
\hline 41 & 6 & 0 & -1.167584 & -4.841591 & -1.648575 \\
\hline 42 & 1 & 0 & 0.178099 & -3.946227 & -0.267940 \\
\hline 43 & 6 & 0 & -1.078276 & -4.103775 & -3.951602 \\
\hline 44 & 6 & 0 & 0.562093 & -2.381645 & -4.593498 \\
\hline 45 & 6 & 0 & 2.177284 & -0.649670 & -5.274255 \\
\hline 46 & 1 & 0 & 3.644290 & -0.568423 & -1.669522 \\
\hline 47 & 6 & 0 & 3.740441 & 0.210341 & -3.642664 \\
\hline 48 & 6 & 0 & -1.640130 & -4.898302 & -2.996058 \\
\hline 49 & 1 & 0 & -1.649653 & -5.448556 & -0.888573 \\
\hline 50 & 1 & 0 & -1.440775 & -4.124260 & -4.976115 \\
\hline 51 & 1 & 0 & 0.177341 & -2.408731 & -5.611303 \\
\hline 52 & 6 & 0 & 3.209825 & 0.187968 & -4.968431 \\
\hline 53 & 1 & 0 & 1.768477 & -0.682180 & -6.280972 \\
\hline 54 & 1 & 0 & 4.568313 & 0.873368 & -3.410579 \\
\hline 55 & 1 & 0 & -2.459440 & -5.564124 & -3.249660 \\
\hline 56 & 1 & 0 & 3.635042 & 0.836462 & -5.728189 \\
\hline 57 & 1 & 0 & -0.342276 & 4.137442 & 3.583871 \\
\hline 58 & 6 & 0 & -1.862566 & 2.106130 & 2.653239 \\
\hline 59 & 6 & 0 & -2.503882 & 2.948136 & 1.721817 \\
\hline 60 & 6 & 0 & -2.619716 & 1.234506 & 3.463430 \\
\hline 61 & 6 & 0 & -1.777315 & 3.821226 & 0.841521 \\
\hline 62 & 6 & 0 & -3.936431 & 2.918499 & 1.607814 \\
\hline 63 & 6 & 0 & -4.048712 & 1.202615 & 3.329981 \\
\hline 64 & 6 & 0 & -2.017704 & 0.373603 & 4.439748 \\
\hline 65 & 6 & 0 & -2.429868 & 4.617034 & -0.056846 \\
\hline 66 & 1 & 0 & -0.692879 & 3.821610 & 0.877030 \\
\hline 67 & 6 & 0 & -4.582296 & 3.775605 & 0.658133 \\
\hline 68 & 6 & 0 & -4.673698 & 2.045931 & 2.410303 \\
\hline 69 & 6 & 0 & -4.810794 & 0.307321 & 4.148474 \\
\hline 70 & 1 & 0 & -0.941028 & 0.396928 & 4.573424 \\
\hline 71 & 6 & 0 & -2.777042 & -0.464367 & 5.204238 \\
\hline 72 & 6 & 0 & -3.856464 & 4.603944 & -0.146714 \\
\hline 73 & 1 & 0 & -1.858683 & 5.260016 & -0.719360 \\
\hline 74 & 1 & 0 & -5.666611 & 3.745558 & 0.590347 \\
\hline 75 & 1 & 0 & -5.757436 & 2.021093 & 2.314612 \\
\hline 76 & 6 & 0 & -4.197058 & -0.505090 & 5.055500 \\
\hline 77 & 1 & 0 & -5.890882 & 0.291069 & 4.029031 \\
\hline 78 & 1 & 0 & -2.299915 & -1.108794 & 5.936058 \\
\hline 79 & 1 & 0 & -4.354743 & 5.246844 & -0.865578 \\
\hline 80 & 1 & 0 & -4.782529 & -1.181491 & 5.670278 \\
\hline 81 & 1 & 0 & -1.672627 & 1.274015 & -0.408686 \\
\hline
\end{tabular}

Frequencies -- $\quad 17.2797$

18.6374

26.9829 


\begin{tabular}{|c|c|c|c|}
\hline Frequencies -- & 31.1196 & 34.6001 & 45.8512 \\
\hline Frequencies -- & 55.4137 & 79.1476 & 84.6839 \\
\hline Frequencies -- & 91.1969 & 98.0679 & 105.4735 \\
\hline Frequencies -- & 122.2634 & 128.6238 & 138.8232 \\
\hline Frequencies -- & 140.0306 & 151.4566 & 176.2440 \\
\hline Frequencies -- & 176.8859 & 184.8938 & 197.3522 \\
\hline Frequencies -- & 206.0175 & 225.0428 & 230.3841 \\
\hline Frequencies -- & 234.4971 & 235.4947 & 249.8301 \\
\hline Frequencies -- & 271.5364 & 290.9841 & 292.4331 \\
\hline Frequencies -- & 295.8339 & 315.1145 & 329.0085 \\
\hline Frequencies -- & 340.8255 & 357.0824 & 363.7999 \\
\hline Frequencies -- & 368.0488 & 377.2281 & 390.4262 \\
\hline Frequencies -- & 401.5205 & 402.2687 & 414.9394 \\
\hline Frequencies -- & 419.4050 & 423.0265 & 425.7213 \\
\hline Frequencies -- & 433.4373 & 473.8577 & 482.8759 \\
\hline Frequencies -- & 487.8742 & 494.4587 & 497.5971 \\
\hline Frequencies -- & 497.7551 & 516.5549 & 524.8932 \\
\hline Frequencies -- & 539.8712 & 547.2890 & 554.7937 \\
\hline Frequencies -- & 559.3417 & 560.7609 & 569.2127 \\
\hline Frequencies -- & 571.1492 & 605.9515 & 608.4595 \\
\hline Frequencies -- & 615.6467 & 620.8618 & 623.2840 \\
\hline Frequencies -- & 639.8284 & 648.7340 & 649.4679 \\
\hline Frequencies -- & 650.3158 & 657.7776 & 662.8051 \\
\hline Frequencies -- & 675.2406 & 688.7008 & 705.6030 \\
\hline Frequencies -- & 707.3900 & 732.9997 & 748.4554 \\
\hline Frequencies -- & 753.9718 & 754.7425 & 756.4660 \\
\hline Frequencies -- & 757.5740 & 771.0109 & 778.6942 \\
\hline Frequencies -- & 795.2747 & 797.1568 & 808.8854 \\
\hline Frequencies -- & 809.5219 & 824.5988 & 833.2283 \\
\hline Frequencies -- & 842.6399 & 845.6957 & 847.0200 \\
\hline Frequencies -- & 866.1721 & 869.6048 & 870.7232 \\
\hline Frequencies -- & 870.8389 & 877.1128 & 880.1008 \\
\hline Frequencies -- & 881.0554 & 887.4461 & 902.8653 \\
\hline Frequencies -- & 921.5627 & 921.9452 & 939.3238 \\
\hline Frequencies -- & 939.8103 & 943.0637 & 947.6921 \\
\hline Frequencies -- & 955.4509 & 971.6029 & 972.7482 \\
\hline Frequencies -- & 982.8719 & 984.6692 & 989.8165 \\
\hline Frequencies -- & 991.0666 & 993.6883 & 994.4009 \\
\hline Frequencies -- & 1000.4959 & 1000.7867 & 1004.6221 \\
\hline Frequencies -- & 1005.7542 & 1014.4957 & 1020.8784 \\
\hline Frequencies -- & 1035.3713 & 1045.0489 & 1045.5161 \\
\hline Frequencies -- & 1047.3483 & 1047.5504 & 1049.1324 \\
\hline Frequencies -- & 1061.2444 & 1081.0801 & 1092.4476 \\
\hline Frequencies -- & 1103.2493 & 1139.1704 & 1140.3618 \\
\hline Frequencies -- & 1159.1298 & 1159.4924 & 1164.6924 \\
\hline Frequencies -- & 1168.6164 & 1178.3829 & 1186.4150 \\
\hline Frequencies -- & 1193.0399 & 1198.0830 & 1203.2723 \\
\hline Frequencies -- & 1206.5601 & 1207.1698 & 1207.5317 \\
\hline Frequencies -- & 1210.6692 & 1220.1341 & 1251.2436 \\
\hline Frequencies -- & 1252.9871 & 1253.4608 & 1257.2441 \\
\hline Frequencies -- & 1259.2153 & 1268.0727 & 1279.3609 \\
\hline Frequencies -- & 1295.3888 & 1296.3742 & 1304.4706 \\
\hline Frequencies -- & 1316.4070 & 1321.4690 & 1321.9581 \\
\hline Frequencies -- & 1324.9162 & 1344.1507 & 1347.9004 \\
\hline Frequencies -- & 1348.6212 & 1351.8509 & 1357.4634 \\
\hline Frequencies -- & 1362.3925 & 1371.2531 & 1409.6532 \\
\hline Frequencies -- & 1412.7543 & 1413.2735 & 1415.3051 \\
\hline Frequencies -- & 1442.7563 & 1443.1252 & 1482.6660 \\
\hline Frequencies -- & 1483.4934 & 1486.4844 & 1489.2073 \\
\hline Frequencies -- & 1489.9074 & 1493.0566 & 1498.2440 \\
\hline Frequencies -- & 1499.7011 & 1500.4743 & 1504.7434 \\
\hline Frequencies -- & 1512.5303 & 1518.0510 & 1529.4453 \\
\hline Frequencies -- & 1531.2780 & 1550.0117 & 1555.0333 \\
\hline Frequencies -- & 1602.1029 & 1604.2682 & 1648.9586 \\
\hline Frequencies -- & 1650.2780 & 1657.2535 & 1659.1745 \\
\hline Frequencies -- & 1661.9151 & 1665.4060 & 1706.2256 \\
\hline
\end{tabular}

$\begin{array}{llll}\text { Frequencies -- } 1711.6739 & 1713.0722 & 1714.4358 \\ \text { Frequencies -- } 1718.0701 & 1719.6582 & 3076.1116 \\ \text { Frequencies -- } 3077.4972 & 3084.0342 & 3091.6913 \\ \text { Frequencies -- } 3140.5574 & 3145.1610 & 3155.9108 \\ \text { Frequencies -- } 3160.2232 & 3193.5982 & 3200.0272 \\ \text { Frequencies -- } 3204.2979 & 3205.6318 & 3206.8308 \\ \text { Frequencies -- } 3209.7512 & 3209.8432 & 3210.7514 \\ \text { Frequencies -- } 3214.7164 & 3218.3407 & 3219.5089 \\ \text { Frequencies -- } 3223.0951 & 3223.5955 & 3227.3226 \\ \text { Frequencies -- } 3230.5413 & 3232.3320 & 3233.0831 \\ \text { Frequencies -- } 3234.4238 & 3236.1983 & 3241.8853 \\ \text { Frequencies -- } 3243.0049 & 3247.4516 & 3803.0299\end{array}$

SCF Done: $E(R M 062 X / D G D Z V P)=-2375.13878930$ Sum of electronic and zero-point Energies $=\quad-2374.494913$ Sum of electronic and thermal Energies $=\quad-2374.448765$ Sum of electronic and thermal Free Energies $=\quad-2374.576069$ SCF Done: $E(R M 062 X / D G T Z V P / S M D)=-2375.67807820$

\section{Cat a 10_1}

Center Atomic Atomic Coordinates (Angstroms) Number Number Type $\quad X \quad$ Y $\quad$ Z

\begin{tabular}{cccccc}
1 & 15 & 0 & -0.167938 & -0.020550 & 0.097086 \\
2 & 8 & 0 & -0.080758 & 0.012287 & 1.698012 \\
3 & 8 & 0 & 1.373657 & -0.036155 & -0.385163 \\
4 & 6 & 0 & 0.586379 & 1.087547 & 2.284045 \\
5 & 8 & 0 & -0.964151 & -1.178396 & -0.351347 \\
6 & 8 & 0 & -0.610085 & 1.404779 & -0.347686 \\
7 & 6 & 0 & 2.100202 & -1.172870 & -0.038591 \\
8 & 6 & 0 & 1.961823 & 1.025555 & 2.442435 \\
9 & 6 & 0 & -0.154142 & 2.210507 & 2.675248 \\
10 & 6 & 0 & 2.731330 & -1.208124 & 1.195791 \\
11 & 6 & 0 & 2.144671 & -2.258249 & -0.921822 \\
12 & 6 & 0 & 2.936568 & -0.119527 & 2.233900 \\
13 & 6 & 0 & 2.638180 & 2.155809 & 2.914081 \\
14 & 6 & 0 & 0.544389 & 3.301028 & 3.203114 \\
15 & 6 & 0 & -1.640558 & 2.215366 & 2.551185 \\
16 & 6 & 0 & 3.339412 & -2.394970 & 1.614818 \\
17 & 6 & 0 & 2.822862 & -3.409312 & -0.501455 \\
18 & 6 & 0 & 1.517501 & -2.198838 & -2.273957 \\
19 & 6 & 0 & 4.247528 & 0.677978 & 1.975483 \\
20 & 6 & 0 & 3.089529 & -0.987095 & 3.515864 \\
21 & 6 & 0 & 4.131588 & 1.916490 & 2.884098 \\
22 & 6 & 0 & 1.937671 & 3.292295 & 3.300819 \\
23 & 1 & 0 & -0.020961 & 4.175411 & 3.514226 \\
24 & 6 & 0 & -2.278250 & 3.066589 & 1.628310 \\
25 & 6 & 0 & -2.399010 & 1.343043 & 3.359034 \\
26 & 6 & 0 & 3.829785 & -2.251947 & 3.039141 \\
27 & 6 & 0 & 3.396471 & -3.496193 & 0.767894 \\
28 & 1 & 0 & 2.868236 & -4.257117 & -1.179723 \\
29 & 6 & 0 & 0.470987 & -3.083117 & -2.605168 \\
30 & 6 & 0 & 2.012174 & -1.289833 & -3.235476 \\
31 & 1 & 0 & 5.141991 & 0.077910 & 2.165470 \\
32 & 1 & 0 & 4.261947 & 0.992678 & 0.926433 \\
33 & 1 & 0 & 3.605832 & -0.453002 & 4.318553 \\
34 & 1 & 0 & 2.090435 & -1.259948 & 3.872530 \\
35 & 1 & 0 & 4.681272 & 2.778177 & 2.497253 \\
36 & 1 & 0 & 4.511973 & 1.710929 & 3.891181 \\
37 & 1 & 0 & 2.463416 & 4.168582 & 3.670044 \\
38 & 6 & 0 & -1.549557 & 3.945482 & 0.758041 \\
39 & 6 & 0 & -3.709279 & 3.036193 & 1.509464 \\
40 & 6 & 0 & -1.798647 & 0.472349 & 4.327906 \\
41 & 6 & 0 & -3.827974 & 1.316679 & 3.227610 \\
& & & & & \\
\hline & & & &
\end{tabular}




\begin{tabular}{|c|c|c|c|c|c|}
\hline 42 & 1 & 0 & 3.597341 & -3.130595 & 3.645755 \\
\hline 43 & 1 & 0 & 4.916631 & -2.114341 & 3.064684 \\
\hline 44 & 1 & 0 & 3.872602 & -4.418812 & 1.088230 \\
\hline 45 & 6 & 0 & -0.125860 & -3.967030 & -1.644058 \\
\hline 46 & 6 & 0 & -0.053063 & -3.094547 & -3.943364 \\
\hline 47 & 6 & 0 & 3.075687 & -0.369243 & -2.955503 \\
\hline 48 & 6 & 0 & 1.442115 & -1.273209 & -4.553906 \\
\hline 49 & 6 & 0 & -2.197115 & 4.741799 & -0.141853 \\
\hline 50 & 1 & 0 & -0.465431 & 3.944163 & 0.798426 \\
\hline 51 & 6 & 0 & -4.449578 & 2.161977 & 2.307082 \\
\hline 52 & 6 & 0 & -4.352034 & 3.889640 & 0.554014 \\
\hline 53 & 6 & 0 & -2.559351 & -0.366809 & 5.090229 \\
\hline 54 & 1 & 0 & -0.721335 & 0.490693 & 4.459595 \\
\hline 55 & 6 & 0 & -4.591643 & 0.420687 & 4.043813 \\
\hline 56 & 6 & 0 & -1.110989 & -4.838448 & -2.011135 \\
\hline 57 & 1 & 0 & 0.193123 & -3.912401 & -0.608435 \\
\hline 58 & 6 & 0 & 0.434214 & -2.183594 & -4.881701 \\
\hline 59 & 6 & 0 & -1.072439 & -4.038357 & -4.292195 \\
\hline 60 & 6 & 0 & 3.508464 & 0.519255 & -3.897975 \\
\hline 61 & 1 & 0 & 3.544069 & -0.382915 & -1.976701 \\
\hline 62 & 6 & 0 & 1.920534 & -0.323900 & -5.513906 \\
\hline 63 & 6 & 0 & -3.622955 & 4.719972 & -0.245436 \\
\hline 64 & 1 & 0 & -1.624164 & 5.388151 & -0.799556 \\
\hline 65 & 1 & 0 & -5.533082 & 2.136717 & 2.207168 \\
\hline 66 & 1 & 0 & -5.435896 & 3.855665 & 0.478584 \\
\hline 67 & 6 & 0 & -3.979899 & -0.399579 & 4.945497 \\
\hline 68 & 1 & 0 & -2.083118 & -1.016911 & 5.817723 \\
\hline 69 & 1 & 0 & -5.672215 & 0.410104 & 3.927196 \\
\hline 70 & 6 & 0 & -1.576060 & -4.895837 & -3.360772 \\
\hline 71 & 1 & 0 & -1.559297 & -5.487848 & -1.265491 \\
\hline 72 & 1 & 0 & 0.016012 & -2.179463 & -5.886871 \\
\hline 73 & 1 & 0 & -1.441962 & -4.043029 & -5.314625 \\
\hline 74 & 6 & 0 & 2.917936 & 0.551935 & -5.197580 \\
\hline 75 & 1 & 0 & 4.312448 & 1.208942 & -3.660205 \\
\hline 76 & 1 & 0 & 1.470078 & -0.319640 & -6.503385 \\
\hline 77 & 1 & 0 & -4.118150 & 5.360064 & -0.969147 \\
\hline 78 & 1 & 0 & -4.567293 & -1.075776 & 5.558749 \\
\hline 79 & 1 & 0 & -2.352570 & -5.605672 & -3.630021 \\
\hline 80 & 1 & 0 & 3.273443 & 1.268655 & -5.931341 \\
\hline 81 & 8 & 0 & -1.982811 & 1.159521 & -2.472679 \\
\hline 82 & 6 & 0 & -3.195119 & 0.414358 & -2.157598 \\
\hline 83 & 6 & 0 & -1.446066 & 0.048153 & -3.253850 \\
\hline 84 & 6 & 0 & -2.756273 & -0.765895 & -3.064704 \\
\hline 85 & 1 & 0 & -3.228294 & 0.148086 & -1.096271 \\
\hline 86 & 1 & 0 & -4.081950 & 0.977835 & -2.454009 \\
\hline 87 & 1 & 0 & -1.200981 & 0.374039 & -4.266963 \\
\hline 88 & 1 & 0 & -0.572789 & -0.402086 & -2.770315 \\
\hline 89 & 6 & 0 & -3.560131 & -0.932780 & -4.333190 \\
\hline 90 & 8 & 0 & -2.643124 & -1.999307 & -2.428487 \\
\hline 91 & 6 & 0 & -3.820404 & 0.174158 & -5.149493 \\
\hline 92 & 6 & 0 & -4.036932 & -2.188493 & -4.711413 \\
\hline 93 & 1 & 0 & -2.058467 & -1.898227 & -1.648796 \\
\hline 94 & 6 & 0 & -4.543900 & 0.027442 & -6.330163 \\
\hline 95 & 1 & 0 & -3.454061 & 1.159281 & -4.862543 \\
\hline 96 & 6 & 0 & -4.760134 & -2.334815 & -5.896612 \\
\hline 97 & 1 & 0 & -3.831868 & -3.038203 & -4.068604 \\
\hline 98 & 6 & 0 & -5.014706 & -1.231400 & -6.709043 \\
\hline 99 & 1 & 0 & -4.741024 & 0.893372 & -6.954936 \\
\hline 100 & 1 & 0 & -5.127892 & -3.315455 & -6.184461 \\
\hline 101 & 1 & 0 & -5.577457 & -1.347909 & -7.630257 \\
\hline 102 & 1 & 0 & -1.161411 & 1.430208 & -1.215094 \\
\hline
\end{tabular}

$\begin{array}{cccc}\text { Frequencies -- } & 9.5389 & 18.9454 & 21.2552 \\ \text { Frequencies -- } & 29.6134 & 34.5037 & 35.5763 \\ \text { Frequencies -- } & 39.7977 & 52.1634 & 58.2085\end{array}$

\begin{tabular}{|c|c|c|c|}
\hline Frequencies -- & 61.4754 & 70.9651 & 76.5223 \\
\hline Frequencies -- & 81.4427 & 85.1005 & 93.8216 \\
\hline Frequencies -- & 100.1702 & 104.5680 & 110.2234 \\
\hline Frequencies -- & 120.2570 & 127.3986 & 135.8356 \\
\hline Frequencies -- & 141.8057 & 148.3270 & 153.7387 \\
\hline Frequencies -- & 172.6106 & 180.4064 & 181.2176 \\
\hline Frequencies -- & 190.5951 & 198.2905 & 221.6034 \\
\hline Frequencies -- & 229.3779 & 232.1071 & 236.6821 \\
\hline Frequencies -- & 241.8748 & 252.5578 & 274.8007 \\
\hline Frequencies -- & 289.2293 & 291.7385 & 294.4843 \\
\hline Frequencies -- & 296.1676 & 318.3300 & 331.4455 \\
\hline Frequencies -- & 336.7802 & 338.7773 & 359.8981 \\
\hline Frequencies -- & 363.7300 & 367.5401 & 376.2329 \\
\hline Frequencies -- & 381.9502 & 389.5873 & 402.2467 \\
\hline Frequencies -- & 402.6394 & 414.9935 & 417.1529 \\
\hline Frequencies -- & 421.5063 & 423.6669 & 424.2948 \\
\hline Frequencies -- & 431.2422 & 437.2453 & 441.9471 \\
\hline Frequencies -- & 474.5468 & 484.7386 & 485.1725 \\
\hline Frequencies -- & 495.9337 & 497.4306 & 502.0881 \\
\hline Frequencies -- & 517.2713 & 526.4251 & 529.2856 \\
\hline Frequencies -- & 539.7820 & 547.4317 & 554.5175 \\
\hline Frequencies -- & 559.1704 & 560.8963 & 563.9671 \\
\hline Frequencies -- & 568.2392 & 570.1834 & 593.6620 \\
\hline Frequencies -- & 605.4558 & 607.9697 & 615.2002 \\
\hline Frequencies -- & 620.9389 & 623.1878 & 627.8079 \\
\hline Frequencies -- & 639.3576 & 648.4667 & 648.8709 \\
\hline Frequencies -- & 650.1274 & 657.5237 & 662.7647 \\
\hline Frequencies -- & 675.2206 & 688.5203 & 703.8424 \\
\hline Frequencies -- & 706.8561 & 707.0925 & 719.4828 \\
\hline Frequencies -- & 733.3411 & 749.1575 & 751.9993 \\
\hline Frequencies -- & 753.4905 & 754.3007 & 756.5143 \\
\hline Frequencies -- & 772.4252 & 775.0033 & 775.4142 \\
\hline Frequencies -- & 795.3903 & 796.3364 & 807.7172 \\
\hline Frequencies -- & 808.4147 & 819.6267 & 824.8808 \\
\hline Frequencies -- & 834.6350 & 841.3451 & 844.4166 \\
\hline Frequencies -- & 853.4819 & 865.7377 & 868.1303 \\
\hline Frequencies -- & 869.8578 & 872.3462 & 874.1104 \\
\hline Frequencies -- & 875.7196 & 879.3476 & 880.8969 \\
\hline Frequencies -- & 888.9133 & 909.7676 & 915.3319 \\
\hline Frequencies -- & 916.5358 & 920.0635 & 938.9004 \\
\hline Frequencies -- & 939.5823 & 942.7430 & 944.0511 \\
\hline Frequencies -- & 951.7471 & 952.9773 & 971.9648 \\
\hline Frequencies -- & 975.7025 & 977.4554 & 985.2695 \\
\hline Frequencies -- & 987.5664 & 987.6971 & 988.7604 \\
\hline Frequencies -- & 991.2567 & 993.8155 & 1000.4172 \\
\hline Frequencies -- & 1000.7511 & 1003.4033 & 1003.9397 \\
\hline Frequencies -- & 1003.9614 & 1007.0316 & 1009.8133 \\
\hline Frequencies -- & 1014.3147 & 1019.6283 & 1020.1366 \\
\hline Frequencies -- & 1037.1540 & 1042.6964 & 1045.9372 \\
\hline Frequencies -- & 1047.0557 & 1047.5151 & 1048.3831 \\
\hline Frequencies -- & 1060.6314 & 1063.0866 & 1067.4524 \\
\hline Frequencies -- & 1080.6115 & 1083.1498 & 1088.5012 \\
\hline Frequencies -- & 1093.5377 & 1103.7436 & 1113.1222 \\
\hline Frequencies -- & 1139.0993 & 1139.7367 & 1158.7979 \\
\hline Frequencies -- & 1159.0603 & 1165.5220 & 1170.0105 \\
\hline Frequencies -- & 1173.0025 & 1177.7132 & 1181.6085 \\
\hline Frequencies -- & 1185.5017 & 1192.4194 & 1198.7963 \\
\hline Frequencies -- & 1204.1521 & 1204.3418 & 1206.3371 \\
\hline Frequencies -- & 1207.3051 & 1208.3575 & 1208.4363 \\
\hline Frequencies -- & 1211.0745 & 1220.2705 & 1240.4438 \\
\hline Frequencies -- & 1251.1225 & 1253.0014 & 1254.0293 \\
\hline Frequencies -- & 1257.9041 & 1260.0264 & 1268.6192 \\
\hline Frequencies -- & 1279.2232 & 1284.8242 & 1295.1203 \\
\hline Frequencies -- & 1296.1337 & 1299.3776 & 1305.1617 \\
\hline Frequencies -- & 1314.4940 & 1316.7907 & 1320.7975 \\
\hline Frequencies -- & 1321.1714 & 1325.6535 & 1335.7305 \\
\hline
\end{tabular}




$\begin{array}{llll}\text { Frequencies -- } & 1344.6744 & 1344.8648 & 1347.9544 \\ \text { Frequencies -- } 1348.4927 & 1351.0762 & 1362.4273 \\ \text { Frequencies -- } 1365.9352 & 1370.4499 & 1391.2632 \\ \text { Frequencies -- } 1408.8217 & 1412.4243 & 1412.5822 \\ \text { Frequencies -- } 1413.9985 & 1440.1692 & 1443.9033 \\ \text { Frequencies -- } 1471.9635 & 1482.7263 & 1483.6486 \\ \text { Frequencies -- } 1485.6328 & 1488.8134 & 1489.9031 \\ \text { Frequencies -- } 1492.6766 & 1498.3690 & 1498.7160 \\ \text { Frequencies -- } 1500.3676 & 1500.6415 & 1504.4685 \\ \text { Frequencies -- } 1510.8894 & 1511.6042 & 1517.6507 \\ \text { Frequencies -- } 1529.7025 & 1531.1945 & 1532.4228 \\ \text { Frequencies -- } 1549.6504 & 1551.2513 & 1554.0514 \\ \text { Frequencies -- } 1601.5401 & 1603.2427 & 1646.2605 \\ \text { Frequencies -- } 1649.8555 & 1654.7495 & 1658.1547 \\ \text { Frequencies -- } 1660.9614 & 1664.9519 & 1676.3810 \\ \text { Frequencies -- } 1700.7930 & 1705.7929 & 1711.7151 \\ \text { Frequencies -- } 1712.8807 & 1714.6821 & 1718.1492 \\ \text { Frequencies -- } 1718.2602 & 2687.7483 & 3077.7720 \\ \text { Frequencies -- } 3079.6166 & 3085.4366 & 3091.4218 \\ \text { Frequencies -- } 3109.7294 & 3116.8666 & 3141.8112 \\ \text { Frequencies -- } 3148.0339 & 3158.0454 & 3161.2295 \\ \text { Frequencies -- } 3183.1854 & 3189.7243 & 3192.6622 \\ \text { Frequencies -- } 3194.8214 & 3197.7361 & 3201.5269 \\ \text { Frequencies -- } 3202.8802 & 3207.5707 & 3208.3729 \\ \text { Frequencies -- } 3208.5408 & 3209.6629 & 3209.8256 \\ \text { Frequencies -- } 3216.4462 & 3217.7717 & 3217.9127 \\ \text { Frequencies -- } 3221.8387 & 3222.0628 & 3224.1469 \\ \text { Frequencies -- } 3228.4532 & 3230.7213 & 3231.3116 \\ \text { Frequencies -- } 3232.1358 & 3233.7670 & 3234.5883 \\ \text { Frequencies -- } 3240.9200 & 3241.9417 & 3242.8146 \\ \text { Frequencies -- } 3245.3835 & 3250.3741 & 3649.4400\end{array}$

SCF Done: $E($ RM062X/DGDZVP $)=-2874.40395778$

Sum of electronic and zero-point Energies $=\quad-2873.583004$

Sum of electronic and thermal Energies $=\quad-2873.524884$

Sum of electronic and thermal Free Energies $=\quad-2873.678420$

SCF Done: $E(R M 062 X / D G T Z V P / S M D)=-2875.05444715$

\section{Cat a 10_2}

Center Atomic Atomic Coordinates (Angstroms)

\begin{tabular}{cccccc} 
Number & Number & Type & $X$ & $Y$ & $Z$ \\
\hline 1 & 15 & 0 & -0.250624 & -0.048558 & 0.273059 \\
2 & 8 & 0 & -0.046887 & -0.002601 & 1.862649 \\
3 & 8 & 0 & 1.245970 & 0.064787 & -0.324646 \\
4 & 8 & 0 & -0.845221 & 1.331828 & -0.131597 \\
5 & 8 & 0 & -0.976358 & -1.272712 & -0.114785 \\
6 & 6 & 0 & 0.574862 & 1.120140 & 2.407590 \\
7 & 6 & 0 & 2.091634 & -1.006401 & -0.046045 \\
8 & 6 & 0 & 1.959058 & 1.158257 & 2.475925 \\
9 & 6 & 0 & -0.217854 & 2.188666 & 2.846442 \\
10 & 6 & 0 & 2.812442 & -0.996766 & 1.139914 \\
11 & 6 & 0 & 2.163064 & -2.078571 & -0.943627 \\
12 & 6 & 0 & 3.001368 & 0.094252 & 2.179945 \\
13 & 6 & 0 & 2.580844 & 2.331000 & 2.918121 \\
14 & 6 & 0 & 0.433544 & 3.319884 & 3.349341 \\
15 & 6 & 0 & 3.551188 & -2.129585 & 1.495355 \\
16 & 6 & 0 & 2.973495 & -3.166066 & -0.593171 \\
17 & 6 & 0 & 3.312364 & -0.771907 & 3.433280 \\
18 & 6 & 0 & 4.224824 & 0.993957 & 1.842474 \\
19 & 6 & 0 & 4.082130 & 2.207696 & 2.779870 \\
20 & 6 & 0 & 1.826742 & 3.408558 & 3.367406 \\
21 & 1 & 0 & -0.172112 & 4.152179 & 3.697913 \\
22 & 6 & 0 & 4.124011 & -1.962220 & 2.885965
\end{tabular}

\begin{tabular}{|c|c|c|c|c|c|}
\hline 23 & 6 & 0 & 3.646522 & -3.212008 & 0.628008 \\
\hline 24 & 1 & 0 & 3.038596 & -4.002533 & -1.283719 \\
\hline 25 & 1 & 0 & 3.833819 & -0.206172 & 4.210574 \\
\hline 26 & 1 & 0 & 2.366028 & -1.133970 & 3.849106 \\
\hline 27 & 1 & 0 & 5.173829 & 0.462292 & 1.954392 \\
\hline 28 & 1 & 0 & 4.136980 & 1.322898 & 0.801386 \\
\hline 29 & 1 & 0 & 4.548571 & 2.020110 & 3.753518 \\
\hline 30 & 1 & 0 & 5.194371 & -1.731286 & 2.841799 \\
\hline 31 & 1 & 0 & -1.456287 & 1.315401 & -0.959328 \\
\hline 32 & 8 & 0 & -2.334424 & 0.991548 & -2.159568 \\
\hline 33 & 6 & 0 & -1.758644 & -0.077588 & -2.970855 \\
\hline 34 & 6 & 0 & -2.984208 & -0.993919 & -2.703047 \\
\hline 35 & 1 & 0 & -1.600962 & 0.261520 & -3.996892 \\
\hline 36 & 1 & 0 & -0.824043 & -0.450184 & -2.540271 \\
\hline 37 & 8 & 0 & -2.733750 & -2.207089 & -2.066496 \\
\hline 38 & 6 & 0 & -3.468128 & 0.157221 & -1.780537 \\
\hline 39 & 6 & 0 & -3.844458 & -1.239672 & -3.920803 \\
\hline 40 & 1 & 0 & -2.101911 & -2.055991 & -1.332385 \\
\hline 41 & 1 & 0 & -3.431571 & -0.099748 & -0.716961 \\
\hline 42 & 1 & 0 & -4.408870 & 0.647578 & -2.038902 \\
\hline 43 & 6 & 0 & -4.273359 & -2.528414 & -4.240768 \\
\hline 44 & 6 & 0 & -4.212330 & -0.170257 & -4.746316 \\
\hline 45 & 6 & 0 & -5.053486 & -2.744592 & -5.378175 \\
\hline 46 & 1 & 0 & -3.990755 & -3.350032 & -3.591251 \\
\hline 47 & 6 & 0 & -4.991423 & -0.386937 & -5.879740 \\
\hline 48 & 1 & 0 & -3.888401 & 0.841076 & -4.502836 \\
\hline 49 & 6 & 0 & -5.412573 & -1.678788 & -6.201460 \\
\hline 50 & 1 & 0 & -5.383566 & -3.750757 & -5.619832 \\
\hline 51 & 1 & 0 & -5.269731 & 0.450752 & -6.512026 \\
\hline 52 & 1 & 0 & -6.018604 & -1.850599 & -7.085846 \\
\hline 53 & 1 & 0 & 4.534884 & 3.114021 & 2.370432 \\
\hline 54 & 1 & 0 & 2.310222 & 4.316632 & 3.717044 \\
\hline 55 & 1 & 0 & 4.009732 & -2.865668 & 3.490082 \\
\hline 56 & 1 & 0 & 4.225696 & -4.090626 & 0.898143 \\
\hline 57 & 6 & 0 & -1.705726 & 2.117742 & 2.768713 \\
\hline 58 & 6 & 0 & -2.402578 & 1.194006 & 3.573033 \\
\hline 59 & 6 & 0 & -2.405418 & 2.957735 & 1.879450 \\
\hline 60 & 6 & 0 & -1.737762 & 0.328684 & 4.503729 \\
\hline 61 & 6 & 0 & -3.832055 & 1.105106 & 3.476155 \\
\hline 62 & 6 & 0 & -3.835654 & 2.864801 & 1.795186 \\
\hline 63 & 6 & 0 & -1.740219 & 3.879699 & 1.003468 \\
\hline 64 & 6 & 0 & -2.439641 & -0.562190 & 5.264009 \\
\hline 65 & 1 & 0 & -0.658823 & 0.392152 & 4.606126 \\
\hline 66 & 6 & 0 & -4.532699 & 0.156046 & 4.288362 \\
\hline 67 & 6 & 0 & -4.515020 & 1.941625 & 2.591841 \\
\hline 68 & 6 & 0 & -4.541288 & 3.705297 & 0.873143 \\
\hline 69 & 1 & 0 & -0.656480 & 3.924486 & 1.011530 \\
\hline 70 & 6 & 0 & -2.445650 & 4.660806 & 0.134071 \\
\hline 71 & 6 & 0 & -3.861048 & -0.656283 & 5.154088 \\
\hline 72 & 1 & 0 & -1.915238 & -1.207649 & 5.961905 \\
\hline 73 & 1 & 0 & -5.614325 & 0.098089 & 4.198064 \\
\hline 74 & 1 & 0 & -5.598602 & 1.869376 & 2.518658 \\
\hline 75 & 6 & 0 & -3.871337 & 4.579875 & 0.068963 \\
\hline 76 & 1 & 0 & -5.624278 & 3.624495 & 0.825841 \\
\hline 77 & 1 & 0 & -1.919177 & 5.339918 & -0.529508 \\
\hline 78 & 1 & 0 & -4.400721 & -1.374200 & 5.763806 \\
\hline 79 & 1 & 0 & -4.414036 & 5.208931 & -0.629889 \\
\hline 80 & 6 & 0 & 1.408933 & -2.090469 & -2.231276 \\
\hline 81 & 6 & 0 & 0.417672 & -3.070531 & -2.445666 \\
\hline 82 & 6 & 0 & 1.719815 & -1.160752 & -3.246665 \\
\hline 83 & 6 & 0 & -0.010950 & -3.971368 & -1.413697 \\
\hline 84 & 6 & 0 & -0.226382 & -3.163668 & -3.726684 \\
\hline 85 & 6 & 0 & 1.025063 & -1.225678 & -4.502626 \\
\hline 86 & 6 & 0 & 2.707265 & -0.134162 & -3.080267 \\
\hline 87 & 6 & 0 & -0.944407 & -4.934691 & -1.667860 \\
\hline 88 & 1 & 0 & 0.391278 & -3.854366 & -0.412988 \\
\hline
\end{tabular}




$\begin{array}{cccccc}89 & 6 & 0 & -1.182967 & -4.204311 & -3.960034 \\ 90 & 6 & 0 & 0.082597 & -2.234768 & -4.720353 \\ 91 & 6 & 0 & 1.307601 & -0.250606 & -5.512053 \\ 92 & 1 & 0 & 3.269331 & -0.084191 & -2.153198 \\ 93 & 6 & 0 & 2.950737 & 0.778497 & -4.067033 \\ 94 & 6 & 0 & -1.520780 & -5.074937 & -2.967832 \\ 95 & 1 & 0 & -1.266690 & -5.594675 & -0.868341 \\ 96 & 1 & 0 & -1.641031 & -4.272901 & -4.943635 \\ 97 & 1 & 0 & -0.429006 & -2.291920 & -5.679688 \\ 98 & 6 & 0 & 2.234315 & 0.728897 & -5.301478 \\ 99 & 1 & 0 & 0.762887 & -0.308147 & -6.451099 \\ 100 & 1 & 0 & 3.697724 & 1.551359 & -3.914813 \\ 101 & 1 & 0 & -2.251148 & -5.857928 & -3.148189 \\ 102 & 1 & 0 & 2.436638 & 1.467600 & -6.070811\end{array}$

\begin{tabular}{|c|c|c|c|}
\hline Frequencies -- & 15.2964 & 20.4916 & 25.5952 \\
\hline Frequencies -- & 31.2979 & 36.1362 & 40.5977 \\
\hline Frequencies -- & 43.4503 & 53.5314 & 59.8824 \\
\hline Frequencies -- & 63.0021 & 77.5221 & 81.6741 \\
\hline Frequencies -- & 87.2661 & 90.0945 & 97.4116 \\
\hline Frequencies -- & 100.6777 & 106.7585 & 109.1354 \\
\hline Frequencies -- & 123.6902 & 129.9232 & 140.2707 \\
\hline Frequencies -- & 143.0274 & 149.1809 & 155.3125 \\
\hline Frequencies -- & 171.7583 & 179.9138 & 184.1336 \\
\hline Frequencies -- & 191.6083 & 199.7200 & 225.7101 \\
\hline Frequencies -- & 230.7831 & 234.0027 & 239.5721 \\
\hline Frequencies -- & 244.2438 & 256.7259 & 275.1834 \\
\hline Frequencies -- & 287.0984 & 292.4542 & 294.4029 \\
\hline Frequencies -- & 297.4924 & 318.7974 & 331.8800 \\
\hline Frequencies -- & 336.3952 & 338.8227 & 361.1432 \\
\hline Frequencies -- & 366.1028 & 368.0646 & 376.7084 \\
\hline Frequencies -- & 384.4082 & 390.9592 & 402.7446 \\
\hline Frequencies -- & 403.0708 & 416.7906 & 419.5525 \\
\hline Frequencies -- & 422.8254 & 424.9041 & 425.8031 \\
\hline Frequencies -- & 432.9640 & 438.2402 & 442.6153 \\
\hline Frequencies -- & 476.2355 & 486.8542 & 488.5785 \\
\hline Frequencies -- & 497.4518 & 497.8164 & 503.7318 \\
\hline Frequencies -- & 517.5524 & 522.9141 & 529.2770 \\
\hline Frequencies -- & 539.6893 & 548.9251 & 556.3322 \\
\hline Frequencies -- & 560.2396 & 561.8442 & 564.7629 \\
\hline Frequencies -- & 568.9650 & 570.3269 & 605.7156 \\
\hline Frequencies -- & 608.1911 & 615.2870 & 621.1966 \\
\hline Frequencies -- & 624.1869 & 630.0048 & 639.7954 \\
\hline Frequencies -- & 649.3582 & 650.6696 & 650.9453 \\
\hline Frequencies -- & 658.7821 & 663.6966 & 675.6432 \\
\hline Frequencies -- & 688.8950 & 704.4134 & 706.6762 \\
\hline Frequencies -- & 707.4138 & 709.5794 & 723.3327 \\
\hline Frequencies -- & 733.3395 & 751.0587 & 753.4458 \\
\hline Frequencies -- & 754.6629 & 754.7702 & 757.1993 \\
\hline Frequencies -- & 773.9899 & 776.3050 & 777.8670 \\
\hline Frequencies -- & 794.6829 & 796.1422 & 807.6902 \\
\hline Frequencies -- & 809.5105 & 819.9379 & 825.7232 \\
\hline Frequencies -- & 836.6827 & 843.4688 & 846.6454 \\
\hline Frequencies -- & 854.6448 & 867.6034 & 867.7468 \\
\hline Frequencies -- & 872.7077 & 873.6517 & 875.6529 \\
\hline Frequencies -- & 881.0611 & 881.3192 & 885.4636 \\
\hline Frequencies -- & 889.4618 & 911.2060 & 915.1502 \\
\hline Frequencies -- & 917.4603 & 919.7669 & 940.4940 \\
\hline Frequencies -- & 941.0407 & 942.8776 & 947.4187 \\
\hline Frequencies -- & 952.9775 & 955.8399 & 972.4383 \\
\hline Frequencies -- & 974.8989 & 980.5476 & 985.2079 \\
\hline Frequencies -- & 986.5556 & 990.9603 & 992.8520 \\
\hline Frequencies -- & 994.4467 & 994.9010 & 1001.7251 \\
\hline Frequencies -- & 1002.6486 & 1004.5309 & 1007.5628 \\
\hline Frequencies -- & 1009.6847 & 1010.3933 & 1013.2028 \\
\hline
\end{tabular}

\begin{tabular}{|c|c|c|}
\hline Frequencies -- 1019.2844 & 1019.7945 & 1022.7574 \\
\hline Frequencies -- 1037.2922 & 1044.8872 & 1046.4610 \\
\hline Frequencies -- 1047.7159 & 1050.5242 & 1050.9160 \\
\hline Frequencies -- 1060.8764 & 1063.9229 & 1071.3167 \\
\hline Frequencies -- 1080.6779 & 1084.6679 & 1089.7662 \\
\hline Frequencies -- 1093.4623 & 1103.9351 & 1116.4516 \\
\hline Frequencies -- 1138.0548 & 1143.2314 & 1160.8785 \\
\hline Frequencies -- 1164.0108 & 1166.7115 & 1172.7782 \\
\hline Frequencies -- 1174.9263 & 1175.8182 & 1178.7306 \\
\hline Frequencies -- 1187.6091 & 1193.2668 & 1199.2483 \\
\hline Frequencies -- 1202.3067 & 1202.6178 & 1206.6107 \\
\hline Frequencies -- 1207.2083 & 1210.8134 & 1214.0622 \\
\hline Frequencies -- 1214.6847 & 1220.9282 & 1239.8541 \\
\hline Frequencies -- 1251.5560 & 1254.3277 & 1255.8884 \\
\hline Frequencies -- 1259.3113 & 1260.5403 & 1269.7218 \\
\hline Frequencies -- 1279.7527 & 1285.0497 & 1297.4802 \\
\hline Frequencies -- 1299.4251 & 1301.0446 & 1305.4922 \\
\hline Frequencies -- 1313.4548 & 1316.6794 & 1321.4120 \\
\hline Frequencies -- 1324.9962 & 1326.9590 & 1338.7936 \\
\hline Frequencies -- 1345.9114 & 1346.2126 & 1349.4834 \\
\hline Frequencies -- 1351.8946 & 1354.3222 & 1361.6800 \\
\hline Frequencies -- 1367.6821 & 1370.3094 & 1389.6139 \\
\hline Frequencies -- 1408.5975 & 1410.9857 & 1413.7558 \\
\hline Frequencies -- 1418.4360 & 1444.0625 & 1444.8785 \\
\hline Frequencies -- 1463.5771 & 1484.5273 & 1485.4335 \\
\hline Frequencies -- 1487.1152 & 1490.1317 & 1491.9576 \\
\hline Frequencies -- 1494.3253 & 1497.7805 & 1498.1502 \\
\hline Frequencies -- 1500.6006 & 1502.2865 & 1507.3061 \\
\hline Frequencies -- 1511.0354 & 1511.4172 & 1519.0978 \\
\hline Frequencies -- 1529.3446 & 1530.9573 & 1534.2249 \\
\hline Frequencies -- 1549.8474 & 1554.0763 & 1555.1601 \\
\hline Frequencies -- 1603.4648 & 1604.6358 & 1648.1402 \\
\hline Frequencies -- 1650.8824 & 1657.2248 & 1659.8511 \\
\hline Frequencies -- 1662.6430 & 1665.6474 & 1676.6225 \\
\hline Frequencies -- 1701.9965 & 1706.5972 & 1711.8048 \\
\hline Frequencies -- 1715.0207 & 1715.7857 & 1718.9536 \\
\hline Frequencies -- 1720.6685 & 2678.4506 & 3079.3725 \\
\hline Frequencies -- 3081.8296 & 3084.4409 & 3091.9911 \\
\hline Frequencies -- 3102.2676 & 3117.4605 & 3141.1073 \\
\hline Frequencies -- 3142.9388 & 3154.6252 & 3160.5531 \\
\hline Frequencies -- 3186.6064 & 3187.5528 & 3193.6743 \\
\hline Frequencies -- 3195.1369 & 3202.9146 & 3205.9538 \\
\hline Frequencies -- 3207.6973 & 3208.1340 & 3208.2010 \\
\hline Frequencies -- 3209.3406 & 3211.3992 & 3214.4655 \\
\hline Frequencies -- 3218.2737 & 3218.4871 & 3218.9327 \\
\hline Frequencies -- 3223.5851 & 3224.6399 & 3225.3565 \\
\hline Frequencies -- 3230.8543 & 3231.1489 & 3232.5647 \\
\hline Frequencies -- 3234.0879 & 3236.1989 & 3236.8766 \\
\hline Frequencies -- 3239.5189 & 3242.0796 & 3247.0661 \\
\hline Frequencies -- 3249.5843 & 3249.9395 & 3642.5239 \\
\hline
\end{tabular}

SCF Done: $E(R M 062 X / D G D Z V P)=-2874.40451469$

Sum of electronic and zero-point Energies $=\quad-2873.582442$ Sum of electronic and thermal Energies $=\quad-2873.524644$ Sum of electronic and thermal Free Energies $=\quad-2873.676204$ SCF Done: $E(R M 062 X / D G T Z V P / S M D)=-2875.05432386$

\section{Cat a TS1a_R}

\begin{tabular}{|c|c|c|c|c|c|}
\hline Center & Aton & & omic & Coordinate & s (Angstroms) \\
\hline Number & & ber & Type & $\begin{array}{ll}X & Y\end{array}$ & Z \\
\hline 1 & 15 & 0 & 0.028777 & -0.002382 & 0.012552 \\
\hline 2 & 8 & 0 & 0.008829 & 0.000246 & 1.633286 \\
\hline 3 & 8 & 0 & 1.634993 & 0.015650 & -0.306234 \\
\hline
\end{tabular}




\begin{tabular}{|c|c|c|c|c|c|}
\hline 4 & 8 & 0 & -0.442304 & 1.313309 & -0.544620 \\
\hline 5 & 8 & 0 & -0.613887 & -1.275633 & -0.432269 \\
\hline 6 & 6 & 0 & 0.738349 & 0.992150 & 2.272316 \\
\hline 7 & 6 & 0 & 2.284602 & -1.182081 & -0.045958 \\
\hline 8 & 6 & 0 & 2.061677 & 0.729596 & 2.615320 \\
\hline 9 & 6 & 0 & 0.150074 & 2.240113 & 2.532796 \\
\hline 10 & 6 & 0 & 2.732075 & -1.446389 & 1.235718 \\
\hline 11 & 6 & 0 & 2.401440 & -2.121049 & -1.077989 \\
\hline 12 & 6 & 0 & 2.900594 & -0.525432 & 2.427464 \\
\hline 13 & 6 & 0 & 2.822695 & 1.738024 & 3.215842 \\
\hline 14 & 6 & 0 & 0.925541 & 3.206726 & 3.186708 \\
\hline 15 & 6 & 0 & 3.177072 & -2.733402 & 1.549030 \\
\hline 16 & 6 & 0 & 2.929079 & -3.375922 & -0.759733 \\
\hline 17 & 6 & 0 & 2.775921 & -1.551171 & 3.586678 \\
\hline 18 & 6 & 0 & 4.330092 & 0.095901 & 2.419670 \\
\hline 19 & 6 & 0 & 4.249483 & 1.275954 & 3.401665 \\
\hline 20 & 6 & 0 & 2.257752 & 2.971130 & 3.522668 \\
\hline 21 & 1 & 0 & 0.472531 & 4.171987 & 3.396729 \\
\hline 22 & 6 & 0 & 3.443707 & -2.832436 & 3.037491 \\
\hline 23 & 6 & 0 & 3.282170 & -3.700954 & 0.554992 \\
\hline 24 & 1 & 0 & 3.030598 & -4.115482 & -1.549577 \\
\hline 25 & 1 & 0 & 3.228748 & -1.188658 & 4.514222 \\
\hline 26 & 1 & 0 & 1.713696 & -1.739992 & 3.772929 \\
\hline 27 & 1 & 0 & 5.101419 & -0.636953 & 2.672935 \\
\hline 28 & 1 & 0 & 4.539082 & 0.474360 & 1.411907 \\
\hline 29 & 1 & 0 & 4.416374 & 0.948023 & 4.434310 \\
\hline 30 & 1 & 0 & 4.520000 & -2.843241 & 3.242893 \\
\hline 31 & 1 & 0 & -1.554593 & -1.299235 & -1.711715 \\
\hline 32 & 8 & 0 & -2.193882 & -1.005093 & -2.444793 \\
\hline 33 & 6 & 0 & -3.122243 & -0.049346 & -1.871251 \\
\hline 34 & 6 & 0 & -2.736943 & 1.160192 & -2.740428 \\
\hline 35 & 1 & 0 & -4.144505 & -0.409855 & -1.989301 \\
\hline 36 & 1 & 0 & -2.892377 & 0.122315 & -0.815917 \\
\hline 37 & 8 & 0 & -2.506777 & 2.328792 & -2.022894 \\
\hline 38 & 6 & 0 & -1.477667 & 0.454079 & -3.327662 \\
\hline 39 & 6 & 0 & -3.727607 & 1.447465 & -3.852147 \\
\hline 40 & 1 & 0 & -1.899093 & 2.122561 & -1.280655 \\
\hline 41 & 1 & 0 & -0.523624 & 0.440929 & -2.811392 \\
\hline 42 & 1 & 0 & -1.504172 & -0.001179 & -4.310435 \\
\hline 43 & 6 & 0 & -4.144840 & 2.751739 & -4.113356 \\
\hline 44 & 6 & 0 & -4.206555 & 0.395478 & -4.640771 \\
\hline 45 & 6 & 0 & -5.036666 & 3.000459 & -5.157792 \\
\hline 46 & 1 & 0 & -3.756264 & 3.558343 & -3.500244 \\
\hline 47 & 6 & 0 & -5.094461 & 0.646669 & -5.684051 \\
\hline 48 & 1 & 0 & -3.881951 & -0.626345 & -4.441667 \\
\hline 49 & 6 & 0 & -5.512454 & 1.952784 & -5.944527 \\
\hline 50 & 1 & 0 & -5.355862 & 4.018395 & -5.360354 \\
\hline 51 & 1 & 0 & -5.460083 & -0.175138 & -6.292159 \\
\hline 52 & 1 & 0 & -6.204334 & 2.150804 & -6.757440 \\
\hline 53 & 1 & 0 & 4.312294 & 3.662280 & 0.647327 \\
\hline 54 & 6 & 0 & 3.831711 & 3.831927 & -0.311625 \\
\hline 55 & 6 & 0 & 2.717821 & 3.072330 & -0.651539 \\
\hline 56 & 6 & 0 & 4.341922 & 4.805819 & -1.184218 \\
\hline 57 & 6 & 0 & 2.117545 & 3.320774 & -1.888018 \\
\hline 58 & 1 & 0 & 2.327594 & 2.298177 & 0.006214 \\
\hline 59 & 6 & 0 & 3.740253 & 5.046638 & -2.417142 \\
\hline 60 & 7 & 0 & 1.008525 & 2.665715 & -2.411746 \\
\hline 61 & 6 & 0 & 2.616680 & 4.294048 & -2.759520 \\
\hline 62 & 1 & 0 & 4.133603 & 5.799823 & -3.092264 \\
\hline 63 & 6 & 0 & 0.611894 & 3.053606 & -3.633831 \\
\hline 64 & 1 & 0 & 0.437149 & 2.051150 & -1.793318 \\
\hline 65 & 16 & 0 & 1.643165 & 4.350837 & $7-4.214932$ \\
\hline 66 & 16 & 0 & -0.651262 & 2.452952 & $2-4.559847$ \\
\hline 67 & 1 & 0 & 5.215345 & 5.382171 & -0.897412 \\
\hline 68 & 1 & 0 & 4.978533 & 2.062027 & 3.187443 \\
\hline & 1 & 0 & 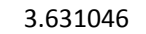 & $-4,702748$ & 0790 \\
\hline
\end{tabular}

\begin{tabular}{cccccc}
70 & 1 & 0 & 3.021699 & -3.742964 & 3.469912 \\
71 & 1 & 0 & 2.847643 & 3.750838 & 3.996915 \\
72 & 6 & 0 & 1.971644 & -1.729356 & -2.450671 \\
73 & 6 & 0 & 2.625602 & -0.651223 & -3.088694 \\
74 & 6 & 0 & 0.890890 & -2.375876 & -3.083238 \\
75 & 6 & 0 & 3.734158 & 0.040147 & -2.494993 \\
76 & 6 & 0 & 2.172098 & -0.205811 & -4.375007 \\
77 & 6 & 0 & 0.484429 & -1.959241 & -4.397674 \\
78 & 6 & 0 & 0.132764 & -3.413200 & -2.441921 \\
79 & 6 & 0 & 4.313364 & 1.111897 & -3.112362 \\
80 & 1 & 0 & 4.119481 & -0.296913 & -1.538110 \\
81 & 6 & 0 & 2.794707 & 0.929974 & -4.986463 \\
82 & 6 & 0 & 1.124127 & -0.878865 & -5.007699 \\
83 & 6 & 0 & -0.613192 & -2.624673 & -5.036775 \\
84 & 1 & 0 & 0.376905 & -3.685945 & -1.421000 \\
85 & 6 & 0 & -0.915768 & -4.012965 & -3.078319 \\
86 & 6 & 0 & 3.830306 & 1.574636 & -4.374254 \\
87 & 1 & 0 & 5.141819 & 1.628669 & -2.635884 \\
88 & 1 & 0 & 2.415559 & 1.272479 & -5.946324 \\
89 & 1 & 0 & 0.787382 & -0.537359 & -5.985088 \\
90 & 6 & 0 & -1.290358 & -3.623743 & -4.401769 \\
91 & 1 & 0 & -0.898889 & -2.306831 & -6.036855 \\
92 & 1 & 0 & -1.486028 & -4.782259 & -2.567104 \\
93 & 1 & 0 & 4.294964 & 2.437090 & -4.843587 \\
94 & 1 & 0 & -2.125085 & -4.116975 & -4.890154 \\
95 & 6 & 0 & -1.232654 & 2.571086 & 2.081836 \\
96 & 6 & 0 & -1.418275 & 3.580067 & 1.110582 \\
97 & 6 & 0 & -2.339012 & 1.868469 & 2.593959 \\
98 & 6 & 0 & -0.322670 & 4.251561 & 0.472070 \\
99 & 6 & 0 & -2.743297 & 3.899302 & 0.662364 \\
100 & 6 & 0 & -3.660024 & 2.183316 & 2.123758 \\
101 & 6 & 0 & -2.206349 & 0.834976 & 3.580870 \\
102 & 6 & 0 & -0.530440 & 5.170104 & -0.517687 \\
103 & 1 & 0 & 0.692632 & 4.002579 & 0.759681 \\
104 & 6 & 0 & -2.923916 & 4.892468 & -0.354835 \\
105 & 6 & 0 & -3.832705 & 3.193765 & 1.177195 \\
106 & 6 & 0 & -4.779163 & 1.446993 & 2.633413 \\
107 & 1 & 0 & -1.219649 & 0.594685 & 3.963629 \\
108 & 6 & 0 & -3.296521 & 0.157197 & 4.044155 \\
109 & 6 & 0 & -1.854320 & 5.509642 & -0.933090 \\
110 & 1 & 0 & 0.323619 & 5.641083 & -0.999907 \\
111 & 1 & 0 & -3.936490 & 5.112805 & -0.682570 \\
112 & 1 & 0 & -4.833525 & 3.422977 & 0.816456 \\
113 & 6 & 0 & -4.606700 & 0.461701 & 3.560290 \\
114 & 1 & 0 & -5.770704 & 1.693741 & 2.262504 \\
115 & 1 & 0 & -3.170984 & -0.622816 & 4.788653 \\
116 & 1 & 0 & -2.000323 & 6.240934 & -1.721897 \\
117 & 1 & 0 & -5.460850 & -0.091676 & 3.938360 \\
---------------------------------------------------------- \\
\hline
\end{tabular}

Frequencies -- -545.3428

Frequencies -- 22.3433

Frequencies -- 37.1673

Frequencies -- 49.4121

Frequencies -- 62.4213

Frequencies -- 85.3774

Frequencies -- 93.5688

Frequencies -- 108.2065

Frequencies -- 132.0524

Frequencies -- 149.4075

Frequencies -- 173.0974

Frequencies -- 190.4021

Frequencies -- 205.8072

Frequencies -- 232.1643

Frequencies -- 248.0895

Frequencies -- 279.9255
13.0686

30.7183

40.6320

53.2587

67.5637

88.7007

99.3714

114.7835

139.0702

154.0602

183.0577

196.7584

215.4573

236.4757

252.6617

287.0010
19.3787

31.5672

43.2414

57.3500

77.4505

92.1538

105.2506

124.3812

143.0026

163.0739

184.6848

202.3947

225.3004

242.4943

274.8286

294.4315 


\begin{tabular}{|c|c|c|c|}
\hline Frequencies -- & 299.0326 & 303.1460 & 307.0105 \\
\hline Frequencies -- & 321.6500 & 333.0835 & 345.1780 \\
\hline Frequencies -- & 354.0148 & 364.6750 & 376.6255 \\
\hline Frequencies -- & 377.6432 & 389.4284 & 393.0495 \\
\hline Frequencies -- & 396.7759 & 400.8930 & 403.8120 \\
\hline Frequencies -- & 404.4392 & 413.3813 & 417.7550 \\
\hline Frequencies -- & 419.0297 & 423.8157 & 428.3316 \\
\hline Frequencies -- & 435.6665 & 439.3429 & 441.0052 \\
\hline Frequencies -- & 449.7799 & 455.3183 & 482.7301 \\
\hline Frequencies -- & 485.2823 & 489.6858 & 497.9085 \\
\hline Frequencies -- & 499.2553 & 508.3466 & 509.2716 \\
\hline Frequencies -- & 523.5575 & 529.4015 & 530.5267 \\
\hline Frequencies -- & 530.9840 & 540.4115 & 550.7007 \\
\hline Frequencies -- & 556.9598 & 562.4034 & 566.7613 \\
\hline Frequencies -- & 567.6344 & 573.5979 & 580.4616 \\
\hline Frequencies -- & 583.2074 & 604.4310 & 608.3179 \\
\hline Frequencies -- & 615.9578 & 617.8662 & 621.6110 \\
\hline Frequencies -- & 624.9017 & 630.1379 & 644.5607 \\
\hline Frequencies -- & 648.9939 & 651.2365 & 652.2610 \\
\hline Frequencies -- & 658.4692 & 660.1275 & 662.5609 \\
\hline Frequencies -- & 676.4937 & 677.3892 & 690.2776 \\
\hline Frequencies -- & 702.4137 & 709.5928 & 710.3515 \\
\hline Frequencies -- & 719.4178 & 726.1771 & 732.9877 \\
\hline Frequencies -- & 734.8042 & 746.6765 & 748.1032 \\
\hline Frequencies -- & 753.5368 & 755.0932 & 755.4532 \\
\hline Frequencies -- & 756.1865 & 768.7037 & 776.8972 \\
\hline Frequencies -- & 777.9541 & 779.0570 & 795.6426 \\
\hline Frequencies -- & 799.8287 & 809.0886 & 812.0090 \\
\hline Frequencies -- & 825.3245 & 837.6450 & 841.8442 \\
\hline Frequencies -- & 844.9400 & 847.9493 & 855.5326 \\
\hline Frequencies -- & 863.5191 & 867.1516 & 869.0197 \\
\hline Frequencies -- & 872.1844 & 873.5473 & 877.7945 \\
\hline Frequencies -- & 880.1503 & 880.7080 & 882.2957 \\
\hline Frequencies -- & 884.2520 & 888.9004 & 889.8441 \\
\hline Frequencies -- & 900.9755 & 916.1362 & 920.6941 \\
\hline Frequencies -- & 935.6982 & 939.8078 & 940.2265 \\
\hline Frequencies -- & 943.3663 & 950.8053 & 951.1129 \\
\hline Frequencies -- & 964.6730 & 970.5873 & 978.7092 \\
\hline Frequencies -- & 981.1565 & 981.7766 & 988.1825 \\
\hline Frequencies -- & 988.7714 & 990.4595 & 995.5259 \\
\hline Frequencies -- & 1000.5727 & 1001.0653 & 1002.1596 \\
\hline Frequencies -- & 1003.1481 & 1004.8136 & 1012.3330 \\
\hline Frequencies -- & 1013.0557 & 1015.9707 & 1019.3578 \\
\hline Frequencies -- & 1019.9464 & 1029.9354 & 1033.7567 \\
\hline Frequencies -- & 1045.7737 & 1047.8860 & 1049.1924 \\
\hline Frequencies -- & 1051.1236 & 1053.5605 & 1054.3243 \\
\hline Frequencies -- & 1055.6990 & 1064.1933 & 1067.0998 \\
\hline Frequencies -- & 1074.6658 & 1077.5781 & 1090.8993 \\
\hline Frequencies -- & 1100.7796 & 1103.6085 & 1111.2619 \\
\hline Frequencies -- & 1114.7965 & 1115.6274 & 1135.4064 \\
\hline Frequencies -- & 1140.3623 & 1144.2135 & 1159.2384 \\
\hline Frequencies -- & 1160.7660 & 1167.1528 & 1168.9791 \\
\hline Frequencies -- & 1170.2351 & 1179.3103 & 1180.4885 \\
\hline Frequencies -- & 1184.8604 & 1187.9393 & 1194.4837 \\
\hline Frequencies -- & 1196.1337 & 1200.4414 & 1201.2596 \\
\hline Frequencies -- & 1203.6151 & 1206.8915 & 1208.6642 \\
\hline Frequencies -- & 1208.8211 & 1212.8062 & 1218.9804 \\
\hline Frequencies -- & 1222.6208 & 1236.7855 & 1250.2405 \\
\hline Frequencies -- & 1253.9274 & 1257.2375 & 1259.5395 \\
\hline Frequencies -- & 1261.5924 & 1271.9142 & 1277.5507 \\
\hline Frequencies -- & 1281.5678 & 1287.4830 & 1297.6008 \\
\hline Frequencies -- & 1298.7629 & 1303.5074 & 1304.1590 \\
\hline Frequencies -- & 1313.3890 & 1315.7092 & 1321.4201 \\
\hline Frequencies -- & 1323.4789 & 1326.4641 & 1341.4811 \\
\hline Frequencies -- & 1344.1455 & 1347.6028 & 1350.7033 \\
\hline Frequencies -- & 1351.6129 & 1355.5794 & 1362.1149 \\
\hline
\end{tabular}

Frequencies -- 1366.0151

Frequencies -- 1388.2682

Frequencies -- 1414.8185

Frequencies -- 1437.0111

Frequencies -- 1466.0552

Frequencies -- 1482.8106

Frequencies -- 1491.9993

Frequencies -- 1499.4670

Frequencies -- 1505.4537

Frequencies -- 1520.9583

Frequencies -- 1532.2877

Frequencies -- 1552.3907

Frequencies -- 1604.0196

Frequencies -- 1655.5841

Frequencies -- 1665.4848

Frequencies -- 1711.8217

Frequencies -- 1717.4116

Frequencies -- 3074.6326

Frequencies -- 3086.9863

Frequencies -- 3137.5245

Frequencies -- 3155.6628

Frequencies -- 3196.0625

Frequencies -- 3203.2224

Frequencies -- 3208.5082

Frequencies -- 3212.9356

Frequencies -- 3222.2254

Frequencies -- 3225.1952

Frequencies -- 3228.9450

Frequencies -- 3233.8935

Frequencies -- 3240.7932

Frequencies -- 3260.3955
Frequencies -- 1684.5327

Frequencies -- 3245.6764

$\begin{array}{ll}1369.1738 & 1370.5200 \\ 1408.6437 & 1412.9957 \\ 1418.8431 & 1421.2150 \\ 1440.9366 & 1447.1294 \\ 1480.9077 & 1482.1855 \\ 1484.8704 & 1490.7202 \\ 1494.5806 & 1497.0991 \\ 1500.5827 & 1501.2609 \\ 1511.0508 & 1520.1419 \\ 1525.0569 & 1528.1889 \\ 1547.9206 & 1551.1493 \\ 1557.8522 & 1601.9084 \\ 1646.6572 & 1648.8410 \\ 1658.0177 & 1661.3248 \\ 1672.2490 & 1679.5298 \\ 1697.0425 & 1700.9002 \\ 1713.6938 & 1714.2033 \\ 1717.5309 & 2928.7796 \\ 3076.9232 & 3080.2647 \\ 3096.6966 & 3121.5395 \\ 3141.9943 & 3150.3414 \\ 3181.6567 & 3195.6915 \\ 3200.2142 & 3200.9995 \\ 3205.5771 & 3208.1651 \\ 3209.2096 & 3211.0684 \\ 3213.0925 & 3219.1271 \\ 3222.3658 & 3222.8045 \\ 3226.4533 & 3227.4526 \\ 3230.3753 & 3230.6845 \\ 3236.5428 & 3239.6049 \\ 3241.5884 & 3244.6900 \\ 3254.4246 & 3256.4000 \\ 3351.6554 & 3632.3211\end{array}$

SCF Done: $E(R M 062 X / D G D Z V P)=-3995.08586802$

Sum of electronic and zero-point Energies $=\quad-3994.157130$ Sum of electronic and thermal Energies $=\quad-3994.088648$

Sum of electronic and thermal Free Energies $=\quad-3994.262406$ SCF Done: $E(R M 062 X / D G T Z V P / S M D)=-3995.88703288$

\section{Cat a I1a_R}

Center Atomic Atomic Coordinates (Angstroms) Number Number Type $\quad X \quad Y \quad$ Z

$\begin{array}{cccccc}1 & 15 & 0 & -0.017129 & -0.010930 & 0.008492 \\ 2 & 8 & 0 & -0.000580 & -0.000692 & 1.631943 \\ 3 & 8 & 0 & 1.590770 & -0.004269 & -0.334890 \\ 4 & 8 & 0 & -0.442739 & 1.351203 & -0.506582 \\ 5 & 8 & 0 & -0.693870 & -1.245899 & -0.447141 \\ 6 & 6 & 0 & 0.755487 & 0.965741 & 2.273478 \\ 7 & 6 & 0 & 2.221635 & -1.215427 & -0.095792 \\ 8 & 6 & 0 & 2.079039 & 0.674574 & 2.593766 \\ 9 & 6 & 0 & 0.192935 & 2.218037 & 2.574116 \\ 10 & 6 & 0 & 2.690683 & -1.497166 & 1.174974 \\ 11 & 6 & 0 & 2.293362 & -2.152843 & -1.132963 \\ 12 & 6 & 0 & 2.894768 & -0.590511 & 2.372253 \\ 13 & 6 & 0 & 2.864303 & 1.654532 & 3.210500 \\ 14 & 6 & 0 & 0.988815 & 3.150161 & 3.252502 \\ 15 & 6 & 0 & 3.117997 & -2.794629 & 1.470376 \\ 16 & 6 & 0 & 2.802314 & -3.420022 & -0.833959 \\ 17 & 6 & 0 & 2.773586 & -1.625316 & 3.524028 \\ 18 & 6 & 0 & 4.332618 & 0.009924 & 2.349813 \\ 19 & 6 & 0 & 4.287122 & 1.166988 & 3.361073 \\ 20 & 6 & 0 & 2.321626 & 2.884707 & 3.565026\end{array}$




\begin{tabular}{|c|c|c|c|c|c|}
\hline 21 & 1 & 0 & 0.552510 & 4.114158 & 3.500918 \\
\hline 22 & 6 & 0 & 3.412711 & -2.910631 & 2.952089 \\
\hline 23 & 6 & 0 & 3.181383 & -3.758637 & 0.469671 \\
\hline 24 & 1 & 0 & 2.866322 & -4.157833 & -1.629388 \\
\hline 25 & 1 & 0 & 3.246394 & -1.278548 & 4.447635 \\
\hline 26 & 1 & 0 & 1.711674 & -1.799929 & 3.725028 \\
\hline 27 & 1 & 0 & 5.098450 & -0.739263 & 2.569504 \\
\hline 28 & 1 & 0 & 4.526042 & 0.409684 & 1.347071 \\
\hline 29 & 1 & 0 & 4.466776 & 0.811248 & 4.382250 \\
\hline 30 & 1 & 0 & 4.492638 & -2.937652 & 3.136225 \\
\hline 31 & 1 & 0 & -2.062566 & -1.517614 & -1.747999 \\
\hline 32 & 8 & 0 & -2.839552 & -1.227074 & -2.267961 \\
\hline 33 & 6 & 0 & -3.192327 & 0.054545 & -1.787912 \\
\hline 34 & 6 & 0 & -2.759185 & 1.199356 & -2.748643 \\
\hline 35 & 1 & 0 & -4.279054 & 0.102488 & -1.667310 \\
\hline 36 & 1 & 0 & -2.737859 & 0.238341 & -0.809369 \\
\hline 37 & 8 & 0 & -2.561214 & 2.392225 & -2.019957 \\
\hline 38 & 6 & 0 & -1.467118 & 0.780664 & -3.486579 \\
\hline 39 & 6 & 0 & -3.831071 & 1.462642 & -3.795759 \\
\hline 40 & 1 & 0 & -2.008895 & 2.178307 & -1.240512 \\
\hline 41 & 1 & 0 & -0.670653 & 0.431993 & -2.821792 \\
\hline 42 & 1 & 0 & -1.670358 & -0.029208 & -4.189970 \\
\hline 43 & 6 & 0 & -4.286876 & 2.755451 & -4.055588 \\
\hline 44 & 6 & 0 & -4.353635 & 0.387341 & -4.525891 \\
\hline 45 & 6 & 0 & -5.254046 & 2.972566 & -5.039794 \\
\hline 46 & 1 & 0 & -3.879566 & 3.580670 & -3.480202 \\
\hline 47 & 6 & 0 & -5.318799 & 0.607393 & -5.505946 \\
\hline 48 & 1 & 0 & -4.011400 & -0.623836 & -4.308636 \\
\hline 49 & 6 & 0 & -5.770518 & 1.902562 & -5.768025 \\
\hline 50 & 1 & 0 & -5.604742 & 3.981578 & -5.236342 \\
\hline 51 & 1 & 0 & -5.723853 & -0.233230 & -6.061602 \\
\hline 52 & 1 & 0 & -6.523624 & 2.073086 & -6.531427 \\
\hline 53 & 1 & 0 & 4.203627 & 3.709336 & 0.504258 \\
\hline 54 & 6 & 0 & 3.680262 & 3.833867 & -0.438852 \\
\hline 55 & 6 & 0 & 2.583133 & 3.028657 & -0.711467 \\
\hline 56 & 6 & 0 & 4.122579 & 4.800167 & -1.359205 \\
\hline 57 & 6 & 0 & 1.931922 & 3.222175 & -1.932159 \\
\hline 58 & 1 & 0 & 2.240633 & 2.262738 & -0.019066 \\
\hline 59 & 6 & 0 & 3.472437 & 4.987143 & -2.574855 \\
\hline 60 & 7 & 0 & 0.822568 & 2.511282 & -2.385624 \\
\hline 61 & 6 & 0 & 2.364894 & 4.182399 & -2.849328 \\
\hline 62 & 1 & 0 & 3.816644 & 5.732498 & -3.284323 \\
\hline 63 & 6 & 0 & 0.406130 & 2.854759 & -3.587945 \\
\hline 64 & 1 & 0 & 0.265795 & 1.905752 & -1.656549 \\
\hline 65 & 16 & 0 & 1.338429 & 4.160152 & -4.266609 \\
\hline 66 & 16 & 0 & -0.843630 & 2.149216 & -4.530337 \\
\hline 67 & 1 & 0 & 4.985911 & 5.412485 & -1.120479 \\
\hline 68 & 1 & 0 & 5.024038 & 1.947502 & 3.153462 \\
\hline 69 & 1 & 0 & 3.514622 & -4.768623 & 0.692116 \\
\hline 70 & 1 & 0 & 2.986676 & -3.818974 & 3.385092 \\
\hline 71 & 1 & 0 & 2.928073 & 3.639060 & 4.059132 \\
\hline 72 & 6 & 0 & 1.835305 & -1.753716 & -2.493830 \\
\hline 73 & 6 & 0 & 2.491902 & -0.689195 & -3.151330 \\
\hline 74 & 6 & 0 & 0.727724 & -2.382258 & -3.096129 \\
\hline 75 & 6 & 0 & 3.612128 & 0.000411 & -2.577753 \\
\hline 76 & 6 & 0 & 2.028933 & -0.255390 & -4.438075 \\
\hline 77 & 6 & 0 & 0.305908 & -1.973929 & -4.407782 \\
\hline 78 & 6 & 0 & -0.045866 & -3.386654 & -2.422380 \\
\hline 79 & 6 & 0 & 4.191365 & 1.064219 & -3.209166 \\
\hline 80 & 1 & 0 & 4.006566 & -0.331263 & -1.622781 \\
\hline 81 & 6 & 0 & 2.658526 & 0.865693 & -5.069646 \\
\hline 82 & 6 & 0 & 0.963021 & -0.921906 & -5.047615 \\
\hline 83 & 6 & 0 & -0.827346 & -2.611513 & -5.011263 \\
\hline 84 & 1 & 0 & 0.210725 & -3.649558 & -1.402211 \\
\hline 85 & 6 & 0 & -1.130392 & -3.957338 & -3.023450 \\
\hline 86 & 6 & 0 & 3.701085 & 1.514733 & -4.472538 \\
\hline
\end{tabular}

$\begin{array}{cccccc}87 & 1 & 0 & 5.028913 & 1.578571 & -2.746328 \\ 88 & 1 & 0 & 2.283715 & 1.190789 & -6.037783 \\ 89 & 1 & 0 & 0.620237 & -0.594087 & -6.027783 \\ 90 & 6 & 0 & -1.524887 & -3.573029 & -4.342418 \\ 91 & 1 & 0 & -1.130037 & -2.294922 & -6.006622 \\ 92 & 1 & 0 & -1.720563 & -4.691235 & -2.483988 \\ 93 & 1 & 0 & 4.170993 & 2.365194 & -4.958889 \\ 94 & 1 & 0 & -2.396238 & -4.034400 & -4.796256 \\ 95 & 6 & 0 & -1.179600 & 2.579639 & 2.118430 \\ 96 & 6 & 0 & -1.341005 & 3.615394 & 1.170870 \\ 97 & 6 & 0 & -2.295613 & 1.849081 & 2.566270 \\ 98 & 6 & 0 & -0.234502 & 4.332613 & 0.605413 \\ 99 & 6 & 0 & -2.649728 & 3.913842 & 0.664074 \\ 100 & 6 & 0 & -3.597566 & 2.140344 & 2.033347 \\ 101 & 6 & 0 & -2.187772 & 0.799892 & 3.539188 \\ 102 & 6 & 0 & -0.417571 & 5.275455 & -0.367130 \\ 103 & 1 & 0 & 0.771651 & 4.109122 & 0.941226 \\ 104 & 6 & 0 & -2.805875 & 4.924136 & -0.339786 \\ 105 & 6 & 0 & -3.745497 & 3.164322 & 1.098151 \\ 106 & 6 & 0 & -4.721128 & 1.360072 & 2.461162 \\ 107 & 1 & 0 & -1.216690 & 0.579681 & 3.971039 \\ 108 & 6 & 0 & -3.281873 & 0.083965 & 3.928318 \\ 109 & 6 & 0 & -1.726718 & 5.588035 & -0.844697 \\ 110 & 1 & 0 & 0.445645 & 5.789563 & -0.784493 \\ 111 & 1 & 0 & -3.805503 & 5.119856 & -0.718420 \\ 112 & 1 & 0 & -4.728224 & 3.366912 & 0.677174 \\ 113 & 6 & 0 & -4.571070 & 0.359684 & 3.375223 \\ 114 & 1 & 0 & -5.696181 & 1.583158 & 2.035902 \\ 115 & 1 & 0 & -3.175312 & -0.707769 & 4.663338 \\ 116 & 1 & 0 & -1.854570 & 6.336619 & -1.620477 \\ 117 & 1 & 0 & -5.427087 & -0.229640 & 3.688835\end{array}$

$\begin{array}{cccc}\text { Frequencies -- } & 11.6326 & 15.5303 & 22.9722 \\ \text { Frequencies -- } & 27.3323 & 32.4390 & 36.6823 \\ \text { Frequencies -- } & 37.8316 & 45.9283 & 47.4365 \\ \text { Frequencies -- } & 49.0452 & 54.5346 & 58.4733 \\ \text { Frequencies -- } & 62.7541 & 71.5539 & 84.0371 \\ \text { Frequencies -- } & 87.7365 & 94.4851 & 96.9311 \\ \text { Frequencies -- } & 99.6006 & 106.9127 & 110.3230 \\ \text { Frequencies -- } & 115.9825 & 121.4741 & 127.8502 \\ \text { Frequencies -- } & 139.6278 & 142.5808 & 148.1334 \\ \text { Frequencies -- } & 156.3592 & 172.3025 & 176.1057 \\ \text { Frequencies -- } & 182.5215 & 187.2258 & 188.7017 \\ \text { Frequencies -- } & 192.4408 & 201.5207 & 206.5749 \\ \text { Frequencies -- } & 215.7504 & 220.2057 & 234.9888 \\ \text { Frequencies -- } & 235.7463 & 240.7418 & 242.8057 \\ \text { Frequencies -- } & 253.1689 & 256.7226 & 279.8168 \\ \text { Frequencies -- } & 283.6892 & 294.3630 & 297.2574 \\ \text { Frequencies -- } & 299.7804 & 306.4398 & 320.7110 \\ \text { Frequencies -- } & 330.7708 & 339.6517 & 349.4056 \\ \text { Frequencies -- } & 364.5699 & 369.7942 & 373.7247 \\ \text { Frequencies -- } & 377.4044 & 385.9135 & 387.3915 \\ \text { Frequencies -- } & 399.0295 & 403.3189 & 403.7019 \\ \text { Frequencies -- } & 406.9531 & 409.4293 & 415.2423 \\ \text { Frequencies -- } & 419.2106 & 424.2279 & 429.7880 \\ \text { Frequencies -- } & 435.5265 & 435.8522 & 444.2038 \\ \text { Frequencies -- } & 455.8077 & 485.1638 & 487.5737 \\ \text { Frequencies -- } & 490.1609 & 497.0432 & 497.4898 \\ \text { Frequencies -- } & 506.3313 & 510.1930 & 521.3213 \\ \text { Frequencies -- } & 528.8636 & 529.9758 & 533.2380 \\ \text { Frequencies -- } & 539.4733 & 550.8410 & 556.9829 \\ \text { Frequencies -- } & 558.9217 & 566.6834 & 567.4018 \\ \text { Frequencies -- } & 573.3226 & 580.4066 & 586.2013 \\ \text { Frequencies -- } & 595.4291 & 603.7923 & 606.7574 \\ \text { Frequencies -- } & 608.7547 & 615.4941 & 620.4353\end{array}$




\begin{tabular}{|c|c|c|c|}
\hline Frequencies -- & 625.2857 & 629.7307 & 630.5485 \\
\hline Frequencies -- & 643.6940 & 648.7236 & 651.0569 \\
\hline Frequencies -- & 652.4461 & 655.2185 & 658.3237 \\
\hline Frequencies -- & 661.9406 & 672.1969 & 681.5430 \\
\hline Frequencies -- & 690.2958 & 710.0485 & 710.5736 \\
\hline Frequencies -- & 719.7548 & 724.3549 & 730.3102 \\
\hline Frequencies -- & 731.3244 & 733.7902 & 749.8353 \\
\hline Frequencies -- & 751.6088 & 754.5775 & 754.8011 \\
\hline Frequencies -- & 756.1501 & 762.7385 & 770.2861 \\
\hline Frequencies -- & 774.1965 & 779.8739 & 788.9567 \\
\hline Frequencies -- & 796.1951 & 800.2051 & 810.1642 \\
\hline Frequencies -- & 813.1307 & 824.7597 & 836.1865 \\
\hline Frequencies -- & 837.6040 & 843.4646 & 845.7457 \\
\hline Frequencies -- & 855.7479 & 865.1896 & 866.7859 \\
\hline Frequencies -- & 869.3491 & 871.1163 & 873.6192 \\
\hline Frequencies -- & 875.4788 & 878.9996 & 880.3230 \\
\hline Frequencies -- & 881.6232 & 884.7550 & 887.3205 \\
\hline Frequencies -- & 889.5525 & 917.8162 & 919.6475 \\
\hline Frequencies -- & 926.3545 & 939.4644 & 939.6296 \\
\hline Frequencies -- & 943.0554 & 950.7845 & 956.8818 \\
\hline Frequencies -- & 965.9515 & 970.6613 & 974.8009 \\
\hline Frequencies -- & 978.0095 & 985.9140 & 986.7148 \\
\hline Frequencies -- & 988.8908 & 990.5030 & 997.4308 \\
\hline Frequencies -- & 998.3041 & 1000.2750 & 1001.8055 \\
\hline Frequencies -- & 1005.1802 & 1005.4769 & 1005.9882 \\
\hline Frequencies -- & 1013.5579 & 1013.7124 & 1018.0684 \\
\hline Frequencies -- & 1019.2798 & 1020.2156 & 1034.4798 \\
\hline Frequencies -- & 1039.1052 & 1045.8675 & 1049.8724 \\
\hline Frequencies -- & 1051.8967 & 1053.4483 & 1055.1421 \\
\hline Frequencies -- & 1058.7087 & 1069.9434 & 1070.3099 \\
\hline Frequencies -- & 1077.7813 & 1090.9367 & 1103.4782 \\
\hline Frequencies -- & 1108.2952 & 1114.3399 & 1121.6167 \\
\hline Frequencies -- & 1126.1502 & 1137.7617 & 1140.9447 \\
\hline Frequencies -- & 1143.5382 & 1157.5710 & 1159.7283 \\
\hline Frequencies -- & 1162.8992 & 1164.3897 & 1165.8526 \\
\hline Frequencies -- & 1168.6547 & 1179.6724 & 1180.9442 \\
\hline Frequencies -- & 1186.8823 & 1188.3209 & 1195.8000 \\
\hline Frequencies -- & 1199.9423 & 1200.7537 & 1203.6421 \\
\hline Frequencies -- & 1206.5608 & 1208.2262 & 1210.3527 \\
\hline Frequencies -- & 1212.4787 & 1218.1453 & 1222.1254 \\
\hline Frequencies -- & 1245.8207 & 1250.4976 & 1252.8727 \\
\hline Frequencies -- & 1256.8286 & 1258.9281 & 1261.4087 \\
\hline Frequencies -- & 1266.4525 & 1272.6209 & 1282.1305 \\
\hline Frequencies -- & 1285.9804 & 1292.5834 & 1298.0571 \\
\hline Frequencies -- & 1303.1597 & 1303.3383 & 1308.1709 \\
\hline Frequencies -- & 1316.2309 & 1317.0135 & 1322.3961 \\
\hline Frequencies -- & 1322.9361 & 1326.6444 & 1341.5311 \\
\hline Frequencies -- & 1343.5479 & 1347.9448 & 1350.7866 \\
\hline Frequencies -- & 1355.1936 & 1361.6938 & 1365.2042 \\
\hline Frequencies -- & 1368.2552 & 1369.2902 & 1406.7381 \\
\hline Frequencies -- & 1408.9657 & 1413.7476 & 1415.9173 \\
\hline Frequencies -- & 1418.2550 & 1420.0170 & 1441.9771 \\
\hline Frequencies -- & 1442.2480 & 1446.8622 & 1454.2601 \\
\hline Frequencies -- & 1464.6665 & 1482.1099 & 1482.6799 \\
\hline Frequencies -- & 1485.3938 & 1490.8954 & 1492.5173 \\
\hline Frequencies -- & 1494.7654 & 1494.9577 & 1496.7964 \\
\hline Frequencies -- & 1498.0600 & 1499.0007 & 1500.9097 \\
\hline Frequencies -- & 1505.4731 & 1511.0343 & 1515.3059 \\
\hline Frequencies -- & 1515.6021 & 1520.5641 & 1527.9812 \\
\hline Frequencies -- & 1532.1497 & 1546.7023 & 1547.0475 \\
\hline Frequencies -- & 1552.6224 & 1581.9355 & 1601.0525 \\
\hline Frequencies -- & 1604.6209 & 1646.2124 & 1649.5092 \\
\hline Frequencies -- & 1656.7385 & 1658.7985 & 1662.2540 \\
\hline Frequencies -- & 1666.3665 & 1671.7851 & 1675.7991 \\
\hline Frequencies -- & 1684.4796 & 1694.3011 & 1694.7391 \\
\hline Frequencies -- & 1712.0689 & 1713.0809 & 1713.5741 \\
\hline
\end{tabular}

$\begin{array}{llll}\text { Frequencies -- } & 1717.9030 & 1719.5507 & 2159.9890 \\ \text { Frequencies -- } 3075.2232 & 3076.1558 & 3080.6910 \\ \text { Frequencies -- } 3086.9311 & 3101.1239 & 3128.7127 \\ \text { Frequencies -- } 3138.3662 & 3141.4823 & 3151.9548 \\ \text { Frequencies -- } 3155.7895 & 3163.4789 & 3190.6651 \\ \text { Frequencies -- } 3194.5646 & 3196.8792 & 3197.2018 \\ \text { Frequencies -- } 3199.3572 & 3201.2707 & 3203.2174 \\ \text { Frequencies -- } 3204.6136 & 3208.2699 & 3209.4735 \\ \text { Frequencies -- } 3212.3152 & 3213.2784 & 3217.0503 \\ \text { Frequencies -- } 3218.3291 & 3218.9282 & 3219.7406 \\ \text { Frequencies -- } 3220.1301 & 3223.4605 & 3224.8455 \\ \text { Frequencies -- } 3228.7705 & 3230.2050 & 3233.9839 \\ \text { Frequencies -- } 3235.6677 & 3236.9797 & 3237.2551 \\ \text { Frequencies -- } 3241.5700 & 3243.0320 & 3245.7878 \\ \text { Frequencies -- } 3246.1768 & 3248.9819 & 3252.9901 \\ \text { Frequencies -- } 3262.8209 & 3665.5673 & 3684.2073\end{array}$

SCF Done: $E(R M 062 X / D G D Z V P)=-3995.13807371$

Sum of electronic and zero-point Energies $=\quad-3994.209234$ Sum of electronic and thermal Energies = $\quad-3994.140233$ Sum of electronic and thermal Free Energies $=\quad-3994.315529$ SCF Done: $E(R M 062 X / D G T Z V P / S M D)=-3995.94025335$

\section{Cat a TS1b_S}

\begin{tabular}{|c|c|c|c|c|c|}
\hline \multirow{2}{*}{$\begin{array}{l}\text { Center } \\
\text { Number }\end{array}$} & \multicolumn{2}{|c|}{ Atomic } & Atomic & \multicolumn{2}{|c|}{ Coordinates (Angstroms) } \\
\hline & & ber & Type & $X \quad Y$ & Z \\
\hline 1 & 15 & 0 & -0.038321 & 0.182197 & 0.423642 \\
\hline 2 & 8 & 0 & 0.097787 & -0.023876 & 2.028931 \\
\hline 3 & 8 & 0 & 1.518892 & 0.281446 & -0.035246 \\
\hline 4 & 8 & 0 & -0.649251 & 1.496504 & 0.074721 \\
\hline 5 & 8 & 0 & -0.685563 & -1.082549 & -0.078289 \\
\hline 6 & 6 & 0 & 0.741979 & 0.974122 & 2.742312 \\
\hline 7 & 6 & 0 & 2.276230 & -0.867993 & 0.117814 \\
\hline 8 & 6 & 0 & 2.110351 & 0.858613 & 2.955682 \\
\hline 9 & 6 & 0 & 0.021834 & 2.093406 & 3.190054 \\
\hline 10 & 6 & 0 & 2.910778 & -1.111894 & 1.327616 \\
\hline 11 & 6 & 0 & 2.341549 & -1.778984 & -0.947124 \\
\hline 12 & 6 & 0 & 3.087013 & -0.238511 & 2.559915 \\
\hline 13 & 6 & 0 & 2.786090 & 1.888334 & 3.618441 \\
\hline 14 & 6 & 0 & 0.714462 & 3.060741 & 3.929432 \\
\hline 15 & 6 & 0 & 3.555461 & -2.338796 & 1.518035 \\
\hline 16 & 6 & 0 & 3.077361 & -2.954050 & -0.757200 \\
\hline 17 & 6 & 0 & 3.236268 & -1.333777 & 3.651999 \\
\hline 18 & 6 & 0 & 4.402174 & 0.588730 & 2.469322 \\
\hline 19 & 6 & 0 & 4.277497 & 1.639015 & 3.588614 \\
\hline 20 & 6 & 0 & 2.090389 & 2.973143 & 4.140448 \\
\hline 21 & 6 & 0 & 4.018338 & -2.464889 & 2.953860 \\
\hline 22 & 6 & 0 & 3.662403 & -3.252773 & 0.475328 \\
\hline 23 & 1 & 0 & 3.151626 & -3.660320 & -1.579971 \\
\hline 24 & 1 & 0 & 3.726361 & -0.956598 & 4.554188 \\
\hline 25 & 1 & 0 & 2.238266 & -1.690413 & 3.927129 \\
\hline 26 & 1 & 0 & 5.294224 & -0.038607 & 2.551841 \\
\hline 27 & 1 & 0 & 4.428460 & 1.093047 & 1.497192 \\
\hline 28 & 1 & 0 & 4.629870 & 1.245766 & 4.548887 \\
\hline 29 & 1 & 0 & 5.100565 & -2.310615 & 3.031954 \\
\hline 30 & 1 & 0 & -2.250979 & -1.401823 & 0.453332 \\
\hline 31 & 8 & 0 & -3.220861 & -1.529252 & 0.229708 \\
\hline 32 & 6 & 0 & -3.219358 & -2.008385 & -1.144149 \\
\hline 33 & 6 & 0 & -3.370304 & -0.650978 & -1.845632 \\
\hline 34 & 1 & 0 & -2.290329 & -2.530907 & -1.379055 \\
\hline 35 & 1 & 0 & -4.079441 & -2.665546 & -1.269278 \\
\hline 36 & 8 & 0 & -2.165064 & -0.147155 & -2.351475 \\
\hline 37 & 6 & 0 & -3.751111 & 0.073295 & -0.528065 \\
\hline
\end{tabular}




\begin{tabular}{|c|c|c|c|c|c|}
\hline 38 & 6 & 0 & -4.417007 & -0.638850 & -2.937713 \\
\hline 39 & 1 & 0 & -1.434727 & -0.466454 & -1.780908 \\
\hline 40 & 1 & 0 & -4.756980 & 0.043625 & -0.123277 \\
\hline 41 & 1 & 0 & -3.032975 & 0.715122 & -0.022593 \\
\hline 42 & 6 & 0 & -4.075920 & -0.247683 & -4.232174 \\
\hline 43 & 6 & 0 & -5.729042 & -1.033301 & -2.661953 \\
\hline 44 & 6 & 0 & -5.042974 & -0.242096 & -5.237116 \\
\hline 45 & 1 & 0 & -3.056113 & 0.063618 & -4.433684 \\
\hline 46 & 6 & 0 & -6.695518 & -1.021918 & -3.664556 \\
\hline 47 & 1 & 0 & -6.011710 & -1.341831 & -1.656740 \\
\hline 48 & 6 & 0 & -6.353746 & -0.625113 & -4.957802 \\
\hline 49 & 1 & 0 & -4.772323 & 0.070953 & -6.241047 \\
\hline 50 & 1 & 0 & -7.713922 & -1.321029 & -3.436571 \\
\hline 51 & 1 & 0 & -7.105688 & -0.613585 & -5.740782 \\
\hline 52 & 1 & 0 & -1.664479 & 2.133521 & -1.090106 \\
\hline 53 & 7 & 0 & -1.972215 & 2.861790 & -1.765653 \\
\hline 54 & 6 & 0 & -3.242156 & 3.084062 & -2.104432 \\
\hline 55 & 6 & 0 & -1.028045 & 3.736329 & -2.282451 \\
\hline 56 & 16 & 0 & -4.601565 & 2.217809 & -1.626538 \\
\hline 57 & 16 & 0 & -3.341996 & 4.477063 & -3.169702 \\
\hline 58 & 6 & 0 & -1.608901 & 4.725121 & -3.083913 \\
\hline 59 & 6 & 0 & 0.347558 & 3.688155 & -2.051387 \\
\hline 60 & 6 & 0 & -0.819474 & 5.713664 & -3.671214 \\
\hline 61 & 6 & 0 & 1.127026 & 4.676107 & -2.640296 \\
\hline 62 & 1 & 0 & 0.766244 & 2.904384 & -1.423765 \\
\hline 63 & 6 & 0 & 0.553595 & 5.679476 & -3.437313 \\
\hline 64 & 1 & 0 & -1.260685 & 6.487533 & -4.291141 \\
\hline 65 & 1 & 0 & 2.200608 & 4.668569 & -2.478306 \\
\hline 66 & 1 & 0 & 1.186663 & 6.440731 & -3.881830 \\
\hline 67 & 1 & 0 & 4.177329 & -4.198967 & 0.617706 \\
\hline 68 & 1 & 0 & 2.612296 & 3.765677 & 4.669695 \\
\hline 69 & 1 & 0 & 3.799416 & -3.450842 & 3.371452 \\
\hline 70 & 1 & 0 & 4.844538 & 2.549664 & 3.380100 \\
\hline 71 & 6 & 0 & 1.597854 & -1.502041 & -2.209801 \\
\hline 72 & 6 & 0 & 0.574668 & -2.379535 & -2.632689 \\
\hline 73 & 6 & 0 & 1.881355 & -0.338978 & -2.956378 \\
\hline 74 & 6 & 0 & 0.197852 & -3.548260 & -1.887728 \\
\hline 75 & 6 & 0 & -0.162348 & -2.086539 & -3.829697 \\
\hline 76 & 6 & 0 & 1.106400 & -0.033254 & -4.126519 \\
\hline 77 & 6 & 0 & 2.932756 & 0.569953 & -2.597334 \\
\hline 78 & 6 & 0 & -0.780372 & -4.390174 & -2.338438 \\
\hline 79 & 1 & 0 & 0.680993 & -3.747004 & -0.937712 \\
\hline 80 & 6 & 0 & -1.200322 & -2.977853 & -4.255044 \\
\hline 81 & 6 & 0 & 0.110092 & -0.916523 & -4.540576 \\
\hline 82 & 6 & 0 & 1.370608 & 1.175551 & -4.850811 \\
\hline 83 & 1 & 0 & 3.557881 & 0.342111 & -1.740133 \\
\hline 84 & 6 & 0 & 3.166001 & 1.703504 & -3.320945 \\
\hline 85 & 6 & 0 & -1.490464 & -4.107837 & -3.546243 \\
\hline 86 & 1 & 0 & -1.044801 & -5.267752 & -1.755918 \\
\hline 87 & 1 & 0 & -1.754832 & -2.732222 & -5.157155 \\
\hline 88 & 1 & 0 & -0.474016 & -0.684292 & -5.428921 \\
\hline 89 & 6 & 0 & 2.364126 & 2.023305 & -4.460450 \\
\hline 90 & 1 & 0 & 0.757049 & 1.402833 & -5.718607 \\
\hline 91 & 1 & 0 & 3.972848 & 2.370617 & -3.031208 \\
\hline 92 & 1 & 0 & -2.273838 & -4.780447 & -3.882023 \\
\hline 93 & 1 & 0 & 2.552612 & 2.939523 & -5.011885 \\
\hline 94 & 1 & 0 & 0.162611 & 3.924276 & 4.291368 \\
\hline 95 & 6 & 0 & -1.398046 & 2.317895 & 2.790531 \\
\hline 96 & 6 & 0 & -2.408477 & 1.404734 & 3.147388 \\
\hline 97 & 6 & 0 & -1.704033 & 3.427217 & 1.968814 \\
\hline 98 & 6 & 0 & -2.148777 & 0.245211 & 3.952116 \\
\hline 99 & 6 & 0 & -3.752451 & 1.609881 & 2.681959 \\
\hline 100 & 6 & 0 & -3.058024 & 3.646122 & 1.547290 \\
\hline 101 & 6 & 0 & -0.698826 & 4.307242 & 1.442940 \\
\hline 102 & 6 & 0 & -3.135373 & -0.655316 & 4.234605 \\
\hline 103 & 1 & 0 & -1.145883 & 0.085601 & 4.334702 \\
\hline
\end{tabular}

$\begin{array}{rrrrrr}104 & 6 & 0 & -4.762383 & 0.645137 & 3.004829 \\ 105 & 6 & 0 & -4.050988 & 2.733489 & 1.909775 \\ 106 & 6 & 0 & -3.368744 & 4.767762 & 0.709782 \\ 107 & 1 & 0 & 0.344501 & 4.109116 & 1.662133 \\ 108 & 6 & 0 & -1.026499 & 5.354522 & 0.629304 \\ 109 & 6 & 0 & -4.465005 & -0.459960 & 3.747045 \\ 110 & 1 & 0 & -2.910648 & -1.530666 & 4.836035 \\ 111 & 1 & 0 & -5.773299 & 0.816159 & 2.641753 \\ 112 & 1 & 0 & -5.069904 & 2.891779 & 1.561791 \\ 113 & 6 & 0 & -2.385967 & 5.605263 & 0.268841 \\ 114 & 1 & 0 & -0.243033 & 5.987541 & 0.221935 \\ 115 & 1 & 0 & -5.234239 & -1.190701 & 3.977062 \\ 116 & 1 & 0 & -4.403634 & 4.916344 & 0.410411 \\ 117 & 1 & 0 & -2.626634 & 6.445401 & -0.376536 \\ ----------------------------------------------------------------\end{array}$

Frequencies -- -531.2406

$14.5734 \quad 18.9315$

Frequencies -- 22.1102

$24.2096 \quad 33.7156$

Frequencies -- 34.3665

$38.8776 \quad 42.9261$

Frequencies -- 51.0846

$55.3673 \quad 59.6475$

Frequencies -- $60.9170 \quad 67.3060 \quad 74.4629$

Frequencies -- $77.8545 \quad 85.1113 \quad 91.8592$

Frequencies -- $94.5607 \quad 100.7795 \quad 102.6982$

Frequencies -- $108.4418 \quad 114.2368 \quad 119.0114$

Frequencies -- $130.0863 \quad 135.0727 \quad 139.2735$

Frequencies -- $145.2596 \quad 150.0827 \quad 161.0544$

Frequencies -- $167.4518 \quad 177.4402 \quad 183.4110$

Frequencies -- $191.9038 \quad 196.5879 \quad 198.1568$

Frequencies -- $200.9183 \quad 211.9438 \quad 232.5400$

Frequencies -- $236.7068 \quad 237.7189 \quad 241.0075$

Frequencies -- $245.0021 \quad 249.4786 \quad 272.2226$

Frequencies -- $277.9178 \quad 286.3218 \quad 293.4582$

Frequencies -- $294.7420 \quad 296.4382 \quad 302.2075$

Frequencies -- $322.3779 \quad 335.2823 \quad 341.8188$

Frequencies -- $346.8290 \quad 365.0653 \quad 374.0852$

Frequencies -- $376.7450 \quad 378.1090 \quad 385.8975$

Frequencies -- $393.1320 \quad 402.1469 \quad 403.2616$

Frequencies -- $403.7223 \quad 405.8479 \quad 417.8633$

Frequencies -- $419.0800 \quad 421.1941 \quad 427.2165$

Frequencies -- $432.4333 \quad 433.0683 \quad 436.5095$

Frequencies -- $448.1754 \quad 465.2322 \quad 484.4477$

Frequencies -- $486.5917 \quad 490.2685 \quad 491.8064$

Frequencies -- $498.3139 \quad 499.1894 \quad 504.6645$

Frequencies -- $506.7133 \quad 529.0340 \quad 529.9693$

Frequencies -- $530.4589 \quad 538.9596 \quad 551.6724$

Frequencies -- $557.8843 \quad 559.8996 \quad 565.3912$

Frequencies -- $567.0782 \quad 568.5815 \quad 570.3863$

Frequencies -- $578.6827 \quad 604.3133 \quad 607.6287$

Frequencies -- $615.7960 \quad 619.0857 \quad 620.8455$

Frequencies -- $625.2290 \quad 626.7540 \quad 644.3702$

Frequencies -- $648.9620 \quad 649.7279 \quad 654.7304$

Frequencies -- $657.7573 \quad 659.2153 \quad 666.5046$

Frequencies -- $675.3377 \quad 679.5000 \quad 682.2013$

Frequencies -- $690.3848 \quad 709.3698 \quad 712.6773$

Frequencies -- $715.0360 \quad 723.6000 \quad 730.5625$

Frequencies -- $736.1035 \quad 746.0297 \quad 751.8423$

Frequencies -- $753.4182 \quad 755.7765 \quad 756.9862$

Frequencies -- $765.1873 \quad 765.5738 \quad 772.5474$

Frequencies -- $773.3920 \quad 774.5352 \quad 795.7623$

Frequencies -- $798.6547 \quad 809.6576 \quad 814.9713$

Frequencies -- $821.9942 \quad 826.6432 \quad 838.3596$

Frequencies -- $845.4811 \quad 846.3518 \quad 850.4844$

Frequencies -- $861.1697 \quad 864.6307 \quad 870.0663$

Frequencies -- $871.4353 \quad 873.6697 \quad 875.1949$

Frequencies -- $879.2132 \quad 881.5549 \quad 881.7495$

Frequencies -- $888.8458 \quad 889.7789 \quad 891.3972$ 


\begin{tabular}{|c|c|c|c|}
\hline Frequencies - & 904.0686 & 915.8512 & 916.8031 \\
\hline Frequencies - & 932.2278 & 939.5908 & 941.0604 \\
\hline Frequencies - & 942.7470 & 946.1373 & 951.5148 \\
\hline Frequencies - & 963.8929 & 971.1811 & 980.9954 \\
\hline Frequencies - & 981.7461 & 983.5953 & 985.8061 \\
\hline Frequencies - & 990.4486 & 991.7024 & 992.5732 \\
\hline Frequencies - & 995.5739 & 1000.1454 & 1002.7458 \\
\hline Frequencies - & 1004.2675 & 1004.5745 & 1005.0214 \\
\hline Frequencies - & 1006.1581 & 1008.9797 & 1015.8350 \\
\hline Frequencies - & 1018.8292 & 1019.7718 & 1030.1213 \\
\hline Frequencies - & 1035.3821 & 1046.2880 & 1047.3928 \\
\hline Frequencies - & 1048.3388 & 1049.8752 & 1051.0282 \\
\hline Frequencies - & 1052.8895 & 1058.9932 & 1064.0885 \\
\hline Frequencies - & 1073.7059 & 1079.3908 & 1090.6934 \\
\hline Frequencies -- & 1091.1209 & 1103.1836 & 1109.6575 \\
\hline Frequencies - & 1111.5665 & 1117.4121 & 1136.9626 \\
\hline Frequencies - & 1140.0204 & 1144.6351 & 1159.4081 \\
\hline Frequencies - & 1162.4531 & 1163.6942 & 1166.3776 \\
\hline Frequencies - & 1170.0281 & 1173.0824 & 1174.8999 \\
\hline Frequencies - & 1177.8068 & 1180.7040 & 1188.2920 \\
\hline Frequencies - & 1189.2042 & 1196.4592 & 1200.3344 \\
\hline Frequencies - & 1203.0352 & 1206.0068 & 1207.1828 \\
\hline Frequencies - & 1211.9715 & 1212.4567 & 1214.4515 \\
\hline Frequencies - & 1222.2553 & 1242.1075 & 1249.5416 \\
\hline Frequencies - & 1253.1329 & 1256.4405 & 1257.9286 \\
\hline Frequencies - & 1260.7425 & 1270.6378 & 1272.6211 \\
\hline Frequencies - & 1282.7745 & 1287.8697 & 1297.2292 \\
\hline Frequencies - & 1298.9039 & 1302.5321 & 1302.9714 \\
\hline Frequencies - & 1312.8098 & 1315.8253 & 1320.8691 \\
\hline Frequencies - & 1324.4011 & 1327.7716 & 1341.2892 \\
\hline Frequencies - & 1342.4437 & 1346.2282 & 1349.1842 \\
\hline Frequencies - & 1351.9101 & 1356.1534 & 1360.0928 \\
\hline Frequencies - & 1360.7092 & 1365.5092 & 1367.9781 \\
\hline Frequencies - & 1394.6754 & 1408.5138 & 1414.1448 \\
\hline Frequencies - & 1415.6258 & 1419.1491 & 1427.6876 \\
\hline Frequencies - & 1433.0782 & 1442.4568 & 1448.9410 \\
\hline Frequencies - & 1466.2316 & 1480.6000 & 1482.3875 \\
\hline Frequencies - & 1485.8216 & 1487.1624 & 1489.8902 \\
\hline Frequencies - & 1492.2772 & 1494.3736 & 1497.6071 \\
\hline Frequencies - & 1499.1308 & 1500.4223 & 1502.8719 \\
\hline Frequencies - & 1508.1311 & 1508.5868 & 1513.2541 \\
\hline Frequencies - & 1518.2025 & 1519.4706 & 1528.3990 \\
\hline Frequencies - & 1531.4898 & 1546.9259 & 1550.7992 \\
\hline Frequencies - & 1552.2881 & 1557.6777 & 1601.2693 \\
\hline Frequencies - & 1604.3437 & 1646.2019 & 1651.8510 \\
\hline Frequencies - & 1654.9199 & 1658.3813 & 1662.4099 \\
\hline Frequencies - & 1665.6589 & 1673.4850 & 1679.4412 \\
\hline Frequencies - & 1686.7829 & 1700.3020 & 1701.7821 \\
\hline Frequencies - & 1706.0048 & 1713.2359 & 1715.6255 \\
\hline Frequencies - & 1716.6599 & 1719.8868 & 3079.5832 \\
\hline Frequencies - & 3082.1627 & 3084.8301 & 3086.4848 \\
\hline Frequencies - & 3140.1082 & 3140.2448 & 3142.8699 \\
\hline Frequencies - & 3152.1100 & 3156.3222 & 3167.0094 \\
\hline Frequencies - & 3189.6546 & 3196.0316 & 3196.8312 \\
\hline Frequencies - & 3199.2840 & 3200.7708 & 3203.8062 \\
\hline Frequencies - & 3206.1352 & 3206.4547 & 3210.2509 \\
\hline Frequencies - & 3210.3970 & 3212.4265 & 3214.1187 \\
\hline Frequencies - & 3214.2007 & 3215.5911 & 3215.6824 \\
\hline Frequencies - & 3220.6996 & 3222.2758 & 3222.6286 \\
\hline Frequencies - & 3225.7491 & 3227.3397 & 3227.4921 \\
\hline Frequencies - & 3227.8171 & 3228.8399 & 3231.5623 \\
\hline Frequencies - & 3231.6462 & 3232.9165 & 3236.4592 \\
\hline Frequencies - & 3238.1972 & 3238.3202 & 3243.7518 \\
\hline Frequencies - & 3246.3445 & 3247.1751 & 3254.2640 \\
\hline Frequencies - & 3255.8545 & 3330.4644 & 3643.1582 \\
\hline
\end{tabular}

SCF Done: $E(R M 062 X / D G D Z V P)=-3995.07424617$

Sum of electronic and zero-point Energies $=\quad-3994.145600$ Sum of electronic and thermal Energies $=\quad-3994.076824$

Sum of electronic and thermal Free Energies $=\quad-3994.251549$ SCF Done: $E(R M 062 X / D G T Z V P / S M D)=-3995.87663314$

\section{Cat a I1b_S}

\begin{tabular}{lllll} 
Center Atomic & Atomic & \multicolumn{3}{c}{ Coordinates (Angstroms) } \\
Number Number Type & $X$ & Y & Z
\end{tabular}

\begin{tabular}{|c|c|c|c|c|c|}
\hline 1 & 15 & 0 & -0.149419 & 0.073272 & 0.432866 \\
\hline 2 & 8 & 0 & 0.115333 & 0.040713 & 2.033840 \\
\hline 3 & 8 & 0 & 1.365984 & 0.251649 & -0.154298 \\
\hline 4 & 8 & 0 & -0.850897 & 1.339792 & 0.047446 \\
\hline 5 & 8 & 0 & -0.729171 & -1.255860 & 0.070054 \\
\hline 6 & 6 & 0 & 0.772539 & 1.119791 & 2.598879 \\
\hline 7 & 6 & 0 & 2.188020 & -0.853271 & 0.008506 \\
\hline 8 & 6 & 0 & 2.154993 & 1.054598 & 2.731428 \\
\hline 9 & 6 & 0 & 0.055126 & 2.262232 & 2.991644 \\
\hline 10 & 6 & 0 & 2.910263 & -0.998792 & 1.186148 \\
\hline 11 & 6 & 0 & 2.198716 & -1.841598 & -0.986409 \\
\hline 12 & 6 & 0 & 3.1329 & -0.047195 & 2.351896 \\
\hline 13 & 6 & 0 & 2.84 & 2.143 & \\
\hline 14 & 6 & 0 & 0.76 & & 3.607694 \\
\hline 15 & 6 & 0 & 3.5 & & 1.416294 \\
\hline 16 & 6 & 0 & & & -0.768992 \\
\hline 17 & 6 & 0 & & & 3.498777 \\
\hline 18 & 6 & 0 & 4.4 & & 7976 \\
\hline 19 & 6 & 0 & 4.334 & & 3.192376 \\
\hline 20 & 6 & 0 & 2.152850 & & 3.751440 \\
\hline 21 & 6 & 0 & 4.140150 & -2.222 & 2.829106 \\
\hline 22 & 6 & 0 & 3.660525 & -3.177746 & 0.434595 \\
\hline 23 & 1 & 0 & 3.001975 & -3.749665 & -1.537941 \\
\hline 24 & 1 & 0 & 3.891631 & -0.625176 & 4.349569 \\
\hline 25 & 1 & 0 & 2.393230 & -1.430821 & 3.846248 \\
\hline 26 & 1 & 0 & 5.332482 & 0.193569 & 2.212155 \\
\hline 27 & 1 & 0 & 4.387143 & 1.237680 & 1.135676 \\
\hline 28 & 1 & 0 & 4.732261 & 1.583818 & 4.158671 \\
\hline 29 & 1 & 0 & 5.220868 & -2.041132 & 2.840490 \\
\hline 30 & 1 & 0 & -2.389927 & -1.687748 & 0.712466 \\
\hline 31 & 8 & 0 & -3.365780 & -1.756664 & 0.678623 \\
\hline 32 & 6 & 0 & -3.745472 & -1.986943 & -0.657567 \\
\hline 33 & 6 & 0 & -3.722715 & -0.716939 & -1.544648 \\
\hline 34 & 1 & 0 & -3.105513 & -2.732834 & -1.151043 \\
\hline 35 & 1 & 0 & -4.764229 & -2.381881 & -0.627975 \\
\hline 36 & 8 & 0 & -2.414138 & -0.418633 & -1.994948 \\
\hline 37 & 6 & 0 & -4.217980 & 0.466764 & -0.682983 \\
\hline 38 & 6 & 0 & -4.597603 & -0.942942 & -2.769709 \\
\hline 39 & 1 & 0 & -1.762525 & -0.836445 & -1.390365 \\
\hline 40 & 1 & 0 & -5.106560 & 0.203602 & -0.099719 \\
\hline 41 & 1 & 0 & -3.448307 & 0.768908 & 0.033618 \\
\hline 42 & 6 & 0 & -4.037343 & -0.905039 & -4.047573 \\
\hline 43 & 6 & 0 & -5.972829 & -1.169785 & -2.639392 \\
\hline 44 & 6 & 0 & -4.837568 & -1.095088 & -5.175382 \\
\hline 45 & 1 & 0 & -2.971474 & -0.721986 & -4.138821 \\
\hline 46 & 6 & 0 & -6.772013 & -1.359937 & -3.764337 \\
\hline 47 & 1 & 0 & -6.433204 & -1.198077 & -1.653425 \\
\hline 48 & 6 & 0 & -6.205450 & -1.323876 & -5.039617 \\
\hline 49 & 1 & 0 & -4.389674 & -1.060753 & -6.164805 \\
\hline 50 & 1 & 0 & -7.836933 & -1.535956 & -3.645977 \\
\hline 51 & 1 & 0 & -6.826967 & -1.472141 & -5.917501 \\
\hline 52 & 1 & 0 & -1.750202 & 1.781878 & -1.061013 \\
\hline 53 & 7 & 0 & -2.102714 & 2.454769 & -1.830492 \\
\hline 54 & 6 & 0 & -3.339994 & 2.667202 & -2.205186 \\
\hline
\end{tabular}




\begin{tabular}{|c|c|c|c|c|c|}
\hline 55 & 6 & 0 & -1.155432 & 3.261490 & -2.452903 \\
\hline 56 & 16 & 0 & -4.786345 & 1.923613 & -1.632582 \\
\hline 57 & 16 & 0 & -3.465134 & 3.911666 & -3.412677 \\
\hline 58 & 6 & 0 & -1.734465 & 4.162991 & -3.351134 \\
\hline 59 & 6 & 0 & 0.222255 & 3.226471 & -2.226896 \\
\hline 60 & 6 & 0 & -0.949810 & 5.085391 & -4.044771 \\
\hline 61 & 6 & 0 & 0.996892 & 4.147398 & -2.917417 \\
\hline 62 & 1 & 0 & 0.643313 & 2.506464 & -1.529021 \\
\hline 63 & 6 & 0 & 0.421296 & 5.068142 & -3.809858 \\
\hline 64 & 1 & 0 & -1.390282 & 5.791785 & -4.740474 \\
\hline 65 & 1 & 0 & 2.070760 & 4.155037 & -2.762180 \\
\hline 66 & 1 & 0 & 1.055924 & 5.777075 & -4.331769 \\
\hline 67 & 1 & 0 & 4.204133 & -4.102380 & 0.608635 \\
\hline 68 & 1 & 0 & 2.684537 & 4.091742 & 4.193254 \\
\hline 69 & 1 & 0 & 3.966845 & -3.185403 & 3.315434 \\
\hline 70 & 1 & 0 & 4.879557 & 2.817037 & 2.904890 \\
\hline 71 & 6 & 0 & 1.330946 & -1.720771 & -2.194855 \\
\hline 72 & 6 & 0 & 0.297187 & -2.662451 & -2.397401 \\
\hline 73 & 6 & 0 & 1.516253 & -0.669352 & -3.114632 \\
\hline 74 & 6 & 0 & -0.012278 & -3.685602 & -1.439033 \\
\hline 75 & 6 & 0 & -0.526052 & -2.576278 & -3.569970 \\
\hline 76 & 6 & 0 & 0.654805 & -0.565230 & -4.259560 \\
\hline 77 & 6 & 0 & 2.554661 & 0.310888 & -2.966823 \\
\hline 78 & 6 & 0 & -1.016322 & -4.583283 & -1.663838 \\
\hline 79 & 1 & 0 & 0.533579 & -3.710886 & -0.502891 \\
\hline 80 & 6 & 0 & -1.568388 & -3.538644 & -3.773722 \\
\hline 81 & 6 & 0 & -0.328956 & -1.530803 & -4.473108 \\
\hline 82 & 6 & 0 & 0.826750 & 0.528393 & -5.170014 \\
\hline 83 & 1 & 0 & 3.237968 & 0.231632 & -2.126789 \\
\hline 84 & 6 & 0 & 2.699247 & 1.328101 & -3.866394 \\
\hline 85 & 6 & 0 & -1.800071 & -4.522425 & -2.857412 \\
\hline 86 & 1 & 0 & -1.241861 & -5.335867 & -0.914415 \\
\hline 87 & 1 & 0 & -2.181175 & -3.458929 & -4.668436 \\
\hline 88 & 1 & 0 & -0.959633 & -1.464824 & -5.358402 \\
\hline 89 & 6 & 0 & 1.811976 & 1.452068 & -4.980572 \\
\hline 90 & 1 & 0 & 0.151045 & 0.604088 & -6.018199 \\
\hline 91 & 1 & 0 & 3.499588 & 2.051615 & -3.738934 \\
\hline 92 & 1 & 0 & -2.596846 & -5.242749 & -3.015983 \\
\hline 93 & 1 & 0 & 1.932306 & 2.277941 & -5.675498 \\
\hline 94 & 1 & 0 & 0.215828 & 4.179916 & 3.930345 \\
\hline 95 & 6 & 0 & -1.390891 & 2.436242 & 2.665749 \\
\hline 96 & 6 & 0 & -2.365523 & 1.563479 & 3.185210 \\
\hline 97 & 6 & 0 & -1.762780 & 3.457275 & 1.761953 \\
\hline 98 & 6 & 0 & -2.037816 & 0.489715 & 4.078488 \\
\hline 99 & 6 & 0 & -3.741886 & 1.722221 & 2.802834 \\
\hline 100 & 6 & 0 & -3.143535 & 3.624826 & 1.408903 \\
\hline 101 & 6 & 0 & -0.803486 & 4.289471 & 1.092253 \\
\hline 102 & 6 & 0 & -2.996443 & -0.371577 & 4.526801 \\
\hline 103 & 1 & 0 & -1.006948 & 0.361875 & 4.391760 \\
\hline 104 & 6 & 0 & -4.719943 & 0.802619 & 3.303440 \\
\hline 105 & 6 & 0 & -4.101882 & 2.756286 & 1.938098 \\
\hline 106 & 6 & 0 & -3.517064 & 4.655060 & 0.484136 \\
\hline 107 & 1 & 0 & 0.253652 & 4.126088 & 1.272011 \\
\hline 108 & 6 & 0 & -1.191652 & 5.248418 & 0.199362 \\
\hline 109 & 6 & 0 & -4.360493 & -0.219195 & 4.130479 \\
\hline 110 & 1 & 0 & -2.722556 & -1.187468 & 5.188287 \\
\hline 111 & 1 & 0 & -5.755658 & 0.934244 & 2.999541 \\
\hline 112 & 1 & 0 & -5.146546 & 2.879410 & 1.655257 \\
\hline 113 & 6 & 0 & -2.573562 & 5.450531 & -0.100632 \\
\hline 114 & 1 & 0 & -4.572122 & 4.779599 & 0.250299 \\
\hline 115 & 1 & 0 & -0.441260 & 5.846296 & -0.310887 \\
\hline 116 & 1 & 0 & -5.104700 & -0.922426 & 4.490621 \\
\hline 117 & 1 & 0 & -2.865116 & 6.227865 & -0.801694 \\
\hline
\end{tabular}

Frequencies -- $\quad 14.0147$

15.8284

22.8076

\begin{tabular}{|c|c|c|c|}
\hline Frequencies -- & 25.2503 & 28.9004 & 34.4874 \\
\hline Frequencies -- & 35.8041 & 40.9923 & 48.7082 \\
\hline Frequencies -- & 53.8889 & 58.8675 & 59.7560 \\
\hline Frequencies -- & 68.9002 & 74.7161 & 78.0438 \\
\hline Frequencies -- & 89.1407 & 93.2714 & 99.9695 \\
\hline Frequencies -- & 101.6435 & 105.6160 & 108.5441 \\
\hline Frequencies -- & 121.5778 & 124.1187 & 130.3490 \\
\hline Frequencies -- & 134.9677 & 142.4531 & 144.3060 \\
\hline Frequencies -- & 149.2435 & 159.8402 & 168.4175 \\
\hline Frequencies -- & 169.1983 & 176.8990 & 192.9388 \\
\hline Frequencies -- & 196.8698 & 204.4776 & 211.6967 \\
\hline Frequencies -- & 222.6019 & 231.4157 & 237.3680 \\
\hline Frequencies -- & 240.5447 & 243.3308 & 245.9412 \\
\hline Frequencies -- & 257.9571 & 265.5196 & 280.2033 \\
\hline Frequencies -- & 290.7070 & 294.2723 & 296.8566 \\
\hline Frequencies -- & 301.0897 & 306.4822 & 322.6892 \\
\hline Frequencies -- & 328.7583 & 337.9434 & 345.3259 \\
\hline Frequencies -- & 348.3670 & 366.0541 & 373.6064 \\
\hline Frequencies -- & 377.0385 & 380.4397 & 385.4371 \\
\hline Frequencies -- & 398.7614 & 403.3158 & 403.6231 \\
\hline Frequencies -- & 404.3912 & 409.8420 & 417.8913 \\
\hline Frequencies -- & 419.0266 & 424.8567 & 430.6590 \\
\hline Frequencies -- & 433.5424 & 436.4469 & 444.5135 \\
\hline Frequencies -- & 468.6841 & 480.7300 & 485.0965 \\
\hline Frequencies -- & 487.4117 & 490.9482 & 497.0651 \\
\hline Frequencies -- & 498.0854 & 504.6321 & 507.9635 \\
\hline Frequencies -- & 523.3886 & 527.0955 & 531.8814 \\
\hline Frequencies -- & 539.9023 & 550.6803 & 556.4770 \\
\hline Frequencies -- & 558.5814 & 563.6848 & 566.7682 \\
\hline Frequencies -- & 569.7773 & 581.4710 & 586.7067 \\
\hline Frequencies -- & 605.1777 & 608.2487 & 610.0580 \\
\hline Frequencies -- & 615.9927 & 619.3426 & 621.3285 \\
\hline Frequencies -- & 626.5303 & 631.0217 & 635.9069 \\
\hline Frequencies -- & 643.9080 & 648.7313 & 650.6627 \\
\hline Frequencies -- & 654.7437 & 658.2900 & 661.1301 \\
\hline Frequencies -- & 670.4533 & 682.2171 & 690.4767 \\
\hline Frequencies -- & 705.5479 & 710.4319 & 712.7737 \\
\hline Frequencies -- & 716.6684 & 722.1947 & 727.6976 \\
\hline Frequencies -- & 733.5340 & 733.8253 & 744.4070 \\
\hline Frequencies -- & 747.3399 & 750.5997 & 754.3810 \\
\hline Frequencies -- & 758.7431 & 769.9173 & 771.3833 \\
\hline Frequencies -- & 773.5407 & 776.5816 & 792.3163 \\
\hline Frequencies -- & 796.8645 & 798.7539 & 809.9916 \\
\hline Frequencies -- & 812.6276 & 827.9617 & 835.9681 \\
\hline Frequencies -- & 842.1888 & 842.9113 & 851.5771 \\
\hline Frequencies -- & 859.5112 & 860.5386 & 861.7245 \\
\hline Frequencies -- & 865.9849 & 870.1400 & 871.0876 \\
\hline Frequencies -- & 875.4881 & 879.6818 & 880.9817 \\
\hline Frequencies -- & 888.1109 & 890.8290 & 891.9631 \\
\hline Frequencies -- & 892.9491 & 906.9007 & 915.7306 \\
\hline Frequencies -- & 934.0655 & 938.0771 & 940.1771 \\
\hline Frequencies -- & 942.9924 & 949.1601 & 951.9711 \\
\hline Frequencies -- & 957.6317 & 971.0269 & 974.1404 \\
\hline Frequencies -- & 975.6019 & 976.5324 & 982.3544 \\
\hline Frequencies -- & 983.7643 & 985.8483 & 990.4991 \\
\hline Frequencies -- & 991.8439 & 995.7905 & 997.6673 \\
\hline Frequencies -- & 999.8394 & 1000.3159 & 1002.0320 \\
\hline Frequencies -- & 1002.4359 & 1004.9299 & 1019.1057 \\
\hline Frequencies -- & 1019.6847 & 1021.9731 & 1023.5042 \\
\hline Frequencies -- & 1035.1227 & 1046.2433 & 1046.5738 \\
\hline Frequencies -- & 1047.8325 & 1050.1570 & 1052.8019 \\
\hline Frequencies -- & 1059.6325 & 1067.1067 & 1071.8121 \\
\hline Frequencies -- & 1077.8930 & 1092.2151 & 1104.0049 \\
\hline Frequencies -- & 1108.5973 & 1113.2745 & 1118.5394 \\
\hline Frequencies -- & 1124.2534 & 1136.8564 & 1140.9641 \\
\hline Frequencies -- & 1145.5867 & 1155.6763 & 1160.6462 \\
\hline
\end{tabular}




\begin{tabular}{|c|c|c|c|}
\hline Frequencies - & 1163.9552 & 1164.8836 & 1168.8641 \\
\hline Frequencies - & 1171.1334 & 1173.9098 & 1177.7343 \\
\hline Frequencies - & 1182.5962 & 1184.7773 & 1194.8119 \\
\hline Frequencies & 1199.1274 & 1203.1920 & 1203.6276 \\
\hline Frequencies - & 1204.3963 & 1206.9333 & 1210.6147 \\
\hline Frequencies - & 1211.4578 & 1213.0664 & 1222.4409 \\
\hline Frequencies - & 1247.3130 & 1250.2195 & 1251.6968 \\
\hline Frequencies - & 1255.1418 & 1256.7610 & 1258.7885 \\
\hline Frequencies - & 1272.2177 & 1274.5292 & 1282.6298 \\
\hline Frequencies - & 1293.7369 & 1297.0922 & 1298.8346 \\
\hline Frequencies - & 1300.4849 & 1303.6081 & 1312.1135 \\
\hline Frequencies - & 1313.2284 & 1315.7047 & 1318.1394 \\
\hline Frequencies - & 1321.5145 & 1328.2065 & 1342.5431 \\
\hline Frequencies - & 1342.6145 & 1344.4972 & 1347.7094 \\
\hline Frequencies & 1351.0458 & 1360.0406 & 1365.2547 \\
\hline Frequencies - & 1366.4955 & 1368.2320 & 1400.7100 \\
\hline Frequencies - & 1402.8783 & 1407.2686 & 1411.3608 \\
\hline Frequencies - & 1414.4669 & 1415.2122 & 1431.8478 \\
\hline Frequencies - & 1439.8745 & 1444.7341 & 1455.8023 \\
\hline Frequencies - & 1481.5266 & 1481.9858 & 1484.0866 \\
\hline Frequencies - & 1486.6500 & 1489.7186 & 1490.7638 \\
\hline Frequencies - & 1492.9567 & 1494.8441 & 1496.8184 \\
\hline Frequencies - & 1498.5560 & 1499.3682 & 1501.1443 \\
\hline Frequencies - & 1507.2116 & 1507.9733 & 1511.2734 \\
\hline Frequencies & 1514.7943 & 1522.1430 & 1527.1889 \\
\hline Frequencies & 1529.3211 & 1546.1508 & 1546.5943 \\
\hline Frequencies & 1549.9399 & 1596.4914 & 1599.9271 \\
\hline Frequencies & 1601.6020 & 1646.2112 & 1648.6819 \\
\hline Frequencies & 1654.5556 & 1656.5258 & 1660.8943 \\
\hline Frequencies & 1664.1487 & 1674.2952 & 1676.2879 \\
\hline Frequencies - & 1687.7474 & 1698.1256 & 1698.8025 \\
\hline Frequencies & 1704.5523 & 1709.9566 & 1713.2529 \\
\hline Frequencies - & 1716.1078 & 1718.7304 & 2469.1413 \\
\hline Frequencies - & 3077.4116 & 3077.4567 & 3081.4469 \\
\hline Frequencies - & 3086.3918 & 3089.5409 & 3108.8860 \\
\hline Frequencies - & 3141.4172 & 3144.0313 & 3154.3335 \\
\hline Frequencies - & 3154.6229 & 3157.9989 & 3178.4374 \\
\hline Frequencies - & 3180.7546 & 3190.0949 & 3191.8220 \\
\hline Frequencies - & 3194.7439 & 3198.4453 & 3201.6228 \\
\hline Frequencies - & 3204.3077 & 3204.6249 & 3205.5072 \\
\hline Frequencies - & 3207.1690 & 3209.1707 & 3211.8454 \\
\hline Frequencies - & 3217.5151 & 3219.3555 & 3219.8412 \\
\hline Frequencies - & 3220.2725 & 3220.3865 & 3221.5893 \\
\hline Frequencies - & 3223.1767 & 3226.9176 & 3232.3719 \\
\hline Frequencies - & 3233.0739 & 3235.3671 & 3235.4058 \\
\hline Frequencies - & 3235.4819 & 3242.5825 & 3243.5178 \\
\hline Frequencies - & 3243.8505 & 3245.2268 & 3250.8034 \\
\hline Frequencies - & 3253.4663 & 3574.0066 & 3644.6630 \\
\hline
\end{tabular}

SCF Done: $E(R M 062 X / D G D Z V P)=-3995.13267500$

Sum of electronic and zero-point Energies $=\quad-3994.204104$ Sum of electronic and thermal Energies $=\quad-3994.135081$ Sum of electronic and thermal Free Energies $=\quad-3994.310404$ SCF Done: $E($ RM062X/DGTZVP/SMD $)=-3995.93630212$

\section{Cat a TS1c_S}

Center Atomic Atomic Coordinates (Angstroms)

\begin{tabular}{cccccc} 
Number & Number & Type & $X$ & $Y$ & $Z$ \\
\hline 1 & 15 & 0 & 0.079566 & 0.042670 & -0.105938 \\
2 & 8 & 0 & -0.001430 & -0.003626 & 1.518292 \\
3 & 8 & 0 & 1.681414 & 0.006321 & -0.363655 \\
4 & 8 & 0 & -0.379849 & 1.378290 & -0.630508 \\
5 & 8 & 0 & -0.595709 & -1.197448 & -0.582863
\end{tabular}

\begin{tabular}{|c|c|c|c|c|c|}
\hline 6 & 6 & 0 & 0.500990 & 1.099926 & 2.188063 \\
\hline 7 & 6 & 0 & 2.369263 & -1.099660 & 0.110059 \\
\hline 8 & 6 & 0 & 1.850631 & 1.143459 & 2.510894 \\
\hline 9 & 6 & 0 & -0.360632 & 2.169435 & 2.478254 \\
\hline 10 & 6 & 0 & 2.917690 & -1.041278 & 1.384959 \\
\hline 11 & 6 & 0 & 2.461470 & -2.260208 & -0.675911 \\
\hline 12 & 6 & 0 & 2.941144 & 0.088599 & 2.402188 \\
\hline 13 & 6 & 0 & 2.379411 & 2.315306 & 3.062218 \\
\hline 14 & 6 & 0 & 0.181240 & 3.285268 & 3.127549 \\
\hline 15 & 6 & 0 & 3.563720 & -2.168631 & 1.901504 \\
\hline 16 & 6 & 0 & 3.192020 & -3.338794 & -0.161755 \\
\hline 17 & 6 & 0 & 3.055963 & -0.733328 & 3.719471 \\
\hline 18 & 6 & 0 & 4.189076 & 1.001692 & 2.250939 \\
\hline 19 & 6 & 0 & 3.886820 & 2.212600 & 3.156815 \\
\hline 20 & 6 & 0 & 1.547622 & 3.377576 & 3.398786 \\
\hline 21 & 1 & 0 & -0.478849 & 4.111808 & 3.377206 \\
\hline 22 & 6 & 0 & 3.938601 & -1.939065 & 3.348385 \\
\hline 23 & 6 & 0 & 3.736045 & -3.307110 & 1.122444 \\
\hline 24 & 1 & 0 & 3.282540 & -4.235597 & -0.769049 \\
\hline 25 & 1 & 0 & 3.451239 & -0.137728 & 4.547162 \\
\hline 26 & 1 & 0 & 2.056341 & -1.083748 & 3.998310 \\
\hline 27 & 1 & 0 & 5.116582 & 0.482642 & 2.509005 \\
\hline 28 & 1 & 0 & 4.256918 & 1.329542 & 1.208326 \\
\hline 29 & 1 & 0 & 4.204526 & 2.029662 & 4.189601 \\
\hline 30 & 1 & 0 & 5.004103 & -1.701020 & 3.444718 \\
\hline 31 & 1 & 0 & -0.347338 & 1.707924 & -2.250116 \\
\hline 32 & 8 & 0 & -0.831896 & 1.892629 & -3.112854 \\
\hline 33 & 6 & 0 & -2.092350 & 2.491382 & -2.704168 \\
\hline 34 & 6 & 0 & -2.929458 & 1.205374 & -2.638931 \\
\hline 35 & 1 & 0 & -1.991094 & 3.007913 & -1.748077 \\
\hline 36 & 1 & 0 & -2.406077 & 3.181333 & -3.487068 \\
\hline 37 & 8 & 0 & -3.086859 & 0.711161 & -1.336370 \\
\hline 38 & 6 & 0 & -1.860283 & 0.382345 & -3.405577 \\
\hline 39 & 6 & 0 & -4.288152 & 1.312850 & -3.295999 \\
\hline 40 & 1 & 0 & -2.299962 & 0.969215 & -0.813513 \\
\hline 41 & 1 & 0 & -1.771423 & 0.419664 & -4.485642 \\
\hline 42 & 1 & 0 & -1.227313 & -0.327003 & -2.876799 \\
\hline 43 & 6 & 0 & -5.439450 & 0.993146 & -2.575864 \\
\hline 44 & 6 & 0 & -4.403432 & 1.735136 & -4.623210 \\
\hline 45 & 6 & 0 & -6.691541 & 1.085123 & -3.183004 \\
\hline 46 & 1 & 0 & -5.339930 & 0.655888 & -1.549443 \\
\hline 47 & 6 & 0 & -5.653898 & 1.821811 & -5.230394 \\
\hline 48 & 1 & 0 & -3.515249 & 1.988058 & -5.200058 \\
\hline 49 & 6 & 0 & -6.803801 & 1.496124 & -4.510170 \\
\hline 50 & 1 & 0 & -7.582165 & 0.825464 & -2.618613 \\
\hline 51 & 1 & 0 & -5.730476 & 2.142389 & -6.264759 \\
\hline 52 & 1 & 0 & -7.779120 & 1.562104 & -4.982330 \\
\hline 53 & 1 & 0 & -2.056694 & -1.679779 & -1.237886 \\
\hline 54 & 7 & 0 & -2.881240 & -2.304277 & -1.350935 \\
\hline 55 & 6 & 0 & -3.571945 & -2.436577 & -2.482662 \\
\hline 56 & 6 & 0 & -3.264371 & -3.112525 & -0.291646 \\
\hline 57 & 16 & 0 & -3.340736 & -1.618455 & -3.934070 \\
\hline 58 & 16 & 0 & -4.829814 & -3.643760 & -2.281019 \\
\hline 59 & 6 & 0 & -4.335277 & -3.948343 & -0.627261 \\
\hline 60 & 6 & 0 & -2.677423 & -3.139894 & 0.974130 \\
\hline 61 & 6 & 0 & -4.847536 & -4.851907 & 0.303842 \\
\hline 62 & 6 & 0 & -3.192965 & -4.043237 & 1.896245 \\
\hline 63 & 1 & 0 & -1.846947 & -2.475136 & 1.201354 \\
\hline 64 & 6 & 0 & -4.262617 & -4.891053 & 1.567426 \\
\hline 65 & 1 & 0 & -5.677093 & -5.504751 & 0.052067 \\
\hline 66 & 1 & 0 & -2.757622 & -4.092669 & 2.889622 \\
\hline 67 & 1 & 0 & -4.643531 & -5.588338 & 2.306641 \\
\hline 68 & 1 & 0 & 4.382424 & 3.126787 & 2.820497 \\
\hline 69 & 1 & 0 & 3.741978 & -2.815638 & 3.970848 \\
\hline 70 & 1 & 0 & 1.953767 & 4.280865 & 3.845816 \\
\hline 71 & 1 & 0 & 4.252816 & -4.177657 & 1.517206 \\
\hline
\end{tabular}




\begin{tabular}{|c|c|c|c|c|c|}
\hline 72 & 6 & 0 & -1.788340 & 2.134760 & 2.047551 \\
\hline 73 & 6 & 0 & -2.277094 & 3.125681 & 1.164102 \\
\hline 74 & 6 & 0 & -2.644966 & 1.105415 & 2.490337 \\
\hline 75 & 6 & 0 & -1.441704 & 4.154673 & 0.611544 \\
\hline 76 & 6 & 0 & -3.652603 & 3.094983 & 0.751995 \\
\hline 77 & 6 & 0 & -4.001939 & 1.051559 & 2.020740 \\
\hline 78 & 6 & 0 & -2.218781 & 0.089630 & 3.411448 \\
\hline 79 & 6 & 0 & -1.953925 & 5.117070 & -0.214414 \\
\hline 80 & 1 & 0 & -0.380486 & 4.147471 & 0.832648 \\
\hline 81 & 6 & 0 & -4.148832 & 4.108325 & -0.130869 \\
\hline 82 & 6 & 0 & -4.479774 & 2.054858 & 1.180256 \\
\hline 83 & 6 & 0 & -4.843710 & -0.037993 & 2.421645 \\
\hline 84 & 1 & 0 & -1.214615 & 0.135630 & 3.819640 \\
\hline 85 & 6 & 0 & -3.054684 & -0.922606 & 3.784174 \\
\hline 86 & 6 & 0 & -3.333525 & 5.105238 & -0.585374 \\
\hline 87 & 1 & 0 & -1.297802 & 5.881826 & -0.619089 \\
\hline 88 & 1 & 0 & -5.191573 & 4.061735 & -0.434795 \\
\hline 89 & 1 & 0 & -5.511264 & 2.018920 & 0.835141 \\
\hline 90 & 6 & 0 & -4.385134 & -1.004014 & 3.266472 \\
\hline 91 & 1 & 0 & -5.856924 & -0.078002 & 2.030345 \\
\hline 92 & 1 & 0 & -2.707055 & -1.677360 & 4.483815 \\
\hline 93 & 1 & 0 & -3.720803 & 5.871411 & -1.250023 \\
\hline 94 & 1 & 0 & -5.025111 & -1.832467 & 3.554607 \\
\hline 95 & 6 & 0 & 1.716120 & -2.383818 & -1.962070 \\
\hline 96 & 6 & 0 & 0.685849 & -3.345966 & -2.067113 \\
\hline 97 & 6 & 0 & 1.967042 & -1.498935 & -3.028334 \\
\hline 98 & 6 & 0 & 0.292972 & -4.189170 & -0.973518 \\
\hline 99 & 6 & 0 & -0.075737 & -3.439156 & -3.279854 \\
\hline 100 & 6 & 0 & 1.162192 & -1.565591 & -4.216578 \\
\hline 101 & 6 & 0 & 2.995559 & -0.499838 & -2.971926 \\
\hline 102 & 6 & 0 & -0.716439 & -5.100472 & -1.106409 \\
\hline 103 & 1 & 0 & 0.781602 & -4.072693 & -0.012475 \\
\hline 104 & 6 & 0 & -1.115138 & -4.420283 & -3.390547 \\
\hline 105 & 6 & 0 & 0.169756 & -2.541263 & -4.321725 \\
\hline 106 & 6 & 0 & 1.385105 & -0.617862 & -5.269216 \\
\hline 107 & 1 & 0 & 3.634997 & -0.452401 & -2.096541 \\
\hline 108 & 6 & 0 & 3.179108 & 0.385466 & -3.994400 \\
\hline 109 & 6 & 0 & -1.421762 & -5.237140 & -2.341159 \\
\hline 110 & 1 & 0 & -1.008887 & -5.709300 & -0.255402 \\
\hline 111 & 1 & 0 & -1.670120 & -4.480081 & -4.323537 \\
\hline 112 & 1 & 0 & -0.438069 & -2.594821 & -5.223065 \\
\hline 113 & 6 & 0 & 2.354271 & 0.335785 & -5.160848 \\
\hline 114 & 1 & 0 & 0.762019 & -0.678149 & -6.158525 \\
\hline 115 & 1 & 0 & 3.959029 & 1.137286 & -3.923669 \\
\hline 116 & 1 & 0 & -2.215558 & -5.973320 & -2.430637 \\
\hline 117 & 1 & 0 & 2.509966 & 1.054538 & -5.959351 \\
\hline
\end{tabular}

$\begin{array}{cccc}\text { Frequencies -- } & -535.3208 & 10.9249 & 16.5920 \\ \text { Frequencies -- } & 18.6192 & 26.2941 & 30.5180 \\ \text { Frequencies -- } & 34.1267 & 35.9212 & 39.6361 \\ \text { Frequencies -- } & 50.4122 & 51.1282 & 54.8654 \\ \text { Frequencies -- } & 60.5481 & 63.7729 & 66.4964 \\ \text { Frequencies -- } & 75.6647 & 83.5788 & 91.8862 \\ \text { Frequencies -- } & 94.8326 & 100.3533 & 104.1125 \\ \text { Frequencies -- } & 108.4730 & 115.2352 & 120.7578 \\ \text { Frequencies -- } & 129.0967 & 136.9571 & 144.4449 \\ \text { Frequencies -- } & 145.6585 & 150.0602 & 161.2617 \\ \text { Frequencies -- } & 169.6435 & 177.4161 & 184.8873 \\ \text { Frequencies -- } & 194.2494 & 196.8967 & 199.6933 \\ \text { Frequencies -- } & 202.5984 & 211.1434 & 232.1451 \\ \text { Frequencies -- } & 237.3704 & 238.5775 & 240.6267 \\ \text { Frequencies -- } & 245.5252 & 248.7801 & 274.7479 \\ \text { Frequencies -- } & 275.9927 & 283.8720 & 293.9267 \\ \text { Frequencies -- } & 294.6644 & 298.6829 & 302.1923 \\ \text { Frequencies -- } & 323.8088 & 335.1502 & 342.6756\end{array}$

Frequencies -- 348.0302

Frequencies -- 376.3722

Frequencies -- 390.4264

Frequencies -- 403.4456

Frequencies -- 418.2840

Frequencies -- 431.5860

Frequencies -- 443.6898

Frequencies -- 489.2393

Frequencies -- 499.9300

Frequencies -- 507.3494

Frequencies -- 531.4561

Frequencies -- 553.7024

Frequencies -- 567.8648

Frequencies -- 581.7082

Frequencies -- 607.2191

Frequencies -- 621.7822

Frequencies -- 646.8146

Frequencies -- 655.9609

Frequencies -- 675.2953

Frequencies -- 690.6130

Frequencies -- 717.5818

Frequencies -- 737.3670

Frequencies -- 755.5741

Frequencies -- 766.1829

Frequencies -- 777.8940

Frequencies -- 799.2568

Frequencies -- 826.0069

Frequencies -- 843.5602

Frequencies -- 863.4429

Frequencies -- 875.1516

Frequencies -- 880.3871

Frequencies -- 887.6031

Frequencies -- 904.4554

Frequencies -- 935.4737

Frequencies -- 942.2707

Frequencies -- 970.5262

Frequencies -- 980.1683

Frequencies -- 991.0763

Frequencies -- 1000.0455

Frequencies -- 1004.2143

Frequencies -- 1012.1540

Frequencies -- 1017.7724

Frequencies -- 1035.6325

Frequencies -- 1048.9068

Frequencies -- 1051.8947

Frequencies -- 1076.0615

Frequencies -- 1092.7190

Frequencies -- 1113.6231

Frequencies -- 1140.4876

Frequencies -- 1162.6507

Frequencies -- 1168.4236

Frequencies -- 1180.2334

Frequencies -- 1191.6763

Frequencies -- 1202.1511

Frequencies -- 1212.5203

Frequencies -- 1221.8054

Frequencies -- 1251.9850

Frequencies -- 1260.0260

Frequencies -- 1281.8947

Frequencies -- 1299.6526

Frequencies -- 1311.6935

Frequencies -- 1322.2652

Frequencies -- 1343.6299

Frequencies -- 1351.5245

Frequencies -- 1360.9678

Frequencies -- 1394.8377
364.2459

378.1480

401.6233

404.4144

421.7637

435.0911

465.8340

489.6744

500.3220

527.2446

540.2328

558.0702

569.4662

598.9080

615.6744

627.5294

648.6060

657.7031

678.1739

708.7502

724.6583

753.3469

756.9753

769.5945

780.0878

811.7829

834.2957

845.6387

870.4640

876.8740

882.7335

889.1928

919.8430

939.6506

949.9769

970.7437

987.1069

991.5181

1003.3715

1005.7877

1015.3630

1021.7243

1045.8136

1050.2112

1058.9168

1078.9306

1101.1077

1122.1658

1141.6130

1164.2103

1175.6100

1182.3552

1195.9131

1204.8382

1213.7271

1243.8686

1255.1677

1270.9080

1286.0454

1300.3937

1316.5070

1328.1579

1346.0561

1355.6557

1367.5345

1407.7174
373.1023

385.6251

402.9252

414.6054

427.0511

438.3692

483.0436

491.4057

502.2567

531.0383

547.9104

565.0884

571.5046

604.5727

618.5900

629.5915

649.4355

661.8259

686.4954

712.0162

731.5878

753.9480

758.2258

772.7788

796.9663

813.5778

836.8434

851.7299

872.7083

877.3987

883.6530

893.0453

928.6232

940.2788

951.0033

979.0682

987.5912

992.0898

1004.1092

1006.7803

1017.5280

1032.2921

1048.1576

1051.3898

1062.9968

1089.6712

1108.6022

1137.9498

1155.8897

1165.1614

1179.8685

1187.2681

1199.9572

1211.5697

1215.5173

1249.0284

1257.2835

1272.2353

1294.7059

1301.6764

1322.1293

1341.0673

1349.3671

1359.7230

1368.8165

1412.8703 


$\begin{array}{llll}\text { Frequencies -- } & 1415.7861 & 1417.8767 & 1436.0030 \\ \text { Frequencies -- } & 1441.7336 & 1443.5532 & 1445.7203 \\ \text { Frequencies -- } 1460.7261 & 1481.2293 & 1483.1770 \\ \text { Frequencies -- } 1483.8697 & 1484.9589 & 1489.7749 \\ \text { Frequencies -- } 1492.5178 & 1493.7722 & 1497.9932 \\ \text { Frequencies -- } 1498.6049 & 1499.5742 & 1500.5380 \\ \text { Frequencies -- } 1503.7740 & 1508.2880 & 1513.8350 \\ \text { Frequencies -- } 1515.9158 & 1517.5794 & 1527.6354 \\ \text { Frequencies -- } 1530.4281 & 1546.2147 & 1549.2087 \\ \text { Frequencies -- } 1553.5968 & 1558.9588 & 1600.6840 \\ \text { Frequencies -- } 1602.7423 & 1647.3838 & 1648.2485 \\ \text { Frequencies -- } 1655.7441 & 1657.9689 & 1662.5323 \\ \text { Frequencies -- } 1665.2244 & 1670.0484 & 1679.8832 \\ \text { Frequencies -- } 1685.5860 & 1698.9403 & 1701.5258 \\ \text { Frequencies -- } 1704.8056 & 1712.2133 & 1713.2606 \\ \text { Frequencies -- } 1717.1468 & 1718.5471 & 3076.4086 \\ \text { Frequencies -- } 3078.3718 & 3083.7863 & 3086.8961 \\ \text { Frequencies -- } 3128.0533 & 3139.7793 & 3142.2891 \\ \text { Frequencies -- } 3150.5401 & 3151.9059 & 3155.7279 \\ \text { Frequencies -- } 3181.9445 & 3197.9959 & 3199.5882 \\ \text { Frequencies -- } 3203.9197 & 3204.0706 & 3204.5608 \\ \text { Frequencies -- } 3205.1085 & 3205.8216 & 3205.9754 \\ \text { Frequencies -- } 3206.3165 & 3211.0690 & 3216.1964 \\ \text { Frequencies -- } 3217.2868 & 3219.9388 & 3220.0349 \\ \text { Frequencies -- } 3221.5546 & 3221.5697 & 3222.1056 \\ \text { Frequencies -- } 3222.9758 & 3226.8328 & 3229.6248 \\ \text { Frequencies -- } 3231.4966 & 3231.6866 & 3232.9464 \\ \text { Frequencies -- } 3233.0624 & 3234.4867 & 3237.0426 \\ \text { Frequencies -- } 3238.9283 & 3241.5722 & 3246.9153 \\ \text { Frequencies -- } 3248.7281 & 3250.6719 & 3252.5307 \\ \text { Frequencies -- } 3254.8938 & 3338.3821 & 3661.2363\end{array}$

SCF Done: $E(R M 062 X / D G D Z V P)=-3995.07397851$

Sum of electronic and zero-point Energies $=\quad-3994.145241$ Sum of electronic and thermal Energies= $\quad-3994.076408$

Sum of electronic and thermal Free Energies $=\quad-3994.252244$ SCF Done: $E(R M 062 X / D G T Z V P / S M D)=-3995.87720105$

\section{Cat a I1c_S}

Center Atomic Atomic Coordinates (Angstroms) Number Number Type $X \quad Y \quad Z$

$\begin{array}{cccccc}1 & 15 & 0 & -0.099252 & -0.038836 & 0.170419 \\ 2 & 8 & 0 & 0.003327 & 0.013208 & 1.799988 \\ 3 & 8 & 0 & 1.462845 & 0.030777 & -0.266241 \\ 4 & 8 & 0 & -0.748575 & 1.188764 & -0.382423 \\ 5 & 8 & 0 & -0.659940 & -1.396600 & -0.127868 \\ 6 & 6 & 0 & 0.418006 & 1.233524 & 2.311737 \\ 7 & 6 & 0 & 2.309689 & -0.938509 & 0.243492 \\ 8 & 6 & 0 & 1.775298 & 1.490269 & 2.445968 \\ 9 & 6 & 0 & -0.547331 & 2.208529 & 2.600964 \\ 10 & 6 & 0 & 2.990912 & -0.663574 & 1.423585 \\ 11 & 6 & 0 & 2.440789 & -2.177994 & -0.403553 \\ 12 & 6 & 0 & 2.984257 & 0.579297 & 2.301601 \\ 13 & 6 & 0 & 2.190615 & 2.780321 & 2.792307 \\ 14 & 6 & 0 & -0.102316 & 3.461119 & 3.037433 \\ 15 & 6 & 0 & 3.826599 & -1.639530 & 1.974285 \\ 16 & 6 & 0 & 3.345381 & -3.103025 & 0.134366 \\ 17 & 6 & 0 & 3.365194 & -0.053751 & 3.672321 \\ 18 & 6 & 0 & 4.063089 & 1.619322 & 1.890665 \\ 19 & 6 & 0 & 3.697792 & 2.884778 & 2.693546 \\ 20 & 6 & 0 & 1.259677 & 3.762502 & 3.111093 \\ 21 & 1 & 0 & -0.842354 & 4.222500 & 3.269944 \\ 22 & 6 & 0 & 4.348484 & -1.182855 & 3.317887\end{array}$

\begin{tabular}{|c|c|c|c|c|c|}
\hline 23 & 6 & 0 & 4.036994 & -2.847072 & 1.318124 \\
\hline 24 & 1 & 0 & 3.464878 & -4.058716 & -0.369258 \\
\hline 25 & 1 & 0 & 3.772978 & 0.683359 & 4.369581 \\
\hline 26 & 1 & 0 & 2.461706 & -0.483021 & 4.118722 \\
\hline 27 & 1 & 0 & 5.079535 & 1.261180 & 2.078722 \\
\hline 28 & 1 & 0 & 3.962382 & 1.821083 & 0.819174 \\
\hline 29 & 1 & 0 & 4.159409 & 2.875942 & 3.687595 \\
\hline 30 & 1 & 0 & 5.373944 & -0.806009 & 3.230047 \\
\hline 31 & 1 & 0 & -0.765563 & 1.341977 & -2.210454 \\
\hline 32 & 8 & 0 & -1.158810 & 1.252342 & -3.101969 \\
\hline 33 & 6 & 0 & -2.548214 & 1.451959 & -2.991198 \\
\hline 34 & 6 & 0 & -3.319173 & 0.225725 & -2.440123 \\
\hline 35 & 1 & 0 & -2.797598 & 2.306112 & -2.344982 \\
\hline 36 & 1 & 0 & -2.910954 & 1.672981 & -3.998422 \\
\hline 37 & 8 & 0 & -3.259051 & 0.152719 & -1.027609 \\
\hline 38 & 6 & 0 & -2.653458 & -1.043827 & -3.017758 \\
\hline 39 & 6 & 0 & -4.784342 & 0.334152 & -2.839178 \\
\hline 40 & 1 & 0 & -2.469370 & 0.643915 & -0.710957 \\
\hline 41 & 1 & 0 & -2.445541 & -0.944817 & -4.088338 \\
\hline 42 & 1 & 0 & -1.694773 & -1.236286 & -2.525801 \\
\hline 43 & 6 & 0 & -5.773868 & 0.427661 & -1.859322 \\
\hline 44 & 6 & 0 & -5.164302 & 0.311204 & -4.186505 \\
\hline 45 & 6 & 0 & -7.120333 & 0.501516 & -2.220584 \\
\hline 46 & 1 & 0 & -5.468345 & 0.434407 & -0.817778 \\
\hline 47 & 6 & 0 & -6.507549 & 0.383366 & -4.547734 \\
\hline 48 & 1 & 0 & -4.410459 & 0.229936 & -4.967438 \\
\hline 49 & 6 & 0 & -7.492569 & 0.479527 & -3.563221 \\
\hline 50 & 1 & 0 & -7.881374 & 0.573888 & -1.448523 \\
\hline 51 & 1 & 0 & -6.786286 & 0.363667 & -5.597012 \\
\hline 52 & 1 & 0 & -8.540156 & 0.535049 & -3.843126 \\
\hline 53 & 1 & 0 & -1.999544 & -1.952090 & -0.434560 \\
\hline 54 & 7 & 0 & -2.821857 & -2.653963 & -0.354942 \\
\hline 55 & 6 & 0 & -3.635437 & -3.047460 & -1.303526 \\
\hline 56 & 6 & 0 & -2.996607 & -3.292279 & 0.869198 \\
\hline 57 & 16 & 0 & -3.696985 & -2.545828 & $\begin{array}{ll}3 & -2.950824\end{array}$ \\
\hline 58 & 16 & 0 & -4.744536 & -4.272353 & $\begin{array}{ll}3 & -0.763513\end{array}$ \\
\hline 59 & 6 & 0 & -4.012344 & -4.251999 & 0.825898 \\
\hline 60 & 6 & 0 & -2.260212 & -3.049582 & 2.031283 \\
\hline 61 & 6 & 0 & -4.317311 & -5.022949 & 1.948594 \\
\hline 62 & 6 & 0 & -2.565982 & -3.821466 & 3.143271 \\
\hline 63 & 1 & 0 & -1.483007 & -2.288492 & 2.038542 \\
\hline 64 & 6 & 0 & -3.576460 & -4.797426 & 3.103950 \\
\hline 65 & 1 & 0 & -5.102298 & -5.771511 & 1.922139 \\
\hline 66 & 1 & 0 & -2.013996 & -3.660545 & 4.063659 \\
\hline 67 & 1 & 0 & -3.790750 & -5.383550 & 3.991763 \\
\hline 68 & 1 & 0 & 4.012985 & 3.805469 & 2.196112 \\
\hline 69 & 1 & 0 & 4.357724 & -1.988161 & 4.056719 \\
\hline 70 & 1 & 0 & 1.581035 & 4.762241 & 3.390267 \\
\hline 71 & 1 & 0 & 4.700642 & -3.598866 & 1.736426 \\
\hline 72 & 6 & 0 & -1.996399 & 1.922441 & 2.387362 \\
\hline 73 & 6 & 0 & -2.716189 & 2.644877 & 1.411490 \\
\hline 74 & 6 & 0 & -2.639521 & 0.916437 & 3.137328 \\
\hline 75 & 6 & 0 & -2.094002 & 3.615956 & 0.556503 \\
\hline 76 & 6 & 0 & -4.112003 & 2.374034 & 1.210967 \\
\hline 77 & 6 & 0 & -4.024592 & 0.623035 & 2.896400 \\
\hline 78 & 6 & 0 & -1.966030 & 0.170472 & 4.162122 \\
\hline 79 & 6 & 0 & -2.817974 & 4.300175 & -0.377361 \\
\hline 80 & 1 & 0 & -1.023496 & 3.770382 & 0.629834 \\
\hline 81 & 6 & 0 & -4.839135 & 3.116488 & 0.224048 \\
\hline 82 & 6 & 0 & -4.731326 & 1.365560 & 1.949475 \\
\hline 83 & 6 & 0 & -4.663118 & -0.417416 & 3.646638 \\
\hline 84 & 1 & 0 & -0.927023 & 0.396528 & 4.381537 \\
\hline 85 & 6 & 0 & -2.614510 & -0.798592 & 4.873626 \\
\hline 86 & 6 & 0 & -4.217616 & 4.058581 & -0.541287 \\
\hline 87 & 1 & 0 & -2.321184 & 5.017898 & -1.023314 \\
\hline 88 & 1 & 0 & -5.897144 & 2.905052 & 0.089647 \\
\hline
\end{tabular}




\begin{tabular}{|c|c|c|c|c|c|}
\hline 89 & 1 & 0 & -5.785648 & 1.151575 & 1.781481 \\
\hline 90 & 6 & 0 & -3.983972 & -1.109422 & 4.605951 \\
\hline 91 & 1 & 0 & -5.706213 & -0.639449 & 3.436133 \\
\hline 92 & 1 & 0 & -2.087396 & -1.336707 & 5.656570 \\
\hline 93 & 1 & 0 & -4.775169 & 4.608727 & -1.293233 \\
\hline 94 & 1 & 0 & -4.479035 & -1.894009 & 5.170734 \\
\hline 95 & 6 & 0 & 1.582036 & -2.555811 & -1.564219 \\
\hline 96 & 6 & 0 & 0.653555 & -3.610312 & -1.411157 \\
\hline 97 & 6 & 0 & 1.649021 & -1.844693 & -2.776817 \\
\hline 98 & 6 & 0 & 0.442747 & -4.282297 & -0.160271 \\
\hline 99 & 6 & 0 & -0.188612 & -3.980009 & -2.512551 \\
\hline 100 & 6 & 0 & 0.781409 & -2.204188 & -3.864482 \\
\hline 101 & 6 & 0 & 2.554574 & -0.749941 & -2.975212 \\
\hline 102 & 6 & 0 & -0.482217 & -5.280561 & -0.035437 \\
\hline 103 & 1 & 0 & 1.005478 & -3.964273 & 0.710946 \\
\hline 104 & 6 & 0 & -1.133863 & -5.046211 & -2.351645 \\
\hline 105 & 6 & 0 & -0.103335 & -3.272750 & -3.715066 \\
\hline 106 & 6 & 0 & 0.832252 & -1.449684 & -5.081391 \\
\hline 107 & 1 & 0 & 3.232909 & -0.476140 & -2.173967 \\
\hline 108 & 6 & 0 & 2.568682 & -0.050382 & -4.146609 \\
\hline 109 & 6 & 0 & -1.274293 & -5.685999 & -1.153366 \\
\hline 110 & 1 & 0 & -0.635281 & -5.757539 & 0.928883 \\
\hline 111 & 1 & 0 & -1.743704 & -5.327538 & -3.207086 \\
\hline 112 & 1 & 0 & -0.750476 & -3.552586 & -4.545293 \\
\hline 113 & 6 & 0 & 1.689499 & -0.398770 & -5.217487 \\
\hline 114 & 1 & 0 & 0.164409 & -1.730842 & -5.892083 \\
\hline 115 & 1 & 0 & 3.253482 & 0.782823 & -4.269515 \\
\hline 116 & 1 & 0 & -1.993164 & -6.492742 & -1.040312 \\
\hline 117 & 1 & 0 & 1.709269 & 0.178282 & -6.136557 \\
\hline
\end{tabular}

Frequencies -- $\quad 17.7067$

Frequencies -- 29.4337

Frequencies -- 41.0607

Frequencies -- 57.3580

Frequencies -- 72.0528

Frequencies -- 89.0162

Frequencies -- 102.3017

Frequencies -- 120.8958

Frequencies -- 142.8368

Frequencies -- $\quad 152.1104$

Frequencies -- 170.2345

Frequencies -- 194.3112

Frequencies -- 217.8155

Frequencies -- 240.3169

Frequencies -- 255.3940

Frequencies -- 290.6072

Frequencies -- 301.8843

Frequencies -- 331.3119

Frequencies -- 349.9681

Frequencies -- 377.7075

Frequencies -- 397.0550

Frequencies -- 404.0633

Frequencies -- 421.1999

Frequencies -- 431.6659

Frequencies -- 467.4185

Frequencies -- 490.1071

Frequencies -- 501.1755

Frequencies -- 523.9279

Frequencies -- 540.3479

Frequencies -- 558.0520

Frequencies -- 571.2824

Frequencies -- 605.1959

Frequencies -- 616.0223

Frequencies -- 623.7576

Frequencies -- 644.9710

$\begin{array}{ll}20.3034 & 23.5363 \\ 32.4795 & 37.7710 \\ 45.9336 & 54.0417 \\ 60.0555 & 62.5943 \\ 77.2387 & 85.1183 \\ 92.4961 & 97.2450 \\ 109.4057 & 111.4690 \\ 123.9491 & 134.2329 \\ 143.4978 & 144.7883 \\ 160.1031 & 168.6409 \\ 176.5500 & 188.2395 \\ 205.8561 & 214.3964 \\ 230.9298 & 233.5958 \\ 244.1640 & 246.1656 \\ 267.4112 & 275.0492 \\ 294.9565 & 296.8315 \\ 307.7581 & 322.7314 \\ 333.9378 & 343.9169 \\ 362.1675 & 372.2262 \\ 379.4176 & 384.6112 \\ 402.7821 & 403.1799 \\ 408.5516 & 419.2939 \\ 425.6878 & 428.3397 \\ 437.7124 & 441.9903 \\ 483.1294 & 488.0064 \\ 492.5282 & 500.0736 \\ 502.8606 & 507.7678 \\ 527.7251 & 531.3632 \\ 550.1233 & 555.2115 \\ 564.3496 & 568.2656 \\ 579.4006 & 588.1415 \\ 607.2949 & 608.1151 \\ 616.4104 & 621.2442 \\ 627.7764 & 630.5833 \\ 649.5693 & 650.4373\end{array}$

Frequencies -- 654.1157

Frequencies -- 672.8317

Frequencies -- 703.6430

Frequencies -- 718.2129

Frequencies -- 733.3267

Frequencies -- 754.5557

Frequencies -- 757.3406

Frequencies -- 779.8534

Frequencies -- 798.1157

Frequencies -- 816.2122

Frequencies -- 842.1079

Frequencies -- 859.8108

Frequencies -- 873.7095

Frequencies -- 880.1509

Frequencies -- 884.6191

Frequencies -- 891.0197

Frequencies -- 934.6615

Frequencies -- 941.2169

Frequencies -- 969.0912

Frequencies -- 981.1025

Frequencies -- 991.1270

Frequencies -- 1002.1714

Frequencies -- 1005.7021

Frequencies -- 1018.5445

Frequencies -- 1025.0433

Frequencies -- 1036.7408

Frequencies -- 1049.3565

Frequencies -- 1059.1701

Frequencies -- 1079.3639

Frequencies -- 1104.6263

Frequencies -- 1121.2229

Frequencies -- 1148.2461

Frequencies -- 1164.2855

Frequencies -- 1171.1509

Frequencies -- 1180.7355

Frequencies -- 1200.2566

Frequencies -- 1207.1550

Frequencies -- 1212.8382

Frequencies -- 1248.9804

Frequencies -- 1256.3861

Frequencies -- 1272.6885

Frequencies -- 1290.1189

Frequencies -- 1302.1820

Frequencies -- 1312.1740

Frequencies -- 1323.0861

Frequencies -- 1343.5545

Frequencies -- 1352.2601

Frequencies -- 1362.3607

Frequencies -- 1404.0720

Frequencies -- 1415.0078

Frequencies -- 1444.1477

Frequencies -- 1484.3918

Frequencies -- 1486.3361

Frequencies -- 1493.8166

Frequencies -- 1498.4668

Frequencies -- 1502.9040

Frequencies -- 1512.6528

Frequencies -- 1531.9389

Frequencies -- 1551.4047

Frequencies -- 1602.6773

Frequencies -- 1656.4039

Frequencies -- 1665.6812

Frequencies -- 1681.3211

Frequencies -- 1707.4701

Frequencies -- 1718.9801

Frequencies -- 3072.7485
657.5029

683.0997

709.2591

724.9513

736.8818

755.4388

776.2065

785.6584

800.2219

826.0879

845.2287

866.2272

874.5451

881.5021

887.3333

914.4330

939.2582

951.5230

969.6228

986.2333

992.9552

1002.8804

1009.5022

1019.5086

1025.9906

1046.9303

1049.7033

1061.0216

1091.2707

1112.4053

1140.2438

1160.7418

1164.5267

1174.2646

1188.4656

1203.5895

1209.6311

1213.7444

1251.8591

1258.2068

1276.0782

1297.5501

1304.2379

1316.8218

1329.6168

1347.7028

1360.1582

1368.8648

1408.2206

1417.8403

1444.6316

1485.0456

1491.0494

1494.0345

1500.0726

1507.3528

1517.8811

1546.2806

1593.5452

1648.6729

1659.2419

1671.3855

1696.5692

1711.4762

1720.1492

3074.8432
661.8400

690.6838

711.5789

727.8205

751.6204

756.4150

779.0810

790.0162

812.1534

836.2815

851.1409

868.7592

879.3053

883.1622

890.7536

917.3020

940.1202

958.2114

973.6672

987.6148

996.0670

1004.2316

1009.6217

1021.3043

1030.3883

1047.2493

1053.1506

1070.7730

1103.7624

1115.0310

1141.5319

1163.8284

1165.3065

1177.6041

1194.8736

1206.9168

1212.1879

1223.6773

1255.0077

1260.6628

1283.6305

1298.8231

1308.6938

1322.0877

1342.1242

1349.0850

1361.3052

1398.8179

1412.7859

1433.0186

1456.3063

1485.5712

1492.5772

1495.0923

1500.4292

1510.0287

1529.2841

1547.9073

1602.0226

1649.1908

1662.6274

1672.8371

1701.8355

1715.4550

2430.1352

3076.7494 


$\begin{array}{llll}\text { Frequencies -- } 3085.6595 & 3086.8005 & 3103.9014 \\ \text { Frequencies -- } 3138.4607 & 3140.3148 & 3154.2514 \\ \text { Frequencies -- } 3156.1886 & 3158.9734 & 3172.0989 \\ \text { Frequencies -- } 3185.8552 & 3190.4496 & 3198.2909 \\ \text { Frequencies -- } 3200.7418 & 3202.3765 & 3205.7315 \\ \text { Frequencies -- } 3207.2067 & 3209.8473 & 3212.4919 \\ \text { Frequencies -- } 3214.8121 & 3215.8662 & 3216.2893 \\ \text { Frequencies -- } 3221.1924 & 3221.4576 & 3221.6093 \\ \text { Frequencies -- } 3224.3598 & 3225.5645 & 3229.9295 \\ \text { Frequencies -- } 3230.8342 & 3231.0604 & 3231.9582 \\ \text { Frequencies -- } 3234.5858 & 3235.1611 & 3237.4464 \\ \text { Frequencies -- } 3240.5980 & 3243.5009 & 3243.8301 \\ \text { Frequencies -- } 3246.9208 & 3251.8443 & 3253.8019 \\ \text { Frequencies -- } 3254.8918 & 3566.0728 & 3657.3390\end{array}$

SCF Done: $E(R M 062 X / D G D Z V P)=-3995.13332598$

Sum of electronic and zero-point Energies $=\quad-3994.203812$

Sum of electronic and thermal Energies= $\quad-3994.134984$

Sum of electronic and thermal Free Energies $=\quad-3994.308549$

SCF Done: $E(R M 062 X / D G T Z V P / S M D)=-3995.93678555$

\section{Cat a TS1d_R}

\begin{tabular}{|c|c|c|c|c|c|}
\hline \multirow{2}{*}{$\begin{array}{l}\text { Center } \\
\text { Number }\end{array}$} & \multicolumn{2}{|c|}{ Atomic } & Atomic & \multicolumn{2}{|c|}{ Coordinates (Angstroms } \\
\hline & & & Type & $\begin{array}{ll}X & Y\end{array}$ & z \\
\hline 1 & 15 & 0 & 0.040215 & -0.015199 & -0.102558 \\
\hline 2 & 8 & 0 & -0.035096 & -0.043652 & 1.533309 \\
\hline 3 & 8 & 0 & 1.640125 & -0.042180 & -0.365208 \\
\hline 4 & 8 & 0 & -0.480113 & 1.266343 & -0.665617 \\
\hline 5 & 8 & 0 & -0.599425 & -1.323303 & -0.478485 \\
\hline 6 & 6 & 0 & 0.375990 & 1.125025 & 2.154508 \\
\hline 7 & 6 & 0 & 2.349454 & -1.080472 & 0.220799 \\
\hline 8 & 6 & 0 & 1.721690 & 1.321588 & 2.412362 \\
\hline 9 & 6 & 0 & -0.581022 & 2.102485 & 2.444880 \\
\hline 10 & 6 & 0 & 2.892857 & -0.882548 & 1.486275 \\
\hline 11 & 6 & 0 & 2.474653 & -2.311248 & -0.443860 \\
\hline 12 & 6 & 0 & 2.880361 & 0.345227 & 2.383646 \\
\hline 13 & 6 & 0 & 2.156011 & 2.578103 & 2.841702 \\
\hline 14 & 6 & 0 & -0.130264 & 3.325713 & 2.952782 \\
\hline 15 & 6 & 0 & 3.552053 & -1.941668 & 2.120102 \\
\hline 16 & 6 & 0 & 3.191282 & -3.331071 & 0.196425 \\
\hline 17 & 6 & 0 & 3.043398 & -0.329422 & 3.778069 \\
\hline 18 & 6 & 0 & 4.058781 & 1.323463 & 2.126282 \\
\hline 19 & 6 & 0 & 3.669705 & 2.603883 & 2.899007 \\
\hline 20 & 6 & 0 & 1.234398 & 3.582773 & 3.118994 \\
\hline 21 & 1 & 0 & -0.862077 & 4.095028 & 3.185139 \\
\hline 22 & 6 & 0 & 3.954455 & -1.541462 & 3.521552 \\
\hline 23 & 6 & 0 & 3.721071 & -3.162204 & 1.475575 \\
\hline 24 & 1 & 0 & 3.298281 & -4.283660 & -0.315498 \\
\hline 25 & 1 & 0 & 3.431441 & 0.363486 & 4.529898 \\
\hline 26 & 1 & 0 & 2.058875 & -0.678109 & 4.112224 \\
\hline 27 & 1 & 0 & 5.021376 & 0.905550 & 2.435237 \\
\hline 28 & 1 & 0 & 4.104752 & 1.539833 & 1.054042 \\
\hline 29 & 1 & 0 & 4.021518 & 2.568387 & 3.936185 \\
\hline 30 & 1 & 0 & 5.013310 & -1.258909 & 3.551425 \\
\hline 31 & 1 & 0 & -1.959780 & 1.297876 & -1.275443 \\
\hline 32 & 8 & 0 & -2.822945 & 1.013042 & -1.727052 \\
\hline 33 & 6 & 0 & -2.490208 & 0.047436 & -2.757659 \\
\hline 34 & 6 & 0 & -3.265718 & -1.150824 & -2.180059 \\
\hline 35 & 1 & 0 & -2.835586 & 0.408746 & -3.726501 \\
\hline 36 & 1 & 0 & -1.412812 & -0.136589 & -2.774796 \\
\hline 37 & 8 & 0 & -2.522299 & -2.322330 & -2.086264 \\
\hline 38 & 6 & 0 & -3.570925 & -0.423779 & -0.835346 \\
\hline 39 & 6 & 0 & -4.558212 & -1.444758 & -2.917512 \\
\hline
\end{tabular}




$\begin{array}{llllll}106 & 6 & 0 & 1.957234 & -1.251680 & -5.293505 \\ 107 & 1 & 0 & 3.773348 & -0.642059 & -1.903743 \\ 108 & 6 & 0 & 3.601022 & -0.094815 & -3.948316 \\ 109 & 6 & 0 & -1.315854 & -5.394403 & -2.143501 \\ 110 & 1 & 0 & -1.099664 & -5.651799 & 0.012528 \\ 111 & 1 & 0 & -1.335629 & -4.879205 & -4.214309 \\ 112 & 1 & 0 & 0.078911 & -3.167264 & -5.209349 \\ 113 & 6 & 0 & 2.938397 & -0.309497 & -5.196315 \\ 114 & 1 & 0 & 1.450689 & -1.430476 & -6.238513 \\ 115 & 1 & 0 & 4.385760 & 0.652466 & -3.882458 \\ 116 & 1 & 0 & -2.132778 & -6.104689 & -2.223922 \\ 117 & 1 & 0 & 3.223542 & 0.277586 & -6.063810\end{array}$

Frequencies -- -546.9229

Frequencies -- 21.6014

Frequencies -- 38.8182

Frequencies -- 52.5249

Frequencies -- 63.5817

Frequencies -- 83.1890

Frequencies -- 95.1214

Frequencies -- 107.7791

Frequencies -- 131.5560

Frequencies -- 146.1605

Frequencies -- 171.8629

Frequencies -- 191.6912

Frequencies -- 207.1293

Frequencies -- 236.9797

Frequencies -- 248.8517

Frequencies -- 282.3679

Frequencies -- 297.9338

Frequencies -- 322.7981

Frequencies -- 359.7975

Frequencies -- 380.4817

Frequencies -- 395.9938

Frequencies -- 404.0273

Frequencies -- 419.1563

Frequencies -- 431.7535

Frequencies -- 450.6535

Frequencies -- 487.7457

Frequencies -- 501.7243

Frequencies -- 525.0763

Frequencies -- 534.4854

Frequencies -- 556.9225

Frequencies -- 568.7078

Frequencies -- 583.4612

Frequencies -- 615.6400

Frequencies -- 626.4422

Frequencies -- 648.6918

Frequencies -- 657.4070

Frequencies -- 674.8181

Frequencies -- 690.8013

Frequencies -- 720.2814

Frequencies -- 735.2365

Frequencies -- 753.8190

Frequencies -- 758.1267

Frequencies -- 776.1747

Frequencies -- 800.8283

Frequencies -- 826.9421

Frequencies -- 847.5680

Frequencies -- 860.7560

Frequencies -- 873.1820

Frequencies -- 876.6378

Frequencies -- 887.7009

Frequencies -- 911.3640

Frequencies -- 939.2143
33.6200

$43.3286 \quad 43.9461$

$56.1444 \quad 58.7776$

$67.5095 \quad 77.9058$

$87.1121 \quad 92.3002$

$100.4146 \quad 105.6313$

$118.5499 \quad 127.5688$

$139.3343 \quad 144.7295$

$155.8061 \quad 160.9130$

$181.6779 \quad 187.2917$

$197.2324 \quad 202.1441$

$215.6561 \quad 225.0466$

$239.4253 \quad 244.3256$

$255.0354 \quad 273.8406$

$291.1119 \quad 294.7927$

$299.2538 \quad 308.7122$

$336.2838 \quad 348.3247$

$366.9339 \quad 377.4699$

$386.6753 \quad 394.1331$

$400.8031 \quad 403.7217$

$411.9814 \quad 418.5308$

$424.8620 \quad 427.4271$

$434.8668 \quad 440.7849$

$455.0650 \quad 486.9556$

$488.1478 \quad 498.8205$

$506.9579 \quad 508.2196$

$531.1117 \quad 532.8191$

$540.9834 \quad 549.8774$

$565.0236 \quad 568.6685$

$574.8850 \quad 579.9001$

$604.8318 \quad 607.1723$

$616.7434 \quad 621.7609$

$628.5935 \quad 645.0383$

$649.5756 \quad 652.1529$

$660.7255 \quad 662.7607$

$676.9875 \quad 689.9115$

$708.7356 \quad 711.0703$

$724.3674 \quad 732.0070$

$748.8889 \quad 753.0827$

$757.1932 \quad 757.4821$

$764.7614 \quad 774.9465$

$780.6825 \quad 796.6152$

$813.0824 \quad 813.3449$

$839.1210 \quad 842.0058$

$850.3228 \quad 857.5618$

$869.8352 \quad 871.1598$

$874.3833 \quad 875.2627$

$881.4425 \quad 882.3449$

$890.1350 \quad 899.5462$

$921.0727 \quad 926.2399$

$939.6217 \quad 943.3880$
Frequencies -- 946.1901

Frequencies -- 953.8575

Frequencies -- 985.9604

Frequencies -- 990.7057

Frequencies -- 995.9844

Frequencies -- 1005.4284

Frequencies -- 1013.0204

Frequencies -- 1020.7455

Frequencies -- 1044.0822

Frequencies -- 1050.0271

Frequencies -- 1056.3430

Frequencies -- 1078.1064

Frequencies -- 1098.5760

Frequencies -- 1114.6912

Frequencies -- 1140.1223

Frequencies -- 1161.8705

Frequencies -- 1169.3548

Frequencies -- 1180.3776

Frequencies -- 1193.3419

Frequencies -- 1201.6289

Frequencies -- 1210.2721

Frequencies -- 1221.8202

Frequencies -- 1253.1537

Frequencies -- 1260.1675

Frequencies -- 1282.5077

Frequencies -- 1293.9827

Frequencies -- 1309.4834

Frequencies -- 1322.6929

Frequencies -- 1343.4629

Frequencies -- 1348.9559

Frequencies -- 1367.7728

Frequencies -- 1385.0309

Frequencies -- 1413.4474

Frequencies -- 1436.8421

Frequencies -- 1459.5104

Frequencies -- 1483.1451

Frequencies -- 1490.9439

Frequencies -- 1498.4463

Frequencies -- 1510.9920

Frequencies -- 1523.2056

Frequencies -- 1531.6304

Frequencies -- 1551.8375

Frequencies -- 1602.2635

Frequencies -- 1655.1054

Frequencies -- 1665.5339

Frequencies -- 1684.4831

Frequencies -- 1710.7812

Frequencies -- 1717.7226

Frequencies -- 3077.8180

Frequencies -- 3088.8371

Frequencies -- 3141.6677

Frequencies -- 3169.0914

Frequencies -- 3199.2470

Frequencies -- 3207.8865

Frequencies -- 3209.6673

Frequencies -- 3215.4781

Frequencies -- 3218.4139

Frequencies -- 3223.7147

Frequencies -- 3231.1537

Frequencies -- 3235.9170

Frequencies -- 3239.2738

Frequencies -- 3246.2140

Frequencies -- 3256.2916
951.6654

972.1999

987.9375

993.2396

1003.0952

1008.0665

1015.7820

1027.0848

1046.4607

1050.8931

1060.8714

1080.2263

1105.3131

1119.4645

1140.3856

1163.8397

1177.2246

1187.4562

1197.6683

1205.9032

1212.2382

1234.1596

1255.6527

1271.2864

1288.3598

1298.2896

1317.4977

1328.2282

1346.4320

1352.0330

1369.3603

1407.4067

1414.9464

1443.3679

1474.7218

1487.3420

1493.1777

1500.6867

1513.0573

1529.8654

1548.0203

1552.6978

1646.9706

1657.7309

1672.6008

1699.9978

1712.8065

1717.8359

3079.7952

3125.6237

3154.0847

3188.8066

3200.2636

3208.3791

3215.0886

3217.7116

3218.7636

3226.3795

3231.2254

3236.0284

3243.9853

3247.2497

3350.2118

953.2255

975.9932

989.2715

993.5957

1004.9705

1010.9292

1019.3691

1034.7297

1048.1567

1053.2985

1064.2871

1092.3435

1109.3828

1133.3169

1160.8780

1165.1361

1180.2008

1188.0090

1201.2396

1207.1580

1213.9897

1251.0058

1258.5542

1277.8198

1293.0393

1304.4194

1322.1083

1343.1491

1347.0432

1361.4580

1373.7664

1412.1946

1424.9054

1443.6232

1482.7498

1490.0895

1498.0098

1501.0940

1516.7820

1530.7163

1550.6114

1601.4263

1648.6168

1661.0823

1677.3630

1701.7496

1713.2086

2961.2414

3081.5772

3137.9989

3158.3254

3191.2731

3201.3377

3208.9531

3215.3963

3218.2905

3220.1341

3227.3060

3235.1310 
Sum of electronic and thermal Energies=

Sum of electronic and thermal Free Energies $=\quad-3994.261394$ SCF Done: E(RM062X/DGTZVP/SMD $)=-3995.88726983$

\section{Cat a I1d_R}

Center Atomic Atomic Coordinates (Angstroms) Number Number Type $X \quad$ Y Z

\begin{tabular}{|c|c|c|c|c|c|}
\hline 1 & 15 & 0 & 0.034660 & -0.036579 & -0.092191 \\
\hline 2 & 8 & 0 & -0.038372 & -0.070895 & 1.551206 \\
\hline 3 & 8 & 0 & 1.639692 & -0.061156 & -0.347674 \\
\hline 4 & 8 & 0 & -0.514356 & 1.211113 & -0.667099 \\
\hline 5 & 8 & 0 & -0.555157 & -1.392033 & -0.429948 \\
\hline 6 & 6 & 0 & 0.364393 & 1.104047 & 2.164419 \\
\hline 7 & 6 & 0 & 2.356501 & -1.090720 & 0.237083 \\
\hline 8 & 6 & 0 & 1.709225 & 1.308837 & 2.424313 \\
\hline 9 & 6 & 0 & -0.595529 & 2.082728 & 2.440032 \\
\hline 10 & 6 & 0 & 2.896624 & -0.889778 & 1.503642 \\
\hline 11 & 6 & 0 & 2.498128 & -2.319425 & -0.430110 \\
\hline 12 & 6 & 0 & 2.873845 & 0.338629 & 2.399734 \\
\hline 13 & 6 & 0 & 2.137587 & 2.570375 & 2.844635 \\
\hline 14 & 6 & 0 & -0.150546 & 3.312901 & 2.936398 \\
\hline 15 & 6 & 0 & 3.566434 & -1.942391 & 2.137429 \\
\hline 16 & 6 & 0 & 3.226557 & -3.331478 & 0.208795 \\
\hline 17 & 6 & 0 & 3.043009 & -0.332818 & 3.794664 \\
\hline 18 & 6 & 0 & 4.046967 & 1.322517 & 2.138613 \\
\hline 19 & 6 & 0 & 3.650802 & 2.603577 & 2.905872 \\
\hline 20 & 6 & 0 & 1.212122 & 3.575321 & 3.107650 \\
\hline 21 & 1 & 0 & -0.885820 & 4.083263 & 3.153712 \\
\hline 22 & 6 & 0 & 3.963976 & -1.537984 & 3.539358 \\
\hline 23 & 6 & 0 & 3.751115 & -3.159518 & 1.490309 \\
\hline 24 & 1 & 0 & 3.349847 & -4.280453 & -0.306290 \\
\hline 25 & 1 & 0 & 3.425949 & 0.364000 & 4.545492 \\
\hline 26 & 1 & 0 & 2.061458 & -0.688803 & 4.130001 \\
\hline 27 & 1 & 0 & 5.012071 & 0.911125 & 2.448648 \\
\hline 28 & 1 & 0 & 4.090977 & 1.534891 & 1.065503 \\
\hline 29 & 1 & 0 & 4.000204 & 2.572884 & 3.944101 \\
\hline 30 & 1 & 0 & 5.020409 & -1.246774 & 3.570033 \\
\hline 31 & 1 & 0 & -2.137362 & 1.516772 & -1.639919 \\
\hline 32 & 8 & 0 & -2.850395 & 1.228673 & -2.246014 \\
\hline 33 & 6 & 0 & -2.490790 & -0.055183 & -2.717299 \\
\hline 34 & 6 & 0 & -3.313495 & -1.196746 & -2.050549 \\
\hline 35 & 1 & 0 & -2.661687 & -0.097001 & -3.797356 \\
\hline 36 & 1 & 0 & -1.429065 & -0.248379 & -2.536333 \\
\hline 37 & 8 & 0 & -2.567680 & -2.394989 & -2.044049 \\
\hline 38 & 6 & 0 & -3.696812 & -0.767664 & -0.614931 \\
\hline 39 & 6 & 0 & -4.599825 & -1.458161 & -2.820450 \\
\hline 40 & 1 & 0 & -1.666591 & -2.184074 & -1.725696 \\
\hline 41 & 1 & 0 & -2.851577 & -0.424470 & -0.009313 \\
\hline 42 & 1 & 0 & -4.418242 & 0.050946 & -0.641237 \\
\hline 43 & 6 & 0 & -4.958946 & -2.751474 & -3.202393 \\
\hline 44 & 6 & 0 & -5.442825 & -0.385697 & -3.139426 \\
\hline 45 & 6 & 0 & -6.152587 & -2.972147 & -3.892428 \\
\hline 46 & 1 & 0 & -4.292322 & -3.572717 & -2.959472 \\
\hline 47 & 6 & 0 & -6.632014 & -0.609312 & -3.829743 \\
\hline 48 & 1 & 0 & -5.151103 & 0.626442 & -2.861235 \\
\hline 49 & 6 & 0 & -6.992590 & -1.905057 & -4.206045 \\
\hline 50 & 1 & 0 & -6.425510 & -3.981474 & -4.186553 \\
\hline 51 & 1 & 0 & -7.275226 & 0.228986 & -4.080394 \\
\hline 52 & 1 & 0 & -7.919746 & -2.079019 & -4.743854 \\
\hline 53 & 1 & 0 & 1.602594 & -3.823804 & 3.762172 \\
\hline 54 & 6 & 0 & 0.545394 & -3.910715 & 3.528538 \\
\hline 55 & 6 & 0 & 0.013766 & -3.111619 & 2.525231 \\
\hline 56 & 6 & 0 & -0.254879 & -4.823606 & 4.236080 \\
\hline
\end{tabular}

\begin{tabular}{|c|c|c|c|c|c|}
\hline 57 & 6 & 0 & -1.347478 & -3.252906 & 2.244035 \\
\hline 58 & 1 & 0 & 0.621128 & -2.394123 & 1.978625 \\
\hline 59 & 6 & 0 & -1.609425 & -4.963082 & 3.951190 \\
\hline 60 & 7 & 0 & -2.063699 & -2.541080 & 1.284015 \\
\hline 61 & 6 & 0 & -2.146461 & -4.163149 & 2.941385 \\
\hline 62 & 1 & 0 & -2.226334 & -5.668204 & 4.498444 \\
\hline 63 & 6 & 0 & -3.348814 & -2.830900 & 1.231283 \\
\hline 64 & 1 & 0 & -1.502318 & -1.954750 & 0.549319 \\
\hline 65 & 16 & 0 & -3.792324 & -4.087355 & 2.351429 \\
\hline 66 & 16 & 0 & -4.570838 & -2.113327 & 0.263429 \\
\hline 67 & 1 & 0 & 0.187787 & -5.432995 & 5.017101 \\
\hline 68 & 1 & 0 & 4.061485 & 3.508609 & 2.451573 \\
\hline 69 & 1 & 0 & 1.538205 & 4.557734 & 3.437811 \\
\hline 70 & 1 & 0 & 3.827280 & -2.341986 & 4.267894 \\
\hline 71 & 1 & 0 & 4.280211 & -3.974053 & 1.977896 \\
\hline 72 & 6 & 0 & -2.038875 & 1.787388 & 2.212144 \\
\hline 73 & 6 & 0 & -2.664429 & 0.763111 & 2.957236 \\
\hline 74 & 6 & 0 & -2.771269 & 2.512526 & 1.250860 \\
\hline 75 & 6 & 0 & -1.956697 & -0.049035 & 3.906200 \\
\hline 76 & 6 & 0 & -4.064109 & 0.504480 & 2.769561 \\
\hline 77 & 6 & 0 & -4.175282 & 2.255837 & 1.083652 \\
\hline 78 & 6 & 0 & -2.152826 & 3.469223 & 0.377384 \\
\hline 79 & 6 & 0 & -2.582804 & -1.053763 & 4.588422 \\
\hline 80 & 1 & 0 & -0.902299 & 0.136478 & 4.083060 \\
\hline 81 & 6 & 0 & -4.691434 & -0.544195 & 3.516835 \\
\hline 82 & 6 & 0 & -4.791498 & 1.269498 & 1.854623 \\
\hline 83 & 6 & 0 & -4.909784 & 2.984921 & 0.091964 \\
\hline 84 & 1 & 0 & -1.080709 & 3.619343 & 0.439233 \\
\hline 85 & 6 & 0 & -2.883527 & 4.130349 & -0.567313 \\
\hline 86 & 6 & 0 & -3.976284 & -1.305720 & 4.395623 \\
\hline 87 & 1 & 0 & -2.019250 & -1.662217 & 5.290634 \\
\hline 88 & 1 & 0 & -5.752202 & -0.724813 & 3.360532 \\
\hline 89 & 1 & 0 & -5.853237 & 1.069725 & 1.717709 \\
\hline 90 & 6 & 0 & -4.285690 & 3.894084 & -0.709151 \\
\hline 91 & 1 & 0 & -5.971652 & 2.779790 & -0.020174 \\
\hline 92 & 1 & 0 & -2.390537 & 4.824458 & -1.240548 \\
\hline 93 & 1 & 0 & -4.462702 & -2.099196 & 4.955606 \\
\hline 94 & 1 & 0 & -4.842691 & 4.424615 & -1.474834 \\
\hline 95 & 6 & 0 & 1.857729 & -2.561842 & -1.755022 \\
\hline 96 & 6 & 0 & 0.850256 & -3.544298 & -1.873203 \\
\hline 97 & 6 & 0 & 2.223768 & -1.784438 & -2.870943 \\
\hline 98 & 6 & 0 & 0.373845 & -4.307456 & -0.755083 \\
\hline 99 & 6 & 0 & 0.201825 & -3.747884 & -3.137263 \\
\hline 100 & 6 & 0 & 1.555853 & -1.984603 & -4.126641 \\
\hline 101 & 6 & 0 & 3.251168 & -0.784537 & -2.810197 \\
\hline 102 & 6 & 0 & -0.642315 & -5.210982 & -0.890663 \\
\hline 103 & 1 & 0 & 0.817486 & -4.149986 & 0.221590 \\
\hline 104 & 6 & 0 & -0.842163 & -4.723634 & -3.246811 \\
\hline 105 & 6 & 0 & 0.563907 & -2.960002 & -4.231984 \\
\hline 106 & 6 & 0 & 1.917476 & -1.173269 & -5.251128 \\
\hline 107 & 1 & 0 & 3.779637 & -0.626302 & -1.875694 \\
\hline 108 & 6 & 0 & 3.571343 & -0.034189 & -3.903864 \\
\hline 109 & 6 & 0 & -1.255333 & -5.436830 & -2.161082 \\
\hline 110 & 1 & 0 & -0.989332 & -5.762412 & -0.020009 \\
\hline 111 & 1 & 0 & -1.321280 & -4.856557 & -4.213096 \\
\hline 112 & 1 & 0 & 0.053928 & -3.102233 & -5.182683 \\
\hline 113 & 6 & 0 & 2.891541 & -0.224988 & -5.146417 \\
\hline 114 & 1 & 0 & 1.395798 & -1.332451 & -6.191236 \\
\hline 115 & 1 & 0 & 4.349433 & 0.719443 & -3.831741 \\
\hline 116 & 1 & 0 & -2.061406 & -6.158282 & -2.250768 \\
\hline 117 & 1 & 0 & 3.156862 & 0.386546 & -6.003158 \\
\hline
\end{tabular}

Frequencies -- 13.2831

Frequencies -- 28.3357

Frequencies -- 43.0725

18.5508

22.5658 32.1970 45.0577
36.7233 48.4185 


\begin{tabular}{|c|c|c|c|}
\hline Frequencies -- & 51.8675 & 54.9403 & 61.1328 \\
\hline Frequencies -- & 63.0492 & 67.8369 & 80.7959 \\
\hline Frequencies -- & 82.4011 & 92.9023 & 95.9382 \\
\hline Frequencies -- & 97.5343 & 102.4921 & 108.1257 \\
\hline Frequencies -- & 117.9589 & 123.2193 & 125.4334 \\
\hline Frequencies -- & 135.5158 & 142.9148 & 147.2731 \\
\hline Frequencies -- & 154.1226 & 170.8782 & 174.1575 \\
\hline Frequencies -- & 180.6490 & 184.5313 & 186.7979 \\
\hline Frequencies -- & 192.7458 & 199.6531 & 203.2844 \\
\hline Frequencies -- & 216.5547 & 223.7112 & 234.6891 \\
\hline Frequencies -- & 238.2826 & 239.3226 & 243.7340 \\
\hline Frequencies -- & 255.5812 & 257.0233 & 281.2737 \\
\hline Frequencies -- & 284.1071 & 294.4234 & 295.5072 \\
\hline Frequencies -- & 297.4189 & 304.7240 & 320.8453 \\
\hline Frequencies -- & 331.9028 & 339.9356 & 349.8562 \\
\hline Frequencies -- & 365.4690 & 366.4767 & 375.9728 \\
\hline Frequencies -- & 378.4805 & 382.7744 & 388.1463 \\
\hline Frequencies -- & 397.6608 & 402.6855 & 403.7197 \\
\hline Frequencies -- & 406.4266 & 412.3291 & 414.5464 \\
\hline Frequencies -- & 419.1666 & 425.4100 & 429.7189 \\
\hline Frequencies -- & 434.0792 & 436.1273 & 445.5776 \\
\hline Frequencies -- & 454.6289 & 487.0120 & 488.1764 \\
\hline Frequencies -- & 489.6446 & 497.6685 & 497.7420 \\
\hline Frequencies -- & 505.8649 & 510.0719 & 520.4784 \\
\hline Frequencies -- & 525.8091 & 528.4337 & 532.2295 \\
\hline Frequencies -- & 539.9951 & 549.1862 & 556.9346 \\
\hline Frequencies -- & 561.9114 & 567.3606 & 567.7481 \\
\hline Frequencies -- & 573.0362 & 576.4382 & 584.9287 \\
\hline Frequencies -- & 595.1433 & 604.5397 & 606.4211 \\
\hline Frequencies -- & 608.2221 & 615.5194 & 618.7585 \\
\hline Frequencies -- & 623.0120 & 626.6565 & 628.2683 \\
\hline Frequencies -- & 644.5863 & 648.7635 & 649.7929 \\
\hline Frequencies -- & 652.5475 & 657.1812 & 660.7090 \\
\hline Frequencies -- & 663.3770 & 675.5247 & 688.1377 \\
\hline Frequencies -- & 690.9143 & 708.7889 & 711.5970 \\
\hline Frequencies -- & 718.5679 & 723.6175 & 729.0661 \\
\hline Frequencies -- & 732.5740 & 736.7345 & 750.2064 \\
\hline Frequencies -- & 751.7995 & 755.0574 & 756.0057 \\
\hline Frequencies -- & 756.9291 & 758.5970 & 764.4414 \\
\hline Frequencies -- & 771.6510 & 774.9737 & 790.0946 \\
\hline Frequencies -- & 797.1969 & 801.2731 & 812.4479 \\
\hline Frequencies -- & 814.0193 & 825.9785 & 837.1424 \\
\hline Frequencies -- & 839.4403 & 845.8214 & 849.0937 \\
\hline Frequencies -- & 855.8531 & 866.1867 & 867.5204 \\
\hline Frequencies -- & 868.3545 & 871.2634 & 873.4241 \\
\hline Frequencies -- & 874.5640 & 879.1272 & 881.1035 \\
\hline Frequencies -- & 881.2992 & 882.9863 & 886.8571 \\
\hline Frequencies -- & 889.8525 & 919.3433 & 921.2350 \\
\hline Frequencies -- & 923.0916 & 938.9765 & 940.0848 \\
\hline Frequencies -- & 943.5741 & 951.7608 & 955.5814 \\
\hline Frequencies -- & 961.8051 & 972.1674 & 976.9088 \\
\hline Frequencies -- & 983.3597 & 987.7720 & 987.9456 \\
\hline Frequencies -- & 990.6619 & 991.4248 & 992.3926 \\
\hline Frequencies -- & 999.3527 & 1002.2632 & 1002.7591 \\
\hline Frequencies -- & 1003.3577 & 1005.6468 & 1008.9306 \\
\hline Frequencies -- & 1010.4913 & 1015.2498 & 1015.9650 \\
\hline Frequencies -- & 1019.6943 & 1020.7488 & 1034.9040 \\
\hline Frequencies -- & 1038.3593 & 1044.1885 & 1047.1036 \\
\hline Frequencies -- & 1049.9730 & 1051.3412 & 1053.6472 \\
\hline Frequencies -- & 1058.6518 & 1066.8814 & 1069.7624 \\
\hline Frequencies -- & 1080.4790 & 1092.5116 & 1105.1570 \\
\hline Frequencies -- & 1107.4187 & 1110.1285 & 1114.5105 \\
\hline Frequencies -- & 1119.4631 & 1137.4068 & 1139.8457 \\
\hline Frequencies -- & 1140.9755 & 1155.4280 & 1156.8022 \\
\hline Frequencies -- & 1161.8215 & 1163.1387 & 1164.8119 \\
\hline Frequencies -- & 1169.3277 & 1174.5520 & 1179.5351 \\
\hline
\end{tabular}

\begin{tabular}{|c|c|c|}
\hline Frequencies -- 1180.0363 & 1187.6568 & 1193.7329 \\
\hline Frequencies -- 1197.3850 & 1200.6281 & 1201.3582 \\
\hline Frequencies -- 1206.1990 & 1208.5782 & 1209.7694 \\
\hline Frequencies -- 1210.1152 & 1212.6376 & 1222.0301 \\
\hline Frequencies -- 1245.4401 & 1251.6721 & 1253.8078 \\
\hline Frequencies -- 1255.7929 & 1258.5717 & 1260.6965 \\
\hline Frequencies -- 1268.2444 & 1273.1130 & 1283.3365 \\
\hline Frequencies -- 1284.2156 & 1289.3937 & 1295.0327 \\
\hline Frequencies -- 1299.1992 & 1304.3674 & 1308.0150 \\
\hline Frequencies -- 1316.1894 & 1318.2860 & 1322.3265 \\
\hline Frequencies -- 1322.7251 & 1329.0613 & 1341.7294 \\
\hline Frequencies -- 1343.4835 & 1348.0023 & 1348.9586 \\
\hline Frequencies -- 1352.4782 & 1361.5964 & 1364.5246 \\
\hline Frequencies -- 1366.9214 & 1369.5852 & 1406.6958 \\
\hline Frequencies -- 1408.0652 & 1413.1065 & 1414.2812 \\
\hline Frequencies -- 1414.6459 & 1417.5915 & 1427.1202 \\
\hline Frequencies -- 1443.2646 & 1444.0432 & 1455.2035 \\
\hline Frequencies -- 1460.6192 & 1482.7501 & 1483.8415 \\
\hline Frequencies -- 1487.6221 & 1490.3352 & 1491.4208 \\
\hline Frequencies -- 1493.1537 & 1493.3085 & 1495.7874 \\
\hline Frequencies -- 1498.2977 & 1499.2485 & 1501.4135 \\
\hline Frequencies -- 1510.3877 & 1510.8785 & 1512.7523 \\
\hline Frequencies -- 1519.7037 & 1523.0833 & 1530.1430 \\
\hline Frequencies -- 1531.4225 & 1547.2670 & 1548.1465 \\
\hline Frequencies -- 1552.3984 & 1580.4755 & 1600.5622 \\
\hline Frequencies -- 1602.8816 & 1647.2631 & 1648.4522 \\
\hline Frequencies -- 1655.2275 & 1658.4248 & 1661.4000 \\
\hline Frequencies -- 1665.8027 & 1672.9261 & 1673.3200 \\
\hline Frequencies -- 1684.6622 & 1698.9887 & 1699.1498 \\
\hline Frequencies -- 1709.9421 & 1711.4972 & 1713.7710 \\
\hline Frequencies -- 1717.2505 & 1719.0193 & 2219.0128 \\
\hline Frequencies -- 3078.2756 & 3080.1674 & 3081.4184 \\
\hline Frequencies -- 3088.5041 & 3097.5407 & 3133.5768 \\
\hline Frequencies -- 3137.6344 & 3141.5772 & 3154.4149 \\
\hline Frequencies -- 3158.0983 & 3161.5657 & 3189.2214 \\
\hline Frequencies -- 3199.3264 & 3199.6558 & 3200.3361 \\
\hline Frequencies -- 3203.2748 & 3203.5591 & 3203.9999 \\
\hline Frequencies -- 3207.3991 & 3208.9804 & 3211.4100 \\
\hline Frequencies -- 3211.7949 & 3212.7602 & 3218.9261 \\
\hline Frequencies -- 3219.6625 & 3220.2598 & 3223.9206 \\
\hline Frequencies -- 3224.4506 & 3224.7319 & 3228.4011 \\
\hline Frequencies -- 3228.7069 & 3232.3650 & 3233.2481 \\
\hline Frequencies -- 3234.2766 & 3235.2461 & 3237.0489 \\
\hline Frequencies -- 3240.0311 & 3240.1319 & 3240.7049 \\
\hline Frequencies -- 3242.1439 & 3246.3191 & 3253.6744 \\
\hline Frequencies -- 3263.6757 & 3656.1665 & 3665.3294 \\
\hline
\end{tabular}

SCF Done: $E(R M 062 X / D G D Z V P)=-3995.13801731$ Sum of electronic and zero-point Energies $=\quad-3994.209289$ Sum of electronic and thermal Energies $=\quad-3994.140240$ Sum of electronic and thermal Free Energies $=\quad-3994.315334$ SCF Done: $E(R M 062 X / D G T Z V P / S M D)=-3995.94092534$

\section{Cat b}

Center Atomic Atomic Coordinates (Angstroms) Number Number Type $\quad X \quad Y \quad Z$

$\begin{array}{llllll}1 & 15 & 0 & 0.004905 & 0.010892 & 0.009161 \\ 2 & 8 & 0 & 0.011245 & 0.004531 & 1.604864 \\ 3 & 8 & 0 & 1.586463 & 0.010491 & -0.281518 \\ 4 & 1 & 0 & -0.450677 & 0.779296 & 1.964546 \\ 5 & 8 & 0 & -0.461429 & -1.468517 & -0.397255 \\ 6 & 8 & 0 & -0.787901 & 1.064101 & -0.627253 \\ 7 & 6 & 0 & 1.928479 & -0.110262 & -1.635880\end{array}$




$\begin{array}{rrrrrr}74 & 1 & 0 & 7.159668 & -4.794182 & 3.684291 \\ 75 & 1 & 0 & 4.004049 & -8.732171 & 3.959524 \\ 76 & 1 & 0 & 1.845516 & 2.002932 & -1.966470 \\ 77 & 1 & 0 & -1.334603 & -2.670333 & 1.742329\end{array}$

Frequencies -- $\quad 20.2108$

Frequencies -- 34.1742

Frequencies -- 61.6626

Frequencies -- 77.7903

Frequencies -- 100.8890

Frequencies -- 120.9797

Frequencies -- 174.5716

Frequencies -- 209.9349

Frequencies -- 233.9209

Frequencies -- 273.9592

Frequencies -- 301.2567

Frequencies -- 350.0751

Frequencies -- 399.2926

Frequencies -- 408.5321

Frequencies -- 427.1995

Frequencies -- 486.1137

Frequencies -- 529.5344

Frequencies -- 549.6887

Frequencies -- 592.4695

Frequencies -- 621.8231

Frequencies -- 629.5302

Frequencies -- 654.3101

Frequencies -- 677.5773

Frequencies -- 708.9549

Frequencies -- 720.6152

Frequencies -- 772.5676

Frequencies -- 789.6032

Frequencies -- 825.3590

Frequencies -- 857.0536

Frequencies -- 867.8415

Frequencies -- 895.5598

Frequencies -- 931.1212

Frequencies -- 941.3765

Frequencies -- 970.2045

Frequencies -- 990.2886

Frequencies -- 999.2855

Frequencies -- 1010.4227

Frequencies -- 1019.0394

Frequencies -- 1020.0258

Frequencies -- 1049.7793

Frequencies -- 1066.7658

Frequencies -- 1085.9446

Frequencies -- 1109.7194

Frequencies -- 1122.1745

Frequencies -- 1175.2610

Frequencies -- 1188.0174

Frequencies -- 1204.4053

Frequencies -- 1213.1441

Frequencies -- 1235.5923

Frequencies -- 1272.4359

Frequencies -- 1297.6846

Frequencies -- 1325.1807

Frequencies -- 1335.7495

Frequencies -- 1357.1604

Frequencies -- 1362.4827

Frequencies -- 1377.4999

Frequencies -- 1458.8017

Frequencies -- 1492.6280

Frequencies -- 1504.0219

Frequencies -- 1530.9773
$24.1348 \quad 32.6538$

$53.7400 \quad 57.6414$

$63.4364 \quad 70.4344$

$82.4323 \quad 94.8072$

$101.3679 \quad 108.3793$

$146.7988 \quad 159.3810$

$178.3907 \quad 186.1831$

$222.0607 \quad 232.4510$

$241.8311 \quad 249.5028$

$281.1523 \quad 287.1439$

$306.8007 \quad 326.4657$

$383.3047 \quad 392.7842$

$399.9569 \quad 408.3370$

$409.9830 \quad 413.8676$

$447.6436 \quad 463.6975$

$500.0977 \quad 504.3065$

$536.4151 \quad 541.6408$

$555.0252 \quad 576.1959$

$608.6947 \quad 613.6769$

$625.0454 \quad 629.4034$

$635.1476 \quad 638.8324$

$664.0253 \quad 669.1718$

$689.0892 \quad 708.4454$

$715.2742 \quad 716.3788$

$729.9644 \quad 736.5471$

$774.8792 \quad 778.8098$

$801.4722 \quad 817.1119$

$844.1629 \quad 849.5884$

$858.1233 \quad 865.8104$

$871.0639 \quad 871.3464$

$919.7475 \quad 929.1157$

$935.8550 \quad 936.0283$

$943.2493 \quad 953.4896$

$973.8824 \quad 989.4807$

$997.1993 \quad 997.7985$

$1001.4018 \quad 1005.9831$

$1013.2684 \quad 1015.9801$

$1019.5133 \quad 1019.7114$

$1024.3514 \quad 1042.9734$

$1062.5726 \quad 1065.9672$

$1067.3869 \quad 1069.7582$

$1099.4246 \quad 1104.2607$

$1113.0434 \quad 1120.8771$

$1138.6609 \quad 1174.8458$

$1175.4087 \quad 1178.6650$

$1198.6594 \quad 1203.2830$

$1205.9489 \quad 1206.2132$

$1224.1460 \quad 1232.2743$

$1242.9416 \quad 1252.7690$

$1282.0792 \quad 1292.9087$

$1307.1994 \quad 1312.2353$

$1327.4904 \quad 1334.3190$

$1340.9499 \quad 1343.0208$

$1358.6469 \quad 1358.8339$

$1363.7536 \quad 1368.8618$

$1383.9015 \quad 1390.2696$

$1472.5565 \quad 1491.9148$

$1496.6738 \quad 1498.3783$

$1507.6912 \quad 1527.9676$

$1545.0939 \quad 1547.0007$ 


$\begin{array}{llll}\text { Frequencies -- } 1564.8312 & 1568.9399 & 1668.3016 \\ \text { Frequencies -- } 1668.6756 & 1675.2494 & 1675.6281 \\ \text { Frequencies -- } 1682.6271 & 1684.5852 & 1686.1277 \\ \text { Frequencies -- } 1689.8627 & 1696.6797 & 1698.2858 \\ \text { Frequencies -- } 1699.8321 & 1702.4500 & 3072.2571 \\ \text { Frequencies -- } 3072.3917 & 3088.1390 & 3092.3703 \\ \text { Frequencies -- } 3152.9383 & 3157.5151 & 3190.8579 \\ \text { Frequencies -- } 3198.6322 & 3200.0128 & 3201.2069 \\ \text { Frequencies -- } 3204.9031 & 3209.1872 & 3212.5214 \\ \text { Frequencies -- } 3215.2635 & 3215.2762 & 3217.1074 \\ \text { Frequencies -- } 3217.7799 & 3218.6773 & 3221.5964 \\ \text { Frequencies -- } 3225.6776 & 3225.8091 & 3227.2365 \\ \text { Frequencies -- } 3230.0167 & 3235.6721 & 3236.6377 \\ \text { Frequencies -- } 3237.0269 & 3243.9202 & 3249.0682 \\ \text { Frequencies -- } 3249.8791 & 3252.5042 & 3823.0952\end{array}$

SCF Done: $E(R M 062 X / D G D Z V P)=-2222.74132429$

Sum of electronic and zero-point Energies $=\quad-2222.122488$

Sum of electronic and thermal Energies $=\quad-2222.078367$

Sum of electronic and thermal Free Energies $=\quad-2222.202490$

SCF Done: $E(R M 062 X / D G T Z V P / S M D)=-2223.24177564$

\section{Cat b 101}

Center Atomic Atomic Coordinates (Angstroms) Number Number Type $X \quad Y \quad$ Z

\begin{tabular}{|c|c|c|c|c|c|}
\hline 1 & 15 & 0 & 0.000000 & 0.000000 & 0.000000 \\
\hline 2 & 8 & 0 & 0.000000 & 0.000000 & 1.601596 \\
\hline 3 & 8 & 0 & 1.559282 & 0.000000 & -0.400122 \\
\hline 4 & 8 & 0 & -0.686096 & 1.135169 & -0.649638 \\
\hline 5 & 8 & 0 & -0.559922 & -1.429269 & -0.301656 \\
\hline 6 & 6 & 0 & 0.564892 & 1.137234 & 2.196230 \\
\hline 7 & 6 & 0 & 2.340001 & -1.065847 & 0.062448 \\
\hline 8 & 6 & 0 & 1.925731 & 1.168612 & 2.461450 \\
\hline 9 & 6 & 0 & -0.240330 & 2.242748 & 2.448670 \\
\hline 10 & 6 & 0 & 2.903628 & -1.023803 & 1.329525 \\
\hline 11 & 6 & 0 & 2.484969 & -2.189264 & -0.745156 \\
\hline 12 & 6 & 0 & 2.984335 & 0.092577 & 2.349356 \\
\hline 13 & 6 & 0 & 2.489755 & 2.352358 & 2.943172 \\
\hline 14 & 6 & 0 & 0.327907 & 3.388536 & 3.002406 \\
\hline 15 & 6 & 0 & 3.562794 & -2.159443 & 1.809267 \\
\hline 16 & 6 & 0 & 3.215272 & -3.280643 & -0.281038 \\
\hline 17 & 6 & 0 & 3.105856 & -0.724908 & 3.661997 \\
\hline 18 & 6 & 0 & 4.252878 & 0.960747 & 2.137360 \\
\hline 19 & 6 & 0 & 4.010238 & 2.258095 & 2.947627 \\
\hline 20 & 6 & 0 & 1.701991 & 3.462765 & 3.258053 \\
\hline 21 & 1 & 0 & -0.298955 & 4.247402 & 3.225294 \\
\hline 22 & 6 & 0 & 3.899827 & -2.000206 & 3.286299 \\
\hline 23 & 6 & 0 & 3.760668 & -3.286223 & 1.007842 \\
\hline 24 & 1 & 0 & 3.348673 & -4.150997 & -0.917528 \\
\hline 25 & 1 & 0 & 3.579512 & -0.154006 & 4.466569 \\
\hline 26 & 1 & 0 & 2.103287 & -1.009337 & 3.997082 \\
\hline 27 & 1 & 0 & 5.167812 & 0.439893 & 2.435887 \\
\hline 28 & 1 & 0 & 4.339265 & 1.209946 & 1.074883 \\
\hline 29 & 1 & 0 & 4.380389 & 2.136695 & 3.973216 \\
\hline 30 & 6 & 0 & 4.670987 & 3.478918 & 2.339693 \\
\hline 31 & 6 & 0 & 2.317440 & 4.688316 & 3.836102 \\
\hline 32 & 1 & 0 & 4.975743 & -1.827495 & 3.413512 \\
\hline 33 & 6 & 0 & 3.507210 & -3.213458 & 4.105374 \\
\hline 34 & 6 & 0 & 4.520076 & -4.459086 & 1.520749 \\
\hline 35 & 6 & 0 & 4.305175 & 3.906557 & 1.057393 \\
\hline 36 & 6 & 0 & 5.609431 & 4.223796 & 3.054269 \\
\hline 37 & 6 & 0 & 2.350733 & 5.884473 & 3.112221 \\
\hline 38 & 6 & 0 & 2.912292 & 4.639433 & 5.101089 \\
\hline
\end{tabular}

\begin{tabular}{|c|c|c|c|c|c|}
\hline & 6 & 0 & 2.195211 & -3.699702 & 4.047848 \\
\hline & 6 & 0 & 4.441597 & -3.891918 & 4.888337 \\
\hline 41 & 6 & 0 & 3.878145 & -5.678482 & 1.759279 \\
\hline 42 & 6 & 0 & 5.882270 & -4.336505 & 1.813781 \\
\hline 43 & 6 & 0 & 4.863555 & 5.056807 & 0.506394 \\
\hline 44 & 1 & 0 & 3.556275 & 3.349444 & 0.496506 \\
\hline 45 & 6 & 0 & 6.168306 & 5.380095 & 2.508145 \\
\hline 46 & 1 & 0 & 5.888351 & 3.911981 & 4.058242 \\
\hline 47 & 6 & 0 & 2.988310 & 7.007471 & 3.636970 \\
\hline 48 & 1 & 0 & 1.905933 & 5.920493 & 2.121015 \\
\hline 49 & 6 & 0 & 3.540424 & 5.765312 & 5.631547 \\
\hline 50 & 1 & 0 & 2.877901 & 3.712813 & 5.669432 \\
\hline 51 & 6 & 0 & 1.830563 & -4.843891 & 4.752278 \\
\hline 52 & 1 & 0 & 1.458986 & -3.192834 & 3.425756 \\
\hline 53 & 6 & 0 & 4.081804 & -5.041950 & 5.592527 \\
\hline 54 & 1 & 0 & 5.467692 & -3.533522 & 4.928699 \\
\hline 55 & 6 & 0 & 4.582914 & -6.750997 & 2.302988 \\
\hline 56 & 1 & 0 & 2.815920 & -5.771743 & 1.548514 \\
\hline 57 & 6 & 0 & 6.591176 & -5.412561 & 2.346063 \\
\hline 58 & 1 & 0 & 6.385197 & -3.391804 & 1.620055 \\
\hline 59 & 6 & 0 & 5.795487 & 5.801089 & 1.233005 \\
\hline 60 & 1 & 0 & 4.563659 & 5.379655 & -0.486080 \\
\hline 61 & 1 & 0 & 6.884957 & 5.956606 & 3.085372 \\
\hline 62 & 6 & 0 & 3.586884 & 6.949953 & 4.896136 \\
\hline 63 & 1 & 0 & 3.028331 & 7.923926 & 3.056195 \\
\hline 64 & 1 & 0 & 3.995511 & 5.716426 & 6.616355 \\
\hline 65 & 6 & 0 & 2.775603 & -5.522879 & 5.524626 \\
\hline 66 & 1 & 0 & 0.810817 & -5.212244 & 4.691552 \\
\hline 67 & 1 & 0 & 4.826596 & -5.566302 & 6.183698 \\
\hline 68 & 6 & 0 & 5.940032 & -6.619884 & 2.600344 \\
\hline 69 & 1 & 0 & 4.069063 & -7.685711 & 2.505702 \\
\hline 70 & 1 & 0 & 7.649393 & -5.307032 & 2.565851 \\
\hline 71 & 1 & 0 & 6.223870 & 6.704054 & 0.808853 \\
\hline 72 & 1 & 0 & 4.085184 & 7.824829 & 5.302430 \\
\hline 73 & 1 & 0 & 2.494768 & -6.420095 & 6.067666 \\
\hline 74 & 1 & 0 & 6.486884 & -7.455272 & 3.026917 \\
\hline 75 & 1 & 0 & -1.130304 & -1.457247 & -1.140690 \\
\hline 76 & 8 & 0 & -1.895843 & -1.173237 & -2.505772 \\
\hline 77 & 6 & 0 & -2.915220 & -0.132323 & -2.431474 \\
\hline 78 & 6 & 0 & -2.183357 & 0.717643 & -3.503831 \\
\hline 79 & 1 & 0 & -3.893643 & -0.533755 & -2.701894 \\
\hline 80 & 1 & 0 & -2.931279 & 0.336675 & -1.444372 \\
\hline 81 & 8 & 0 & -1.772438 & 1.986934 & -3.098858 \\
\hline 82 & 6 & 0 & -1.100782 & -0.393285 & -3.443445 \\
\hline 83 & 6 & 0 & -2.902358 & 0.814304 & -4.829219 \\
\hline 84 & 1 & 0 & -1.328583 & 1.900699 & -2.230046 \\
\hline 85 & 1 & 0 & -0.160661 & -0.054069 & -2.994626 \\
\hline 86 & 1 & 0 & -0.917259 & -0.958329 & -4.359591 \\
\hline 87 & 6 & 0 & -3.057149 & 2.042057 & -5.472734 \\
\hline 88 & 6 & 0 & -3.418555 & -0.342639 & -5.425411 \\
\hline 89 & 6 & 0 & -3.723204 & 2.110513 & -6.697938 \\
\hline 90 & 1 & 0 & -2.654198 & 2.934253 & -5.006771 \\
\hline 91 & 6 & 0 & -4.083324 & -0.273052 & -6.646470 \\
\hline 92 & 1 & 0 & -3.301766 & -1.308285 & -4.934419 \\
\hline 93 & 6 & 0 & -4.237882 & 0.957787 & -7.287625 \\
\hline 94 & 1 & 0 & -3.839521 & 3.070533 & -7.191785 \\
\hline 95 & 1 & 0 & -4.480380 & -1.177316 & -7.097492 \\
\hline 96 & 1 & 0 & -4.755946 & 1.014782 & -8.239973 \\
\hline 97 & 1 & 0 & -1.294657 & 2.196619 & 2.196089 \\
\hline 98 & 1 & 0 & 2.014705 & -2.198784 & -1.723656 \\
\hline
\end{tabular}

Frequencies -- $1.5230 \quad 7.1214 \quad 18.5931$ Frequencies -- $26.3407 \quad 28.6203 \quad 37.5423$ Frequencies -- $39.6425 \quad 53.0236 \quad 56.2762$ Frequencies -- $58.5006 \quad 62.1873 \quad 62.9376$ 


\begin{tabular}{|c|c|c|c|}
\hline Frequencies -- & 68.6424 & 74.8127 & 75.2746 \\
\hline Frequencies -- & 79.9946 & 92.6386 & 93.4719 \\
\hline Frequencies -- & 95.6179 & 101.9084 & 111.0114 \\
\hline Frequencies -- & 122.2369 & 126.0720 & 155.2031 \\
\hline Frequencies -- & 166.1652 & 168.5883 & 177.0836 \\
\hline Frequencies -- & 184.1034 & 198.6934 & 210.7253 \\
\hline Frequencies -- & 221.7196 & 234.0492 & 241.7045 \\
\hline Frequencies -- & 245.8420 & 254.8054 & 275.0152 \\
\hline Frequencies -- & 279.1382 & 281.1535 & 287.4597 \\
\hline Frequencies -- & 303.9633 & 307.7620 & 324.5156 \\
\hline Frequencies -- & 334.9213 & 351.3339 & 365.9124 \\
\hline Frequencies -- & 390.0711 & 391.9972 & 400.6796 \\
\hline Frequencies -- & 408.5489 & 411.1455 & 412.8910 \\
\hline Frequencies -- & 413.6037 & 416.6352 & 421.2921 \\
\hline Frequencies -- & 425.4401 & 426.2635 & 435.6215 \\
\hline Frequencies -- & 449.2960 & 466.5795 & 488.3988 \\
\hline Frequencies -- & 504.5541 & 505.0946 & 526.4303 \\
\hline Frequencies -- & 532.1096 & 537.5544 & 542.4073 \\
\hline Frequencies -- & 550.9636 & 559.4357 & 559.9755 \\
\hline Frequencies -- & 575.9817 & 591.5102 & 608.3690 \\
\hline Frequencies -- & 614.1339 & 622.7263 & 624.7085 \\
\hline Frequencies -- & 628.2575 & 629.3211 & 631.1226 \\
\hline Frequencies -- & 636.2820 & 638.4802 & 654.1950 \\
\hline Frequencies -- & 663.3660 & 670.2903 & 677.7151 \\
\hline Frequencies -- & 684.6799 & 688.4106 & 704.0136 \\
\hline Frequencies -- & 706.0936 & 713.8204 & 714.4043 \\
\hline Frequencies -- & 718.9790 & 720.1303 & 721.2527 \\
\hline Frequencies -- & 730.5591 & 737.1163 & 771.7592 \\
\hline Frequencies -- & 774.2599 & 776.5305 & 777.9053 \\
\hline Frequencies -- & 787.7798 & 800.4477 & 817.3449 \\
\hline Frequencies -- & 824.0522 & 825.4780 & 837.5774 \\
\hline Frequencies -- & 849.4240 & 850.8037 & 863.1577 \\
\hline Frequencies -- & 866.7549 & 868.3303 & 872.5005 \\
\hline Frequencies -- & 874.5156 & 878.7965 & 895.8669 \\
\hline Frequencies -- & 913.9678 & 917.9221 & 924.7160 \\
\hline Frequencies -- & 931.5492 & 934.2116 & 935.9925 \\
\hline Frequencies -- & 940.2927 & 943.8799 & 946.4736 \\
\hline Frequencies -- & 948.6311 & 958.4029 & 968.6741 \\
\hline Frequencies -- & 974.5109 & 986.4523 & 992.9708 \\
\hline Frequencies -- & 995.2554 & 996.0314 & 998.2979 \\
\hline Frequencies -- & 1001.0645 & 1005.2253 & 1006.5505 \\
\hline Frequencies -- & 1007.6854 & 1008.0296 & 1009.7296 \\
\hline Frequencies -- & 1013.4152 & 1017.8576 & 1019.1916 \\
\hline Frequencies -- & 1019.3041 & 1019.6955 & 1019.8484 \\
\hline Frequencies -- & 1019.9313 & 1024.1814 & 1034.9307 \\
\hline Frequencies -- & 1042.8611 & 1052.3890 & 1060.5724 \\
\hline Frequencies -- & 1062.3750 & 1064.0056 & 1067.7510 \\
\hline Frequencies -- & 1068.9215 & 1072.1709 & 1086.6725 \\
\hline Frequencies -- & 1089.8824 & 1090.1603 & 1100.3937 \\
\hline Frequencies -- & 1103.2177 & 1107.7794 & 1111.7507 \\
\hline Frequencies -- & 1116.2398 & 1121.8237 & 1126.4101 \\
\hline Frequencies -- & 1134.6386 & 1172.1159 & 1174.1896 \\
\hline Frequencies -- & 1175.9424 & 1178.2593 & 1179.0888 \\
\hline Frequencies -- & 1183.2665 & 1184.7302 & 1192.4636 \\
\hline Frequencies -- & 1200.1211 & 1201.1532 & 1202.0721 \\
\hline Frequencies -- & 1207.4232 & 1211.9900 & 1214.2846 \\
\hline Frequencies -- & 1214.4553 & 1223.2245 & 1230.9000 \\
\hline Frequencies -- & 1233.9011 & 1235.5292 & 1242.4274 \\
\hline Frequencies -- & 1252.0597 & 1254.0078 & 1270.6809 \\
\hline Frequencies -- & 1278.1864 & 1292.4882 & 1296.3972 \\
\hline Frequencies -- & 1297.7286 & 1306.1837 & 1310.9357 \\
\hline Frequencies -- & 1315.2133 & 1323.0692 & 1326.2838 \\
\hline Frequencies -- & 1329.4400 & 1336.0114 & 1336.1395 \\
\hline Frequencies -- & 1341.6947 & 1344.6739 & 1345.9381 \\
\hline Frequencies -- & 1356.6713 & 1359.1670 & 1365.7590 \\
\hline Frequencies -- & 1367.3296 & 1368.8841 & 1370.6753 \\
\hline
\end{tabular}

$\begin{array}{llll}\text { Frequencies -- } & 1376.0367 & 1382.2531 & 1387.7275 \\ \text { Frequencies -- } 1393.6966 & 1457.0870 & 1469.1381 \\ \text { Frequencies -- } 1470.6278 & 1489.0590 & 1492.3859 \\ \text { Frequencies -- } 1499.4004 & 1500.4612 & 1500.9175 \\ \text { Frequencies -- } 1506.5289 & 1510.0319 & 1513.5700 \\ \text { Frequencies -- } 1526.4895 & 1528.7279 & 1538.0248 \\ \text { Frequencies -- } 1546.9956 & 1551.4374 & 1555.5072 \\ \text { Frequencies -- } 1563.6022 & 1567.2844 & 1664.2900 \\ \text { Frequencies -- } 1669.2068 & 1677.0267 & 1678.0206 \\ \text { Frequencies -- } 1679.6804 & 1682.3887 & 1684.1353 \\ \text { Frequencies -- } 1685.3293 & 1691.7462 & 1697.7627 \\ \text { Frequencies -- } 1701.0679 & 1701.4340 & 1702.8930 \\ \text { Frequencies -- } 1703.1442 & 2908.0890 & 3072.2951 \\ \text { Frequencies -- } 3076.6858 & 3088.5436 & 3089.6066 \\ \text { Frequencies -- } 3104.5627 & 3122.9037 & 3153.1238 \\ \text { Frequencies -- } 3155.5995 & 3185.7581 & 3186.2935 \\ \text { Frequencies -- } 3190.0073 & 3190.3926 & 3200.2727 \\ \text { Frequencies -- } 3200.4754 & 3200.9593 & 3203.7095 \\ \text { Frequencies -- } 3204.1364 & 3208.9983 & 3210.2783 \\ \text { Frequencies -- } 3212.4118 & 3214.7877 & 3216.4124 \\ \text { Frequencies -- } 3216.6717 & 3217.4378 & 3217.4848 \\ \text { Frequencies -- } 3223.1245 & 3223.5641 & 3226.0116 \\ \text { Frequencies -- } 3226.4941 & 3228.8568 & 3228.9825 \\ \text { Frequencies -- } 3229.6540 & 3236.6202 & 3236.6623 \\ \text { Frequencies -- } 3238.6932 & 3239.0938 & 3239.1173 \\ \text { Frequencies -- } 3248.0873 & 3251.2192 & 3670.9629\end{array}$

SCF Done: $E($ RM062X/DGDZVP $)=-2721.99270329$

Sum of electronic and zero-point Energies $=\quad-2721.196670$ Sum of electronic and thermal Energies $=\quad-2721.140300$ Sum of electronic and thermal Free Energies $=\quad-2721.296791$ SCF Done: $E(R M 062 X / D G T Z V P / S M D)=-2722.60970473$

\section{Cat b 10_2}

Center Atomic Atomic Coordinates (Angstroms)

Number Number Type $\quad X \quad Y \quad$ Z

$\begin{array}{cccccc}1 & 15 & 0 & -0.032029 & 0.038638 & 0.121493 \\ 2 & 8 & 0 & 0.000433 & 0.040447 & 1.730151 \\ 3 & 8 & 0 & 1.511035 & 0.067296 & -0.307985 \\ 4 & 8 & 0 & -0.504719 & 1.457952 & -0.336495 \\ 5 & 8 & 0 & -0.815512 & -1.113931 & -0.366789 \\ 6 & 6 & 0 & 0.655035 & 1.105043 & 2.360411 \\ 7 & 6 & 0 & 2.244872 & -1.070240 & 0.059388 \\ 8 & 6 & 0 & 2.026542 & 1.058081 & 2.568301 \\ 9 & 6 & 0 & -0.081951 & 2.229114 & 2.719436 \\ 10 & 6 & 0 & 2.859484 & -1.119391 & 1.301446 \\ 11 & 6 & 0 & 2.280738 & -2.162145 & -0.801000 \\ 12 & 6 & 0 & 3.030233 & -0.058207 & 2.366738 \\ 13 & 6 & 0 & 2.664909 & 2.186652 & 3.088893 \\ 14 & 6 & 0 & 0.561422 & 3.314316 & 3.310931 \\ 15 & 6 & 0 & 3.472736 & -2.308633 & 1.702210 \\ 16 & 6 & 0 & 2.966448 & -3.312669 & -0.415814 \\ 17 & 6 & 0 & 3.158708 & -0.942818 & 3.634809 \\ 18 & 6 & 0 & 4.330606 & 0.759495 & 2.147691 \\ 19 & 6 & 0 & 4.178372 & 2.028433 & 3.022011 \\ 20 & 6 & 0 & 1.947522 & 3.311930 & 3.501801 \\ 21 & 1 & 0 & -0.014781 & 4.183856 & 3.614521 \\ 22 & 6 & 0 & 3.873664 & -2.236004 & 3.170363 \\ 23 & 6 & 0 & 3.571730 & -3.405796 & 0.842813 \\ 24 & 1 & 0 & 3.017890 & -4.160799 & -1.092807 \\ 25 & 1 & 0 & 3.688666 & -0.433396 & 4.445515 \\ 26 & 1 & 0 & 2.155996 & -1.194199 & 3.995060 \\ 27 & 1 & 0 & 5.229130 & 0.183745 & 2.389812\end{array}$




\begin{tabular}{|c|c|c|c|c|c|}
\hline 28 & 1 & 0 & 4.392316 & 1.052308 & 1.094747 \\
\hline & 1 & 0 & 4.589264 & 1.852172 & 4.023803 \\
\hline 30 & 6 & 0 & 4.857632 & 3.247006 & 2.429317 \\
\hline 31 & 6 & 0 & 2.650599 & 4.464199 & 4.129196 \\
\hline 32 & 1 & 0 & 4.960843 & -2.120860 & 3.262579 \\
\hline 33 & 6 & 0 & 3.451516 & -3.466163 & 3.948592 \\
\hline 34 & 6 & 0 & 4.292984 & -4.635579 & 1.269182 \\
\hline 35 & 6 & 0 & 4.434213 & 3.741821 & 1.189485 \\
\hline 36 & 6 & 0 & 5.870233 & 3.920923 & 3.112688 \\
\hline 37 & 6 & 0 & 2.744206 & 5.698620 & 3.479049 \\
\hline 38 & 6 & 0 & 3.274033 & 4.297290 & 5.370617 \\
\hline 39 & 6 & 0 & 2.114678 & -3.882672 & 3.924338 \\
\hline 40 & 6 & 0 & 4.379732 & -4.227430 & 4.659531 \\
\hline 41 & 6 & 0 & 3.607972 & -5.837177 & 1.474636 \\
\hline 42 & 6 & 0 & 5.668728 & -4.583151 & 1.517343 \\
\hline 43 & 6 & 0 & 5.007124 & 4.889768 & 0.649746 \\
\hline 44 & 1 & 0 & 3.629878 & 3.238429 & 0.655088 \\
\hline 45 & 6 & 0 & 6.445042 & 5.074672 & 2.576848 \\
\hline 46 & 1 & 0 & 6.196482 & 3.556169 & 4.084006 \\
\hline 47 & 6 & 0 & 3.468429 & 6.742628 & 4.052800 \\
\hline 48 & 1 & 0 & 2.279036 & 5.824879 & 2.504810 \\
\hline 49 & 6 & 0 & 3.989341 & 5.343996 & 5.950184 \\
\hline 50 & 1 & 0 & 3.192393 & 3.340477 & 5.881213 \\
\hline 51 & 6 & 0 & 1.718586 & -5.038290 & 4.591972 \\
\hline 52 & 1 & 0 & 1.382255 & -3.310861 & 3.356417 \\
\hline 53 & 6 & 0 & 3.988205 & -5.389129 & 5.326561 \\
\hline 54 & 1 & 0 & 5.424243 & -3.924137 & 4.670894 \\
\hline 55 & 6 & 0 & 4.285066 & -6.962188 & 1.942808 \\
\hline 56 & 1 & 0 & 2.536002 & -5.876143 & 1.299180 \\
\hline 57 & 6 & 0 & 6.349051 & -5.710782 & 1.974211 \\
\hline 58 & 1 & 0 & 6.204090 & -3.651866 & 1.347089 \\
\hline 59 & 6 & 0 & 6.013533 & 5.563919 & 1.345331 \\
\hline 60 & 1 & 0 & 4.662353 & 5.264928 & -0.309212 \\
\hline 61 & 1 & 0 & 7.221594 & 5.595999 & 3.128444 \\
\hline 62 & 6 & 0 & 4.095426 & 6.566786 & 5.287247 \\
\hline 63 & 1 & 0 & 3.555336 & 7.689508 & 3.528650 \\
\hline 64 & 1 & 0 & 4.465146 & 5.204390 & 6.916275 \\
\hline 65 & 6 & 0 & 2.656743 & -5.799291 & 5.292966 \\
\hline 66 & 1 & 0 & 0.679343 & -5.351535 & 4.558088 \\
\hline 67 & 1 & 0 & 4.727108 & -5.977801 & 5.861974 \\
\hline 68 & 6 & 0 & 5.655966 & -6.900739 & 2.196727 \\
\hline 69 & 1 & 0 & 3.739468 & -7.883541 & 2.122314 \\
\hline 70 & 1 & 0 & 7.417782 & -5.659762 & 2.159844 \\
\hline 71 & 1 & 0 & 6.454907 & 6.464814 & 0.930148 \\
\hline 72 & 1 & 0 & 4.661749 & 7.379888 & 5.731055 \\
\hline 73 & 1 & 0 & 2.351172 & -6.705458 & 5.807096 \\
\hline 74 & 1 & 0 & 6.180946 & -7.776978 & 2.564731 \\
\hline 75 & 1 & 0 & -1.457611 & 1.457389 & -0.685048 \\
\hline 76 & 8 & 0 & -2.960062 & 1.126125 & -1.102820 \\
\hline 77 & 6 & 0 & -3.109371 & 0.097253 & -2.125488 \\
\hline 78 & 6 & 0 & -3.946673 & -0.791605 & -1.167682 \\
\hline 79 & 1 & 0 & -3.622560 & 0.498816 & -3.001283 \\
\hline 80 & 1 & 0 & -2.144349 & -0.342037 & -2.391434 \\
\hline 81 & 8 & 0 & -3.409354 & -2.045771 & -0.879218 \\
\hline 82 & 6 & 0 & -3.665532 & 0.317236 & -0.119151 \\
\hline 83 & 6 & 0 & -5.400152 & -0.941280 & -1.552887 \\
\hline 84 & 1 & 0 & -2.465908 & -1.927133 & -0.644935 \\
\hline 85 & 1 & 0 & -3.000933 & -0.011026 & 0.687052 \\
\hline 86 & 1 & 0 & -4.528677 & 0.855763 & 0.277396 \\
\hline 87 & 6 & 0 & -6.003734 & -2.198102 & -1.584076 \\
\hline 88 & 6 & 0 & -6.154844 & 0.190856 & -1.883691 \\
\hline 89 & 6 & 0 & -7.347479 & -2.320639 & -1.942390 \\
\hline 90 & 1 & 0 & -5.413862 & -3.070206 & -1.326049 \\
\hline 91 & 6 & 0 & -7.494977 & 0.067492 & -2.240427 \\
\hline 92 & 1 & 0 & -5.695154 & 1.178580 & -1.863989 \\
\hline 93 & 6 & 0 & -8.096120 & -1.192174 & -2.270483 \\
\hline
\end{tabular}

$\begin{array}{lllrrr}94 & 1 & 0 & -7.809390 & -3.303120 & -1.964955 \\ 95 & 1 & 0 & -8.069903 & 0.952369 & -2.495689 \\ 96 & 1 & 0 & -9.140786 & -1.290626 & -2.549031 \\ 97 & 1 & 0 & -1.149812 & 2.243349 & 2.525147 \\ 98 & 1 & 0 & 1.759594 & -2.102874 & -1.750970\end{array}$

\begin{tabular}{|c|c|c|c|}
\hline Frequencies -- & 8.3217 & 14.3967 & 24.6890 \\
\hline Frequencies -- & 26.8680 & 30.1279 & 34.4649 \\
\hline Frequencies -- & 39.9360 & 57.7758 & 60.7013 \\
\hline Frequencies -- & 61.4472 & 64.0926 & 66.1560 \\
\hline Frequencies -- & 70.4235 & 75.3722 & 78.8713 \\
\hline Frequencies -- & 80.0374 & 88.5692 & 92.2220 \\
\hline Frequencies -- & 95.5411 & 98.2983 & 115.2854 \\
\hline Frequencies -- & 123.0360 & 126.7227 & 156.8571 \\
\hline Frequencies -- & 165.3816 & 168.1554 & 180.7965 \\
\hline Frequencies -- & 186.5963 & 200.6144 & 212.3613 \\
\hline Frequencies -- & 223.1028 & 234.0689 & 242.7512 \\
\hline Frequencies -- & 246.6981 & 256.3885 & 275.2250 \\
\hline Frequencies -- & 279.0048 & 281.9186 & 287.0393 \\
\hline Frequencies -- & 304.8369 & 308.4643 & 325.9598 \\
\hline Frequencies -- & 331.5200 & 351.4566 & 364.7929 \\
\hline Frequencies -- & 389.9736 & 393.7433 & 401.1693 \\
\hline Frequencies -- & 409.1075 & 411.5680 & 412.3089 \\
\hline Frequencies -- & 414.9880 & 416.1157 & 421.0541 \\
\hline Frequencies -- & 424.5452 & 427.0591 & 438.6201 \\
\hline Frequencies -- & 451.1544 & 467.9518 & 489.3522 \\
\hline Frequencies -- & 504.9295 & 506.2769 & 521.1513 \\
\hline Frequencies -- & 533.0518 & 538.8416 & 543.1955 \\
\hline Frequencies -- & 551.4166 & 559.5573 & 560.4969 \\
\hline Frequencies -- & 577.3237 & 591.9856 & 609.2372 \\
\hline Frequencies -- & 614.6432 & 623.0631 & 625.4629 \\
\hline Frequencies -- & 629.6324 & 630.5925 & 631.3927 \\
\hline Frequencies -- & 636.8949 & 640.1782 & 654.3233 \\
\hline Frequencies -- & 664.0756 & 670.3377 & 678.4581 \\
\hline Frequencies -- & 679.1963 & 689.9705 & 704.7786 \\
\hline Frequencies -- & 708.1434 & 714.2588 & 714.9698 \\
\hline Frequencies -- & 718.8945 & 720.9156 & 722.9795 \\
\hline Frequencies -- & 730.8200 & 738.4918 & 772.8017 \\
\hline Frequencies -- & 775.4325 & 777.4520 & 777.5829 \\
\hline Frequencies -- & 789.4340 & 801.9367 & 818.9639 \\
\hline Frequencies -- & 824.5817 & 827.3409 & 841.9501 \\
\hline Frequencies -- & 849.6216 & 859.4826 & 864.2728 \\
\hline Frequencies -- & 869.2436 & 869.9932 & 871.1681 \\
\hline Frequencies -- & 876.1026 & 878.8703 & 895.7946 \\
\hline Frequencies -- & 916.7173 & 919.4143 & 929.9260 \\
\hline Frequencies -- & 932.9921 & 936.5695 & 938.9904 \\
\hline Frequencies -- & 941.7927 & 945.4250 & 947.5346 \\
\hline Frequencies -- & 950.9145 & 965.1946 & 970.9047 \\
\hline Frequencies -- & 975.1350 & 991.7135 & 992.6255 \\
\hline Frequencies -- & 996.1919 & 997.9799 & 998.6567 \\
\hline Frequencies -- & 1003.5512 & 1005.0479 & 1006.7625 \\
\hline Frequencies -- & 1009.2059 & 1011.2904 & 1012.1971 \\
\hline Frequencies -- & 1014.3310 & 1017.2873 & 1019.0846 \\
\hline Frequencies -- & 1019.3642 & 1019.7109 & 1020.1248 \\
\hline Frequencies -- & 1020.2890 & 1026.4517 & 1034.1444 \\
\hline Frequencies -- & 1044.0648 & 1052.7256 & 1061.8591 \\
\hline Frequencies -- & 1063.9136 & 1065.0410 & 1065.8993 \\
\hline Frequencies -- & 1069.7142 & 1071.2623 & 1087.8288 \\
\hline Frequencies -- & 1090.7156 & 1091.6043 & 1102.4219 \\
\hline Frequencies -- & 1105.8677 & 1110.9809 & 1112.1342 \\
\hline Frequencies -- & 1121.0582 & 1121.5278 & 1127.5710 \\
\hline Frequencies -- & 1138.2531 & 1173.1197 & 1174.3469 \\
\hline Frequencies -- & 1176.4924 & 1177.5289 & 1177.6608 \\
\hline Frequencies -- & 1184.3473 & 1187.2523 & 1191.7921 \\
\hline Frequencies -- & 1201.9716 & 1203.5971 & 1203.9156 \\
\hline
\end{tabular}




\begin{tabular}{|c|c|c|c|c|c|c|c|c|c|c|c|}
\hline \multicolumn{4}{|c|}{ Frequencies -- 1206.0112} & 1215.1487 & 1216.6946 & 17 & 6 & 0 & 2.866483 & -1.096563 & 3.694342 \\
\hline \multicolumn{2}{|c|}{ Frequencies -- } & 1220 & 0.0465 & 1225.0706 & 1230.8228 & 18 & 6 & 0 & 4.284335 & 0.544853 & 2.369417 \\
\hline \multicolumn{2}{|c|}{ Frequencies -- } & 1233 & 3.9399 & 1237.9755 & 1244.7962 & 19 & 6 & 0 & 4.124366 & 1.800551 & 3.257459 \\
\hline \multicolumn{2}{|c|}{ Frequencies -- } & 1256 & 6.6524 & 1260.4840 & 1273.4394 & 20 & 6 & 0 & 1.921754 & 3.266755 & 3.433256 \\
\hline \multicolumn{2}{|c|}{ Frequencies -- } & 1280 & 0.8282 & 1292.6944 & 1298.3234 & 21 & 1 & 0 & -0.013482 & 4.204672 & 3.344981 \\
\hline \multicolumn{2}{|c|}{ Frequencies -- } & 1300 & 0.4250 & 1309.9648 & 1314.4183 & 22 & 6 & 0 & 3.579192 & -2.408572 & 3.279682 \\
\hline \multicolumn{2}{|c|}{ Frequencies -- } & 1315 & 5.9783 & 1327.1241 & 1327.4086 & 23 & 6 & 0 & 3.496843 & -3.520124 & 0.909313 \\
\hline \multicolumn{2}{|c|}{ Frequencies -- } & 1330 & 0.4412 & 1336.6257 & 1338.0367 & 24 & 1 & 0 & 3.125849 & -4.222101 & -1.089260 \\
\hline \multicolumn{2}{|c|}{ Frequencies -- } & 1342 & 2.9000 & 1346.0748 & 1348.2330 & 25 & 1 & 0 & 3.320182 & -0.629299 & 4.573901 \\
\hline \multicolumn{2}{|c|}{ Frequencies -- } & 1358 & 8.8486 & 1359.3659 & 1362.5845 & 26 & 1 & 0 & 1.819460 & -1.312356 & 3.928842 \\
\hline Frequen & cies -- 1 & 1367 & 7.5700 & 1370.1764 & 1372.1630 & 27 & 1 & 0 & 5.120210 & -0.087731 & 2.683704 \\
\hline Frequen & cies -- 1 & 1376 & 6.1835 & 1381.2781 & 1387.3518 & 28 & 1 & 0 & 4.466503 & 0.853944 & 1.335092 \\
\hline Frequen & cies -- 1 & 1393 & 3.8722 & 1460.0234 & 1471.2433 & 29 & 1 & 0 & 4.342860 & 1.531457 & 4.298556 \\
\hline Frequen & cies -- 1 r a & 1473 & 3.3878 & 1491.7871 & 1492.8766 & 30 & 6 & 0 & 5.057827 & 2.919138 & 2.848034 \\
\hline Frequen & cies -- 1 r r r & 1497 & 7.3934 & 1502.5469 & 1502.9229 & 31 & 6 & 0 & 2.528513 & 4.419227 & 4.150738 \\
\hline Frequen & cies -- 1 r r r & 1505 & 5.6931 & 1510.7745 & 1510.8969 & 32 & 1 & 0 & 4.652135 & -2.341940 & 3.498573 \\
\hline Frequen & cies -- 1 r r r & 1528 & 8.3354 & 1530.3555 & 1531.1496 & 33 & 6 & 0 & 3.022557 & -3.637700 & 3.969803 \\
\hline Frequen & cies -- 1 & 1543 & 3.7125 & 1550.5156 & 1556.9158 & 34 & 6 & 0 & 4.141238 & -4.776585 & 1.378668 \\
\hline Frequen & cies -- 1 & 1564 & 4.5486 & 1568.3048 & 1666.6337 & 35 & 6 & 0 & 4.966018 & 3.471084 & 1.566540 \\
\hline Frequen & cies -- 1 & 1670 & 0.1920 & 1674.3682 & 1676.2323 & 36 & 6 & 0 & 6.034843 & 3.406705 & 3.718261 \\
\hline Frequen & cies -- 1 & 1678 & 8.8431 & 1682.6035 & 1684.8533 & 37 & 6 & 0 & 2.275476 & 5.736548 & 3.746482 \\
\hline Frequen & cies -- 1 & 1687 & 7.5536 & 1691.6455 & 1697.4074 & 38 & 6 & 0 & 3.314185 & 4.212112 & 5.290584 \\
\hline Frequen & cies -- 1 & 1700 & 0.3954 & 1701.0483 & 1702.8085 & 39 & 6 & 0 & 1.682030 & -3.995471 & 3.779284 \\
\hline Frequen & cies -- 1 & 1703 & 3.7819 & 2936.7385 & 3080.2570 & 40 & 6 & 0 & 3.832476 & -4.457386 & 4.756541 \\
\hline Frequen & cies -- 3 & 3082 & 2.1437 & 3090.6986 & 3094.2517 & 41 & 6 & 0 & 3.411965 & -5.964975 & 1.485497 \\
\hline Frequen & cies -- 3 & 3098 & 8.9664 & 3119.8970 & 3156.0623 & 42 & 6 & 0 & 5.486205 & -4.765296 & 1.764035 \\
\hline Frequen & cies -- 3 & 3159 & 9.3233 & 3181.3821 & 3184.7336 & 43 & 6 & 0 & 5.814720 & 4.501838 & 1.170738 \\
\hline Frequen & cies -- & 3189 & 9.4426 & 3198.5317 & 3199.8185 & 44 & 1 & 0 & 4.218663 & 3.093764 & 0.871274 \\
\hline Frequen & cies -- & 3202 & 2.5366 & 3204.9690 & 3206.3241 & 45 & 6 & 0 & 6.889609 & 4.437206 & 3.327502 \\
\hline Frequen & cies -- 3 & 3206 & 6.8751 & 3207.7105 & 3212.5021 & 46 & 1 & 0 & 6.118657 & 2.986606 & 4.718374 \\
\hline Frequen & cies -- & 3215 & 5.8539 & 3216.0215 & 3218.5717 & 47 & 6 & 0 & 2.823145 & 6.813910 & 4.440263 \\
\hline Frequen & cies -- 3 & 3218 & 8.6653 & 3219.0622 & 3224.9726 & 48 & 1 & 0 & 1.645106 & 5.922312 & 2.880151 \\
\hline Frequen & cies -- & 3225 & 5.0623 & 3226.6364 & 3227.3491 & 49 & 6 & 0 & 3.853625 & 5.286839 & 5.992560 \\
\hline Frequen & cies -- & 3228 & 3.5278 & 3234.6106 & 3235.9046 & 50 & 1 & 0 & 3.482442 & 3.198452 & 5.644557 \\
\hline Frequen & cies -- 3 & 3235 & 5.9924 & 3238.9789 & 3239.0507 & 51 & 6 & 0 & 1.166210 & -5.150513 & 4.360703 \\
\hline Frequen & cies -- 3 & 3241 & 1.4360 & 3242.3698 & 3243.2464 & 52 & 1 & 0 & 1.044391 & -3.378525 & 3.147599 \\
\hline Frequen & cies -- 3 & 3243 & 3.2665 & 3270.7599 & 3659.9581 & 53 & 6 & 0 & 3.320743 & -5.618821 & 5.337317 \\
\hline & & & & & & 54 & 1 & 0 & 4.879823 & -4.199995 & 4.897146 \\
\hline SCF Don & : $E(R M$ & 1062〉 & $\mathrm{X} /$ DGDZVP) $=$ & -2721.9930 & 05799 & 55 & 6 & 0 & 4.013593 & -7.117963 & 1.987603 \\
\hline Sum of $e$ & lectronic & ic an & d zero-point $E$ & Energies= & -2721.196148 & 56 & 1 & 0 & 2.361653 & -5.971530 & 1.205740 \\
\hline Sum of & electroni & ic an & d thermal En & ergies= & -2721.139938 & 57 & 6 & 0 & 6.092044 & -5.920371 & 2.256136 \\
\hline Sum of & electroni & ic an & d thermal Fre & ee Energies= & $=\quad-2721.293115$ & 58 & 1 & 0 & 6.056913 & -3.843898 & 1.672744 \\
\hline SCF Don & e: $E(R M$ & 1062〉 & X/DGTZVP/SN & MD) $=-2722$. & .60955718 & 59 & 6 & 0 & 6.779132 & 4.991709 & 2.053069 \\
\hline & & & & & & 60 & 1 & 0 & 5.721431 & 4.919151 & 0.171734 \\
\hline Cat b & TS1a & & & & & 61 & 1 & 0 & 7.636346 & 4.811299 & 4.021302 \\
\hline & & & & & & 62 & 6 & 0 & 3.619102 & 6.592999 & 5.563620 \\
\hline & & & & & & 63 & 1 & 0 & 2.626248 & 7.826940 & 4.102785 \\
\hline Center & Atomic & & Atomic & Coordinates & (Angstroms) & 64 & 1 & 0 & 4.455463 & 5.103577 & 6.877758 \\
\hline Number & Numb & nber & Type & $\mathrm{x}$ & Z & 65 & 6 & 0 & 1.986755 & -5.970048 & 5.139347 \\
\hline 1 & & 0 & & & 0.000000 & 66 & 1 & 0 & 0.126442 & -5.417706 & 4.196981 \\
\hline 1 & 15 & 0 & 0.000000 & 0.000000 & 0.000000 & 67 & 1 & 0 & 3.968624 & -6.253542 & 5.934478 \\
\hline 2 & 8 & 0 & 0.000000 & 0.000000 & 1.618772 & 68 & 6 & 0 & 5.353670 & -7.097841 & 2.376789 \\
\hline 3 & 8 & 0 & 1.592226 & 0.000000 & -0.355332 & 69 & 1 & 0 & 3.431972 & -8.029475 & 2.086715 \\
\hline 4 & 8 & 0 & -0.509173 & 1.314963 & -0.534852 & 70 & 1 & 0 & 7.137844 & -5.900631 & 2.548292 \\
\hline 5 & 8 & 0 & -0.652970 & -1.270953 & -0.449718 & 71 & 1 & 0 & 7.440393 & 5.797082 & 1.748451 \\
\hline 6 & 6 & 0 & 0.632436 & 1.075949 & 2.231535 & 72 & 1 & 0 & 4.046125 & 7.431578 & 6.104783 \\
\hline 7 & 6 & 0 & 2.294394 & -1.135914 & 0.049513 & 73 & 1 & 0 & 1.588505 & -6.876952 & 5.584132 \\
\hline 8 & 6 & 0 & 1.980827 & 0.993145 & 2.551352 & 74 & 1 & 0 & 5.819150 & -7.995959 & 2.771187 \\
\hline 9 & 6 & 0 & -0.082931 & 2.241570 & 2.487632 & 75 & 1 & 0 & -1.480501 & -1.365878 & -1.718523 \\
\hline 10 & 6 & 0 & 2.801607 & -1.221533 & 1.339756 & 76 & 8 & 0 & -2.042320 & -1.168400 & -2.557538 \\
\hline 11 & 6 & 0 & 2.398951 & -2.215965 & -0.822333 & 77 & 6 & 0 & -3.047496 & -0.191010 & -2.199641 \\
\hline 12 & 6 & 0 & 2.921829 & -0.186703 & 2.440575 & 78 & 6 & 0 & -2.537838 & 0.968578 & -3.076805 \\
\hline 13 & 6 & 0 & 2.631847 & 2.099956 & 3.112724 & 79 & 1 & 0 & -4.039093 & -0.568300 & -2.450337 \\
\hline 14 & 6 & 0 & 0.558190 & 3.313589 & 3.101150 & 80 & 1 & 0 & -2.983468 & 0.044810 & -1.133853 \\
\hline 15 & 6 & 0 & 3.340457 & -2.435321 & 1.774667 & 81 & 8 & 0 & -2.346508 & 2.161232 & -2.380567 \\
\hline 16 & 6 & 0 & 3.024304 & -3.387204 & -0.401366 & 82 & 6 & 0 & -1.228969 & 0.216874 & -3.462602 \\
\hline
\end{tabular}




$\begin{array}{cccccc}83 & 6 & 0 & -3.395627 & 1.229348 & -4.298460 \\ 84 & 1 & 0 & -1.878744 & 1.954498 & -1.543779 \\ 85 & 1 & 0 & -0.346055 & 0.266089 & -2.835207 \\ 86 & 1 & 0 & -1.138680 & -0.317836 & -4.399789 \\ 87 & 6 & 0 & -3.782583 & 2.526078 & -4.632679 \\ 88 & 6 & 0 & -3.793391 & 0.158492 & -5.106151 \\ 89 & 6 & 0 & -4.562195 & 2.748773 & -5.767771 \\ 90 & 1 & 0 & -3.463443 & 3.349454 & -4.003728 \\ 91 & 6 & 0 & -4.568233 & 0.383385 & -6.241474 \\ 92 & 1 & 0 & -3.500037 & -0.859432 & -4.849956 \\ 93 & 6 & 0 & -4.955005 & 1.682171 & -6.574810 \\ 94 & 1 & 0 & -4.858305 & 3.761147 & -6.024880 \\ 95 & 1 & 0 & -4.870832 & -0.453644 & -6.862959 \\ 96 & 1 & 0 & -5.558739 & 1.860614 & -7.459264 \\ 97 & 1 & 0 & 2.720612 & 5.111015 & 1.137689 \\ 98 & 6 & 0 & 2.491881 & 5.035831 & 0.078791 \\ 99 & 6 & 0 & 1.713102 & 3.977346 & -0.375335 \\ 100 & 6 & 0 & 3.011291 & 5.991215 & -0.807802 \\ 101 & 6 & 0 & 1.467376 & 3.904786 & -1.746568 \\ 102 & 1 & 0 & 1.306238 & 3.227341 & 0.301194 \\ 103 & 6 & 0 & 2.764380 & 5.909517 & -2.175754 \\ 104 & 7 & 0 & 0.711359 & 2.933980 & -2.395104 \\ 105 & 6 & 0 & 1.983006 & 4.851250 & -2.637828 \\ 106 & 1 & 0 & 3.167456 & 6.647565 & -2.861657 \\ 107 & 6 & 0 & 0.595465 & 3.056116 & -3.724614 \\ 108 & 1 & 0 & 0.234805 & 2.205275 & -1.832788 \\ 109 & 16 & 0 & 1.487442 & 4.463549 & -4.273097 \\ 110 & 16 & 0 & -0.243585 & 2.070003 & -4.794176 \\ 111 & 1 & 0 & -1.128952 & 2.291870 & 2.203336 \\ 112 & 1 & 0 & 1.970449 & -2.130086 & -1.815844 \\ 113 & 1 & 0 & 3.618148 & 6.804035 & -0.421285\end{array}$

Frequencies -- -549.7239

Frequencies -- 19.3456

Frequencies -- 30.2262

Frequencies -- 43.7776

Frequencies -- $\quad 51.2076$

Frequencies -- 65.3092

Frequencies -- 82.7495

Frequencies -- 94.0883

Frequencies -- 99.5185

Frequencies -- 130.0576

Frequencies -- 161.8679

Frequencies -- 185.1235

Frequencies -- 207.7187

Frequencies -- 226.0023

Frequencies -- 251.9956

Frequencies -- 276.9322

Frequencies -- 293.8888

Frequencies -- 324.8935

Frequencies -- 365.2607

Frequencies -- 398.1744

Frequencies -- 412.1627

Frequencies -- 415.4702

Frequencies -- 421.9875

Frequencies -- 445.5222

Frequencies -- 471.8565

Frequencies -- 508.9477

Frequencies -- 531.5615

Frequencies -- 542.6686

Frequencies -- 576.3773

Frequencies -- 597.7045

Frequencies -- 618.9233

Frequencies -- 628.9191

Frequencies -- 637.9256
23.8691

30.7234

44.8968

57.9965

73.0161

85.6103

97.4307

113.6584

131.8091

171.2765

193.2343

210.3814

231.3263

261.8995

284.1573

304.7843

330.0821

385.4473

406.0363

412.9732

418.2602

433.6511

452.8911

493.7147

511.9447

536.4820

555.2510

576.7275

609.2676

623.3498

629.5585

639.9197
13.3012

28.8128

38.7619

48.9787

61.7455

77.0646

90.3757

98.6858

122.8922

156.9583

181.3600

194.2303

218.1021

241.2168

273.3999

289.2470

313.7676

351.7046

387.7907

411.1028

414.9469

421.6718

438.7661

461.3236

507.9045

523.9004

538.6139

567.6414

586.1916

614.8005

627.0654

632.3067

653.8270
Frequencies -- 663.9226

Frequencies -- 681.2067

Frequencies -- 704.6898

Frequencies -- 717.4250

Frequencies -- 722.2896

Frequencies -- 736.7359

Frequencies -- 769.8775

Frequencies -- 779.8519

Frequencies -- 799.4480

Frequencies -- 841.6531

Frequencies -- 864.3374

Frequencies -- 872.6014

Frequencies -- 881.0133

Frequencies -- 896.7844

Frequencies -- 933.1828

Frequencies -- 939.9291

Frequencies -- 944.2371

Frequencies -- 967.6953

Frequencies -- 995.6968

Frequencies -- 998.8910

Frequencies -- 1010.2259

Frequencies -- 1011.3027

Frequencies -- 1019.1216

Frequencies -- 1020.8501

Frequencies -- 1042.5095

Frequencies -- 1053.3305

Frequencies -- 1064.7651

Frequencies -- 1071.5099

Frequencies -- 1089.2725

Frequencies -- 1105.7129

Frequencies -- 1114.3686

Frequencies -- 1124.1738

Frequencies -- 1153.7644

Frequencies -- 1176.2361

Frequencies -- 1178.9751

Frequencies -- 1198.1238

Frequencies -- 1207.5547

Frequencies -- 1210.8214

Frequencies -- 1224.7701

Frequencies -- 1241.0772

Frequencies -- 1264.9370

Frequencies -- 1291.0639

Frequencies -- 1300.3763

Frequencies -- 1312.1854

Frequencies -- 1333.6003

Frequencies -- 1343.8922

Frequencies -- 1357.2361

Frequencies -- 1367.0073

Frequencies -- 1371.5801

Frequencies -- 1385.6343

Frequencies -- 1451.7902

Frequencies -- 1474.6896

Frequencies -- 1494.2118

Frequencies -- 1500.9803

Frequencies -- 1514.9389

Frequencies -- 1530.5881

Frequencies -- 1552.9174

Frequencies -- 1567.8984

Frequencies -- 1673.5065

Frequencies -- 1678.3727

Frequencies -- 1684.7438

Frequencies -- 1698.4869

Frequencies -- 1702.0148

Frequencies -- 3074.7061

Frequencies -- 3092.8142

Frequencies -- 3157.8132
664.8229

683.8269

712.2917

719.3055

725.7112

739.8658

771.9357

780.4396

817.6459

849.8458

866.1514

873.3777

885.4618

901.2759

934.7806

942.1533

954.0321

974.7973

997.4508

1001.8149

1010.4612

1017.4616

1019.2921

1022.6789

1045.5146

1058.7613

1068.2401

1079.2893

1102.0676

1109.4813

1117.6495

1125.6342

1158.9257

1176.2917

1180.3117

1202.0466

1209.4407

1214.1901

1230.9172

1246.8046

1277.4318

1295.4487

1306.0305

1324.7021

1337.2053

1346.9664

1358.6251

1368.0774

1376.9730

1394.7940

1465.0351

1478.2164

1499.5537

1506.4187

1523.7025

1547.9568

1555.5070

1662.8643

1675.6312

1679.5446

1686.4080

1698.7700

1702.9815

3077.5782

3121.3975

3189.8592

673.0238

693.2976

713.0174

720.7706

732.1741

748.8462

775.5007

783.7047

825.0657

862.7805

868.5120

879.2431

890.4430

904.1417

939.4745

943.3234

965.8221

993.3685

998.2229

1006.1902

1011.1586

1018.0736

1019.9908

1027.5733

1051.5983

1063.4426

1069.8021

1087.2321

1103.2958

1111.1481

1119.8422

1139.0890

1175.2888

1177.6660

1189.0403

1206.3841

1209.8179

1214.6140

1232.8730

1257.1405

1289.9534

1296.7475

1308.3665

1327.4939

1343.3444

1349.7928

1366.1566

1370.0194

1382.6429

1441.8145

1468.4369

1490.6592

1500.6174

1510.6853

1527.6211

1550.7214 


\begin{tabular}{llll} 
Frequencies -- 3201.9876 & 3202.3093 & 3202.9041 \\
Frequencies -- 3204.5087 & 3205.3540 & 3207.3949 \\
Frequencies -- 3207.8445 & 3213.0652 & 3213.7088 \\
Frequencies -- 3215.3823 & 3216.8504 & 3217.7117 \\
Frequencies -- 3218.2762 & 3218.8263 & 3218.9176 \\
Frequencies -- 3221.8018 & 3224.9566 & 3226.0826 \\
Frequencies -- 3226.6571 & 3229.2161 & 3229.7456 \\
Frequencies -- 3229.8253 & 3233.1269 & 3235.1881 \\
Frequencies -- 3237.4690 & 3239.6311 & 3239.7516 \\
Frequencies -- 3241.4530 & 3241.6534 & 3242.8627 \\
Frequencies -- 3243.7242 & 3247.5105 & 3250.0473 \\
Frequencies -- 3258.8232 & 3349.5619 & 3608.6537 \\
\multicolumn{3}{c}{} \\
SCF Done: E(RM062X/DGDZVP) = -3842.65727870 \\
Sum of electronic and zero-point Energies= -3841.753818 \\
Sum of electronic and thermal Energies= & -3841.686876 \\
Sum of electronic and thermal Free Energies= & -3841.863287 \\
SCF Done: E(RM062X/DGTZVP/SMD) = -3843.43434433
\end{tabular}

\section{Cat b TS1b_S}

Center Atomic Atomic Coordinates (Angstroms) Number Number Type $X \quad Y \quad Z$

$\begin{array}{cccccc}1 & 15 & 0 & 0.000000 & 0.000000 & 0.000000 \\ 2 & 8 & 0 & 0.000000 & 0.000000 & 1.635973 \\ 3 & 8 & 0 & 1.571144 & 0.000000 & -0.374553 \\ 4 & 8 & 0 & -0.546272 & 1.310179 & -0.510647 \\ 5 & 8 & 0 & -0.688819 & -1.270068 & -0.395588 \\ 6 & 6 & 0 & 0.535663 & 1.122420 & 2.256090 \\ 7 & 6 & 0 & 2.337882 & -1.057994 & 0.108730 \\ 8 & 6 & 0 & 1.907113 & 1.234624 & 2.447734 \\ 9 & 6 & 0 & -0.312789 & 2.158626 & 2.635878 \\ 10 & 6 & 0 & 2.940708 & -0.955128 & 1.355287 \\ 11 & 6 & 0 & 2.448355 & -2.224865 & -0.640780 \\ 12 & 6 & 0 & 3.009281 & 0.203187 & 2.328028 \\ 13 & 6 & 0 & 2.431152 & 2.443657 & 2.913597 \\ 14 & 6 & 0 & 0.219109 & 3.335395 & 3.160188 \\ 15 & 6 & 0 & 3.623184 & -2.060445 & 1.867900 \\ 16 & 6 & 0 & 3.199522 & -3.289413 & -0.146103 \\ 17 & 6 & 0 & 3.182914 & -0.561612 & 3.668910 \\ 18 & 6 & 0 & 4.233921 & 1.118492 & 2.074371 \\ 19 & 6 & 0 & 3.954336 & 2.409325 & 2.884567 \\ 20 & 6 & 0 & 1.602630 & 3.503831 & 3.291269 \\ 21 & 1 & 0 & -0.446075 & 4.140842 & 3.464668 \\ 22 & 6 & 0 & 3.995200 & -1.834992 & 3.327383 \\ 23 & 6 & 0 & 3.800417 & -3.224691 & 1.116139 \\ 24 & 1 & 0 & 3.308773 & -4.194073 & -0.738139 \\ 25 & 1 & 0 & 3.660315 & 0.049443 & 4.441299 \\ 26 & 1 & 0 & 2.194701 & -0.854132 & 4.037677 \\ 27 & 1 & 0 & 5.177042 & 0.640045 & 2.356112 \\ 28 & 1 & 0 & 4.283607 & 1.359164 & 1.007601 \\ 29 & 1 & 0 & 4.337762 & 2.296409 & 3.906517 \\ 30 & 6 & 0 & 4.576134 & 3.653962 & 2.285649 \\ 31 & 6 & 0 & 2.170936 & 4.772649 & 3.821995 \\ 32 & 1 & 0 & 5.070066 & -1.636595 & 3.423068 \\ 33 & 6 & 0 & 3.642037 & -3.018852 & 4.205443 \\ 34 & 6 & 0 & 4.594167 & -4.361959 & 1.656327 \\ 35 & 6 & 0 & 4.258155 & 4.048564 & 0.980818 \\ 36 & 6 & 0 & 5.451392 & 4.446588 & 3.030366 \\ 37 & 6 & 0 & 2.121208 & 5.939270 & 3.053065 \\ 38 & 6 & 0 & 2.785872 & 4.807063 & 5.077059 \\ 39 & 6 & 0 & 2.337228 & -3.527493 & 4.195333 \\ 40 & 6 & 0 & 4.602724 & -3.649550 & 4.996124 \\ 41 & 6 & 0 & 3.984708 & -5.581745 & 1.967130\end{array}$

\begin{tabular}{|c|c|c|c|c|c|}
\hline 42 & 6 & 0 & 5.961957 & -4.202404 & 1.902758 \\
\hline 43 & 6 & 0 & 4.808813 & 5.206580 & 0.436277 \\
\hline 44 & 1 & 0 & 3.567630 & 3.446977 & 0.391456 \\
\hline 45 & 6 & 0 & 5.990389 & 5.616548 & 2.495428 \\
\hline 46 & 1 & 0 & 5.697382 & 4.157818 & 4.049569 \\
\hline 47 & 6 & 0 & 2.700542 & 7.117203 & 3.520665 \\
\hline 48 & 1 & 0 & 1.654187 & 5.906819 & 2.071281 \\
\hline 49 & 6 & 0 & 3.354543 & 5.988879 & 5.551885 \\
\hline 50 & 1 & 0 & 2.814610 & 3.903459 & 5.681696 \\
\hline 51 & 6 & 0 & 2.004206 & -4.644363 & 4.956607 \\
\hline 52 & 1 & 0 & 1.582861 & -3.060790 & 3.563548 \\
\hline 53 & 6 & 0 & 4.274724 & -4.772808 & 5.757451 \\
\hline 54 & 1 & 0 & 5.624338 & -3.276335 & 4.997544 \\
\hline 55 & 6 & 0 & 4.726735 & -6.616250 & 2.534810 \\
\hline 56 & 1 & 0 & 2.918550 & -5.703734 & 1.794003 \\
\hline 57 & 6 & 0 & 6.708088 & -5.240482 & 2.459010 \\
\hline 58 & 1 & 0 & 6.439976 & -3.257957 & 1.652897 \\
\hline 59 & 6 & 0 & 5.670839 & 6.000613 & 1.194471 \\
\hline 60 & 1 & 0 & 4.573770 & 5.489946 & -0.585578 \\
\hline 61 & 1 & 0 & 6.654389 & 6.228821 & 3.098162 \\
\hline 62 & 6 & 0 & 3.320370 & 7.144734 & 4.770820 \\
\hline 63 & 1 & 0 & 2.676692 & 8.010644 & 2.903216 \\
\hline 64 & 1 & 0 & 3.826373 & 6.007137 & 6.529925 \\
\hline 65 & 6 & 0 & 2.974793 & -5.274685 & 5.738566 \\
\hline 66 & 1 & 0 & 0.989564 & -5.030639 & 4.931685 \\
\hline 67 & 1 & 0 & 5.039509 & -5.260957 & 6.354163 \\
\hline 68 & 6 & 0 & 6.089275 & -6.447632 & 2.784787 \\
\hline 69 & 1 & 0 & 4.237215 & -7.550226 & 2.793728 \\
\hline 70 & 1 & 0 & 7.770042 & -5.105333 & 2.641755 \\
\hline 71 & 1 & 0 & 6.091927 & 6.907987 & 0.772451 \\
\hline 72 & 1 & 0 & 3.772469 & 8.062136 & 5.135475 \\
\hline 73 & 1 & 0 & 2.718802 & -6.151875 & 6.324940 \\
\hline 74 & 1 & 0 & 6.664578 & -7.253145 & 3.230979 \\
\hline 75 & 1 & 0 & -2.252159 & -1.304869 & -0.242882 \\
\hline 76 & 8 & 0 & -3.238918 & -1.048958 & -0.315514 \\
\hline 77 & 6 & 0 & -3.421630 & -0.434324 & -1.612538 \\
\hline 78 & 6 & 0 & -3.773061 & 0.991326 & -1.144266 \\
\hline 79 & 1 & 0 & -2.483386 & -0.456599 & -2.173331 \\
\hline 80 & 1 & 0 & -4.210122 & -0.951938 & -2.159081 \\
\hline 81 & 8 & 0 & -2.947503 & 1.975254 & -1.690961 \\
\hline 82 & 6 & 0 & -3.490383 & 0.679740 & 0.352937 \\
\hline 83 & 6 & 0 & -5.223490 & 1.367080 & -1.367703 \\
\hline 84 & 1 & 0 & -2.016216 & 1.689086 & -1.574824 \\
\hline 85 & 1 & 0 & -4.288134 & 0.395712 & 1.028744 \\
\hline 86 & 1 & 0 & -2.497824 & 0.787029 & 0.769815 \\
\hline 87 & 6 & 0 & -5.564139 & 2.576423 & -1.970993 \\
\hline 88 & 6 & 0 & -6.232908 & 0.490706 & -0.956813 \\
\hline 89 & 6 & 0 & -6.906529 & 2.907682 & -2.156510 \\
\hline 90 & 1 & 0 & -4.774759 & 3.249491 & -2.286503 \\
\hline 91 & 6 & 0 & -7.572709 & 0.825317 & -1.137707 \\
\hline 92 & 1 & 0 & -5.976015 & -0.461508 & -0.492871 \\
\hline 93 & 6 & 0 & -7.912471 & 2.037708 & -1.739333 \\
\hline 94 & 1 & 0 & -7.166142 & 3.851816 & -2.625527 \\
\hline 95 & 1 & 0 & -8.349297 & 0.140228 & -0.812107 \\
\hline 96 & 1 & 0 & -8.956029 & 2.300371 & -1.882476 \\
\hline 97 & 1 & 0 & -1.229040 & 2.747167 & 0.069163 \\
\hline 98 & 7 & 0 & -1.524899 & 3.714627 & 0.307594 \\
\hline 99 & 6 & 0 & -2.705312 & 4.006900 & 0.860179 \\
\hline 100 & 6 & 0 & -0.653146 & 4.775364 & 0.110357 \\
\hline 101 & 16 & 0 & -3.964884 & 2.956672 & 1.241458 \\
\hline 102 & 16 & 0 & -2.800600 & 5.726941 & 1.192250 \\
\hline 103 & 6 & 0 & -1.194914 & 5.990508 & 0.541437 \\
\hline 104 & 6 & 0 & 0.614843 & 4.698139 & -0.465661 \\
\hline 105 & 6 & 0 & -0.476388 & 7.177131 & 0.395693 \\
\hline 106 & 6 & 0 & 1.319451 & 5.886292 & -0.621499 \\
\hline 107 & 1 & 0 & 1.008400 & 3.736676 & -0.784844 \\
\hline
\end{tabular}




$\begin{array}{rrrrrr}108 & 6 & 0 & 0.781862 & 7.111560 & -0.197554 \\ 109 & 1 & 0 & -0.886748 & 8.124412 & 0.729920 \\ 110 & 1 & 0 & 2.304540 & 5.865274 & -1.073909 \\ 111 & 1 & 0 & -1.388354 & 2.034194 & 2.524605 \\ 112 & 1 & 0 & 1.932432 & -2.286363 & -1.593439 \\ 113 & 1 & 0 & 1.356476 & 8.022799 & -0.327555\end{array}$

Frequencies -- -551.3082

Frequencies -- 18.8554

Frequencies -- 33.1426

Frequencies -- 45.1772

Frequencies -- 56.5464

Frequencies -- $\quad 67.2065$

Frequencies -- $\quad 77.4810$

Frequencies -- 90.6767

Frequencies -- 107.6663

Frequencies -- 128.9696

Frequencies -- 163.0046

Frequencies -- 185.5186

Frequencies -- 205.7507

Frequencies -- 232.1842

Frequencies -- 249.7436

Frequencies -- 275.8069

Frequencies -- 297.5127

Frequencies -- 309.9414

Frequencies -- 354.0328

Frequencies -- 394.9181

Frequencies -- 408.6948

Frequencies -- 413.4232

Frequencies -- 428.6219

Frequencies -- 438.7741

Frequencies -- 472.9498

Frequencies -- 509.5132

Frequencies -- 531.0801

Frequencies -- 543.5424

Frequencies -- 574.0243

Frequencies -- 595.9591

Frequencies -- 617.3478

Frequencies -- 628.8878

Frequencies -- 637.1481

Frequencies -- 659.9733

Frequencies -- 680.5521

Frequencies -- 695.3628

Frequencies -- 713.4651

Frequencies -- 719.8810

Frequencies -- 733.6054

Frequencies -- 759.7774

Frequencies -- 777.1179

Frequencies -- 799.5768

Frequencies -- 845.9614

Frequencies -- 862.2181

Frequencies -- 869.9251

Frequencies -- 875.2745

Frequencies -- 896.7789

Frequencies -- 932.5394

Frequencies -- 939.8742

Frequencies -- 950.1128

Frequencies -- 967.7672

Frequencies -- 989.1193

Frequencies -- 997.5887

Frequencies -- 1005.7330

Frequencies -- 1008.8285

Frequencies -- 1018.9957

Frequencies -- 1019.9979

Frequencies -- 1041.2700
11.4255

24.8882

16.8705

29.1649

$36.4211 \quad 36.8854$

$50.8296 \quad 55.6331$

$61.3090 \quad 63.0220$

$69.9126 \quad 77.2147$

$82.2687 \quad 83.0798$

$93.4105 \quad 95.2208$

$114.1708 \quad 121.1726$

$133.3957 \quad 150.3469$

$168.1643 \quad 178.8419$

$192.2169 \quad 193.4189$

$214.4970 \quad 226.9680$

$240.3713 \quad 241.7539$

$256.5519 \quad 270.5496$

$280.1772 \quad 293.2751$

$300.1951 \quad 307.6656$

$335.1026 \quad 351.0721$

$385.4807 \quad 390.9976$

$405.8226 \quad 407.2998$

$409.5498 \quad 411.4163$

$418.2831 \quad 418.4471$

$429.4348 \quad 433.3777$

$454.0561 \quad 456.0592$

$492.9442 \quad 507.4842$

$516.1330 \quad 520.2876$

$534.3359 \quad 538.4504$

$558.2160 \quad 565.9310$

$575.7059 \quad 584.1726$

$608.3766 \quad 613.5775$

$623.0307 \quad 625.2530$

$628.9317 \quad 631.8394$

$639.3562 \quad 654.7941$

$664.5618 \quad 670.4184$

$683.0100 \quad 693.3374$

$707.9606 \quad 712.2936$

$716.6396 \quad 718.3757$

$725.2742 \quad 729.2203$

$737.2771 \quad 747.8982$

$772.6654 \quad 775.4707$

$777.3845 \quad 787.9007$

$817.7798 \quad 825.0761$

$847.8729 \quad 852.3920$

$864.3638 \quad 868.5190$

$872.3074 \quad 874.0717$

$885.8297 \quad 893.4606$

$899.0820 \quad 926.5214$

$934.3675 \quad 936.9247$

$940.3512 \quad 944.1633$

$953.4337 \quad 959.2908$

$983.1536 \quad 986.5824$

$992.2010 \quad 995.8840$

$998.0719 \quad 999.5123$

$1007.7338 \quad 1008.7501$

$1010.0845 \quad 1014.7203$

$1019.9298 \quad 1019.9810$

$1020.0430 \quad 1023.4793$

$1042.9230 \quad 1049.0641$
Frequencies -- 1051.9927

Frequencies -- 1061.7586

Frequencies -- 1069.6853

Frequencies -- 1085.0225

Frequencies -- 1104.0999

Frequencies -- 1109.9586

Frequencies -- 1122.4037

Frequencies -- 1137.7863

Frequencies -- 1175.6987

Frequencies -- 1181.3359

Frequencies -- 1187.1170

Frequencies -- 1201.4153

Frequencies -- 1210.3298

Frequencies -- 1224.6148

Frequencies -- 1236.8016

Frequencies -- 1263.0338

Frequencies -- 1292.4414

Frequencies -- 1297.9771

Frequencies -- 1312.0900

Frequencies -- 1335.4437

Frequencies -- 1344.1779

Frequencies -- 1357.6857

Frequencies -- 1366.5958

Frequencies -- 1374.1234

Frequencies -- 1392.1873

Frequencies -- 1454.9264

Frequencies -- 1470.1141

Frequencies -- 1491.1811

Frequencies -- 1502.2060

Frequencies -- 1518.3160

Frequencies -- 1529.5736

Frequencies -- 1553.4980

Frequencies -- 1568.3828

Frequencies -- 1672.5210

Frequencies -- 1679.7818

Frequencies -- 1682.9827

Frequencies -- 1696.8653

Frequencies -- 1702.5301

Frequencies -- 3069.7329

Frequencies -- 3089.6319

Frequencies -- 3155.1438

Frequencies -- 3188.9759

Frequencies -- 3198.1601

Frequencies -- 3207.8112

Frequencies -- 3213.3659

Frequencies -- 3217.4738

Frequencies -- 3219.1517

Frequencies -- 3223.3603

Frequencies -- 3226.3477

Frequencies -- 3231.6576

Frequencies -- 3240.7487

Frequencies -- 3244.6144

Frequencies -- 3249.7938
1057.3489

1067.9060

1072.1586

1098.4365

1108.5637

1115.6916

1123.0426

1155.7893

1176.2400

1182.7264

1199.4478

1208.2060

1210.7373

1230.6229

1243.3241

1278.3067

1292.9983

1309.4287

1324.9780

1336.7659

1344.9289

1357.7241

1367.9115

1375.5855

1392.8890

1456.4374

1481.5467

1499.3092

1505.7840

1525.9928

1547.7294

1561.3521

1667.0979

1676.0932

1681.5600

1689.5634

1698.7932

1703.2226

3072.8123

3120.3374

3168.4648

3191.8674

3199.1163

3209.1790

3214.4475

3217.6261

3222.3777

3224.9021

3228.2440

3235.9143

3242.0259

3245.2420

3362.3208
1061.5735

1068.5907

1077.6421

1100.4180

1109.4309

1118.0006

1133.8906

1173.2853

1178.6003

1183.8491

1199.8760

1208.5668

1212.8417

1232.9914

1252.8488

1286.9818

1295.4441

1310.6529

1325.6429

1340.5262

1356.7796

1366.0276

1368.1613

1382.8033

1437.9232

1468.3001

1490.6522

1500.6891

1509.3049

1527.3101

1548.0121

1563.9334

1668.0577

1678.6774

1681.6725

1689.9591

1699.4342

2812.0922

3088.5223

3152.5139

3178.3653

3196.8089

3205.5653

3209.8980

3215.1686

3219.1102

3222.7520

3225.5544

3228.9897

3237.5038

3243.1585

3249.1612

3602.2240

SCF Done: $E(R M 062 X / D G D Z V P)=-3842.65836648$

Sum of electronic and zero-point Energies $=\quad-3841.755729$

Sum of electronic and thermal Energies $=\quad-3841.688686$

Sum of electronic and thermal Free Energies $=\quad-3841.864539$ SCF Done: $E(R M 062 X / D G T Z V P / S M D)=-3843.43328230$

\section{Cat b TS1c_S}

Center Atomic Atomic Coordinates (Angstroms)

Number Number Type $\quad X \quad$ Y $\quad$ Z




$\begin{array}{llllll}67 & 1 & 0 & 4.976768 & -5.993616 & 5.798955 \\ 68 & 6 & 0 & 5.773925 & -6.920991 & 2.167585 \\ 69 & 1 & 0 & 3.859029 & -7.906641 & 2.058822 \\ 70 & 1 & 0 & 7.533617 & -5.676927 & 2.163449 \\ 71 & 1 & 0 & 6.499567 & 6.363884 & 1.018682 \\ 72 & 1 & 0 & 4.541536 & 7.340868 & 5.738121 \\ 73 & 1 & 0 & 2.625991 & -6.802739 & 5.895779 \\ 74 & 1 & 0 & 6.297703 & -7.800580 & 2.529551 \\ 75 & 1 & 0 & -0.811877 & 1.237116 & -2.144171 \\ 76 & 8 & 0 & -1.129899 & 0.950254 & -3.071442 \\ 77 & 6 & 0 & -2.420891 & 0.317848 & -2.904052 \\ 78 & 6 & 0 & -2.037250 & -1.106092 & -3.349990 \\ 79 & 1 & 0 & -2.718380 & 0.346812 & -1.852638 \\ 80 & 1 & 0 & -3.162208 & 0.818192 & -3.527507 \\ 81 & 8 & 0 & -2.343624 & -2.085186 & -2.403812 \\ 82 & 6 & 0 & -0.521604 & -0.772664 & -3.461811 \\ 83 & 6 & 0 & -2.613749 & -1.506197 & -4.692788 \\ 84 & 1 & 0 & -2.001580 & -1.783475 & -1.535341 \\ 85 & 1 & 0 & -0.076871 & -0.498527 & -4.411152 \\ 86 & 1 & 0 & 0.137401 & -0.858178 & -2.608017 \\ 87 & 6 & 0 & -3.227659 & -2.746212 & -4.862782 \\ 88 & 6 & 0 & -2.515350 & -0.628546 & -5.777051 \\ 89 & 6 & 0 & -3.736029 & -3.105695 & -6.110832 \\ 90 & 1 & 0 & -3.295193 & -3.421315 & -4.017103 \\ 91 & 6 & 0 & -3.020187 & -0.990646 & -7.023874 \\ 92 & 1 & 0 & -2.042540 & 0.345449 & -5.652481 \\ 93 & 6 & 0 & -3.632928 & -2.233063 & -7.192875 \\ 94 & 1 & 0 & -4.209355 & -4.074357 & -6.238614 \\ 95 & 1 & 0 & -2.937820 & -0.303562 & -7.860259 \\ 96 & 1 & 0 & -4.026759 & -2.518115 & -8.163533 \\ 97 & 1 & 0 & -0.163316 & -2.749108 & -1.161905 \\ 98 & 7 & 0 & 0.015541 & -3.724365 & -1.475847 \\ 99 & 6 & 0 & 0.233928 & -4.062691 & -2.749498 \\ 100 & 6 & 0 & 0.083399 & -4.746241 & -0.541331 \\ 101 & 16 & 0 & 0.248483 & -3.059994 & -4.101157 \\ 102 & 16 & 0 & 0.549642 & -5.785361 & -2.862535 \\ 103 & 6 & 0 & 0.365548 & -5.982692 & -1.129977 \\ 104 & 6 & 0 & -0.112227 & -4.610700 & 0.833075 \\ 105 & 6 & 0 & 0.441110 & -7.136332 & -0.349164 \\ 106 & 6 & 0 & -0.047585 & -5.765968 & 1.603342 \\ 107 & 1 & 0 & -0.315804 & -3.630702 & 1.257955 \\ 108 & 6 & 0 & 0.222320 & -7.014196 & 1.020905 \\ 109 & 1 & 0 & 0.657140 & -8.101403 & -0.795713 \\ 110 & 1 & 0 & -0.205307 & -5.701213 & 2.674014 \\ 111 & 1 & 0 & 2.169504 & -2.042006 & -1.913172 \\ 112 & 1 & 0 & -1.157818 & 2.249246 & 2.263298 \\ 113 & 1 & 0 & 0.268908 & -7.898590 & 1.647894\end{array}$

$\begin{array}{cccc}\text { Frequencies -- } & -550.4680 & 15.0536 & 18.3299 \\ \text { Frequencies -- } & 21.3497 & 25.8595 & 32.5099 \\ \text { Frequencies -- } & 35.9578 & 37.5800 & 43.7581 \\ \text { Frequencies -- } & 43.9935 & 51.6410 & 58.0689 \\ \text { Frequencies -- } & 59.4875 & 65.3430 & 67.4357 \\ \text { Frequencies -- } & 70.3083 & 72.8181 & 78.5221 \\ \text { Frequencies -- } & 80.2129 & 84.1298 & 91.3177 \\ \text { Frequencies -- } & 93.4051 & 97.4063 & 99.5105 \\ \text { Frequencies -- } & 114.1915 & 117.1154 & 121.5759 \\ \text { Frequencies -- } & 130.5529 & 137.5501 & 150.0111 \\ \text { Frequencies -- } & 163.5884 & 169.6330 & 181.7332 \\ \text { Frequencies -- } & 189.1263 & 193.2901 & 197.6020 \\ \text { Frequencies -- } & 206.7836 & 215.3612 & 226.9782 \\ \text { Frequencies -- } & 232.9651 & 240.2553 & 243.0599 \\ \text { Frequencies -- } & 251.3020 & 257.7945 & 271.3404 \\ \text { Frequencies -- } & 276.2701 & 280.9448 & 292.7332 \\ \text { Frequencies -- } & 297.3359 & 302.3314 & 308.8142\end{array}$




\begin{tabular}{|c|c|c|c|}
\hline Frequencies -- & 309.4999 & 333.9401 & 352.9562 \\
\hline Frequencies -- & 354.7359 & 386.6714 & 392.0680 \\
\hline Frequencies -- & 396.0379 & 406.8722 & 408.8800 \\
\hline Frequencies -- & 410.7192 & 412.1718 & 413.6843 \\
\hline Frequencies -- & 416.5310 & 420.1607 & 421.1822 \\
\hline Frequencies -- & 428.9706 & 431.5573 & 435.7274 \\
\hline Frequencies -- & 438.7534 & 454.1363 & 458.4675 \\
\hline Frequencies -- & 473.2010 & 493.2484 & 507.7284 \\
\hline Frequencies -- & 510.3631 & 516.7122 & 523.9324 \\
\hline Frequencies -- & 529.7062 & 536.2959 & 539.6585 \\
\hline Frequencies -- & 544.3247 & 558.2039 & 566.1188 \\
\hline Frequencies -- & 575.4236 & 576.5009 & 584.4632 \\
\hline Frequencies -- & 596.2445 & 609.3712 & 614.1290 \\
\hline Frequencies -- & 618.4841 & 623.4565 & 625.8917 \\
\hline Frequencies -- & 627.9074 & 629.5186 & 631.8093 \\
\hline Frequencies -- & 637.0754 & 640.9574 & 655.0182 \\
\hline Frequencies -- & 659.7231 & 665.9626 & 670.6371 \\
\hline Frequencies -- & 681.4934 & 684.4364 & 695.0432 \\
\hline Frequencies -- & 704.6601 & 712.7759 & 715.4162 \\
\hline Frequencies -- & 717.6469 & 721.3465 & 721.4669 \\
\hline Frequencies -- & 723.9074 & 725.3109 & 730.0078 \\
\hline Frequencies -- & 736.2047 & 738.0943 & 748.2267 \\
\hline Frequencies -- & 762.6645 & 773.8548 & 775.8796 \\
\hline Frequencies -- & 779.2043 & 780.2688 & 789.0648 \\
\hline Frequencies -- & 801.0697 & 818.5425 & 826.3998 \\
\hline Frequencies -- & 849.8636 & 852.5034 & 866.7285 \\
\hline Frequencies -- & 867.6684 & 870.8805 & 871.3971 \\
\hline Frequencies -- & 878.0748 & 880.2655 & 881.6525 \\
\hline Frequencies -- & 881.8348 & 887.7669 & 895.2239 \\
\hline Frequencies -- & 898.1323 & 900.9121 & 930.9715 \\
\hline Frequencies -- & 936.3198 & 938.3345 & 940.5586 \\
\hline Frequencies -- & 942.9611 & 947.6575 & 949.5457 \\
\hline Frequencies -- & 954.3918 & 955.6182 & 963.2474 \\
\hline Frequencies -- & 974.9825 & 991.6251 & 993.9779 \\
\hline Frequencies -- & 995.1508 & 997.0208 & 999.5323 \\
\hline Frequencies -- & 1000.6649 & 1005.3458 & 1005.5216 \\
\hline Frequencies -- & 1008.4775 & 1008.6954 & 1010.6864 \\
\hline Frequencies -- & 1013.4948 & 1014.6656 & 1019.4386 \\
\hline Frequencies -- & 1019.5704 & 1019.7573 & 1020.3496 \\
\hline Frequencies -- & 1020.6296 & 1021.2672 & 1026.2052 \\
\hline Frequencies -- & 1041.9652 & 1043.8727 & 1050.3781 \\
\hline Frequencies -- & 1052.5357 & 1057.4462 & 1061.7466 \\
\hline Frequencies -- & 1064.3276 & 1069.0294 & 1069.9192 \\
\hline Frequencies -- & 1071.8533 & 1074.8850 & 1080.0159 \\
\hline Frequencies -- & 1086.8045 & 1100.8203 & 1102.9558 \\
\hline Frequencies -- & 1105.6547 & 1108.9848 & 1110.1538 \\
\hline Frequencies -- & 1112.7034 & 1116.6512 & 1117.5322 \\
\hline Frequencies -- & 1122.3959 & 1125.6169 & 1136.5497 \\
\hline Frequencies -- & 1142.0949 & 1158.2197 & 1174.8166 \\
\hline Frequencies -- & 1176.1612 & 1178.0450 & 1178.2553 \\
\hline Frequencies -- & 1180.2613 & 1182.6249 & 1186.8881 \\
\hline Frequencies -- & 1190.5793 & 1200.6017 & 1203.2749 \\
\hline Frequencies -- & 1204.9759 & 1206.3914 & 1211.5738 \\
\hline Frequencies -- & 1211.6601 & 1214.6031 & 1214.7989 \\
\hline Frequencies -- & 1225.4941 & 1230.7506 & 1235.2275 \\
\hline Frequencies -- & 1235.3737 & 1243.3796 & 1253.8790 \\
\hline Frequencies -- & 1263.7100 & 1280.7892 & 1289.7946 \\
\hline Frequencies -- & 1293.3427 & 1294.0413 & 1295.6762 \\
\hline Frequencies -- & 1297.9398 & 1311.4818 & 1313.9081 \\
\hline Frequencies -- & 1315.3835 & 1325.7605 & 1327.5503 \\
\hline Frequencies -- & 1336.0423 & 1338.0007 & 1342.1936 \\
\hline Frequencies -- & 1344.5503 & 1344.8031 & 1358.3037 \\
\hline Frequencies -- & 1358.9311 & 1359.0630 & 1365.2258 \\
\hline Frequencies -- & 1366.4143 & 1367.2165 & 1368.1391 \\
\hline Frequencies -- & 1375.2452 & 1376.1382 & 1382.1371 \\
\hline Frequencies -- & 1389.8583 & 1394.3887 & 1438.3808 \\
\hline
\end{tabular}

$\begin{array}{llll}\text { Frequencies -- } & 1457.8875 & 1458.9870 & 1472.4070 \\ \text { Frequencies -- } & 1474.9892 & 1482.7436 & 1490.3304 \\ \text { Frequencies -- } 1493.2546 & 1499.1861 & 1500.2616 \\ \text { Frequencies -- } 1501.5202 & 1505.6211 & 1509.7058 \\ \text { Frequencies -- } 1518.2821 & 1527.6411 & 1529.8650 \\ \text { Frequencies -- } 1531.4205 & 1545.6182 & 1548.0625 \\ \text { Frequencies -- } 1554.4498 & 1564.5365 & 1566.5045 \\ \text { Frequencies -- } 1569.3913 & 1666.3653 & 1669.5559 \\ \text { Frequencies -- } 1673.0069 & 1675.2055 & 1677.3423 \\ \text { Frequencies -- } 1678.4810 & 1681.2255 & 1683.3366 \\ \text { Frequencies -- } 1684.0246 & 1687.6015 & 1690.7479 \\ \text { Frequencies -- } 1697.9182 & 1699.5005 & 1700.2056 \\ \text { Frequencies -- } 1701.9976 & 1702.2818 & 2832.2765 \\ \text { Frequencies -- } 3072.3430 & 3075.6490 & 3090.5037 \\ \text { Frequencies -- } 3093.1120 & 3128.5516 & 3155.6189 \\ \text { Frequencies -- } 3157.3672 & 3158.0864 & 3192.7904 \\ \text { Frequencies -- } 3196.0775 & 3196.9260 & 3197.8182 \\ \text { Frequencies -- } 3198.8232 & 3199.0994 & 3205.7587 \\ \text { Frequencies -- } 3206.7544 & 3207.1018 & 3208.2968 \\ \text { Frequencies -- } 3210.9531 & 3213.5779 & 3215.6917 \\ \text { Frequencies -- } 3216.2714 & 3217.4602 & 3218.2383 \\ \text { Frequencies -- } 3223.2282 & 3224.2102 & 3224.7446 \\ \text { Frequencies -- } 3227.5016 & 3227.9670 & 3228.1609 \\ \text { Frequencies -- } 3228.6049 & 3231.7326 & 3232.1609 \\ \text { Frequencies -- } 3233.7300 & 3235.1146 & 3236.4430 \\ \text { Frequencies -- } 3241.2955 & 3241.3883 & 3243.8859 \\ \text { Frequencies -- } 3245.4994 & 3253.6201 & 3255.3749 \\ \text { Frequencies -- } 3256.1954 & 3362.0819 & 3626.9469\end{array}$

SCF Done: $E($ RM062X/DGDZVP) $=-3842.65863965$ Sum of electronic and zero-point Energies= $\quad-3841.754507$ Sum of electronic and thermal Energies $=\quad-3841.687804$ Sum of electronic and thermal Free Energies $=\quad-3841.861642$ SCF Done: E(RM062X/DGTZVP/SMD) = -3843.43304769

\section{Cat b TS1d_R}

Center Atomic Atomic Coordinates (Angstroms) Number Number Type $X \quad Y$

$\begin{array}{cccccc}1 & 15 & 0 & 0.000000 & 0.000000 & 0.000000 \\ 2 & 8 & 0 & 0.000000 & 0.000000 & 1.625213 \\ 3 & 8 & 0 & 1.583630 & 0.000000 & -0.353781 \\ 4 & 8 & 0 & -0.580426 & 1.272123 & -0.538482 \\ 5 & 8 & 0 & -0.624872 & -1.311154 & -0.406602 \\ 6 & 6 & 0 & 0.611963 & 1.092086 & 2.239017 \\ 7 & 6 & 0 & 2.286071 & -1.124241 & 0.070825 \\ 8 & 6 & 0 & 1.977425 & 1.068599 & 2.491754 \\ 9 & 6 & 0 & -0.141630 & 2.224439 & 2.533390 \\ 10 & 6 & 0 & 2.857518 & -1.153403 & 1.335596 \\ 11 & 6 & 0 & 2.359780 & -2.238936 & -0.759540 \\ 12 & 6 & 0 & 2.997337 & -0.043554 & 2.354324 \\ 13 & 6 & 0 & 2.592672 & 2.217941 & 2.991856 \\ 14 & 6 & 0 & 0.475049 & 3.333145 & 3.110734 \\ 15 & 6 & 0 & 3.464336 & -2.329668 & 1.797967 \\ 16 & 6 & 0 & 3.029777 & -3.375569 & -0.320305 \\ 17 & 6 & 0 & 3.111124 & -0.867922 & 3.658317 \\ 18 & 6 & 0 & 4.290757 & 0.782484 & 2.126066 \\ 19 & 6 & 0 & 4.108948 & 2.072831 & 2.963079 \\ 20 & 6 & 0 & 1.853906 & 3.349073 & 3.347845 \\ 21 & 1 & 0 & -0.116579 & 4.207575 & 3.367385 \\ 22 & 6 & 0 & 3.865966 & -2.160085 & 3.264024 \\ 23 & 6 & 0 & 3.593916 & -3.449903 & 0.963561 \\ 24 & 1 & 0 & 3.122948 & -4.226738 & -0.988885 \\ 25 & 1 & 0 & 3.611816 & -0.319846 & 4.462460\end{array}$




\begin{tabular}{|c|c|c|c|c|c|}
\hline 26 & 1 & 0 & 2.104920 & -1.127229 & 4.003328 \\
\hline 27 & 1 & 0 & 5.195617 & 0.228197 & 2.394055 \\
\hline 28 & 1 & 0 & 4.359254 & 1.046387 & 1.065886 \\
\hline 29 & 1 & 0 & 4.501106 & 1.924920 & 3.976979 \\
\hline 30 & 6 & 0 & 4.791574 & 3.281858 & 2.355576 \\
\hline 31 & 6 & 0 & 2.529401 & 4.523685 & 3.963499 \\
\hline 32 & 1 & 0 & 4.945830 & -1.980286 & 3.334782 \\
\hline 33 & 6 & 0 & 3.513779 & -3.324138 & 4.165129 \\
\hline 34 & 6 & 0 & 4.325522 & -4.682102 & 1.360262 \\
\hline 35 & 6 & 0 & 4.394246 & 3.743488 & 1.094483 \\
\hline 36 & 6 & 0 & 5.779473 & 3.982637 & 3.047991 \\
\hline 37 & 6 & 0 & 2.620005 & 5.746450 & 3.291160 \\
\hline 38 & 6 & 0 & 3.132496 & 4.390121 & 5.219193 \\
\hline 39 & 6 & 0 & 2.198176 & -3.795748 & 4.210173 \\
\hline 40 & 6 & 0 & 4.470897 & -3.932584 & 4.979211 \\
\hline 41 & 6 & 0 & 3.806428 & -5.950401 & 1.068281 \\
\hline 42 & 6 & 0 & 5.577188 & -4.602362 & 1.982189 \\
\hline 43 & 6 & 0 & 4.969958 & 4.884347 & 0.542500 \\
\hline 44 & 1 & 0 & 3.606884 & 3.220696 & 0.553547 \\
\hline 45 & 6 & 0 & 6.356373 & 5.129570 & 2.500201 \\
\hline 46 & 1 & 0 & 6.083318 & 3.644767 & 4.036216 \\
\hline 47 & 6 & 0 & 3.320925 & 6.810692 & 3.856930 \\
\hline 48 & 1 & 0 & 2.170400 & 5.847650 & 2.306772 \\
\hline 49 & 6 & 0 & 3.824467 & 5.456657 & 5.790640 \\
\hline 50 & 1 & 0 & 3.053593 & 3.442373 & 5.746898 \\
\hline 51 & 6 & 0 & 1.848776 & -4.861330 & 5.035939 \\
\hline 52 & 1 & 0 & 1.438328 & -3.326439 & 3.587110 \\
\hline 53 & 6 & 0 & 4.127588 & -4.999031 & 5.810051 \\
\hline 54 & 1 & 0 & 5.499014 & -3.577575 & 4.954116 \\
\hline 55 & 6 & 0 & 4.502218 & -7.105292 & 1.420263 \\
\hline 56 & 1 & 0 & 2.846363 & -6.035215 & 0.564905 \\
\hline 57 & 6 & 0 & 6.279030 & -5.754425 & 2.327628 \\
\hline 58 & 1 & 0 & 6.014066 & -3.626326 & 2.176030 \\
\hline 59 & 6 & 0 & 5.951859 & 5.585047 & 1.246781 \\
\hline 60 & 1 & 0 & 4.644963 & 5.234099 & -0.432844 \\
\hline 61 & 1 & 0 & 7.112438 & 5.672333 & 3.059631 \\
\hline 62 & 6 & 0 & 3.927678 & 6.667509 & 5.105637 \\
\hline 63 & 1 & 0 & 3.405415 & 7.748090 & 3.315534 \\
\hline 64 & 1 & 0 & 4.284904 & 5.341528 & 6.767431 \\
\hline 65 & 6 & 0 & 2.815636 & -5.470125 & 5.837621 \\
\hline 66 & 1 & 0 & 0.821384 & -5.215331 & 5.050122 \\
\hline 67 & 1 & 0 & 4.886911 & -5.465968 & 6.430075 \\
\hline 68 & 6 & 0 & 5.739893 & -7.011915 & 2.056316 \\
\hline 69 & 1 & 0 & 4.076382 & -8.078890 & 1.197100 \\
\hline 70 & 1 & 0 & 7.250709 & -5.669589 & 2.805053 \\
\hline 71 & 1 & 0 & 6.394212 & 6.481015 & 0.821991 \\
\hline 72 & 1 & 0 & 4.475924 & 7.496357 & 5.543083 \\
\hline 73 & 1 & 0 & 2.547657 & -6.303702 & 6.479450 \\
\hline 74 & 1 & 0 & 6.283227 & -7.910644 & 2.330797 \\
\hline 75 & 1 & 0 & -1.886548 & 1.325503 & -1.312147 \\
\hline 76 & 8 & 0 & -2.729697 & 1.111933 & -1.861465 \\
\hline 77 & 6 & 0 & -2.351922 & 0.202117 & -2.919760 \\
\hline 78 & 6 & 0 & -3.155402 & -1.025440 & -2.447956 \\
\hline 79 & 1 & 0 & -2.648824 & 0.611552 & -3.885581 \\
\hline 80 & 1 & 0 & -1.274169 & 0.018482 & -2.897578 \\
\hline 81 & 8 & 0 & -2.390173 & -2.184986 & -2.319145 \\
\hline 82 & 6 & 0 & -3.557319 & -0.357688 & -1.099871 \\
\hline 83 & 6 & 0 & -4.373067 & -1.316622 & -3.301536 \\
\hline 84 & 1 & 0 & -1.573758 & -1.956606 & -1.826156 \\
\hline 85 & 1 & 0 & -2.908414 & -0.414137 & -0.234093 \\
\hline 86 & 1 & 0 & -4.516321 & 0.128499 & -0.973475 \\
\hline 87 & 6 & 0 & -4.642422 & -2.611860 & -3.740100 \\
\hline 88 & 6 & 0 & -5.241731 & -0.275337 & -3.644424 \\
\hline 89 & 6 & 0 & -5.773601 & -2.862495 & -4.516883 \\
\hline 90 & 1 & 0 & -3.966345 & -3.412816 & -3.462892 \\
\hline 91 & 6 & 0 & -6.373248 & -0.528201 & -4.416394 \\
\hline
\end{tabular}

$\begin{array}{cccccc}92 & 1 & 0 & -5.036867 & 0.741682 & -3.310377 \\ 93 & 6 & 0 & -6.641338 & -1.825370 & -4.855194 \\ 94 & 1 & 0 & -5.979561 & -3.873765 & -4.854024 \\ 95 & 1 & 0 & -7.042350 & 0.286078 & -4.676538 \\ 96 & 1 & 0 & -7.522306 & -2.025674 & -5.457122 \\ 97 & 1 & 0 & 1.375398 & -5.421738 & 2.176572 \\ 98 & 6 & 0 & 0.310240 & -5.379609 & 1.969031 \\ 99 & 6 & 0 & -0.227882 & -4.230328 & 1.400273 \\ 100 & 6 & 0 & -0.500630 & -6.476096 & 2.299534 \\ 101 & 6 & 0 & -1.604429 & -4.211804 & 1.173537 \\ 102 & 1 & 0 & 0.386481 & -3.371031 & 1.133538 \\ 103 & 6 & 0 & -1.873672 & -6.449895 & 2.068747 \\ 104 & 7 & 0 & -2.330815 & -3.163628 & 0.620034 \\ 105 & 6 & 0 & -2.419617 & -5.300951 & 1.497834 \\ 106 & 1 & 0 & -2.499004 & -7.298791 & 2.325489 \\ 107 & 6 & 0 & -3.646733 & -3.362102 & 0.468455 \\ 108 & 1 & 0 & -1.828962 & -2.320192 & 0.286078 \\ 109 & 16 & 0 & -4.083325 & -4.948984 & 1.071372 \\ 110 & 16 & 0 & -4.786790 & -2.314896 & -0.182878 \\ 111 & 1 & 0 & -1.199887 & 2.226507 & 2.293846 \\ 112 & 1 & 0 & 1.887725 & -2.201501 & -1.735903 \\ 113 & 1 & 0 & -0.050494 & -7.357418 & 2.745355\end{array}$

Frequencies -- -552.0767

Frequencies -- 20.5026

Frequencies -- 29.4406

Frequencies -- 38.9704

Frequencies -- 54.1368

Frequencies -- 65.0232

Frequencies -- 81.0418

Frequencies -- 92.9733

Frequencies -- 103.4471

Frequencies -- 126.1241

Frequencies -- 162.3587

Frequencies -- 185.7412

Frequencies -- 209.9996

Frequencies -- 227.9607

Frequencies -- 250.4036

Frequencies -- 278.2090

Frequencies -- 294.6652

Frequencies -- 322.7372

Frequencies -- 365.3297

Frequencies -- 395.5273

Frequencies -- 412.2042

Frequencies -- 416.1511

Frequencies -- 422.2806

Frequencies -- 443.9155

Frequencies -- 472.5053

Frequencies -- 508.8360

Frequencies -- 532.1587

Frequencies -- 544.0565

Frequencies -- 573.7267

Frequencies -- 597.7316

Frequencies -- 619.5166

Frequencies -- 628.5213

Frequencies -- 638.3831

Frequencies -- 662.7901

Frequencies -- 680.9273

Frequencies -- 704.5586

Frequencies -- 717.3349

Frequencies -- 723.6399

Frequencies -- 738.2858

Frequencies -- 772.1379

Frequencies -- 777.9921

Frequencies -- 800.1086
22.0478

31.3085

43.5088

57.7411

69.1898

89.4592

98.9127

113.9562

131.6957

170.1609

191.3260

215.0181

232.3487

260.3142

283.4552

305.5375

334.3929

385.6892

405.0751

413.0679

418.5159

435.0274

452.9219

495.8658

513.4525

537.6251

555.0042

576.8240

609.9332

624.3396

629.6341

640.8278

664.2446

685.2686

714.8066

720.3519

725.4091

740.2221

775.2002

784.4132

818.7377
17.2492

24.8205

33.1234

46.9708

62.7327

78.0490

91.3351

99.1619

125.2369

157.9127

182.4592

196.3646

219.4835

241.0595

271.7328

291.7094

312.5884

352.3720

386.7269

408.9836

414.2908

419.4532

437.8154

462.7903

507.3898

524.1191

539.4909

567.3071

587.3243

614.6525

626.6218

633.1819

653.2095

672.8698

694.1679

716.6594

722.6744

732.9486

748.8472

776.1659

785.0616

826.2736 


\begin{tabular}{|c|c|c|c|}
\hline Frequencies -- & 844.1977 & 848.0302 & 866.6704 \\
\hline Frequencies -- & 866.7909 & 869.4607 & 870.5377 \\
\hline Frequencies -- & 871.8296 & 872.7397 & 878.7300 \\
\hline Frequencies -- & 885.6925 & 890.2396 & 894.2034 \\
\hline Frequencies -- & 896.2999 & 901.6227 & 912.5996 \\
\hline Frequencies -- & 932.5332 & 935.7453 & 940.3284 \\
\hline Frequencies -- & 943.2844 & 944.1883 & 948.7679 \\
\hline Frequencies -- & 949.5946 & 950.0910 & 969.3717 \\
\hline Frequencies -- & 971.3497 & 975.8408 & 994.0403 \\
\hline Frequencies -- & 995.5971 & 997.6475 & 998.5582 \\
\hline Frequencies -- & 1000.5369 & 1001.0064 & 1005.9970 \\
\hline Frequencies -- & 1008.9735 & 1011.1846 & 1012.5121 \\
\hline Frequencies -- & 1012.9272 & 1015.3172 & 1018.4893 \\
\hline Frequencies -- & 1019.2298 & 1019.5737 & 1020.1698 \\
\hline Frequencies -- & 1020.5231 & 1026.3977 & 1030.6214 \\
\hline Frequencies -- & 1043.4067 & 1045.7303 & 1050.8543 \\
\hline Frequencies -- & 1052.5386 & 1059.4411 & 1062.2270 \\
\hline Frequencies -- & 1064.4672 & 1068.0749 & 1070.3401 \\
\hline Frequencies -- & 1072.1129 & 1079.1700 & 1086.8482 \\
\hline Frequencies -- & 1089.9906 & 1101.4962 & 1102.6195 \\
\hline Frequencies -- & 1106.1342 & 1109.9996 & 1111.4735 \\
\hline Frequencies -- & 1117.5803 & 1117.8191 & 1120.3948 \\
\hline Frequencies -- & 1122.4462 & 1127.4279 & 1140.4012 \\
\hline Frequencies -- & 1152.4574 & 1158.4741 & 1175.7218 \\
\hline Frequencies -- & 1177.9434 & 1178.3823 & 1178.5829 \\
\hline Frequencies -- & 1181.6376 & 1184.6526 & 1189.5452 \\
\hline Frequencies -- & 1196.3664 & 1203.2017 & 1204.8305 \\
\hline Frequencies -- & 1207.3941 & 1209.7159 & 1213.1332 \\
\hline Frequencies -- & 1213.3276 & 1213.8663 & 1215.5551 \\
\hline Frequencies -- & 1226.8625 & 1231.8132 & 1234.4464 \\
\hline Frequencies -- & 1235.5785 & 1244.5622 & 1256.5957 \\
\hline Frequencies -- & 1261.5993 & 1278.7585 & 1287.8941 \\
\hline Frequencies -- & 1289.0637 & 1293.9297 & 1295.5501 \\
\hline Frequencies -- & 1298.1862 & 1305.5631 & 1309.6652 \\
\hline Frequencies -- & 1313.4025 & 1324.5014 & 1326.3205 \\
\hline Frequencies -- & 1334.2558 & 1338.1032 & 1342.7207 \\
\hline Frequencies -- & 1344.9393 & 1345.5050 & 1347.9965 \\
\hline Frequencies -- & 1357.4379 & 1362.0709 & 1364.8432 \\
\hline Frequencies -- & 1367.9251 & 1369.4705 & 1371.1113 \\
\hline Frequencies -- & 1372.7405 & 1378.2756 & 1383.9419 \\
\hline Frequencies -- & 1386.8263 & 1388.8221 & 1441.1532 \\
\hline Frequencies -- & 1451.3884 & 1464.1632 & 1469.1224 \\
\hline Frequencies -- & 1475.3377 & 1479.4434 & 1490.3460 \\
\hline Frequencies -- & 1495.2060 & 1498.8656 & 1501.4713 \\
\hline Frequencies -- & 1501.5537 & 1505.4269 & 1509.3208 \\
\hline Frequencies -- & 1512.6071 & 1528.6286 & 1529.3758 \\
\hline Frequencies -- & 1531.5548 & 1546.3705 & 1551.2592 \\
\hline Frequencies -- & 1555.2368 & 1559.3871 & 1563.5578 \\
\hline Frequencies -- & 1568.7252 & 1662.3953 & 1664.5643 \\
\hline Frequencies -- & 1672.2432 & 1676.7472 & 1678.2448 \\
\hline Frequencies -- & 1679.2131 & 1682.0114 & 1682.1528 \\
\hline Frequencies -- & 1684.1337 & 1687.1233 & 1691.7958 \\
\hline Frequencies -- & 1698.2335 & 1699.3204 & 1701.0107 \\
\hline Frequencies -- & 1702.3032 & 1702.5795 & 2698.8358 \\
\hline Frequencies -- & 3073.5321 & 3074.8948 & 3089.1919 \\
\hline Frequencies -- & 3090.3582 & 3119.1289 & 3153.9746 \\
\hline Frequencies -- & 3156.4886 & 3184.3325 & 3192.3029 \\
\hline Frequencies -- & 3197.6784 & 3198.1548 & 3200.6265 \\
\hline Frequencies -- & 3204.5064 & 3205.1120 & 3207.5609 \\
\hline Frequencies -- & 3210.3682 & 3214.2512 & 3214.3311 \\
\hline Frequencies -- & 3214.9813 & 3215.3476 & 3216.5764 \\
\hline Frequencies -- & 3216.8092 & 3220.2040 & 3221.9524 \\
\hline Frequencies -- & 3223.0664 & 3224.5383 & 3224.6312 \\
\hline Frequencies -- & 3225.4291 & 3226.7463 & 3230.1254 \\
\hline Frequencies -- & 3230.9078 & 3232.2186 & 3235.0888 \\
\hline Frequencies -- & 3236.6774 & 3237.6071 & 3237.9849 \\
\hline
\end{tabular}

$\begin{array}{llll}\text { Frequencies -- } & 3239.1073 & 3239.6288 & 3242.9018 \\ \text { Frequencies -- } 3243.8772 & 3246.4005 & 3248.6883 \\ \text { Frequencies -- } 3251.1052 & 3358.3602 & 3618.2220\end{array}$

SCF Done: $E(R M 062 X / D G D Z V P)=-3842.65693498$

Sum of electronic and zero-point Energies $=\quad-3841.753274$

Sum of electronic and thermal Energies $=\quad-3841.686352$

Sum of electronic and thermal Free Energies $=\quad-3841.863671$

SCF Done: $E(R M 062 X / D G T Z V P / S M D)=-3843.43435353$

\section{Cat $f$}

Center Atomic Atomic Coordinates (Angstroms)

Number Number Type $\quad X \quad Y \quad Z$

$\begin{array}{cccccc}1 & 15 & 0 & 0.604621 & 0.259730 & 0.141339 \\ 2 & 8 & 0 & 0.409991 & 0.469504 & 1.698272 \\ 3 & 8 & 0 & 2.168469 & -0.102282 & 0.187089 \\ 4 & 1 & 0 & -0.473725 & 0.784098 & 1.978407 \\ 5 & 8 & 0 & -0.087600 & -1.160711 & -0.242096 \\ 6 & 8 & 0 & 0.146839 & 1.327412 & -0.748551 \\ 7 & 6 & 0 & 2.727037 & -0.868472 & -0.836261 \\ 8 & 6 & 0 & -0.135067 & -2.069568 & 0.812939 \\ 9 & 6 & 0 & 2.747688 & -2.252972 & -0.724016 \\ 10 & 6 & 0 & 3.219831 & -0.234957 & -1.977510 \\ 11 & 6 & 0 & 0.970421 & -2.821418 & 1.153816 \\ 12 & 6 & 0 & -1.290106 & -2.079597 & 1.598339 \\ 13 & 6 & 0 & 2.216578 & -3.160719 & 0.367917 \\ 14 & 6 & 0 & 3.287369 & -3.002794 & -1.769496 \\ 15 & 6 & 0 & 3.823955 & -1.003929 & -2.972969 \\ 16 & 6 & 0 & 3.002156 & 1.223259 & -2.147958 \\ 17 & 6 & 0 & 0.982448 & -3.474621 & 2.386890 \\ 18 & 6 & 0 & -1.290899 & -2.801871 & 2.789506 \\ 19 & 6 & 0 & -2.470720 & -1.365844 & 1.040400 \\ 20 & 6 & 0 & 3.220228 & -3.421027 & 1.517774 \\ 21 & 6 & 0 & 1.949487 & -4.461284 & -0.443717 \\ 22 & 6 & 0 & 2.976200 & -4.482434 & -1.599253 \\ 23 & 6 & 0 & 3.887398 & -2.394560 & -2.876817 \\ 24 & 1 & 0 & 4.233592 & -0.505189 & -3.847736 \\ 25 & 6 & 0 & 3.800515 & 2.164446 & -1.507250 \\ 26 & 6 & 0 & 1.915483 & 1.669942 & -2.889034 \\ 27 & 6 & 0 & 2.376318 & -4.020077 & 2.677834 \\ 28 & 6 & 0 & -0.140885 & -3.481233 & 3.217611 \\ 29 & 1 & 0 & -2.188925 & -2.828102 & 3.401523 \\ 30 & 6 & 0 & -2.951097 & -0.157466 & 1.528488 \\ 31 & 6 & 0 & -3.052312 & -1.878308 & -0.120957 \\ 32 & 1 & 0 & 4.040458 & -4.077665 & 1.211501 \\ 33 & 1 & 0 & 3.651858 & -2.467159 & 1.837178 \\ 34 & 1 & 0 & 1.992538 & -5.357427 & 0.182917 \\ 35 & 1 & 0 & 0.941650 & -4.400329 & -0.867557 \\ 36 & 6 & 0 & 2.411622 & -5.084748 & -2.872511 \\ 37 & 1 & 0 & 3.873531 & -5.041635 & -1.308841 \\ 38 & 6 & 0 & 4.530410 & -3.215927 & -3.935706 \\ 39 & 6 & 0 & 3.503674 & 3.517400 & -1.591163 \\ 40 & 8 & 0 & 4.880477 & 1.715797 & -0.783145 \\ 41 & 6 & 0 & 1.586959 & 3.024832 & -2.959233 \\ 42 & 8 & 0 & 1.107745 & 0.731404 & -3.487284 \\ 43 & 6 & 0 & 2.862968 & -3.610941 & 4.053644 \\ 44 & 1 & 0 & 2.375069 & -5.115264 & 2.618942 \\ 45 & 6 & 0 & -0.110780 & -4.197509 & 4.521836 \\ 46 & 6 & 0 & -4.013973 & 0.514782 & 0.917998 \\ 47 & 8 & 0 & -2.283046 & 0.422662 & 2.597135 \\ 48 & 6 & 0 & -4.103791 & -1.221748 & -0.744294 \\ 49 & 8 & 0 & -2.544405 & -3.052792 & -0.615695 \\ 50 & 6 & 0 & 1.339401 & -4.455446 & -3.518888\end{array}$




\begin{tabular}{|c|c|c|c|c|c|}
\hline 51 & 6 & 0 & 2.973856 & -6.220356 & -3.454796 \\
\hline 52 & 6 & 0 & 4.043667 & -3.210750 & -5.246678 \\
\hline 53 & 6 & 0 & 5.608594 & -4.045780 & -3.610197 \\
\hline 54 & 6 & 0 & 2.383169 & 3.962759 & -2.301379 \\
\hline 55 & 8 & 0 & 4.306446 & 4.447795 & -0.973283 \\
\hline 56 & 6 & 0 & 5.327281 & 2.595193 & 0.182215 \\
\hline 57 & 8 & 0 & 0.460203 & 3.397904 & -3.647738 \\
\hline 58 & 6 & 0 & -0.224798 & 1.099219 & -3.558526 \\
\hline 59 & 6 & 0 & 2.879915 & -2.256709 & 4.411009 \\
\hline 60 & 6 & 0 & 3.245200 & -4.563138 & 4.998666 \\
\hline 61 & 6 & 0 & -0.129891 & -3.484631 & 5.724726 \\
\hline 62 & 6 & 0 & -0.010074 & -5.592014 & 4.555505 \\
\hline 63 & 6 & 0 & -4.607808 & -0.025316 & -0.223209 \\
\hline 64 & 8 & 0 & -4.447674 & 1.713599 & 1.419267 \\
\hline 65 & 6 & 0 & -3.039073 & 1.327316 & 3.332610 \\
\hline 66 & 8 & 0 & -4.692706 & -1.729672 & -1.871990 \\
\hline 67 & 6 & 0 & -2.912244 & -3.363805 & -1.908045 \\
\hline 68 & 6 & 0 & 0.852469 & -4.943878 & -4.728337 \\
\hline 69 & 1 & 0 & 0.907401 & -3.549295 & -3.094060 \\
\hline 70 & 6 & 0 & 2.484959 & -6.717433 & -4.664472 \\
\hline 71 & 1 & 0 & 3.820374 & -6.705752 & -2.974726 \\
\hline 72 & 6 & 0 & 4.612680 & -4.041502 & -6.210748 \\
\hline 73 & 1 & 0 & 3.193593 & -2.581144 & -5.497442 \\
\hline 74 & 6 & 0 & 6.185615 & -4.867620 & -4.576346 \\
\hline 75 & 1 & 0 & 5.997069 & -4.038719 & -2.594437 \\
\hline 76 & 6 & 0 & 2.109913 & 5.448514 & -2.283622 \\
\hline 77 & 6 & 0 & 5.040783 & 3.956078 & 0.088808 \\
\hline 78 & 6 & 0 & 6.087149 & 2.108078 & 1.234817 \\
\hline 79 & 6 & 0 & -0.550577 & 2.447739 & -3.631000 \\
\hline 80 & 6 & 0 & -1.216059 & 0.130683 & -3.536071 \\
\hline 81 & 6 & 0 & 3.270957 & -1.866815 & 5.689318 \\
\hline 82 & 1 & 0 & 2.559864 & -1.503955 & 3.691147 \\
\hline 83 & 6 & 0 & 3.632243 & -4.176476 & 6.282588 \\
\hline 84 & 1 & 0 & 3.217176 & -5.618657 & 4.737982 \\
\hline 85 & 6 & 0 & -0.030925 & -4.155107 & 6.942444 \\
\hline 86 & 1 & 0 & -0.188585 & -2.399517 & 5.698882 \\
\hline 87 & 6 & 0 & 0.077643 & -6.264674 & 5.773673 \\
\hline 88 & 1 & 0 & -0.005365 & -6.148800 & 3.621320 \\
\hline 89 & 6 & 0 & -5.787355 & 0.589568 & -0.942882 \\
\hline 90 & 6 & 0 & -4.114955 & 1.971174 & 2.732205 \\
\hline 91 & 6 & 0 & -2.684547 & 1.601119 & 4.643788 \\
\hline 92 & 6 & 0 & -3.974079 & -2.711569 & -2.530149 \\
\hline 93 & 6 & 0 & -2.222952 & -4.367995 & -2.572129 \\
\hline 94 & 6 & 0 & 1.425846 & -6.078651 & -5.306989 \\
\hline 95 & 1 & 0 & 0.042054 & -4.422245 & -5.229773 \\
\hline 96 & 1 & 0 & 2.945332 & -7.592967 & -5.112223 \\
\hline 97 & 6 & 0 & 5.681243 & -4.874519 & -5.877158 \\
\hline 98 & 1 & 0 & 4.212832 & -4.047293 & -7.220126 \\
\hline 99 & 1 & 0 & 7.026397 & -5.502759 & -4.314002 \\
\hline 100 & 9 & 0 & 1.036069 & 5.803233 & -2.992640 \\
\hline 101 & 9 & 0 & 1.913490 & 5.886258 & -1.025790 \\
\hline 102 & 9 & 0 & 3.153333 & 6.138335 & -2.781060 \\
\hline 103 & 6 & 0 & 5.514090 & 4.840759 & 1.046243 \\
\hline 104 & 6 & 0 & 6.579464 & 2.996093 & 2.191975 \\
\hline 105 & 1 & 0 & 6.287235 & 1.042441 & 1.280614 \\
\hline 106 & 6 & 0 & -1.873533 & 2.853708 & -3.684304 \\
\hline 107 & 6 & 0 & -2.552583 & 0.531407 & -3.602123 \\
\hline 108 & 1 & 0 & -0.931890 & -0.915184 & -3.456317 \\
\hline 109 & 6 & 0 & 3.645168 & -2.827285 & 6.631824 \\
\hline 110 & 1 & 0 & 3.276112 & -0.813405 & 5.953181 \\
\hline 111 & 1 & 0 & 3.913271 & -4.931246 & 7.011035 \\
\hline 112 & 6 & 0 & 0.075903 & -5.546319 & 6.969525 \\
\hline 113 & 1 & 0 & -0.024295 & -3.590299 & 7.869587 \\
\hline 114 & 1 & 0 & 0.150315 & -7.347995 & 5.789032 \\
\hline 115 & 9 & 0 & -6.834603 & -0.254404 & -0.939001 \\
\hline 116 & 9 & 0 & -5.493983 & 0.845264 & -2.230848 \\
\hline
\end{tabular}

$\begin{array}{llllll}117 & 9 & 0 & -6.205294 & 1.735076 & -0.402231 \\ 118 & 6 & 0 & -4.852449 & 2.907840 & 3.441397 \\ 119 & 6 & 0 & -3.414735 & 2.552850 & 5.356446 \\ 120 & 1 & 0 & -1.840051 & 1.077705 & 5.081000 \\ 121 & 6 & 0 & -4.347539 & -3.047329 & -3.822703 \\ 122 & 6 & 0 & -2.607041 & -4.724431 & -3.865191 \\ 123 & 1 & 0 & -1.403809 & -4.868010 & -2.063802 \\ 124 & 1 & 0 & 1.057163 & -6.453797 & -6.256870 \\ 125 & 1 & 0 & 6.120593 & -5.523384 & -6.628599 \\ 126 & 6 & 0 & 6.294404 & 4.357630 & 2.097375 \\ 127 & 1 & 0 & 5.270601 & 5.893353 & 0.944954 \\ 128 & 1 & 0 & 7.183517 & 2.620103 & 3.010823 \\ 129 & 6 & 0 & -2.879223 & 1.885850 & -3.676471 \\ 130 & 1 & 0 & -2.094724 & 3.915210 & -3.726236 \\ 131 & 1 & 0 & -3.342540 & -0.214891 & -3.593602 \\ 132 & 1 & 0 & 3.942395 & -2.524985 & 7.631345 \\ 133 & 1 & 0 & 0.156019 & -6.067417 & 7.918584 \\ 134 & 6 & 0 & -4.492761 & 3.202912 & 4.756177 \\ 135 & 1 & 0 & -5.684757 & 3.397847 & 2.947131 \\ 136 & 1 & 0 & -3.139038 & 2.783707 & 6.379579 \\ 137 & 6 & 0 & -3.662847 & -4.063308 & -4.491006 \\ 138 & 1 & 0 & -5.175930 & -2.514983 & -4.279348 \\ 139 & 1 & 0 & -2.077410 & -5.523135 & -4.373736 \\ 140 & 1 & 0 & 6.675571 & 5.048605 & 2.841582 \\ 141 & 1 & 0 & -3.918568 & 2.192085 & -3.719925 \\ 142 & 1 & 0 & -5.059014 & 3.943147 & 5.310884 \\ 143 & 1 & 0 & -3.960616 & -4.336360 & -5.497501 \\ & 1 & & & & \\ 135 & & 1 & \end{array}$

$\begin{array}{llll}\text { Frequencies -- } & 6.5949 & 11.0183 & 12.8363 \\ \text { Frequencies -- } & 21.3281 & 22.4683 & 24.6758 \\ \text { Frequencies -- } & 26.8277 & 29.8956 & 32.9820 \\ \text { Frequencies -- } & 35.5990 & 40.0641 & 41.5891 \\ \text { Frequencies -- } & 44.1985 & 45.7741 & 49.1107 \\ \text { Frequencies -- } & 54.5376 & 56.4049 & 59.8903 \\ \text { Frequencies -- } & 61.8095 & 68.7883 & 70.1778 \\ \text { Frequencies -- } & 73.1274 & 74.3869 & 76.7110 \\ \text { Frequencies -- } & 79.5871 & 83.1208 & 87.4512 \\ \text { Frequencies -- } & 93.9860 & 98.4450 & 98.9431 \\ \text { Frequencies -- } & 101.8198 & 107.5072 & 118.4638 \\ \text { Frequencies -- } & 121.7342 & 123.2292 & 127.2160 \\ \text { Frequencies -- } & 129.7576 & 134.8964 & 139.2514 \\ \text { Frequencies -- } & 154.7359 & 156.6357 & 163.2739 \\ \text { Frequencies -- } & 170.1021 & 177.1527 & 181.8198 \\ \text { Frequencies -- } & 190.2485 & 199.4558 & 203.8871 \\ \text { Frequencies -- } & 211.9638 & 225.5579 & 230.3783 \\ \text { Frequencies -- } & 232.6531 & 239.2671 & 244.2146 \\ \text { Frequencies -- } & 247.1443 & 252.1636 & 268.0910 \\ \text { Frequencies -- } & 269.2060 & 273.6640 & 275.2405 \\ \text { Frequencies -- } & 277.9295 & 287.6285 & 290.4372 \\ \text { Frequencies -- } & 291.2595 & 293.2154 & 295.0864 \\ \text { Frequencies -- } & 299.4554 & 301.8184 & 302.3786 \\ \text { Frequencies -- } & 307.2308 & 311.2750 & 316.8949 \\ \text { Frequencies -- } & 321.7025 & 322.6261 & 327.2434 \\ \text { Frequencies -- } & 330.0108 & 334.4525 & 347.3854 \\ \text { Frequencies -- } & 367.0957 & 391.6637 & 403.8178 \\ \text { Frequencies -- } & 406.6181 & 407.7330 & 409.6516 \\ \text { Frequencies -- } & 414.3334 & 415.1751 & 417.1762 \\ \text { Frequencies -- } & 418.1336 & 421.6280 & 441.9236 \\ \text { Frequencies -- } & 443.0772 & 446.1157 & 447.7454 \\ \text { Frequencies -- } & 449.8407 & 455.9824 & 456.6273 \\ \text { Frequencies -- } & 466.5782 & 469.8898 & 484.3002 \\ \text { Frequencies -- } & 490.7770 & 492.7089 & 501.0407 \\ \text { Frequencies -- } & 505.5321 & 511.9629 & 516.5010 \\ \text { Frequencies -- } & 521.2834 & 527.0236 & 530.1533 \\ \text { Frequencies -- } & 536.4919 & 540.0263 & 543.4772\end{array}$




\begin{tabular}{|c|c|c|c|}
\hline Frequencies -- & 549.6209 & 555.5957 & 557.1799 \\
\hline Frequencies -- & 558.3109 & 559.1892 & 564.4425 \\
\hline Frequencies -- & 567.5656 & 569.4623 & 582.5970 \\
\hline Frequencies -- & 588.5968 & 596.5473 & 598.2975 \\
\hline Frequencies -- & 600.6716 & 602.4829 & 603.7161 \\
\hline Frequencies -- & 605.5184 & 607.3441 & 608.9022 \\
\hline Frequencies -- & 610.4493 & 617.5090 & 623.2335 \\
\hline Frequencies -- & 624.8420 & 626.4982 & 631.2370 \\
\hline Frequencies -- & 632.0580 & 633.9965 & 635.7907 \\
\hline Frequencies -- & 646.2771 & 648.5785 & 653.5649 \\
\hline Frequencies -- & 662.5912 & 665.6055 & 670.1387 \\
\hline Frequencies -- & 680.1460 & 683.0841 & 702.8534 \\
\hline Frequencies -- & 705.8721 & 712.8522 & 715.2799 \\
\hline Frequencies -- & 715.4815 & 715.8693 & 716.4818 \\
\hline Frequencies -- & 717.2009 & 720.7738 & 723.0324 \\
\hline Frequencies -- & 727.4106 & 731.1988 & 734.9414 \\
\hline Frequencies -- & 740.2823 & 743.6513 & 748.9917 \\
\hline Frequencies -- & 755.1728 & 757.3442 & 768.9814 \\
\hline Frequencies -- & 769.6638 & 770.4806 & 771.5483 \\
\hline Frequencies -- & 774.2550 & 776.9325 & 779.4664 \\
\hline Frequencies -- & 780.5170 & 784.9989 & 790.9371 \\
\hline Frequencies -- & 797.0331 & 800.3661 & 805.5392 \\
\hline Frequencies -- & 807.7348 & 820.0842 & 825.3152 \\
\hline Frequencies -- & 828.3472 & 830.1484 & 857.8641 \\
\hline Frequencies -- & 859.2484 & 862.9967 & 864.1081 \\
\hline Frequencies -- & 866.3855 & 868.5618 & 870.4225 \\
\hline Frequencies -- & 871.7789 & 873.0949 & 882.5993 \\
\hline Frequencies -- & 887.6485 & 889.2213 & 890.0199 \\
\hline Frequencies -- & 896.6662 & 909.9282 & 913.4304 \\
\hline Frequencies -- & 915.5053 & 922.7787 & 931.6894 \\
\hline Frequencies -- & 933.8809 & 934.3274 & 938.4076 \\
\hline Frequencies -- & 942.5893 & 944.1054 & 945.6448 \\
\hline Frequencies -- & 946.9729 & 948.4015 & 948.8867 \\
\hline Frequencies -- & 955.8890 & 960.0686 & 966.2978 \\
\hline Frequencies -- & 971.7662 & 986.7648 & 990.5078 \\
\hline Frequencies -- & 991.1095 & 992.9956 & 993.7390 \\
\hline Frequencies -- & 996.8167 & 998.3259 & 1002.1234 \\
\hline Frequencies -- & 1003.3838 & 1011.7790 & 1013.5386 \\
\hline Frequencies -- & 1013.6754 & 1017.5557 & 1018.5743 \\
\hline Frequencies -- & 1019.2779 & 1019.6315 & 1020.4973 \\
\hline Frequencies -- & 1026.5671 & 1031.5309 & 1036.9290 \\
\hline Frequencies -- & 1037.9864 & 1042.6136 & 1054.5464 \\
\hline Frequencies -- & 1057.4283 & 1063.0334 & 1063.6586 \\
\hline Frequencies -- & 1066.1856 & 1067.0541 & 1069.9331 \\
\hline Frequencies -- & 1071.5412 & 1074.3837 & 1091.8312 \\
\hline Frequencies -- & 1101.9385 & 1107.0587 & 1110.3921 \\
\hline Frequencies -- & 1113.5487 & 1114.9835 & 1123.0742 \\
\hline Frequencies -- & 1127.2927 & 1129.5526 & 1130.4976 \\
\hline Frequencies -- & 1135.3233 & 1136.6601 & 1166.5229 \\
\hline Frequencies -- & 1167.7378 & 1169.6214 & 1172.8851 \\
\hline Frequencies -- & 1173.6055 & 1175.7303 & 1177.6267 \\
\hline Frequencies -- & 1179.0590 & 1179.3450 & 1180.6952 \\
\hline Frequencies -- & 1186.7441 & 1195.6406 & 1202.8287 \\
\hline Frequencies -- & 1204.9089 & 1207.1270 & 1209.7368 \\
\hline Frequencies -- & 1212.3017 & 1215.8834 & 1216.4496 \\
\hline Frequencies -- & 1218.6044 & 1223.7747 & 1228.2745 \\
\hline Frequencies -- & 1231.5229 & 1232.6070 & 1233.4326 \\
\hline Frequencies -- & 1234.8829 & 1241.3943 & 1246.0046 \\
\hline Frequencies -- & 1249.0915 & 1257.2029 & 1258.3583 \\
\hline Frequencies -- & 1267.0314 & 1275.9097 & 1276.4589 \\
\hline Frequencies -- & 1291.8037 & 1293.2931 & 1296.1357 \\
\hline Frequencies -- & 1302.1897 & 1303.1495 & 1303.1950 \\
\hline Frequencies -- & 1306.8071 & 1307.4246 & 1311.6617 \\
\hline Frequencies -- & 1313.0541 & 1314.1207 & 1316.3567 \\
\hline Frequencies -- & 1320.3910 & 1325.2393 & 1327.1716 \\
\hline Frequencies -- & 1330.4838 & 1335.0306 & 1337.4924 \\
\hline
\end{tabular}

\begin{tabular}{llll} 
Frequencies -- & 1340.5085 & 1350.7364 & 1357.2523 \\
Frequencies -- 1357.8801 & 1358.7861 & 1359.0495 \\
Frequencies -- 1362.0177 & 1362.5147 & 1364.2242 \\
Frequencies -- 1364.3292 & 1370.8719 & 1372.1259 \\
Frequencies -- 1375.1553 & 1376.4896 & 1385.0931 \\
Frequencies -- 1395.3010 & 1411.4124 & 1452.6015 \\
Frequencies -- 1463.6624 & 1472.9155 & 1475.0095 \\
Frequencies -- 1490.9047 & 1494.0902 & 1498.2658 \\
Frequencies -- 1499.7118 & 1506.1244 & 1507.1753 \\
Frequencies -- 1508.8995 & 1509.3158 & 1510.9408 \\
Frequencies -- 1515.7461 & 1519.7845 & 1523.3571 \\
Frequencies -- 1529.9924 & 1534.8767 & 1544.8847 \\
Frequencies -- 1550.7662 & 1555.9609 & 1557.6548 \\
Frequencies -- 1560.5221 & 1563.2439 & 1564.1028 \\
Frequencies -- 1565.6688 & 1569.4587 & 1578.2293 \\
Frequencies -- 1666.7503 & 1667.5712 & 1671.0349 \\
Frequencies -- 1671.5842 & 1673.5741 & 1674.2515 \\
Frequencies -- 1676.2378 & 1679.2519 & 1691.3981 \\
Frequencies -- 1693.1502 & 1695.8643 & 1696.9360 \\
Frequencies -- 1698.4637 & 1699.1823 & 1701.7636 \\
Frequencies -- 1703.0104 & 1703.7451 & 1704.0154 \\
Frequencies -- 1704.9391 & 1707.1902 & 1722.7488 \\
Frequencies -- 1722.8746 & 1740.1099 & 1743.2582 \\
Frequencies -- 3076.5421 & 3084.2504 & 3088.5154 \\
Frequencies -- 3089.0875 & 3151.9215 & 3155.8392 \\
Frequencies -- 3178.7407 & 3187.5701 & 3200.8481 \\
Frequencies -- 3201.5006 & 3205.2214 & 3206.6827 \\
Frequencies -- 3211.0629 & 3211.3322 & 3211.7900 \\
Frequencies -- 3214.4338 & 3218.6416 & 3219.7303 \\
Frequencies -- 3221.7487 & 3222.3420 & 3225.2449 \\
Frequencies -- 3225.5625 & 3227.4548 & 3228.6386 \\
Frequencies -- 3229.5177 & 3230.2360 & 3231.3578 \\
Frequencies -- 3231.8069 & 3234.4326 & 3235.0655 \\
Frequencies -- 3237.0613 & 3237.4737 & 3239.4862 \\
\hline
\end{tabular}

SCF Done: $E(R M 062 X / D G D Z V P)=-4879.40731991$

Sum of electronic and zero-point Energies= $\quad-4878.341618$ Sum of electronic and thermal Energies $=\quad-4878.250170$ Sum of electronic and thermal Free Energies $=\quad-4878.478168$ SCF Done: $E(R M 062 X / D G T Z V P / S M D)=-4880.43072127$

\section{Cat $f 10 \_1$}

Center Atomic Atomic Coordinates (Angstroms)

\begin{tabular}{cccccc} 
Number & Number & Type & $X$ & $Y$ & $Z$ \\
\hline 1 & 15 & 0 & 0.225185 & 0.018377 & 0.027214 \\
2 & 8 & 0 & 0.188651 & 0.254195 & 1.620224 \\
3 & 8 & 0 & 1.802781 & -0.000227 & -0.308670 \\
4 & 8 & 0 & -0.464117 & 1.038332 & -0.791645 \\
5 & 8 & 0 & -0.286949 & -1.449833 & -0.067253 \\
6 & 6 & 0 & 0.833603 & 1.388863 & 2.113007 \\
7 & 6 & 0 & 2.512193 & -1.081054 & 0.221307 \\
8 & 6 & 0 & 2.173521 & 1.310311 & 2.462953 \\
9 & 6 & 0 & 0.146254 & 2.602969 & 2.175213 \\
10 & 6 & 0 & 2.988511 & -1.033013 & 1.519379 \\
11 & 6 & 0 & 2.620189 & -2.245556 & -0.545464 \\
12 & 6 & 0 & 3.112995 & 0.123051 & 2.488271 \\
13 & 6 & 0 & 2.839619 & 2.469727 & 2.860595 \\
14 & 6 & 0 & 0.817514 & 3.734192 & 2.644294 \\
15 & 6 & 0 & 3.501251 & -2.198769 & 2.091338
\end{tabular}




\begin{tabular}{|c|c|c|c|c|c|}
\hline 16 & 6 & 0 & 3.202845 & -3.377645 & 0.022869 \\
\hline 17 & 6 & 0 & 3.062480 & -0.627411 & 3.843891 \\
\hline 18 & 6 & 0 & 4.481102 & 0.832151 & 2.294738 \\
\hline 19 & 6 & 0 & 4.339966 & 2.230073 & 2.945728 \\
\hline 20 & 6 & 0 & 2.165307 & 3.686313 & 3.002507 \\
\hline 21 & 1 & 0 & 0.281211 & 4.677492 & 2.706846 \\
\hline 22 & 6 & 0 & 3.712482 & -2.012919 & 3.588002 \\
\hline 23 & 6 & 0 & 3.641161 & -3.374975 & 1.351172 \\
\hline 24 & 1 & 0 & 3.294381 & -4.284045 & -0.569818 \\
\hline 25 & 1 & 0 & 3.555926 & -0.069086 & 4.645401 \\
\hline 26 & 1 & 0 & 2.014881 & -0.768226 & 4.129335 \\
\hline 27 & 1 & 0 & 5.310398 & 0.250948 & 2.710020 \\
\hline 28 & 1 & 0 & 4.663547 & 0.953742 & 1.221747 \\
\hline 29 & 1 & 0 & 4.668357 & 2.198636 & 3.991634 \\
\hline 30 & 6 & 0 & 5.122364 & 3.307988 & 2.220717 \\
\hline 31 & 6 & 0 & 2.874118 & 4.906083 & 3.472336 \\
\hline 32 & 1 & 0 & 4.781970 & -1.984625 & 3.828669 \\
\hline 33 & 6 & 0 & 3.063475 & -3.131083 & 4.380836 \\
\hline 34 & 6 & 0 & 4.213770 & -4.596795 & 1.977095 \\
\hline 35 & 6 & 0 & 4.788240 & 3.641273 & 0.901266 \\
\hline 36 & 6 & 0 & 6.131197 & 4.027014 & 2.860648 \\
\hline 37 & 6 & 0 & 3.024317 & 6.008521 & 2.625024 \\
\hline 38 & 6 & 0 & 3.433388 & 4.946943 & 4.753450 \\
\hline 39 & 6 & 0 & 1.721321 & -3.456553 & 4.144570 \\
\hline 40 & 6 & 0 & 3.785629 & -3.884084 & 5.306133 \\
\hline 41 & 6 & 0 & 3.422829 & -5.732772 & 2.175341 \\
\hline 42 & 6 & 0 & 5.539067 & -4.599763 & 2.424076 \\
\hline 43 & 6 & 0 & 5.445795 & 4.677166 & 0.243541 \\
\hline 44 & 1 & 0 & 3.976901 & 3.112710 & 0.400798 \\
\hline 45 & 6 & 0 & 6.793811 & 5.065602 & 2.203774 \\
\hline 46 & 1 & 0 & 6.384480 & 3.791924 & 3.891814 \\
\hline 47 & 6 & 0 & 3.746275 & 7.124410 & 3.043971 \\
\hline 48 & 1 & 0 & 2.598322 & 5.973387 & 1.625037 \\
\hline 49 & 6 & 0 & 4.142646 & 6.069296 & 5.178803 \\
\hline 50 & 1 & 0 & 3.304258 & 4.097067 & 5.419713 \\
\hline 51 & 6 & 0 & 1.119206 & -4.514755 & 4.818700 \\
\hline 52 & 1 & 0 & 1.155834 & -2.896894 & 3.399463 \\
\hline 53 & 6 & 0 & 3.186173 & -4.949363 & 5.980715 \\
\hline 54 & 1 & 0 & 4.833585 & -3.654016 & 5.484014 \\
\hline 55 & 6 & 0 & 3.943385 & -6.845888 & 2.832858 \\
\hline 56 & 1 & 0 & 2.388354 & -5.725907 & 1.840720 \\
\hline 57 & 6 & 0 & 6.064454 & -5.717449 & 3.070337 \\
\hline 58 & 1 & 0 & 6.158964 & -3.721855 & 2.256901 \\
\hline 59 & 6 & 0 & 6.451169 & 5.395781 & 0.894474 \\
\hline 60 & 1 & 0 & 5.156123 & 4.943998 & -0.768739 \\
\hline 61 & 1 & 0 & 7.566396 & 5.624913 & 2.722559 \\
\hline 62 & 6 & 0 & 4.309496 & 7.156220 & 4.320202 \\
\hline 63 & 1 & 0 & 3.875674 & 7.965637 & 2.369873 \\
\hline 64 & 1 & 0 & 4.566667 & 6.094540 & 6.178180 \\
\hline 65 & 6 & 0 & 1.851760 & -5.268527 & 5.738706 \\
\hline 66 & 1 & 0 & 0.081252 & -4.763978 & 4.618027 \\
\hline 67 & 1 & 0 & 3.767729 & -5.535890 & 6.685493 \\
\hline 68 & 6 & 0 & 5.263663 & -6.839431 & 3.284925 \\
\hline 69 & 1 & 0 & 3.314704 & -7.714738 & 3.002351 \\
\hline 70 & 1 & 0 & 7.096507 & -5.711625 & 3.407964 \\
\hline 71 & 1 & 0 & 6.955937 & 6.211930 & 0.386880 \\
\hline 72 & 1 & 0 & 4.872262 & 8.026170 & 4.644520 \\
\hline 73 & 1 & 0 & 1.385549 & -6.100519 & 6.257347 \\
\hline 74 & 1 & 0 & 5.667383 & -7.706839 & 3.798080 \\
\hline 75 & 1 & 0 & -1.108209 & -1.583600 & -0.689538 \\
\hline 76 & 8 & 0 & -2.269000 & -1.584412 & -1.593776 \\
\hline 77 & 6 & 0 & -3.284462 & -0.639696 & -1.140977 \\
\hline 78 & 6 & 0 & -3.204864 & 0.144785 & -2.475988 \\
\hline 79 & 1 & 0 & -4.218307 & -1.156422 & -0.911876 \\
\hline 80 & 1 & 0 & -2.929058 & -0.065075 & -0.278366 \\
\hline 81 & 8 & 0 & -2.809853 & 1.480866 & -2.362551 \\
\hline
\end{tabular}

\begin{tabular}{|c|c|c|c|c|c|}
\hline 82 & 6 & 0 & -2.071884 & -0.851433 & -2.840370 \\
\hline 83 & 6 & 0 & -4.435441 & 0.041869 & -3.341661 \\
\hline 84 & 1 & 0 & -1.954418 & 1.502141 & -1.884890 \\
\hline 85 & 1 & 0 & -1.079894 & -0.387283 & -2.881628 \\
\hline 86 & 1 & 0 & -2.246383 & -1.505150 & -3.700678 \\
\hline 87 & 6 & 0 & -5.013708 & 1.177327 & -3.905914 \\
\hline 88 & 6 & 0 & -5.010346 & -1.212583 & -3.574008 \\
\hline 89 & 6 & 0 & -6.157725 & 1.058189 & -4.695920 \\
\hline 90 & 1 & 0 & -4.566580 & 2.146709 & -3.714739 \\
\hline 91 & 6 & 0 & -6.150283 & -1.330597 & -4.363975 \\
\hline 92 & 1 & 0 & -4.565013 & -2.104622 & -3.131972 \\
\hline 93 & 6 & 0 & -6.728314 & -0.191777 & -4.928091 \\
\hline 94 & 1 & 0 & -6.606020 & 1.947592 & -5.128233 \\
\hline 95 & 1 & 0 & -6.589768 & -2.308157 & -4.538383 \\
\hline 96 & 1 & 0 & -7.618892 & -0.279772 & -5.542727 \\
\hline 97 & 6 & 0 & -1.234177 & 2.689332 & 1.651641 \\
\hline 98 & 6 & 0 & -1.520305 & 3.512262 & 0.565908 \\
\hline 99 & 6 & 0 & -2.254224 & 1105 & 2.124830 \\
\hline 100 & 6 & 0 & -2.769171 & 3.508625 & -0.057799 \\
\hline 101 & 8 & 0 & -0.503686 & 4.262524 & 0.020149 \\
\hline 102 & 6 & 0 & -3.500681 & 1.854545 & 1.512031 \\
\hline 103 & 8 & 0 & -2.006191 & 1.015575 & 3.175763 \\
\hline 104 & 6 & 0 & -3.773146 & 2.654466 & 0.400225 \\
\hline 105 & 8 & 0 & -2.944824 & 4.297382 & -1.168399 \\
\hline 106 & 6 & 0 & -0.563995 & 4.315027 & -1.363199 \\
\hline 107 & 8 & 0 & -4.458294 & 0.973199 & 1.965477 \\
\hline 108 & 6 & 0 & -2.657696 & -0.194734 & 3.028319 \\
\hline 109 & 6 & 0 & -5.120095 & 2.483340 & -0.258896 \\
\hline 110 & 6 & 0 & -1.813970 & 4.343772 & -1.970034 \\
\hline 111 & 6 & 0 & 0.596086 & 4.325588 & -2.121841 \\
\hline 112 & 6 & 0 & -3.912234 & -0.214419 & 2.422254 \\
\hline 113 & 6 & 0 & -2.072015 & -1.368305 & 3.477472 \\
\hline 114 & 9 & 0 & -6.115499 & 2.765865 & 0.599983 \\
\hline 115 & 9 & 0 & -5.298426 & 1.201822 & -0.649231 \\
\hline 116 & 9 & 0 & -5.297878 & 3.249804 & -1.333432 \\
\hline 117 & 6 & 0 & -1.931942 & 4.398433 & -3.349520 \\
\hline 118 & 6 & 0 & 0.485432 & 4.400964 & -3.510887 \\
\hline 119 & 1 & 0 & 1.558281 & 4.260811 & -1.622727 \\
\hline 120 & 6 & 0 & -4.608078 & -1.403589 & 2.267682 \\
\hline 121 & 6 & 0 & -2.774031 & -2.567118 & 3.340105 \\
\hline 122 & 1 & 0 & -1.085563 & -1.321366 & 3.927962 \\
\hline 123 & 6 & 0 & -0.769587 & 4.440160 & -4.120332 \\
\hline 124 & 1 & 0 & -2.921952 & 4.392804 & -3.792984 \\
\hline 125 & 1 & 0 & 1.386263 & 4.409987 & -4.115333 \\
\hline 126 & 6 & 0 & -4.034177 & -2.584690 & 2.741712 \\
\hline 127 & 1 & 0 & -5.582029 & -1.383933 & 1.788672 \\
\hline 128 & 1 & 0 & -2.332451 & -3.489677 & 3.702331 \\
\hline 129 & 1 & 0 & -0.847991 & 4.481207 & -5.201512 \\
\hline 130 & 1 & 0 & -4.575772 & -3.519450 & 2.640735 \\
\hline 131 & 6 & 0 & 2.067117 & -2.223552 & -1.918874 \\
\hline 132 & 6 & 0 & 1.050113 & -3.085141 & -2.318890 \\
\hline 133 & 6 & 0 & 2.503182 & -1.262129 & -2.830388 \\
\hline 134 & 6 & 0 & 0.436373 & -2.962748 & -3.567488 \\
\hline 135 & 8 & 0 & 0.606364 & -4.042167 & -1.436950 \\
\hline 136 & 6 & 0 & 1.889380 & -1.114930 & -4.068270 \\
\hline 137 & 8 & 0 & 3.548559 & -0.455649 & -2.460311 \\
\hline 138 & 6 & 0 & 0.830994 & -1.948457 & -4.443566 \\
\hline 139 & 8 & 0 & -0.582528 & -3.828414 & -3.890669 \\
\hline 140 & 6 & 0 & -0.749392 & -4.293780 & -1.548237 \\
\hline 141 & 8 & 0 & 2.304006 & -0.148664 & -4.948279 \\
\hline 142 & 6 & 0 & 3.637233 & 0.737814 & -3.144924 \\
\hline 143 & 6 & 0 & 0.154067 & -1.647096 & -5.760291 \\
\hline 144 & 6 & 0 & -1.349003 & -4.205707 & -2.799810 \\
\hline 145 & 6 & 0 & -1.497944 & -4.603873 & -0.424130 \\
\hline 146 & 6 & 0 & 3.019735 & 0.891368 & -4.384771 \\
\hline 147 & 6 & 0 & 4.392915 & 1.766917 & -2.603948 \\
\hline
\end{tabular}




$\begin{array}{rllrrr}148 & 9 & 0 & 1.008970 & -1.746482 & -6.789657 \\ 149 & 9 & 0 & -0.327055 & -0.388465 & -5.759923 \\ 150 & 9 & 0 & -0.878404 & -2.453472 & -6.024205 \\ 151 & 6 & 0 & -2.701564 & -4.460597 & -2.959167 \\ 152 & 6 & 0 & -2.860795 & -4.865002 & -0.575681 \\ 153 & 1 & 0 & -1.007704 & -4.623518 & 0.544223 \\ 154 & 6 & 0 & 3.151337 & 2.074505 & -5.094863 \\ 155 & 6 & 0 & 4.544006 & 2.953359 & -3.322610 \\ 156 & 1 & 0 & 4.872318 & 1.614367 & -1.641466 \\ 157 & 6 & 0 & -3.457235 & -4.801940 & -1.835633 \\ 158 & 1 & 0 & -3.137927 & -4.384438 & -3.950702 \\ 159 & 1 & 0 & -3.456325 & -5.115409 & 0.295755 \\ 160 & 6 & 0 & 3.925548 & 3.106991 & -4.563166 \\ 161 & 1 & 0 & 2.657918 & 2.160669 & -6.057660 \\ 162 & 1 & 0 & 5.160191 & 3.748588 & -2.917046 \\ 163 & 1 & 0 & -4.516275 & -5.010825 & -1.946949 \\ 164 & 1 & 0 & 4.049060 & 4.027553 & -5.124378\end{array}$

\begin{tabular}{|c|c|c|c|}
\hline Frequencies -- & 15.0586 & 17.3505 & 17.5656 \\
\hline Frequencies -- & 21.2992 & 22.2803 & 25.7151 \\
\hline Frequencies -- & 28.0341 & 29.4685 & 30.9976 \\
\hline Frequencies -- & 33.6934 & 36.1080 & 38.6201 \\
\hline Frequencies -- & 40.9607 & 41.9264 & 42.1176 \\
\hline Frequencies -- & 44.7181 & 52.8974 & 55.3088 \\
\hline Frequencies -- & 56.7875 & 61.6772 & 63.4852 \\
\hline Frequencies -- & 64.4061 & 66.8842 & 68.8113 \\
\hline Frequencies -- & 73.0031 & 73.3683 & 77.7801 \\
\hline Frequencies -- & 78.2200 & 81.4453 & 86.6609 \\
\hline Frequencies -- & 91.7249 & 92.3858 & 94.9593 \\
\hline Frequencies -- & 97.9237 & 100.3179 & 104.9519 \\
\hline Frequencies -- & 106.9101 & 113.1710 & 116.0094 \\
\hline Frequencies -- & 119.2255 & 123.3963 & 126.8867 \\
\hline Frequencies -- & 132.9618 & 135.1651 & 143.3184 \\
\hline Frequencies -- & 144.8866 & 155.0913 & 159.2866 \\
\hline Frequencies -- & 163.9471 & 171.0830 & 179.1515 \\
\hline Frequencies -- & 183.8827 & 195.8791 & 197.7571 \\
\hline Frequencies -- & 197.9333 & 204.8663 & 212.2355 \\
\hline Frequencies -- & 217.9689 & 227.6721 & 237.4993 \\
\hline Frequencies -- & 241.0942 & 244.9354 & 248.0462 \\
\hline Frequencies -- & 253.0668 & 262.1912 & 270.9953 \\
\hline Frequencies -- & 273.3648 & 275.0350 & 278.5445 \\
\hline Frequencies -- & 282.5253 & 291.2098 & 292.5240 \\
\hline Frequencies -- & 294.8600 & 297.0955 & 298.8239 \\
\hline Frequencies -- & 304.7809 & 310.6201 & 311.0855 \\
\hline Frequencies -- & 315.5745 & 316.1844 & 323.5768 \\
\hline Frequencies -- & 327.2962 & 330.2860 & 332.5741 \\
\hline Frequencies -- & 336.2482 & 337.3636 & 339.7138 \\
\hline Frequencies -- & 346.9937 & 358.1172 & 369.0445 \\
\hline Frequencies -- & 372.1082 & 403.6278 & 404.9467 \\
\hline Frequencies -- & 408.3130 & 409.0986 & 411.1875 \\
\hline Frequencies -- & 412.3846 & 415.4040 & 416.0888 \\
\hline Frequencies -- & 419.7126 & 424.2988 & 433.4024 \\
\hline Frequencies -- & 435.7794 & 437.4526 & 439.8937 \\
\hline Frequencies -- & 441.3944 & 444.0572 & 447.1312 \\
\hline Frequencies -- & 449.1953 & 451.4251 & 456.6806 \\
\hline Frequencies -- & 466.5410 & 474.2607 & 486.7288 \\
\hline Frequencies -- & 491.7529 & 501.0682 & 512.4864 \\
\hline Frequencies -- & 516.0950 & 516.5742 & 521.0950 \\
\hline Frequencies -- & 522.8553 & 529.0175 & 531.7190 \\
\hline Frequencies -- & 536.4528 & 539.6241 & 543.2070 \\
\hline Frequencies -- & 548.0926 & 555.4643 & 559.2820 \\
\hline Frequencies -- & 561.5231 & 563.5135 & 563.7656 \\
\hline Frequencies -- & 565.5937 & 566.8811 & 570.9788 \\
\hline Frequencies -- & 583.1423 & 588.4774 & 596.5233 \\
\hline Frequencies -- & 597.7494 & 600.9678 & 601.6026 \\
\hline
\end{tabular}

\begin{tabular}{|c|c|c|c|}
\hline Frequencies -- & 603.8427 & 607.7970 & 609.6840 \\
\hline Frequencies -- & 610.2600 & 617.3708 & 618.4805 \\
\hline Frequencies -- & 620.8805 & 624.4944 & 626.6328 \\
\hline Frequencies -- & 629.5229 & 630.3079 & 630.5365 \\
\hline Frequencies -- & 632.2177 & 633.3281 & 635.1315 \\
\hline Frequencies -- & 646.4380 & 649.8022 & 654.5384 \\
\hline Frequencies -- & 669.9417 & 673.2325 & 680.9496 \\
\hline Frequencies -- & 684.3933 & 691.2520 & 701.2297 \\
\hline Frequencies -- & 704.8629 & 705.8566 & 708.3002 \\
\hline Frequencies -- & 709.9230 & 714.5549 & 714.8257 \\
\hline Frequencies -- & 718.9381 & 721.7519 & 723.7115 \\
\hline Frequencies -- & 725.7556 & 728.7668 & 730.2308 \\
\hline Frequencies -- & 733.0960 & 737.6789 & 741.1310 \\
\hline Frequencies -- & 745.1639 & 747.3506 & 750.8984 \\
\hline Frequencies -- & 763.5565 & 768.1764 & 769.3057 \\
\hline Frequencies -- & 770.7712 & 772.9864 & 775.7955 \\
\hline Frequencies -- & 776.8005 & 778.7688 & 780.7033 \\
\hline Frequencies -- & 782.6429 & 787.7604 & 795.9955 \\
\hline Frequencies -- & 800.3295 & 801.3288 & 804.1713 \\
\hline Frequencies -- & 805.5643 & 820.3164 & 823.8014 \\
\hline Frequencies -- & 825.7864 & 827.9831 & 829.8032 \\
\hline Frequencies -- & 856.9067 & 857.6712 & 858.8975 \\
\hline Frequencies -- & 862.1523 & 863.4392 & 864.8139 \\
\hline Frequencies -- & 866.7608 & 867.4748 & 870.3444 \\
\hline Frequencies -- & 874.0959 & 883.9506 & 885.6014 \\
\hline Frequencies -- & 887.9306 & 890.1916 & 905.2601 \\
\hline Frequencies -- & 916.2019 & 918.4625 & 919.0076 \\
\hline Frequencies -- & 921.4004 & 923.8869 & 930.6513 \\
\hline Frequencies -- & 932.2823 & 933.4043 & 938.0923 \\
\hline Frequencies -- & 940.0770 & 942.1746 & 943.9883 \\
\hline Frequencies -- & 945.1060 & 945.9973 & 948.7185 \\
\hline Frequencies -- & 950.3730 & 954.5745 & 954.9240 \\
\hline Frequencies -- & 970.5364 & 974.2678 & 986.5446 \\
\hline Frequencies -- & 987.8880 & 988.5253 & 990.4508 \\
\hline Frequencies -- & 990.7747 & 991.0952 & 995.1526 \\
\hline Frequencies -- & 999.0324 & 1000.5665 & 1000.9352 \\
\hline Frequencies -- & 1006.8166 & 1008.3871 & 1009.8565 \\
\hline Frequencies -- & 1011.6415 & 1012.5492 & 1014.0509 \\
\hline Frequencies -- & 1018.8546 & 1019.0730 & 1019.5518 \\
\hline Frequencies -- & 1019.7808 & 1019.8600 & 1021.9618 \\
\hline Frequencies -- & 1023.0522 & 1029.3200 & 1033.6202 \\
\hline Frequencies -- & 1035.5542 & 1042.7093 & 1044.2502 \\
\hline Frequencies -- & 1053.8663 & 1055.0482 & 1056.6102 \\
\hline Frequencies -- & 1059.4738 & 1063.1208 & 1063.9225 \\
\hline Frequencies -- & 1066.8380 & 1069.2936 & 1072.0374 \\
\hline Frequencies -- & 1073.1969 & 1084.7665 & 1093.0950 \\
\hline Frequencies -- & 1093.7718 & 1100.6238 & 1107.9395 \\
\hline Frequencies -- & 1108.6584 & 1110.0215 & 1114.3961 \\
\hline Frequencies -- & 1116.4507 & 1122.7761 & 1125.9356 \\
\hline Frequencies -- & 1126.7919 & 1128.1093 & 1130.1983 \\
\hline Frequencies -- & 1132.1706 & 1162.6181 & 1167.4445 \\
\hline Frequencies -- & 1169.2957 & 1169.5771 & 1170.8104 \\
\hline Frequencies -- & 1171.1086 & 1171.8963 & 1172.3558 \\
\hline Frequencies -- & 1174.5660 & 1176.3075 & 1178.2108 \\
\hline Frequencies -- & 1181.3565 & 1183.8697 & 1192.7415 \\
\hline Frequencies -- & 1195.5758 & 1198.7185 & 1199.1905 \\
\hline Frequencies -- & 1202.9503 & 1210.1223 & 1212.8674 \\
\hline Frequencies -- & 1213.2845 & 1214.7639 & 1216.1698 \\
\hline Frequencies -- & 1221.4598 & 1223.3878 & 1224.6439 \\
\hline Frequencies -- & 1228.1749 & 1230.3011 & 1232.0992 \\
\hline Frequencies -- & 1233.9955 & 1236.5671 & 1241.3162 \\
\hline Frequencies -- & 1243.0894 & 1247.1884 & 1255.8997 \\
\hline Frequencies -- & 1259.4137 & 1266.2113 & 1273.7727 \\
\hline Frequencies -- & 1277.1246 & 1282.0329 & 1289.8495 \\
\hline Frequencies -- & 1290.5519 & 1291.7072 & 1296.0340 \\
\hline Frequencies -- & 1297.1621 & 1300.1367 & 1301.6125 \\
\hline
\end{tabular}




\begin{tabular}{|c|c|c|c|c|c|c|c|c|c|c|}
\hline \multicolumn{3}{|c|}{ Frequencies -- 1304.5104} & 1307.1324 & 1308.0947 & 5 & 8 & 0 & -0.862674 & -1.196666 & 0.023676 \\
\hline \multicolumn{2}{|c|}{ Frequencies -- } & 08.9848 & 1311.1095 & 1313.0418 & 6 & 6 & 0 & 0.838989 & 1.142777 & 2.504785 \\
\hline \multicolumn{2}{|c|}{ Frequencies -- } & 14.6013 & 1318.3526 & 1324.5575 & 7 & 6 & 0 & 2.239007 & -1.236937 & 0.232542 \\
\hline \multicolumn{2}{|c|}{ Frequencies -- } & 25.1005 & 1326.6540 & 1328.6839 & 8 & 6 & 0 & 2.213895 & 1.035253 & 2.664025 \\
\hline \multicolumn{2}{|c|}{ Frequencies -- } & 36.4606 & 1339.7434 & 1341.1403 & 9 & 6 & 0 & 0.171357 & 2.333483 & 2.819820 \\
\hline \multicolumn{2}{|c|}{ Frequencies -- } & 45.4665 & 1346.8459 & 1357.0047 & 10 & 6 & 0 & 2.923007 & -1.235314 & 1.439445 \\
\hline \multicolumn{2}{|c|}{ Frequencies -- } & 57.1985 & 1359.9600 & 1362.8508 & 11 & 6 & 0 & 2.202047 & -2.382038 & -0.573479 \\
\hline \multicolumn{2}{|c|}{ Frequencies -- } & 66.2705 & 1366.7279 & 1368.0001 & 12 & 6 & 0 & 3.158963 & -0.133250 & 2.454501 \\
\hline \multicolumn{2}{|c|}{ Frequencies -- } & 68.3893 & 1370.3702 & 1371.7671 & 13 & 6 & 0 & 2.930506 & 2.143078 & 3.121507 \\
\hline \multicolumn{2}{|c|}{ Frequencies -- } & 72.4276 & 1377.8782 & 1383.0772 & 14 & 6 & 0 & 0.904803 & 3.392109 & 3.362264 \\
\hline \multicolumn{2}{|c|}{ Frequencies -- } & 83.8657 & 1394.4831 & 1410.3863 & 15 & 6 & 0 & 3.548294 & -2.408466 & 1.864508 \\
\hline Frequen & cies -- 145 & 50.7909 & 1459.6268 & 1461.8143 & 16 & 6 & 0 & 2.928942 & -3.505738 & -0.169853 \\
\hline Frequen & cies -- 146 & 68.9015 & 1471.6976 & 1490.4514 & 17 & 6 & 0 & 3.306447 & -0.968323 & 3.753777 \\
\hline Frequen & cies -- 149 & 91.6375 & 1495.7035 & 1498.1596 & 18 & 6 & 0 & 4.480212 & 0.618824 & 2.144428 \\
\hline Frequen & cies -- 149 & 99.6621 & 1499.9606 & 1505.1755 & 19 & 6 & 0 & 4.428832 & 1.919477 & 2.981318 \\
\hline Frequen & cies -- 150 & 06.0904 & 1508.0555 & 1508.8069 & 20 & 6 & 0 & 2.287090 & 3.313860 & 3.529098 \\
\hline Frequen & cies -- 151 & 12.5762 & 1512.7593 & 1516.1803 & 21 & 1 & 0 & 0.387031 & 4.307785 & 3.634375 \\
\hline Frequen & cies -- 151 & 18.0218 & 1527.6113 & 1530.5183 & 22 & 6 & 0 & 3.963913 & -2.304011 & 3.325112 \\
\hline Frequen & cies -- 153 & 30.7247 & 1548.8591 & 1549.4658 & 23 & 6 & 0 & 3.611185 & -3.539109 & 1.046158 \\
\hline Frequen & cies -- 155 & 53.5087 & 1555.0029 & 1555.1627 & 24 & 1 & 0 & 2.927221 & -4.389025 & -0.802204 \\
\hline Frequen & cies -- 155 & 55.8198 & 1560.6204 & 1562.2836 & 25 & 1 & 0 & 3.879926 & -0.441486 & 4.522605 \\
\hline Frequen & cies -- 156 & 53.4762 & 1570.4679 & 1575.9468 & 26 & 1 & 0 & 2.309788 & -1.167704 & 4.161033 \\
\hline Frequen & cies -- 166 & 54.9959 & 1665.6942 & 1668.5939 & 27 & 1 & 0 & 5.363832 & 0.010154 & 2.359431 \\
\hline Frequen & cies -- 166 & 59.5727 & 1674.7456 & 1675.2259 & 28 & 1 & 0 & 4.500104 & 0.873689 & 1.079591 \\
\hline Frequen & cies -- 167 & 76.9498 & 1677.2002 & 1677.6907 & 29 & 1 & 0 & 4.888000 & 1.758429 & 3.964217 \\
\hline Frequen & cies -- 168 & 38.9312 & 1690.8408 & 1694.8390 & 30 & 6 & 0 & 5.115374 & 3.095204 & 2.314515 \\
\hline Frequen & cies -- 169 & 96.7275 & 1696.9427 & 1698.8796 & 31 & 6 & 0 & 3.063725 & 4.450466 & 4.091151 \\
\hline Frequen & cies -- 169 & 99.5025 & 1701.2443 & 1701.5184 & 32 & 1 & 0 & 5.054400 & -2.241419 & 3.420793 \\
\hline Frequen & cies -- 170 & 2.7181 & 1702.8059 & 1705.4699 & 33 & 6 & 0 & 3.470923 & -3.497793 & 4.120844 \\
\hline Frequen & cies -- 170 & 07.2217 & 1720.1828 & 1723.0785 & 34 & 6 & 0 & 4.333250 & -4.758369 & 1.493698 \\
\hline Frequen & cies -- 173 & 33.7308 & 1734.2595 & 2476.8812 & 35 & 6 & 0 & 4.632046 & 3.583545 & 1.093849 \\
\hline Frequen & cies -- 308 & 32.3016 & 3083.4024 & 3087.5789 & 36 & 6 & 0 & 6.193889 & 3.741920 & 2.917366 \\
\hline Frequen & cies -- 308 & 39.3498 & 3096.3185 & 3110.0339 & 37 & 6 & 0 & 3.129845 & 5.676565 & 3.422380 \\
\hline Frequen & cies -- 315 & 51.3111 & 3155.7250 & 3171.8656 & 38 & 6 & 0 & 3.778425 & 4.281845 & 5.281580 \\
\hline Frequen & cies -- 318 & 30.3715 & 3183.2231 & 3192.0362 & 39 & 6 & 0 & 2.117208 & -3.854342 & 4.064573 \\
\hline Frequen & cies -- 319 & 94.5391 & 3197.7578 & 3200.9900 & 40 & 6 & 0 & 4.344130 & -4.289644 & 4.865995 \\
\hline Frequen & cies -- 320 & 04.0423 & 3205.3580 & 3210.3889 & 41 & 6 & 0 & 3.647695 & -5.960872 & 1.691613 \\
\hline Frequen & cies -- 321 & 14.5410 & 3215.4609 & 3217.8993 & 42 & 6 & 0 & 5.703016 & -4.698857 & 1.771036 \\
\hline Frequen & cies -- 321 & 18.4653 & 3218.9946 & 3219.3106 & 43 & 6 & 0 & 5.214569 & 4.695539 & 0.492336 \\
\hline Frequen & cies -- 322 & 20.2219 & 3221.0137 & 3221.8544 & 44 & 1 & 0 & 3.771949 & 3.103783 & 0.628136 \\
\hline Frequen & cies -- 322 & 23.3377 & 3223.5576 & 3224.9943 & 45 & 6 & 0 & 6.776288 & 4.861941 & 2.322086 \\
\hline Frequen & cies -- 322 & 27.5234 & 3228.2147 & 3228.7927 & 46 & 1 & 0 & 6.565534 & 3.384322 & 3.874762 \\
\hline Frequen & cies -- 323 & 30.0824 & 3231.9785 & 3232.5387 & 47 & 6 & 0 & 3.920559 & 6.709110 & 3.923532 \\
\hline Frequen & cies -- 323 & 32.7792 & 3233.0250 & 3234.3384 & 48 & 1 & 0 & 2.583312 & 5.805888 & 2.491346 \\
\hline Frequen & cies -- 323 & 37.5205 & 3238.2484 & 3240.2957 & 49 & 6 & 0 & 4.557889 & 5.318605 & 5.791487 \\
\hline Frequen & cies -- 324 & 40.6899 & 3241.2959 & 3243.0468 & 50 & 1 & 0 & 3.714765 & 3.333515 & 5.810313 \\
\hline Frequen & cies -- 324 & 43.1895 & 3243.3979 & 3247.2444 & 51 & 6 & 0 & 1.649227 & -4.977854 & 4.739565 \\
\hline Frequen & cies -- 324 & 47.7246 & 3249.8742 & 3255.7207 & 52 & 1 & 0 & 1.432535 & -3.261298 & 3.458795 \\
\hline Frequen & cies -- 325 & 56.6455 & 3258.4149 & 3647.2515 & 53 & 6 & 0 & 3.880837 & -5.422798 & 5.536883 \\
\hline & & & & & 54 & 1 & 0 & 5.401185 & -4.036027 & 4.899457 \\
\hline SCF Done & : $E(R M 062$ & 2X/DGDZVP) = & -5378.6889 & 98726 & 55 & 6 & 0 & 4.317025 & -7.079348 & 2.185059 \\
\hline Sum of $\mathrm{e}$ & lectronic ar & ad zero-point $\mathrm{E}$ & Energies= & -5377.446857 & 56 & 1 & 0 & 2.581013 & -6.005522 & 1.484999 \\
\hline Sum of $e$ & electronic a & nd thermal En & lergies= & -5377.343581 & 57 & 6 & 0 & 6.376020 & -5.820100 & 2.252651 \\
\hline Sum of $e$ & electronic a & nd thermal Fre & ee Energies= & $=-5377.594377$ & 58 & 1 & 0 & 6.240532 & -3.768816 & 1.600416 \\
\hline SCF Done & e: $E(R M 062$ & XX/DGTZVP/SN & MD) $=-5379.8$ & 82096920 & 59 & 6 & 0 & 6.287795 & 5.342893 & 1.108775 \\
\hline & & & & & 60 & 1 & 0 & 4.828202 & 5.064563 & -0.453558 \\
\hline & 02 & & & & 61 & 1 & 0 & 7.604633 & 5.363564 & 2.813028 \\
\hline Iac I & $0-2$ & & & & 62 & 6 & 0 & 4.638782 & 6.531455 & 5.106781 \\
\hline & & & & & 63 & 1 & 0 & 3.983067 & 7.649988 & 3.385377 \\
\hline Center & Atomic & Atomic & Coordinates & s (Angstroms) & 64 & 1 & 0 & 5.102675 & 5.179266 & 6.720410 \\
\hline Number & Number & Type & $\mathrm{x}$ & Z & 65 & 6 & 0 & 2.533017 & -5.770653 & 5.475446 \\
\hline & & & & & 66 & 1 & 0 & 0.596869 & -5.242963 & 4.687259 \\
\hline 1 & 15 & -0.013767 & -0.036707 & 0.365803 & 67 & 1 & 0 & 4.576575 & -6.038326 & 6.099071 \\
\hline 2 & 8 & 0.123276 & 0.075134 & 1.964628 & 68 & 6 & 0 & 5.681279 & -7.010147 & 2.470857 \\
\hline 3 & 8 & 1.507870 & -0.105658 & -0.136253 & 69 & 1 & 0 & 3.771762 & -8.002424 & 2.356131 \\
\hline 4 & 8 & -0.437830 & 1.361693 & -0.180642 & 70 & 1 & 0 & 7.440679 & -5.765102 & 2.459158 \\
\hline
\end{tabular}




\begin{tabular}{|c|c|c|c|c|c|}
\hline 71 & 1 & 0 & 6.737444 & 6.216274 & 0.646645 \\
\hline 72 & & 0 & 5.255289 & 7.336397 & 5.494913 \\
\hline 73 & & 0 & 2.172870 & -6.653992 & 5.993736 \\
\hline 74 & 1 & 0 & 6.200862 & -7.881576 & 2.857316 \\
\hline 75 & 1 & 0 & -1.417907 & 1.395299 & -0.489643 \\
\hline 76 & 8 & 0 & -2.822805 & 1.183231 & -1.075247 \\
\hline 77 & 6 & 0 & -2.637603 & 0.425271 & -2.310226 \\
\hline 78 & 6 & 0 & -3.572512 & -0.706291 & -1.809373 \\
\hline 79 & 1 & 0 & -2.967497 & 1.004570 & -3.173933 \\
\hline 80 & 1 & 0 & -1.593931 & 0.110418 & -2.423285 \\
\hline 81 & 8 & 0 & -2.983797 & -1.970041 & -1.678348 \\
\hline 82 & 6 & 0 & -3.642329 & 0.119493 & -0.501177 \\
\hline 83 & 6 & 0 & -4.876407 & -0.824760 & -2.558489 \\
\hline 84 & 1 & 0 & -2.223170 & -1.881868 & -1.067178 \\
\hline 85 & 1 & 0 & -3.133228 & -0.365398 & 0.336480 \\
\hline 86 & 1 & 0 & -4.628371 & 0.490803 & -0.212595 \\
\hline 87 & 6 & 0 & -5.304144 & -2.050957 & -3.065980 \\
\hline 88 & 6 & 0 & 4550 & & -2.753198 \\
\hline 89 & 6 & 0 & 1076 & 5509 & -3.761941 \\
\hline 90 & 1 & 0 & -4.684845 & 8619 & -2.915370 \\
\hline 91 & 6 & 0 & -6.870047 & 0.228050 & -3.444206 \\
\hline 92 & 1 & 0 & -5.339792 & 1.276937 & -2.359138 \\
\hline 93 & 6 & 0 & -7.296893 & -1.000537 & -3.951589 \\
\hline 94 & 1 & 0 & -6.836404 & -3.092480 & 8905 \\
\hline 95 & 1 & 0 & -7.476101 & 1.117760 & -3.5 \\
\hline 96 & 1 & 0 & -8.235963 & -1.070109 & 2025 \\
\hline 97 & 6 & 0 & 1.294788 & -2.441901 & 2121 \\
\hline 98 & 6 & 0 & 0.332 & 0906 & 2853 \\
\hline 99 & 6 & 0 & 1.26 & 1952 & 591 \\
\hline 100 & 6 & 0 & -0.663029 & -3.449477 & -2.800831 \\
\hline 101 & 8 & 0 & 0.328384 & -4.434150 & -0.856915 \\
\hline 102 & 6 & 0 & 0.261025 & -1.425909 & -3.68 \\
\hline 103 & 8 & 0 & 2.212467 & -0.452983 & -2.7 \\
\hline 104 & 6 & 0 & -0.725610 & -2.410488 & 3. -3.730592 \\
\hline 105 & 8 & 0 & -1.611573 & -4.442236 & -2.792453 \\
\hline 106 & 6 & 0 & -0.927717 & -4.918707 & -0.550174 \\
\hline 107 & 8 & 0 & 0.198701 & -0.359690 & -4.551319 \\
\hline 108 & 6 & 0 & 1.654639 & 0.776431 & -3.030914 \\
\hline 109 & 6 & 0 & -1.819992 & -2.246823 & $3-4.757697$ \\
\hline 110 & 6 & 0 & -1.912477 & -4.920503 & -1.532054 \\
\hline 111 & 6 & 0 & -1.183550 & -5.430073 & 0.712174 \\
\hline 112 & 6 & 0 & 0.621429 & 0.823766 & -3.961942 \\
\hline 113 & 6 & 0 & 2.103408 & 1.932467 & -2.412785 \\
\hline 114 & 9 & 0 & -1.309099 & -2.201509 & -6.000312 \\
\hline 115 & 9 & 0 & -2.484501 & -1.086414 & -4.569130 \\
\hline 116 & 9 & 0 & -2.727421 & -3.222253 & -4.740523 \\
\hline 117 & 6 & 0 & -3.174634 & -5.426073 & -1.263754 \\
\hline 118 & 6 & 0 & -2.443908 & -5.966692 & 0.980950 \\
\hline 119 & 1 & 0 & -0.391383 & -5.407709 & 1.455158 \\
\hline 120 & 6 & 0 & 0.004941 & 2.024702 & -4.280237 \\
\hline 121 & 6 & 0 & 1.502536 & 3.146577 & -2.742631 \\
\hline 122 & 1 & 0 & 2.898461 & 1.856531 & -1.678521 \\
\hline 123 & 6 & 0 & -3.434619 & -5.961796 & -0.001311 \\
\hline 124 & 1 & 0 & -3.925661 & -5.403491 & -2.046395 \\
\hline 125 & 1 & 0 & -2.649687 & -6.388838 & 1.960012 \\
\hline 126 & 6 & 0 & 0.454631 & 3.193566 & -3.663029 \\
\hline 127 & 1 & 0 & -0.810822 & 2.024992 & -4.996460 \\
\hline 128 & 1 & 0 & & 4.059494 & -2.265762 \\
\hline 129 & 1 & 0 & -4.415265 & -6.374775 & 0.211192 \\
\hline 130 & 1 & 0 & -0.024690 & 4.140501 & -3.890753 \\
\hline 131 & 6 & 0 & -1.255999 & 2.506637 & 2.465825 \\
\hline 132 & 6 & 0 & -2.236243 & 1.592227 & 2.839921 \\
\hline 133 & 6 & 0 & -1.646463 & 3.572343 & 1.648574 \\
\hline 134 & 6 & 0 & -3.542945 & 1.665346 & 2.347146 \\
\hline 135 & 8 & 0 & -1.906760 & 0.569279 & 3.698718 \\
\hline 136 & 6 & 0 & -2.950117 & 3.675484 & 1.182208 \\
\hline
\end{tabular}

$\begin{array}{rlrrrr}137 & 8 & 0 & -0.703291 & 4.498976 & 1.265746 \\ 138 & 6 & 0 & -3.912935 & 2.715817 & 1.505525 \\ 139 & 8 & 0 & -4.424082 & 0.664436 & 2.678182 \\ 140 & 6 & 0 & -2.519526 & -0.620948 & 3.346380 \\ 141 & 8 & 0 & -3.312394 & 4.710307 & 0.355422 \\ 142 & 6 & 0 & -0.965773 & 5.122549 & 0.062759 \\ 143 & 6 & 0 & -5.290266 & 2.903794 & 0.915233 \\ 144 & 6 & 0 & -3.809481 & -0.571803 & 2.832002 \\ 145 & 6 & 0 & -1.855794 & -1.829647 & 3.484649 \\ 146 & 6 & 0 & -2.274729 & 5.220363 & -0.401716 \\ 147 & 6 & 0 & 0.078980 & 5.684177 & -0.653918 \\ 148 & 9 & 0 & -5.241032 & 2.933349 & -0.431625 \\ 149 & 9 & 0 & -5.832864 & 4.065370 & 1.313133 \\ 150 & 9 & 0 & -6.146248 & 1.932944 & 1.248563 \\ 151 & 6 & 0 & -4.461516 & -1.729246 & 2.433274 \\ 152 & 6 & 0 & -2.508873 & -2.999324 & 3.098447 \\ 153 & 1 & 0 & -0.843326 & -1.830040 & 3.874354 \\ 154 & 6 & 0 & -2.552593 & 5.858302 & -1.600741 \\ 155 & 6 & 0 & -0.195555 & 6.350959 & -1.849100 \\ 156 & 1 & 0 & 1.086202 & 5.595480 & -0.258028 \\ 157 & 6 & 0 & -3.799765 & -2.950402 & 2.570170 \\ 158 & 1 & 0 & -5.458193 & -1.655933 & 2.009496 \\ 159 & 1 & 0 & -1.998808 & -3.952755 & 3.188766 \\ 160 & 6 & 0 & -1.504874 & 6.432531 & -2.323076 \\ 161 & 1 & 0 & -3.582594 & 5.909331 & -1.938192 \\ 162 & 1 & 0 & 0.616062 & 6.805528 & -2.408488 \\ 163 & 1 & 0 & -4.286264 & -3.862804 & 2.241116 \\ 164 & 1 & 0 & -1.716678 & 6.947726 & -3.254027 \\ -------------------------------------------------------\end{array}$

Frequencies -- 10.5971

Frequencies -23.9448

Frequencies -- 28.6361

Frequencies -- 40.1437

Frequencies -- 45.8657

Frequencies -- 51.9092

Frequencies -- 61.0369

Frequencies -- 68.8889

Frequencies -- 74.8818

Frequencies -- 84.2855

Frequencies -- 88.0580

Frequencies -- 97.8718

Frequencies -- 109.5045

Frequencies -- 126.5676

Frequencies -- 133.4991

Frequencies -- 148.7991

Frequencies -- 170.0752

Frequencies -- 192.9021

Frequencies -- 201.6174

Frequencies -- 218.4412

Frequencies -- 240.3816

Frequencies -- 253.2009

Frequencies -- 272.8420

Frequencies -- 286.2421

Frequencies -- 294.3827

Frequencies -- 304.9047

Frequencies -- 317.0490

Frequencies -- 328.4785

Frequencies -- 337.6510

Frequencies -- 351.9375

Frequencies -- 377.3686

Frequencies -- 411.4537

Frequencies -- 416.1798

Frequencies -- 418.9438

Frequencies -- 431.8085

Frequencies -- 444.2120
$25.3216 \quad 27.1427$

$32.5539 \quad 34.6652$

$41.6126 \quad 43.8277$

$49.0653 \quad 51.2733$

$56.8259 \quad 59.8138$

$63.3113 \quad 67.1341$

$69.3511 \quad 73.6333$

$\begin{array}{ll}78.2776 & 80.6437\end{array}$

$85.1788 \quad 86.1571$

$94.1481 \quad 96.2555$

$100.4169 \quad 106.2258$

$122.1419 \quad 125.9967$

$128.0770 \quad 131.4409$

$140.5596 \quad 144.8193$

$157.1670 \quad 164.2800$

$173.3680 \quad 182.8238$

$193.5243 \quad 198.2582$

$206.1875 \quad 211.3806$

$225.1259 \quad 236.8547$

$241.9896 \quad 247.2060$

$261.9979 \quad 271.5909$

$276.4164 \quad 279.5568$

$288.5038 \quad 290.7619$

$299.0567 \quad 302.8022$

$309.2639 \quad 310.3690$

$318.6678 \quad 324.6691$

$329.7098 \quad 334.4486$

$342.2825 \quad 347.6485$

$358.9425 \quad 372.0355$

$403.3039 \quad 407.1352$

$412.7912 \quad 413.7780$

$416.7538 \quad 417.3103$

$424.8722 \quad 430.3126$

$435.3158 \quad 438.7144$

$446.5720 \quad 449.2418$ 
Frequencies -- 1198.0563

Frequencies -- 1207.3740

Frequencies -- 1215.3717

Frequencies -- 1219.0453

Frequencies -- 1229.2767

Frequencies -- 1235.5262

Frequencies -- 1243.4946

Frequencies -- 1258.5542

Frequencies -- 1280.7118

Frequencies -- 1289.0216

Frequencies -- 1296.9867

Frequencies -- 1305.9642

Frequencies -- 1309.8789

Frequencies -- 1315.7792

Frequencies -- 1324.3900

Frequencies -- 1335.5070

Frequencies -- 1344.2248

Frequencies -- 1360.6895

Frequencies -- 1363.9383

Frequencies -- 1367.8059

Frequencies -- 1371.9480

Frequencies -- 1389.6147

Frequencies -- 1448.1094

Frequencies -- 1467.1069

Frequencies -- 1494.6264

Frequencies -- 1502.2110

Frequencies -- 1506.5074

Frequencies -- 1511.8403

Frequencies -- 1519.1554

Frequencies -- 1539.1330

Frequencies -- 1553.3568

Frequencies -- 1557.5124

Frequencies -- 1562.9631

Frequencies -- 1661.6095

Frequencies -- 1669.4830

Frequencies -- 1675.2768

Frequencies -- 1690.2965

Frequencies -- 1695.7667

Frequencies -- 1701.6274

Frequencies -- 1702.9565

Frequencies -- 1706.1791

Frequencies -- 1728.1166

Frequencies -- 3083.0040

Frequencies -- 3094.3248

Frequencies -- 3152.0592

Frequencies -- 3184.2605

Frequencies -- 3201.4671

Frequencies -- 3205.7402

Frequencies -- 3213.4967

Frequencies -- 3215.3557

Frequencies -- 3219.3467

Frequencies -- 3223.3095

Frequencies -- 3225.6926

Frequencies -- 3227.7287

Frequencies -- 3235.1132

Frequencies -- 3235.5254

Frequencies -- 3236.4627

Frequencies -- 3241.8891

Frequencies -- 3246.2000

Frequencies -- 3248.6386
1211.3597

1216.1463

1222.4179

1230.3717

1237.0025

1248.0052

1266.1581

1283.3220

1294.8650

1297.6403

1307.6476

1310.8156

1318.2185

1327.9827

1337.5958

1345.2425

1362.1312

1365.3080

1369.5297

1378.4975

1392.3669

1456.7764

1470.2545

1497.2476

1502.4541

1510.5067

1512.5619

1526.2816

1543.9058

1553.9076

1559.1774

1567.3767

1662.4284

1673.0478

1677.6671

1691.0246

1698.6767

1701.7142

1703.3207

1718.9599

1731.9928

3088.7664

3112.9653

3159.2564

3195.7094

3201.4829

3208.1166

3214.1469

3216.5644

3219.8140

3224.6333

3226.2064

3230.6069

3235.4159

3235.6232

3237.3689

3243.0192

3248.2219

3248.9677
1204.4227

1211.5791

1217.3483

1224.5862

1232.7775

1242.0549

1254.8798

1272.7511

1285.5161

1295.5525

1301.8267

1308.0907

1312.9237

1321.8574

1329.1369

1341.0946

1356.9377

1362.9011

1366.1636

1370.1260

1384.0495

1405.6908

1457.7559

1490.3047

1499.9226

1505.1042

1510.6253

1515.0292

1527.4770

1551.8688

1554.3248

1561.2501

1570.1448

1667.6493

1673.6543

1678.4738

1694.4818

1700.4436

1702.2182

1703.8252

1719.7596

2673.2284

3089.6723

3126.8326

3183.7734

3198.8130

3205.0839

3208.1910

3214.9412

3216.8817

3221.1890

3225.6112

3226.6100

3232.9637

3235.5151

3235.7711

3238.5769

3244.6588

3248.5239

3646.0000

SCF Done: $E($ RM062X/DGDZVP) $=-5378.68958244$

Sum of electronic and zero-point Energies $=\quad-5377.446451$

Sum of electronic and thermal Energies $=\quad-5377.343614$

Sum of electronic and thermal Free Energies $=\quad-5377.591446$ SCF Done: $E(R M 062 X / D G T Z V P / S M D)=-5379.82032726$ 


\section{Cat $\mathrm{f} 10 \mathrm{a} \_R$}

Center Atomic Atomic Coordinates (Angstroms) Number Number Type $\quad X \quad$ Y $\quad$ Z

\begin{tabular}{|c|c|c|c|c|c|}
\hline 1 & 15 & 0 & 0.234062 & -0.033140 & -0.307778 \\
\hline 2 & 8 & 0 & 0.121367 & -0.090182 & 1.294472 \\
\hline 3 & 8 & 0 & 1.802982 & -0.225662 & -0.602047 \\
\hline 4 & 8 & 0 & -0.168995 & 1.269992 & -0.897968 \\
\hline 5 & 8 & 0 & -0.528920 & -1.300523 & -0.745181 \\
\hline 6 & 6 & 0 & 0.821813 & 0.927603 & 1.944575 \\
\hline 7 & 6 & 0 & 2.338951 & -1.446816 & -0.182740 \\
\hline 8 & 6 & 0 & 2.140448 & 0.707406 & 2.298573 \\
\hline 9 & 6 & 0 & 0.203545 & 2.164607 & 2.149671 \\
\hline 10 & 6 & 0 & 2.745746 & -1.600308 & 1.129545 \\
\hline 11 & 6 & 0 & 2.328120 & -2.517773 & -1.079028 \\
\hline 12 & 6 & 0 & 2.928494 & -0.585253 & 2.241211 \\
\hline 13 & 6 & 0 & 2.901927 & 1.756985 & 2.826370 \\
\hline 14 & 6 & 0 & 0.967902 & 3.189288 & 2.702807 \\
\hline 15 & 6 & 0 & -1.226655 & 2.321895 & 1.790663 \\
\hline 16 & 6 & 0 & 3.076728 & -2.878367 & 1.579728 \\
\hline 17 & 6 & 0 & 2.742587 & -3.770049 & -0.626810 \\
\hline 18 & 6 & 0 & 1.871846 & -2.256604 & -2.465589 \\
\hline 19 & 6 & 0 & 2.670569 & -1.479231 & 3.482286 \\
\hline 20 & 6 & 0 & 4.375556 & -0.035215 & 2.232608 \\
\hline 21 & 6 & 0 & 4.339950 & 1.284074 & 3.041333 \\
\hline 22 & 6 & 0 & 2.318669 & 3.017080 & 3.043307 \\
\hline 23 & 1 & 0 & 0.493116 & 4.148393 & 2.887784 \\
\hline 24 & 6 & 0 & -1.678043 & 3.325658 & 0.933193 \\
\hline 25 & 6 & 0 & -2.159212 & 1.414044 & 2.294527 \\
\hline 26 & 6 & 0 & 3.174940 & -2.897214 & 3.100351 \\
\hline 27 & 6 & 0 & 3.115320 & -3.970355 & 0.707502 \\
\hline 28 & 1 & 0 & 2.757250 & -4.607700 & -1.319250 \\
\hline 29 & 6 & 0 & 0.747784 & -2.868887 & -3.014370 \\
\hline 30 & 6 & 0 & 2.533758 & -1.306701 & -3.240118 \\
\hline 31 & 1 & 0 & 3.149276 & -1.087434 & 4.385110 \\
\hline 32 & 1 & 0 & 1.591466 & -1.518902 & 3.664422 \\
\hline 33 & 1 & 0 & 5.100388 & -0.753099 & 2.628779 \\
\hline 34 & 1 & 0 & 4.659662 & 0.191963 & 1.199449 \\
\hline 35 & 1 & 0 & 4.492205 & 1.058491 & 4.104394 \\
\hline 36 & 6 & 0 & 5.419859 & 2.241461 & 2.587521 \\
\hline 37 & 6 & 0 & 3.050179 & 4.173655 & 3.623012 \\
\hline 38 & 6 & 0 & -3.012385 & 3.359941 & 0.529504 \\
\hline 39 & 8 & 0 & -0.768548 & 4.248404 & 0.484953 \\
\hline 40 & 6 & 0 & -3.491188 & 1.428090 & 1.885795 \\
\hline 41 & 8 & 0 & -1.726668 & 0.503923 & 3.230651 \\
\hline 42 & 1 & 0 & 4.211758 & -3.033884 & 3.429503 \\
\hline 43 & 6 & 0 & 2.325431 & -4.014768 & 3.677251 \\
\hline 44 & 6 & 0 & 3.513320 & -5.314014 & 1.203773 \\
\hline 45 & 6 & 0 & 0.291612 & -2.512768 & -4.278689 \\
\hline 46 & 8 & 0 & 0.046144 & -3.795099 & -2.278557 \\
\hline 47 & 6 & 0 & 2.086153 & -0.936335 & -4.511245 \\
\hline 48 & 8 & 0 & 3.662135 & -0.733162 & -2.707760 \\
\hline 49 & 6 & 0 & 5.341262 & 2.817403 & 1.315875 \\
\hline 50 & 6 & 0 & 6.516071 & 2.544540 & 3.395864 \\
\hline 51 & 6 & 0 & 2.862980 & 5.460592 & 3.098975 \\
\hline 52 & 6 & 0 & 3.903039 & 4.016802 & 4.720611 \\
\hline 53 & 6 & 0 & -3.921650 & 2.395414 & 0.974524 \\
\hline 54 & 8 & 0 & -3.483783 & 4.317504 & -0.318430 \\
\hline 55 & 6 & 0 & -1.202616 & 5.079180 & -0.536421 \\
\hline 56 & 8 & 0 & -4.367486 & 0.493496 & 2.377586 \\
\hline 57 & 6 & 0 & -2.460155 & -0.661783 & 3.280544 \\
\hline 58 & 6 & 0 & 0.998422 & -4.163286 & 3.251386 \\
\hline 59 & 6 & 0 & 2.858151 & -4.954333 & 4.559738 \\
\hline
\end{tabular}

\begin{tabular}{|c|c|c|c|c|c|}
\hline 60 & 6 & 0 & 2.618013 & -6.387262 & 1.163902 \\
\hline 61 & 6 & 0 & 4.780413 & -5.501398 & 1.766876 \\
\hline 62 & 6 & 0 & 0.940127 & -1.535708 & -5.039610 \\
\hline 63 & 8 & 0 & -0.848330 & -3.106425 & -4.772283 \\
\hline 64 & 6 & 0 & -1.318361 & -3.727823 & -2.508137 \\
\hline 65 & 8 & 0 & 2.792760 & -0.008079 & -5.229340 \\
\hline 66 & 6 & 0 & 3.938152 & 0.531540 & -3.187291 \\
\hline 67 & 6 & 0 & 6.317714 & 3.706002 & 0.874931 \\
\hline 68 & 1 & 0 & 4.497590 & 2.576642 & 0.669985 \\
\hline 69 & 6 & 0 & 7.506364 & 3.422159 & 2.953073 \\
\hline 70 & 1 & 0 & 6.590715 & 2.102697 & 4.387534 \\
\hline 71 & 6 & 0 & 3.535659 & 6.554291 & 3.638238 \\
\hline 72 & 1 & 0 & 2.190980 & 5.605103 & 2.255619 \\
\hline 73 & 6 & 0 & 4.571730 & 5.109874 & 5.265840 \\
\hline 74 & 1 & 0 & 4.027949 & 3.033256 & 5.165311 \\
\hline 75 & 6 & 0 & -5.314649 & 2.454554 & 0.390426 \\
\hline 76 & 6 & 0 & -2.531911 & 5.089834 & -0.949104 \\
\hline 77 & 6 & 0 & -0.280683 & 5.925760 & -1.131506 \\
\hline 78 & 6 & 0 & -3.783133 & -0.666923 & 2.847724 \\
\hline 79 & 6 & 0 & -1.890083 & -1.808145 & 3.813010 \\
\hline 80 & 6 & 0 & 0.232405 & -5.242041 & 3.684189 \\
\hline 81 & 1 & 0 & 0.576919 & -3.457453 & 2.535262 \\
\hline 82 & 6 & 0 & 2.088611 & -6.030960 & 5.004435 \\
\hline 83 & 1 & 0 & 3.893440 & -4.862984 & 4.880090 \\
\hline 84 & 6 & 0 & 2.976606 & -7.621930 & 1.702475 \\
\hline 85 & 1 & 0 & 1.628553 & -6.240242 & 0.737974 \\
\hline 86 & 6 & 0 & 5.144039 & -6.738746 & 2.294458 \\
\hline 87 & 1 & 0 & 5.483648 & -4.671895 & 1.782440 \\
\hline 88 & 6 & 0 & 0.357742 & -1.226734 & -6.400999 \\
\hline 89 & 6 & 0 & -1.773053 & -3.387391 & -3.779242 \\
\hline 90 & 6 & 0 & -2.213653 & -3.968859 & -1.477386 \\
\hline 91 & 6 & 0 & 3.505975 & 0.893175 & -4.460391 \\
\hline 92 & 6 & 0 & 4.679188 & 1.410342 & -2.412240 \\
\hline 93 & 6 & 0 & 7.405651 & 4.012677 & 1.694422 \\
\hline 94 & 1 & 0 & 6.223885 & 4.165574 & -0.105614 \\
\hline 95 & 1 & 0 & 8.350141 & 3.653465 & 3.595996 \\
\hline 96 & 6 & 0 & 4.398281 & 6.381650 & 4.720566 \\
\hline 97 & 1 & 0 & 3.386024 & 7.541277 & 3.211361 \\
\hline 98 & 1 & 0 & 5.229491 & 4.968111 & 6.118041 \\
\hline 99 & 9 & 0 & -5.931290 & 3.605751 & 0.696985 \\
\hline 100 & 9 & 0 & -5.264935 & 2.370431 & -0.953608 \\
\hline 101 & 9 & 0 & -6.106285 & 1.460093 & 0.806634 \\
\hline 102 & 6 & 0 & -2.940379 & 5.913277 & -1.986430 \\
\hline 103 & 1 & 0 & 0.741104 & 5.927849 & -0.763258 \\
\hline 104 & 6 & 0 & -0.685698 & 6.757405 & -2.175861 \\
\hline 105 & 6 & 0 & -4.548217 & -1.820820 & 2.926897 \\
\hline 106 & 1 & 0 & -0.861362 & -1.774278 & 4.159082 \\
\hline 107 & 6 & 0 & -2.662950 & -2.964720 & 3.921970 \\
\hline 108 & 6 & 0 & 0.775825 & -6.182123 & 4.562888 \\
\hline 109 & 1 & 0 & -0.779026 & -5.367078 & 3.308344 \\
\hline 110 & 1 & 0 & 2.523792 & -6.761824 & 5.679230 \\
\hline 111 & 6 & 0 & 4.237775 & -7.799196 & 2.272895 \\
\hline 112 & 1 & 0 & 2.266888 & -8.443393 & 1.685650 \\
\hline 113 & 1 & 0 & 6.132111 & -6.874694 & 2.723771 \\
\hline 114 & 9 & 0 & 1.051849 & -0.322554 & -7.087117 \\
\hline 115 & 9 & 0 & -0.905008 & -0.769556 & -6.293849 \\
\hline 116 & 9 & 0 & 0.304634 & -2.341429 & -7.153560 \\
\hline 117 & 6 & 0 & -3.129986 & -3.305544 & -4.049956 \\
\hline 118 & 1 & 0 & -1.827533 & -4.193086 & -0.487773 \\
\hline 119 & 6 & 0 & -3.581881 & -3.894591 & -1.743521 \\
\hline 120 & 6 & 0 & 3.816244 & 2.139450 & -4.980939 \\
\hline 121 & 1 & 0 & 5.001864 & 1.095332 & -1.424076 \\
\hline 122 & 6 & 0 & 5.009577 & 2.660488 & -2.936651 \\
\hline 123 & 1 & 0 & 8.167881 & 4.706169 & 1.353253 \\
\hline 124 & 1 & 0 & 4.924342 & 7.232739 & 5.141533 \\
\hline 125 & 1 & 0 & -3.984137 & 5.887428 & -2.283697 \\
\hline
\end{tabular}




\begin{tabular}{|c|c|c|c|c|c|}
\hline 126 & 6 & 0 & -2.009966 & 6.744544 & -2.609545 \\
\hline 127 & 1 & 0 & 0.042057 & 7.410080 & -2.648001 \\
\hline 128 & 1 & 0 & -5.576694 & -1.788510 & 2.582060 \\
\hline 129 & 6 & 0 & -3.983987 & -2.973813 & 3.474707 \\
\hline 130 & 1 & 0 & -2.228314 & -3.851111 & 4.371452 \\
\hline 131 & 1 & 0 & 0.183700 & -7.031592 & 4.889277 \\
\hline 132 & 1 & 0 & 4.514455 & -8.761099 & 2.693497 \\
\hline 133 & 1 & 0 & -3.449820 & -3.034666 & -5.051569 \\
\hline 134 & 6 & 0 & -4.037103 & -3.570953 & -3.022630 \\
\hline 135 & 1 & 0 & -4.291879 & -4.083452 & -0.945036 \\
\hline 136 & 1 & 0 & 3.454893 & 2.397334 & -5.971821 \\
\hline 137 & 6 & 0 & 4.582184 & 3.020741 & -4.214245 \\
\hline 138 & 1 & 0 & 5.593238 & 3.355393 & -2.341494 \\
\hline 139 & 1 & 0 & -2.324652 & 7.383152 & -3.427849 \\
\hline 140 & 1 & 0 & -4.583258 & -3.874122 & 3.560038 \\
\hline 141 & 1 & 0 & -5.102319 & -3.517750 & -3.222780 \\
\hline 142 & 1 & 0 & 4.831305 & 3.997850 & -4.615928 \\
\hline 143 & 1 & 0 & -1.389110 & -1.148327 & -1.372903 \\
\hline 144 & 8 & 0 & -2.441657 & -0.792226 & -2.202880 \\
\hline 145 & 6 & 0 & -3.282113 & 0.254088 & -1.627189 \\
\hline 146 & 6 & 0 & -2.887976 & 1.253431 & -2.747757 \\
\hline 147 & 1 & 0 & -4.322466 & -0.073510 & -1.580036 \\
\hline 148 & 1 & 0 & -2.913099 & 0.543596 & -0.637111 \\
\hline 149 & 8 & 0 & -2.239282 & 2.407599 & -2.298973 \\
\hline 150 & 6 & 0 & -1.978153 & 0.102633 & -3.270713 \\
\hline 151 & 1 & 0 & -1.489237 & 2.130024 & -1.738158 \\
\hline 152 & 1 & 0 & -0.902706 & 0.287388 & -3.195650 \\
\hline 153 & 1 & 0 & -2.228103 & -0.311518 & -4.249747 \\
\hline 154 & 1 & 0 & 3.467341 & 4.595984 & 0.619402 \\
\hline 155 & 6 & 0 & 3.100058 & 4.657353 & -0.400533 \\
\hline 156 & 6 & 0 & 2.304862 & 3.631945 & -0.907653 \\
\hline 157 & 6 & 0 & 3.436802 & 5.771082 & -1.182720 \\
\hline 158 & 6 & 0 & 1.852091 & 3.754427 & -2.220819 \\
\hline 159 & 1 & 0 & 2.029449 & 2.764599 & -0.308779 \\
\hline 160 & 6 & 0 & 2.971067 & 5.890006 & -2.493759 \\
\hline 161 & 1 & 0 & 4.061559 & 6.552536 & -0.760924 \\
\hline 162 & 7 & 0 & 1.068011 & 2.846086 & -2.911800 \\
\hline 163 & 6 & 0 & 2.168875 & 4.873169 & -3.004940 \\
\hline 164 & 1 & 0 & 3.224892 & 6.755407 & -3.098608 \\
\hline 165 & 6 & 0 & 0.737422 & 3.150872 & -4.192741 \\
\hline 166 & 1 & 0 & 0.696228 & 2.012056 & -2.452202 \\
\hline 167 & 16 & 0 & 1.426778 & 4.733004 & -4.584785 \\
\hline 168 & 16 & 0 & -0.127994 & 2.217996 & -5.242330 \\
\hline 169 & 6 & 0 & -3.997116 & 1.637530 & -3.694071 \\
\hline 170 & 6 & 0 & -4.150251 & 2.960705 & -4.104693 \\
\hline 171 & 6 & 0 & -4.866139 & 0.656705 & -4.182251 \\
\hline 172 & 6 & 0 & -5.176963 & 3.300721 & -4.985925 \\
\hline 173 & 1 & 0 & -3.455522 & 3.708064 & -3.735144 \\
\hline 174 & 6 & 0 & -5.890307 & 0.997573 & -5.061516 \\
\hline 175 & 1 & 0 & -4.740702 & -0.382668 & -3.877184 \\
\hline 176 & 6 & 0 & -6.050451 & 2.324842 & -5.462841 \\
\hline 177 & 1 & 0 & -5.291511 & 4.332308 & -5.305483 \\
\hline 178 & 1 & 0 & -6.562842 & 0.231058 & -5.434338 \\
\hline 179 & 1 & 0 & -6.849225 & 2.593588 & -6.147250 \\
\hline
\end{tabular}

$\begin{array}{cccc}\text { Frequencies -- } & 11.4603 & 14.1677 & 20.7109 \\ \text { Frequencies -- } & 22.2228 & 23.7521 & 26.4235 \\ \text { Frequencies -- } & 29.6828 & 32.3005 & 33.3021 \\ \text { Frequencies -- } & 33.9932 & 34.4809 & 38.7470 \\ \text { Frequencies -- } & 40.4525 & 41.4294 & 43.9544 \\ \text { Frequencies -- } & 45.6289 & 48.8468 & 53.6625 \\ \text { Frequencies -- } & 55.3574 & 56.7206 & 58.0755 \\ \text { Frequencies -- } & 61.0398 & 61.7049 & 65.0268 \\ \text { Frequencies -- } & 66.8568 & 68.1225 & 73.6565 \\ \text { Frequencies -- } & 75.5159 & 77.4056 & 78.5194\end{array}$

\begin{tabular}{|c|c|c|c|}
\hline Frequencies -- & 82.7116 & 84.0719 & 88.1624 \\
\hline Frequencies -- & 91.1401 & 93.6033 & 98.2724 \\
\hline Frequencies -- & 103.5222 & 104.4990 & 106.2233 \\
\hline Frequencies -- & 109.0384 & 111.9642 & 116.7571 \\
\hline Frequencies -- & 118.9373 & 124.9710 & 126.1757 \\
\hline Frequencies -- & 127.0431 & 129.4082 & 131.1878 \\
\hline Frequencies -- & 137.7968 & 144.2597 & 144.7308 \\
\hline Frequencies -- & 147.3497 & 151.9952 & 154.8852 \\
\hline Frequencies -- & 166.0615 & 172.7669 & 177.2434 \\
\hline Frequencies -- & 181.8517 & 188.2311 & 192.7668 \\
\hline Frequencies -- & 195.9698 & 201.5120 & 207.6472 \\
\hline Frequencies -- & 213.5600 & 222.5552 & 224.5380 \\
\hline Frequencies -- & 230.9186 & 233.2361 & 237.6871 \\
\hline Frequencies -- & 244.5970 & 246.0123 & 248.5664 \\
\hline Frequencies -- & 251.3776 & 264.0551 & 269.0864 \\
\hline Frequencies -- & 271.6450 & 273.6263 & 278.0599 \\
\hline Frequencies -- & 281.1321 & 286.3964 & 289.9845 \\
\hline Frequencies -- & 295.7989 & 297.8423 & 298.8428 \\
\hline Frequencies -- & 301.1013 & 304.0234 & 305.7531 \\
\hline Frequencies -- & 309.1151 & 312.5313 & 316.5532 \\
\hline Frequencies -- & 319.4440 & 323.7337 & 324.8600 \\
\hline Frequencies -- & 331.9898 & 333.4998 & 335.8664 \\
\hline Frequencies -- & 338.1709 & 344.8225 & 359.4046 \\
\hline Frequencies -- & 368.5948 & 379.3210 & 386.0761 \\
\hline Frequencies -- & 402.6220 & 404.5269 & 408.3785 \\
\hline Frequencies -- & 409.6839 & 410.8549 & 414.5196 \\
\hline Frequencies -- & 415.0078 & 419.3148 & 420.5509 \\
\hline Frequencies -- & 420.9095 & 426.1002 & 428.1752 \\
\hline Frequencies -- & 432.9159 & 440.6678 & 441.2476 \\
\hline Frequencies -- & 444.5407 & 446.0611 & 449.6416 \\
\hline Frequencies -- & 451.4791 & 455.4276 & 459.5471 \\
\hline Frequencies -- & 467.8086 & 470.3936 & 474.7155 \\
\hline Frequencies -- & 485.8163 & 489.4662 & 502.2173 \\
\hline Frequencies -- & 506.2026 & 507.5398 & 511.9564 \\
\hline Frequencies -- & 516.6863 & 520.2577 & 521.1532 \\
\hline Frequencies -- & 524.0535 & 529.6250 & 531.5686 \\
\hline Frequencies -- & 535.6321 & 540.2032 & 540.6454 \\
\hline Frequencies -- & 547.0885 & 550.8613 & 555.0038 \\
\hline Frequencies -- & 556.3865 & 558.6393 & 559.2022 \\
\hline Frequencies -- & 561.0123 & 564.6384 & 569.2978 \\
\hline Frequencies -- & 571.0098 & 583.5942 & 584.3958 \\
\hline Frequencies -- & 588.4576 & 593.4685 & 597.2447 \\
\hline Frequencies -- & 599.7767 & 602.7477 & 603.2534 \\
\hline Frequencies -- & 604.5318 & 604.7982 & 611.3075 \\
\hline Frequencies -- & 612.7363 & 619.0510 & 621.1051 \\
\hline Frequencies -- & 622.3730 & 625.4138 & 626.8697 \\
\hline Frequencies -- & 629.7922 & 630.4840 & 631.0660 \\
\hline Frequencies -- & 633.3393 & 635.4349 & 645.6690 \\
\hline Frequencies -- & 649.3088 & 656.1811 & 657.7626 \\
\hline Frequencies -- & 665.7509 & 673.4503 & 675.2688 \\
\hline Frequencies -- & 681.3122 & 683.3972 & 701.3493 \\
\hline Frequencies -- & 704.5649 & 708.0145 & 711.5718 \\
\hline Frequencies -- & 717.5217 & 717.8107 & 719.6407 \\
\hline Frequencies -- & 719.7782 & 721.1895 & 721.2464 \\
\hline Frequencies -- & 722.1151 & 723.9332 & 727.1912 \\
\hline Frequencies -- & 727.9068 & 732.5318 & 733.4289 \\
\hline Frequencies -- & 737.6718 & 740.1279 & 741.7542 \\
\hline Frequencies -- & 744.0829 & 747.4161 & 752.6918 \\
\hline Frequencies -- & 760.8397 & 762.6652 & 771.0243 \\
\hline Frequencies -- & 771.1670 & 772.8805 & 773.1655 \\
\hline Frequencies -- & 775.1029 & 776.3607 & 779.9493 \\
\hline Frequencies -- & 783.4416 & 783.5460 & 784.9913 \\
\hline Frequencies -- & 791.1160 & 796.1403 & 802.0103 \\
\hline Frequencies -- & 804.9743 & 805.8985 & 819.6422 \\
\hline Frequencies -- & 820.5358 & 825.2619 & 827.8208 \\
\hline Frequencies -- & 829.8068 & 866.7256 & 867.2710 \\
\hline
\end{tabular}




\begin{tabular}{|c|c|c|c|}
\hline Frequencies -- & 868.4121 & 868.8866 & 870.3532 \\
\hline Frequencies -- & 872.5442 & 874.8434 & 875.6665 \\
\hline Frequencies -- & 879.1985 & 880.8223 & 883.6605 \\
\hline Frequencies -- & 886.6776 & 887.3568 & 889.4925 \\
\hline Frequencies -- & 890.0080 & 891.0597 & 909.7850 \\
\hline Frequencies -- & 913.4587 & 914.0498 & 920.5922 \\
\hline Frequencies -- & 922.1122 & 925.8503 & 930.4163 \\
\hline Frequencies -- & 936.6271 & 939.0516 & 939.8496 \\
\hline Frequencies -- & 941.4966 & 942.1276 & 945.9833 \\
\hline Frequencies -- & 947.2016 & 948.7446 & 951.7383 \\
\hline Frequencies -- & 954.2371 & 954.5643 & 955.1893 \\
\hline Frequencies -- & 960.5977 & 964.4194 & 970.1424 \\
\hline Frequencies -- & 980.2166 & 988.3822 & 989.8655 \\
\hline Frequencies -- & 992.5236 & 993.5626 & 994.8009 \\
\hline Frequencies -- & 997.6630 & 1002.5432 & 1002.7409 \\
\hline Frequencies -- & 1005.2151 & 1006.5050 & 1009.8475 \\
\hline Frequencies -- & 1012.0562 & 1013.2890 & 1016.9793 \\
\hline Frequencies -- & 1018.1020 & 1018.4889 & 1018.9815 \\
\hline Frequencies -- & 1019.0997 & 1019.6186 & 1020.6795 \\
\hline Frequencies -- & 1022.5698 & 1027.7103 & 1029.7070 \\
\hline Frequencies -- & 1034.0384 & 1036.3204 & 1040.3212 \\
\hline Frequencies -- & 1044.4818 & 1051.0312 & 1051.9384 \\
\hline Frequencies -- & 1055.9414 & 1058.2317 & 1059.3494 \\
\hline Frequencies -- & 1060.1606 & 1066.3177 & 1068.3403 \\
\hline Frequencies -- & 1069.5418 & 1070.8512 & 1071.6771 \\
\hline Frequencies -- & 1072.4549 & 1073.8876 & 1080.9508 \\
\hline Frequencies -- & 1085.9293 & 1094.4352 & 1100.3799 \\
\hline Frequencies -- & 1109.1974 & 1112.7222 & 1114.1949 \\
\hline Frequencies -- & 1114.8558 & 1117.5417 & 1120.4188 \\
\hline Frequencies -- & 1124.2452 & 1125.4542 & 1127.2888 \\
\hline Frequencies -- & 1129.7048 & 1132.6477 & 1157.3180 \\
\hline Frequencies -- & 1162.1010 & 1163.7626 & 1167.3439 \\
\hline Frequencies -- & 1168.3135 & 1169.9112 & 1171.4693 \\
\hline Frequencies -- & 1171.8230 & 1173.2772 & 1174.4150 \\
\hline Frequencies -- & 1175.6271 & 1175.7757 & 1177.3329 \\
\hline Frequencies -- & 1178.4105 & 1182.3058 & 1189.8388 \\
\hline Frequencies -- & 1198.0656 & 1201.5684 & 1204.8002 \\
\hline Frequencies -- & 1207.0280 & 1207.3469 & 1208.5467 \\
\hline Frequencies -- & 1208.8254 & 1209.8404 & 1210.6681 \\
\hline Frequencies -- & 1213.0768 & 1221.6218 & 1223.4953 \\
\hline Frequencies -- & 1225.5658 & 1227.5171 & 1229.8426 \\
\hline Frequencies -- & 1230.9504 & 1233.3285 & 1236.3619 \\
\hline Frequencies -- & 1240.9963 & 1245.2848 & 1251.3323 \\
\hline Frequencies -- & 1257.1050 & 1257.5487 & 1262.8796 \\
\hline Frequencies -- & 1268.3852 & 1278.2172 & 1279.1906 \\
\hline Frequencies -- & 1282.5382 & 1285.1628 & 1287.9242 \\
\hline Frequencies -- & 1294.1624 & 1294.5768 & 1297.6934 \\
\hline Frequencies -- & 1298.1432 & 1300.5568 & 1302.6414 \\
\hline Frequencies -- & 1302.7764 & 1304.6607 & 1306.3054 \\
\hline Frequencies -- & 1309.3473 & 1309.7561 & 1311.0599 \\
\hline Frequencies -- & 1314.9231 & 1323.4390 & 1324.7010 \\
\hline Frequencies -- & 1326.8099 & 1329.4769 & 1331.1501 \\
\hline Frequencies -- & 1332.4589 & 1334.2332 & 1339.1964 \\
\hline Frequencies -- & 1340.4705 & 1344.3054 & 1344.8310 \\
\hline Frequencies -- & 1356.5643 & 1358.7676 & 1360.5133 \\
\hline Frequencies -- & 1361.0699 & 1362.5333 & 1363.4408 \\
\hline Frequencies -- & 1365.5921 & 1366.2886 & 1366.7406 \\
\hline Frequencies -- & 1371.1873 & 1371.9410 & 1374.9923 \\
\hline Frequencies -- & 1378.0677 & 1381.0631 & 1381.3719 \\
\hline Frequencies -- & 1393.7755 & 1408.2744 & 1446.1556 \\
\hline Frequencies -- & 1457.9505 & 1461.6298 & 1464.6102 \\
\hline Frequencies -- & 1470.3236 & 1471.2682 & 1491.9135 \\
\hline Frequencies -- & 1495.3799 & 1496.2912 & 1496.7535 \\
\hline Frequencies -- & 1498.8024 & 1499.8752 & 1503.4661 \\
\hline Frequencies -- & 1504.3698 & 1504.7190 & 1506.6935 \\
\hline Frequencies -- & 1509.9027 & 1510.9447 & 1517.1492 \\
\hline
\end{tabular}

Frequencies -- 1517.9036 Frequencies -- 1526.4357 Frequencies -- 1543.3113 Frequencies -- 1554.6662 Frequencies -- 1559.0862 Frequencies -- 1571.6980 Frequencies -- 1665.3326 Frequencies -- 1668.0533 Frequencies -- 1674.8919 Frequencies -- 1682.4064 Frequencies -- 1696.2440 Frequencies -- 1699.2985 Frequencies -- 1702.5687 Frequencies -- 1705.4110 Frequencies -- 1722.5862 Frequencies -- 2026.7079 Frequencies -- 3085.2355 Frequencies -- 3130.0328 Frequencies -- 3166.7806 Frequencies -- 3195.8879 Frequencies -- 3200.3250 Frequencies -- 3210.6866 Frequencies -- 3213.7189 Frequencies -- 3217.5682 Frequencies -- 3221.0845 Frequencies -- 3222.9721 Frequencies -- 3227.1110 Frequencies -- 3231.7538 Frequencies -- 3233.0613 Frequencies -- 3236.7832 Frequencies -- 3238.4133 Frequencies -- 3241.5996 Frequencies -- 3243.4967 Frequencies -- 3245.7849 Frequencies -- 3250.3553

$\begin{array}{ll}1520.3499 & 1522.2939 \\ 1532.7563 & 1536.9366 \\ 1545.7890 & 1552.1682 \\ 1555.9413 & 1558.1653 \\ 1559.7328 & 1566.3863 \\ 1576.2811 & 1652.8574 \\ 1667.5184 & 1667.6066 \\ 1671.8385 & 1674.1204 \\ 1677.1553 & 1679.5854 \\ 1688.8080 & 1690.5533 \\ 1696.5661 & 1698.9018 \\ 1699.9540 & 1700.6972 \\ 1702.6609 & 1703.1622 \\ 1708.8866 & 1721.6180 \\ 1732.1861 & 1734.9530 \\ 3068.3208 & 3082.2497 \\ 3090.4511 & 3104.3729 \\ 3146.5841 & 3156.5508 \\ 3189.4020 & 3191.9924 \\ 3198.7252 & 3199.5415 \\ 3200.8348 & 3209.0071 \\ 3211.6614 & 3212.1418 \\ 3213.8306 & 3216.2597 \\ 3217.9775 & 3219.0441 \\ 3221.8871 & 3222.0312 \\ 3223.8740 & 3225.8298 \\ 3228.9319 & 3230.0541 \\ 3232.6443 & 3232.7910 \\ 3234.1365 & 3234.5693 \\ 3236.8359 & 3237.5133 \\ 3241.0637 & 3241.5955 \\ 3241.6125 & 3243.4495 \\ 3243.8529 & 3244.6170 \\ 3248.7428 & 3250.2793 \\ 3479.2680 & 3686.7433 \\ & \end{array}$

SCF Done: $E(R M 062 X / D G D Z V P)=-6499.39950806$ Sum of electronic and zero-point Energies $=\quad-6498.049855$ Sum of electronic and thermal Energies $=\quad-6497.935546$ Sum of electronic and thermal Free Energies $=\quad-6498.208075$ SCF Done: $E(R M 062 X / D G T Z V P / S M D)=-6500.68639466$

\section{Cat $\mathrm{f}$ TS1a_R}

Center Atomic Atomic Coordinates (Angstroms) Number Number Type $\quad X \quad Y \quad$ Z

$\begin{array}{cccccc}1 & 15 & 0 & -0.026717 & 0.008552 & 0.003297 \\ 2 & 8 & 0 & -0.013331 & 0.020778 & 1.626204 \\ 3 & 8 & 0 & 1.568125 & 0.011530 & -0.349501 \\ 4 & 8 & 0 & -0.498933 & 1.330399 & -0.539897 \\ 5 & 8 & 0 & -0.702605 & -1.257077 & -0.421269 \\ 6 & 6 & 0 & 0.721910 & 1.043110 & 2.212010 \\ 7 & 6 & 0 & 2.215095 & -1.177756 & -0.043589 \\ 8 & 6 & 0 & 2.050483 & 0.803841 & 2.530196 \\ 9 & 6 & 0 & 0.146163 & 2.305682 & 2.408317 \\ 10 & 6 & 0 & 2.654482 & -1.421238 & 1.246049 \\ 11 & 6 & 0 & 2.258494 & -2.175438 & -1.017977 \\ 12 & 6 & 0 & 2.825874 & -0.491472 & 2.429026 \\ 13 & 6 & 0 & 2.836706 & 1.840391 & 3.034825 \\ 14 & 6 & 0 & 0.945981 & 3.324290 & 2.933319 \\ 15 & 6 & 0 & -1.274869 & 2.505047 & 2.037166 \\ 16 & 6 & 0 & 3.029309 & -2.716092 & 1.599331 \\ 17 & 6 & 0 & 2.733995 & -3.440706 & -0.669838 \\ 18 & 6 & 0 & 1.742432 & -1.861258 & -2.370397\end{array}$




\begin{tabular}{|c|c|c|c|c|c|}
\hline 19 & 6 & 0 & 2.555084 & -1.472193 & 3.596165 \\
\hline 20 & 6 & 0 & 4.290108 & 0.026599 & 2.465947 \\
\hline 21 & 6 & 0 & 4.276033 & 1.374737 & 3.226788 \\
\hline 22 & 6 & 0 & 2.289124 & 3.110013 & 3.262101 \\
\hline 23 & 1 & 0 & 0.507355 & 4.303952 & 3.102939 \\
\hline 24 & 6 & 0 & -1.685917 & 3.511179 & 1.160306 \\
\hline 25 & 6 & 0 & -2.238167 & 1.605012 & 2.489767 \\
\hline 26 & 6 & 0 & 3.104290 & -2.845566 & 3.117706 \\
\hline 27 & 6 & 0 & 3.109989 & -3.735036 & 0.645395 \\
\hline 28 & 1 & 0 & 2.779378 & -4.221201 & -1.425174 \\
\hline 29 & 6 & 0 & 0.697696 & -2.592926 & -2.927248 \\
\hline 30 & 6 & 0 & 2.264916 & -0.797486 & -3.109870 \\
\hline 31 & 1 & 0 & 3.008000 & -1.138024 & 4.534996 \\
\hline 32 & 1 & 0 & 1.473580 & -1.549663 & 3.748095 \\
\hline 33 & 1 & 0 & 4.973903 & -0.700027 & 2.915184 \\
\hline 34 & 1 & 0 & 4.626190 & 0.196345 & 1.439167 \\
\hline 35 & 1 & 0 & 4.462272 & 1.205820 & 4.294734 \\
\hline 36 & 6 & 0 & 5.321308 & 2.339566 & 2.702507 \\
\hline 37 & 6 & 0 & 3.093970 & 4.204088 & 3.868927 \\
\hline 38 & 6 & 0 & -2.996755 & 3.550620 & 0.693405 \\
\hline 39 & 8 & 0 & -0.757416 & 4.427186 & 0.724995 \\
\hline 40 & 6 & 0 & -3.539473 & 1.600934 & 1.986848 \\
\hline 41 & 8 & 0 & -1.877686 & 0.688713 & 3.450913 \\
\hline 42 & 1 & 0 & 4.137923 & -2.980054 & 3.457361 \\
\hline 43 & 6 & 0 & 2.278882 & -4.026712 & 3.592465 \\
\hline 44 & 6 & 0 & 3.543039 & -5.101333 & 1.038691 \\
\hline 45 & 6 & 0 & 0.214033 & -2.291097 & -4.193767 \\
\hline 46 & 8 & 0 & 0.114282 & -3.606760 & -2.206811 \\
\hline 47 & 6 & 0 & 1.792139 & -0.486014 & -4.390283 \\
\hline 48 & 8 & 0 & 3.272715 & -0.080455 & -2.515435 \\
\hline 49 & 6 & 0 & 5.326214 & 2.688394 & 1.348022 \\
\hline 50 & 6 & 0 & 6.287787 & 2.899973 & 3.539118 \\
\hline 51 & 6 & 0 & 3.311139 & 5.404577 & 3.185682 \\
\hline 52 & 6 & 0 & 3.643395 & 4.041018 & 5.145502 \\
\hline 53 & 6 & 0 & -3.924956 & 2.570324 & 1.059353 \\
\hline 54 & 8 & 0 & -3.411912 & 4.531085 & -0.166747 \\
\hline 55 & 6 & 0 & -1.087143 & 5.087958 & -0.447875 \\
\hline 56 & 8 & 0 & -4.418404 & 0.632843 & 2.405706 \\
\hline 57 & 6 & 0 & -2.533369 & -0.519621 & 3.341610 \\
\hline 58 & 6 & 0 & 0.958216 & -4.175273 & 3.146026 \\
\hline 59 & 6 & 0 & 2.833714 & -5.020964 & 4.397796 \\
\hline 60 & 6 & 0 & 2.677602 & -6.191759 & 0.906036 \\
\hline 61 & 6 & 0 & 4.809174 & -5.299129 & 1.600624 \\
\hline 62 & 6 & 0 & 0.752999 & -1.242970 & -4.944649 \\
\hline 63 & 8 & 0 & -0.832454 & -3.023747 & -4.705035 \\
\hline 64 & 6 & 0 & -1.244795 & -3.708977 & -2.447474 \\
\hline 65 & 8 & 0 & 2.332532 & 0.545145 & -5.114614 \\
\hline 66 & 6 & 0 & 3.852389 & 0.913010 & -3.266917 \\
\hline 67 & 6 & 0 & 6.264106 & 3.585383 & 0.842495 \\
\hline 68 & 1 & 0 & 4.576038 & 2.263715 & 0.681945 \\
\hline 69 & 6 & 0 & 7.230025 & 3.799765 & 3.039720 \\
\hline 70 & 1 & 0 & 6.294954 & 2.643312 & 4.595823 \\
\hline 71 & 6 & 0 & 4.096562 & 6.408153 & 3.751679 \\
\hline 72 & 1 & 0 & 2.865653 & 5.547735 & 2.203898 \\
\hline 73 & 6 & 0 & 4.416494 & 5.048233 & 5.718686 \\
\hline 74 & 1 & 0 & 3.452879 & 3.120788 & 5.693057 \\
\hline 75 & 6 & 0 & -5.281232 & 2.621372 & 0.394421 \\
\hline 76 & 6 & 0 & -2.405264 & 5.132723 & -0.893473 \\
\hline 77 & 6 & 0 & -0.088388 & 5.736613 & -1.159970 \\
\hline 78 & 6 & 0 & -3.817548 & -0.547503 & 2.807371 \\
\hline 79 & 6 & 0 & -1.933354 & -1.683947 & 3.794745 \\
\hline 80 & 6 & 0 & 0.221587 & -5.306995 & 3.485825 \\
\hline 81 & 1 & 0 & 0.519038 & -3.423813 & 2.488532 \\
\hline 82 & 6 & 0 & 2.094245 & -6.152679 & 4.746412 \\
\hline 83 & 1 & 0 & 3.864637 & -4.926675 & 4.731183 \\
\hline 84 & 6 & 0 & 3.064092 & -7.454891 & 1.351696 \\
\hline
\end{tabular}

\begin{tabular}{|c|c|c|c|c|c|}
\hline 85 & 1 & 0 & 1.687708 & -6.036559 & 0.483611 \\
\hline 86 & 6 & 0 & 5.200876 & -6.563720 & 2.035689 \\
\hline 87 & 1 & 0 & 5.488854 & -4.454782 & 1.690425 \\
\hline 88 & 6 & 0 & 0.187363 & -1.047982 & -6.334322 \\
\hline 89 & 6 & 0 & -1.725344 & -3.430866 & -3.724098 \\
\hline 90 & 6 & 0 & -2.112668 & -4.064492 & -1.426734 \\
\hline 91 & 6 & 0 & 3.406418 & 1.204411 & -4.551067 \\
\hline 92 & 6 & 0 & 4.904997 & 1.627895 & -2.709435 \\
\hline 93 & 6 & 0 & 7.220585 & 4.147436 & 1.689523 \\
\hline 94 & 1 & 0 & 6.239758 & 3.850466 & -0.212234 \\
\hline 95 & 1 & 0 & 7.966397 & 4.233945 & 3.709146 \\
\hline 96 & 6 & 0 & 4.656193 & 6.230147 & 5.017093 \\
\hline 97 & 1 & 0 & 4.272222 & 7.329184 & 3.204353 \\
\hline 98 & 1 & 0 & 4.832901 & 4.911177 & 6.712214 \\
\hline 99 & 9 & 0 & -5.909787 & 3.780971 & 0.639737 \\
\hline 100 & 9 & 0 & -5.161832 & 2.507184 & -0.943990 \\
\hline 101 & 9 & 0 & -6.099788 & 1.639496 & 0.788455 \\
\hline 102 & 6 & 0 & -2.735262 & 5.804159 & -2.060393 \\
\hline 103 & 1 & 0 & 0.929244 & 5.711379 & -0.781432 \\
\hline 104 & 6 & 0 & -0.413806 & 6.411459 & -2.337235 \\
\hline 105 & 6 & 0 & -4.517882 & -1.739672 & 2.705085 \\
\hline 106 & 1 & 0 & -0.931733 & -1.634074 & 4.210407 \\
\hline 107 & 6 & 0 & -2.643005 & -2.883238 & 3.723487 \\
\hline 108 & 6 & 0 & 0.787560 & -6.301804 & 4.286556 \\
\hline 109 & 1 & 0 & -0.786709 & -5.427639 & 3.099568 \\
\hline 110 & 1 & 0 & 2.548258 & -6.925038 & 5.359892 \\
\hline 111 & 6 & 0 & 4.324153 & -7.643003 & 1.921246 \\
\hline 112 & 1 & 0 & 2.375791 & -8.290071 & 1.264720 \\
\hline 113 & 1 & 0 & 6.187617 & -6.706163 & 2.466145 \\
\hline 114 & 9 & 0 & 0.729982 & -0.033599 & -7.003656 \\
\hline 115 & 9 & 0 & -1.145158 & -0.833805 & -6.295636 \\
\hline 116 & 9 & 0 & 0.375245 & -2.156589 & -7.073646 \\
\hline 117 & 6 & 0 & -3.076453 & -3.533591 & -4.013421 \\
\hline 118 & 1 & 0 & -1.710704 & -4.219871 & -0.430369 \\
\hline 119 & 6 & 0 & -3.474312 & -4.180489 & -1.712795 \\
\hline 120 & 6 & 0 & 4.035105 & 2.187351 & -5.304427 \\
\hline 121 & 1 & 0 & 5.223380 & 1.377815 & -1.701586 \\
\hline 122 & 6 & 0 & 5.523416 & 2.631119 & -3.452855 \\
\hline 123 & 1 & 0 & 7.951209 & 4.849968 & 1.300648 \\
\hline 124 & 1 & 0 & 5.266956 & 7.011968 & 5.457795 \\
\hline 125 & 1 & 0 & -3.775334 & 5.819367 & -2.370753 \\
\hline 126 & 6 & 0 & -1.732320 & 6.442394 & -2.789367 \\
\hline 127 & 1 & 0 & 0.371378 & 6.913000 & -2.894606 \\
\hline 128 & 1 & 0 & -5.517384 & -1.725340 & 2.282490 \\
\hline 129 & 6 & 0 & -3.926240 & -2.912623 & 3.176958 \\
\hline 130 & 1 & 0 & -2.187738 & -3.790508 & 4.106455 \\
\hline 131 & 1 & 0 & 0.217127 & -7.190095 & 4.540084 \\
\hline 132 & 1 & 0 & 4.622638 & -8.626783 & 2.270194 \\
\hline 133 & 1 & 0 & -3.417566 & -3.308360 & -5.019245 \\
\hline 134 & 6 & 0 & -3.952059 & -3.923700 & -2.998460 \\
\hline 135 & 1 & 0 & -4.162691 & -4.461812 & -0.922833 \\
\hline 136 & 1 & 0 & 3.668173 & 2.379604 & -6.307685 \\
\hline 137 & 6 & 0 & 5.093664 & 2.906131 & -4.752023 \\
\hline 138 & 1 & 0 & 6.344550 & 3.190962 & -3.017296 \\
\hline 139 & 1 & 0 & -1.984812 & 6.965399 & -3.705556 \\
\hline 140 & 1 & 0 & -4.474453 & -3.847371 & 3.123065 \\
\hline 141 & 1 & 0 & -5.011758 & -4.014965 & -3.212575 \\
\hline 142 & 1 & 0 & 5.581200 & 3.678192 & -5.337983 \\
\hline 143 & 1 & 0 & -1.949055 & -1.232294 & -1.409053 \\
\hline 144 & 8 & 0 & -2.649407 & -0.951295 & -2.086062 \\
\hline 145 & 6 & 0 & -3.382280 & 0.190177 & -1.572328 \\
\hline 146 & 6 & 0 & -2.921536 & 1.214986 & -2.627636 \\
\hline 147 & 1 & 0 & -4.449817 & -0.031171 & -1.559574 \\
\hline 148 & 1 & 0 & -3.029008 & 0.452072 & -0.569900 \\
\hline 149 & 8 & 0 & -2.412076 & 2.396933 & -2.090939 \\
\hline 150 & 6 & 0 & -1.851117 & 0.250806 & -3.227665 \\
\hline
\end{tabular}




$\begin{array}{llllll}151 & 1 & 0 & -1.807573 & 2.148033 & -1.360271 \\ 152 & 1 & 0 & -0.855708 & 0.160829 & -2.801043 \\ 153 & 1 & 0 & -2.045422 & -0.310856 & -4.136008 \\ 154 & 1 & 0 & 3.581823 & 4.334712 & 0.395963 \\ 155 & 6 & 0 & 3.186971 & 4.280119 & -0.613396 \\ 156 & 6 & 0 & 2.244748 & 3.303830 & -0.923331 \\ 157 & 6 & 0 & 3.626276 & 5.203292 & -1.573777 \\ 158 & 6 & 0 & 1.735615 & 3.297790 & -2.222386 \\ 159 & 1 & 0 & 1.885564 & 2.590519 & -0.183774 \\ 160 & 6 & 0 & 3.118543 & 5.182575 & -2.871616 \\ 161 & 1 & 0 & 4.365951 & 5.949632 & -1.300094 \\ 162 & 7 & 0 & 0.741688 & 2.456689 & -2.712092 \\ 163 & 6 & 0 & 2.156590 & 4.223840 & -3.183506 \\ 164 & 1 & 0 & 3.452618 & 5.900214 & -3.614660 \\ 165 & 6 & 0 & 0.314489 & 2.699743 & -3.966007 \\ 166 & 1 & 0 & 0.231224 & 1.854836 & -2.044353 \\ 167 & 16 & 0 & 1.246878 & 4.011040 & -4.663564 \\ 168 & 16 & 0 & -0.905594 & 1.928025 & -4.809847 \\ 169 & 6 & 0 & -3.979764 & 1.537684 & -3.660951 \\ 170 & 6 & 0 & -4.232145 & 2.857529 & -4.027987 \\ 171 & 6 & 0 & -4.697578 & 0.499334 & -4.262252 \\ 172 & 6 & 0 & -5.204063 & 3.137644 & -4.987868 \\ 173 & 1 & 0 & -3.656794 & 3.648655 & -3.558466 \\ 174 & 6 & 0 & -5.664739 & 0.780949 & -5.224182 \\ 175 & 1 & 0 & -4.502544 & -0.535773 & -3.978917 \\ 176 & 6 & 0 & -5.921536 & 2.103565 & -5.587574 \\ 177 & 1 & 0 & -5.399082 & 4.167527 & -5.271644 \\ 178 & 1 & 0 & -6.217532 & -0.029526 & -5.689143 \\ 179 & 1 & 0 & -6.676369 & 2.324386 & -6.335978\end{array}$

Frequencies -- -543.8153

Frequencies -- 18.2324

Frequencies -- 26.2233

Frequencies -- 34.3215

Frequencies -- 39.9412

Frequencies -- 43.7443

Frequencies -- 50.2729

Frequencies -- 59.8156

Frequencies -- 66.4591

Frequencies -- 75.0899

Frequencies -- 83.9804

Frequencies -- 92.4877

Frequencies -- 98.2597

Frequencies -- 109.3033

Frequencies -- 119.0002

Frequencies -- 127.0014

Frequencies -- 136.7112

Frequencies -- 150.8336

Frequencies -- 167.4185

Frequencies -- 187.5222

Frequencies -- 195.7078

Frequencies -- 216.5480

Frequencies -- 227.9613

Frequencies -- 232.7892

Frequencies -- 252.6916

Frequencies -- 271.6604

Frequencies -- 278.2295

Frequencies -- 291.9502

Frequencies -- 299.6578

Frequencies -- 306.9544

Frequencies -- 315.6957

Frequencies -- 321.4841

Frequencies -- 338.7599

Frequencies -- 362.2807

Frequencies -- 399.5360

\begin{tabular}{cc}
10.0599 & 15.9684 \\
20.3338 & 24.5509 \\
28.4622 & 31.3556 \\
35.9874 & 37.7787 \\
40.5073 & 41.4971 \\
45.5274 & 47.2644 \\
52.3670 & 58.5803 \\
63.2984 & 64.5206 \\
68.6765 & 74.7620 \\
78.9614 & 81.1879 \\
85.6960 & 91.3872 \\
93.4292 & 96.4668 \\
101.7933 & 106.5896 \\
112.1100 & 113.8695 \\
119.6186 & 122.2850 \\
131.5961 & 134.4501 \\
139.8112 & 146.7820 \\
155.5844 & 163.1330 \\
170.9407 & 174.9303 \\
188.9673 & 192.9908 \\
203.9153 & 209.8729 \\
218.8343 & 226.1028 \\
229.6503 & 232.1187 \\
244.4593 & 249.3967 \\
261.2427 & 269.7950 \\
274.1688 & 275.2418 \\
284.6152 & 289.4405 \\
292.4395 & 296.8329 \\
302.0215 & 302.6551 \\
308.6280 & 309.6916 \\
317.5247 & 320.6299 \\
331.6523 & 334.0125 \\
342.6018 & 351.6656 \\
377.8670 & 385.1959 \\
407.1587 & 409.5930 \\
& \\
\hline
\end{tabular}

\begin{tabular}{|c|c|c|c|}
\hline Frequencies -- & 410.9346 & 412.1076 & 413.1918 \\
\hline Frequencies -- & 415.8853 & 417.2507 & 419.9970 \\
\hline Frequencies -- & 421.8847 & 424.1463 & 424.5886 \\
\hline Frequencies -- & 435.1620 & 435.9464 & 442.7173 \\
\hline Frequencies -- & 445.0173 & 449.0529 & 452.4711 \\
\hline Frequencies -- & 454.9931 & 460.4990 & 463.3727 \\
\hline Frequencies -- & 464.4240 & 466.8878 & 469.9622 \\
\hline Frequencies -- & 475.8676 & 485.6376 & 490.0740 \\
\hline Frequencies -- & 500.7194 & 505.8125 & 509.2466 \\
\hline Frequencies -- & 512.1159 & 520.1731 & 522.1187 \\
\hline Frequencies -- & 526.6107 & 530.7741 & 534.1029 \\
\hline Frequencies -- & 537.6442 & 539.7053 & 545.8639 \\
\hline Frequencies -- & 548.1001 & 552.2494 & 552.5609 \\
\hline Frequencies -- & 557.4303 & 558.6468 & 561.7319 \\
\hline Frequencies -- & 563.2629 & 569.2045 & 570.5132 \\
\hline Frequencies -- & 577.8996 & 582.3944 & 584.2641 \\
\hline Frequencies -- & 586.2311 & 590.9848 & 596.6580 \\
\hline Frequencies -- & 596.8505 & 599.1571 & 601.3404 \\
\hline Frequencies -- & 603.3286 & 609.3339 & 609.7914 \\
\hline Frequencies -- & 610.7205 & 613.9654 & 618.4903 \\
\hline Frequencies -- & 620.8624 & 624.7716 & 626.6676 \\
\hline Frequencies -- & 630.2509 & 630.9638 & 632.0704 \\
\hline Frequencies -- & 634.9337 & 635.2035 & 646.1418 \\
\hline Frequencies -- & 648.0722 & 656.2915 & 658.8931 \\
\hline Frequencies -- & 663.6839 & 664.5644 & 674.6204 \\
\hline Frequencies -- & 677.3013 & 677.7158 & 682.2374 \\
\hline Frequencies -- & 684.5655 & 698.7308 & 706.9220 \\
\hline Frequencies -- & 714.0327 & 714.8256 & 715.1876 \\
\hline Frequencies -- & 716.2271 & 718.9011 & 721.8649 \\
\hline Frequencies -- & 722.8857 & 724.6964 & 726.4352 \\
\hline Frequencies -- & 726.6500 & 729.4250 & 731.6919 \\
\hline Frequencies -- & 733.4872 & 736.5559 & 740.2195 \\
\hline Frequencies -- & 745.5292 & 745.9743 & 751.1390 \\
\hline Frequencies -- & 752.5164 & 762.5788 & 765.2598 \\
\hline Frequencies -- & 768.1780 & 768.3550 & 771.4910 \\
\hline Frequencies -- & 775.5227 & 776.2979 & 779.7318 \\
\hline Frequencies -- & 780.1890 & 781.7088 & 782.7049 \\
\hline Frequencies -- & 789.1739 & 794.4900 & 798.9287 \\
\hline Frequencies -- & 801.5408 & 804.3111 & 804.7308 \\
\hline Frequencies -- & 822.5611 & 825.3063 & 826.9545 \\
\hline Frequencies -- & 831.9396 & 858.4870 & 866.9145 \\
\hline Frequencies -- & 868.3082 & 868.6584 & 869.5116 \\
\hline Frequencies -- & 871.4536 & 871.8443 & 873.8475 \\
\hline Frequencies -- & 874.8061 & 876.5094 & 878.7008 \\
\hline Frequencies -- & 884.0624 & 886.7652 & 887.6554 \\
\hline Frequencies -- & 888.1683 & 889.4424 & 897.2506 \\
\hline Frequencies -- & 900.0715 & 901.8452 & 912.7162 \\
\hline Frequencies -- & 918.2185 & 919.5318 & 923.8272 \\
\hline Frequencies -- & 928.6366 & 935.6488 & 936.9902 \\
\hline Frequencies -- & 940.4739 & 941.8618 & 946.6357 \\
\hline Frequencies -- & 946.8174 & 948.0873 & 951.2701 \\
\hline Frequencies -- & 952.8153 & 953.8867 & 956.1125 \\
\hline Frequencies -- & 958.0579 & 961.7330 & 968.3835 \\
\hline Frequencies -- & 974.9717 & 984.9991 & 986.2857 \\
\hline Frequencies -- & 990.7737 & 992.3633 & 996.9463 \\
\hline Frequencies -- & 997.0614 & 1001.5242 & 1002.9144 \\
\hline Frequencies -- & 1004.3197 & 1006.3163 & 1006.8618 \\
\hline Frequencies -- & 1009.4057 & 1012.9364 & 1013.7538 \\
\hline Frequencies -- & 1015.0759 & 1015.9510 & 1018.1634 \\
\hline Frequencies -- & 1018.5437 & 1019.9916 & 1020.7949 \\
\hline Frequencies -- & 1021.1202 & 1023.3198 & 1024.4769 \\
\hline Frequencies -- & 1028.0176 & 1036.1027 & 1036.2522 \\
\hline Frequencies -- & 1043.0803 & 1048.9286 & 1051.7157 \\
\hline Frequencies -- & 1054.7520 & 1056.4733 & 1057.6442 \\
\hline Frequencies -- & 1058.7856 & 1062.8574 & 1065.1424 \\
\hline Frequencies -- & 1065.3972 & 1067.6662 & 1068.2405 \\
\hline
\end{tabular}




\begin{tabular}{|c|c|c|c|}
\hline Frequencies -- & 1071.2681 & 1073.9437 & 1078.7389 \\
\hline Frequencies -- & 1092.2564 & 1094.4459 & 1099.3242 \\
\hline Frequencies -- & 1108.9911 & 1109.3094 & 1111.3366 \\
\hline Frequencies -- & 1112.4304 & 1113.9862 & 1115.8409 \\
\hline Frequencies -- & 1121.9863 & 1124.7658 & 1126.4721 \\
\hline Frequencies -- & 1128.2152 & 1129.2607 & 1130.4555 \\
\hline Frequencies -- & 1132.4655 & 1160.5353 & 1164.3031 \\
\hline Frequencies -- & 1165.3845 & 1167.1245 & 1170.4661 \\
\hline Frequencies -- & 1171.2072 & 1171.8633 & 1172.8981 \\
\hline Frequencies -- & 1174.6931 & 1175.3196 & 1177.2628 \\
\hline Frequencies -- & 1177.6115 & 1180.8072 & 1180.8623 \\
\hline Frequencies -- & 1186.8321 & 1194.6528 & 1196.7266 \\
\hline Frequencies -- & 1201.6441 & 1203.6604 & 1204.4951 \\
\hline Frequencies -- & 1207.1623 & 1209.3338 & 1209.8891 \\
\hline Frequencies -- & 1210.0793 & 1210.9503 & 1218.6449 \\
\hline Frequencies -- & 1222.3684 & 1226.1312 & 1227.5466 \\
\hline Frequencies -- & 1230.4024 & 1231.8493 & 1233.5582 \\
\hline Frequencies -- & 1237.8112 & 1242.0991 & 1248.4289 \\
\hline Frequencies -- & 1251.6018 & 1255.4723 & 1257.2483 \\
\hline Frequencies -- & 1268.3027 & 1272.3266 & 1279.4785 \\
\hline Frequencies -- & 1281.1369 & 1284.2255 & 1286.8201 \\
\hline Frequencies -- & 1289.4205 & 1292.7264 & 1296.9907 \\
\hline Frequencies -- & 1297.7847 & 1298.7260 & 1300.7534 \\
\hline Frequencies -- & 1302.4373 & 1305.2449 & 1306.7200 \\
\hline Frequencies -- & 1307.9203 & 1309.9967 & 1310.9932 \\
\hline Frequencies -- & 1313.5116 & 1321.5498 & 1324.7771 \\
\hline Frequencies -- & 1327.0590 & 1328.5434 & 1333.6781 \\
\hline Frequencies -- & 1337.1650 & 1340.2375 & 1340.6824 \\
\hline Frequencies -- & 1341.9326 & 1346.5961 & 1357.0386 \\
\hline Frequencies -- & 1358.2425 & 1360.5440 & 1361.4950 \\
\hline Frequencies -- & 1362.8705 & 1363.9039 & 1364.2943 \\
\hline Frequencies -- & 1365.9020 & 1366.7486 & 1368.5533 \\
\hline Frequencies -- & 1369.7395 & 1370.1428 & 1375.3453 \\
\hline Frequencies -- & 1382.0852 & 1383.4832 & 1392.7903 \\
\hline Frequencies -- & 1407.7710 & 1438.5960 & 1447.8536 \\
\hline Frequencies -- & 1452.7362 & 1459.9143 & 1466.0527 \\
\hline Frequencies -- & 1468.8645 & 1470.4815 & 1481.4026 \\
\hline Frequencies -- & 1490.4666 & 1494.7287 & 1496.8107 \\
\hline Frequencies -- & 1498.5727 & 1502.7408 & 1503.0991 \\
\hline Frequencies -- & 1503.7506 & 1505.5835 & 1506.3209 \\
\hline Frequencies -- & 1510.5002 & 1511.4018 & 1512.8702 \\
\hline Frequencies -- & 1516.5652 & 1517.6792 & 1524.8469 \\
\hline Frequencies -- & 1526.1039 & 1528.7913 & 1540.6590 \\
\hline Frequencies -- & 1544.2945 & 1547.1640 & 1552.1723 \\
\hline Frequencies -- & 1555.2430 & 1557.2026 & 1557.3927 \\
\hline Frequencies -- & 1559.1727 & 1560.9513 & 1561.6739 \\
\hline Frequencies -- & 1568.8871 & 1572.8729 & 1657.0064 \\
\hline Frequencies -- & 1662.7883 & 1665.6089 & 1666.9161 \\
\hline Frequencies -- & 1668.3969 & 1672.7198 & 1674.3773 \\
\hline Frequencies -- & 1675.2555 & 1677.7653 & 1678.8615 \\
\hline Frequencies -- & 1682.0462 & 1688.1044 & 1689.5761 \\
\hline Frequencies -- & 1694.1778 & 1695.3793 & 1697.3103 \\
\hline Frequencies -- & 1698.8802 & 1700.0645 & 1700.2263 \\
\hline Frequencies -- & 1700.8066 & 1701.3908 & 1702.2237 \\
\hline Frequencies -- & 1704.2268 & 1705.6893 & 1718.1462 \\
\hline Frequencies -- & 1719.8847 & 1730.5691 & 1731.1433 \\
\hline Frequencies -- & 2957.2369 & 3072.4779 & 3082.7544 \\
\hline Frequencies -- & 3088.5799 & 3093.4304 & 3118.1340 \\
\hline Frequencies -- & 3152.9324 & 3155.5223 & 3177.4440 \\
\hline Frequencies -- & 3177.5739 & 3189.8996 & 3199.5083 \\
\hline Frequencies -- & 3201.7773 & 3202.4241 & 3203.1910 \\
\hline Frequencies -- & 3203.8165 & 3211.5375 & 3212.9189 \\
\hline Frequencies -- & 3213.0326 & 3213.9782 & 3215.9208 \\
\hline Frequencies -- & 3216.1715 & 3216.4372 & 3217.7495 \\
\hline Frequencies -- & 3219.5642 & 3220.4931 & 3221.7935 \\
\hline Frequencies -- & 3222.1381 & 3223.1203 & 3223.3711 \\
\hline
\end{tabular}

$\begin{array}{lll}\text { Frequencies -- } 3223.5569 & 3225.2824 & 3229.2385 \\ \text { Frequencies -- } 3229.5726 & 3230.7391 & 3231.3946 \\ \text { Frequencies -- } 3231.5937 & 3232.6342 & 3232.9204 \\ \text { Frequencies -- } 3233.0211 & 3233.3467 & 3234.8630 \\ \text { Frequencies -- } 3238.1756 & 3238.2304 & 3240.2514 \\ \text { Frequencies -- } 3240.7121 & 3240.9133 & 3241.5480 \\ \text { Frequencies -- } 3243.1767 & 3243.3329 & 3243.5354 \\ \text { Frequencies -- } 3246.4141 & 3246.9661 & 3247.4004 \\ \text { Frequencies -- } 3248.0780 & 3250.5248 & 3252.9636 \\ \text { Frequencies -- } 3266.6789 & 3331.0438 & 3596.5203\end{array}$

SCF Done: $E($ RM062X/DGDZVP $)=-6499.37479158$

Sum of electronic and zero-point Energies $=\quad-6498.024768$ Sum of electronic and thermal Energies $=\quad-6497.911090$

Sum of electronic and thermal Free Energies $=\quad-6498.181743$ SCF Done: $E(R M 062 X / D G T Z V P / S M D)=-6500.65713238$

\section{Cat f I1a_R}

Center Atomic Atomic Coordinates (Angstroms)

Number Number Type $X \quad$ Y $\quad$ Z

\begin{tabular}{|c|c|c|c|c|c|}
\hline 1 & 15 & 0 & 0.044349 & 0.060565 & -0.042029 \\
\hline 2 & 8 & 0 & 0.002249 & 0.033213 & 1.580893 \\
\hline 3 & 8 & 0 & 1.655957 & 0.079396 & -0.343740 \\
\hline 4 & 8 & 0 & -0.368704 & 1.437691 & -0.523767 \\
\hline 5 & 8 & 0 & -0.625911 & -1.159164 & -0.555962 \\
\hline 6 & 6 & 0 & 0.707196 & 1.044370 & 2.217809 \\
\hline 7 & 6 & 0 & 2.300360 & -1.112263 & -0.050734 \\
\hline 8 & 6 & 0 & 2.030071 & 0.809332 & 2.565635 \\
\hline 9 & 6 & 0 & 0.114669 & 2.295983 & 2.434029 \\
\hline 10 & 6 & 0 & 2.708611 & -1.378942 & 1.246899 \\
\hline 11 & 6 & 0 & 2.366219 & -2.096884 & -1.037205 \\
\hline 12 & 6 & 0 & 2.826878 & -0.473258 & 2.455963 \\
\hline 13 & 6 & 0 & 2.788383 & 1.838929 & 3.122785 \\
\hline 14 & 6 & 0 & 0.889515 & 3.306502 & 3.012190 \\
\hline 15 & 6 & 0 & -1.292352 & 2.518436 & 2.024058 \\
\hline 16 & 6 & 0 & 3.083309 & -2.677009 & 1.587234 \\
\hline 17 & 6 & 0 & 2.849325 & -3.362962 & -0.698765 \\
\hline 18 & 6 & 0 & 1.809815 & -1.812255 & -2.379975 \\
\hline 19 & 6 & 0 & 2.527421 & -1.482443 & 3.591668 \\
\hline 20 & 6 & 0 & 4.283049 & 0.061671 & 2.555919 \\
\hline 21 & 6 & 0 & 4.225061 & 1.387049 & 3.351800 \\
\hline 22 & 6 & 0 & 2.223913 & 3.095453 & 3.370106 \\
\hline 23 & 1 & 0 & 0.439105 & 4.279013 & 3.191824 \\
\hline 24 & 6 & 0 & -1.664091 & 3.573080 & 1.184923 \\
\hline 25 & 6 & 0 & -2.285690 & 1.620919 & 2.408330 \\
\hline 26 & 6 & 0 & 3.105975 & -2.839748 & 3.103627 \\
\hline 27 & 6 & 0 & 3.201145 & -3.677482 & 0.616827 \\
\hline 28 & 1 & 0 & 2.905377 & -4.133793 & -1.463297 \\
\hline 29 & 6 & 0 & 0.766476 & -2.592618 & -2.872959 \\
\hline 30 & 6 & 0 & 2.251876 & -0.743122 & -3.161013 \\
\hline 31 & 1 & 0 & 2.944352 & -1.164329 & 4.552526 \\
\hline 32 & 1 & 0 & 1.442177 & -1.573473 & 3.705359 \\
\hline 33 & 1 & 0 & 4.959913 & -0.669473 & 3.008358 \\
\hline 34 & 1 & 0 & 4.651016 & 0.262086 & 1.545872 \\
\hline 35 & 1 & 0 & 4.373978 & 1.191864 & 4.421380 \\
\hline 36 & 6 & 0 & 5.268372 & 2.389502 & 2.898632 \\
\hline 37 & 6 & 0 & 3.019885 & 4.179623 & 4.008752 \\
\hline 38 & 6 & 0 & -2.968680 & 3.680123 & 0.716981 \\
\hline 39 & 8 & 0 & -0.704978 & 4.480246 & 0.785734 \\
\hline 40 & 6 & 0 & -3.582772 & 1.684409 & 1.892659 \\
\hline 41 & 8 & 0 & -1.969063 & 0.639014 & 3.319258 \\
\hline 42 & 1 & 0 & 4.127815 & -2.974682 & 3.477039 \\
\hline 43 & 6 & 0 & 2.274298 & -4.037054 & 3.524327 \\
\hline
\end{tabular}




\begin{tabular}{|c|c|c|c|c|c|}
\hline 44 & 6 & 0 & 3.621825 & -5.051811 & 0.994160 \\
\hline 45 & 6 & 0 & 0.210931 & -2.342566 & -4.121936 \\
\hline 46 & 8 & 0 & 0.253042 & -3.602101 & -2.101018 \\
\hline 47 & 6 & 0 & 1.712256 & -0.490939 & -4.426618 \\
\hline 48 & 8 & 0 & 3.234546 & 0.058423 & -2.624853 \\
\hline 49 & 6 & 0 & 5.337402 & 2.768128 & 1.553228 \\
\hline 50 & 6 & 0 & 6.161064 & 2.969589 & 3.801261 \\
\hline 51 & 6 & 0 & 3.414179 & 5.303547 & 3.276151 \\
\hline 52 & 6 & 0 & 3.408344 & 4.068074 & 5.347406 \\
\hline 53 & 6 & 0 & -3.936200 & 2.721606 & 1.030750 \\
\hline 54 & 8 & 0 & -3.335901 & 4.713284 & -0.106462 \\
\hline 55 & 6 & 0 & -0.991208 & 5.143627 & -0.395412 \\
\hline 56 & 8 & 0 & -4.484181 & 0.708750 & 2.234728 \\
\hline 57 & 6 & 0 & -2.636299 & -0.549519 & 3.099809 \\
\hline 58 & 6 & 0 & 0.972153 & -4.190480 & 3.027148 \\
\hline 59 & 6 & 0 & 2.808558 & -5.039397 & 4.333302 \\
\hline 60 & 6 & 0 & 2.759834 & -6.136435 & 0.802047 \\
\hline 61 & 6 & 0 & 4.864661 & -5.266407 & 1.599684 \\
\hline 62 & 6 & 0 & 0.684828 & -1.301305 & -4.923144 \\
\hline 63 & 8 & 0 & -0.833328 & -3.115325 & -4.564191 \\
\hline 64 & 6 & 0 & -1.111867 & -3.761467 & -2.273786 \\
\hline 65 & 8 & 0 & 2.162175 & 0.563918 & -5.185662 \\
\hline 66 & 6 & 0 & 3.837444 & 0.942003 & -3.485558 \\
\hline 67 & 6 & 0 & 6.269460 & 3.709343 & 1.122315 \\
\hline 68 & 1 & 0 & 4.647484 & 2.325794 & 0.835160 \\
\hline 69 & 6 & 0 & 7.089423 & 3.920946 & 3.377750 \\
\hline 70 & 1 & 0 & 6.115002 & 2.692048 & 4.851583 \\
\hline 71 & 6 & 0 & 4.208524 & 6.286525 & 3.864107 \\
\hline 72 & 1 & 0 & 3.107093 & 5.393288 & 2.236622 \\
\hline 73 & 6 & 0 & 4.191718 & 5.057020 & 5.941198 \\
\hline 74 & 1 & 0 & 3.090489 & 3.202177 & 5.923485 \\
\hline 75 & 6 & 0 & -5.305010 & 2.894521 & 0.411846 \\
\hline 76 & 6 & 0 & -2.304837 & 5.246771 & -0.847581 \\
\hline 77 & 6 & 0 & 0.038934 & 5.739821 & -1.109109 \\
\hline 78 & 6 & 0 & -3.908542 & -0.513105 & 2.539053 \\
\hline 79 & 6 & 0 & -2.059179 & -1.755533 & 3.464063 \\
\hline 80 & 6 & 0 & 0.234691 & -5.333857 & 3.322784 \\
\hline 81 & 1 & 0 & 0.551489 & -3.433115 & 2.364251 \\
\hline 82 & 6 & 0 & 2.068082 & -6.183197 & 4.637056 \\
\hline 83 & 1 & 0 & 3.825330 & -4.940631 & 4.706399 \\
\hline 84 & 6 & 0 & 3.126323 & -7.411019 & 1.231242 \\
\hline 85 & 1 & 0 & 1.786422 & -5.968181 & 0.346852 \\
\hline 86 & 6 & 0 & 5.236806 & -6.542533 & 2.018348 \\
\hline 87 & 1 & 0 & 5.541473 & -4.426299 & 1.737924 \\
\hline 88 & 6 & 0 & 0.051598 & -1.153471 & -6.288410 \\
\hline 89 & 6 & 0 & -1.663947 & -3.535680 & -3.530794 \\
\hline 90 & 6 & 0 & -1.912393 & -4.138971 & -1.207127 \\
\hline 91 & 6 & 0 & 3.323624 & 1.173691 & -4.757410 \\
\hline 92 & 6 & 0 & 4.967587 & 1.625399 & -3.053120 \\
\hline 93 & 6 & 0 & 7.147140 & 4.295253 & 2.036264 \\
\hline 94 & 1 & 0 & 6.310585 & 3.986120 & 0.071134 \\
\hline 95 & 1 & 0 & 7.764268 & 4.372030 & 4.098955 \\
\hline 96 & 6 & 0 & 4.602177 & 6.163398 & 5.197276 \\
\hline 97 & 1 & 0 & 4.523074 & 7.146640 & 3.280928 \\
\hline 98 & 1 & 0 & 4.483182 & 4.963144 & 6.983089 \\
\hline 99 & 9 & 0 & -5.863122 & 4.055639 & 0.800702 \\
\hline 100 & 9 & 0 & -5.238259 & 2.917248 & -0.929240 \\
\hline 101 & 9 & 0 & -6.158848 & 1.920196 & 0.741893 \\
\hline 102 & 6 & 0 & -2.598150 & 5.909650 & -2.029203 \\
\hline 103 & 1 & 0 & 1.051592 & 5.678893 & -0.722555 \\
\hline 104 & 6 & 0 & -0.250701 & 6.416618 & -2.295540 \\
\hline 105 & 6 & 0 & -4.618115 & -1.681886 & 2.311975 \\
\hline 106 & 1 & 0 & -1.065927 & -1.755377 & 3.902346 \\
\hline 107 & 6 & 0 & -2.782026 & -2.933718 & 3.273043 \\
\hline 108 & 6 & 0 & 0.780396 & -6.336219 & 4.128010 \\
\hline 109 & 1 & 0 & -0.757893 & -5.459534 & 2.899491 \\
\hline
\end{tabular}

\begin{tabular}{|c|c|c|c|c|c|}
\hline 110 & 1 & 0 & 2.506673 & -6.960941 & 5.254922 \\
\hline 111 & 6 & 0 & 4.363263 & -7.616357 & 1.843760 \\
\hline 112 & 1 & 0 & 2.439581 & -8.241373 & 1.098139 \\
\hline 113 & 1 & 0 & 6.206014 & -6.698148 & 2.482765 \\
\hline 114 & 9 & 0 & 0.541512 & -0.138302 & -7.004629 \\
\hline 115 & 9 & 0 & -1.280741 & -0.957061 & -6.192860 \\
\hline 116 & 9 & 0 & 0.226565 & -2.269085 & -7.015184 \\
\hline 117 & 6 & 0 & -3.019164 & -3.713420 & -3.755607 \\
\hline 118 & 1 & 0 & -1.460037 & -4.250668 & -0.226731 \\
\hline 119 & 6 & 0 & -3.276809 & -4.331393 & -1.428401 \\
\hline 120 & 6 & 0 & 3.951590 & 2.061675 & -5.620439 \\
\hline 121 & 1 & 0 & 5.339658 & 1.428729 & -2.052026 \\
\hline 122 & 6 & 0 & 5.586566 & 2.537752 & -3.905899 \\
\hline 123 & 1 & 0 & 7.870576 & 5.033515 & 1.704183 \\
\hline 124 & 1 & 0 & 5.220200 & 6.929540 & 5.655295 \\
\hline 125 & 1 & 0 & -3.634178 & 5.962605 & -2.348370 \\
\hline 126 & 6 & 0 & -1.563541 & 6.494406 & -2.759147 \\
\hline 127 & 1 & 0 & 0.556588 & 6.888103 & -2.848040 \\
\hline 128 & 1 & 0 & -5.601356 & -1.618093 & 1.857540 \\
\hline 129 & 6 & 0 & -4.050306 & -2.898440 & 2.692945 \\
\hline 130 & 1 & 0 & -2.348712 & -3.877281 & 3.587643 \\
\hline 131 & 1 & 0 & 0.208990 & -7.232858 & 4.347644 \\
\hline 132 & 1 & 0 & 4.646019 & -8.609123 & 2.180184 \\
\hline 133 & 1 & 0 & -3.419318 & -3.521248 & -4.746103 \\
\hline 134 & 6 & 0 & -3.824958 & -4.127743 & -2.694556 \\
\hline 135 & 1 & 0 & -3.915167 & -4.615693 & -0.598961 \\
\hline 136 & 1 & 0 & 3.527767 & 2.204871 & -6.609615 \\
\hline 137 & 6 & 0 & 5.084771 & 2.751179 & -5.191078 \\
\hline 138 & 1 & 0 & 6.465579 & 3.075708 & -3.566181 \\
\hline 139 & 1 & 0 & -1.787960 & 7.018118 & -3.682373 \\
\hline 140 & 1 & 0 & -4.605052 & -3.818306 & 2.539599 \\
\hline 141 & 1 & 0 & -4.889198 & -4.262858 & -2.853905 \\
\hline 142 & 1 & 0 & 5.574163 & 3.449490 & -5.861817 \\
\hline 143 & 1 & 0 & -2.332189 & -1.408535 & -1.388179 \\
\hline 144 & 8 & 0 & -3.165544 & -1.094297 & -1.794088 \\
\hline 145 & 6 & 0 & -3.298957 & 0.267143 & -1.448326 \\
\hline 146 & 6 & 0 & -2.842300 & 1.239626 & -2.576963 \\
\hline 147 & 1 & 0 & -4.352344 & 0.470556 & -1.228838 \\
\hline 148 & 1 & 0 & -2.714532 & 0.493293 & -0.548740 \\
\hline 149 & 8 & 0 & -2.428240 & 2.468355 & -2.008089 \\
\hline 150 & 6 & 0 & -1.676215 & 0.594400 & -3.363928 \\
\hline 151 & 1 & 0 & -1.832070 & 2.240391 & -1.266921 \\
\hline 152 & 1 & 0 & -0.852097 & 0.256578 & -2.726927 \\
\hline 153 & 1 & 0 & -2.026700 & -0.275271 & -3.926681 \\
\hline 154 & 1 & 0 & 3.845977 & 4.368515 & 0.083855 \\
\hline 155 & 6 & 0 & 3.364767 & 4.262107 & -0.883084 \\
\hline 156 & 6 & 0 & 2.371352 & 3.302713 & -1.045786 \\
\hline 157 & 6 & 0 & 3.741361 & 5.113455 & -1.934931 \\
\hline 158 & 6 & 0 & 1.752803 & 3.240534 & 5856 \\
\hline 159 & 1 & 0 & 2.054181 & 694 & -0.238139 \\
\hline 160 & 6 & 0 & 3.126445 & 5.036661 & -3.181178 \\
\hline 161 & 1 & 0 & 4.521968 & 5.850144 & -1.773079 \\
\hline 162 & 7 & 0 & & & \\
\hline 163 & 6 & 0 & & & \\
\hline 164 & 1 & 0 & 3.416418 & 5.695537 & -3.993205 \\
\hline 165 & 6 & 0 & 0.224603 & 2.574479 & -3.865356 \\
\hline 166 & 1 & 0 & 0.217023 & 1.854917 & -1.846799 \\
\hline 167 & 16 & 0 & 1.087508 & 3.807550 & -4.732201 \\
\hline 168 & 16 & 0 & -1.040674 & 1.725481 & -4.652645 \\
\hline 169 & 6 & 0 & -3.976768 & 1.520719 & -3.547068 \\
\hline 170 & 6 & 0 & -4.314566 & 2.827278 & -3.899495 \\
\hline 171 & 6 & 0 & -4.677366 & 0.448822 & -4.112229 \\
\hline 172 & 6 & 0 & -5.346521 & 3.061573 & -4.808837 \\
\hline 173 & 1 & 0 & -3.769486 & 3.649145 & -3.446022 \\
\hline 174 & 6 & 0 & -5.706658 & 0.686256 & -5.020720 \\
\hline 175 & 1 & 0 & -4.425027 & -0.570580 & -3.823215 \\
\hline
\end{tabular}




$\begin{array}{llllll}176 & 6 & 0 & -6.043214 & 1.994302 & -5.373281 \\ 177 & 1 & 0 & -5.608273 & 4.081450 & -5.075334 \\ 178 & 1 & 0 & -6.248931 & -0.149918 & -5.451914 \\ 179 & 1 & 0 & -6.845962 & 2.178685 & -6.080741\end{array}$

Frequencies -- 12.3355

Frequencies -- 23.2482

Frequencies -- 29.6561

Frequencies -- 36.2451

Frequencies -- 40.6167

Frequencies -- 46.8128

Frequencies -- 57.9242

Frequencies -- 62.3020

Frequencies -- 68.1853

Frequencies -- 78.7961

Frequencies -- 86.8627

Frequencies -- 94.1844

Frequencies -- 104.2701

Frequencies -- 111.2814

Frequencies -- 119.6304

Frequencies -- 132.0800

Frequencies -- 140.2413

Frequencies -- 156.2171

Frequencies -- 171.4842

Frequencies -- 188.4637

Frequencies -- 201.1304

Frequencies -- 217.4483

Frequencies -- 228.6235

Frequencies -- 245.0071

Frequencies -- 260.4188

Frequencies -- 273.9516

Frequencies -- 285.4822

Frequencies -- 292.1632

Frequencies -- 299.9622

Frequencies -- 308.8657

Frequencies -- 316.8870

Frequencies -- 330.0312

Frequencies -- 341.7748

Frequencies -- 370.0581

Frequencies -- 406.3332

Frequencies -- 410.6917

Frequencies -- 415.1334

Frequencies -- 422.1971

Frequencies -- 432.1442

Frequencies -- 445.1901

Frequencies -- 457.5082

Frequencies -- 465.5574

Frequencies -- 487.2805

Frequencies -- 507.6977

Frequencies -- 520.4826

Frequencies -- 528.9614

Frequencies -- 537.5301

Frequencies -- 551.0619

Frequencies -- 559.1197

Frequencies -- 566.7676

Frequencies -- 576.0736

Frequencies -- 589.0087

Frequencies -- 596.9983

Frequencies -- 601.9157

Frequencies -- 610.1745

Frequencies -- 617.7860

Frequencies -- 625.8680

Frequencies -- 632.2545

Frequencies -- 645.7985

Frequencies -- 663.8458
$17.7047 \quad 21.9561$

$23.7798 \quad 28.7654$

$31.3176 \quad 35.7652$

$36.9302 \quad 39.0885$

$43.0241 \quad 46.0745$

$49.7203 \quad 55.3701$

$58.6943 \quad 60.7509$

$65.9268 \quad 66.9975$

$70.5986 \quad 73.6158$

$80.8268 \quad 81.8787$

$90.7283 \quad 92.4656$

$96.2791 \quad 98.5037$

$104.7427 \quad 109.4344$

$113.1262 \quad 118.2921$

$126.4481 \quad 129.7021$

$135.3187 \quad 136.8440$

$146.0130 \quad 152.5852$

$164.1442 \quad 167.2591$

$184.5054 \quad 186.7448$

$194.3274 \quad 195.8607$

$211.4605 \quad 215.2485$

$220.6530 \quad 226.2928$

$231.7870 \quad 233.8279$

$249.2438 \quad 255.3837$

$268.2033 \quad 271.7208$

$275.2980 \quad 277.5362$

$288.3743 \quad 289.8965$

$293.9414 \quad 298.5480$

$304.0270 \quad 307.2278$

$311.0336 \quad 313.7029$

$320.0723 \quad 322.4935$

$333.3019 \quad 338.6369$

$346.0636 \quad 354.2065$

$380.9735 \quad 388.8152$

$408.4914 \quad 409.4854$

$411.4127 \quad 413.0075$

$417.4171 \quad 420.4070$

$423.2802 \quad 428.4926$

$439.8899 \quad 442.8048$

$446.7370 \quad 452.5895$

$460.3912 \quad 463.6997$

$466.3201 \quad 474.5753$

$491.3845 \quad 500.8799$

$509.1552 \quad 513.4269$

$521.9446 \quad 525.3800$

$530.7297 \quad 533.6114$

$539.0071 \quad 546.2548$

$553.8656 \quad 555.6006$

$560.1226 \quad 561.9207$

$570.9509 \quad 574.6180$

$584.8036 \quad 586.3641$

$592.8838 \quad 595.7270$

$597.5234 \quad 599.1145$

$604.9787 \quad 609.0366$

$611.0273 \quad 613.0488$

$620.8224 \quad 624.0874$

$630.1009 \quad 630.8535$

$634.2005 \quad 634.7839$

$648.0759 \quad 656.5591$

$672.5687 \quad 676.9330$
Frequencies -- $\quad 678.7100$

Frequencies -- 698.0584

Frequencies -- 712.8388

Frequencies -- 715.6644

Frequencies -- 721.8183

Frequencies -- 726.3907

Frequencies -- 732.5617

Frequencies -- 740.9920

Frequencies -- 751.9343

Frequencies -- 764.1449

Frequencies -- 771.3361

Frequencies -- 780.1450

Frequencies -- 786.7072

Frequencies -- 799.5947

Frequencies -- 805.4312

Frequencies -- 827.0117

Frequencies -- 863.6386

Frequencies -- 868.6590

Frequencies -- 872.5595

Frequencies -- 876.3818

Frequencies -- 887.4247

Frequencies -- 889.8755

Frequencies -- 912.3191

Frequencies -- 929.4998

Frequencies -- 936.1610

Frequencies -- 942.6870

Frequencies -- 950.9236

Frequencies -- 958.5512

Frequencies -- 970.2813

Frequencies -- 990.3697

Frequencies -- 997.1011

Frequencies -- 1001.5278

Frequencies -- 1008.7854

Frequencies -- 1013.4229

Frequencies -- 1018.5499

Frequencies -- 1021.1094

Frequencies -- 1028.4487

Frequencies -- 1040.7475

Frequencies -- 1052.5917

Frequencies -- 1062.5822

Frequencies -- 1068.5374

Frequencies -- 1071.0408

Frequencies -- 1099.1036

Frequencies -- 1109.5357

Frequencies -- 1113.3809

Frequencies -- 1125.6502

Frequencies -- 1130.3114

Frequencies -- 1158.0867

Frequencies -- 1164.5408

Frequencies -- 1171.4200

Frequencies -- 1174.6526

Frequencies -- 1177.7471

Frequencies -- 1195.7057

Frequencies -- 1202.7675

Frequencies -- 1211.9626

Frequencies -- 1215.5560

Frequencies -- 1226.7022

Frequencies -- 1232.0788

Frequencies -- 1246.8902

Frequencies -- 1255.3678

Frequencies -- 1275.5001

Frequencies -- 1280.0520

Frequencies -- 1293.2978

Frequencies -- 1300.4926

Frequencies -- 1307.8740

Frequencies -- 1311.4623
681.9739

703.9048

714.0924

718.7810

722.5254

728.5836

733.7408

746.0538

762.2173

767.2309

773.9447

783.2198

787.4043

800.6261

823.4382

832.2505

866.0950

869.4892

874.5806

883.1545

887.8591

892.5942

918.5958

930.3462

938.9208

944.6723

953.2948

968.0548

986.6061

991.9491

998.0927

1006.0132

1012.6065

1015.0877

1019.2553

1023.2070

1035.0885

1044.4518

1057.0575

1062.8343

1069.1141

1073.2561

1105.2459

1109.5913

1117.9249

1127.1553

1132.2306

1162.8079

1166.2681

1171.9798

1175.1844

1181.4651

1197.7310

1204.5236

1212.3040

1220.6682

1228.8415

1238.1603

1250.3638

1258.4753

1278.1272

1285.9269

1297.1647

1301.1735

1309.1600

1312.2436

685.4854

711.2516

715.2816

718.8667

724.5886

730.8308

736.9013

749.3397

764.0165

770.3158

779.4878

784.1992

797.2940

804.6163

824.7434

855.3410

867.6183

871.7347

876.0175

885.4555

888.6984

909.3995

923.5091

934.0608

941.9670

945.1029

953.4041

968.5065

988.5305

994.7884

998.4843

1007.9073

1013.1396

1017.2343

1019.4308

1024.4169

1036.2278 


\begin{tabular}{|c|c|c|}
\hline Frequencies -- 1314.8538 & 1321.2948 & 1324.5792 \\
\hline Frequencies -- 1325.7919 & 1327.5504 & 1333.9641 \\
\hline Frequencies -- 1335.4258 & 1342.7026 & 1342.9425 \\
\hline Frequencies -- 1345.4352 & 1356.2334 & 1357.3404 \\
\hline Frequencies -- 1358.2943 & 1360.6299 & 1361.6342 \\
\hline Frequencies -- 1363.3090 & 1364.3983 & 1366.6091 \\
\hline Frequencies -- 1367.2172 & 1368.8185 & 1370.1125 \\
\hline Frequencies -- 1370.4773 & 1377.0491 & 1383.9653 \\
\hline Frequencies -- 1390.5642 & 1405.4779 & 1408.1608 \\
\hline Frequencies -- 1409.6333 & 1427.5394 & 1448.7831 \\
\hline Frequencies -- 1458.9740 & 1463.6159 & 1468.9019 \\
\hline Frequencies -- 1470.3631 & 1478.8837 & 1489.8311 \\
\hline Frequencies -- 1491.5848 & 1492.5167 & 1495.7805 \\
\hline Frequencies -- 1496.0553 & 1498.6757 & 1503.5434 \\
\hline Frequencies -- 1504.6071 & 1506.0024 & 1506.8284 \\
\hline Frequencies -- 1510.4595 & 1512.9191 & 1513.9255 \\
\hline Frequencies -- 1516.4664 & 1518.4955 & 1519.8830 \\
\hline Frequencies -- 1527.6875 & 1528.0852 & 1545.2620 \\
\hline Frequencies -- 1548.1745 & 1549.3430 & 1554.2157 \\
\hline Frequencies -- 1555.7476 & 1558.0155 & 1560.1740 \\
\hline Frequencies -- 1561.1702 & 1562.0815 & 1569.3346 \\
\hline Frequencies -- 1573.1807 & 1575.2568 & 1659.0827 \\
\hline Frequencies -- 1664.1336 & 1666.7056 & 1668.1408 \\
\hline Frequencies -- 1671.7783 & 1672.0179 & 1673.4016 \\
\hline Frequencies -- 1674.4733 & 1674.9614 & 1678.4708 \\
\hline Frequencies -- 1680.8067 & 1686.8525 & 1689.4725 \\
\hline Frequencies -- 1693.2663 & 1695.9860 & 1696.9794 \\
\hline Frequencies -- 1698.1889 & 1698.3713 & 1698.7466 \\
\hline Frequencies -- 1701.2828 & 1702.0158 & 1702.2539 \\
\hline Frequencies -- 1704.2093 & 1704.6172 & 1719.4872 \\
\hline Frequencies -- 1721.6325 & 1728.7645 & 1731.9945 \\
\hline Frequencies -- 2474.8630 & 3069.7673 & 3083.3826 \\
\hline Frequencies -- 3090.2035 & 3093.0962 & 3093.3578 \\
\hline Frequencies -- 3120.4555 & 3153.6682 & 3154.9500 \\
\hline Frequencies -- 3155.9384 & 3178.7085 & 3186.4777 \\
\hline Frequencies -- 3190.6885 & 3198.4543 & 3200.8447 \\
\hline Frequencies -- 3201.4204 & 3203.3525 & 3208.5265 \\
\hline Frequencies -- 3208.8397 & 3210.4908 & 3213.1708 \\
\hline Frequencies -- 3213.5763 & 3215.9521 & 3217.9985 \\
\hline Frequencies -- 3218.3355 & 3220.0055 & 3221.4061 \\
\hline Frequencies -- 3221.6401 & 3222.5006 & 3224.6137 \\
\hline Frequencies -- 3225.9349 & 3226.1010 & 3226.5779 \\
\hline Frequencies -- 3226.7596 & 3228.1420 & 3229.9152 \\
\hline Frequencies -- 3230.7643 & 3230.7 & 3230.8224 \\
\hline Frequencies -- 3233.0805 & 3234.4907 & 3235.8543 \\
\hline Frequencies -- 3239.9359 & 3240.5587 & 3240.6748 \\
\hline Frequencies -- 3240.7737 & 3241.7861 & 3242.4114 \\
\hline Frequencies -- 3242.5345 & 3243.1380 & 3243.8802 \\
\hline Frequencies -- 3245.4045 & 3247.8421 & 3248.0912 \\
\hline Frequencies -- 3249.4349 & 3250.4502 & 3251.2078 \\
\hline Frequencies -- 3257.1589 & 3663.8535 & 3672.7923 \\
\hline \multicolumn{3}{|c|}{ SCF Done: E(RM062X/DGDZVP) = -6499.42943890 } \\
\hline \multicolumn{2}{|c|}{ Sum of electronic and zero-point Energies= } & -6498.078550 \\
\hline \multicolumn{2}{|c|}{ Sum of electronic and thermal Energies= } & -6497.964524 \\
\hline \multicolumn{2}{|c|}{ Sum of electronic and thermal Free Energies= } & -6498.23467 \\
\hline
\end{tabular}

\section{Cat f 10b_S}

Center Atomic Atomic Coordinates (Angstroms) Number Number Type $\quad X \quad Y \quad Z$

$\begin{array}{cccccc}1 & 15 & 0 & 0.104248 & 0.082937 & -0.034561 \\ 2 & 8 & 0 & 0.022003 & 0.002543 & 1.573980\end{array}$

\begin{tabular}{|c|c|c|c|c|c|}
\hline 3 & 8 & 0 & 1.675752 & 0.086588 & -0.342175 \\
\hline 4 & 8 & 0 & -0.517608 & 1.311107 & -0.572550 \\
\hline 5 & 8 & 0 & -0.447447 & -1.300796 & -0.453068 \\
\hline 6 & 6 & 0 & 0.525352 & 1.094646 & 2.268783 \\
\hline 7 & 6 & 0 & 2.435686 & -1.006997 & 0.073064 \\
\hline 8 & 6 & 0 & 1.869337 & 1.077041 & 2.617974 \\
\hline 9 & 6 & 0 & -0.287402 & 2.201990 & 2.549848 \\
\hline 10 & 6 & 0 & 2.909009 & -1.057876 & 1.376901 \\
\hline 11 & 6 & 0 & 2.625304 & -2.078856 & -0.808662 \\
\hline 12 & 6 & 0 & 2.890272 & -0.039653 & 2.504486 \\
\hline 13 & 6 & 0 & 2.433052 & 2.191854 & 3.238858 \\
\hline 14 & 6 & 0 & 0.290390 & 3.273282 & 3.245261 \\
\hline 15 & 6 & 0 & -1.699763 & 2.266191 & 2.086998 \\
\hline 16 & 6 & 0 & 3.537593 & -2.223879 & 1.818083 \\
\hline 17 & 6 & 0 & 3.367018 & -3.179290 & -0.370361 \\
\hline 18 & 6 & 0 & 1.906050 & -2.102305 & -2.103774 \\
\hline 19 & 6 & 0 & 2.834219 & -0.984337 & 3.733291 \\
\hline 20 & 6 & 0 & 4.209665 & 0.774013 & 2.519437 \\
\hline 21 & 6 & 0 & 3.936108 & 2.009121 & 3.409764 \\
\hline 22 & 6 & 0 & 1.640568 & 3.286401 & 3.601395 \\
\hline 23 & 1 & 0 & -0.329676 & 4.124377 & 3.511007 \\
\hline 24 & 6 & 0 & -2.172948 & 3.389110 & 1.398237 \\
\hline 25 & 6 & 0 & -2.594487 & 1.211339 & 2.273574 \\
\hline 26 & 6 & 0 & 3.667918 & -2.229001 & 5592 \\
\hline 27 & 6 & 0 & 3.832145 & -3.271433 & $\$ 2000$ \\
\hline 28 & 1 & 0 & 566 & -4.002322 & -1.056795 \\
\hline 29 & 6 & 0 & 3541 & -3.169063 & -2.398776 \\
\hline 30 & 6 & 0 & 8780 & -1.038455 & -3.000418 \\
\hline 31 & 1 & 0 & 1671 & -0.503271 & 44989 \\
\hline 32 & 1 & 0 & 1.794096 & -1.280602 & 66145 \\
\hline 33 & 1 & 0 & 5.060238 & 0.182005 & 2.870622 \\
\hline 34 & 1 & 0 & 4.432923 & 1.099809 & 1.498926 \\
\hline 35 & 1 & 0 & 4.152642 & 1.765386 & 4.457533 \\
\hline 36 & 6 & 0 & 4.768797 & 3.216230 & 3.026462 \\
\hline 37 & 6 & 0 & 2.218382 & 4.427879 & 4.359721 \\
\hline 38 & 6 & 0 & -3.468455 & 3.447910 & 0.872567 \\
\hline 39 & 8 & 0 & -1.291521 & 4.429556 & 1.211099 \\
\hline 40 & 6 & 0 & -3.888988 & 1.265443 & 1.769099 \\
\hline 41 & 8 & 0 & -2.216386 & 0.097615 & 2.986041 \\
\hline 42 & 1 & 0 & 4.712538 & -2.102792 & 3.644115 \\
\hline 43 & 6 & 0 & 3.140360 & -3.522507 & 3.926387 \\
\hline 44 & 6 & 0 & 4.569637 & -4.469593 & 1.420545 \\
\hline 45 & 6 & 0 & 0.229944 & -3.139486 & -3.514450 \\
\hline 46 & 8 & 0 & 0.991941 & -4.233541 & -1.524931 \\
\hline 47 & 6 & 0 & 1.071209 & -0.972855 & -4.094693 \\
\hline 48 & 8 & 0 & 2.844200 & -0.028179 & -2.778521 \\
\hline 49 & 6 & 0 & 4.743695 & 3.711172 & 1.717962 \\
\hline 50 & 6 & 0 & 5.579190 & 3.857100 & 3.966206 \\
\hline 51 & 6 & 0 & 2.275904 & 5.706949 & 3.799102 \\
\hline 52 & 6 & 0 & 2.735806 & 4.222099 & 5.642813 \\
\hline 53 & 6 & 0 & -4.350414 & 2.382149 & 1.071766 \\
\hline 54 & 8 & 0 & -3.882119 & 4.520885 & 0.131898 \\
\hline 55 & 6 & 0 & -1.834749 & 5.617766 & 0.767526 \\
\hline 56 & 8 & 0 & -4.728253 & 0.192466 & 1.950474 \\
\hline 57 & 6 & 0 & -2.757647 & -1.062738 & 2.454635 \\
\hline 58 & 6 & 0 & 1.861114 & -3.972135 & 3.575556 \\
\hline 59 & 6 & 0 & 3.922841 & -4.313030 & 4.767719 \\
\hline 60 & 6 & 0 & 3.975689 & -5.735042 & 1.395911 \\
\hline 61 & 6 & 0 & 5.856230 & -4.329404 & 1.951980 \\
\hline 62 & 6 & 0 & 0.204539 & -2.030366 & -4.363402 \\
\hline 63 & 8 & 0 & -0.638365 & -4.181277 & -3.764928 \\
\hline 64 & 6 & 0 & -0.221772 & -4.885911 & -1.506784 \\
\hline 65 & 8 & 0 & 1.070371 & 0.141908 & -4.888020 \\
\hline 66 & 6 & 0 & 2.425566 & 1.211491 & -3.217936 \\
\hline 67 & 6 & 0 & 5.518247 & 4.812414 & 1.356703 \\
\hline 68 & 1 & 0 & 4.110741 & 3.234651 & 0.969965 \\
\hline
\end{tabular}




\begin{tabular}{|c|c|c|c|c|c|}
\hline 69 & 6 & 0 & 6.340018 & 4.971846 & 3.615668 \\
\hline 70 & 1 & 0 & 5.605463 & 3.488253 & 4.988725 \\
\hline 71 & 6 & 0 & 2.881729 & 6.754429 & 4.491651 \\
\hline 72 & 1 & 0 & 1.860253 & 5.869711 & 2.807220 \\
\hline 73 & 6 & 0 & 3.324083 & 5.272543 & 6.344733 \\
\hline 74 & 1 & 0 & 2.669112 & 3.233608 & 6.091670 \\
\hline 75 & 6 & 0 & -5.796199 & 2.368855 & 0.628125 \\
\hline 76 & 6 & 0 & -3.107720 & 5.655348 & 0.207564 \\
\hline 77 & 6 & 0 & -1.074535 & 6.774020 & 0.860378 \\
\hline 78 & 6 & 0 & -4.054328 & -1.020054 & 1.950221 \\
\hline 79 & 6 & 0 & -2.026983 & -2.239548 & 2.424967 \\
\hline 80 & 6 & 0 & 1.376971 & -5.185638 & 4.055141 \\
\hline 81 & 1 & 0 & 1.253735 & -3.377733 & 2.894086 \\
\hline 82 & 6 & 0 & 3.445454 & -5.535839 & 5.243361 \\
\hline 83 & 1 & 0 & 4.925838 & -3.985614 & 5.031601 \\
\hline 84 & 6 & 0 & 4.647141 & -6.837754 & 1.921201 \\
\hline 85 & 1 & 0 & 2.973030 & -5.844596 & 0.989588 \\
\hline 86 & 6 & 0 & 6.534292 & -5.433541 & 2.464712 \\
\hline 87 & 1 & 0 & 6.325895 & -3.348326 & 1.956174 \\
\hline 88 & 6 & 0 & -0.777795 & -2.055912 & -5.511960 \\
\hline 89 & 6 & 0 & -1.041133 & -4.864854 & -2.633347 \\
\hline 90 & 6 & 0 & -0.599340 & -5.592115 & -0.374853 \\
\hline 91 & 6 & 0 & 1.519812 & 1.294572 & -4.271709 \\
\hline 92 & 6 & 0 & 2.953192 & 2.360944 & -2.649436 \\
\hline 93 & 6 & 0 & 6.314831 & 5.451916 & 2.307017 \\
\hline 94 & 1 & 0 & 5.495577 & 5.173849 & 0.332016 \\
\hline 95 & 1 & 0 & 6.951939 & 5.463440 & 4.365899 \\
\hline 96 & 6 & 0 & 3.412265 & 6.537981 & 5.763711 \\
\hline 97 & 1 & 0 & 2.941779 & 7.738775 & 4.037281 \\
\hline 98 & 1 & 0 & 3.718176 & 5.103373 & 7.342403 \\
\hline 99 & 9 & 0 & -6.607529 & 2.178781 & 1.683966 \\
\hline 100 & 9 & 0 & -6.189439 & 3.492006 & 0.030012 \\
\hline 101 & 9 & 0 & -6.040822 & 1.358790 & -0.233570 \\
\hline 102 & 6 & 0 & -3.621495 & 6.842114 & -0.294255 \\
\hline 103 & 1 & 0 & -0.081464 & 6.712005 & 1.296886 \\
\hline 104 & 6 & 0 & -1.582267 & 7.970017 & 0.353145 \\
\hline 105 & 6 & 0 & -4.659784 & -2.156603 & 1.440285 \\
\hline 106 & 1 & 0 & -1.008261 & -2.232733 & 2.797855 \\
\hline 107 & 6 & 0 & -2.629923 & -3.390036 & 1.913487 \\
\hline 108 & 6 & 0 & 2.172374 & -5.976400 & 4.887864 \\
\hline 109 & 1 & 0 & 0.382035 & -5.520518 & 3.775078 \\
\hline 110 & 1 & 0 & 4.074548 & -6.147683 & 5.882694 \\
\hline 111 & 6 & 0 & 5.925784 & -6.688883 & 2.460467 \\
\hline 112 & 1 & 0 & 4.168321 & -7.812206 & 1.917262 \\
\hline 113 & 1 & 0 & 7.534417 & -5.314006 & 2.870376 \\
\hline 114 & 9 & 0 & -2.045657 & -2.206795 & -5.075914 \\
\hline 115 & 9 & 0 & -0.526145 & -3.096982 & -6.326935 \\
\hline 116 & 9 & 0 & -0.754445 & -0.954980 & -6.262899 \\
\hline 117 & 6 & 0 & -2.252151 & -5.541187 & -2.636972 \\
\hline 118 & 1 & 0 & 0.062286 & -5.585191 & 0.486528 \\
\hline 119 & 6 & 0 & -1.806290 & -6.292887 & -0.379334 \\
\hline 120 & 6 & 0 & 1.107952 & 2.527363 & -4.754669 \\
\hline 121 & 1 & 0 & 3.660227 & 2.261257 & -1.831405 \\
\hline 122 & 6 & 0 & 2.582856 & 3.604724 & -3.164779 \\
\hline 123 & 1 & 0 & & 6.314635 & 2.028502 \\
\hline 124 & 1 & 0 & 3.884257 & 7.353750 & 6.302464 \\
\hline 125 & 1 & 0 & -4.613031 & 6.831015 & -0.734842 \\
\hline 126 & 6 & 0 & -2.848477 & 8.001796 & -0.230106 \\
\hline 127 & 1 & 0 & -0.980014 & 8.870881 & 0.408148 \\
\hline 128 & 1 & 0 & -5.669930 & -2.086106 & 1.049809 \\
\hline 129 & 6 & 0 & -3.938244 & -3.351331 & 1.430954 \\
\hline 130 & 1 & 0 & -2.070654 & -4.319251 & 1.886466 \\
\hline 131 & 1 & 0 & 1.801550 & -6.928546 & 5.254465 \\
\hline 132 & 1 & 0 & 6.446280 & -7.548664 & 2.871032 \\
\hline 133 & 1 & 0 & -2.869048 & -5.502595 & -3.529235 \\
\hline 134 & 6 & 0 & -2.630313 & -6.265322 & -1.504419 \\
\hline
\end{tabular}

$\begin{array}{llllll}135 & 1 & 0 & -2.099838 & -6.861461 & 0.497413 \\ 136 & 1 & 0 & 0.388229 & 2.559852 & -5.566619 \\ 137 & 6 & 0 & 1.657296 & 3.687659 & -4.204830 \\ 138 & 1 & 0 & 3.009452 & 4.509763 & -2.741044 \\ 139 & 1 & 0 & -3.242225 & 8.929054 & -0.632337 \\ 140 & 1 & 0 & -4.392607 & -4.250455 & 1.027352 \\ 141 & 1 & 0 & -3.568252 & -6.810339 & -1.507238 \\ 142 & 1 & 0 & 1.362491 & 4.655498 & -4.597221 \\ 143 & 1 & 0 & -1.484790 & -1.329940 & -0.635736 \\ 144 & 8 & 0 & -2.876953 & -1.302591 & -1.009796 \\ 145 & 6 & 0 & -2.924099 & -1.744270 & -2.400882 \\ 146 & 6 & 0 & -2.964529 & -0.288022 & -2.898324 \\ 147 & 1 & 0 & -2.028437 & -2.304425 & -2.667067 \\ 148 & 1 & 0 & -3.834454 & -2.320688 & -2.581395 \\ 149 & 8 & 0 & -1.640272 & 0.082368 & -3.217419 \\ 150 & 6 & 0 & -3.293678 & 0.043367 & -1.424736 \\ 151 & 1 & 0 & -1.582725 & 1.057785 & -3.129685 \\ 152 & 1 & 0 & -4.353089 & 0.172234 & -1.201352 \\ 153 & 1 & 0 & -2.683239 & 0.825115 & -0.967150 \\ 154 & 1 & 0 & -0.466429 & 2.898916 & -1.172164 \\ 155 & 7 & 0 & -0.439910 & 3.858382 & -1.557070 \\ 156 & 6 & 0 & -1.317340 & 4.233278 & -2.510239 \\ 157 & 6 & 0 & 0.505007 & 4.791767 & -1.173184 \\ 158 & 16 & 0 & -2.538880 & 3.307300 & -3.158213 \\ 159 & 16 & 0 & -0.972476 & 5.895585 & -2.989857 \\ 160 & 6 & 0 & 0.385725 & 5.989052 & -1.890002 \\ 161 & 6 & 0 & 1.472322 & 4.631638 & -0.180349 \\ 162 & 6 & 0 & 1.261437 & 7.047518 & -1.655898 \\ 163 & 6 & 0 & 2.338650 & 5.695086 & 0.055637 \\ 164 & 1 & 0 & 1.527653 & 3.703897 & 0.388330 \\ 165 & 6 & 0 & 2.242750 & 6.887515 & -0.678845 \\ 166 & 1 & 0 & 1.171460 & 7.976694 & -2.209930 \\ 167 & 1 & 0 & 3.099592 & 5.600731 & 0.823906 \\ 168 & 1 & 0 & 2.936269 & 7.698118 & -0.478400 \\ 169 & 6 & 0 & -3.971615 & 0.080803 & -3.961201 \\ 170 & 6 & 0 & -5.341227 & -0.107457 & -3.741802 \\ 171 & 6 & 0 & -3.546044 & 0.601190 & -5.184942 \\ 172 & 6 & 0 & -6.268814 & 0.224112 & -4.726282 \\ 173 & 1 & 0 & -5.697915 & -0.512273 & -2.797102 \\ 174 & 6 & 0 & -4.474413 & 0.934027 & -6.170218 \\ 175 & 1 & 0 & -2.484512 & 0.740112 & -5.363682 \\ 176 & 6 & 0 & -5.837136 & 0.748023 & -5.945230 \\ 177 & 1 & 0 & -7.328232 & 0.078673 & -4.538975 \\ 178 & 1 & 0 & -4.128665 & 1.339668 & -7.115984 \\ 179 & 1 & 0 & -6.559348 & 1.010415 & -6.712267\end{array}$

$\begin{array}{cccc}\text { Frequencies -- } & 8.8639 & 12.4468 & 19.0733 \\ \text { Frequencies -- } & 20.7079 & 23.6541 & 24.3654 \\ \text { Frequencies -- } & 27.8710 & 28.7406 & 31.4018 \\ \text { Frequencies -- } & 32.9612 & 35.9684 & 37.1273 \\ \text { Frequencies -- } & 40.2870 & 41.0594 & 42.6379 \\ \text { Frequencies -- } & 44.9607 & 46.5297 & 48.2715 \\ \text { Frequencies -- } & 53.3008 & 54.1272 & 58.4884 \\ \text { Frequencies -- } & 61.1485 & 62.2120 & 64.7427 \\ \text { Frequencies -- } & 66.0417 & 67.5098 & 70.1412 \\ \text { Frequencies -- } & 72.5810 & 77.3656 & 78.6369 \\ \text { Frequencies -- } & 79.5837 & 81.4691 & 82.1158 \\ \text { Frequencies -- } & 84.0282 & 90.5929 & 95.4302 \\ \text { Frequencies -- } & 98.5766 & 99.3080 & 101.7792 \\ \text { Frequencies -- } & 104.7669 & 110.8497 & 113.1186 \\ \text { Frequencies -- } & 117.5956 & 120.0250 & 126.0408 \\ \text { Frequencies -- } & 130.1342 & 131.9988 & 136.0778 \\ \text { Frequencies -- } & 138.7324 & 140.4523 & 143.9839 \\ \text { Frequencies -- } & 146.8277 & 154.9668 & 158.7079 \\ \text { Frequencies -- } & 165.7554 & 171.9356 & 175.0380\end{array}$




\begin{tabular}{|c|c|c|c|}
\hline Frequencies -- & 179.6764 & 186.2973 & 189.3457 \\
\hline Frequencies -- & 195.3081 & 198.8426 & 206.9547 \\
\hline Frequencies -- & 215.4331 & 224.4213 & 226.1610 \\
\hline Frequencies -- & 230.3882 & 235.1114 & 236.8572 \\
\hline Frequencies -- & 241.1885 & 244.6586 & 250.1392 \\
\hline Frequencies -- & 251.1847 & 258.6324 & 272.3469 \\
\hline Frequencies -- & 274.1437 & 276.7194 & 277.5691 \\
\hline Frequencies -- & 282.3177 & 285.7947 & 291.7571 \\
\hline Frequencies -- & 294.2071 & 295.0353 & 300.6213 \\
\hline Frequencies -- & 302.2189 & 303.9389 & 306.5432 \\
\hline Frequencies -- & 308.5324 & 312.4475 & 316.3440 \\
\hline Frequencies -- & 318.1150 & 321.7699 & 323.0277 \\
\hline Frequencies -- & 323.3363 & 330.7383 & 335.8306 \\
\hline Frequencies -- & 339.4067 & 348.4409 & 355.1380 \\
\hline Frequencies -- & 371.2257 & 377.3021 & 389.2043 \\
\hline Frequencies -- & 400.9643 & 403.4869 & 410.1970 \\
\hline Frequencies -- & 411.1996 & 412.7589 & 413.4878 \\
\hline Frequencies -- & 415.1941 & 417.2754 & 417.8015 \\
\hline Frequencies -- & 419.8508 & 422.3105 & 425.6836 \\
\hline Frequencies -- & 430.8036 & 434.2108 & 438.3118 \\
\hline Frequencies -- & 440.6582 & 443.2063 & 449.9082 \\
\hline Frequencies -- & 450.8966 & 453.4987 & 455.9291 \\
\hline Frequencies -- & 457.0789 & 466.2324 & 472.4140 \\
\hline Frequencies -- & 486.9576 & 490.6359 & 497.8756 \\
\hline Frequencies -- & 501.6636 & 507.8430 & 511.8355 \\
\hline Frequencies -- & 512.3619 & 517.4182 & 521.7659 \\
\hline Frequencies -- & 524.3686 & 533.0716 & 534.2641 \\
\hline Frequencies -- & 536.1613 & 537.0254 & 543.8043 \\
\hline Frequencies -- & 545.7201 & 550.7249 & 552.0930 \\
\hline Frequencies -- & 555.7321 & 560.7003 & 561.7806 \\
\hline Frequencies -- & 563.0137 & 566.9911 & 570.8743 \\
\hline Frequencies -- & 572.7012 & 583.3968 & 586.0388 \\
\hline Frequencies -- & 589.6277 & 592.8451 & 597.4831 \\
\hline Frequencies -- & 599.0667 & 601.0102 & 603.0891 \\
\hline Frequencies -- & 605.3285 & 610.0044 & 610.5799 \\
\hline Frequencies -- & 612.3506 & 617.4690 & 619.8061 \\
\hline Frequencies -- & 621.7242 & 625.2757 & 627.4335 \\
\hline Frequencies -- & 629.5436 & 631.0210 & 632.9013 \\
\hline Frequencies -- & 633.5787 & 636.9334 & 644.2098 \\
\hline Frequencies -- & 651.2874 & 656.9185 & 670.0924 \\
\hline Frequencies -- & 671.4520 & 673.2529 & 677.5024 \\
\hline Frequencies -- & 680.2808 & 682.9621 & 695.8880 \\
\hline Frequencies -- & 699.6193 & 703.0498 & 709.9706 \\
\hline Frequencies -- & 715.5252 & 716.0877 & 716.3840 \\
\hline Frequencies -- & 717.7242 & 717.8713 & 720.4657 \\
\hline Frequencies -- & 723.3167 & 724.0469 & 724.8955 \\
\hline Frequencies -- & 725.6640 & 730.9962 & 731.7921 \\
\hline Frequencies -- & 735.3484 & 740.3404 & 745.0385 \\
\hline Frequencies -- & 746.9130 & 753.2294 & 764.6785 \\
\hline Frequencies -- & 768.6633 & 768.7816 & 770.6090 \\
\hline Frequencies -- & 771.3943 & 774.8672 & 776.0820 \\
\hline Frequencies -- & 776.6636 & 781.0513 & 781.6448 \\
\hline Frequencies -- & 784.6882 & 785.3807 & 795.3186 \\
\hline Frequencies -- & 798.5620 & 801.3412 & 805.3892 \\
\hline Frequencies -- & 805.7323 & 819.7635 & 826.0275 \\
\hline Frequencies -- & 827.3045 & 829.5847 & 847.2916 \\
\hline Frequencies -- & 849.1823 & 858.8392 & 861.2395 \\
\hline Frequencies -- & 863.4733 & 865.4695 & 867.837 \\
\hline Frequencies -- & 868.3038 & 870.8085 & 873.147 \\
\hline Frequencies -- & 873.3373 & 875.1123 & 876.601 \\
\hline Frequencies -- & 876.8197 & 888.0856 & 888.301 \\
\hline Frequencies -- & 890.0613 & 892.9309 & 907.997 \\
\hline Frequencies -- & 916.4550 & 917.8934 & 922.954 \\
\hline Frequencies -- & 925.5912 & 931.8167 & 933.999 \\
\hline Frequencies -- & 935.3082 & 939.8801 & 940.051 \\
\hline Frequencies -- & 941.9417 & 943.0684 & 945.255 \\
\hline
\end{tabular}

Frequencies -- 947.2982

Frequencies -- 952.1098

Frequencies -- 958.4751

Frequencies -- 975.8853

Frequencies -- 995.4756

Frequencies -- 996.6565

Frequencies -- 1001.8168

Frequencies -- 1011.5606

Frequencies -- 1015.1061

Frequencies -- 1019.5525

Frequencies -- 1023.6624

Frequencies -- 1032.9296

Frequencies -- 1044.7901

Frequencies -- 1053.2684

Frequencies -- 1060.7701

Frequencies -- 1065.0298

Frequencies -- 1069.8736

Frequencies -- 1074.7722

Frequencies -- 1096.9848

Frequencies -- 1111.9038

Frequencies -- 1123.8284

Frequencies -- 1129.6848

Frequencies -- 1160.5256

Frequencies -- 1167.3132

Frequencies -- 1172.5352

Frequencies -- 1176.4716

Frequencies -- 1182.1129

Frequencies -- 1194.8463

Frequencies -- 1202.7846

Frequencies -- 1209.6132

Frequencies -- 1214.7722

Frequencies -- 1224.7784

Frequencies -- 1235.3969

Frequencies -- 1245.1344

Frequencies -- 1255.5453

Frequencies -- 1280.0883

Frequencies -- 1293.3430

Frequencies -- 1298.2902

Frequencies -- 1303.7995

Frequencies -- 1308.0717

Frequencies -- 1311.5952

Frequencies -- 1320.3689

Frequencies -- 1328.7750

Frequencies -- 1336.7199

Frequencies -- 1347.7585

Frequencies -- 1359.6325

Frequencies -- 1364.0758

Frequencies -- 1366.9989

Frequencies -- 1372.7142

Frequencies -- 1384.5218

Frequencies -- 1403.5941

Frequencies -- 1448.6862

Frequencies -- 1470.2378

Frequencies -- 1493.7204

Frequencies -- 1497.4374

Frequencies -- 1504.9924

Frequencies -- 1510.5666

Frequencies -- 1518.6936

Frequencies -- 1529.0648

Frequencies -- 1549.7185

Frequencies -- 1553.6994

Frequencies -- 1560.2950

Frequencies -- 1566.0879

Frequencies -- 1662.8068

Frequencies -- 1669.8393

Frequencies -- 1674.1752

$\begin{array}{ll}950.9344 & 951.7257 \\ 952.7903 & 957.8553 \\ 963.2502 & 971.1121 \\ 987.9274 & 993.7823 \\ 995.5670 & 996.3419 \\ 997.2444 & 999.3588 \\ 1008.2028 & 1010.6563 \\ 1012.1324 & 1014.6677 \\ 1018.8285 & 1019.2145 \\ 1019.8003 & 1020.2287 \\ 1024.2857 & 1028.1704 \\ 1035.7098 & 1037.2595 \\ 1046.1336 & 1049.8245 \\ 1057.0975 & 1058.5136 \\ 1061.0276 & 1062.9311 \\ 1068.0141 & 1069.3028 \\ 1071.2688 & 1072.3422 \\ 1092.8761 & 1096.2382 \\ 1110.7247 & 1110.8138 \\ 1113.2460 & 1119.3202 \\ 1124.9168 & 1127.6323 \\ 1130.8499 & 1132.5087 \\ 1166.7667 & 1167.0442 \\ 1169.3626 & 1170.4566 \\ 1173.2174 & 1175.3861 \\ 1178.0699 & 1178.3936 \\ 1183.9059 & 1185.0389 \\ 1199.7958 & 1202.5429 \\ 1204.9973 & 1207.4620 \\ 1211.6196 & 1211.9408 \\ 1215.6969 & 1221.8507 \\ 1228.8719 & 1232.9537 \\ 1238.3590 & 1243.3933 \\ 1247.5741 & 1253.3930 \\ 1257.4884 & 1267.4136 \\ 1282.1759 & 1289.1011 \\ 1293.9958 & 1297.9532 \\ 1299.3847 & 1301.4000 \\ 1305.5104 & 1307.0279 \\ 1309.4024 & 1311.0813 \\ 1314.5320 & 1317.0460 \\ 1324.2121 & 1327.3556 \\ 1332.2027 & 1333.6326 \\ 1341.1374 & 1343.6939 \\ 1348.5545 & 1358.6647 \\ 1360.6031 & 1363.6676 \\ 1365.0300 & 1366.7501 \\ 1368.6214 & 1370.8484 \\ 1373.6412 & 1376.6747 \\ 1386.7297 & 1389.0296 \\ 1424.1407 & 1435.6512 \\ 1458.1858 & 1464.9056 \\ 1479.7415 & 1493.1092 \\ 1494.1011 & 1497.1513 \\ 1500.5494 & 1502.7562 \\ 1506.6894 & 1508.0464 \\ 1511.2182 & 1516.2168 \\ 1520.4817 & 1526.8059 \\ 1532.0254 & 1548.3391 \\ 1550.9857 & 1552.7700 \\ 1554.8674 & 1559.4326 \\ 1562.4744 & 1564.1866 \\ 1572.5263 & 1657.1603 \\ 1668.3782 & 1668.5542 \\ 1672.1207 & 1674.1038 \\ 1678.7281 & 1679.4580\end{array}$




$\begin{array}{llcc}\text { Frequencies -- } & 1683.6334 & 1687.7375 & 1690.6082 \\ \text { Frequencies -- } 1691.0565 & 1694.9062 & 1696.7421 \\ \text { Frequencies -- } 1697.9183 & 1698.9899 & 1699.2656 \\ \text { Frequencies -- } 1700.5866 & 1701.5046 & 1702.7881 \\ \text { Frequencies -- } 1703.7733 & 1704.3639 & 1719.2121 \\ \text { Frequencies -- } 1722.5182 & 1730.0656 & 1737.0533 \\ \text { Frequencies -- } 2281.6037 & 3079.7842 & 3079.9190 \\ \text { Frequencies -- } 3086.8408 & 3092.4186 & 3132.1048 \\ \text { Frequencies -- } 3145.3900 & 3151.0634 & 3154.5246 \\ \text { Frequencies -- } 3183.0963 & 3186.9339 & 3195.5326 \\ \text { Frequencies -- } 3197.9503 & 3202.1843 & 3204.3047 \\ \text { Frequencies -- } 3206.3708 & 3206.4822 & 3208.2920 \\ \text { Frequencies -- } 3213.8072 & 3214.3723 & 3214.6986 \\ \text { Frequencies -- } 3216.4166 & 3217.5214 & 3218.0879 \\ \text { Frequencies -- } 3218.5845 & 3219.9541 & 3223.2718 \\ \text { Frequencies -- } 3224.0448 & 3224.4385 & 3225.6282 \\ \text { Frequencies -- } 3225.8925 & 3228.5209 & 3229.6452 \\ \text { Frequencies -- } 3230.7426 & 3231.6861 & 3232.4811 \\ \text { Frequencies -- } 3232.4913 & 3232.8526 & 3232.8912 \\ \text { Frequencies -- } 3233.4465 & 3233.5426 & 3235.3700 \\ \text { Frequencies -- } 3236.3856 & 3236.8651 & 3237.1021 \\ \text { Frequencies -- } 3238.7110 & 3241.2543 & 3241.6132 \\ \text { Frequencies -- } 3244.2895 & 3245.8851 & 3246.4224 \\ \text { Frequencies -- } 3247.5767 & 3248.4736 & 3249.0617 \\ \text { Frequencies -- } 3251.6502 & 3251.9492 & 3252.8465 \\ \text { Frequencies -- } 3257.6975 & 3278.2045 & 3635.0847 \\ & & \\ \text { SCF Done: E(RM062X/DGDZVP) } & -6499.39696055 \\ \text { Sum of electronic and zero-point Energies= } & -6498.046728 \\ \text { Sum of electronic and thermal Energies= } & -6497.932205 \\ \text { Sum of electronic and thermal } & \text { Free Energies= } & -6498.206737 \\ \text { SCF Done: E(RM062X/DGTZVP/SMD) }=-6500.68236596 \\ \end{array}$

\section{Cat f TS1b_S}

Center Atomic Atomic Coordinates (Angstroms) Number Number Type $\quad X \quad$ Y $\quad Z$

$\begin{array}{cccccc}1 & 15 & 0 & 0.052661 & 0.008195 & -0.056709 \\ 2 & 8 & 0 & 0.020621 & 0.009593 & 1.567332 \\ 3 & 8 & 0 & 1.646649 & -0.005739 & -0.361149 \\ 4 & 8 & 0 & -0.414758 & 1.325591 & -0.610821 \\ 5 & 8 & 0 & -0.674495 & -1.228451 & -0.476816 \\ 6 & 6 & 0 & 0.561473 & 1.085653 & 2.238522 \\ 7 & 6 & 0 & 2.382594 & -1.096974 & 0.066472 \\ 8 & 6 & 0 & 1.913912 & 1.067670 & 2.551731 \\ 9 & 6 & 0 & -0.246456 & 2.184675 & 2.573297 \\ 10 & 6 & 0 & 2.902791 & -1.114933 & 1.353150 \\ 11 & 6 & 0 & 2.516325 & -2.205996 & -0.778022 \\ 12 & 6 & 0 & 2.928968 & -0.055924 & 2.440563 \\ 13 & 6 & 0 & 2.493259 & 2.186430 & 3.152242 \\ 14 & 6 & 0 & 0.347293 & 3.261563 & 3.243225 \\ 15 & 6 & 0 & -1.680772 & 2.183981 & 2.185095 \\ 16 & 6 & 0 & 3.519883 & -2.278360 & 1.814145 \\ 17 & 6 & 0 & 3.236697 & -3.314662 & -0.326764 \\ 18 & 6 & 0 & 1.762339 & -2.223708 & -2.053189 \\ 19 & 6 & 0 & 2.902519 & -0.957757 & 3.703668 \\ 20 & 6 & 0 & 4.247862 & 0.756778 & 2.395011 \\ 21 & 6 & 0 & 4.001656 & 2.004778 & 3.276920 \\ 22 & 6 & 0 & 1.712475 & 3.280988 & 3.541020 \\ 23 & 1 & 0 & -0.267634 & 4.105150 & 3.541920 \\ 24 & 6 & 0 & -2.266404 & 3.279329 & 1.537023 \\ 25 & 6 & 0 & -2.487194 & 1.064143 & 2.399042 \\ 26 & 6 & 0 & 3.704373 & -2.229973 & 3.324927 \\ 27 & 6 & 0 & 3.751138 & -3.368175 & 0.969932\end{array}$

$3.364057-4.168335-0.987113$ $\begin{array}{llll}0.816802 & -3.225472 & -2.280586\end{array}$ $\begin{array}{llll}1.851093 & -1.192933 & -2.984858\end{array}$ $\begin{array}{lll}3.303553 & -0.452643 & 4.587941\end{array}$ $\begin{array}{lll}1.864345 & -1.233944 & 3.917735\end{array}$ $\begin{array}{llll}5.110955 & 0.171091 & 2.725799\end{array}$ $\begin{array}{lll}4.433711 & 1.067018 & 1.361943\end{array}$ $\begin{array}{llll}4.253699 & 1.774771 & 4.319779\end{array}$ $\begin{array}{lll}4.810348 & 3.213884 & 2.852377\end{array}$ $\begin{array}{llll}2.313067 & 4.419698 & 4.286381\end{array}$ $\begin{array}{llll}-3.575835 & 3.234850 & 1.044880\end{array}$ $\begin{array}{llll}-1.479932 & 4.397151 & 1.369704\end{array}$ $\begin{array}{lll}-3.766886 & 0.985386 & 1.859429\end{array}$ $\begin{array}{lll}-2.035327 & 0.014353 & 3.161030\end{array}$ $\begin{array}{llll}4.760992 & -2.109059 & 3.592509\end{array}$ $\begin{array}{lll}3.176213 & -3.488314 & 3.986691\end{array}$ $\begin{array}{llll}4.475270 & -4.565313 & 1.472241\end{array}$ $\begin{array}{llll}-0.043904 & -3.161210 & -3.368287\end{array}$ $0.725402-4.249439-1.366506$ $\begin{array}{llll}0.981694 & -1.109367 & -4.073722\end{array}$ $\begin{array}{llll}2.808664 & -0.219484 & -2.810907\end{array}$ $\begin{array}{llll}4.725455 & 3.700463 & 1.543303\end{array}$ $\begin{array}{llll}5.643771 & 3.875350 & 3.756893\end{array}$ $\begin{array}{llll}2.346090 & 5.705340 & 3.738804\end{array}$ $\begin{array}{llll}2.875082 & 4.203511 & 5.549314\end{array}$ $\begin{array}{lll}-4.331795 & 2.064546 & 1.179802\end{array}$ $\begin{array}{lll}-4.133472 & 4.303942 & 0.397382\end{array}$ $\begin{array}{lll}-2.103903 & 5.522610 & 0.876065\end{array}$ $\begin{array}{lll}-4.482072 & -0.174429 & 1.996490\end{array}$ $\begin{array}{llll}-2.426583 & -1.209056 & 2.640089\end{array}$ $\begin{array}{llll}1.888179 & -3.944000 & 3.676674\end{array}$ $\begin{array}{lll}3.961570 & -4.240320 & 4.859596\end{array}$ $\begin{array}{llll}3.844863 & -5.811645 & 1.538806\end{array}$ $\begin{array}{lll}5.785882 & -4.438907 & 1.945321\end{array}$ $\begin{array}{llll}0.021686 & -2.100330 & -4.276313\end{array}$ $\begin{array}{llll}-1.023260 & -4.109289 & -3.556863\end{array}$ $\begin{array}{llll}-0.463684 & -4.939870 & -1.363632\end{array}$ $\begin{array}{llll}1.078011 & -0.030849 & -4.915484\end{array}$ $\begin{array}{llll}2.385849 & 1.031816 & -3.216541\end{array}$ $\begin{array}{llll}5.461947 & 4.815902 & 1.147243\end{array}$ $\begin{array}{llll}4.070756 & 3.207255 & 0.825442\end{array}$ $\begin{array}{lll}6.366975 & 5.003669 & 3.372253\end{array}$ $\begin{array}{llll}5.714587 & 3.513688 & 4.779853\end{array}$ $\begin{array}{llll}2.972563 & 6.747630 & 4.421225\end{array}$ $\begin{array}{lll}1.893534 & 5.879119 & 2.765183\end{array}$ $\begin{array}{llll}3.484079 & 5.248335 & 6.241737\end{array}$ $\begin{array}{llll}2.826475 & 3.210308 & 5.990125\end{array}$ $\begin{array}{llll}-5.746848 & 1.887480 & 0.677126\end{array}$ $\begin{array}{llll}-3.404530 & 5.470715 & 0.381978\end{array}$ $\begin{array}{llll}-1.396041 & 6.715818 & 0.863218\end{array}$ $\begin{array}{lll}-3.669865 & -1.300775 & 2.025837\end{array}$ $\begin{array}{lll}-1.586895 & -2.308793 & 2.707107\end{array}$ $\begin{array}{llll}1.394832 & -5.119482 & 4.234644\end{array}$ $\begin{array}{llll}1.284717 & -3.388934 & 2.959088\end{array}$ $\begin{array}{lll}3.475554 & -5.427036 & 5.412565\end{array}$ $\begin{array}{llll}4.972369 & -3.911598 & 5.090178\end{array}$ $\begin{array}{llll}4.504753 & -6.906644 & 2.093821\end{array}$ $\begin{array}{llll}2.821704 & -5.908741 & 1.183809\end{array}$ $\begin{array}{llll}6.452290 & -5.536053 & 2.487943\end{array}$ $\begin{array}{llll}6.283246 & -3.473860 & 1.879710\end{array}$ $\begin{array}{llll}-0.970307 & -2.094865 & -5.414344\end{array}$ $\begin{array}{llll}-1.338878 & -4.863336 & -2.444550\end{array}$ $\begin{array}{llll}-0.757079 & -5.758060 & -0.282646\end{array}$ $\begin{array}{llll}1.495534 & 1.125751 & -4.281256\end{array}$ $\begin{array}{llll}2.867543 & 2.175074 & -2.595368\end{array}$ $\begin{array}{llll}6.280962 & 5.476714 & 2.063523\end{array}$ 


\begin{tabular}{|c|c|c|c|c|c|}
\hline 94 & 1 & 0 & 5.392196 & 5.172346 & 0.122709 \\
\hline 95 & 1 & 0 & 6.996936 & 5.511918 & 4.096012 \\
\hline 96 & 6 & 0 & 3.548495 & 6.520017 & 5.671397 \\
\hline 97 & 1 & 0 & 3.013532 & 7.737010 & 3.975725 \\
\hline 98 & 1 & 0 & 3.912391 & 5.070199 & 7.223663 \\
\hline 99 & 9 & 0 & -6.596138 & 1.676861 & 1.694343 \\
\hline 100 & 9 & 0 & -6.207366 & 2.929765 & -0.017879 \\
\hline 101 & 9 & 0 & -5.841028 & 0.813913 & -0.139249 \\
\hline 102 & 6 & 0 & -4.001206 & 6.606581 & -0.146078 \\
\hline 103 & 1 & 0 & -0.378688 & 6.721369 & 1.246518 \\
\hline 104 & 6 & 0 & -1.991503 & 7.861780 & 0.336355 \\
\hline 105 & 6 & 0 & -4.086143 & -2.481839 & 1.431094 \\
\hline 106 & 1 & 0 & -0.620417 & -2.196988 & 3.186863 \\
\hline 107 & 6 & 0 & -2.007140 & -3.507048 & 2.133279 \\
\hline 108 & 6 & 0 & 2.191335 & -5.869605 & 5.103127 \\
\hline 109 & 1 & 0 & 0.392790 & -5.458764 & 3.987033 \\
\hline 110 & 1 & 0 & 6738 & -6.01 & 6.076337 \\
\hline 111 & 6 & 0 & 8307 & -6.7 & 2.573328 \\
\hline 112 & 1 & 0 & 7297 & -7.8 & 2.162234 \\
\hline 113 & 1 & . & 0941 & -5.426825 & 2.847936 \\
\hline 114 & 9 & 0 & -2.239278 & -2.017025 & -4.956523 \\
\hline 115 & 9 & 0 & -0.887420 & -3.229165 & -6.131408 \\
\hline 116 & 9 & 0 & 2101 & -1.083302 & -6.265847 \\
\hline 117 & 6 & 0 & -2.526412 & 30467 & 9110 \\
\hline 118 & 1 & 0 & -0.043872 & 7856 & 4864 \\
\hline 119 & 6 & 0 & -1.938330 & -6.500343 & -0.2 \\
\hline 120 & 6 & 0 & 147 & 1077 & 2778 \\
\hline 121 & 1 & 0 & 1368 & 8991 & 7610 \\
\hline 122 & 6 & 0 & 7227 & 081 & 6812 \\
\hline 123 & 1 & 0 & 6.848818 & 6.350452 & 1.759166 \\
\hline 124 & 1 & 0 & 4.036348 & 7.33 & 1932 \\
\hline 125 & 1 & 0 & -5.010824 & 4777 & 35085 \\
\hline 126 & 6 & 0 & -3.289236 & 7.805848 & 71447 \\
\hline 127 & 1 & 0 & -1.433700 & 8.792306 & 0.318997 \\
\hline 128 & 1 & 0 & -5.045636 & -2.506631 & 0.925144 \\
\hline 129 & 6 & 0 & -3.243129 & -3.592021 & 1.488894 \\
\hline 130 & 1 & 0 & -1.355382 & -4.373951 & 2.165229 \\
\hline 131 & 1 & 0 & 1.813582 & -6.793551 & 5.530018 \\
\hline 132 & 1 & 0 & 6.319562 & -7.623746 & 3.008826 \\
\hline 133 & 1 & 0 & -3.186777 & -5.497208 & -3.296346 \\
\hline 134 & 6 & 0 & -2.824518 & -6.406318 & -1.353925 \\
\hline 135 & 1 & 0 & -2.163395 & -7.149791 & 0.559000 \\
\hline 136 & 1 & 0 & 0.330091 & 2.400612 & -5.537707 \\
\hline 137 & 6 & 0 & 1.539858 & 3.515585 & -4.115148 \\
\hline 138 & 1 & 0 & 2.845779 & 4.326963 & -2.606329 \\
\hline 139 & 1 & 0 & -3.751811 & 8.693289 & -0.589762 \\
\hline 140 & 1 & 0 & -3.544346 & -4.520374 & 1.013823 \\
\hline 141 & 1 & 0 & -3.744738 & -6.980703 & -1.355292 \\
\hline 142 & 1 & 0 & 1.209694 & 4.488650 & -4.466038 \\
\hline 143 & 1 & 0 & -2.375112 & -0.810941 & -0.567067 \\
\hline 144 & 8 & 0 & -3.214074 & -0.579198 & -1.060650 \\
\hline 145 & 6 & 0 & -2.957419 & -0.987791 & -2.437088 \\
\hline 146 & 6 & 0 & -2.707956 & 0.406231 & -3.044958 \\
\hline 147 & 1 & 0 & -2.078847 & -1.631119 & -2.476619 \\
\hline 148 & 1 & 0 & -3.843836 & & -2.817428 \\
\hline 149 & 8 & 0 & & & -3.338758 \\
\hline 150 & 6 & 0 & & & -1.773713 \\
\hline 151 & 1 & 0 & & & -2.508010 \\
\hline 152 & 1 & 0 & -4.218841 & 1.356008 & -1.618098 \\
\hline 153 & 1 & 0 & -2.456377 & 1.537129 & -1.057325 \\
\hline 154 & 1 & 0 & -0.736517 & 2.858134 & -1.099155 \\
\hline 155 & 7 & 0 & -0.782106 & 3.831218 & -1.455812 \\
\hline 156 & 6 & 0 & -1.723015 & 4.266522 & -2.308426 \\
\hline 157 & 6 & 0 & 0.226003 & 4.727347 & -1.135989 \\
\hline 158 & 16 & 0 & -3.095979 & 3.463596 & -2.839859 \\
\hline 159 & 16 & 0 & -1.334000 & 5.898563 & -2.83426 \\
\hline
\end{tabular}

$\begin{array}{rrrrrr}160 & 6 & 0 & 0.101551 & 5.933049 & -1.832020 \\ 161 & 6 & 0 & 1.240559 & 4.518497 & -0.201605 \\ 162 & 6 & 0 & 1.019712 & 6.964410 & -1.635610 \\ 163 & 6 & 0 & 2.142244 & 5.557165 & 0.005284 \\ 164 & 1 & 0 & 1.304410 & 3.572699 & 0.335648 \\ 165 & 6 & 0 & 2.040870 & 6.762249 & -0.709491 \\ 166 & 1 & 0 & 0.929281 & 7.902857 & -2.173375 \\ 167 & 1 & 0 & 2.940037 & 5.433588 & 0.730819 \\ 168 & 1 & 0 & 2.765545 & 7.550933 & -0.532951 \\ 169 & 6 & 0 & -3.554616 & 0.697166 & -4.266448 \\ 170 & 6 & 0 & -4.945126 & 0.572884 & -4.195347 \\ 171 & 6 & 0 & -2.958213 & 1.075487 & -5.467757 \\ 172 & 6 & 0 & -5.733994 & 0.838278 & -5.312563 \\ 173 & 1 & 0 & -5.427559 & 0.273497 & -3.265497 \\ 174 & 6 & 0 & -3.747221 & 1.325547 & -6.589328 \\ 175 & 1 & 0 & -1.878987 & 1.169704 & -5.513404 \\ 176 & 6 & 0 & -5.134754 & 1.213051 & -6.515195 \\ 177 & 1 & 0 & -6.813709 & 0.749679 & -5.243569 \\ 178 & 1 & 0 & -3.274541 & 1.612024 & -7.523827 \\ 179 & 1 & 0 & -5.746777 & 1.416211 & -7.388541 \\ ------------------------------------\end{array}$

Frequencies -- -536.1650

Frequencies -- 20.2953

$14.5663 \quad 18.3956$

Frequencies -- 29.6366

Frequencies -- 35.4727

Frequencies -- 40.0524

Frequencies -- 47.3657

Frequencies -- 52.1250

Frequencies -- 58.2618

Frequencies -- 67.9417

Frequencies -- 74.8677

Frequencies -- 78.5734

Frequencies -- 88.2508

Frequencies -- 97.3509

Frequencies -- 103.5594

Frequencies -- 116.1108

Frequencies -- 127.9022

Frequencies -- 138.8387

Frequencies -- 153.2783

Frequencies -- 165.3926

Frequencies -- 176.6739

Frequencies -- 197.0608

Frequencies -- 208.6689

Frequencies -- 227.5815

Frequencies -- 237.2120

Frequencies -- 254.3597

Frequencies -- 271.9452

Frequencies -- 277.9311

Frequencies -- 287.7637

Frequencies -- 295.6019

Frequencies -- 306.2166

Frequencies -- 317.1396

Frequencies -- 328.4353

Frequencies -- 339.0081

Frequencies -- 353.2126

Frequencies -- 398.5064

Frequencies -- 410.6089

Frequencies -- 414.2209

Frequencies -- 419.0847

Frequencies -- 428.1765

Frequencies -- 441.5531

Frequencies -- 450.8172

Frequencies -- 464.9060

Frequencies -- 473.6206

Frequencies -- 500.7041
$21.5980 \quad 26.5025$

$32.2953 \quad 35.0943$

$37.6086 \quad 38.2773$

$41.4346 \quad 43.5995$

$49.4088 \quad 50.8097$

$55.0032 \quad 55.9538$

$62.1292 \quad 65.2789$

$70.1692 \quad 72.6024$

$76.8797 \quad 78.1724$

$84.7349 \quad 86.7235$

$92.1646 \quad 95.4583$

$99.1617 \quad 102.2562$

$106.5100 \quad 113.0879$

$119.2134 \quad 125.6296$

$130.4621 \quad 136.1459$

$141.5651 \quad 147.1759$

$157.6317 \quad 163.1577$

$168.8911 \quad 172.8180$

$188.3196 \quad 190.8608$

$197.2792 \quad 200.4621$

$217.3079 \quad 224.1307$

$230.5316 \quad 232.8871$

$243.2696 \quad 249.1655$

$256.4596 \quad 267.5147$

$274.6500 \quad 276.8527$

$279.9278 \quad 281.0791$

$290.1021 \quad 293.2671$

$297.5423 \quad 300.3679$

$312.8670 \quad 315.0235$

$321.4446 \quad 324.2815$

$331.2804 \quad 335.8448$

$344.2211 \quad 348.6694$

$375.5528 \quad 387.4340$

$404.8472 \quad 408.9945$

$411.3197 \quad 412.6543$

$414.5066 \quad 415.1763$

$421.1546 \quad 421.8479$

$434.7383 \quad 437.8572$

$444.1168 \quad 448.5571$

$452.8415 \quad 460.2049$

$466.6527 \quad 468.3511$

$486.9808 \quad 490.4285$

$508.2077 \quad 510.3366$ 
Frequencies -- 1175.9663 Frequencies -- 1178.7757 Frequencies -- 1187.1648 Frequencies -- 1200.1071 Frequencies -- 1204.6639 Frequencies -- 1209.0120 Frequencies -- 1220.1271 Frequencies -- 1234.2522 Frequencies -- 1239.6489 Frequencies -- 1252.1664 Frequencies -- 1275.2338 Frequencies -- 1283.1138 Frequencies -- 1293.1946 Frequencies -- 1298.2585 Frequencies -- 1306.9205 Frequencies -- 1311.7056 Frequencies -- 1318.6729 Frequencies -- 1326.9743 Frequencies -- 1338.4204 Frequencies -- 1343.5930 Frequencies -- 1359.7943 Frequencies -- 1362.3859 Frequencies -- 1365.2851 Frequencies -- 1370.2616 Frequencies -- 1386.3717 Frequencies -- 1404.7398 Frequencies -- 1449.9054 Frequencies -- 1467.7011 Frequencies -- 1492.2415 Frequencies -- 1496.9707 Frequencies -- 1501.4092 Frequencies -- 1507.7585 Frequencies -- 1514.1971 Frequencies -- 1530.1467 Frequencies -- 1546.7101 Frequencies -- 1554.4362 Frequencies -- 1560.0152 Frequencies -- 1568.3205 Frequencies -- 1662.8322 Frequencies -- 1671.8334 Frequencies -- 1677.9492 Frequencies -- 1685.5518 Frequencies -- 1692.9071 Frequencies -- 1697.2391 Frequencies -- 1700.9668 Frequencies -- 1704.1748 Frequencies -- 1722.9416 Frequencies -- 3077.8636 Frequencies -- 3092.9530 Frequencies -- 3155.7979 Frequencies -- 3181.9449 Frequencies -- 3202.7441 Frequencies -- 3203.8200 Frequencies -- 3211.3595 Frequencies -- 3214.7946 Frequencies -- 3217.5966 Frequencies -- 3222.7164 Frequencies -- 3227.4336 Frequencies -- 3229.8993 Frequencies -- 3232.0386 Frequencies -- 3237.2563 Frequencies -- 3238.6696 Frequencies -- 3241.3194 Frequencies -- 3243.9505 Frequencies -- 3247.8091 Frequencies -- 3250.1148

$\begin{array}{ll}1176.8717 & 1178.1974 \\ 1179.0138 & 1179.2105 \\ 1193.7343 & 1193.9592 \\ 1201.4618 & 1204.5631 \\ 1205.2304 & 1206.4375 \\ 1212.0440 & 1215.6090 \\ 1225.5277 & 1228.0452 \\ 1237.5865 & 1239.4283 \\ 1244.3983 & 1246.0619 \\ 1255.9814 & 1262.2438 \\ 1276.8542 & 1280.5306 \\ 1286.8228 & 1287.6140 \\ 1294.1335 & 1294.6765 \\ 1299.3743 & 1305.8154 \\ 1308.0141 & 1310.5626 \\ 1313.0375 & 1317.6051 \\ 1321.9598 & 1325.7976 \\ 1329.0145 & 1334.8341 \\ 1340.7894 & 1340.8941 \\ 1354.2158 & 1358.6084 \\ 1361.1378 & 1361.9541 \\ 1363.3955 & 1365.1177 \\ 1365.5047 & 1366.8805 \\ 1373.5315 & 1374.1444 \\ 1388.5903 & 1391.9082 \\ 1420.5694 & 1447.3758 \\ 1458.3766 & 1461.8742 \\ 1472.6708 & 1482.0069 \\ 1493.6845 & 1494.5614 \\ 1498.8240 & 1499.6461 \\ 1504.0374 & 1507.2619 \\ 1510.5413 & 1511.8056 \\ 1520.2868 & 1520.9507 \\ 1533.2350 & 1544.6532 \\ 1550.7644 & 1553.5890 \\ 1556.9591 & 1557.6192 \\ 1561.9115 & 1566.1805 \\ 1573.0786 & 1655.5437 \\ 1667.4393 & 1669.8764 \\ 1672.6809 & 1674.5166 \\ 1677.9655 & 1679.1566 \\ 1687.1663 & 1690.3320 \\ 1694.6223 & 1696.0159 \\ 1699.2037 & 1700.0464 \\ 1701.3607 & 1703.6079 \\ 1704.4057 & 1718.7163 \\ 1725.0694 & 1738.9729 \\ 3080.4097 & 3086.7217 \\ 3149.6094 & 3150.4892 \\ 3181.0384 & 3181.8454 \\ 3184.8552 & 3196.9825 \\ 3203.2148 & 3203.7146 \\ 3206.0299 & 3210.2123 \\ 3213.4097 & 3214.7142 \\ 3216.4583 & 3217.1448 \\ 3220.4437 & 3221.7891 \\ 3226.2978 & 3227.4004 \\ 3228.4018 & 3228.6988 \\ 3230.1696 & 3231.0759 \\ 3235.0939 & 3236.7504 \\ 3238.0607 & 3238.0667 \\ 3239.2289 & 3239.6033 \\ 3242.9975 & 3243.7977 \\ 3244.2388 & 3246.1314 \\ 3248.8624 & 3249.2033 \\ 3256.8112 & 3260.6075\end{array}$


Frequencies -- $3279.1630 \quad 3377.3051 \quad 3662.0271$

SCF Done: $E($ RM062X/DGDZVP $)=-6499.36618258$

Sum of electronic and zero-point Energies $=\quad-6498.015868$

Sum of electronic and thermal Energies $=\quad-6497.901949$

Sum of electronic and thermal Free Energies $=\quad-6498.171987$

SCF Done: $E(R M 062 X / D G T Z V P / S M D)=-6500.64666827$

\section{Cat f I1b_S}

Center Atomic Atomic Coordinates (Angstroms) Number Number Type $X \quad$ Y $\quad Z$

\begin{tabular}{|c|c|c|c|c|c|}
\hline 1 & 15 & 0 & 0.013282 & 0.020951 & 0.056719 \\
\hline 2 & 8 & 0 & 0.028359 & -0.009813 & 1.686511 \\
\hline 3 & 8 & 0 & 1.612016 & 0.097306 & -0.268632 \\
\hline 4 & 8 & 0 & -0.528634 & 1.340211 & -0.429276 \\
\hline 5 & 8 & 0 & -0.614070 & -1.248949 & -0.395705 \\
\hline 6 & 6 & 0 & 0.566446 & 1.071851 & 2.347402 \\
\hline 7 & 6 & 0 & 2.369465 & -1.001760 & 0.100872 \\
\hline 8 & 6 & 0 & 1.925855 & 1.074880 & 2.637234 \\
\hline 9 & 6 & 0 & -0.246044 & 2.165805 & 2.684071 \\
\hline 10 & 6 & 0 & 2.920494 & -1.057997 & 1.374929 \\
\hline 11 & 6 & 0 & 2.478709 & -2.096194 & -0.767829 \\
\hline 12 & 6 & 0 & 2.947879 & -0.037511 & 2.498226 \\
\hline 13 & 6 & 0 & 2.502702 & 2.201485 & 3.225597 \\
\hline 14 & 6 & 0 & 0.346888 & 3.258355 & 3.328714 \\
\hline 15 & 6 & 0 & -1.677541 & 2.153894 & 2.286426 \\
\hline 16 & 6 & 0 & 3.555830 & -2.227736 & 1.792027 \\
\hline 17 & 6 & 0 & 3.228390 & -3.205159 & -0.366510 \\
\hline 18 & 6 & 0 & 1.663055 & -2.128768 & -2.004023 \\
\hline 19 & 6 & 0 & 2.934720 & -0.980353 & 3.730151 \\
\hline 20 & 6 & 0 & 4.264710 & 0.778063 & 2.471442 \\
\hline 21 & 6 & 0 & 4.012460 & 2.021014 & 3.357049 \\
\hline 22 & 6 & 0 & 1.715504 & 3.294032 & 3.610100 \\
\hline 23 & 1 & 0 & -0.272755 & 4.101117 & 3.621703 \\
\hline 24 & 6 & 0 & -2.228888 & 3.212562 & 1.557180 \\
\hline 25 & 6 & 0 & -2.490309 & 1.041976 & 2.501732 \\
\hline 26 & 6 & 0 & 3.749894 & -2.228736 & 3.302875 \\
\hline 27 & 6 & 0 & 3.781936 & -3.288921 & 0.911659 \\
\hline 28 & 1 & 0 & 3.340149 & -4.042287 & -1.050477 \\
\hline 29 & 6 & 0 & 0.732220 & -3.153732 & -2.182084 \\
\hline 30 & 6 & 0 & 1.676813 & -1.094625 & -2.934680 \\
\hline 31 & 1 & 0 & 3.335482 & -0.500423 & 4.628669 \\
\hline 32 & 1 & 0 & 1.900723 & -1.275060 & 3.938961 \\
\hline 33 & 1 & 0 & 5.125048 & 0.191259 & 2.807203 \\
\hline 34 & 1 & 0 & 4.461310 & 1.090606 & 1.441750 \\
\hline 35 & 1 & 0 & 4.253024 & 1.785263 & 4.401634 \\
\hline 36 & 6 & 0 & 4.832188 & 3.228117 & 2.947519 \\
\hline 37 & 6 & 0 & 2.311313 & 4.444070 & 4.344066 \\
\hline 38 & 6 & 0 & -3.506095 & 3.142640 & 1.002829 \\
\hline 39 & 8 & 0 & -1.416220 & 4.305841 & 1.322410 \\
\hline 40 & 6 & 0 & -3.751728 & 0.946123 & 1.917894 \\
\hline 41 & 8 & 0 & -2.054765 & 0.001644 & 3.285190 \\
\hline 42 & 1 & 0 & 4.806057 & -2.104513 & 3.571011 \\
\hline 43 & 6 & 0 & 3.239903 & -3.516312 & 3.921440 \\
\hline 44 & 6 & 0 & 4.525676 & -4.494638 & 1.360043 \\
\hline 45 & 6 & 0 & -0.163458 & -3.123493 & -3.241826 \\
\hline 46 & 8 & 0 & 0.692808 & -4.165216 & -1.250700 \\
\hline 47 & 6 & 0 & 0.767907 & -1.041877 & -3.991874 \\
\hline 48 & 8 & 0 & 2.620501 & -0.100389 & -2.792874 \\
\hline 49 & 6 & 0 & 4.809336 & 3.684798 & 1.625786 \\
\hline 50 & 6 & 0 & 5.616159 & 3.918322 & 3.875117 \\
\hline 51 & 6 & 0 & 2.382869 & 5.716186 & 3.768566 \\
\hline 52 & 6 & 0 & 2.829774 & 4.250585 & 5.629089 \\
\hline
\end{tabular}

\begin{tabular}{|c|c|c|c|c|c|}
\hline 53 & 6 & 0 & -4.286467 & 1.995557 & 1.176305 \\
\hline 54 & 8 & 0 & -3.968715 & 4.145262 & 0.175919 \\
\hline 55 & 6 & 0 & -2.036073 & 5.427293 & 0.825648 \\
\hline 56 & 8 & 0 & -4.464932 & -0.211223 & 2.053038 \\
\hline 57 & 6 & 0 & -2.423108 & -1.229772 & 2.759218 \\
\hline 58 & 6 & 0 & 1.952474 & -3.972025 & 3.608069 \\
\hline 59 & 6 & 0 & 4.043715 & -4.294773 & 4.753667 \\
\hline 60 & 6 & 0 & 3.910626 & -5.750240 & 1.381857 \\
\hline 61 & 6 & 0 & 5.840161 & -4.372158 & 1.823521 \\
\hline 62 & 6 & 0 & -0.145777 & -2.080634 & -4.171589 \\
\hline 63 & 8 & 0 & -1.115806 & -4.102513 & -3.396999 \\
\hline 64 & 6 & 0 & -0.465043 & -4.906357 & -1.219724 \\
\hline 65 & 8 & 0 & 0.784754 & 0.036913 & -4.844491 \\
\hline 66 & 6 & 0 & 2.198574 & 1.129962 & -3.242510 \\
\hline 67 & 6 & 0 & 5.539470 & 4.808951 & 1.242576 \\
\hline 68 & 1 & 0 & 4.210376 & 3.157157 & 0.884960 \\
\hline 69 & 6 & 0 & 6.338592 & 5.051036 & 3.502057 \\
\hline 70 & 1 & 0 & 5.647672 & 3.576318 & 4.906579 \\
\hline 71 & 6 & 0 & 3.002515 & 6.765476 & 4.446590 \\
\hline 72 & 1 & 0 & 1.966131 & 5.874271 & 2.776552 \\
\hline 73 & 6 & 0 & 3.431722 & 5.302825 & 6.316888 \\
\hline 74 & 1 & 0 & 2.753576 & 3.268303 & 6.089714 \\
\hline 75 & 6 & 0 & -5.674343 & 1.800479 & 0.611093 \\
\hline 76 & 6 & 0 & -3.300859 & 5.346435 & 0.247105 \\
\hline 77 & 6 & 0 & -1.361683 & 6.639723 & 0.869898 \\
\hline 78 & 6 & 0 & -3.648070 & -1.332905 & 2.112358 \\
\hline 79 & 6 & 0 & -1.578236 & -2.323241 & 2.855326 \\
\hline 80 & 6 & 0 & 1.479873 & -5.175718 & 4.122480 \\
\hline 81 & 1 & 0 & 1.332233 & -3.394007 & 2.923222 \\
\hline 82 & 6 & 0 & 3.577912 & -5.509174 & 5.262126 \\
\hline 83 & 1 & 0 & 5.053605 & -3.964124 & 4.985624 \\
\hline 84 & 6 & 0 & 4.589188 & -6.858948 & 1.884402 \\
\hline 85 & 1 & 0 & 2.884839 & -5.844414 & 1.033592 \\
\hline 86 & 6 & 0 & 6.525155 & -5.482505 & 2.313557 \\
\hline 87 & 1 & 0 & 6.325648 & -3.399333 & 1.791998 \\
\hline 88 & 6 & 0 & -1.098446 & -2.179236 & -5.339423 \\
\hline 89 & 6 & 0 & -1.368830 & -4.867711 & -2.278453 \\
\hline 90 & 6 & 0 & -0.697833 & -5.736539 & -0.133003 \\
\hline 91 & 6 & 0 & 1.270350 & 1.198483 & -4.278902 \\
\hline 92 & 6 & 0 & 2.740701 & 2.287676 & -2.703007 \\
\hline 93 & 6 & 0 & 6.302211 & 5.502190 & 2.182994 \\
\hline 94 & 1 & 0 & 5.512940 & 5.142302 & 0.208160 \\
\hline 95 & 1 & 0 & 6.928335 & 5.581145 & 4.243836 \\
\hline 96 & 6 & 0 & 3.533149 & 6.560016 & 5.720362 \\
\hline 97 & 1 & 0 & 3.074225 & 7.743108 & 3.979471 \\
\hline 98 & 1 & 0 & 3.826230 & 5.141131 & 7.315659 \\
\hline 99 & 9 & 0 & -6.556658 & 1.539477 & 1.587528 \\
\hline 100 & 9 & 0 & -6.133976 & 2.872456 & -0.053077 \\
\hline 101 & 9 & 0 & -5.726115 & 0.770052 & -0.253026 \\
\hline 102 & 6 & 0 & -3.899375 & 6.473362 & -0.298962 \\
\hline 103 & 1 & 0 & -0.372336 & 6.668921 & 1.319580 \\
\hline 104 & 6 & 0 & -1.953237 & 7.773947 & 0.312814 \\
\hline 105 & 6 & 0 & -4.043006 & -2.520038 & 1.517429 \\
\hline 106 & 1 & 0 & -0.624184 & -2.204615 & 3.358322 \\
\hline 107 & 6 & 0 & -1.978551 & -3.528583 & 2.281629 \\
\hline 108 & 6 & 0 & 2.295145 & -5.952969 & 4.948888 \\
\hline 109 & 1 & 0 & 0.478554 & -5.516033 & 3.873480 \\
\hline 110 & 1 & 0 & 4.223293 & -6.111921 & 5.893942 \\
\hline 111 & 6 & 0 & 5.896543 & -6.727266 & 2.354931 \\
\hline 112 & 1 & 0 & 4.093299 & -7.824283 & 1.918779 \\
\hline 113 & 1 & 0 & 7.546586 & -5.375974 & 2.666573 \\
\hline 114 & 9 & 0 & -2.379841 & -2.044597 & -4.952536 \\
\hline 115 & 9 & 0 & -0.996289 & -3.378721 & -5.940957 \\
\hline 116 & 9 & 0 & -0.866256 & -1.264486 & -6.287393 \\
\hline 117 & 6 & 0 & -2.524756 & -5.633540 & -2.244982 \\
\hline 118 & 1 & 0 & 0.034107 & -5.753846 & 0.669348 \\
\hline
\end{tabular}




\begin{tabular}{|c|c|c|c|c|c|}
\hline 119 & 6 & 0 & -1.846881 & -6.528169 & -0.103599 \\
\hline 120 & 6 & 0 & 0.867913 & 2.426072 & -4.783942 \\
\hline 121 & 1 & 0 & 3.454680 & 2.196015 & -1.890205 \\
\hline 122 & 6 & 0 & 2.370777 & 3.523724 & -3.234246 \\
\hline 123 & 1 & 0 & 6.868355 & 6.380687 & 1.889226 \\
\hline 124 & 1 & 0 & 4.015312 & 7.377242 & 6.247823 \\
\hline 125 & 1 & 0 & -4.884488 & 6.371557 & -0.742989 \\
\hline 126 & 6 & 0 & -3.217312 & 7.690577 & -0.271956 \\
\hline 127 & 1 & 0 & -1.423370 & 8.720451 & 0.340135 \\
\hline 128 & 1 & 0 & -4.985644 & -2.550546 & 0.981432 \\
\hline 129 & 6 & 0 & -3.197883 & -3.625691 & 1.608125 \\
\hline 130 & 1 & 0 & -1.323966 & -4.392296 & 2.337971 \\
\hline 131 & 1 & 0 & 1.932440 & -6.898125 & 5.340987 \\
\hline 132 & 1 & 0 & 6.422833 & -7.591525 & 2.748489 \\
\hline 133 & 1 & 0 & -3.209568 & -5.574318 & -3.084689 \\
\hline 134 & 6 & 0 & -2.762054 & -6.471250 & -1.154325 \\
\hline 135 & 1 & 0 & -2.024837 & -7.185298 & 0.741741 \\
\hline 136 & 1 & 0 & 0.140998 & 2.451373 & -5.589681 \\
\hline 137 & 6 & 0 & 1.434843 & 3.592706 & -4.266907 \\
\hline 138 & 1 & 0 & 2.807262 & 4.433879 & -2.830845 \\
\hline 139 & 1 & 0 & -3.678834 & 8.571930 & -0.704251 \\
\hline 140 & 1 & 0 & -3.481639 & -4.559538 & 1.132935 \\
\hline 141 & 1 & 0 & -3.658628 & -7.081583 & -1.133318 \\
\hline 142 & 1 & 0 & 1.149960 & 4.554584 & -4.682611 \\
\hline 143 & 1 & 0 & -2.359849 & -0.889846 & -0.633659 \\
\hline 144 & 8 & 0 & -3.190929 & -0.394864 & -0.800336 \\
\hline 145 & 6 & 0 & -3.501885 & -0.459125 & -2.176291 \\
\hline 146 & 6 & 0 & -3.001902 & 0.785091 & -2.943681 \\
\hline 147 & 1 & 0 & -3.051776 & -1.336550 & -2.656406 \\
\hline 148 & 1 & 0 & -4.589009 & -0.534258 & -2.268030 \\
\hline 149 & 8 & 0 & -1.589827 & 0.774253 & -3.063739 \\
\hline 150 & 6 & 0 & -3.411189 & 2.038734 & -2.152235 \\
\hline 151 & 1 & 0 & -1.215120 & 0.688875 & -2.164624 \\
\hline 152 & 1 & 0 & -4.487153 & 2.070604 & -1.960699 \\
\hline 153 & 1 & 0 & -2.892964 & 2.063865 & -1.190145 \\
\hline 154 & 1 & 0 & -0.832796 & 2.684276 & -1.170321 \\
\hline 155 & 7 & 0 & -0.813658 & 3.673228 & -1.551348 \\
\hline 156 & 6 & 0 & -1.650685 & 4.205835 & -2.424417 \\
\hline 157 & 6 & 0 & 0.233294 & 4.510375 & -1.164744 \\
\hline 158 & 16 & 0 & -3.095498 & 3.573366 & -3.093782 \\
\hline 159 & 16 & 0 & -1.183404 & 5.811419 & -2.891075 \\
\hline 160 & 6 & 0 & 0.203211 & 5.735539 & -1.833307 \\
\hline 161 & 6 & 0 & 1.202364 & 4.224352 & -0.202078 \\
\hline 162 & 6 & 0 & 1.161975 & 6.718581 & -1.587892 \\
\hline 163 & 6 & 0 & 2.140094 & 5.214498 & 0.059828 \\
\hline 164 & 1 & 0 & 1.198945 & 3.265132 & 0.313909 \\
\hline 165 & 6 & 0 & 2.129785 & 6.440656 & -0.629361 \\
\hline 166 & 1 & 0 & 1.138876 & 7.669647 & -2.109777 \\
\hline 167 & 1 & 0 & 2.898683 & 5.041194 & 0.815061 \\
\hline 168 & 1 & 0 & 2.886239 & 7.185711 & -0.402270 \\
\hline 169 & 6 & 0 & -3.599739 & 0.827505 & -4.338281 \\
\hline 170 & 6 & 0 & -4.988742 & 0.855907 & -4.510536 \\
\hline 171 & 6 & 0 & -2.774414 & 0.885526 & -5.461409 \\
\hline 172 & 6 & 0 & -5.541772 & 0.927093 & -5.788002 \\
\hline 173 & 1 & 0 & -5.653102 & 0.822486 & -3.649375 \\
\hline 174 & 6 & 0 & -3.327438 & 0.947532 & -6.739556 \\
\hline 175 & 1 & 0 & -1.698488 & 0.865468 & -5.323734 \\
\hline 176 & 6 & 0 & -4.711039 & 0.969830 & -6.908125 \\
\hline 177 & 1 & 0 & -6.620735 & 0.944254 & -5.907450 \\
\hline 178 & 1 & 0 & -2.672194 & 0.969585 & -7.605332 \\
\hline 179 & 1 & 0 & -5.140747 & 1.017744 & -7.904031 \\
\hline
\end{tabular}

Frequencies -- 16.2088

Frequencies -- 26.9300

Frequencies -- 34.3766

$\begin{array}{cc}23.2722 & 24.5065 \\ 28.5304 & 29.2366 \\ 35.5432 & 36.4923\end{array}$

\begin{tabular}{|c|c|c|c|}
\hline Frequencies -- & 38.6415 & 39.6321 & 40.4153 \\
\hline Frequencies -- & 43.4979 & 46.8181 & 48.2219 \\
\hline Frequencies -- & 50.8122 & 53.9439 & 58.0681 \\
\hline Frequencies -- & 59.4981 & 60.1991 & 63.4720 \\
\hline Frequencies -- & 64.9387 & 68.0709 & 70.7553 \\
\hline Frequencies -- & 73.7054 & 74.8834 & 76.5074 \\
\hline Frequencies -- & 79.6947 & 84.9297 & 87.1915 \\
\hline Frequencies -- & 88.0531 & 90.3564 & 92.9829 \\
\hline Frequencies -- & 96.5624 & 97.7781 & 98.8785 \\
\hline Frequencies -- & 100.4841 & 103.1982 & 108.6668 \\
\hline Frequencies -- & 108.9198 & 114.0983 & 120.0681 \\
\hline Frequencies -- & 120.3279 & 123.6299 & 128.0893 \\
\hline Frequencies -- & 132.1800 & 133.6040 & 140.9894 \\
\hline Frequencies -- & 141.7193 & 147.9132 & 158.0491 \\
\hline Frequencies -- & 161.5647 & 164.8319 & 166.5209 \\
\hline Frequencies -- & 169.2495 & 172.7394 & 177.6006 \\
\hline Frequencies -- & 188.8377 & 191.3830 & 194.9312 \\
\hline Frequencies -- & 195.5624 & 209.3342 & 213.4123 \\
\hline Frequencies -- & 217.5388 & 225.6541 & 229.9606 \\
\hline Frequencies -- & 234.4791 & 236.3078 & 237.0311 \\
\hline Frequencies -- & 242.4258 & 245.0843 & 247.6240 \\
\hline Frequencies -- & 255.5757 & 256.3163 & 271.9500 \\
\hline Frequencies -- & 273.6686 & 276.6969 & 279.7626 \\
\hline Frequencies -- & 282.1588 & 287.1881 & 289.6167 \\
\hline Frequencies -- & 294.0780 & 295.9420 & 298.3067 \\
\hline Frequencies -- & 301.3734 & 304.5664 & 306.2240 \\
\hline Frequencies -- & 311.7989 & 314.9186 & 318.1905 \\
\hline Frequencies -- & 321.1972 & 322.9353 & 328.2217 \\
\hline Frequencies -- & 330.9664 & 331.8824 & 336.6460 \\
\hline Frequencies -- & 339.0924 & 346.9233 & 349.6159 \\
\hline Frequencies -- & 355.1467 & 376.2411 & 388.6437 \\
\hline Frequencies -- & 393.2240 & 404.8733 & 410.5962 \\
\hline Frequencies -- & 411.3833 & 412.6588 & 414.2150 \\
\hline Frequencies -- & 415.6566 & 418.4418 & 419.6647 \\
\hline Frequencies -- & 421.8780 & 423.5720 & 425.8254 \\
\hline Frequencies -- & 434.6682 & 436.3303 & 441.7602 \\
\hline Frequencies -- & 442.6140 & 447.4771 & 449.6066 \\
\hline Frequencies -- & 450.6712 & 455.1990 & 460.8721 \\
\hline Frequencies -- & 467.3937 & 471.1592 & 472.7671 \\
\hline Frequencies -- & 486.2631 & 491.7368 & 500.9330 \\
\hline Frequencies -- & 502.3137 & 510.2633 & 511.2095 \\
\hline Frequencies -- & 512.7163 & 521.1904 & 523.9518 \\
\hline Frequencies -- & 524.6830 & 524.9553 & 530.8869 \\
\hline Frequencies -- & 534.5001 & 536.1975 & 543.4526 \\
\hline Frequencies -- & 548.3756 & 554.2845 & 556.9417 \\
\hline Frequencies -- & 557.3917 & 559.5996 & 563.2096 \\
\hline Frequencies -- & 567.6753 & 569.9178 & 572.5663 \\
\hline Frequencies -- & 584.1965 & 585.3552 & 588.3777 \\
\hline Frequencies -- & 588.8622 & 592.6730 & 596.6535 \\
\hline Frequencies -- & 599.0294 & 600.4266 & 602.1688 \\
\hline Frequencies -- & 605.0500 & 610.0283 & 610.5452 \\
\hline Frequencies -- & 611.6392 & 614.8908 & 618.0024 \\
\hline Frequencies -- & 622.2604 & 625.5449 & 626.7187 \\
\hline Frequencies -- & 630.1758 & 630.9285 & 632.7879 \\
\hline Frequencies -- & 633.9385 & 636.5274 & 640.4598 \\
\hline Frequencies -- & 644.6026 & 650.5051 & 656.8814 \\
\hline Frequencies -- & 669.6288 & 672.5930 & 675.6031 \\
\hline Frequencies -- & 678.2510 & 683.0259 & 694.9611 \\
\hline Frequencies -- & 697.3248 & 703.8961 & 707.9908 \\
\hline Frequencies -- & 714.0214 & 715.6883 & 717.1569 \\
\hline Frequencies -- & 718.9808 & 719.8533 & 721.4315 \\
\hline Frequencies -- & 723.1178 & 723.6041 & 725.7590 \\
\hline Frequencies -- & 729.1826 & 731.6154 & 734.0067 \\
\hline Frequencies -- & 735.1417 & 735.1855 & 738.8411 \\
\hline Frequencies -- & 744.2477 & 748.1645 & 751.0164 \\
\hline Frequencies -- & 755.9045 & 758.6539 & 767.0168 \\
\hline
\end{tabular}




\begin{tabular}{|c|c|c|c|}
\hline Frequencies -- & 767.8565 & 770.0828 & 771.2018 \\
\hline Frequencies -- & 775.1926 & 779.1973 & 780.2482 \\
\hline Frequencies -- & 780.5821 & 781.4739 & 784.3685 \\
\hline Frequencies -- & 795.3084 & 797.2300 & 799.9864 \\
\hline Frequencies -- & 800.7290 & 801.9533 & 804.1706 \\
\hline Frequencies -- & 806.1317 & 823.2887 & 826.4492 \\
\hline Frequencies -- & 829.1135 & 832.0040 & 858.4411 \\
\hline Frequencies -- & 860.0591 & 860.9567 & 862.2527 \\
\hline Frequencies -- & 864.2275 & 866.0392 & 869.6686 \\
\hline Frequencies -- & 870.6736 & 871.8183 & 874.4196 \\
\hline Frequencies -- & 880.1573 & 882.6198 & 883.4875 \\
\hline Frequencies -- & 887.7107 & 889.6480 & 891.4633 \\
\hline Frequencies -- & 894.4607 & 905.8897 & 911.9776 \\
\hline Frequencies -- & 916.4577 & 921.0959 & 924.9136 \\
\hline Frequencies -- & 930.8056 & 934.5160 & 938.6713 \\
\hline Frequencies -- & 939.7632 & 939.9839 & 942.1109 \\
\hline Frequencies -- & 943.7102 & 944.2605 & 946.3744 \\
\hline Frequencies -- & 948.8215 & 952.1844 & 954.3087 \\
\hline Frequencies -- & 955.1821 & 960.5582 & 966.9406 \\
\hline Frequencies -- & 969.9318 & 978.7929 & 984.5928 \\
\hline Frequencies -- & 986.9440 & 987.1187 & 991.5457 \\
\hline Frequencies -- & 992.7422 & 994.1297 & 997.5831 \\
\hline Frequencies -- & 998.7522 & 1000.5154 & 1005.6777 \\
\hline Frequencies -- & 1007.3262 & 1011.1212 & 1012.4816 \\
\hline Frequencies -- & 1014.9912 & 1018.4367 & 1018.8541 \\
\hline Frequencies -- & 1019.6099 & 1019.9327 & 1021.6533 \\
\hline Frequencies -- & 1023.5167 & 1027.8729 & 1028.4617 \\
\hline Frequencies -- & 1030.9341 & 1034.7274 & 1036.0502 \\
\hline Frequencies -- & 1036.6093 & 1044.3788 & 1051.7547 \\
\hline Frequencies -- & 1053.3703 & 1059.7483 & 1059.8062 \\
\hline Frequencies -- & 1062.1569 & 1064.8190 & 1066.0868 \\
\hline Frequencies -- & 1067.4574 & 1069.3509 & 1070.8261 \\
\hline Frequencies -- & 1071.0916 & 1072.5088 & 1091.8119 \\
\hline Frequencies -- & 1096.8882 & 1109.6453 & 1110.8215 \\
\hline Frequencies -- & 1112.2999 & 1115.0045 & 1118.3941 \\
\hline Frequencies -- & 1120.4714 & 1124.1450 & 1125.0938 \\
\hline Frequencies -- & 1126.3693 & 1127.2719 & 1129.6621 \\
\hline Frequencies -- & 1131.3062 & 1132.9075 & 1142.7731 \\
\hline Frequencies -- & 1153.9375 & 1160.9814 & 1163.3937 \\
\hline Frequencies -- & 1163.7585 & 1164.3487 & 1165.9965 \\
\hline Frequencies -- & 1170.6663 & 1173.2528 & 1174.4462 \\
\hline Frequencies -- & 1175.3344 & 1177.8735 & 1178.3301 \\
\hline Frequencies -- & 1179.1563 & 1180.7728 & 1183.0931 \\
\hline Frequencies -- & 1195.6183 & 1197.2861 & 1199.5895 \\
\hline Frequencies -- & 1201.4766 & 1206.6135 & 1207.5311 \\
\hline Frequencies -- & 1211.2903 & 1212.8579 & 1214.1917 \\
\hline Frequencies -- & 1215.2917 & 1217.0496 & 1221.0692 \\
\hline Frequencies -- & 1225.0102 & 1228.5205 & 1235.9709 \\
\hline Frequencies -- & 1237.0706 & 1238.2496 & 1243.8026 \\
\hline Frequencies -- & 1246.9018 & 1249.7064 & 1252.9677 \\
\hline Frequencies -- & 1253.6028 & 1259.1581 & 1271.1374 \\
\hline Frequencies -- & 1276.0275 & 1277.7282 & 1280.1628 \\
\hline Frequencies -- & 1286.7607 & 1290.0530 & 1293.9421 \\
\hline Frequencies -- & 1295.4176 & 1297.0493 & 1298.6082 \\
\hline Frequencies -- & 1300.8130 & 1301.9491 & 1306.0689 \\
\hline Frequencies -- & 1307.0590 & 1308.0970 & 1309.5370 \\
\hline Frequencies -- & 1310.5872 & 1311.9365 & 1313.2002 \\
\hline Frequencies -- & 1317.7623 & 1321.3327 & 1326.1684 \\
\hline Frequencies -- & 1326.8585 & 1327.5414 & 1334.2373 \\
\hline Frequencies -- & 1337.5621 & 1340.8624 & 1342.0867 \\
\hline Frequencies -- & 1347.6790 & 1357.5337 & 1359.0267 \\
\hline Frequencies -- & 1360.7915 & 1361.6856 & 1361.8683 \\
\hline Frequencies -- & 1364.0285 & 1365.4802 & 1365.9907 \\
\hline Frequencies -- & 1366.3202 & 1366.5680 & 1367.3158 \\
\hline Frequencies -- & 1369.9451 & 1374.1154 & 1384.3034 \\
\hline Frequencies -- & 1387.4757 & 1397.2706 & 1400.2007 \\
\hline
\end{tabular}

Frequencies -- 1404.1688

Frequencies -- 1458.5897

Frequencies -- 1471.7690

Frequencies -- 1493.8167

Frequencies -- 1498.6019

Frequencies -- 1502.8337

Frequencies -- 1506.4369

Frequencies -- 1514.4171

Frequencies -- 1529.3768

Frequencies -- 1546.7793

Frequencies -- 1555.2485

Frequencies -- 1562.6485

Frequencies -- 1569.2680

Frequencies -- 1663.0008

Frequencies -- 1672.5339

Frequencies -- 1677.0672

Frequencies -- 1682.1246

Frequencies -- 1691.3284

Frequencies -- 1696.2275

Frequencies -- 1700.2340

Frequencies -- 1702.9925

Frequencies -- 1723.3716

Frequencies -- 2818.9736

Frequencies -- 3085.2208

Frequencies -- 3124.3819

Frequencies -- 3164.5082

Frequencies -- 3202.2738

Frequencies -- 3209.0348

Frequencies -- 3211.8673

Frequencies -- 3214.4184

Frequencies -- 3216.0099

Frequencies -- 3223.4214

Frequencies -- 3225.1552

Frequencies -- 3228.4936

Frequencies -- 3231.9951

Frequencies -- 3234.1890

Frequencies -- 3236.6196

Frequencies -- 3239.8165

Frequencies -- 3246.8880

Frequencies -- 3250.8610

Frequencies -- 3265.8913
Frequencies -- 3199.1284

\begin{tabular}{ll}
1442.3693 & 1448.5951 \\
1462.0239 & 1467.0073 \\
1488.5297 & 1492.2958 \\
1495.9901 & 1496.2122 \\
1498.7399 & 1500.4748 \\
1503.7649 & 1506.1956 \\
1510.4142 & 1510.6758 \\
1515.1783 & 1519.7168 \\
1533.2788 & 1544.9879 \\
1549.1377 & 1553.9641 \\
1559.8018 & 1561.0436 \\
1564.9986 & 1567.9965 \\
1570.6954 & 1655.1933 \\
1669.1037 & 1669.6979 \\
1673.6173 & 1674.2758 \\
1677.2795 & 1677.3236 \\
1686.4708 & 1689.4384 \\
1692.8003 & 1695.4890 \\
1698.9226 & 1699.0150 \\
1700.4837 & 1702.5701 \\
1703.9928 & 1720.9273 \\
1728.4501 & 1739.2016 \\
3077.4628 & 3077.8769 \\
3087.9589 & 3095.3432 \\
3148.5995 & 3156.7781 \\
3183.8506 & 3183.8820 \\
3199.2195 & 3202.2201 \\
3204.9367 & 3206.7981 \\
3209.3903 & 3211.7599 \\
3212.4268 & 3213.8694 \\
3215.5946 & 3215.6903 \\
3216.3645 & 3222.1318 \\
3224.2935 & 3224.5778 \\
3226.4632 & 3227.7556 \\
3229.7159 & 3231.0712 \\
3233.5162 & 3234.0969 \\
3234.6413 & 3236.4960 \\
3237.7401 & 3238.2193 \\
3242.8895 & 3244.3707 \\
3248.0338 & 3249.7087 \\
3253.2864 & 3254.6722 \\
3559.3416 & 3704.0212 \\
\hline
\end{tabular}

SCF Done: $E(R M 062 X / D G D Z V P)=-6499.42805541$

Sum of electronic and zero-point Energies $=\quad-6498.076245$ Sum of electronic and thermal Energies $=\quad-6497.962393$

Sum of electronic and thermal Free Energies $=\quad-6498.230158$ SCF Done: $E(R M 062 X / D G T Z V P / S M D)=-6500.70849985$

\section{Cat f I0c_S}

Center Atomic Atomic Coordinates (Angstroms)

Number Number Type $\quad X \quad Y \quad Z$

$\begin{array}{cccccc}1 & 15 & 0 & -0.039786 & -0.092427 & 0.115302 \\ 2 & 8 & 0 & -0.027364 & -0.094574 & 1.718269 \\ 3 & 8 & 0 & 1.517618 & -0.032500 & -0.292154 \\ 4 & 8 & 0 & -0.543571 & 1.297606 & -0.338799 \\ 5 & 8 & 0 & -0.714684 & -1.310472 & -0.382069 \\ 6 & 6 & 0 & 0.503239 & 1.017258 & 2.372338 \\ 7 & 6 & 0 & 2.328913 & -1.093065 & 0.091575 \\ 8 & 6 & 0 & 1.875345 & 1.113142 & 2.563631 \\ 9 & 6 & 0 & -0.350029 & 2.068342 & 2.731352 \\ 10 & 6 & 0 & 2.950626 & -1.014175 & 1.332146 \\ 11 & 6 & 0 & 2.481161 & -2.224633 & -0.724673\end{array}$




\begin{tabular}{|c|c|c|c|c|c|}
\hline 12 & 6 & 0 & 3.006700 & 0.127392 & 2.329451 \\
\hline 13 & 6 & 0 & 2.402571 & 2.306268 & 3.062933 \\
\hline 14 & 6 & 0 & 0.200693 & 3.191860 & 3.354584 \\
\hline 15 & 6 & 0 & -1.765941 & 2.073232 & 2.296658 \\
\hline 16 & 6 & 0 & 3.723984 & -2.083240 & 1.784068 \\
\hline 17 & 6 & 0 & 3.346522 & -3.235735 & -0.280304 \\
\hline 18 & 6 & 0 & 1.664189 & -2.425381 & -1.952201 \\
\hline 19 & 6 & 0 & 3.319403 & -0.646908 & 3.637309 \\
\hline 20 & 6 & 0 & 4.168178 & 1.099331 & 1.995221 \\
\hline 21 & 6 & 0 & 3.906626 & 2.374119 & 2.837324 \\
\hline 22 & 6 & 0 & 1.577618 & 3.334937 & 3.522383 \\
\hline 23 & 1 & 0 & -0.457016 & 3.999942 & 3.662396 \\
\hline 24 & 6 & 0 & -2.244009 & 3.157245 & 1.552848 \\
\hline 25 & 6 & 0 & -2.635269 & 1.006874 & 2.508360 \\
\hline 26 & 6 & 0 & 4.189818 & -1.851651 & 3.213093 \\
\hline 27 & 6 & 0 & 3.970504 & -3.187778 & 0.965347 \\
\hline 28 & 1 & 0 & 3.514176 & -4.100058 & -0.915109 \\
\hline 29 & 6 & 0 & 0.955331 & -3.621010 & -2.121524 \\
\hline 30 & 6 & 0 & 1.489246 & -1.441474 & -2.926172 \\
\hline 31 & 1 & 0 & 3.805996 & -0.015050 & 4.386631 \\
\hline 32 & 1 & 0 & 2.379046 & -1.007619 & 4.066147 \\
\hline 33 & 1 & 0 & 5.148724 & 0.655342 & 2.192543 \\
\hline 34 & 1 & 0 & 4.124262 & 1.350054 & 0.930492 \\
\hline 35 & 1 & 0 & 4.458734 & 2.334411 & 3.783285 \\
\hline 36 & 6 & 0 & 4.276059 & 3.652598 & 2.106974 \\
\hline 37 & 6 & 0 & 2.162275 & 4.580581 & 4.082362 \\
\hline 38 & 6 & 0 & -3.524838 & 3.152946 & 1.023681 \\
\hline 39 & 8 & 0 & -1.402953 & 4.219617 & 1.286489 \\
\hline 40 & 6 & 0 & -3.905936 & 0.968335 & 1.923521 \\
\hline 41 & 8 & 0 & -2.220400 & -0.025858 & 3.316558 \\
\hline 42 & 1 & 0 & 5.248652 & -1.562943 & 3.205082 \\
\hline 43 & 6 & 0 & 4.036649 & -3.059438 & 4.114546 \\
\hline 44 & 6 & 0 & 4.855255 & -4.295611 & 1.421290 \\
\hline 45 & 6 & 0 & 0.035155 & -3.801939 & -3.157824 \\
\hline 46 & 8 & 0 & 1.146817 & -4.607656 & -1.181569 \\
\hline 47 & 6 & 0 & 0.603483 & -1.623826 & -3.983182 \\
\hline 48 & 8 & 0 & 2.218989 & -0.278980 & -2.857985 \\
\hline 49 & 6 & 0 & 3.597549 & 3.990942 & 0.928622 \\
\hline 50 & 6 & 0 & 5.237020 & 4.531182 & 2.605717 \\
\hline 51 & 6 & 0 & 1.933027 & 5.811797 & 3.459429 \\
\hline 52 & 6 & 0 & 2.997512 & 4.526821 & 5.203118 \\
\hline 53 & 6 & 0 & -4.367146 & 2.049234 & 1.177145 \\
\hline 54 & 8 & 0 & -3.954707 & 4.212135 & 0.251367 \\
\hline 55 & 6 & 0 & -1.646579 & 4.834310 & 0.076203 \\
\hline 56 & 8 & 0 & -4.685309 & -0.144323 & 2.074799 \\
\hline 57 & 6 & 0 & -2.762033 & -1.253540 & 2.991147 \\
\hline 58 & 6 & 0 & 2.777530 & -3.634447 & 4.324846 \\
\hline 59 & 6 & 0 & 5.146818 & -3.633622 & 4.736101 \\
\hline 60 & 6 & 0 & 4.309018 & -5.525079 & 1.802352 \\
\hline 61 & 6 & 0 & 6.236468 & -4.103584 & 1.514832 \\
\hline 62 & 6 & 0 & -0.144398 & -2.796123 & -4.110430 \\
\hline 63 & 8 & 0 & -0.720734 & -4.941515 & -3.233931 \\
\hline 64 & 6 & 0 & 0.673658 & -5.860892 & -1.502366 \\
\hline 65 & 8 & 0 & 0.448920 & -0.631307 & -4.921947 \\
\hline 66 & 6 & 0 & 1.526271 & 0.816403 & -3.344906 \\
\hline 67 & 6 & 0 & 3.878431 & 5.180907 & 0.264170 \\
\hline 68 & 1 & 0 & 2.822123 & 3.327008 & 0.546504 \\
\hline 69 & 6 & 0 & 5.514615 & 5.730989 & 1.947263 \\
\hline 70 & 1 & 0 & 5.755147 & 4.290314 & 3.531113 \\
\hline 71 & 6 & 0 & 2.552380 & 6.966604 & 3.933610 \\
\hline 72 & 1 & 0 & 1.291018 & 5.851321 & 2.582490 \\
\hline 73 & 6 & 0 & 3.607449 & 5.683216 & 5.685773 \\
\hline 74 & 1 & 0 & 3.161901 & 3.572703 & 5.698618 \\
\hline 75 & 6 & 0 & -5.702121 & 2.093246 & 0.471428 \\
\hline 76 & 6 & 0 & -2.937905 & 4.838808 & -0.444812 \\
\hline 77 & 6 & 0 & -0.612585 & 5.468501 & -0.596324 \\
\hline
\end{tabular}

\begin{tabular}{|c|c|c|c|c|c|}
\hline 78 & 6 & 0 & -3.993835 & -1.309230 & 2.344429 \\
\hline 79 & 6 & 0 & -2.110352 & -2.419712 & 3.362553 \\
\hline 80 & 6 & 0 & 2.634238 & -4.752601 & 5.143809 \\
\hline 81 & 1 & 0 & 1.900299 & -3.206659 & 3.840439 \\
\hline 82 & 6 & 0 & 5.011170 & -4.764486 & 5.541100 \\
\hline 83 & 1 & 0 & 6.132142 & -3.203154 & 4.573594 \\
\hline 84 & 6 & 0 & 5.128629 & -6.541915 & 2.288036 \\
\hline 85 & 1 & 0 & 3.231682 & -5.665555 & 1.742876 \\
\hline 86 & 6 & 0 & 7.059808 & -5.124014 & 1.990943 \\
\hline 87 & 1 & 0 & 6.664519 & -3.151735 & 1.209135 \\
\hline 88 & 6 & 0 & -1.065254 & -2.908543 & -5.305590 \\
\hline 89 & 6 & 0 & -0.274668 & -6.021388 & -2.505581 \\
\hline 90 & 6 & 0 & 1.123761 & -6.952560 & -0.774752 \\
\hline 91 & 6 & 0 & 0.659233 & 0.642990 & -4.420607 \\
\hline 92 & 6 & 0 & 1.709918 & 2.070566 & -2.783028 \\
\hline 93 & 6 & 0 & 4.837012 & 6.059542 & 0.775161 \\
\hline 94 & 1 & 0 & 3.346584 & 5.429331 & -0.650345 \\
\hline 95 & 1 & 0 & 6.253488 & 6.412372 & 2.357946 \\
\hline 96 & 6 & 0 & 3.394534 & 6.904139 & 5.044539 \\
\hline 97 & 1 & 0 & 2.385240 & 7.913946 & 3.430331 \\
\hline 98 & 1 & 0 & 4.249197 & 5.632667 & 6.560270 \\
\hline 99 & 9 & 0 & -6.451083 & 1.007824 & 0.664940 \\
\hline 100 & 9 & 0 & -6.424403 & 3.150866 & 0.882390 \\
\hline 101 & 9 & 0 & -5.543955 & 2.227609 & -0.862643 \\
\hline 102 & 6 & 0 & -3.212651 & 5.468694 & -1.649570 \\
\hline 103 & 1 & 0 & 0.383935 & 5.441938 & -0.164937 \\
\hline 104 & 6 & 0 & -0.886865 & 6.125840 & -1.797124 \\
\hline 105 & 6 & 0 & -4.570920 & -2.527795 & 2.022290 \\
\hline 106 & 1 & 0 & -1.155055 & -2.344313 & 3.873330 \\
\hline 107 & 6 & 0 & -2.714734 & -3.650492 & 3.095787 \\
\hline 108 & 6 & 0 & 3.753257 & -5.327639 & 5.748664 \\
\hline 109 & 1 & 0 & 1.647754 & -5.175600 & 5.313769 \\
\hline 110 & 1 & 0 & 5.889405 & -5.205887 & 6.002456 \\
\hline 111 & 6 & 0 & 6.506680 & -6.342233 & 2.385208 \\
\hline 112 & 1 & 0 & 4.691578 & -7.485433 & 2.601106 \\
\hline 113 & 1 & 0 & 8.132384 & -4.967145 & 2.054976 \\
\hline 114 & 9 & 0 & -2.010614 & -1.947787 & -5.289897 \\
\hline 115 & 9 & 0 & -1.697776 & -4.076975 & -5.391700 \\
\hline 116 & 9 & 0 & -0.371144 & -2.750540 & -6.448240 \\
\hline 117 & 6 & 0 & -0.811876 & -7.272030 & -2.771948 \\
\hline 118 & 1 & 0 & 1.873748 & -6.795007 & -0.006103 \\
\hline 119 & 6 & 0 & 0.580035 & -8.210770 & -1.029312 \\
\hline 120 & 6 & 0 & 0.006159 & 1.724990 & -4.988854 \\
\hline 121 & 1 & 0 & 2.368066 & 2.166819 & -1.925638 \\
\hline 122 & 6 & 0 & 1.047315 & 3.163896 & -3.343864 \\
\hline 123 & 1 & 0 & 5.049738 & 6.993498 & 0.264274 \\
\hline 124 & 1 & 0 & 3.877585 & 7.804303 & 5.412030 \\
\hline 125 & 1 & 0 & -4.228242 & 5.445974 & -2.032103 \\
\hline 126 & 6 & 0 & -2.178937 & 6.122911 & -2.322843 \\
\hline 127 & 1 & 0 & -0.087651 & 6.640470 & -2.321082 \\
\hline 128 & 1 & 0 & -5.521451 & -2.536253 & 1.498258 \\
\hline 129 & 6 & 0 & -3.930021 & -3.705107 & 2.414360 \\
\hline 130 & 1 & 0 & -2.224465 & -4.568230 & 8630 \\
\hline 131 & 1 & 0 & 3.643581 & -6.203678 & 0481 \\
\hline 132 & 1 & 0 & & & \\
\hline 133 & 1 & 0 & -1.563808 & -7.359084 & -3.549558 \\
\hline 134 & 6 & 0 & -0.391863 & -8.368276 & -2.018174 \\
\hline 135 & 1 & 0 & 0.912395 & -9.064490 & -0.448117 \\
\hline 136 & 1 & 0 & -0.655818 & 1.555069 & -5.832411 \\
\hline 137 & 6 & 0 & 0.211595 & 2.994553 & -4.447486 \\
\hline 138 & 1 & 0 & 1.180659 & 4.150092 & -2.911314 \\
\hline 139 & 1 & 0 & -2.388068 & 6.631336 & -3.258037 \\
\hline 140 & 1 & 0 & -4.389528 & -4.663336 & 2.194083 \\
\hline 141 & 1 & 0 & -0.820155 & -9.345905 & -2.212400 \\
\hline 142 & 1 & 0 & -0.299027 & 3.849537 & -4.878351 \\
\hline 143 & 1 & 0 & -1.017128 & 1.335205 & -1.284415 \\
\hline
\end{tabular}




\begin{tabular}{|c|c|c|c|c|c|}
\hline 144 & 8 & 0 & -1.750488 & 1.344664 & -2.506987 \\
\hline 145 & 6 & 0 & -3.118268 & 1.765696 & -2.225776 \\
\hline 146 & 6 & 0 & -3.596634 & 0.303529 & -2.189340 \\
\hline 147 & 1 & 0 & -3.178459 & 2.295689 & -1.277099 \\
\hline 148 & 1 & 0 & -3.504116 & 2.365019 & -3.053988 \\
\hline 149 & 8 & 0 & -3.595480 & -0.094501 & -0.834091 \\
\hline 150 & 6 & 0 & -2.234376 & 0.003318 & -2.855359 \\
\hline 151 & 1 & 0 & -3.457187 & -1.065214 & -0.816091 \\
\hline 152 & 1 & 0 & -2.253712 & -0.096358 & -3.941524 \\
\hline 153 & 1 & 0 & -1.644036 & -0.786536 & -2.384810 \\
\hline 154 & 1 & 0 & -1.267338 & -2.904850 & -0.205800 \\
\hline 155 & 7 & 0 & -1.639754 & -3.868288 & -0.137027 \\
\hline 156 & 6 & 0 & -2.750672 & -4.211947 & -0.825843 \\
\hline 157 & 6 & 0 & -1.119917 & -4.816917 & 0.726659 \\
\hline 158 & 16 & 0 & -3.606040 & -3.255059 & -1.883795 \\
\hline 159 & 16 & 0 & -3.186783 & -5.871529 & -0.425288 \\
\hline 160 & 6 & 0 & -1.857423 & -6.007522 & 0.707491 \\
\hline 161 & 6 & 0 & 0.003755 & -4.670228 & 1.541141 \\
\hline 162 & 6 & 0 & -1.504061 & -7.074191 & 1.532820 \\
\hline 163 & 6 & 0 & 0.353802 & -5.739472 & 2.359200 \\
\hline 164 & 1 & 0 & 0.581025 & -3.747219 & 1.517771 \\
\hline 165 & 6 & 0 & -0.395727 & -6.927092 & 2.363630 \\
\hline 166 & 1 & 0 & -2.075130 & -7.997152 & 1.522159 \\
\hline 167 & 1 & 0 & 1.224050 & -5.652899 & 3.003833 \\
\hline 168 & 1 & 0 & -0.103099 & -7.745613 & 3.013457 \\
\hline 169 & 6 & 0 & -4.860560 & -0.065157 & -2.926725 \\
\hline 170 & 6 & 0 & -4.975218 & 0.162290 & -4.302811 \\
\hline 171 & 6 & 0 & -5.939556 & -0.622772 & -2.238640 \\
\hline 172 & 6 & 0 & -6.146643 & -0.167294 & -4.980273 \\
\hline 173 & 1 & 0 & -4.147145 & 0.596908 & -4.858955 \\
\hline 174 & 6 & 0 & -7.111669 & -0.954416 & -2.916542 \\
\hline 175 & 1 & 0 & -5.858090 & -0.789767 & -1.169070 \\
\hline 176 & 6 & 0 & -7.219375 & -0.729383 & -4.287659 \\
\hline 177 & 1 & 0 & -6.219207 & 0.008769 & -6.049096 \\
\hline 178 & 1 & 0 & -7.941772 & -1.391501 & -2.370161 \\
\hline 179 & 1 & 0 & -8.131117 & -0.991417 & -4.815545 \\
\hline
\end{tabular}

Frequencies -- 15.9512

Frequencies -- 22.8275

Frequencies -- 30.4297

Frequencies -- 35.3831

Frequencies -- 41.9673

Frequencies -- 48.9774

Frequencies -- 56.5115

Frequencies -- 63.0108

Frequencies -- 69.0734

Frequencies -- 74.4793

Frequencies -- 85.9914

Frequencies -- 90.3299

Frequencies -- 98.4728

Frequencies -- 106.6276

Frequencies -- 119.8200

Frequencies -- 131.8128

Frequencies -- 141.4101

Frequencies -- 150.6608

Frequencies -- 169.3445

Frequencies -- 178.1950

Frequencies -- 196.4814

Frequencies -- 216.8754

Frequencies -- 231.7278

Frequencies -- 240.7640

Frequencies -- 252.1180

Frequencies -- 275.2753

Frequencies -- 283.3010

Frequencies -- 297.2308

$\begin{array}{cc}17.4436 & 20.6167 \\ 24.8579 & 26.3977 \\ 32.3133 & 33.3978 \\ 39.5374 & 40.9859 \\ 44.0721 & 47.1162 \\ 50.3321 & 55.8603 \\ 57.6028 & 60.2180 \\ 63.9489 & 64.9193 \\ 71.1584 & 73.2443 \\ 80.5813 & 83.9368 \\ 87.5423 & 89.3580 \\ 94.3423 & 95.1056 \\ 101.4901 & 103.1762 \\ 110.3212 & 116.7364 \\ 123.7016 & 129.1505 \\ 134.4373 & 135.1882 \\ 142.9436 & 147.5843 \\ 157.7756 & 165.2203 \\ 171.7398 & 174.6537 \\ 182.3863 & 192.9331 \\ 201.3169 & 208.0064 \\ 224.5820 & 226.9652 \\ 234.6412 & 239.8060 \\ 242.0369 & 250.2215 \\ 258.4218 & 272.8573 \\ 275.7366 & 278.6959 \\ 286.7465 & 290.1472 \\ 297.6970 & 301.9883\end{array}$

\begin{tabular}{|c|c|c|c|}
\hline Frequencies -- & 303.6910 & 307.2088 & 308.2666 \\
\hline Frequencies -- & 311.7391 & 314.5774 & 317.8478 \\
\hline Frequencies -- & 319.6611 & 322.0723 & 323.1387 \\
\hline Frequencies -- & 324.8559 & 330.9046 & 337.4664 \\
\hline Frequencies -- & 342.6706 & 345.3734 & 356.9737 \\
\hline Frequencies -- & 369.5723 & 375.1487 & 388.7025 \\
\hline Frequencies -- & 401.3916 & 402.5688 & 408.5374 \\
\hline Frequencies -- & 410.4431 & 412.4675 & 414.3433 \\
\hline Frequencies -- & 416.0523 & 416.6659 & 419.4416 \\
\hline Frequencies -- & 421.7851 & 423.4710 & 427.0509 \\
\hline Frequencies -- & 435.1226 & 439.9388 & 442.4465 \\
\hline Frequencies -- & 443.3853 & 447.0069 & 449.7600 \\
\hline Frequencies -- & 452.3821 & 454.9739 & 455.1840 \\
\hline Frequencies -- & 457.1328 & 467.3231 & 472.9172 \\
\hline Frequencies -- & 488.2670 & 489.2932 & 491.0137 \\
\hline Frequencies -- & 502.4158 & 506.9264 & 511.0663 \\
\hline Frequencies -- & 513.0652 & 519.1821 & 521.9548 \\
\hline Frequencies -- & 522.5609 & 533.3139 & 536.5722 \\
\hline Frequencies -- & 537.0806 & 538.7258 & 545.2753 \\
\hline Frequencies -- & 546.9862 & 552.2799 & 553.2090 \\
\hline Frequencies -- & 555.2590 & 560.3660 & 564.2287 \\
\hline Frequencies -- & 565.3291 & 569.9976 & 572.2081 \\
\hline Frequencies -- & 574.3307 & 582.5968 & 586.1099 \\
\hline Frequencies -- & 590.0320 & 592.6507 & 597.7496 \\
\hline Frequencies -- & 598.8664 & 601.1826 & 602.3366 \\
\hline Frequencies -- & 606.3982 & 609.0627 & 610.3850 \\
\hline Frequencies -- & 611.6802 & 616.3717 & 621.2553 \\
\hline Frequencies -- & 622.4813 & 624.6443 & 626.1294 \\
\hline Frequencies -- & 628.6873 & 630.3657 & 632.1349 \\
\hline Frequencies -- & 633.1919 & 635.9412 & 643.7134 \\
\hline Frequencies -- & 651.7652 & 656.3959 & 668.8665 \\
\hline Frequencies -- & 672.6748 & 675.6273 & 678.1943 \\
\hline Frequencies -- & 680.7522 & 683.2188 & 694.7988 \\
\hline Frequencies -- & 699.9667 & 702.7082 & 709.3461 \\
\hline Frequencies -- & 711.6709 & 714.7235 & 716.7001 \\
\hline Frequencies -- & 719.0429 & 720.4143 & 720.9140 \\
\hline Frequencies -- & 724.2608 & 724.4201 & 725.1398 \\
\hline Frequencies -- & 728.0251 & 732.9841 & 735.0002 \\
\hline Frequencies -- & 737.3942 & 740.9347 & 745.7396 \\
\hline Frequencies -- & 747.2311 & 751.6373 & 760.1732 \\
\hline Frequencies -- & 762.3193 & 769.1259 & 770.0070 \\
\hline Frequencies -- & 771.1827 & 773.8744 & 775.1341 \\
\hline Frequencies -- & 777.1240 & 780.1681 & 782.2903 \\
\hline Frequencies -- & 783.4508 & 793.7524 & 795.3925 \\
\hline Frequencies -- & 798.9686 & 800.9704 & 804.4460 \\
\hline Frequencies -- & 804.6228 & 820.3409 & 826.3231 \\
\hline Frequencies -- & 827.3589 & 829.7471 & 847.2914 \\
\hline Frequencies -- & 857.9332 & 858.7610 & 861.7044 \\
\hline Frequencies -- & 863.5985 & 864.3396 & 865.6737 \\
\hline Frequencies -- & 866.6864 & 870.0932 & 870.6785 \\
\hline Frequencies -- & 874.2856 & 878.7836 & 879.2448 \\
\hline Frequencies -- & 885.1985 & 887.4987 & 887.9861 \\
\hline Frequencies -- & 889.4345 & 891.8265 & 907.5995 \\
\hline Frequencies -- & 913.2968 & 919.2588 & 921.5782 \\
\hline Frequencies -- & 923.7039 & 929.5504 & 930.1090 \\
\hline Frequencies -- & 933.6673 & 935.3305 & 938.6138 \\
\hline Frequencies -- & 940.3956 & 942.3083 & 943.1606 \\
\hline Frequencies -- & 944.9477 & 945.8220 & 948.0989 \\
\hline Frequencies -- & 951.3647 & 952.6296 & 952.9662 \\
\hline Frequencies -- & 956.8413 & 958.4982 & 971.1087 \\
\hline Frequencies -- & 976.3055 & 987.9087 & 988.7461 \\
\hline Frequencies -- & 991.6825 & 992.1177 & 993.2555 \\
\hline Frequencies -- & 996.0095 & 999.7238 & 1002.9188 \\
\hline Frequencies -- & 1005.8109 & 1007.3777 & 1009.4159 \\
\hline Frequencies -- & 1011.6766 & 1013.8912 & 1014.7703 \\
\hline Frequencies -- & 1017.0795 & 1018.8651 & 1019.2574 \\
\hline
\end{tabular}




\begin{tabular}{|c|c|c|c|}
\hline Frequencies -- & 1019.4528 & 1019.7939 & 1020.1387 \\
\hline Frequencies -- & 1020.4102 & 1020.6120 & 1025.5417 \\
\hline Frequencies -- & 1028.5232 & 1034.5134 & 1036.1937 \\
\hline Frequencies -- & 1040.4755 & 1047.1621 & 1048.0410 \\
\hline Frequencies -- & 1053.7112 & 1056.4688 & 1057.3962 \\
\hline Frequencies -- & 1061.0784 & 1061.9825 & 1063.4257 \\
\hline Frequencies -- & 1064.4387 & 1067.8134 & 1068.2117 \\
\hline Frequencies -- & 1068.5147 & 1070.3340 & 1071.4028 \\
\hline Frequencies -- & 1074.9953 & 1093.2350 & 1094.2838 \\
\hline Frequencies -- & 1097.9565 & 1107.4037 & 1110.2097 \\
\hline Frequencies -- & 1111.4013 & 1113.3691 & 1122.0264 \\
\hline Frequencies -- & 1122.7169 & 1123.8408 & 1126.3500 \\
\hline Frequencies -- & 1128.4530 & 1131.0812 & 1134.1306 \\
\hline Frequencies -- & 1159.5948 & 1161.7934 & 1164.3439 \\
\hline Frequencies -- & 1167.2773 & 1167.7083 & 1169.8972 \\
\hline Frequencies -- & 1172.4087 & 1173.0248 & 1174.2671 \\
\hline Frequencies -- & 1175.1433 & 1175.4741 & 1177.3064 \\
\hline Frequencies -- & 1177.6496 & 1181.0201 & 1181.1680 \\
\hline Frequencies -- & 1195.2315 & 1199.4490 & 1201.1209 \\
\hline Frequencies -- & 1203.6017 & 1204.2500 & 1205.5786 \\
\hline Frequencies -- & 1211.2569 & 1212.4495 & 1214.1174 \\
\hline Frequencies -- & 1214.9759 & 1217.7378 & 1224.5863 \\
\hline Frequencies -- & 1227.4146 & 1229.2466 & 1230.6082 \\
\hline Frequencies -- & 1235.6009 & 1238.4045 & 1243.6085 \\
\hline Frequencies -- & 1244.7138 & 1247.4293 & 1249.3956 \\
\hline Frequencies -- & 1257.6880 & 1260.3476 & 1266.4593 \\
\hline Frequencies -- & 1280.7695 & 1281.9543 & 1286.7033 \\
\hline Frequencies -- & 1292.8350 & 1295.8688 & 1297.1645 \\
\hline Frequencies -- & 1297.6274 & 1298.8676 & 1300.3744 \\
\hline Frequencies -- & 1302.8451 & 1303.0004 & 1305.3385 \\
\hline Frequencies -- & 1306.6528 & 1308.7852 & 1310.0415 \\
\hline Frequencies -- & 1311.1953 & 1314.2259 & 1316.2236 \\
\hline Frequencies -- & 1321.2315 & 1323.6295 & 1325.4509 \\
\hline Frequencies -- & 1329.9608 & 1332.2575 & 1332.5454 \\
\hline Frequencies -- & 1337.3695 & 1342.2242 & 1343.3219 \\
\hline Frequencies -- & 1345.0363 & 1345.7689 & 1355.7506 \\
\hline Frequencies -- & 1360.1288 & 1361.1953 & 1362.2927 \\
\hline Frequencies -- & 1362.5563 & 1363.1707 & 1364.9337 \\
\hline Frequencies -- & 1365.5699 & 1368.1310 & 1368.8973 \\
\hline Frequencies -- & 1370.2562 & 1373.6902 & 1378.0535 \\
\hline Frequencies -- & 1381.3201 & 1384.7471 & 1387.5463 \\
\hline Frequencies -- & 1403.1128 & 1416.2293 & 1418.5767 \\
\hline Frequencies -- & 1447.4465 & 1457.7734 & 1464.2699 \\
\hline Frequencies -- & 1468.9227 & 1474.8708 & 1489.2360 \\
\hline Frequencies -- & 1489.4612 & 1494.5624 & 1495.9603 \\
\hline Frequencies -- & 1496.6706 & 1500.7565 & 1502.3076 \\
\hline Frequencies -- & 1505.5257 & 1505.8386 & 1509.1454 \\
\hline Frequencies -- & 1510.4854 & 1511.5210 & 1515.5298 \\
\hline Frequencies -- & 1516.1925 & 1517.4340 & 1518.7892 \\
\hline Frequencies -- & 1528.1669 & 1529.4134 & 1545.3966 \\
\hline Frequencies -- & 1549.0171 & 1549.1430 & 1550.6814 \\
\hline Frequencies -- & 1552.5853 & 1554.0798 & 1559.4806 \\
\hline Frequencies -- & 1560.2488 & 1561.6748 & 1562.6418 \\
\hline Frequencies -- & 1567.2926 & 1569.0696 & 1656.8502 \\
\hline Frequencies -- & 1663.2799 & 1666.7647 & 1666.9665 \\
\hline Frequencies -- & 1668.5897 & 1669.0855 & 1673.8788 \\
\hline Frequencies -- & 1674.3011 & 1677.3818 & 1679.2595 \\
\hline Frequencies -- & 1683.6049 & 1688.0246 & 1690.6458 \\
\hline Frequencies -- & 1691.5750 & 1694.5940 & 1696.8687 \\
\hline Frequencies -- & 1699.8229 & 1699.8857 & 1700.1921 \\
\hline Frequencies -- & 1700.9744 & 1701.2057 & 1702.4543 \\
\hline Frequencies -- & 1702.7833 & 1703.4342 & 1717.8749 \\
\hline Frequencies -- & 1720.9435 & 1730.4507 & 1735.0926 \\
\hline Frequencies -- & 2228.5931 & 3077.2263 & 3088.2874 \\
\hline Frequencies -- & 3091.0396 & 3093.1378 & 3132.2840 \\
\hline Frequencies -- & 3142.5084 & 3154.3062 & 3157.8723 \\
\hline
\end{tabular}

$\begin{array}{llll}\text { Frequencies -- } 3179.4874 & 3181.5587 & 3196.5133 \\ \text { Frequencies -- } 3197.0597 & 3198.8917 & 3201.9373 \\ \text { Frequencies -- } 3205.3450 & 3207.3249 & 3209.9081 \\ \text { Frequencies -- } 3210.6746 & 3213.0373 & 3215.0523 \\ \text { Frequencies -- } 3215.6456 & 3216.2683 & 3217.9402 \\ \text { Frequencies -- } 3218.2179 & 3218.5397 & 3219.0846 \\ \text { Frequencies -- } 3220.9967 & 3222.8649 & 3225.2299 \\ \text { Frequencies -- } 3225.8714 & 3227.2912 & 3227.3082 \\ \text { Frequencies -- } 3227.9223 & 3228.3672 & 3228.5270 \\ \text { Frequencies -- } 3229.8143 & 3230.1501 & 3231.9810 \\ \text { Frequencies -- } 3233.1943 & 3233.8498 & 3234.9913 \\ \text { Frequencies -- } 3235.9129 & 3236.2924 & 3236.8996 \\ \text { Frequencies -- } 3238.2884 & 3239.0720 & 3239.6340 \\ \text { Frequencies -- } 3240.4984 & 3241.5211 & 3241.6009 \\ \text { Frequencies -- } 3243.3031 & 3243.3332 & 3247.8976 \\ \text { Frequencies -- } 3251.0468 & 3252.0071 & 3255.1308 \\ \text { Frequencies -- } 3256.1861 & 3262.2097 & 3634.8402\end{array}$

SCF Done: $E($ RM062X/DGDZVP $)=-6499.39694452$

Sum of electronic and zero-point Energies $=\quad-6498.046953$ Sum of electronic and thermal Energies $=\quad-6497.932831$

Sum of electronic and thermal Free Energies $=\quad-6498.203599$ SCF Done: E(RM062X/DGTZVP/SMD) $=-6500.6820721$

\section{Cat f TS1c_S}

Center Atomic Atomic Coordinates (Angstroms)

\begin{tabular}{cccccc}
1 & 15 & 0 & -0.014269 & -0.009930 & 0.010924 \\
2 & 8 & 0 & -0.020468 & -0.004823 & 1.635637 \\
3 & 8 & 0 & 1.573248 & 0.000148 & -0.326187 \\
4 & 8 & 0 & -0.580947 & 1.228542 & -0.602597 \\
5 & 8 & 0 & -0.624025 & -1.336574 & -0.352506 \\
6 & 6 & 0 & 0.506645 & 1.107948 & 2.268745 \\
7 & 6 & 0 & 2.344124 & -1.063171 & 0.097777 \\
8 & 6 & 0 & 1.872019 & 1.181984 & 2.510651 \\
9 & 6 & 0 & -0.333291 & 2.187064 & 2.566607 \\
10 & 6 & 0 & 2.917407 & -0.997542 & 1.360971 \\
11 & 6 & 0 & 2.516914 & -2.194033 & -0.717677 \\
12 & 6 & 0 & 2.978562 & 0.156723 & 2.342673 \\
13 & 6 & 0 & 2.409191 & 2.377955 & 2.988308 \\
14 & 6 & 0 & 0.218305 & 3.330145 & 3.151504 \\
15 & 6 & 0 & -1.749593 & 2.149209 & 2.134798 \\
16 & 6 & 0 & 3.635669 & -2.088821 & 1.847954 \\
17 & 6 & 0 & 3.311298 & -3.241284 & -0.231634 \\
18 & 6 & 0 & 1.817839 & -2.280195 & -2.026406 \\
19 & 6 & 0 & 3.220779 & -0.606529 & 3.671311 \\
20 & 6 & 0 & 4.180205 & 1.087476 & 2.030379 \\
21 & 6 & 0 & 3.923630 & 2.393130 & 2.828377 \\
22 & 6 & 0 & 1.592365 & 3.447557 & 3.365911 \\
23 & 1 & 0 & -0.431965 & 4.163339 & 3.404023 \\
24 & 6 & 0 & -2.244239 & 3.153765 & 1.299760 \\
25 & 6 & 0 & -2.605231 & 1.099207 & 2.457250 \\
26 & 6 & 0 & 4.043801 & -1.863411 & 3.297291 \\
27 & 6 & 0 & 3.876171 & -3.208167 & 1.044769 \\
28 & 1 & 0 & 3.488830 & -4.107968 & -0.861042 \\
29 & 6 & 0 & 1.087680 & -3.420514 & -2.390339 \\
30 & 6 & 0 & 1.807994 & -1.205900 & -2.918812 \\
31 & 1 & 0 & 3.718368 & 0.015614 & 4.421703 \\
32 & 1 & 0 & 2.253279 & -0.911093 & 4.082778 \\
33 & 1 & 0 & 5.139900 & 0.621894 & 2.276099 \\
34 & 1 & 0 & 4.182379 & 1.311615 & 0.958457 \\
35 & 1 & 0 & 4.432747 & 2.357476 & 3.798467 \\
36 & 6 & 0 & 4.369927 & 3.638276 & 2.083708 \\
& & & & & \\
\hline & & &
\end{tabular}




\begin{tabular}{|c|c|c|c|c|c|}
\hline 37 & 6 & 0 & 2.190053 & 4.697947 & 3.901951 \\
\hline 38 & 6 & 0 & -3.526744 & 3.081650 & 0.776414 \\
\hline 39 & 8 & 0 & -1.416771 & 4.201546 & 0.963082 \\
\hline 40 & 6 & 0 & -3.895847 & 1.010194 & 1.927031 \\
\hline 41 & 8 & 0 & -2.159075 & 0.121707 & 3.317363 \\
\hline 42 & 1 & 0 & 5.116426 & -1.636580 & 3.343882 \\
\hline 43 & 6 & 0 & 3.770123 & -3.050643 & 4.199141 \\
\hline 44 & 6 & 0 & 4.712696 & -4.334516 & 1.540543 \\
\hline 45 & 6 & 0 & 0.298587 & -3.455634 & -3.545720 \\
\hline 46 & 8 & 0 & 1.147621 & -4.502836 & -1.539507 \\
\hline 47 & 6 & 0 & 0.981932 & -1.209292 & -4.038001 \\
\hline 48 & 8 & 0 & 2.631396 & -0.124854 & -2.714749 \\
\hline 49 & 6 & 0 & 3.734480 & 3.982249 & 0.883015 \\
\hline 50 & 6 & 0 & 5.357830 & 4.483692 & 2.586998 \\
\hline 51 & 6 & 0 & 2.028536 & 5.910765 & 3.224273 \\
\hline 52 & 6 & 0 & 2.977523 & 4.662972 & 5.057819 \\
\hline 53 & 6 & 0 & -4.369194 & 2.005665 & 1.074496 \\
\hline 54 & 8 & 0 & -3.976103 & 4.039748 & -0.104259 \\
\hline 55 & 6 & 0 & -1.693997 & 4.780734 & -0.255077 \\
\hline 56 & 8 & 0 & -4.674718 & -0.072154 & 2.242568 \\
\hline 57 & 6 & 0 & -2.679159 & -1.128543 & 3.043487 \\
\hline 58 & 6 & 0 & 2.466210 & -3.536477 & 4.349869 \\
\hline 59 & 6 & 0 & 4.804409 & -3.691092 & 4.884223 \\
\hline 60 & 6 & 0 & 4.147247 & -5.591066 & 1.777952 \\
\hline 61 & 6 & 0 & 6.070369 & -4.136581 & 1.810966 \\
\hline 62 & 6 & 0 & 0.212633 & -2.326005 & -4.366493 \\
\hline 63 & 8 & 0 & -0.414278 & -4.569465 & -3.893895 \\
\hline 64 & 6 & 0 & 0.542602 & -5.664909 & -1.967838 \\
\hline 65 & 8 & 0 & 0.928102 & -0.099722 & -4.838339 \\
\hline 66 & 6 & 0 & 2.027253 & 1.071092 & -3.066015 \\
\hline 67 & 6 & 0 & 4.087274 & 5.141426 & 0.199035 \\
\hline 68 & 1 & 0 & 2.927706 & 3.353183 & 0.506185 \\
\hline 69 & 6 & 0 & 5.705958 & 5.654530 & 1.909481 \\
\hline 70 & 1 & 0 & 5.842050 & 4.241545 & 3.530355 \\
\hline 71 & 6 & 0 & 2.666595 & 7.063083 & 3.679543 \\
\hline 72 & 1 & 0 & 1.424537 & 5.937088 & 2.320443 \\
\hline 73 & 6 & 0 & 3.606285 & 5.817095 & 5.521308 \\
\hline 74 & 1 & 0 & 3.091052 & 3.723841 & 5.594536 \\
\hline 75 & 6 & 0 & -5.720664 & 1.980079 & 0.403029 \\
\hline 76 & 6 & 0 & -2.974846 & 4.695703 & -0.795524 \\
\hline 77 & 6 & 0 & -0.698696 & 5.486126 & -0.913208 \\
\hline 78 & 6 & 0 & -3.958695 & -1.224714 & 2.504529 \\
\hline 79 & 6 & 0 & -1.955593 & -2.272716 & 3.346324 \\
\hline 80 & 6 & 0 & 2.202423 & -4.626308 & 5.177270 \\
\hline 81 & 1 & 0 & 1.648369 & -3.057832 & 3.811910 \\
\hline 82 & 6 & 0 & 4.549636 & -4.798042 & 5.693772 \\
\hline 83 & 1 & 0 & 5.823731 & -3.329992 & 4.771188 \\
\hline 84 & 6 & 0 & 4.916151 & -6.621301 & 2.316599 \\
\hline 85 & 1 & 0 & 3.093248 & -5.746884 & 1.558926 \\
\hline 86 & 6 & 0 & 6.845700 & -5.171396 & 2.332107 \\
\hline 87 & 1 & 0 & 6.517292 & -3.165691 & 1.609414 \\
\hline 88 & 6 & 0 & -0.608672 & -2.245584 & -5.633095 \\
\hline 89 & 6 & 0 & -0.245535 & -5.689203 & -3.114717 \\
\hline 90 & 6 & 0 & 0.718958 & -6.817219 & -1.215580 \\
\hline 91 & 6 & 0 & 1.141591 & 1.079450 & -4.137973 \\
\hline 92 & 6 & 0 & 2.291675 & 2.233787 & -2.360069 \\
\hline 93 & 6 & 0 & 5.074104 & 5.985947 & 0.713198 \\
\hline 94 & 1 & 0 & 3.587691 & 5.396510 & -0.731378 \\
\hline 95 & 1 & 0 & 6.464719 & 6.311357 & 2.324037 \\
\hline 96 & 6 & 0 & 3.460989 & 7.017957 & 4.825911 \\
\hline 97 & 1 & 0 & 2.552638 & 7.994513 & 3.133424 \\
\hline 98 & 1 & 0 & 4.211520 & 5.779681 & 6.422132 \\
\hline 99 & 9 & 0 & -6.488737 & 0.957050 & 0.777372 \\
\hline 100 & 9 & 0 & -6.413424 & 3.103382 & 0.655480 \\
\hline 101 & 9 & 0 & -5.583527 & 1.901446 & -0.939872 \\
\hline 102 & 6 & 0 & -3.268235 & 5.295692 & -2.010839 \\
\hline
\end{tabular}

\begin{tabular}{|c|c|c|c|c|c|}
\hline 103 & 1 & 0 & 0.286456 & 5.535626 & -0.458971 \\
\hline 104 & 6 & 0 & -0.994535 & 6.117330 & -2.121887 \\
\hline 105 & 6 & 0 & -4.532844 & -2.461421 & 2.255117 \\
\hline 106 & 1 & 0 & -0.958336 & -2.164688 & 3.761610 \\
\hline 107 & 6 & 0 & -2.541785 & -3.521956 & 3.131212 \\
\hline 108 & 6 & 0 & 3.245991 & -5.266968 & 5.847543 \\
\hline 109 & 1 & 0 & 1.180925 & -4.977535 & 5.298702 \\
\hline 110 & 1 & 0 & 5.370794 & -5.292079 & 6.204251 \\
\hline 111 & 6 & 0 & 6.266388 & -6.412110 & 2.599819 \\
\hline 112 & 1 & 0 & 4.460766 & -7.586281 & 2.518016 \\
\hline 113 & 1 & 0 & 7.900054 & -5.007686 & 2.534083 \\
\hline 114 & 9 & 0 & -1.433839 & -1.174455 & -5.607605 \\
\hline 115 & 9 & 0 & -1.381020 & -3.311643 & -5.845065 \\
\hline 116 & 9 & 0 & 0.178983 & -2.099019 & -6.710411 \\
\hline 117 & 6 & 0 & -0.883650 & -6.857504 & -3.505619 \\
\hline 18 & 1 & 0 & 1.335268 & -6.765880 & -0.322130 \\
\hline 19 & 6 & 0 & 0.079014 & -7.994722 & -1.602065 \\
\hline 20 & 6 & 0 & 0.472967 & 2.236404 & -4.504797 \\
\hline 21 & 1 & 0 & 2.986539 & 2.193880 & -1.527591 \\
\hline 122 & 6 & 0 & 1.644056 & 3.408689 & -2.739985 \\
\hline 123 & 1 & 0 & 5.342335 & 6.896663 & 0.186713 \\
\hline 124 & 1 & 0 & 3.960104 & 7.915745 & 5.177479 \\
\hline 125 & 1 & 0 & -4.274444 & 5.205652 & -2.407458 \\
\hline 126 & 6 & 0 & -2.272194 & 6.016871 & -2.672291 \\
\hline 127 & 1 & 0 & -0.223523 & 6.686167 & -2.632379 \\
\hline 128 & 1 & 0 & -5.530342 & -2.503664 & 1.830246 \\
\hline 129 & 6 & 0 & -3.819938 & -3.616082 & 2.580363 \\
\hline 130 & 1 & 0 & -1.994342 & -4.424224 & 3.388446 \\
\hline 131 & 1 & 0 & 3.042212 & -6.121590 & 6.485251 \\
\hline 132 & 1 & 0 & 6.865354 & -7.214402 & 3.019625 \\
\hline 133 & 1 & 0 & -1.500306 & -6.834430 & -4.398165 \\
\hline 134 & 6 & 0 & -0.725937 & -8.012983 & -2.741065 \\
\hline 135 & 1 & 0 & 0.208240 & -8.892212 & -1.006174 \\
\hline 136 & 1 & 0 & -0.239387 & 2.195405 & -5.322035 \\
\hline 137 & 6 & 0 & 0.731948 & 3.409670 & -3.796570 \\
\hline 138 & 1 & 0 & 1.837657 & 4.326089 & -2.193625 \\
\hline 139 & 1 & 0 & -2.500615 & 6.503811 & -3.614422 \\
\hline 140 & 1 & 0 & -4.267619 & -4.589662 & 2.406636 \\
\hline 141 & 1 & 0 & -1.230589 & -8.925239 & -3.040622 \\
\hline 142 & 1 & 0 & 0.210052 & 4.322155 & -4.065157 \\
\hline 143 & 1 & 0 & -1.194286 & 0.914164 & -2.206518 \\
\hline 144 & 8 & 0 & -1.923862 & 0.691382 & -2.853592 \\
\hline 145 & 6 & 0 & -3.165644 & 0.990191 & -2.151885 \\
\hline 146 & 6 & 0 & -3.597531 & -0.451429 & -1.816331 \\
\hline 147 & 1 & 0 & -2.959765 & 1.583795 & -1.260705 \\
\hline 148 & 1 & 0 & -3.830847 & 1.515666 & -2.836209 \\
\hline 149 & 8 & 0 & -3.452640 & -0.774165 & -0.456357 \\
\hline 150 & 6 & 0 & -2.492704 & -1.085208 & -2.684772 \\
\hline 151 & 1 & 0 & -2.507816 & -0.936982 & -0.257455 \\
\hline 152 & 1 & 0 & -2.655771 & -1.232693 & -3.745128 \\
\hline 153 & 1 & 0 & -1.570713 & -1.451276 & -2.252268 \\
\hline 154 & 1 & 0 & -1.147269 & -2.885209 & -0.506397 \\
\hline 155 & 7 & 0 & -1.554380 & -3.837052 & -0.436214 \\
\hline 156 & 6 & 0 & -2.597974 & -4.245702 & -1.177006 \\
\hline 157 & 6 & 0 & -1.095065 & -4.722288 & 0.528017 \\
\hline 158 & 16 & 0 & -3.347081 & -3.461835 & -2.455228 \\
\hline 159 & 16 & 0 & -3.120088 & -5.833262 & -0.638244 \\
\hline 160 & 6 & 0 & -1.859626 & -5.892335 & 0.574274 \\
\hline 161 & 6 & 0 & 0.020449 & -4.543319 & 1.346877 \\
\hline 162 & 6 & 0 & -1.547307 & -6.911561 & 1.472543 \\
\hline 163 & 6 & 0 & 0.337885 & -5.569784 & 2.230550 \\
\hline 164 & 1 & 0 & 0.611993 & -3.631068 & 1.278911 \\
\hline 165 & 6 & 0 & -0.440523 & -6.737365 & 2.300265 \\
\hline 166 & 1 & 0 & -2.137427 & -7.821693 & 1.510661 \\
\hline 167 & 1 & 0 & 1.202786 & -5.465077 & 2.878599 \\
\hline 168 & 1 & 0 & -0.172140 & -7.519047 & 3.003977 \\
\hline
\end{tabular}




$\begin{array}{llllll}169 & 6 & 0 & -5.005812 & -0.782828 & -2.264824 \\ 170 & 6 & 0 & -5.384097 & -0.578029 & -3.594528 \\ 171 & 6 & 0 & -5.939551 & -1.267563 & -1.351259 \\ 172 & 6 & 0 & -6.681905 & -0.867513 & -4.010012 \\ 173 & 1 & 0 & -4.668997 & -0.192595 & -4.320269 \\ 174 & 6 & 0 & -7.241817 & -1.543320 & -1.764081 \\ 175 & 1 & 0 & -5.636083 & -1.419904 & -0.321151 \\ 176 & 6 & 0 & -7.616343 & -1.348776 & -3.092611 \\ 177 & 1 & 0 & -6.963325 & -0.712684 & -5.046965 \\ 178 & 1 & 0 & -7.966182 & -1.911903 & -1.044144 \\ 179 & 1 & 0 & -8.630125 & -1.568598 & -3.412668\end{array}$

Frequencies -- -538.5894

Frequencies -- 19.8835

Frequencies -- 23.8380

Frequencies -- 35.5325

Frequencies -- 40.1047

Frequencies -- 48.0409

Frequencies -- 53.3189

Frequencies -- 60.4880

Frequencies -- 69.1110

Frequencies -- 74.4294

Frequencies -- 80.3261

Frequencies -- 90.4817

Frequencies -- 97.6831

Frequencies -- 102.1170

Frequencies -- 113.8080

Frequencies -- 127.1271

Frequencies -- 138.1700

Frequencies -- 156.3463

Frequencies -- 165.3535

Frequencies -- 182.7637

Frequencies -- 197.2498

Frequencies -- 210.9235

Frequencies -- 224.4681

Frequencies -- 236.6019

Frequencies -- 252.9247

Frequencies -- 272.8635

Frequencies -- 280.2519

Frequencies -- 290.4636

Frequencies -- 296.7070

Frequencies -- 305.7927

Frequencies -- 318.0819

Frequencies -- 325.3823

Frequencies -- 339.4562

Frequencies -- 354.1434

Frequencies -- 396.6766

Frequencies -- 408.5893

Frequencies -- 413.4957

Frequencies -- 418.3352

Frequencies -- 428.5358

Frequencies -- 441.7710

Frequencies -- 450.7667

Frequencies -- 466.4183

Frequencies -- 473.6892

Frequencies -- 500.8144

Frequencies -- 512.0012

Frequencies -- 522.4579

Frequencies -- 534.1118

Frequencies -- 543.7397

Frequencies -- 556.5234

Frequencies -- 564.4113

Frequencies -- 574.8333

Frequencies -- 587.7816

Frequencies -- 595.5787

$\begin{array}{ll}2.3415 & 12.7084 \\ 20.8353 & 22.9470 \\ 28.0338 & 31.6982 \\ 37.1717 & 38.3091 \\ 43.3487 & 44.0209 \\ 51.5601 & 52.8818 \\ 55.1646 & 58.1321 \\ 62.0651 & 63.6796 \\ 69.5195 & 71.6234 \\ 76.1841 & 79.6532 \\ 83.4500 & 83.9574 \\ 91.5305 & 93.9990 \\ 100.0964 & 100.9613 \\ 107.0551 & 109.3873 \\ 118.2896 & 125.4361 \\ 131.9349 & 136.2682 \\ 140.5873 & 149.2239 \\ 158.1003 & 164.3626 \\ 168.1780 & 172.4872 \\ 187.1283 & 189.5298 \\ 200.0484 & 201.2862 \\ 216.6744 & 220.8454 \\ 229.9748 & 233.5997 \\ 241.6229 & 250.3218 \\ 256.9342 & 267.8432 \\ 274.4898 & 276.0129 \\ 281.1279 & 288.7780 \\ 292.5424 & 294.3376 \\ 300.0644 & 302.7050 \\ 310.4171 & 313.9984 \\ 319.0940 & 323.1438 \\ 331.1503 & 337.0493 \\ 343.2849 & 347.4156 \\ 374.9705 & 385.8687 \\ 403.9318 & 408.5137 \\ 411.5381 & 412.0105 \\ 414.3270 & 415.7202 \\ 418.7997 & 419.6207 \\ 438.6625 & 439.4746 \\ 444.5083 & 447.9530 \\ 452.4806 & 459.8010 \\ 467.4431 & 469.3198 \\ 487.7993 & 491.0435 \\ 505.9987 & 507.0283 \\ 513.9506 & 521.4397 \\ 522.6447 & 530.5404 \\ 535.7238 & 538.0652 \\ 546.9458 & 554.4879 \\ 559.9780 & 561.2111 \\ 567.1731 & 571.6457 \\ 583.3612 & 585.9061 \\ 589.6631 & 593.7220 \\ 599.4273 & 601.6283\end{array}$

Frequencies -- 602.7093

Frequencies -- 610.8563

Frequencies -- 620.0853

Frequencies -- 626.1375

Frequencies -- 632.0928

Frequencies -- 644.0808

Frequencies -- 666.6633

Frequencies -- 676.9657

Frequencies -- 684.1847

Frequencies -- 709.8803

Frequencies -- 717.3454

Frequencies -- 720.2990

Frequencies -- 724.3093

Frequencies -- 732.8563

Frequencies -- 744.0436

Frequencies -- 763.5368

Frequencies -- 766.5218

Frequencies -- 772.1297

Frequencies -- 779.7161

Frequencies -- 794.9151

Frequencies -- 800.7702

Frequencies -- 822.9091

Frequencies -- 830.9976

Frequencies -- 865.8142

Frequencies -- 869.7850

Frequencies -- 873.7254

Frequencies -- 881.2119

Frequencies -- 888.9871

Frequencies -- 900.7593

Frequencies -- 923.4478

Frequencies -- 931.5753

Frequencies -- 935.5422

Frequencies -- 943.9116

Frequencies -- 950.4414

Frequencies -- 959.9152

Frequencies -- 970.4349

Frequencies -- 988.5548

Frequencies -- 993.8750

Frequencies -- 999.7710

Frequencies -- 1005.9598

Frequencies -- 1014.2322

Frequencies -- 1019.3654

Frequencies -- 1020.9803

Frequencies -- 1026.0100

Frequencies -- 1038.0397

Frequencies -- 1049.0367

Frequencies -- 1058.7335

Frequencies -- 1066.3762

Frequencies -- 1070.6002

Frequencies -- 1088.3680

Frequencies -- 1106.7492

Frequencies -- 1111.7184

Frequencies -- 1119.8018

Frequencies -- 1129.2270

Frequencies -- 1133.6761

Frequencies -- 1165.8681

Frequencies -- 1171.2372

Frequencies -- 1174.1463

Frequencies -- 1177.1456

Frequencies -- 1189.1441

Frequencies -- 1201.9134

Frequencies -- 1204.3996

Frequencies -- 1212.6853

Frequencies -- 1223.4582

Frequencies -- 1232.3340

Frequencies -- 1242.2424
605.8311

613.7036

621.4898

630.0817

634.4780

651.2025

669.9041

680.8743

695.7241

714.4797

717.9969

722.8794

726.1523

734.6723

748.1123

764.1364

770.3966

774.5392

782.2200

796.1059

804.3502

826.4330

857.6053

866.3028

871.1645

877.1632

886.4070

889.1533

913.7157

924.0078

934.2407

936.1232

946.7657

952.1711

961.0902

970.6935

989.5813

995.6139

1003.3543

1008.7938

1016.3015

1019.8092

1021.3551

1033.6646

1044.0675

1054.6551

1059.2424

1067.3975

1073.2365

1091.6923

1108.0839

1113.3951

1122.7532

1130.7189

1154.3296

1168.0423

1171.8555

1175.7639

1178.8228

1193.6826

1203.4375

1204.7569

1214.3820

1225.6002

1234.1475

1242.6302
609.6877

617.4957

624.2433

630.7719

635.8472

656.7087

673.6694

682.1449

703.6652

714.7008

718.2265

723.6101

732.0525

739.1237

751.7374

765.4389

771.4491

776.4258

783.5029

798.9730

804.9721

827.7029

862.9928

867.6771

871.9702

878.0584

888.0122

894.5442

917.1325

927.0769

935.3453

941.7562

947.1380

956.7392

963.0817

987.5023

991.0769

996.4003

1005.4719

1013.4239

1018.4035

1020.1905

1024.4764

1036.2112

1045.7069

1057.8247

1064.3142

1068.7661

1077.0699

1096.7488

1110.6008

1117.8935

1126.0653

1132.2486

1160.6653

1170.0331

1174.0102

1176.4997

1179.2311

1200.4499

1203.8322

1205.9587

1216.0868

1227.1120

1240.5112

1247.0578 


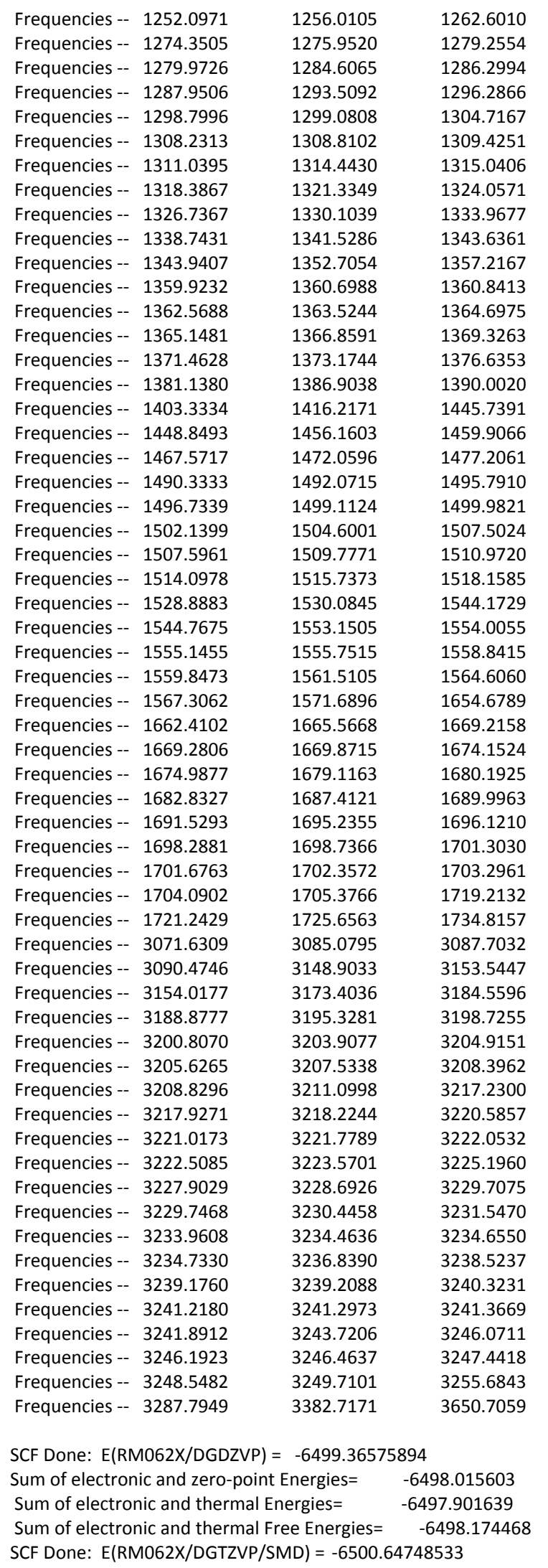

\section{Cat f I1c_S}

\begin{tabular}{|c|c|c|c|c|c|}
\hline \multirow{2}{*}{$\begin{array}{l}\text { Center } \\
\text { Number }\end{array}$} & \multicolumn{2}{|c|}{ Atomic } & Atomic & \multicolumn{2}{|c|}{ Coordinates (Angstroms) } \\
\hline & & ber & Type & $\begin{array}{ll}X & Y\end{array}$ & Z \\
\hline 1 & 15 & 0 & -0.073518 & -0.078696 & 0.088959 \\
\hline 2 & 8 & 0 & -0.024747 & -0.113580 & 1.721330 \\
\hline 3 & 8 & 0 & 1.514372 & -0.013913 & -0.274063 \\
\hline 4 & 8 & 0 & -0.694386 & 1.158395 & -0.454552 \\
\hline 5 & 8 & 0 & -0.636212 & -1.423708 & -0.289108 \\
\hline 6 & 6 & 0 & 0.481850 & 1.007830 & 2.357042 \\
\hline 7 & 6 & 0 & 2.308409 & -1.067028 & 0.125593 \\
\hline 8 & 6 & 0 & 1.848444 & 1.112245 & 2.590311 \\
\hline 9 & 6 & 0 & -0.369697 & 2.080415 & 2.654109 \\
\hline 10 & 6 & 0 & 2.911411 & -1.016164 & 1.376679 \\
\hline 11 & 6 & 0 & 2.469905 & -2.185258 & -0.706060 \\
\hline 12 & 6 & 0 & 2.984698 & 0.130580 & 2.365200 \\
\hline 13 & 6 & 0 & 2.365682 & 2.312622 & 3.079598 \\
\hline 14 & 6 & 0 & 0.160383 & 3.213539 & 3.276228 \\
\hline 15 & 6 & 0 & -1.748789 & 2.090029 & 2.112517 \\
\hline 16 & 6 & 0 & 3.655188 & -2.107068 & 1.830079 \\
\hline 17 & 6 & 0 & 3.261306 & -3.244920 & -0.247735 \\
\hline 18 & 6 & 0 & 1.752944 & -2.239476 & -2.005431 \\
\hline 19 & 6 & 0 & 3.320052 & -0.630045 & 3.673615 \\
\hline 20 & 6 & 0 & 4.133959 & 1.110404 & 2.012864 \\
\hline 21 & 6 & 0 & 3.872074 & 2.381519 & 2.862834 \\
\hline 22 & 6 & 0 & 1.530415 & 3.351120 & 3.499415 \\
\hline 23 & 1 & 0 & -0.503958 & 4.034781 & 3.531677 \\
\hline 24 & 6 & 0 & -2.105509 & 3.101040 & 1.219117 \\
\hline 25 & 6 & 0 & -2.656045 & 1.056522 & 2.318646 \\
\hline 26 & 6 & 0 & 4.157402 & -1.858191 & 3.248630 \\
\hline 27 & 6 & 0 & 3.863583 & -3.223998 & 1.012756 \\
\hline 28 & 1 & 0 & 3.414849 & -4.106919 & -0.891119 \\
\hline 29 & 6 & 0 & 0.932007 & -3.324902 & -2.325960 \\
\hline 30 & 6 & 0 & 1.771805 & -1.171015 & -2.899513 \\
\hline 31 & 1 & 0 & 3.840439 & 0.002007 & 4.399632 \\
\hline 32 & 1 & 0 & 2.384660 & -0.960352 & 4.134604 \\
\hline 33 & 1 & 0 & 5.122425 & 0.675657 & 2.192472 \\
\hline 34 & 1 & 0 & 4.067073 & 1.364481 & 0.949760 \\
\hline 35 & 1 & 0 & 4.422437 & 2.332850 & 3.809658 \\
\hline 36 & 6 & 0 & 4.251284 & 3.663138 & 2.143138 \\
\hline 37 & 6 & 0 & 2.103602 & 4.601270 & 4.060191 \\
\hline 38 & 6 & 0 & -3.317490 & 3.063354 & 0.545466 \\
\hline 39 & 8 & 0 & -1.197838 & 4.106156 & 0.978551 \\
\hline 40 & 6 & 0 & -3.870359 & 0.994760 & 1.632940 \\
\hline 41 & 8 & 0 & -2.329631 & 0.077819 & 3.231614 \\
\hline 42 & 1 & 0 & 5.219385 & -1.583705 & 3.203217 \\
\hline 43 & 6 & 0 & 4.017868 & -3.040304 & 4.186170 \\
\hline 44 & 6 & 0 & 4.742720 & -4.345124 & 1.448558 \\
\hline 45 & 6 & 0 & 0.107560 & -3.321082 & -3.449347 \\
\hline 46 & 8 & 0 & 0.894524 & -4.374753 & -1.428221 \\
\hline 47 & 6 & 0 & 0.926136 & -1.142236 & -4.005962 \\
\hline 48 & 8 & 0 & 2.618672 & -0.110454 & -2.691960 \\
\hline 49 & 6 & 0 & 3.564329 & 4.028753 & 0.977042 \\
\hline 50 & 6 & 0 & 5.230656 & 4.520436 & 2.643115 \\
\hline 51 & 6 & 0 & 1.879652 & 5.830724 & 3.431510 \\
\hline 52 & 6 & 0 & 2.929532 & 4.553838 & 5.188476 \\
\hline 53 & 6 & 0 & -4.224584 & 2.021581 & 0.756415 \\
\hline 54 & 8 & 0 & -3.648720 & 4.033064 & -0.372268 \\
\hline 55 & 6 & 0 & -1.369942 & 4.793046 & -0.200354 \\
\hline 56 & 8 & 0 & -4.703963 & -0.081847 & 1.829800 \\
\hline 57 & 6 & 0 & -2.865465 & -1.155454 & 2.941650 \\
\hline 58 & 6 & 0 & 2.752000 & -3.520783 & 4.537116 \\
\hline 59 & 6 & 0 & 5.141769 & -3.688745 & 4.703372 \\
\hline 60 & 6 & 0 & 4.212440 & -5.596761 & 1.775205 \\
\hline
\end{tabular}




\begin{tabular}{|c|c|c|c|c|c|}
\hline 61 & 6 & 0 & 6.123335 & -4.144898 & 1.550332 \\
\hline 62 & 6 & 0 & 0.093792 & -2.216809 & -4.306801 \\
\hline 63 & 8 & 0 & -0.790474 & -4.346825 & -3.660558 \\
\hline 64 & 6 & 0 & 0.310395 & -5.533869 & -1.879599 \\
\hline 65 & 8 & 0 & 0.890731 & -0.027322 & -4.794199 \\
\hline 66 & 6 & 0 & 2.025864 & 1.101166 & -3.018394 \\
\hline 67 & 6 & 0 & 3.861584 & 5.221817 & 0.325041 \\
\hline 68 & 1 & 0 & 2.764292 & 3.389507 & 0.601355 \\
\hline 69 & 6 & 0 & 5.521548 & 5.724595 & 1.998300 \\
\hline 70 & 1 & 0 & 5.753959 & 4.259079 & 3.560066 \\
\hline 71 & 6 & 0 & 2.495632 & 6.987452 & 3.905338 \\
\hline 72 & 1 & 0 & 1.244477 & 5.866974 & 2.549475 \\
\hline 73 & 6 & 0 & 3.535514 & 5.712038 & 5.671660 \\
\hline 74 & 1 & 0 & 3.090892 & 3.601343 & 5.688106 \\
\hline 75 & 6 & 0 & -5.555404 & 2.112425 & 0.048399 \\
\hline 76 & 6 & 0 & -2.586301 & 4.754024 & -0.877013 \\
\hline 77 & 6 & 0 & -0.326215 & 5.570488 & -0.678798 \\
\hline 78 & 6 & 0 & -4.059448 & -1.235749 & 2.227987 \\
\hline 79 & 6 & 0 & -2.244487 & -2.303405 & 3.412203 \\
\hline 80 & 6 & 0 & 2.611667 & -4.629316 & 5.370514 \\
\hline 81 & 1 & 0 & 1.862728 & -3.024143 & 4.152280 \\
\hline 82 & 6 & 0 & 5.008900 & -4.803743 & 5.529751 \\
\hline 83 & 1 & 0 & 6.133714 & -3.330011 & 4.439894 \\
\hline 84 & 6 & 0 & 5.043152 & -6.620603 & 2.228544 \\
\hline 85 & 1 & 0 & 3.140740 & -5.758705 & 1.687207 \\
\hline 86 & 6 & 0 & 6.957745 & -5.171761 & 1.989899 \\
\hline 87 & 1 & 0 & 6.541634 & -3.178404 & 1.278598 \\
\hline 88 & 6 & 0 & -0.773432 & -2.089737 & -5.536606 \\
\hline 89 & 6 & 0 & -0.535749 & -5.519409 & -2.986048 \\
\hline 90 & 6 & 0 & 0.542230 & -6.715122 & -1.188528 \\
\hline 91 & 6 & 0 & 1.130651 & 1.139311 & -4.080483 \\
\hline 92 & 6 & 0 & 2.313628 & 2.245674 & -2.292636 \\
\hline 93 & 6 & 0 & 4.839864 & 6.077956 & 0.836240 \\
\hline 94 & 1 & 0 & 3.325390 & 5.495177 & -0.579673 \\
\hline 95 & 1 & 0 & 6.274104 & 6.389352 & 2.411541 \\
\hline 96 & 6 & 0 & 3.328806 & 6.930218 & 5.023350 \\
\hline 97 & 1 & 0 & 2.333279 & 7.932353 & 3.395794 \\
\hline 98 & 1 & 0 & 4.170602 & 5.664848 & 6.551265 \\
\hline 99 & 9 & 0 & -6.443934 & 1.207189 & 0.473958 \\
\hline 100 & 9 & 0 & -6.121031 & 3.317070 & 0.247601 \\
\hline 101 & 9 & 0 & -5.426145 & 1.956319 & -1.281158 \\
\hline 102 & 6 & 0 & -2.761251 & 5.472667 & -2.050147 \\
\hline 103 & 1 & 0 & 0.606598 & 5.580968 & -0.121462 \\
\hline 104 & 6 & 0 & -0.503399 & 6.315738 & -1.845233 \\
\hline 105 & 6 & 0 & -4.638446 & -2.468502 & 1.964188 \\
\hline 106 & 1 & 0 & -1.311254 & -2.203161 & 3.957977 \\
\hline 107 & 6 & 0 & -2.845157 & -3.542713 & 3.188399 \\
\hline 108 & 6 & 0 & 3.742140 & -5.280080 & 5.865743 \\
\hline 109 & 1 & 0 & 1.618509 & -4.982190 & 5.636650 \\
\hline 110 & 1 & 0 & 5.896745 & -5.301223 & 5.908391 \\
\hline 111 & 6 & 0 & 6.417725 & -6.409214 & 2.341089 \\
\hline 112 & 1 & 0 & 4.617109 & -7.582326 & 2.498402 \\
\hline 113 & 1 & 0 & 8.028612 & -5.005346 & 2.059660 \\
\hline 114 & 9 & 0 & -1.626266 & -1.052041 & -5.442867 \\
\hline 115 & 9 & 0 & -1.526769 & -3.175392 & -5.771076 \\
\hline 116 & 9 & 0 & -0.027301 & -1.888192 & -6.633197 \\
\hline 117 & 6 & 0 & -1.162545 & -6.682961 & -3.409303 \\
\hline 118 & 1 & 0 & 1.201730 & -6.690570 & -0.324739 \\
\hline 119 & 6 & 0 & -0.095040 & -7.885801 & -1.601103 \\
\hline 120 & 6 & 0 & 0.478043 & 2.312867 & -4.420933 \\
\hline 121 & 1 & 0 & 3.015438 & 2.181275 & -1.467204 \\
\hline 122 & 6 & 0 & 1.679726 & 3.436092 & -2.644714 \\
\hline 123 & 1 & 0 & 5.062369 & 7.014519 & 0.334307 \\
\hline 124 & 1 & 0 & 3.809826 & 7.831861 & 5.390000 \\
\hline 125 & 1 & 0 & -3.719810 & 5.414825 & -2.555653 \\
\hline 126 & 6 & 0 & -1.716196 & 6.263026 & -2.531748 \\
\hline
\end{tabular}

\begin{tabular}{|c|c|c|c|c|c|}
\hline 127 & 1 & 0 & 0.308058 & 6.934722 & -2.215532 \\
\hline 128 & 1 & 0 & -5.563086 & -2.504646 & 1.396649 \\
\hline 129 & 6 & 0 & -4.033815 & -3.625044 & 2.461858 \\
\hline 130 & 1 & 0 & -2.375036 & -4.443958 & 3.573469 \\
\hline 131 & 1 & 0 & 3.636845 & -6.145372 & 6.512947 \\
\hline 132 & 1 & 0 & 7.064985 & -7.206658 & 2.692951 \\
\hline 133 & 1 & 0 & -1.815224 & -6.635587 & -4.275123 \\
\hline 134 & 6 & 0 & -0.946426 & -7.869149 & -2.706562 \\
\hline 135 & 1 & 0 & 0.079363 & -8.808945 & -1.058233 \\
\hline 136 & 1 & 0 & -0.243392 & 2.296240 & -5.231036 \\
\hline 137 & 6 & 0 & 0.760534 & 3.469523 & -3.694556 \\
\hline 138 & 1 & 0 & 1.889941 & 4.340989 & -2.084417 \\
\hline 139 & 1 & 0 & -1.855677 & 6.837583 & -3.441500 \\
\hline 140 & 1 & 0 & -4.503998 & -4.588821 & 2.291756 \\
\hline 141 & 1 & 0 & -1.440509 & -8.779324 & -3.029398 \\
\hline 142 & 1 & 0 & 0.248325 & 4.395238 & -3.936792 \\
\hline 143 & 1 & 0 & -1.252216 & 0.739679 & -2.128477 \\
\hline 144 & 8 & 0 & -1.561824 & 0.201757 & -2.888315 \\
\hline 145 & 6 & 0 & -2.972483 & 0.243570 & -2.945502 \\
\hline 146 & 6 & 0 & -3.621880 & -0.955705 & -2.218590 \\
\hline 147 & 1 & 0 & -3.372414 & 1.154457 & -2.483675 \\
\hline 148 & 1 & 0 & -3.260066 & 0.234567 & -3.999891 \\
\hline 149 & 8 & 0 & -3.463119 & -0.838253 & -0.815586 \\
\hline 150 & 6 & 0 & -2.924632 & -2.246300 & -2.678637 \\
\hline 151 & 1 & 0 & -2.510786 & -0.713993 & -0.637017 \\
\hline 152 & 1 & 0 & -2.948221 & -2.361768 & -3.765732 \\
\hline 153 & 1 & 0 & -1.881233 & -2.245491 & -2.354230 \\
\hline 154 & 1 & 0 & -1.414058 & -2.751635 & -0.335378 \\
\hline 155 & 7 & 0 & -1.762192 & -3.747657 & -0.197961 \\
\hline 156 & 6 & 0 & -2.784994 & -4.326851 & -0.799378 \\
\hline 157 & 6 & 0 & -1.130327 & -4.535280 & 0.763190 \\
\hline 158 & 16 & 0 & -3.785106 & -3.741274 & -2.063573 \\
\hline 159 & 16 & 0 & -3.082522 & -5.928501 & -0.195869 \\
\hline 160 & 6 & 0 & -1.751960 & -5.775164 & 0.923397 \\
\hline 161 & 6 & 0 & 0.010146 & -4.186366 & 1.488279 \\
\hline 162 & 6 & 0 & -1.274539 & -6.709126 & 1.844074 \\
\hline 163 & 6 & 0 & 0.495539 & -5.125617 & 2.388394 \\
\hline 164 & 1 & 0 & 0.485163 & -3.218349 & 1.335595 \\
\hline 165 & 6 & 0 & -0.141488 & -6.366155 & 2.573331 \\
\hline 166 & 1 & 0 & -1.757424 & -7.672478 & 1.970959 \\
\hline 167 & 1 & 0 & 1.390076 & -4.900759 & 2.959095 \\
\hline 168 & 1 & 0 & 0.264951 & -7.071776 & 3.291342 \\
\hline 169 & 6 & 0 & -5.105766 & -1.020950 & -2.529622 \\
\hline 170 & 6 & 0 & -5.544965 & -1.143693 & -3.853513 \\
\hline 171 & 6 & 0 & -6.046126 & -1.004755 & -1.499794 \\
\hline 172 & 6 & 0 & -6.905991 & -1.230804 & -4.140951 \\
\hline 173 & 1 & 0 & -4.829185 & -1.172790 & -4.672869 \\
\hline 174 & 6 & 0 & -7.407891 & -1.082962 & -1.788050 \\
\hline 175 & 1 & 0 & -5.700750 & -0.916659 & -0.475188 \\
\hline 176 & 6 & 0 & -7.842766 & -1.196226 & -3.107626 \\
\hline 177 & 1 & 0 & -7.233866 & -1.320672 & -5.172061 \\
\hline 178 & 1 & 0 & -8.128924 & -1.046320 & -0.976723 \\
\hline 179 & 1 & 0 & -8.903531 & -1.254904 & -3.331437 \\
\hline
\end{tabular}

Frequencies --

Frequencies -- 23.6748

Frequencies -- 30.8569

Frequencies -- 37.6298

Frequencies -- 44.9349

Frequencies -- 52.2965

Frequencies -- 60.8176

Frequencies -- 68.4931

Frequencies -- 73.4892

Frequencies -- 80.2222

Frequencies -- 91.0201
18.4719

25.4271

32.0678

38.6131

47.5925

56.1535

62.0028

69.5594

74.0299

88.0661

92.1741
21.9104 28.1406 35.1082 40.2655 50.3056 59.7080 64.6564 70.8703 78.2005 88.8451 94.1629 


\begin{tabular}{|c|c|c|c|}
\hline Frequencies -- & 95.1515 & 96.5310 & 98.7892 \\
\hline Frequencies -- & 100.1584 & 101.8867 & 104.0110 \\
\hline Frequencies -- & 106.3322 & 113.7882 & 116.4690 \\
\hline Frequencies -- & 120.5235 & 122.1062 & 126.3862 \\
\hline Frequencies -- & 128.8856 & 134.3722 & 139.6385 \\
\hline Frequencies -- & 141.4355 & 146.6303 & 152.5743 \\
\hline Frequencies -- & 158.2629 & 161.7887 & 166.0794 \\
\hline Frequencies -- & 167.4012 & 173.2606 & 179.1358 \\
\hline Frequencies -- & 188.4989 & 191.1984 & 194.6911 \\
\hline Frequencies -- & 198.8595 & 207.6144 & 212.3056 \\
\hline Frequencies -- & 212.9642 & 223.1345 & 227.0583 \\
\hline Frequencies -- & 231.7590 & 236.4527 & 240.2386 \\
\hline Frequencies -- & 240.9065 & 242.9507 & 250.4250 \\
\hline Frequencies -- & 254.1146 & 254.7924 & 269.6198 \\
\hline Frequencies -- & 274.4692 & 277.8436 & 279.9289 \\
\hline Frequencies -- & 282.4031 & 287.0493 & 289.8679 \\
\hline Frequencies -- & 293.1213 & 294.4474 & 298.2748 \\
\hline Frequencies -- & 300.0246 & 303.2468 & 304.8276 \\
\hline Frequencies -- & 308.6638 & 312.5260 & 315.5659 \\
\hline Frequencies -- & 318.6578 & 321.9472 & 328.7685 \\
\hline Frequencies -- & 329.0340 & 332.6802 & 334.9775 \\
\hline Frequencies -- & 337.7357 & 348.5656 & 351.2970 \\
\hline Frequencies -- & 355.4958 & 375.7316 & 382.6894 \\
\hline Frequencies -- & 395.0627 & 404.5587 & 405.6195 \\
\hline Frequencies -- & 413.1681 & 415.5067 & 415.7917 \\
\hline Frequencies -- & 416.5611 & 417.8542 & 420.1459 \\
\hline Frequencies -- & 422.2567 & 423.2662 & 424.0295 \\
\hline Frequencies -- & 433.1470 & 434.5242 & 443.2117 \\
\hline Frequencies -- & 445.2981 & 448.6551 & 448.9718 \\
\hline Frequencies -- & 453.0507 & 454.2881 & 459.2526 \\
\hline Frequencies -- & 462.9679 & 467.5188 & 472.1596 \\
\hline Frequencies -- & 481.8604 & 486.1132 & 491.8438 \\
\hline Frequencies -- & 499.8776 & 501.7238 & 511.2122 \\
\hline Frequencies -- & 511.7108 & 512.8823 & 522.0066 \\
\hline Frequencies -- & 522.5470 & 522.9455 & 525.9692 \\
\hline Frequencies -- & 531.8073 & 533.7357 & 538.3084 \\
\hline Frequencies -- & 544.3293 & 549.3356 & 556.3100 \\
\hline Frequencies -- & 557.6498 & 558.4888 & 562.5129 \\
\hline Frequencies -- & 564.1718 & 568.4138 & 569.9211 \\
\hline Frequencies -- & 583.2778 & 584.6692 & 586.6755 \\
\hline Frequencies -- & 589.4298 & 593.2002 & 597.8280 \\
\hline Frequencies -- & 598.4761 & 600.0248 & 601.9776 \\
\hline Frequencies -- & 604.5258 & 609.6236 & 610.1977 \\
\hline Frequencies -- & 611.2135 & 614.0222 & 617.0902 \\
\hline Frequencies -- & 621.9693 & 624.8202 & 626.1217 \\
\hline Frequencies -- & 628.7775 & 630.4064 & 632.0346 \\
\hline Frequencies -- & 633.8926 & 636.2211 & 640.9251 \\
\hline Frequencies -- & 643.9516 & 650.0556 & 655.9731 \\
\hline Frequencies -- & 667.9062 & 670.4866 & 675.3577 \\
\hline Frequencies -- & 678.4626 & 682.5340 & 693.9841 \\
\hline Frequencies -- & 695.8223 & 701.0041 & 710.7691 \\
\hline Frequencies -- & 712.9933 & 716.3166 & 716.4882 \\
\hline Frequencies -- & 717.6540 & 719.2854 & 720.4154 \\
\hline Frequencies -- & 722.9968 & 725.3125 & 725.6694 \\
\hline Frequencies -- & 727.3059 & 730.8386 & 732.2805 \\
\hline Frequencies -- & 732.7879 & 734.9490 & 739.1115 \\
\hline Frequencies -- & 744.5000 & 748.4578 & 751.5302 \\
\hline Frequencies -- & 755.5151 & 762.0617 & 765.1931 \\
\hline Frequencies -- & 767.5931 & 770.3412 & 771.6685 \\
\hline Frequencies -- & 772.0792 & 773.1230 & 777.0546 \\
\hline Frequencies -- & 779.1194 & 781.8639 & 784.5836 \\
\hline Frequencies -- & 789.2072 & 796.5207 & 798.6071 \\
\hline Frequencies -- & 799.4558 & 800.6029 & 804.1659 \\
\hline Frequencies -- & 805.0761 & 822.7960 & 826.3857 \\
\hline Frequencies -- & 828.6760 & 830.2172 & 858.8719 \\
\hline Frequencies -- & 859.4325 & 861.8011 & 863.1029 \\
\hline
\end{tabular}

Frequencies -- 865.8835 Frequencies -- 869.1702 Frequencies -- 876.4146 Frequencies -- 888.7291 Frequencies -- 893.1932 Frequencies -- 915.6344 Frequencies -- 930.4141 Frequencies -- 936.6615 Frequencies -- 941.3228 Frequencies -- 950.7730 Frequencies -- 955.9626 Frequencies -- 975.6378 Frequencies -- 986.9567 Frequencies -- 992.1712 Frequencies -- 996.7749 Frequencies -- 1004.7634 Frequencies -- 1008.4787 Frequencies -- 1019.1020 Frequencies -- 1020.5093 Frequencies -- 1027.2318 Frequencies -- 1036.9296 Frequencies -- 1053.1623 Frequencies -- 1062.6892 Frequencies -- 1068.3599 Frequencies -- 1071.8136 Frequencies -- 1096.9110 Frequencies -- 1113.2289 Frequencies -- 1120.1227 Frequencies -- 1126.2433 Frequencies -- 1129.7584 Frequencies -- 1157.2710 Frequencies -- 1166.0389 Frequencies -- 1168.9592 Frequencies -- 1175.3740 Frequencies -- 1177.1567 Frequencies -- 1195.9801 Frequencies -- 1203.2140 Frequencies -- 1211.0181 Frequencies -- 1214.9478 Frequencies -- 1225.4258 Frequencies -- 1236.9198 Frequencies -- 1244.6826 Frequencies -- 1253.2940 Frequencies -- 1272.9342 Frequencies -- 1284.4827 Frequencies -- 1297.1451 Frequencies -- 1299.1801 Frequencies -- 1307.1856 Frequencies -- 1311.1559 Frequencies -- 1316.5801 Frequencies -- 1327.6846 Frequencies -- 1336.5052 Frequencies -- 1346.5980 Frequencies -- 1360.7753 Frequencies -- 1365.0114 Frequencies -- 1366.6500 Frequencies -- 1369.9431 Frequencies -- 1387.8990 Frequencies -- 1404.5871 Frequencies -- 1458.2810 Frequencies -- 1470.6932 Frequencies -- 1493.1932 Frequencies -- 1498.8622 Frequencies -- 1502.6986 Frequencies -- 1506.7439 Frequencies -- 1515.7002

\begin{tabular}{ll}
867.2866 & 868.8848 \\
873.2013 & 874.0270 \\
880.8276 & 881.7115 \\
889.2331 & 890.6695 \\
907.1399 & 911.8109 \\
919.5790 & 922.5162 \\
933.5214 & 935.7587 \\
939.3072 & 941.1405 \\
944.2978 & 944.5407 \\
952.1504 & 953.3477 \\
958.5573 & 969.8351 \\
982.7743 & 986.7313 \\
989.1320 & 991.8527 \\
994.3216 & 995.7812 \\
1004.0719 & 1004.5407 \\
1006.3748 & 1007.6882 \\
1012.6035 & 1018.3575 \\
1019.2159 & 1019.7338 \\
1024.3504 & 1025.9532 \\
1034.2691 & 1036.1536 \\
1043.8068 & 1052.3269 \\
1057.0554 & 1059.2331 \\
1062.9803 & 1066.7670 \\
1069.8482 & 1070.2608 \\
1072.3355 & 1091.5792 \\
1108.7507 & 1111.7091 \\
1114.7485 & 1116.5282 \\
1123.4397 & 1126.0404 \\
1128.1523 & 1129.0016 \\
1131.7980 & 1145.4862 \\
1158.6046 & 1161.4399 \\
1166.1257 & 1166.5310 \\
1170.1291 & 1173.7034 \\
1175.8574 & 1176.9718 \\
1178.5003 & 1188.7894 \\
1200.0548 & 1201.9617 \\
1207.7894 & 1207.9415 \\
1211.6886 & 1213.1570 \\
1216.2124 & 1222.7106 \\
1229.9120 & 1235.4265 \\
1238.2333 & 1243.1974 \\
1247.9211 & 1251.1265 \\
1259.3809 & 1271.7237 \\
1275.4259 & 1280.6367 \\
1292.6824 & 1294.6323 \\
1297.4596 & 1298.1973 \\
1303.2326 & 1305.0558 \\
1308.4016 & 1309.6856 \\
1314.5471 & 1316.0651 \\
1322.2743 & 1324.2517 \\
1329.4082 & 1333.0793 \\
1341.2938 & 1342.7602 \\
1356.2687 & 1359.6490 \\
1361.1909 & 1362.8273 \\
1365.2297 & 1366.2422 \\
1367.5991 & 1368.3855 \\
1375.0514 & 1383.8912 \\
1392.9610 & 1402.6748 \\
1433.8025 & 1447.2844 \\
1459.2091 & 1466.3337 \\
1488.6944 & 1490.5037 \\
1495.3218 & 1496.1878 \\
1499.2449 & 1502.2061 \\
1505.1878 & 1506.4695 \\
1507.6267 & 1509.5016 \\
1517.6458 & 1518.4873 \\
& \\
\hline
\end{tabular}




$\begin{array}{llll}\text { Frequencies -- } & 1528.9346 & 1532.3265 & 1546.2673 \\ \text { Frequencies -- } 1547.7616 & 1548.3481 & 1553.3845 \\ \text { Frequencies -- } 1555.0480 & 1559.5209 & 1561.1979 \\ \text { Frequencies -- } 1564.1234 & 1565.0989 & 1568.3422 \\ \text { Frequencies -- } 1570.5119 & 1575.0257 & 1654.7961 \\ \text { Frequencies -- } 1661.8383 & 1667.7931 & 1668.6154 \\ \text { Frequencies -- } 1672.1087 & 1672.5535 & 1673.8815 \\ \text { Frequencies -- } 1677.1508 & 1677.2552 & 1678.1222 \\ \text { Frequencies -- } 1683.4447 & 1687.7644 & 1689.3553 \\ \text { Frequencies -- } 1691.6362 & 1694.7917 & 1697.6152 \\ \text { Frequencies -- } 1698.3998 & 1698.6553 & 1699.1441 \\ \text { Frequencies -- } 1699.7919 & 1701.1245 & 1702.1540 \\ \text { Frequencies -- } 1703.2142 & 1704.2203 & 1721.2905 \\ \text { Frequencies -- } 1723.0625 & 1733.1362 & 1737.3862 \\ \text { Frequencies -- } 2728.4014 & 3070.9951 & 3085.0614 \\ \text { Frequencies -- } 3086.5625 & 3094.1462 & 3101.4561 \\ \text { Frequencies -- } 3126.4783 & 3150.8468 & 3158.3692 \\ \text { Frequencies -- } 3170.8090 & 3174.6135 & 3185.5325 \\ \text { Frequencies -- } 3193.7343 & 3198.7598 & 3200.1867 \\ \text { Frequencies -- } 3203.1756 & 3203.5139 & 3205.1253 \\ \text { Frequencies -- } 3206.2391 & 3207.7171 & 3209.3001 \\ \text { Frequencies -- } 3209.6252 & 3211.8860 & 3212.5723 \\ \text { Frequencies -- } 3212.8800 & 3215.4470 & 3215.9389 \\ \text { Frequencies -- } 3216.2783 & 3217.3163 & 3219.7170 \\ \text { Frequencies -- } 3220.3213 & 3220.4506 & 3222.2005 \\ \text { Frequencies -- } 3222.7440 & 3223.8615 & 3224.2408 \\ \text { Frequencies -- } 3224.4595 & 3228.4246 & 3230.1258 \\ \text { Frequencies -- } 3232.1194 & 3232.2911 & 3232.9517 \\ \text { Frequencies -- } 3233.0401 & 3234.0328 & 3235.2374 \\ \text { Frequencies -- } 3235.3511 & 3236.6170 & 3236.8278 \\ \text { Frequencies -- } 3239.5907 & 3239.9401 & 3240.7039 \\ \text { Frequencies -- } 3241.9591 & 3243.8294 & 3245.9272 \\ \text { Frequencies -- } 3247.4657 & 3248.8134 & 3250.4116 \\ \text { Frequencies -- } 3260.8317 & 3588.0535 & 3714.1814 \\ & & \end{array}$

SCF Done: $E(R M 062 X / D G D Z V P)=-6499.42768961$

Sum of electronic and zero-point Energies $=\quad-6498.076763$ Sum of electronic and thermal Energies $=\quad-6497.962686$ Sum of electronic and thermal Free Energies $=\quad-6498.231810$ SCF Done: $E(R M 062 X / D G T Z V P / S M D)=-6500.70823578$

\section{Cat f IOd $R$}

Center Atomic Atomic Coordinates (Angstroms)

\begin{tabular}{cccccc} 
Number & Number & Type & $X$ & $Y$ & $Z$ \\
\hline 1 & 15 & 0 & 0.380511 & 0.396154 & -0.477466 \\
2 & 8 & 0 & 0.392812 & 0.470910 & 1.129326 \\
3 & 8 & 0 & 1.938575 & 0.428943 & -0.863687 \\
4 & 8 & 0 & -0.139714 & 1.737361 & -1.039921 \\
5 & 8 & 0 & -0.334343 & -0.848339 & -0.871388 \\
6 & 6 & 0 & 0.783993 & 1.708037 & 1.649598 \\
7 & 6 & 0 & 2.742353 & -0.519435 & -0.223267 \\
8 & 6 & 0 & 2.124666 & 2.039175 & 1.734846 \\
9 & 6 & 0 & -0.215024 & 2.650486 & 1.895689 \\
10 & 6 & 0 & 3.362434 & -0.155682 & 0.961253 \\
11 & 6 & 0 & 2.854552 & -1.813432 & -0.745229 \\
12 & 6 & 0 & 3.377673 & 1.191785 & 1.647920 \\
13 & 6 & 0 & 2.471561 & 3.374545 & 1.936417 \\
14 & 6 & 0 & 0.159532 & 3.957491 & 2.211466 \\
15 & 6 & 0 & -1.628885 & 2.243620 & 1.726773 \\
16 & 6 & 0 & 4.110127 & -1.103565 & 1.660553 \\
17 & 6 & 0 & 3.619866 & -2.742414 & -0.032709 \\
18 & 6 & 0 & 2.148625 & -2.131853 & -2.009744 \\
19 & 6 & 0 & 3.829842 & 0.798377 & 3.082004
\end{tabular}

\begin{tabular}{|c|c|c|c|c|c|}
\hline 20 & 6 & 0 & 4.360385 & 2.210185 & 1.021041 \\
\hline 21 & 6 & 0 & 3.942882 & 3.592117 & 1.598618 \\
\hline 22 & 6 & 0 & 1.504000 & 4.344083 & 2.213699 \\
\hline 23 & 1 & 0 & -0.609643 & 4.698670 & 2.411514 \\
\hline 24 & 6 & 0 & -2.459567 & 2.915266 & 0.831187 \\
\hline 25 & 6 & 0 & -2.154996 & 1.147227 & 2.412952 \\
\hline 26 & 6 & 0 & 4.650000 & -0.507257 & 2.957119 \\
\hline 27 & 6 & 0 & 4.263753 & -2.403367 & 1.161933 \\
\hline 28 & 1 & 0 & 3.727972 & -3.748641 & -0.427946 \\
\hline 29 & 6 & 0 & 1.227991 & -3.175967 & -2.111059 \\
\hline 30 & 6 & 0 & 2.304133 & -1.295278 & -3.115644 \\
\hline 31 & 1 & 0 & 4.392401 & 1.600368 & 3.569206 \\
\hline 32 & 1 & 0 & 2.940747 & 0.610973 & 3.689526 \\
\hline 33 & 1 & 0 & 5.405933 & 1.962673 & 1.229158 \\
\hline 34 & 1 & 0 & 4.219751 & 2.216736 & -0.064620 \\
\hline 35 & 1 & 0 & 4.528053 & 3.826436 & 2.495366 \\
\hline 36 & 6 & 0 & 4.095153 & 4.731602 & 0.608395 \\
\hline 37 & 6 & 0 & 1.901363 & 5.755624 & 2.454595 \\
\hline 38 & 6 & 0 & -3.771572 & 2.505423 & 0.633970 \\
\hline 39 & 8 & 0 & -1.963099 & 3.986470 & 0.122863 \\
\hline 40 & 6 & 0 & -3.487830 & 0.750249 & 2.252532 \\
\hline 41 & 8 & 0 & -1.304482 & 0.469258 & 3.251718 \\
\hline 42 & 1 & 0 & 5.713344 & -0.271637 & 2.821504 \\
\hline 43 & 6 & 0 & 4.510083 & -1.406979 & 4.169826 \\
\hline 44 & 6 & 0 & 5.128445 & -3.392917 & 1.861663 \\
\hline 45 & 6 & 0 & 0.400979 & -3.305614 & -3.232295 \\
\hline 46 & 8 & 0 & 1.106618 & -4.042900 & -1.050267 \\
\hline 47 & 6 & 0 & 1.483573 & -1.414890 & -4.228453 \\
\hline 48 & 8 & 0 & 3.265594 & -0.309265 & -3.086842 \\
\hline 49 & 6 & 0 & 3.313027 & 4.750438 & -0.555142 \\
\hline 50 & 6 & 0 & 4.940465 & 5.809474 & 0.870212 \\
\hline 51 & 6 & 0 & 1.471361 & 6.775104 & 1.599386 \\
\hline 52 & 6 & 0 & 2.759066 & 6.068726 & 3.514436 \\
\hline 53 & 6 & 0 & -4.306853 & 1.427078 & 1.343498 \\
\hline 54 & 8 & 0 & -4.559209 & 3.167128 & -0.278792 \\
\hline 55 & 6 & 0 & -2.528378 & 4.115148 & -1.133552 \\
\hline 56 & 8 & 0 & -3.994979 & -0.296997 & 2.974143 \\
\hline 57 & 6 & 0 & -1.898650 & -0.367890 & 4.169149 \\
\hline 58 & 6 & 0 & 3.240026 & -1.774137 & 4.625391 \\
\hline 59 & 6 & 0 & 5.628926 & -1.906271 & 4.839470 \\
\hline 60 & 6 & 0 & 4.584939 & -4.527254 & 2.471706 \\
\hline 61 & 6 & 0 & 6.508992 & -3.177971 & 1.927080 \\
\hline 62 & 6 & 0 & 0.498775 & -2.399828 & -4.289373 \\
\hline 63 & 8 & 0 & -0.541053 & -4.294000 & -3.278071 \\
\hline 64 & 6 & 0 & -0.120598 & -4.678589 & -0.944318 \\
\hline 65 & 8 & 0 & 1.603101 & -0.519483 & -5.265815 \\
\hline 66 & 6 & 0 & 2.889607 & 0.834931 & -3.762479 \\
\hline 67 & 6 & 0 & 3.366601 & 5.838218 & -1.422846 \\
\hline 68 & 1 & 0 & 2.624475 & 3.929795 & -0.762251 \\
\hline 69 & 6 & 0 & 5.002117 & 6.896912 & -0.002886 \\
\hline 70 & 1 & 0 & 5.536198 & 5.813819 & 1.780025 \\
\hline 71 & 6 & 0 & 1.911923 & 8.083874 & 1.788863 \\
\hline 72 & 1 & 0 & 0.817101 & 6.530183 & 0.766031 \\
\hline 73 & 6 & 0 & 3.189637 & 7.379111 & 3.712302 \\
\hline 74 & 1 & 0 & 3.081351 & 5.279136 & 4.189431 \\
\hline 75 & 6 & 0 & -5.765555 & 1.109381 & 1.091460 \\
\hline 76 & 6 & 0 & -3.841234 & 3.700327 & -1.336028 \\
\hline 77 & 6 & 0 & -1.779422 & 4.630482 & -2.179767 \\
\hline 78 & 6 & 0 & -3.235287 & -0.726564 & 4.043854 \\
\hline 79 & 6 & 0 & -1.128020 & -0.867603 & 5.210278 \\
\hline 80 & 6 & 0 & 3.088692 & -2.635399 & 5.709856 \\
\hline 81 & 1 & 0 & 2.353512 & -1.397860 & 4.117743 \\
\hline 82 & 6 & 0 & 5.484929 & -2.771976 & 5.923413 \\
\hline 83 & 1 & 0 & 6.624147 & -1.636054 & 4.494702 \\
\hline 84 & 6 & 0 & 5.405664 & -5.412447 & 3.169410 \\
\hline 85 & 1 & 0 & 3.514594 & -4.708136 & 2.408406 \\
\hline
\end{tabular}




\begin{tabular}{|c|c|c|c|c|c|}
\hline 86 & 6 & 0 & 7.332553 & -4.071513 & 2.609318 \\
\hline 87 & 1 & 0 & 6.936166 & -2.306195 & 1.436636 \\
\hline 88 & 6 & 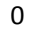 & -0.409863 & -2.393264 & -5.498070 \\
\hline 89 & 6 & 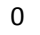 & -0.965063 & -4.753161 & -2.046882 \\
\hline 90 & 6 & 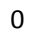 & -0.483297 & -5.271555 & 0.256103 \\
\hline 91 & 6 & 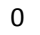 & 2.044785 & 0.728898 & -4.864902 \\
\hline 92 & 6 & 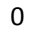 & 3.375461 & 2.071309 & -3.366076 \\
\hline 93 & 6 & $v$ & 4.210162 & 6.917348 & -1.149003 \\
\hline 94 & 1 & 0 & 2.727906 & 5.857681 & -2.301524 \\
\hline 95 & 1 & 0 & 5.653638 & 7.734923 & 0.225293 \\
\hline 96 & 6 & 0 & 2.774349 & 8.388259 & 2.842925 \\
\hline 97 & 1 & 0 & 1.588890 & 8.864750 & 1.107175 \\
\hline 98 & 1 & 0 & 3.849424 & 7.612584 & 4.542528 \\
\hline 99 & 9 & 0 & -6.528966 & 2.180813 & 1.384880 \\
\hline 100 & 9 & 0 & -5.982534 & 0.821622 & -0.203794 \\
\hline 101 & 9 & 0 & -6.228728 & 0.091967 & 1.813543 \\
\hline 102 & 6 & 0 & -4.429515 & 3.796470 & -2.587292 \\
\hline 103 & 1 & 0 & 48542 & 7213 & -1.994671 \\
\hline 104 & 6 & 0 & -2.368155 & 4.740408 & -3.440466 \\
\hline 105 & 6 & 0 & -3.828601 & -1.560924 & 4.981320 \\
\hline 106 & 1 & 0 & -0.084326 & -0.574376 & 5.271280 \\
\hline 107 & 6 & 0 & -1.710999 & -1.729641 & 6.137393 \\
\hline 108 & 6 & 0 & 4.213692 & -3.142272 & 6.361412 \\
\hline 109 & 1 & 0 & 2.089959 & -2.914326 & 6.037687 \\
\hline 110 & 1 & 0 & 6.367890 & -3.163059 & 6.419964 \\
\hline 111 & 6 & 0 & 6.780065 & -5.184488 & 3.243577 \\
\hline 112 & 1 & 0 & 4.970459 & -6.279839 & 3.656059 \\
\hline 113 & 1 & 0 & 8.403728 & -3.897634 & 2.648688 \\
\hline 114 & 9 & 0 & -1.032514 & -1.200380 & -5.622728 \\
\hline 115 & 9 & 0 & -1.356677 & -3.326485 & -5.476909 \\
\hline 116 & 9 & 0 & 0.301671 & -2.573227 & -6.626523 \\
\hline 117 & 6 & 0 & -2.219802 & -5.333348 & -1.942623 \\
\hline 118 & 1 & 0 & 0.205121 & -5.236548 & 1.095567 \\
\hline 119 & 6 & 0 & -1.727173 & -5.896935 & 0.354203 \\
\hline 120 & 6 & 0 & 1.659218 & 1.855879 & -5.575148 \\
\hline 121 & 1 & 0 & 4.038743 & 2.127330 & -2.508495 \\
\hline 122 & 6 & 0 & 3.016701 & 3.206583 & -4.094117 \\
\hline 123 & 1 & 0 & 4.241326 & 7.770601 & -1.819553 \\
\hline 124 & 1 & 0 & 3.117797 & 9.407878 & 2.988043 \\
\hline 125 & 1 & 0 & -5.452542 & 3.454762 & -2.713140 \\
\hline 126 & 6 & 0 & -3.685523 & 4.327680 & -3.642630 \\
\hline 127 & 1 & 0 & -1.791141 & 5.141989 & -4.266844 \\
\hline 128 & 1 & 0 & -4.875704 & -1.816943 & 4.855313 \\
\hline 129 & 6 & 0 & -3.060430 & -2.069651 & 6.027591 \\
\hline 130 & 1 & 0 & -1.109559 & -2.131220 & 6.946330 \\
\hline 131 & 1 & 0 & 4.101200 & -3.819286 & 7.202640 \\
\hline 132 & 1 & 0 & 7.417763 & -5.875160 & 3.786657 \\
\hline 133 & 1 & 0 & -2.864970 & -5.336963 & -2.814571 \\
\hline 134 & 6 & 0 & -2.603232 & -5.905372 & -0.730341 \\
\hline 135 & 1 & 0 & -2.013653 & -6.363084 & 1.291599 \\
\hline 136 & 1 & 0 & 0.993796 & 1.736802 & -6.424438 \\
\hline 137 & 6 & 0 & 2.157458 & 3.101078 & -5.188109 \\
\hline 138 & 1 & 0 & 3.420114 & 4.170255 & -3.801405 \\
\hline 139 & 1 & 0 & -4.138291 & 4.416331 & -4.624913 \\
\hline 140 & 1 & 0 & -3.517633 & -2.732698 & $\begin{array}{l}3 \\
6.754398\end{array}$ \\
\hline 141 & 1 & 0 & -3.584336 & -6.358165 & -0.636234 \\
\hline 142 & 1 & 0 & 1.878796 & 3.986101 & -5.750362 \\
\hline 143 & 1 & 0 & -0.987883 & 1.691019 & -1.689476 \\
\hline 144 & 8 & 0 & -2.063002 & 1.467833 & -2.554598 \\
\hline 145 & 6 & 0 & -1.654082 & 0.596428 & -3.653835 \\
\hline 146 & 6 & 0 & -2.671488 & -0.493481 & -3.225717 \\
\hline 147 & 1 & 0 & -1.810746 & 1.089494 & -4.614481 \\
\hline 148 & 1 & 0 & -0.607977 & 0.288528 & -3.536429 \\
\hline 149 & 8 & 0 & -2.102330 & -1.727059 & -2.900384 \\
\hline 150 & 6 & 0 & -2.994031 & 0.457535 & -2.037273 \\
\hline 151 & 1 & 0 & -1.442597 & -1.575433 & -2.196032 \\
\hline
\end{tabular}

$\begin{array}{rrrrrr}152 & 1 & 0 & -2.678360 & 0.090498 & -1.057007 \\ 153 & 1 & 0 & -4.010355 & 0.857736 & -1.990572 \\ 154 & 1 & 0 & 1.839706 & -3.435832 & 3.297932 \\ 155 & 6 & 0 & 0.762549 & -3.477916 & 3.175940 \\ 156 & 6 & 0 & 0.157111 & -2.675910 & 2.211434 \\ 157 & 6 & 0 & 0.012707 & -4.350690 & 3.974703 \\ 158 & 6 & 0 & -1.221917 & -2.796147 & 2.043026 \\ 159 & 1 & 0 & 0.741440 & -2.008509 & 1.582141 \\ 160 & 6 & 0 & -1.369442 & -4.451642 & 3.812624 \\ 161 & 1 & 0 & 0.512580 & -4.961813 & 4.720188 \\ 162 & 7 & 0 & -1.997176 & -2.161787 & 1.082343 \\ 163 & 6 & 0 & -1.979613 & -3.674248 & 2.831883 \\ 164 & 1 & 0 & -1.955522 & -5.131320 & 4.423803 \\ 165 & 6 & 0 & -3.304868 & -2.535981 & 0.997487 \\ 166 & 1 & 0 & -1.563731 & -1.607916 & 0.335568 \\ 167 & 16 & 0 & -3.648127 & -3.680791 & 2.298695 \\ 168 & 16 & 0 & -4.412653 & -2.027546 & -0.111176 \\ 169 & 6 & 0 & -3.820007 & -0.701680 & -4.180920 \\ 170 & 6 & 0 & -4.228829 & -1.985838 & -4.536193 \\ 171 & 6 & 0 & -4.489842 & 0.407584 & -4.708196 \\ 172 & 6 & 0 & -5.295517 & -2.157061 & -5.419478 \\ 173 & 1 & 0 & -3.708285 & -2.839367 & -4.115541 \\ 174 & 6 & 0 & -5.555245 & 0.235033 & -5.587120 \\ 175 & 1 & 0 & -4.179679 & 1.414515 & -4.426440 \\ 176 & 6 & 0 & -5.959660 & -1.051852 & -5.947499 \\ 177 & 1 & 0 & -5.607856 & -3.159669 & -5.695271 \\ 178 & 1 & 0 & -6.070091 & 1.101329 & -5.991782 \\ 179 & 1 & 0 & -6.788601 & -1.189469 & -6.634940 \\ -----------------------------------------------------\end{array}$

Frequencies -- $\quad 11.9102$

Frequencies -- 23.3587

Frequencies -- 30.2232

Frequencies -- 36.6369

Frequencies -- 42.3241

Frequencies -- 48.9314

Frequencies -- 58.1617

Frequencies -- 64.6192

Frequencies -- 69.0971

Frequencies -- 74.6223

Frequencies -- 85.9017

Frequencies -- 92.7519

Frequencies -- 100.5960

Frequencies -- 107.5343

Frequencies -- 121.0254

Frequencies -- 125.1230

Frequencies -- 137.5497

Frequencies -- 146.7441

Frequencies -- 167.5208

Frequencies -- 184.0242

Frequencies -- 199.6204

Frequencies -- 218.9613

Frequencies -- 227.7326

Frequencies -- 236.0998

Frequencies -- 251.0681

Frequencies -- 271.7375

Frequencies -- 284.9972

Frequencies -- 293.9602

Frequencies -- 302.6654

Frequencies -- 311.5171

Frequencies -- 317.8122

Frequencies -- 329.3276

Frequencies -- 344.4043

Frequencies -- 371.5842

Frequencies -- 404.2285

Frequencies -- 409.4068

$\begin{array}{ll}15.4349 & 20.7485 \\ 25.0632 & 27.7685 \\ 32.7748 & 34.8830 \\ 39.8931 & 41.4741 \\ 43.8269 & 45.6852 \\ 51.7691 & 55.4877 \\ 58.7210 & 62.2207 \\ 65.5908 & 68.1146 \\ 71.6339 & 73.1325 \\ 78.8863 & 81.3304 \\ 88.4777 & 90.3893 \\ 95.0912 & 95.7542 \\ 102.5834 & 105.5284 \\ 114.3219 & 117.1162 \\ 122.9199 & 124.0839 \\ 130.1275 & 134.4293 \\ 142.6331 & 146.2461 \\ 153.8088 & 160.6709 \\ 172.8149 & 174.3989 \\ 187.1821 & 192.4385 \\ 201.9574 & 211.8364 \\ 223.0543 & 226.4657 \\ 231.7741 & 233.3865 \\ 243.0469 & 245.1429 \\ 259.3659 & 269.6757 \\ 276.9692 & 281.3348 \\ 289.1857 & 291.9073 \\ 296.9888 & 299.4220 \\ 303.2964 & 308.5334 \\ 314.6690 & 316.5796 \\ 321.2924 & 325.2965 \\ 334.2452 & 337.0938 \\ 346.0000 & 357.1704 \\ 373.9925 & 385.3285 \\ 405.5978 & 406.2147 \\ 410.8591 & 412.6406\end{array}$




\begin{tabular}{|c|c|c|c|}
\hline Frequencies -- & 413.0773 & 418.0007 & 418.7711 \\
\hline Frequencies -- & 425.5998 & 429.1438 & 436.2433 \\
\hline Frequencies -- & 436.9286 & 437.2065 & 439.8282 \\
\hline Frequencies -- & 445.0715 & 447.0234 & 449.4265 \\
\hline Frequencies -- & 452.9746 & 458.1605 & 461.6106 \\
\hline Frequencies -- & 463.1417 & 467.2943 & 474.9066 \\
\hline Frequencies -- & 486.6456 & 489.7384 & 499.3647 \\
\hline Frequencies -- & 505.2268 & 506.2083 & 513.2143 \\
\hline Frequencies -- & 519.4668 & 519.9857 & 521.6472 \\
\hline Frequencies -- & 530.2682 & 533.5494 & 537.9329 \\
\hline Frequencies -- & 539.7911 & 541.4924 & 544.2286 \\
\hline Frequencies -- & 551.9526 & 554.1445 & 556.4888 \\
\hline Frequencies -- & 557.4314 & 560.6748 & 562.2883 \\
\hline Frequencies -- & 563.6560 & 567.9521 & 568.9067 \\
\hline Frequencies -- & 581.8018 & 583.2615 & 589.4521 \\
\hline Frequencies -- & 593.5800 & 595.7378 & 596.8629 \\
\hline Frequencies -- & 598.0105 & 601.4783 & 604.9697 \\
\hline Frequencies -- & 607.1328 & 609.6235 & 610.0681 \\
\hline Frequencies -- & 611.1173 & 618.6572 & 620.9965 \\
\hline Frequencies -- & 624.7720 & 626.5897 & 627.8033 \\
\hline Frequencies -- & 630.4439 & 631.0220 & 632.4048 \\
\hline Frequencies -- & 633.4991 & 634.7575 & 646.4650 \\
\hline Frequencies -- & 648.3919 & 655.7512 & 659.6941 \\
\hline Frequencies -- & 666.1217 & 673.2781 & 675.8146 \\
\hline Frequencies -- & 682.1149 & 682.9870 & 697.2982 \\
\hline Frequencies -- & 705.5144 & 705.8590 & 711.7072 \\
\hline Frequencies -- & 711.9678 & 714.1571 & 716.5603 \\
\hline Frequencies -- & 717.6500 & 719.7652 & 720.3548 \\
\hline Frequencies -- & 722.8472 & 725.4626 & 725.9741 \\
\hline Frequencies -- & 729.0686 & 730.0903 & 732.5509 \\
\hline Frequencies -- & 735.3851 & 740.9153 & 744.0096 \\
\hline Frequencies -- & 746.1733 & 749.9233 & 753.6841 \\
\hline Frequencies -- & 760.9584 & 764.9087 & 767.8699 \\
\hline Frequencies -- & 769.8586 & 771.0754 & 773.0242 \\
\hline Frequencies -- & 773.2260 & 776.1889 & 779.9017 \\
\hline Frequencies -- & 780.3818 & 782.2357 & 785.0398 \\
\hline Frequencies -- & 793.8312 & 796.9966 & 801.5814 \\
\hline Frequencies -- & 803.0439 & 805.5881 & 820.3861 \\
\hline Frequencies -- & 824.7338 & 825.2949 & 827.5378 \\
\hline Frequencies -- & 830.0429 & 864.9012 & 865.1933 \\
\hline Frequencies -- & 867.1290 & 867.8757 & 868.3209 \\
\hline Frequencies -- & 869.1975 & 871.1403 & 872.1560 \\
\hline Frequencies -- & 872.9772 & 875.8565 & 877.0038 \\
\hline Frequencies -- & 885.8015 & 887.6236 & 888.5309 \\
\hline Frequencies -- & 889.9425 & 891.1129 & 909.4508 \\
\hline Frequencies -- & 918.7935 & 920.0371 & 921.6850 \\
\hline Frequencies -- & 923.3969 & 925.9621 & 932.6144 \\
\hline Frequencies -- & 934.0911 & 937.0986 & 940.0074 \\
\hline Frequencies -- & 941.9675 & 943.1069 & 943.5096 \\
\hline Frequencies -- & 944.1407 & 947.2963 & 951.6412 \\
\hline Frequencies -- & 952.8937 & 953.6766 & 954.2536 \\
\hline Frequencies -- & 964.1531 & 968.1142 & 973.5817 \\
\hline Frequencies -- & 982.5423 & 986.8071 & 987.8303 \\
\hline Frequencies -- & 989.9380 & 993.5849 & 994.6571 \\
\hline Frequencies -- & 997.2153 & 997.9157 & 998.9681 \\
\hline Frequencies -- & 999.4541 & 1008.3874 & 1010.2094 \\
\hline Frequencies -- & 1010.6312 & 1010.7882 & 1011.4864 \\
\hline Frequencies -- & 1011.6466 & 1014.4451 & 1018.0455 \\
\hline Frequencies -- & 1018.6483 & 1019.1621 & 1019.7537 \\
\hline Frequencies -- & 1020.6805 & 1020.8610 & 1025.2056 \\
\hline Frequencies -- & 1030.0968 & 1035.5916 & 1036.0728 \\
\hline Frequencies -- & 1044.0577 & 1051.8603 & 1054.6170 \\
\hline Frequencies -- & 1055.8809 & 1059.5835 & 1061.0872 \\
\hline Frequencies -- & 1062.1281 & 1062.9876 & 1068.4841 \\
\hline Frequencies -- & 1068.8169 & 1071.0305 & 1071.6328 \\
\hline Frequencies -- & 1073.1579 & 1077.5874 & 1080.9561 \\
\hline
\end{tabular}

Frequencies -- 1090.5877 Frequencies -- 1110.7956 Frequencies -- 1114.2186 Frequencies -- 1126.2912 Frequencies -- 1130.6259 Frequencies -- 1164.8330 Frequencies -- 1168.2800 Frequencies -- 1171.4696 Frequencies -- 1176.6341 Frequencies -- 1179.1822 Frequencies -- 1198.9181 Frequencies -- 1203.8910 Frequencies -- 1210.8662 Frequencies -- 1214.0962 Frequencies -- 1227.1581 Frequencies -- 1232.1791 Frequencies -- 1241.9834 Frequencies -- 1256.4659 Frequencies -- 1268.3549 Frequencies -- 1284.1799 Frequencies -- 1294.9657 Frequencies -- 1298.3637 Frequencies -- 1303.0078 Frequencies -- 1308.0879 Frequencies -- 1318.8755 Frequencies -- 1326.0254 Frequencies -- 1335.2568 Frequencies -- 1340.6971 Frequencies -- 1358.8294 Frequencies -- 1362.4214 Frequencies -- 1367.0510 Frequencies -- 1371.2406 Frequencies -- 1378.0851 Frequencies -- 1392.6010 Frequencies -- 1457.3820 Frequencies -- 1471.6849 Frequencies -- 1493.2199 Frequencies -- 1499.9098 Frequencies -- 1504.8302 Frequencies -- 1510.0167 Frequencies -- 1515.6472 Frequencies -- 1526.8032 Frequencies -- 1546.8639 Frequencies -- 1555.2984 Frequencies -- 1560.3231 Frequencies -- 1565.5286 Frequencies -- 1663.5765 Frequencies -- 1669.3801 Frequencies -- 1677.3604 Frequencies -- 1680.1919 Frequencies -- 1696.1458 Frequencies -- 1700.2802 Frequencies -- 1702.6594 Frequencies -- 1704.2991 Frequencies -- 1719.7074 Frequencies -- 2074.7398 Frequencies -- 3090.8013 Frequencies -- 3124.7764 Frequencies -- 3169.1761 Frequencies -- 3194.9113 Frequencies -- 3201.9421 Frequencies -- 3206.6626 Frequencies -- 3210.0565 Frequencies -- 3216.8626 Frequencies -- 3220.7502 Frequencies -- 3222.7066

$\begin{array}{ll}1094.3447 & 1100.4299 \\ 1111.7949 & 1113.1461 \\ 1117.1703 & 1124.1990 \\ 1127.7592 & 1129.5968 \\ 1132.7908 & 1160.7806 \\ 1165.5527 & 1167.2225 \\ 1170.0783 & 1171.2107 \\ 1174.0566 & 1175.0046 \\ 1178.0528 & 1178.2059 \\ 1182.8044 & 1196.1177 \\ 1202.0367 & 1202.9458 \\ 1206.2654 & 1208.1387 \\ 1212.1016 & 1213.3669 \\ 1223.4581 & 1226.1021 \\ 1227.9990 & 1229.1657 \\ 1234.9978 & 1238.1151 \\ 1242.7984 & 1247.7050 \\ 1259.2549 & 1265.4988 \\ 1277.0426 & 1283.0144 \\ 1287.1587 & 1293.5597 \\ 1295.0704 & 1296.7741 \\ 1300.3017 & 1301.5481 \\ 1306.4193 & 1306.9700 \\ 1311.1981 & 1311.6634 \\ 1323.1946 & 1324.3888 \\ 1326.6402 & 1327.7821 \\ 1337.8477 & 1340.0472 \\ 1345.4585 & 1350.5466 \\ 1359.0471 & 1360.2490 \\ 1363.0080 & 1366.3002 \\ 1368.8673 & 1370.3276 \\ 1372.8339 & 1375.8233 \\ 1383.9299 & 1388.0391 \\ 1407.6261 & 1449.1464 \\ 1461.4914 & 1468.4924 \\ 1474.5128 & 1491.9173 \\ 1497.1047 & 1498.5710 \\ 1503.1057 & 1504.2161 \\ 1506.8798 & 1507.3663 \\ 1511.9961 & 1515.3635 \\ 1517.6645 & 1525.0122 \\ 1529.3416 & 1532.8840 \\ 1549.3573 & 1554.9874 \\ 1555.9673 & 1558.1905 \\ 1561.2774 & 1563.2095 \\ 1571.8813 & 1660.6540 \\ 1668.2337 & 1668.6744 \\ 1672.4946 & 1674.7353 \\ 1677.7010 & 1679.5012 \\ 1689.2929 & 1691.1252 \\ 1697.7970 & 1699.8068 \\ 1701.2298 & 1701.6104 \\ 1703.1482 & 1703.6035 \\ 1706.0935 & 1719.1781 \\ 1731.2491 & 1733.2763 \\ 3068.4911 & 3086.1156 \\ 3097.6422 & 3101.6318 \\ 3156.9002 & 3163.2700 \\ 3170.1690 & 3187.5337 \\ 3199.3374 & 3201.5426 \\ 3205.3628 & 3205.4191 \\ 3207.3590 & 3209.5158 \\ 3212.8146 & 3216.0687 \\ 3218.5095 & 3218.5169 \\ 3221.2918 & 3222.4731 \\ 3225.7128 & 3226.1017\end{array}$




$\begin{array}{llll}\text { Frequencies -- } 3226.7027 & 3227.2731 & 3227.4188 \\ \text { Frequencies -- } 3228.0106 & 3228.7419 & 3229.0763 \\ \text { Frequencies -- } 3231.2172 & 3231.8935 & 3232.5486 \\ \text { Frequencies -- } 3232.5863 & 3233.1725 & 3235.5165 \\ \text { Frequencies -- } 3236.6065 & 3236.9852 & 3237.0255 \\ \text { Frequencies -- } 3238.1063 & 3239.6689 & 3241.2972 \\ \text { Frequencies -- } 3246.8391 & 3247.3661 & 3247.6744 \\ \text { Frequencies -- } 3248.2595 & 3248.4988 & 3252.4109 \\ \text { Frequencies -- } 3260.2839 & 3428.6563 & 3710.8780\end{array}$

SCF Done: $E($ RM062X/DGDZVP) $=-6499.40019055$

Sum of electronic and zero-point Energies $=\quad-6498.050144$ Sum of electronic and thermal Energies = $\quad-6497.936044$ Sum of electronic and thermal Free Energies $=\quad-6498.206875$ SCF Done: $E(R M 062 X / D G T Z V P / S M D)=-6500.6845574$

\section{Cat f TS1d_R}

Center Atomic Atomic Coordinates (Angstroms) Number Number Type $X \quad$ Y Z

\begin{tabular}{|c|c|c|c|c|c|}
\hline 1 & 15 & 0 & -0.011846 & -0.020306 & 0.006666 \\
\hline 2 & 8 & 0 & 0.000717 & -0.003940 & 1.639022 \\
\hline 3 & 8 & 0 & 1.570245 & 0.001360 & -0.346108 \\
\hline 4 & 8 & 0 & -0.588988 & 1.241529 & -0.555824 \\
\hline 5 & 8 & 0 & -0.626391 & -1.351673 & -0.336588 \\
\hline 6 & 6 & 0 & 0.371845 & 1.217558 & 2.184252 \\
\hline 7 & 6 & 0 & 2.374164 & -0.949131 & 0.267455 \\
\hline 8 & 6 & 0 & 1.711756 & 1.552530 & 2.302844 \\
\hline 9 & 6 & 0 & -0.626091 & 2.161951 & 2.431320 \\
\hline 10 & 6 & 0 & 2.980775 & -0.614508 & 1.470735 \\
\hline 11 & 6 & 0 & 2.526019 & -2.228358 & -0.286520 \\
\hline 12 & 6 & 0 & 2.967496 & 0.709326 & 2.204348 \\
\hline 13 & 6 & 0 & 2.056397 & 2.880250 & 2.550370 \\
\hline 14 & 6 & 0 & -0.256725 & 3.458449 & 2.796088 \\
\hline 15 & 6 & 0 & -2.042170 & 1.800359 & 2.192538 \\
\hline 16 & 6 & 0 & 3.745151 & -1.565652 & 2.145184 \\
\hline 17 & 6 & 0 & 3.319120 & -3.156519 & 0.397634 \\
\hline 18 & 6 & 0 & 1.792203 & -2.571035 & -1.527859 \\
\hline 19 & 6 & 0 & 3.397463 & 0.271987 & 3.632452 \\
\hline 20 & 6 & 0 & 3.958829 & 1.747742 & 1.625082 \\
\hline 21 & 6 & 0 & 3.529757 & 3.112750 & 2.232718 \\
\hline 22 & 6 & 0 & 1.085965 & 3.842915 & 2.842185 \\
\hline 23 & 1 & 0 & -1.029101 & 4.196779 & 2.995510 \\
\hline 24 & 6 & 0 & -2.806213 & 2.540607 & 1.290681 \\
\hline 25 & 6 & 0 & -2.649192 & 0.708315 & 2.815939 \\
\hline 26 & 6 & 0 & 4.240842 & -1.015345 & 3.477027 \\
\hline 27 & 6 & 0 & 3.943017 & -2.842337 & 1.606988 \\
\hline 28 & 1 & 0 & 3.453577 & -4.147611 & -0.026165 \\
\hline 29 & 6 & 0 & 0.937569 & -3.675044 & -1.589511 \\
\hline 30 & 6 & 0 & 1.824586 & -1.722065 & -2.633486 \\
\hline 31 & 1 & 0 & 3.935747 & 1.065411 & 4.159654 \\
\hline 32 & 1 & 0 & 2.499194 & 0.047532 & 4.213742 \\
\hline 33 & 1 & 0 & 5.001096 & 1.495577 & 1.844542 \\
\hline 34 & 1 & 0 & 3.838221 & 1.782677 & 0.537476 \\
\hline 35 & 1 & 0 & 4.106858 & 3.330080 & 3.139049 \\
\hline 36 & 6 & 0 & 3.682229 & 4.275125 & 1.268965 \\
\hline 37 & 6 & 0 & 1.477936 & 5.248149 & 3.123673 \\
\hline 38 & 6 & 0 & -4.137009 & 2.225666 & 1.048588 \\
\hline 39 & 8 & 0 & -2.230446 & 3.590183 & 0.615893 \\
\hline 40 & 6 & 0 & -3.994549 & 0.391120 & 2.591150 \\
\hline 41 & 8 & 0 & -1.875401 & -0.048510 & 3.663611 \\
\hline 42 & 1 & 0 & 5.305167 & -0.763064 & 3.388619 \\
\hline 43 & 6 & 0 & 4.070590 & -1.972456 & 4.640137 \\
\hline 44 & 6 & 0 & 4.808182 & -3.837574 & 2.298087 \\
\hline
\end{tabular}




\begin{tabular}{|c|c|c|c|c|c|}
\hline 111 & 6 & 0 & 6.447145 & -5.645358 & 3.674989 \\
\hline 112 & 1 & 0 & 4.630529 & -6.732849 & 4.079421 \\
\hline 13 & 1 & 0 & 8.077548 & -4.358911 & 3.098839 \\
\hline 14 & 9 & 0 & -1.661765 & -1.862050 & -4.956482 \\
\hline 15 & 9 & 0 & -1.807392 & -4.006686 & -4.808416 \\
\hline 116 & 9 & 0 & -0.275254 & -3.121162 & -6.035477 \\
\hline 17 & 6 & 0 & -2.352301 & -6.046319 & -1.209778 \\
\hline 118 & 1 & 0 & 0.257932 & -5.794790 & 1.656025 \\
\hline 119 & 6 & 0 & -1.678640 & -6.572054 & 1.052639 \\
\hline 120 & 6 & 0 & 0.800698 & 1.398940 & -4.993807 \\
\hline 121 & 1 & 0 & 3.335051 & 1.800250 & -2.065803 \\
\hline 122 & 6 & 0 & 2.139510 & 2.823878 & -3.565629 \\
\hline 123 & 1 & 0 & 3.832299 & 7.359985 & -1.100824 \\
\hline 124 & 1 & 0 & 2.685399 & 8.889754 & 3.747560 \\
\hline 125 & 1 & 0 & -5.670573 & 3.352416 & -2.315004 \\
\hline 126 & 6 & 0 & -3.803739 & 4.040298 & -3.202828 \\
\hline 127 & 1 & 0 & -1.818426 & 4.637663 & -3.787588 \\
\hline 128 & 1 & 0 & -5.642984 & -2.157479 & 5.044659 \\
\hline 129 & 6 & 0 & -3.918187 & -2.493441 & 6.330005 \\
\hline 130 & 1 & 0 & -2.036563 & -2.635053 & 7.373832 \\
\hline 131 & 1 & 0 & 3.588686 & -4.510055 & 7.557123 \\
\hline 132 & 1 & 0 & 7.080345 & -6.342610 & 4.214997 \\
\hline 133 & 1 & 0 & -3.049907 & -6.100387 & -2.038962 \\
\hline 134 & 6 & 0 & -2.619184 & -6.639860 & 0.024640 \\
\hline 135 & 1 & 0 & -1.871724 & -7.050867 & 2.007267 \\
\hline 136 & 1 & 0 & 0.092524 & 1.244549 & -5.801741 \\
\hline 137 & 6 & 0 & 1.226161 & 2.671655 & -4.609257 \\
\hline 138 & 1 & 0 & 2.480379 & 3.812041 & -3.275841 \\
\hline 139 & 1 & 0 & -4.220885 & 4.164073 & -4.196670 \\
\hline 140 & 1 & 0 & -4.450467 & -3.133660 & 7.025558 \\
\hline 141 & 1 & 0 & -3.558444 & -7.161120 & 0.175770 \\
\hline 142 & 1 & 0 & 0.845955 & 3.542801 & -5.132664 \\
\hline 143 & 1 & 0 & -1.837642 & 1.264549 & -1.481347 \\
\hline 144 & 8 & 0 & -2.653864 & 1.015134 & -2.035702 \\
\hline 145 & 6 & 0 & -2.272338 & -0.022550 & -2.971100 \\
\hline 146 & 6 & 0 & -3.145565 & -1.154121 & -2.389598 \\
\hline 147 & 1 & 0 & -2.513403 & 0.291325 & -3.986309 \\
\hline 148 & 1 & 0 & -1.202860 & -0.240237 & -2.881769 \\
\hline 149 & 8 & 0 & -2.444567 & -2.326904 & -2.105399 \\
\hline 150 & 6 & 0 & -3.550199 & -0.318419 & -1.138051 \\
\hline 151 & 1 & 0 & -1.642636 & -2.076149 & -1.598211 \\
\hline 152 & 1 & 0 & -2.925803 & -0.293212 & -0.249730 \\
\hline 153 & 1 & 0 & -4.495483 & 0.210822 & -1.091312 \\
\hline 154 & 1 & 0 & 0.980932 & -4.004014 & 3.934807 \\
\hline 155 & 6 & 0 & -0.075769 & -3.984235 & 3.686718 \\
\hline 156 & 6 & 0 & -0.516831 & -3.158554 & 2.656016 \\
\hline 157 & 6 & 0 & -0.962673 & -4.812044 & 4.389510 \\
\hline 158 & 6 & 0 & -1.872933 & -3.208274 & 2.334329 \\
\hline 159 & 1 & 0 & 0.161231 & -2.516427 & 2.097326 \\
\hline 160 & 6 & 0 & -2.319239 & -4.842080 & 4.071266 \\
\hline 161 & 1 & 0 & -0.589826 & -5.441139 & 5.192065 \\
\hline 162 & 7 & 0 & -2.501846 & -2.509894 & 1.307140 \\
\hline 163 & 6 & 0 & -2.763793 & -4.038571 & 3.023423 \\
\hline 164 & 1 & 0 & -3.007520 & -5.481332 & 4.615295 \\
\hline 165 & 6 & 0 & -3.811591 & -2.761915 & 1.126139 \\
\hline 166 & 1 & 0 & -1.922222 & -1.977741 & 0.633118 \\
\hline 167 & 16 & 0 & -4.365530 & -3.915306 & 2.32608 \\
\hline 168 & 16 & 0 & -4.840390 & -2.121911 & -0.02944 \\
\hline 169 & 6 & 0 & -4.354373 & -1.478852 & -3.239553 \\
\hline 170 & 6 & 0 & -4.650575 & -2.796305 & -3.582888 \\
\hline 171 & 6 & 0 & -5.182100 & -0.442061 & -3.682519 \\
\hline 172 & 6 & 0 & -5.770801 & -3.073728 & -4.366677 \\
\hline 173 & 1 & 0 & -4.001832 & -3.590755 & -3.229797 \\
\hline 174 & 6 & 0 & -6.303537 & -0.722199 & -4.458909 \\
\hline 175 & 1 & 0 & -4.949896 & 0.591579 & -3.422998 \\
\hline 176 & 6 & 0 & -6.599666 & -2.041938 & -4.803650 \\
\hline
\end{tabular}

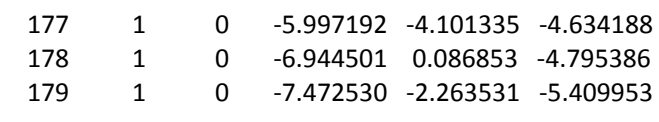

Frequencies -- -552.7541

$12.4527 \quad 20.8232$

Frequencies

$27.1042 \quad 27.7565$

Frequencies -- 29.0629

$31.3692 \quad 32.6736$

Frequencies -- 35.3367

$38.0973 \quad 39.2407$

Frequencies -- 40.4993

$41.3025 \quad 46.3748$

Frequencies -- 49.1444

Frequencies -- 56.3718

53.3944

57.3914

Frequencies -- 61.1203

Frequencies -- 68.6020

59.8365

66.8795

Frequencies -- 76.1126

$73.2119 \quad 73.4533$

$77.7387 \quad 80.1871$

Frequencies -- $84.8460 \quad 86.8601 \quad 89.5372$

Frequencies -- 90.7442

95.2133

101.3712

97.4311

Frequencies -- 99.5382

Frequencies -- 112.2881

114.3535

120.0963

129.4743

Frequencies -- 127.8410

143.9663

156.4510

172.3393

190.4327

202.9501

219.1890

230.7450

244.2379

262.6099

277.2497

284.9554

295.5984

302.8837

312.9422

319.5838

329.9961

345.4777

377.9343

406.0942

412.6880

419.0657

423.8044

436.3501

446.6342

460.8834

463.8507

486.5073

506.4999

519.6707

530.4357

540.3806

553.0228

561.2971

569.0796

584.8029

594.0802

598.1640

608.6163

616.8075

624.8033

630.9860

635.6787

655.7594

671.8712

683.4081
105.7059

115.5224

125.7284

134.9356

148.7049

163.4244

174.9815

194.1920

209.5304

221.5578

233.8772

247.7907

269.4463

278.5555

288.7623

298.5916

304.2531

315.8557

321.5177

336.7100

353.2025

386.8573

406.7691

414.6242

421.0897

429.1359

442.1372

452.0536

461.2751

466.6894

491.4859

508.7153

521.3587

536.1259

541.3060

556.1832

562.3204

571.2145

587.6264

596.0194

601.0530

610.3434

618.9920

626.7164

632.6814

645.7446

665.9646

677.3237

684.9672 


\begin{tabular}{|c|c|c|c|}
\hline Frequencies -- & 696.5411 & 703.9562 & 709.3956 \\
\hline Frequencies -- & 713.4996 & 716.1188 & 719.1995 \\
\hline Frequencies -- & 719.8033 & 720.1593 & 722.2923 \\
\hline Frequencies -- & 723.8206 & 724.6613 & 726.5706 \\
\hline Frequencies -- & 727.6856 & 729.4609 & 731.2745 \\
\hline Frequencies -- & 732.5676 & 738.0177 & 740.1883 \\
\hline Frequencies -- & 745.4872 & 747.2176 & 750.9953 \\
\hline Frequencies -- & 752.1131 & 762.0874 & 764.0903 \\
\hline Frequencies -- & 764.1726 & 769.4941 & 771.9782 \\
\hline Frequencies -- & 772.5770 & 773.8477 & 778.5429 \\
\hline Frequencies -- & 781.2766 & 781.5697 & 782.4285 \\
\hline Frequencies -- & 788.2319 & 795.8983 & 798.3878 \\
\hline Frequencies -- & 801.6277 & 803.5922 & 806.3243 \\
\hline Frequencies -- & 822.9616 & 824.9833 & 826.3525 \\
\hline Frequencies -- & 831.5468 & 859.7779 & 862.0607 \\
\hline Frequencies -- & 863.4151 & 866.8594 & 867.4694 \\
\hline Frequencies -- & 868.6484 & 868.9061 & 869.0300 \\
\hline Frequencies -- & 874.0814 & 875.1169 & 876.0660 \\
\hline Frequencies -- & 880.0921 & 883.3348 & 886.8821 \\
\hline Frequencies -- & 887.5360 & 888.8568 & 890.5430 \\
\hline Frequencies -- & 897.8386 & 912.5686 & 915.7165 \\
\hline Frequencies -- & 916.6477 & 920.4459 & 923.1043 \\
\hline Frequencies -- & 930.7767 & 932.7322 & 937.6827 \\
\hline Frequencies -- & 941.4938 & 943.4598 & 944.0037 \\
\hline Frequencies -- & 944.6593 & 947.5728 & 949.5946 \\
\hline Frequencies -- & 951.2613 & 951.5449 & 952.9327 \\
\hline Frequencies -- & 957.5075 & 959.3462 & 966.7271 \\
\hline Frequencies -- & 968.9655 & 984.3275 & 988.0680 \\
\hline Frequencies -- & 988.6723 & 992.1135 & 995.0516 \\
\hline Frequencies -- & 996.5005 & 997.6095 & 1001.2278 \\
\hline Frequencies -- & 1004.5897 & 1005.4413 & 1005.9168 \\
\hline Frequencies -- & 1007.4534 & 1008.4151 & 1009.8545 \\
\hline Frequencies -- & 1011.0832 & 1011.3082 & 1018.5256 \\
\hline Frequencies -- & 1018.8390 & 1019.5244 & 1019.8215 \\
\hline Frequencies -- & 1020.1602 & 1020.8582 & 1021.3161 \\
\hline Frequencies -- & 1028.9416 & 1034.7917 & 1035.9004 \\
\hline Frequencies -- & 1043.5748 & 1047.0831 & 1053.0941 \\
\hline Frequencies -- & 1056.9672 & 1057.8317 & 1058.8886 \\
\hline Frequencies -- & 1059.5782 & 1060.7658 & 1062.0718 \\
\hline Frequencies -- & 1063.5260 & 1068.3524 & 1068.8152 \\
\hline Frequencies -- & 1071.9820 & 1073.3797 & 1079.3797 \\
\hline Frequencies -- & 1094.5260 & 1098.2800 & 1100.1207 \\
\hline Frequencies -- & 1109.6713 & 1110.4173 & 1111.6474 \\
\hline Frequencies -- & 1113.0374 & 1115.2708 & 1118.5375 \\
\hline Frequencies -- & 1123.6462 & 1125.2770 & 1126.9466 \\
\hline Frequencies -- & 1127.9236 & 1128.8267 & 1130.1631 \\
\hline Frequencies -- & 1132.1420 & 1158.5447 & 1162.3926 \\
\hline Frequencies -- & 1165.0185 & 1165.6808 & 1166.5682 \\
\hline Frequencies -- & 1166.7477 & 1172.3126 & 1173.2423 \\
\hline Frequencies -- & 1175.3248 & 1176.9618 & 1178.0515 \\
\hline Frequencies -- & 1178.7496 & 1178.9547 & 1183.2123 \\
\hline Frequencies -- & 1192.2803 & 1196.5870 & 1200.8777 \\
\hline Frequencies -- & 1201.4139 & 1201.9709 & 1202.9171 \\
\hline Frequencies -- & 1205.2870 & 1207.7316 & 1210.6585 \\
\hline Frequencies -- & 1213.6822 & 1213.8502 & 1214.7732 \\
\hline Frequencies -- & 1222.6709 & 1225.5818 & 1226.6505 \\
\hline Frequencies -- & 1227.0370 & 1231.7282 & 1233.9813 \\
\hline Frequencies -- & 1237.2860 & 1241.5345 & 1246.8189 \\
\hline Frequencies -- & 1249.2234 & 1255.3013 & 1261.7293 \\
\hline Frequencies -- & 1268.1664 & 1271.4173 & 1281.1063 \\
\hline Frequencies -- & 1283.0376 & 1286.4106 & 1286.7627 \\
\hline Frequencies -- & 1293.2139 & 1294.0641 & 1296.3362 \\
\hline Frequencies -- & 1299.4137 & 1299.7570 & 1301.5822 \\
\hline Frequencies -- & 1305.4886 & 1307.5194 & 1308.9858 \\
\hline Frequencies -- & 1309.9115 & 1310.9100 & 1311.9149 \\
\hline Frequencies -- & 1313.0612 & 1320.7134 & 1324.7988 \\
\hline
\end{tabular}

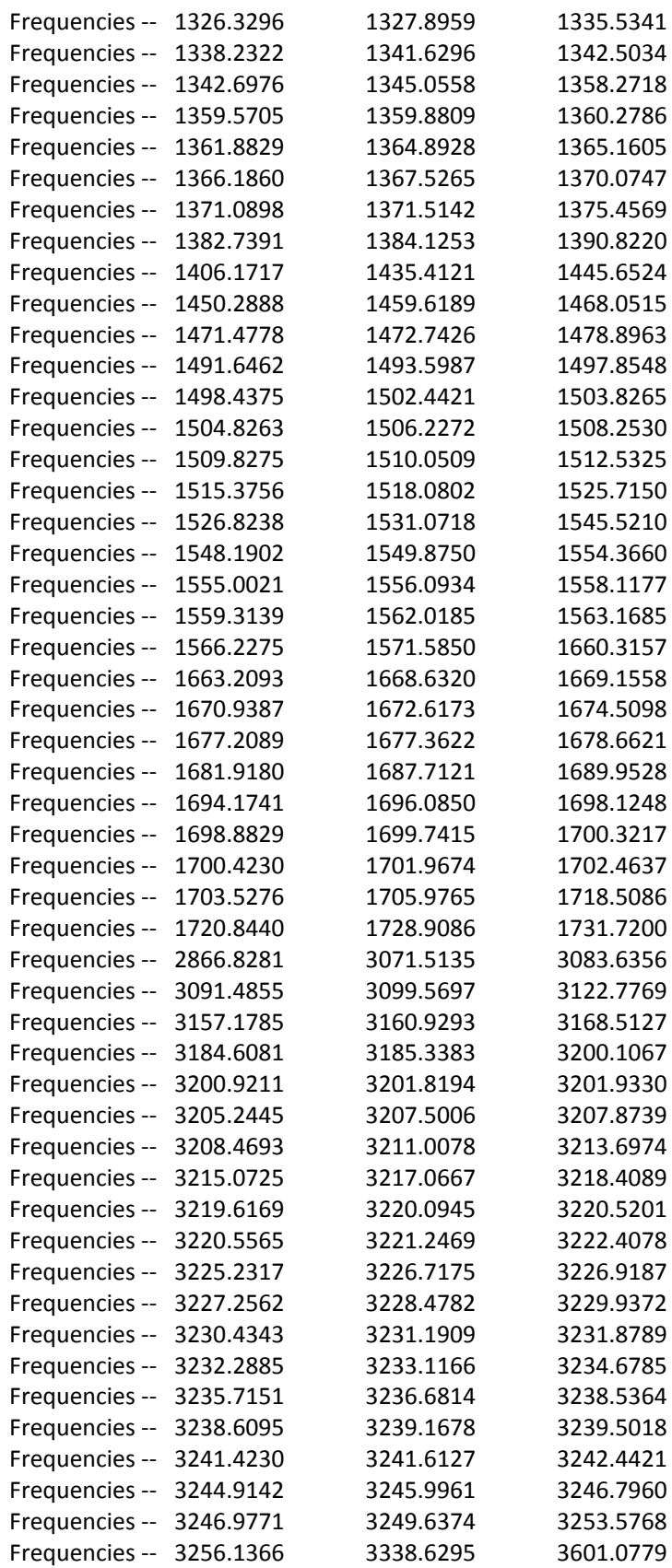

SCF Done: $E(R M 062 X / D G D Z V P)=-6499.37689259$

Sum of electronic and zero-point Energies $=\quad-6498.026708$ Sum of electronic and thermal Energies $=\quad-6497.913330$ Sum of electronic and thermal Free Energies $=\quad-6498.181075$ SCF Done: $E(R M 062 X / D G T Z V P / S M D)=-6500.65858215$

\section{Cat f I1d_R}

Center Atomic Atomic Coordinates (Angstroms)

\begin{tabular}{ccccccc} 
Number & Number & Type & $X$ & $Y$ & \multicolumn{1}{c}{ Z } \\
\hline 1 & 15 & 0 & 0.398421 & 0.435629 & -0.462442 \\
2 & 8 & 0 & 0.455291 & 0.463923 & 1.176477 \\
3 & 8 & 0 & 1.971977 & 0.487221 & -0.855668
\end{tabular}




\begin{tabular}{|c|c|c|c|c|c|}
\hline 4 & 8 & 0 & -0.262634 & 1.640059 & -1.023232 \\
\hline 5 & 8 & 0 & -0.141105 & -0.954415 & -0.743349 \\
\hline 6 & 6 & 0 & 0.824087 & 1.693072 & 1.702121 \\
\hline 7 & 6 & 0 & 2.809199 & -0.452230 & -0.273332 \\
\hline 8 & 6 & 0 & 2.165660 & 2.030608 & 1.805135 \\
\hline 9 & 6 & 0 & -0.170041 & 2.640956 & 1.952453 \\
\hline 10 & 6 & 0 & 3.433876 & -0.124414 & 0.924232 \\
\hline 11 & 6 & 0 & 2.978965 & -1.720606 & -0.847841 \\
\hline 12 & 6 & 0 & 3.418782 & 1.187111 & 1.682137 \\
\hline 13 & 6 & 0 & 2.514387 & 3.355766 & 2.059786 \\
\hline 14 & 6 & 0 & 0.204643 & 3.935188 & 2.321716 \\
\hline 15 & 6 & 0 & -1.588172 & 2.302372 & 1.690018 \\
\hline 16 & 6 & 0 & 4.229522 & -1.070607 & 1.569683 \\
\hline 17 & 6 & 0 & 3.812042 & -2.638068 & -0.196876 \\
\hline 18 & 6 & 0 & 2.224015 & -2.089391 & -2.069185 \\
\hline 19 & 6 & 0 & 3.860806 & 0.725627 & 3.097983 \\
\hline 20 & 6 & 0 & 4.409340 & 2.232179 & 1.112525 \\
\hline 21 & 6 & 0 & 3.986601 & 3.589710 & 1.739932 \\
\hline 22 & 6 & 0 & 1.547359 & 4.317248 & 2.366523 \\
\hline 23 & 1 & 0 & -0.565616 & 4.675444 & 2.522484 \\
\hline 24 & 6 & 0 & -2.309743 & 3.052343 & 0.761891 \\
\hline 25 & 6 & 0 & -2.233000 & 1.225016 & 2.298805 \\
\hline 26 & 6 & 0 & 4.732462 & -0.536373 & 2.904845 \\
\hline 27 & 6 & 0 & 4.450216 & -2.331500 & 1.005947 \\
\hline 28 & 1 & 0 & 3.961604 & -3.618589 & -0.639660 \\
\hline 29 & 6 & 0 & 1.422947 & -3.235218 & -2.104629 \\
\hline 30 & 6 & 0 & 2.189660 & -1.253501 & -3.183797 \\
\hline 31 & 1 & 0 & 4.381656 & 1.517579 & 3.644593 \\
\hline 32 & 1 & 0 & 2.968104 & 0.465212 & 3.673382 \\
\hline 33 & 1 & 0 & 5.452218 & 1.975346 & 1.323723 \\
\hline 34 & 1 & 0 & 4.285290 & 2.281359 & 0.025831 \\
\hline 35 & 1 & 0 & 4.566685 & 3.792322 & 2.647896 \\
\hline 36 & 6 & 0 & 4.143390 & 4.762775 & 0.790299 \\
\hline 37 & 6 & 0 & 1.942227 & 5.720438 & 2.654702 \\
\hline 38 & 6 & 0 & -3.639568 & 2.763070 & 0.480087 \\
\hline 39 & 8 & 0 & -1.689065 & 4.083971 & 0.108231 \\
\hline 40 & 6 & 0 & -3.575362 & 0.934455 & 2.035238 \\
\hline 41 & 8 & 0 & -1.496477 & 0.448593 & 3.166409 \\
\hline 42 & 1 & 0 & 5.788690 & -0.255532 & 2.805829 \\
\hline 43 & 6 & 0 & 4.606039 & -1.522222 & 4.049356 \\
\hline 44 & 6 & 0 & 5.344646 & -3.321082 & 1.667610 \\
\hline 45 & 6 & 0 & 0.542861 & -3.481816 & -3.158251 \\
\hline 46 & 8 & 0 & 1.474168 & -4.104968 & -1.033446 \\
\hline 47 & 6 & 0 & 1.315115 & -1.493515 & -4.239670 \\
\hline 48 & 8 & 0 & 3.020038 & -0.158535 & -3.241158 \\
\hline 49 & 6 & 0 & 3.367832 & 4.815166 & -0.376439 \\
\hline 50 & 6 & 0 & 4.991571 & 5.829901 & 1.084120 \\
\hline 51 & 6 & 0 & 1.518284 & 6.762936 & 1.823989 \\
\hline 52 & 6 & 0 & 2.793192 & 6.005363 & 3.727723 \\
\hline 53 & 6 & 0 & -4.297147 & 1.711700 & 1.124264 \\
\hline 54 & 8 & 0 & -4.322088 & 3.511414 & -0.444855 \\
\hline 55 & 6 & 0 & -2.171745 & 4.288300 & -1.172591 \\
\hline 56 & 8 & 0 & -4.187457 & -0.134224 & 2.647524 \\
\hline 57 & 6 & 0 & -2.223247 & -0.336129 & 4.030517 \\
\hline 58 & 6 & 0 & 3.345869 & -2.002152 & 4.425296 \\
\hline 59 & 6 & 0 & 5.729103 & -1.987469 & 4.734282 \\
\hline 60 & 6 & 0 & 4.819185 & -4.460700 & 2.284326 \\
\hline 61 & 6 & 0 & 6.724925 & -3.100488 & 1.706170 \\
\hline 62 & 6 & 0 & 0.469061 & -2.600898 & -4.238587 \\
\hline 63 & 8 & 0 & -0.297716 & -4.565771 & -3.107369 \\
\hline 64 & 6 & 0 & 0.314911 & -4.816569 & -0.805241 \\
\hline 65 & 8 & 0 & 1.249148 & -0.597331 & -5.278030 \\
\hline 66 & 6 & 0 & 2.422569 & 0.929610 & -3.852675 \\
\hline 67 & 6 & 0 & 3.438379 & 5.918584 & -1.221798 \\
\hline 68 & 1 & 0 & 2.673305 & 4.004452 & -0.601118 \\
\hline & 6 & 0 & 5.0641 & 6.937695 & 02370 \\
\hline
\end{tabular}

\begin{tabular}{|c|c|c|c|c|c|}
\hline 70 & 1 & 0 & 5.581447 & 5.809709 & 1.997656 \\
\hline 71 & 6 & 0 & 1.958906 & 8.065743 & 2.049923 \\
\hline 72 & 1 & 0 & 0.869677 & 6.539343 & 0.980064 \\
\hline 73 & 6 & 0 & 3.223705 & 7.309880 & 3.962438 \\
\hline 74 & 1 & 0 & 3.111848 & 5.197809 & 4.382895 \\
\hline 75 & 6 & 0 & -5.763544 & 1.529040 & 0.804652 \\
\hline 76 & 6 & 0 & -3.508820 & 4.020804 & -1.449494 \\
\hline 77 & 6 & 0 & -1.327309 & 4.766154 & -2.161917 \\
\hline 78 & 6 & 0 & -3.565098 & -0.611114 & 3.784249 \\
\hline 79 & 6 & 0 & -1.590663 & -0.880046 & 5.140355 \\
\hline 80 & 6 & 0 & 3.213676 & -2.928440 & 5.457047 \\
\hline 81 & 1 & 0 & 2.460368 & -1.646304 & 3.899660 \\
\hline 82 & 6 & 0 & 5.603736 & -2.923651 & 5.761454 \\
\hline 83 & 1 & 0 & 6.715662 & -1.631396 & 4.447549 \\
\hline 84 & 6 & 0 & 5.657867 & -5.354833 & 2.947429 \\
\hline 85 & 1 & 0 & 3.745282 & -4.630573 & 2.259359 \\
\hline 86 & 6 & 0 & 7.566825 & -3.999152 & 2.360577 \\
\hline 87 & 1 & 0 & 7.137290 & -2.220844 & 1.217394 \\
\hline 88 & 6 & 0 & -0.455944 & -2.781338 & -5.421319 \\
\hline 89 & 6 & 0 & -0.601373 & -4.999975 & -1.836637 \\
\hline 90 & 6 & 0 & 0.092032 & -5.384019 & 0.440334 \\
\hline 91 & 6 & 0 & 1.518736 & 0.705827 & -4.887838 \\
\hline 92 & 6 & 0 & 2.731645 & 2.219496 & -3.451502 \\
\hline 93 & 6 & 0 & 4.285324 & 6.986667 & -0.916802 \\
\hline 94 & 1 & 0 & 2.815826 & 5.959673 & -2.110792 \\
\hline 95 & 1 & 0 & 5.716738 & 7.767776 & 0.489991 \\
\hline 96 & 6 & 0 & 2.815331 & 8.341711 & 3.116753 \\
\hline 97 & 1 & 0 & 1.642214 & 8.864238 & 1.385866 \\
\hline 98 & 1 & 0 & 3.879720 & 7.521003 & 4.801690 \\
\hline 99 & 9 & 0 & -6.457209 & 2.634403 & 1.122052 \\
\hline 100 & 9 & 0 & -5.956562 & 1.313752 & -0.513473 \\
\hline 101 & 9 & 0 & -6.333686 & 0.509407 & 1.452104 \\
\hline 102 & 6 & 0 & -4.032881 & 4.252746 & -2.709910 \\
\hline 103 & 1 & 0 & -0.279599 & 4.921989 & -1.923879 \\
\hline 104 & 6 & 0 & -1.851769 & 5.015896 & -3.430704 \\
\hline 105 & 6 & 0 & -4.294619 & -1.411022 & 4.651770 \\
\hline 106 & 1 & 0 & -0.540771 & -0.654592 & 5.301559 \\
\hline 107 & 6 & 0 & -2.312639 & -1.701947 & 6.005562 \\
\hline 108 & 6 & 0 & 4.345438 & -3.398474 & 6.126293 \\
\hline 109 & 1 & 0 & 2.226958 & -3.282269 & 5.746411 \\
\hline 110 & 1 & 0 & 6.491757 & -3.285278 & 6.271064 \\
\hline 111 & 6 & 0 & 7.033690 & -5.124177 & 2.989713 \\
\hline 112 & 1 & 0 & 5.236675 & -6.227577 & 3.437132 \\
\hline 113 & 1 & 0 & 8.637790 & -3.820666 & 2.379694 \\
\hline 114 & 9 & 0 & -1.238003 & -1.705699 & -5.608103 \\
\hline 115 & 9 & 0 & -1.265350 & -3.837272 & -5.317155 \\
\hline 116 & 9 & 0 & 0.259822 & -2.943793 & -6.551296 \\
\hline 117 & 6 & 0 & -1.795409 & -5.668508 & -1.610025 \\
\hline 118 & 1 & 0 & 0.844990 & -5.264223 & 1.211611 \\
\hline 119 & 6 & 0 & -1.088173 & -6.094373 & 0.664912 \\
\hline 120 & 6 & 0 & 0.892814 & 1.763947 & -5.527677 \\
\hline 121 & 1 & 0 & 3.438283 & 2.364829 & -2.640520 \\
\hline 122 & 6 & 0 & 2.129005 & 3.290986 & -4.112007 \\
\hline 123 & 1 & 0 & 4.329616 & 7.8 & 0274 \\
\hline 124 & 1 & 0 & 3.160004 & & 3.289472 \\
\hline 125 & 1 & 0 & 77968 & & -2.895155 \\
\hline 126 & 6 & 0 & -3.196317 & 4.765988 & -3.701675 \\
\hline 127 & 1 & 0 & -1.200892 & 5.394313 & -4.211522 \\
\hline 128 & 1 & 0 & -5.341654 & -1.594067 & 4.430868 \\
\hline 129 & 6 & 0 & -3.661991 & -1.964519 & 5.765438 \\
\hline 130 & 1 & 0 & -1.817828 & -2.133315 & 6.869422 \\
\hline 131 & 1 & 0 & 4.245109 & -4.125339 & 6.926395 \\
\hline 132 & 1 & 0 & 7.686793 & -5.820350 & 3.506989 \\
\hline 133 & 1 & 0 & -2.499195 & -5.769848 & -2.429587 \\
\hline 134 & 6 & 0 & -2.039146 & -6.215839 & -0.349000 \\
\hline 135 & 1 & 0 & -1.259189 & -6.548345 & 1.635625 \\
\hline
\end{tabular}




\begin{tabular}{cccccc}
136 & 1 & 0 & 0.175163 & 1.555145 & -6.314422 \\
137 & 6 & 0 & 1.211235 & 3.065445 & -5.138055 \\
138 & 1 & 0 & 2.379930 & 4.305182 & -3.819553 \\
139 & 1 & 0 & -3.595069 & 4.948702 & -4.693624 \\
140 & 1 & 0 & -4.227122 & -2.594100 & 6.444578 \\
141 & 1 & 0 & -2.964443 & -6.753196 & -0.169216 \\
142 & 1 & 0 & 0.736112 & 3.901446 & -5.640101 \\
143 & 1 & 0 & -1.492457 & 1.954140 & -2.404761 \\
144 & 8 & 0 & -2.098658 & 1.668159 & -3.116933 \\
145 & 6 & 0 & -1.734287 & 0.343267 & -3.437461 \\
146 & 6 & 0 & -2.676593 & -0.726918 & -2.802149 \\
147 & 1 & 0 & -1.760781 & 0.226985 & -4.522453 \\
148 & 1 & 0 & -0.710651 & 0.136352 & -3.099117 \\
149 & 8 & 0 & -1.964278 & -1.932610 & -2.588410 \\
150 & 6 & 0 & -3.218289 & -0.177705 & -1.464571 \\
151 & 1 & 0 & -1.139362 & -1.692375 & -2.119064 \\
152 & 1 & 0 & -2.425192 & 0.145770 & -0.782920 \\
153 & 1 & 0 & -3.866028 & 0.684645 & -1.637968 \\
154 & 1 & 0 & 1.260170 & -3.536104 & 3.580176 \\
155 & 6 & 0 & 0.224617 & -3.492641 & 3.257158 \\
156 & 6 & 0 & -0.122588 & -2.656306 & 2.202420 \\
157 & 6 & 0 & -0.729439 & -4.301263 & 3.895264 \\
158 & 6 & 0 & -1.456629 & -2.674175 & 1.794105 \\
159 & 1 & 0 & 0.600911 & -2.022164 & 1.694130 \\
160 & 6 & 0 & -2.061065 & -4.302045 & 3.490733 \\
161 & 1 & 0 & -0.426698 & -4.935946 & 4.722282 \\
162 & 7 & 0 & -1.998442 & -1.941414 & 0.737985 \\
163 & 6 & 0 & -2.410486 & -3.481896 & 2.418761 \\
164 & 1 & 0 & -2.799121 & -4.922921 & 3.987986 \\
165 & 6 & 0 & -3.286271 & -2.142934 & 0.523217 \\
166 & 1 & 0 & -1.316545 & -1.419849 & 0.085737 \\
167 & 16 & 0 & -3.964663 & -3.294092 & 1.637041 \\
168 & 16 & 0 & -4.308272 & -1.386484 & -0.627658 \\
169 & 6 & 0 & -3.848263 & -1.025329 & -3.720838 \\
170 & 6 & 0 & -4.122787 & -2.326338 & -4.142057 \\
171 & 6 & 0 & -4.664457 & 0.027968 & -4.151727 \\
172 & 6 & 0 & -5.209782 & -2.573577 & -4.981917 \\
173 & 1 & 0 & -3.475401 & -3.134540 & -3.817396 \\
174 & 6 & 0 & -5.750571 & -0.223338 & -4.986677 \\
175 & 1 & 0 & -4.432785 & 1.048530 & -3.849233 \\
176 & 6 & 0 & -6.028527 & -1.526869 & -5.401936 \\
177 & 1 & 0 & -5.413735 & -3.588103 & -5.311296 \\
178 & 1 & 0 & -6.377805 & 0.599119 & -5.317258 \\
179 & 1 & 0 & -6.873980 & -1.722930 & -6.054429 \\
---------------------------------------------------------- \\
\hline
\end{tabular}

Frequencies -- 8.3351

Frequencies -- 22.4037

Frequencies -- 26.8980

Frequencies -- 34.6127

Frequencies -- 40.9799

Frequencies -- 52.1221

Frequencies -- 55.3199

Frequencies -- 60.3124

Frequencies -- 70.0698

Frequencies -- 74.7413

Frequencies -- 85.5396

Frequencies -- 92.5220

Frequencies -- 100.0367

Frequencies -- 112.1496

Frequencies -- 121.4827

Frequencies -- 132.7400

Frequencies -- 142.7708

Frequencies -- 157.3947

Frequencies -- 171.7562

Frequencies -- 187.9648

$\begin{array}{cc}17.6539 & 19.6299 \\ 23.7277 & 26.4324 \\ 30.5191 & 32.0641 \\ 36.9726 & 40.2626 \\ 43.2646 & 48.1517 \\ 52.8996 & 54.5355 \\ 58.8957 & 59.5577 \\ 64.5774 & 66.4507 \\ 71.7166 & 73.5414 \\ 77.0846 & 82.7797 \\ 87.4616 & 89.3765 \\ 94.9208 & 98.7999 \\ 103.2383 & 109.2509 \\ 113.9955 & 117.4310 \\ 126.1624 & 128.9620 \\ 134.3416 & 138.6043 \\ 146.1173 & 152.7247 \\ 163.3538 & 167.4488 \\ 181.5937 & 185.9193 \\ 190.9503 & 197.3918\end{array}$

\begin{tabular}{|c|c|c|c|}
\hline Frequencies -- & 199.1105 & 207.2593 & 211.7314 \\
\hline Frequencies -- & 216.8492 & 219.0558 & 224.1393 \\
\hline Frequencies -- & 230.0577 & 233.6162 & 236.2804 \\
\hline Frequencies -- & 244.1374 & 247.4928 & 254.2494 \\
\hline Frequencies -- & 256.6996 & 265.1935 & 270.2410 \\
\hline Frequencies -- & 275.0777 & 276.4343 & 282.0002 \\
\hline Frequencies -- & 285.8577 & 287.3790 & 288.3815 \\
\hline Frequencies -- & 292.5772 & 296.8251 & 297.8201 \\
\hline Frequencies -- & 299.1056 & 302.8000 & 306.0328 \\
\hline Frequencies -- & 309.1162 & 311.6533 & 314.5982 \\
\hline Frequencies -- & 319.6611 & 320.1852 & 322.7764 \\
\hline Frequencies -- & 329.8448 & 335.8785 & 337.0097 \\
\hline Frequencies -- & 338.0826 & 347.0648 & 355.4660 \\
\hline Frequencies -- & 376.9086 & 381.4271 & 391.5916 \\
\hline Frequencies -- & 405.1261 & 407.7200 & 409.7618 \\
\hline Frequencies -- & 412.0914 & 412.8704 & 414.8056 \\
\hline Frequencies -- & 417.6436 & 418.7948 & 419.7210 \\
\hline Frequencies -- & 421.1206 & 422.9367 & 425.1886 \\
\hline Frequencies -- & 428.7947 & 436.9811 & 440.8121 \\
\hline Frequencies -- & 443.4430 & 446.8036 & 452.8169 \\
\hline Frequencies -- & 458.7217 & 460.5763 & 462.2532 \\
\hline Frequencies -- & 464.7623 & 468.3356 & 473.5090 \\
\hline Frequencies -- & 487.6831 & 492.6575 & 501.5958 \\
\hline Frequencies -- & 507.0986 & 509.7123 & 515.7371 \\
\hline Frequencies -- & 521.9541 & 523.1536 & 528.0600 \\
\hline Frequencies -- & 530.4683 & 531.7419 & 534.4927 \\
\hline Frequencies -- & 538.0529 & 540.4745 & 545.9161 \\
\hline Frequencies -- & 552.3168 & 556.1822 & 558.4369 \\
\hline Frequencies -- & 560.8616 & 561.7301 & 563.7376 \\
\hline Frequencies -- & 567.4533 & 572.0760 & 580.0431 \\
\hline Frequencies -- & 585.3755 & 588.5318 & 591.2692 \\
\hline Frequencies -- & 595.3593 & 596.9659 & 597.4746 \\
\hline Frequencies -- & 598.5540 & 598.9516 & 601.6282 \\
\hline Frequencies -- & 607.2558 & 607.4744 & 609.9628 \\
\hline Frequencies -- & 611.8064 & 612.5080 & 614.3660 \\
\hline Frequencies -- & 618.5998 & 621.3454 & 624.7966 \\
\hline Frequencies -- & 626.3855 & 629.8864 & 631.0960 \\
\hline Frequencies -- & 632.5184 & 634.7814 & 635.6451 \\
\hline Frequencies -- & 645.1969 & 648.8903 & 656.2816 \\
\hline Frequencies -- & 664.9115 & 672.2804 & 677.2736 \\
\hline Frequencies -- & 681.4923 & 685.4169 & 685.7365 \\
\hline Frequencies -- & 696.9750 & 703.0297 & 710.0910 \\
\hline Frequencies -- & 713.3949 & 715.7985 & 717.4299 \\
\hline Frequencies -- & 718.1943 & 719.9215 & 720.5333 \\
\hline Frequencies -- & 720.8074 & 724.4780 & 725.8010 \\
\hline Frequencies -- & 727.9511 & 729.0168 & 731.3066 \\
\hline Frequencies -- & 731.8030 & 733.1713 & 737.3529 \\
\hline Frequencies -- & 740.8951 & 746.1605 & 749.1913 \\
\hline Frequencies -- & 751.8032 & 760.7034 & 763.1175 \\
\hline Frequencies -- & 764.7376 & 768.4338 & 769.0539 \\
\hline Frequencies -- & 771.4480 & 772.4400 & 773.2522 \\
\hline Frequencies -- & 778.1928 & 779.9354 & 784.1638 \\
\hline Frequencies -- & 788.5844 & 797.2645 & 798.0201 \\
\hline Frequencies -- & 799.1788 & 801.2515 & 805.5598 \\
\hline Frequencies -- & 806.2566 & 823.2106 & 825.4236 \\
\hline Frequencies -- & 826.8569 & 831.6818 & 853.9857 \\
\hline Frequencies -- & 857.3723 & 863.0403 & 864.4806 \\
\hline Frequencies -- & 864.8697 & 866.3216 & 868.1702 \\
\hline Frequencies -- & 870.2845 & 871.8924 & 873.9528 \\
\hline Frequencies -- & 875.8386 & 880.8249 & 883.8649 \\
\hline Frequencies -- & 887.4147 & 888.7837 & 889.5302 \\
\hline Frequencies -- & 891.0452 & 892.2175 & 911.9719 \\
\hline Frequencies -- & 912.8292 & 919.7630 & 924.7406 \\
\hline Frequencies -- & 930.7244 & 931.6276 & 934.1243 \\
\hline Frequencies -- & 936.7165 & 941.7587 & 942.4656 \\
\hline Frequencies -- & 943.2984 & 944.8755 & 945.6611 \\
\hline
\end{tabular}




\begin{tabular}{|c|c|c|c|}
\hline Frequencies -- & 947.2339 & 954.0790 & 955.6189 \\
\hline Frequencies -- & 957.7743 & 958.4169 & 968.6560 \\
\hline Frequencies -- & 969.7355 & 984.7736 & 989.2410 \\
\hline Frequencies -- & 989.6235 & 992.4402 & 992.7215 \\
\hline Frequencies -- & 994.1857 & 997.3516 & 1000.6231 \\
\hline Frequencies -- & 1006.1278 & 1007.7749 & 1008.2504 \\
\hline Frequencies -- & 1008.5953 & 1011.1672 & 1011.8462 \\
\hline Frequencies -- & 1014.3794 & 1017.5828 & 1017.9971 \\
\hline Frequencies -- & 1018.4891 & 1019.2292 & 1019.4562 \\
\hline Frequencies -- & 1019.6371 & 1020.3208 & 1022.6413 \\
\hline Frequencies -- & 1029.4505 & 1033.9633 & 1036.8377 \\
\hline Frequencies -- & 1042.3246 & 1043.2749 & 1053.1678 \\
\hline Frequencies -- & 1053.5809 & 1058.7458 & 1059.1609 \\
\hline Frequencies -- & 1060.9068 & 1064.4612 & 1065.6798 \\
\hline Frequencies -- & 1068.1097 & 1069.7780 & 1070.5532 \\
\hline Frequencies -- & 1071.7212 & 1072.9499 & 1093.4922 \\
\hline Frequencies -- & 1094.9049 & 1098.8837 & 1108.8941 \\
\hline Frequencies -- & 1109.3504 & 1111.4070 & 1113.0136 \\
\hline Frequencies -- & 1114.4909 & 1118.9038 & 1123.3704 \\
\hline Frequencies -- & 1124.6647 & 1127.0821 & 1127.8416 \\
\hline Frequencies -- & 1129.9666 & 1132.6107 & 1137.1829 \\
\hline Frequencies -- & 1157.5832 & 1161.5399 & 1164.7838 \\
\hline Frequencies -- & 1165.2952 & 1165.8148 & 1167.1259 \\
\hline Frequencies -- & 1170.1646 & 1170.5050 & 1174.4276 \\
\hline Frequencies -- & 1175.8907 & 1177.0129 & 1177.2829 \\
\hline Frequencies -- & 1177.9793 & 1182.9895 & 1185.7393 \\
\hline Frequencies -- & 1196.3476 & 1200.0814 & 1200.8372 \\
\hline Frequencies -- & 1201.6795 & 1204.8877 & 1207.0338 \\
\hline Frequencies -- & 1207.3277 & 1211.6184 & 1212.3671 \\
\hline Frequencies -- & 1215.0899 & 1219.1557 & 1221.7896 \\
\hline Frequencies -- & 1223.1749 & 1225.6017 & 1226.8703 \\
\hline Frequencies -- & 1234.6199 & 1239.4366 & 1242.9391 \\
\hline Frequencies -- & 1247.5324 & 1250.3136 & 1253.2557 \\
\hline Frequencies -- & 1254.4173 & 1261.0055 & 1270.1543 \\
\hline Frequencies -- & 1274.2974 & 1277.1730 & 1282.9908 \\
\hline Frequencies -- & 1285.0627 & 1286.7503 & 1290.7369 \\
\hline Frequencies -- & 1291.0590 & 1298.3583 & 1298.6649 \\
\hline Frequencies -- & 1300.3342 & 1303.0733 & 1303.5816 \\
\hline Frequencies -- & 1304.3098 & 1308.2134 & 1309.3726 \\
\hline Frequencies -- & 1311.2078 & 1313.5073 & 1313.6965 \\
\hline Frequencies -- & 1314.8896 & 1321.5546 & 1325.9551 \\
\hline Frequencies -- & 1326.3881 & 1328.2750 & 1335.3349 \\
\hline Frequencies -- & 1338.2065 & 1341.3402 & 1342.0569 \\
\hline Frequencies -- & 1344.3504 & 1358.2344 & 1358.3370 \\
\hline Frequencies -- & 1359.4744 & 1360.0870 & 1362.8194 \\
\hline Frequencies -- & 1363.8236 & 1364.4491 & 1364.6097 \\
\hline Frequencies -- & 1365.6523 & 1368.6226 & 1369.0801 \\
\hline Frequencies -- & 1371.0095 & 1374.6801 & 1382.1273 \\
\hline Frequencies -- & 1389.4250 & 1404.9680 & 1406.9488 \\
\hline Frequencies -- & 1413.3347 & 1439.2692 & 1449.1266 \\
\hline Frequencies -- & 1458.9795 & 1467.3375 & 1470.4771 \\
\hline Frequencies -- & 1471.3335 & 1476.7087 & 1491.3444 \\
\hline Frequencies -- & 1493.7155 & 1495.1812 & 1497.4943 \\
\hline Frequencies -- & 1497.9815 & 1498.7083 & 1504.2075 \\
\hline Frequencies -- & 1504.9543 & 1506.6806 & 1508.4559 \\
\hline Frequencies -- & 1508.7199 & 1510.6784 & 1513.1543 \\
\hline Frequencies -- & 1515.8812 & 1519.3503 & 1520.7268 \\
\hline Frequencies -- & 1528.2543 & 1531.9673 & 1545.6807 \\
\hline Frequencies -- & 1547.4812 & 1550.0407 & 1554.9435 \\
\hline Frequencies -- & 1555.7399 & 1559.2294 & 1559.6713 \\
\hline Frequencies -- & 1562.6535 & 1564.1562 & 1568.4251 \\
\hline Frequencies -- & 1572.1152 & 1576.4927 & 1660.0961 \\
\hline Frequencies -- & 1664.1617 & 1668.7527 & 1669.6374 \\
\hline Frequencies -- & 1672.2223 & 1674.5947 & 1674.7890 \\
\hline Frequencies -- & 1676.1187 & 1677.0422 & 1677.5652 \\
\hline Frequencies -- & 1681.5639 & 1688.2583 & 1689.6352 \\
\hline
\end{tabular}

$\begin{array}{llll}\text { Frequencies -- } 1693.4878 & 1695.4368 & 1697.5569 \\ \text { Frequencies -- } 1698.2926 & 1698.6092 & 1699.5027 \\ \text { Frequencies -- } 1700.2631 & 1701.8767 & 1702.1780 \\ \text { Frequencies -- } 1703.6454 & 1705.6127 & 1719.7108 \\ \text { Frequencies -- } 1720.0878 & 1729.4579 & 1732.7466 \\ \text { Frequencies -- } 2432.4827 & 3070.9289 & 3082.0548 \\ \text { Frequencies -- } 3091.0329 & 3091.1541 & 3098.3028 \\ \text { Frequencies -- } 3124.9366 & 3156.3999 & 3159.5663 \\ \text { Frequencies -- } 3172.9519 & 3179.3179 & 3181.8973 \\ \text { Frequencies -- } 3192.0812 & 3197.3789 & 3199.5689 \\ \text { Frequencies -- } 3200.7266 & 3201.1288 & 3201.8151 \\ \text { Frequencies -- } 3204.3229 & 3207.1216 & 3207.7832 \\ \text { Frequencies -- } 3212.3704 & 3214.4196 & 3217.0565 \\ \text { Frequencies -- } 3218.3856 & 3220.4757 & 3221.2281 \\ \text { Frequencies -- } 3221.9008 & 3222.5271 & 3222.8662 \\ \text { Frequencies -- } 3223.0289 & 3224.0801 & 3225.6909 \\ \text { Frequencies -- } 3225.7136 & 3226.7835 & 3228.3065 \\ \text { Frequencies -- } 3229.5151 & 3233.0509 & 3234.5906 \\ \text { Frequencies -- } 3234.9438 & 3235.6263 & 3236.3056 \\ \text { Frequencies -- } 3236.4445 & 3236.6922 & 3237.8564 \\ \text { Frequencies -- } 3239.2786 & 3239.9249 & 3241.6503 \\ \text { Frequencies -- } 3242.3829 & 3242.3981 & 3243.4835 \\ \text { Frequencies -- } 3244.1749 & 3245.5774 & 3247.9230 \\ \text { Frequencies -- } 3248.3856 & 3252.2627 & 3253.8564 \\ \text { Frequencies -- } 3262.2893 & 3648.2542 & 3659.4336\end{array}$

SCF Done: $E($ RM062X/DGDZVP $)=-6499.42926344$

Sum of electronic and zero-point Energies $=\quad-6498.078417$ Sum of electronic and thermal Energies $=\quad-6497.964364$

Sum of electronic and thermal Free Energies $=\quad-6498.235515$ SCF Done: $E(R M 062 X / D G T Z V P / S M D)=-6500.71185914873$ 


\section{RRHO- and BSSE-corrected absolute and relative energies}

Table S4. RRHO- and BSSE-corrected thermodynamics for tautomerization $\mathrm{N} \bullet \bullet \cdot \mathrm{H} \bullet \bullet$ S at M06-2X-D3/DGTZVP/SMD//M06-2X-D3/DGDZVP: absolute $H, T S$ and $G$ are presented in Hartrees and relative $\Delta \mathrm{H},-\mathrm{T} \Delta \mathrm{S}$ and $\Delta \mathrm{G}$ are presented in $\mathrm{kcal} \cdot \mathrm{mol}^{-1}$.

\begin{tabular}{|c|c|c|c|c|c|c|}
\hline Structures & H & T·qh-S & qh-G(T) & $\Delta \mathbf{H}$ & $-\mathrm{T} \Delta \mathrm{S}$ & $\Delta \mathbf{G}$ \\
\hline $2 *$ Benzo[d]thiazole-2-thiol & -2241.40287 & 0.09196 & -2241.49483 & 0.0 & 0.0 & 0.0 \\
\hline I0Ha & -2241.41350 & 0.07346 & -2241.48695 & -6.7 & 11.6 & 4.9 \\
\hline TS1Ha & -2241.41494 & 0.07033 & -2241.48527 & -7.6 & 13.6 & 6.0 \\
\hline I1Ha & -2241.44392 & 0.07169 & -2241.51561 & -25.8 & 12.7 & -13.0 \\
\hline $\begin{array}{c}2 * \text { Benzo[d]thiazole-2(3H)- } \\
\text { thione }\end{array}$ & -2241.43109 & 0.09052 & -2241.52161 & -17.7 & 0.9 & -16.8 \\
\hline Benzo[d]thiazole-2-thiol & -1120.70144 & 0.04598 & -1120.74742 & 0.0 & 0.0 & 0.0 \\
\hline TS1Hb & -1120.65510 & 0.04479 & -1120.69989 & 29.1 & 0.7 & 29.8 \\
\hline $\begin{array}{l}\text { Benzo[d]thiazole-2(3H)- } \\
\text { thione }\end{array}$ & -1120.71555 & 0.04526 & -1120.76080 & -8.9 & 0.5 & -8.4 \\
\hline
\end{tabular}

Table S5. RRHO- and BSSE-corrected thermodynamics for reaction of 22a and 23a catalyzed by Cat $\boldsymbol{b}$ at M06-2X-D3/DGTZVP/SMD//M06-2X-D3/DGDZVP: absolute $H$, TS and $G$ are presented in Hartrees and relative $\Delta \mathrm{H},-\mathrm{T} \Delta \mathrm{S}$ and $\Delta \mathrm{G}$ are presented in $\mathrm{kcal} \cdot \mathrm{mol}^{-1}$.

\begin{tabular}{|c|c|c|c|c|c|c|}
\hline Structures & H & $T \cdot q h-S$ & $q h-G(T)$ & $\Delta \mathrm{H}$ & $-\mathrm{T} \Delta \mathrm{S}$ & $\Delta \mathbf{G}$ \\
\hline $\begin{array}{c}\text { Benzo }[d] \text { thiazole- } \\
2(3 H) \text {-thione } \\
\end{array}$ & -1120.71555 & 0.04526 & -1120.76080 & \multirow{2}{*}{\multicolumn{3}{|c|}{ Reactants }} \\
\hline 3-Phenyloxetan-3-ol & -499.14799 & 0.04801 & -499.19600 & & & \\
\hline Catalyst b & -2222.57703 & 0.11320 & -2222.69023 & 0.0 & 0.0 & 0.0 \\
\hline 10_1 & -2721.75349 & 0.13899 & -2721.89248 & -17.9 & 13.9 & -3.9 \\
\hline TS1a_R & -3842.45492 & 0.15747 & -3842.61239 & -9.0 & 30.7 & 21.7 \\
\hline$(R)$-Product & -1619.90535 & 0.07278 & -1619.97813 & -26.2 & 12.9 & -13.4 \\
\hline TS1b_S & -3842.45407 & 0.15729 & -3842.61136 & -8.5 & 30.9 & 22.4 \\
\hline (S)-Product & -1619.90535 & 0.07278 & -1619.97813 & -26.2 & 12.9 & -13.4 \\
\hline $10 \_2$ & -2721.75265 & 0.13732 & -2721.88997 & -17.3 & 15.0 & -2.3 \\
\hline TS1c_S & -3842.45271 & 0.15626 & -3842.60897 & -7.6 & 31.5 & 23.9 \\
\hline (S)-Product & -1619.90535 & 0.07278 & -1619.97813 & -26.2 & 12.9 & -13.4 \\
\hline TS1d_R & -3842.45479 & 0.15787 & -3842.61266 & -8.9 & 30.5 & 21.6 \\
\hline (R)-Product & -1619.90535 & 0.07278 & -1619.97813 & -26.2 & 12.9 & -13.4 \\
\hline
\end{tabular}


Table S6. RRHO- and BSSE-corrected thermodynamics for reaction of 22a and 23a catalyzed by $C$ at a at M06-2X-D3/DGTZVP/SMD//M06-2X-D3/DGDZVP: absolute $H$, TS and $G$ are presented in Hartrees and relative $\Delta \mathrm{H},-\mathrm{T} \Delta \mathrm{S}$ and $\Delta \mathrm{G}$ are presented in $\mathrm{kcal} \cdot \mathrm{mol}^{-1}$.

\begin{tabular}{|c|c|c|c|c|c|c|}
\hline Structures & H & T.qh-S & qh-G(T) & $\Delta H$ & $-\mathrm{T} \Delta \mathrm{S}$ & $\Delta \mathbf{G}$ \\
\hline $\begin{array}{c}\text { Benzo }[d] \text { thiazole- } \\
2(3 H) \text {-thione } \\
\end{array}$ & -1120.71555 & 0.04526 & -1120.76080 & \multirow{2}{*}{\multicolumn{3}{|c|}{ Reactants }} \\
\hline 3-Phenyloxetan-3-ol & -499.14799 & 0.04801 & -499.19600 & & & \\
\hline Catalyst $\mathbf{f}$ & -2374.98632 & 0.11650 & -2375.10282 & 0.0 & 0.0 & 0.0 \\
\hline 10_1 & -2874.16993 & 0.13918 & -2874.30911 & -22.4 & 15.9 & -6.5 \\
\hline TS1a_R & -3994.87786 & 0.15784 & -3995.03570 & -17.6 & 32.6 & 15.0 \\
\hline I1a_R & -3994.92965 & 0.15891 & -3995.08856 & -50.1 & 31.9 & -18.2 \\
\hline (R)-Product & -1619.90535 & 0.07278 & -1619.97813 & -26.2 & 12.9 & -13.4 \\
\hline TS1b_S & -3994.86796 & 0.15855 & -3995.02651 & -11.4 & 32.1 & 20.8 \\
\hline I1b_S & -3994.92658 & 0.15901 & -3995.08559 & -48.1 & 31.9 & -16.3 \\
\hline (S)-Product & -1619.90535 & 0.07278 & -1619.97813 & -26.2 & 12.9 & -13.4 \\
\hline $10 \_2$ & -2874.16898 & 0.13812 & -2874.30709 & -21.8 & 16.6 & -5.2 \\
\hline TS1c_S & -3994.86830 & 0.15898 & -3995.02728 & -11.6 & 31.9 & 20.3 \\
\hline I1c_S & -3994.92641 & 0.15820 & -3995.08461 & -48.0 & 32.4 & -15.7 \\
\hline (S)-Product & -1619.90535 & 0.07278 & -1619.97813 & -26.2 & 12.9 & -13.4 \\
\hline TS1d_R $R$ & -3994.87796 & 0.15742 & -3995.03538 & -17.6 & 32.8 & 15.2 \\
\hline I1d_R $R$ & -3994.93045 & 0.15884 & -3995.08929 & -50.6 & 32.0 & -18.6 \\
\hline (R)-Product & -1619.90535 & 0.07278 & -1619.97813 & -26.2 & 12.9 & -13.4 \\
\hline
\end{tabular}


Table S7. RRHO- and BSSE-corrected thermodynamics for reaction of 22a and 23a catalyzed by Cat $f$ at M06-2X-D3/DGTZVP/SMD//M06-2X-D3/DGDZVP: absolute $H$, TS and $G$ are presented in Hartrees and relative $\Delta \mathrm{H},-\mathrm{T} \Delta \mathrm{S}$ and $\Delta \mathrm{G}$ are presented in $\mathrm{kcal} \cdot \mathrm{mol}^{-1}$.

\begin{tabular}{|c|c|c|c|c|c|c|}
\hline Structures & H & T.qh-S & $q h-G(T)$ & $\Delta H$ & $-\mathrm{T} \Delta \mathrm{S}$ & $\Delta \mathbf{G}$ \\
\hline $\begin{array}{c}\text { Benzo }[d] \text { thiazole- } \\
2(3 H) \text {-thione } \\
\end{array}$ & -1120.71555 & 0.04526 & -1120.76080 & \multirow{2}{*}{\multicolumn{3}{|c|}{ Reactants }} \\
\hline 3-Phenyloxetan-3-ol & -499.14799 & 0.04801 & -499.19600 & & & \\
\hline Catalyst f & -4879.27182 & 0.20274 & -4879.47456 & 0.0 & 0.0 & 0.0 \\
\hline 10_1 & -5378.46810 & 0.22350 & -5378.69160 & -30.3 & 17.1 & -13.2 \\
\hline $10 a_{-} R$ & -6499.20831 & 0.24364 & -6499.45195 & -45.8 & 32.9 & -12.9 \\
\hline TS1a_R & -6499.17765 & 0.24227 & -6499.41992 & -26.5 & 33.7 & 7.2 \\
\hline $\mid 1 a_{-} R$ & -6499.23096 & 0.24238 & -6499.47333 & -60.0 & 33.7 & -26.3 \\
\hline (R)-Product & -1619.90535 & 0.07278 & -1619.97813 & -26.2 & 12.9 & -13.4 \\
\hline $10 b \_s$ & -6499.20325 & 0.24455 & -6499.44779 & -42.6 & 32.3 & -10.3 \\
\hline TS1b_S & -6499.16758 & 0.24222 & -6499.40980 & -20.2 & 33.8 & 13.5 \\
\hline 11b_S & -6499.22711 & 0.24145 & -6499.46856 & -57.6 & 34.2 & -23.3 \\
\hline (S)-Product & -1619.90535 & 0.07278 & -1619.97813 & -26.2 & 12.9 & -13.4 \\
\hline $10 \_2$ & -5378.46681 & 0.22209 & -5378.68890 & -29.5 & 18.0 & -11.5 \\
\hline $10 c \_s$ & -6499.20303 & 0.24279 & -6499.44582 & -42.5 & 33.4 & -9.1 \\
\hline TS1c_S & -6499.16842 & 0.24358 & -6499.41200 & -20.8 & 32.9 & 12.2 \\
\hline 11c_S & -6499.22745 & 0.24238 & -6499.46983 & -57.8 & 33.7 & -24.1 \\
\hline (S)-Product & -1619.90535 & 0.07278 & -1619.97813 & -26.2 & 12.9 & -13.4 \\
\hline $10 \mathrm{~d} \_R$ & -6499.20551 & 0.24287 & -6499.44838 & -44.0 & 33.3 & -10.7 \\
\hline TS1d_R & -6499.17923 & 0.24085 & -6499.42008 & -27.5 & 34.6 & 7.1 \\
\hline I1d_R $R$ & -6499.23044 & 0.24281 & -6499.47325 & -59.7 & 33.4 & -26.3 \\
\hline (R)-Product & -1619.90535 & 0.07278 & -1619.97813 & -26.2 & 12.9 & -13.4 \\
\hline
\end{tabular}


Table S8. Accounting for weak interactions in the principal $(R)$ - and $(S)$-enantiodirecting transition states for $\mathbf{C a t} \mathbf{a}$, Cat $\mathbf{b}$ and $\mathbf{C a t} \mathbf{f}$ : BSSE-corrected $\triangle \mathrm{PE}$ is calculated as difference of single point energies between the aggregated complex (TS) and Fragment 1 (Catalyst) and Fragment $2\left(\mathrm{H}^{+}\right.$-Activated complex of reactants) separated at infinite distance at M06-2X-

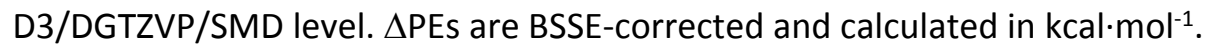

\begin{tabular}{|c|c|c|c|}
\hline Structures & E+BSSE (DGTZVP) & BSSE & E (DGTZVP) \\
\hline Cat b TS1d_R & -3843.42643 & 0.00792 & -3843.43435 \\
\hline Fragment 1 & -2222.77256 & 0.00000 & -2222.77256 \\
\hline Fragment 2 & -1620.53565 & 0.00353 & -1620.53919 \\
\hline$\triangle \mathrm{PE}$ (R-directing) & 74.2 & -2.8 & 76.9 \\
\hline Cat b TS1b_S & -3843.42480 & 0.00848 & -3843.43328 \\
\hline Fragment 1 & -2222.77134 & 0.00000 & -2222.77134 \\
\hline Fragment 2 & -1620.53672 & 0.00353 & -1620.54025 \\
\hline$\triangle \mathrm{PE}$ (S-directing) & 73.3 & -3.1 & 76.4 \\
\hline Cat a TS1a_R $R$ & -3995.87614 & 0.01089 & -3995.88703 \\
\hline Fragment 1 & -2375.19727 & 0.00000 & -2375.19727 \\
\hline Fragment 2 & -1620.53498 & 0.00340 & -1620.53838 \\
\hline$\triangle \mathrm{PE}$ (R-directing) & 90.3 & -4.7 & 95.0 \\
\hline Cat a TS1c_R $R$ & -3995.86693 & 0.01027 & -3995.87720 \\
\hline Fragment 1 & -2375.19594 & 0.00000 & -2375.19594 \\
\hline Fragment 2 & -1620.53738 & 0.00359 & -1620.54097 \\
\hline$\triangle \mathrm{PE}$ (S-directing) & 83.8 & -4.2 & 88.0 \\
\hline Cat $\mathrm{f}$ TS1d_R $R$ & -6500.64385 & 0.01473 & -6500.65858 \\
\hline Fragment 1 & -4879.95945 & 0.00000 & -4879.95945 \\
\hline Fragment 2 & -1620.53502 & 0.00345 & -1620.53847 \\
\hline$\triangle \mathrm{PE}$ (R-directing) & 93.7 & -7.1 & 100.8 \\
\hline Cat f TS1c_S & -6500.63360 & 0.01389 & -6500.64749 \\
\hline Fragment 1 & -4879.95954 & 0.00000 & -4879.95954 \\
\hline Fragment 2 & -1620.53409 & 0.00340 & -1620.53749 \\
\hline$\triangle P E$ (S-directing) & 87.8 & -6.6 & 94.4 \\
\hline
\end{tabular}




\section{Fragmentation schemes for counterpoise corrections}

In order to counterpoise the energies of the structures and ensure the best and most reliable partition of the system in different fragments, thus properly accounting for BSSE corrections, we fragmented the stationary points as follows: three examples are given for reactant, transition state and product.

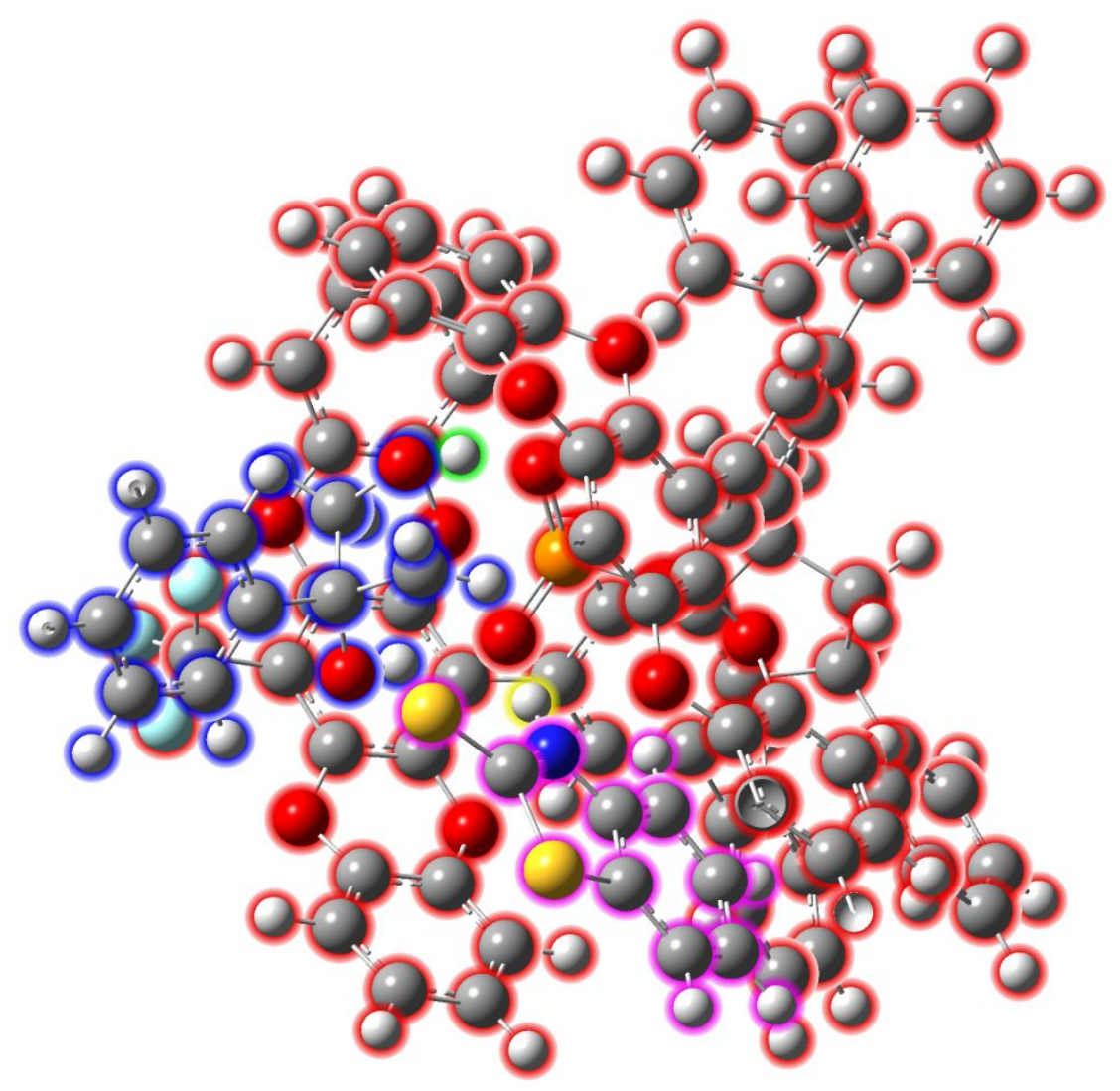

Figure S1. Clipped structure of Cat $\mathbf{f}$ TS1d_ $R$ transition state. Five fragments for counterpoise corrections are defined with their relative assigned charges: Catalyst ${ }^{-1}, \mathrm{H}^{+}, \mathrm{Oxetane}^{0}, \mathrm{H}^{+}$and $\mathrm{R}_{2} \mathrm{~N}^{-}$. 


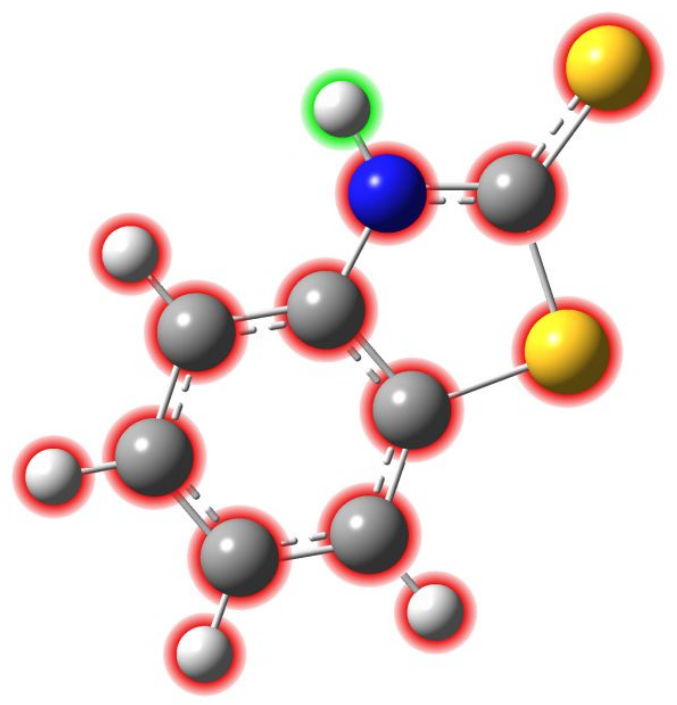

Figure S2. Two fragments are defined for the reactant with their relative assigned charges: $\mathrm{R}_{2} \mathrm{~N}^{-}$and $\mathrm{H}^{+}$.

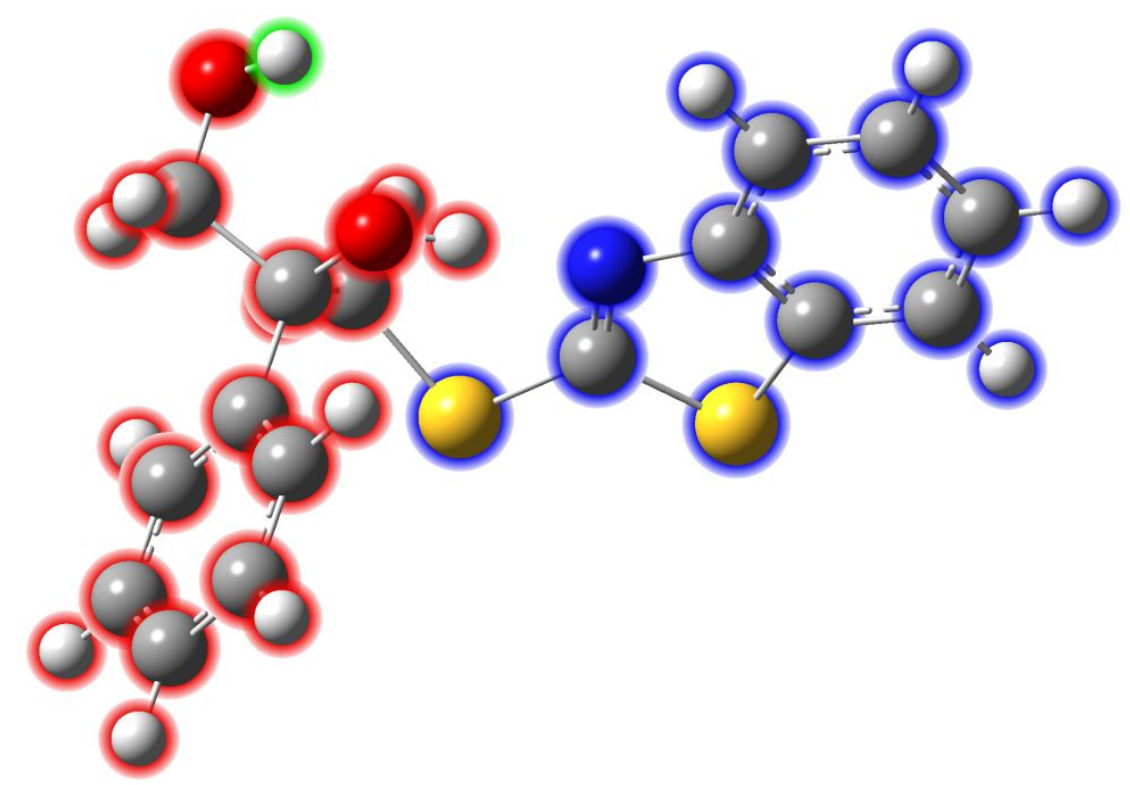

Figure S3. Three fragments are defined for the product with their relative assigned charges: Oxetane $^{0}, \mathrm{R}_{2} \mathrm{~N}^{-}$and $\mathrm{H}^{+}$. 


\section{X-ray diffraction data}

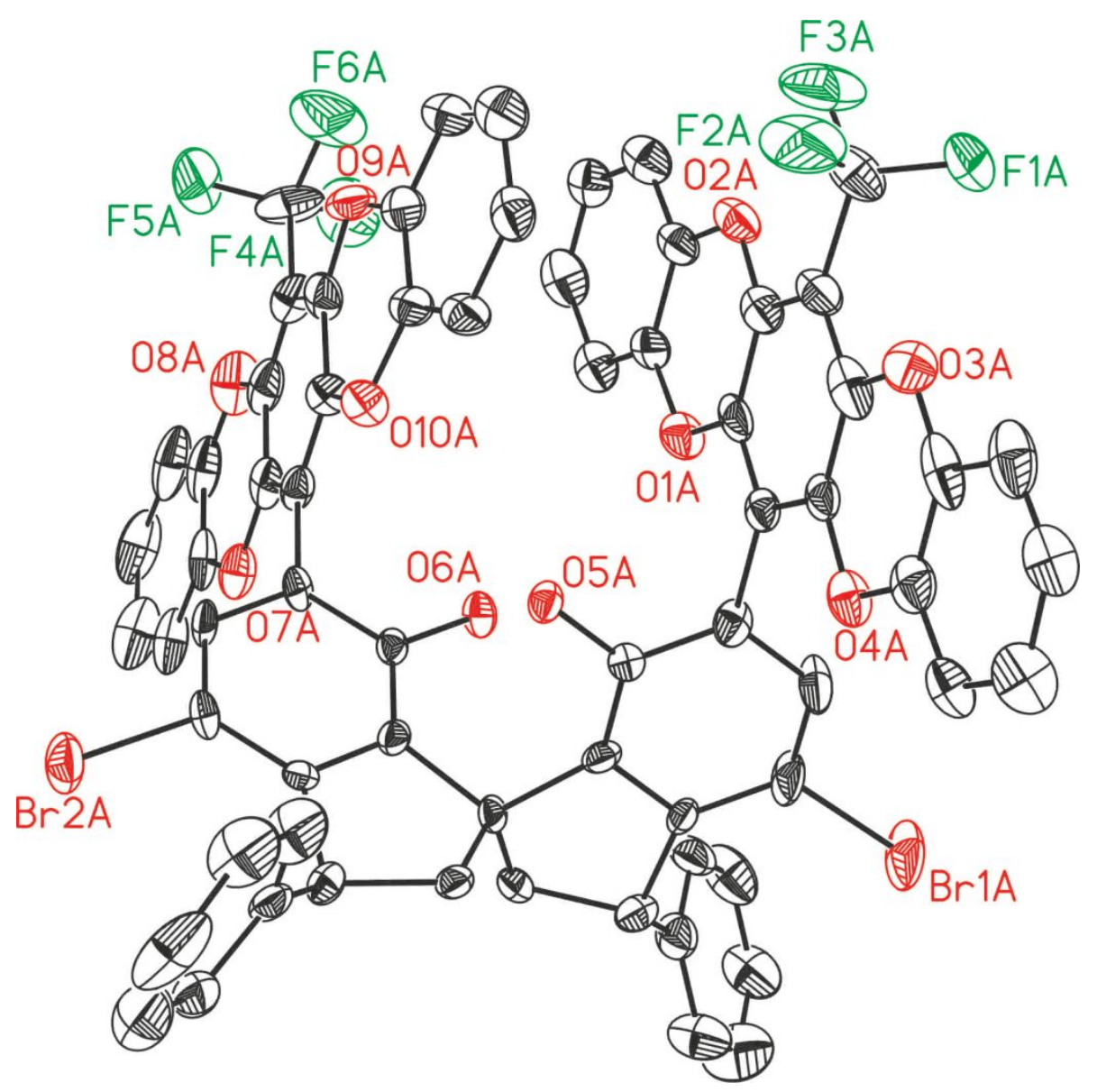

Figure S4. ORTEP X-ray structure of Compound 19 
Table S9. Crystal data and structure refinement for Compound 19.

\begin{tabular}{|c|c|c|}
\hline Empirical formula & $\mathrm{C} 79 \mathrm{H} 45.50 \mathrm{Br} 2 \mathrm{~F} 6 \mathrm{I} 2 \mathrm{O} 10$ & \\
\hline Formula weight & 1682.27 & \\
\hline Temperature & $293(2) \mathrm{K}$ & \\
\hline Wavelength & $0.71073 \AA$ & \\
\hline Crystal system & monoclinic & \\
\hline Space group & P 21 & \\
\hline \multirow{3}{*}{ Unit cell dimensions } & $a=18.5988(4) \AA$ & $\alpha=90^{\circ}$ \\
\hline & $b=13.7054(3) \AA$ & $\beta=104.1331(16)^{\circ}$ \\
\hline & $c=26.5064(4) \AA$ & $\gamma=90^{\circ}$ \\
\hline Volume & $6552.1(2) \AA^{3}$ & \\
\hline Z & 4 & \\
\hline Density (calculated) & $1.705 \mathrm{Mg} / \mathrm{m}^{3}$ & \\
\hline Absorption coefficient & $2.261 \mathrm{~mm}^{-1}$ & \\
\hline$F(000)$ & 3318 & \\
\hline Crystal size & $0.250 \times 0.120 \times 0.050 \mathrm{~mm}^{3}$ & \\
\hline Theta range for data collection & 2.258 to $29.786^{\circ}$. & \\
\hline Index ranges & $\begin{array}{c}-25<=\mathrm{h}<=25,-19<=\mathrm{k}<=19,- \\
36<=\mathrm{l}<=36\end{array}$ & \\
\hline Reflections collected & 52522 & \\
\hline Independent reflections & $52522[\mathrm{R}($ int $)=$ ?] & \\
\hline Completeness to theta $=29.786^{\circ}$ & $95.1 \%$ & \\
\hline Absorption correction & Multi-scan & \\
\hline Max. and min. transmission & 1.00 and 0.91 & \\
\hline Refinement method & Full-matrix least-squares on $\mathrm{F}^{2}$ & \\
\hline Data / restraints / parameters & $52522 / 755 / 2003$ & \\
\hline Goodness-of-fit on $\mathrm{F}^{2}$ & 1.151 & \\
\hline Final R indices [I>2sigma $(I)]$ & $\mathrm{R} 1=0.0676, \mathrm{wR} 2=0.1666$ & \\
\hline $\mathrm{R}$ indices (all data) & $\mathrm{R} 1=0.0993, \mathrm{wR} 2=0.1756$ & \\
\hline Flack parameter & $x=0.002(4)$ & \\
\hline Largest diff. peak and hole & 1.388 and -0.979 e. $\AA^{-3}$ & \\
\hline
\end{tabular}




\section{References}

1. a) Yin, L.; Xing, J.; Wang, Y.; Shen, Y.; Lu, T.; Hayashi, T.; Dou, X. Enantioselective Synthesis of 3,3'-Diaryl-SPINOLs: Rhodium-Catalyzed Asymmetric Arylation/BF ${ }_{3}$-Promoted Spirocyclization Sequence. Angew. Chem. Int. Ed. 2019, 58, 2474-2478; b) St John-Campbell, S.; White, A. J.; Bull, J. A. Single operation palladium catalysed C (sp3)-H functionalisation of tertiary aldehydes: investigations into transient imine directing groups. Chem. Sci. 2017, 8, 4840-4847.

2. Clot-Almenara, L.; Rodríguez-Escrich, C.; Osorio-Planes, L.; Pericàs, M.A. PolystyreneSupported TRIP: A Highly Recyclable Catalyst for Batch and Flow Enantioselective Allylation of Aldehydes. ACS Catalysis 2016, 6, 7647-7651.

3. Xing, C.H.; Liao, Y.X.; Ng, J.; Hu, Q.S. Optically Active 1,1'-Spirobiindane-7,7'-diol (SPINOL)Based Phosphoric Acids as Highly Enantioselective Catalysts for Asymmetric Organocatalysis. J. Org. Chem. 2011, 76, 4125-4131.

4. a) Shih, J.L.; Nguyen, T.S.; May, J.A. Organocatalyzed Asymmetric Conjugate Addition of Heteroaryl and Aryl Trifluoroborates: a Synthetic Strategy for Discoipyrrole D. Angew. Chem. Int. Ed. 2015, 54, 9931-9935; b) Le, P.Q.; Nguyen, T.S.; May, J.A. A General Method for the Enantioselective Synthesis of $\alpha$-Chiral Heterocycles. Org. Lett. 2012, 14, 6104-6107.

5. Wang, Z.; Chen, Z.; Sun, J. Catalytic Enantioselective Intermolecular Desymmetrization of 3-Substituted Oxetanes. Angew. Chem. Int. Ed. 2013, 52, 6685 -6688.

6. Frisch, M. J.; Trucks, G. W.; Schlegel, H. B.; Scuseria, G. E.; Robb, M. A.; Cheeseman, J. R.; Scalmani, G.; Barone, V.; Petersson, G. A.; Nakatsuji, H.; Li, X.; Caricato, M.; Marenich, A.; Bloino, J.; Janesko, B. G.; Gomperts, R.; Mennucci, B.; Hratchian, H. P.; Ortiz, J. V.; Izmaylov, A. F.; Sonnenberg, J. L.; Williams-Young, D.; Ding, F.; Lipparini, F.; Egidi, F.; Goings, J.; Peng, B.; Petrone, A.; Henderson, T.; Ranasinghe, D.; Zakrzewski, V. G.; Gao, J.; Rega, N.; Zheng, G.; Liang, W.; Hada, M.; Ehara, M.; Toyota, K.; Fukuda, R.; Hasegawa, J.; Ishida, M.; Nakajima, T.; Honda, Y.; Kitao, O.; Nakai, H.; Vreven, T.; Throssell, K.; Montgomery, Jr., J. A.; Peralta, J. E.; Ogliaro, F.; Bearpark, M.; Heyd, J. J.; Brothers, E.; Kudin, K. N.; Staroverov, V. N.; Keith, T.; Kobayashi, R.; Normand, J.; Raghavachari, K.; Rendell, A.; Burant, J. C.; Iyengar, S. S.; Tomasi, J.; Cossi, M.; Millam, J. M.; Klene, M.; Adamo, C.; Cammi, R.; Ochterski, J. W.; Martin, R. L.; Morokuma, K.; Farkas, O.; Foresman, J. B.; Fox, D. J. Gaussian 09, Revision D.01, Gaussian, Inc., Wallingford CT, 2016.

7. Zhao, Y.; Truhlar, D. G. The M06 suite of density functionals for main group thermochemistry, thermochemical kinetics, noncovalent interactions, excited states, and transition elements: two new functionals and systematic testing of four M06-class functionals and 12 other functionals. Theor. Chem. Acc. 2008, 120, 215-241. 
8. Fianchini, M.; O'Brien, C. J.; Chass, G. A. Reduction Rate of 1-Phenyl Phospholane 1-Oxide Enhanced by Silanol Byproducts: Comprehensive DFT Study and Kinetic Modeling Linked to Reagent Design. J. Org. Chem. 2019, 84, 10579-10592.

9. Sedlak, R.; Janowski, T.; Pitoňák, M.; Řezáč, J.; Pulay, P.; Hobza, P. Accuracy of Quantum Chemical Methods for Large Noncovalent Complexes. J. Chem. Theory Comput. 2013, 9, 33643374.

10. Dapprich, S.; Komáromi, I.; Byun, K. S.; Morokuma, K.; Frisch, M. J. A new ONIOM implementation in Gaussian98. Part I. The calculation of energies, gradients, vibrational frequencies and electric field derivatives. J. Mol. Struct. (THEOCHEM) 1999, 461-462, 1-21.

11. Maseras, F.; Morokuma, K. IMOMM: A new integrated ab initio + molecular mechanics geometry optimization scheme of equilibrium structures and transition states. J. Comp. Chem. 1995, 16, 1170-1179.

12. Vreven, T.; Byun, K. S.; Komáromi, I.; Dapprich, S.; Montgomery, J. A.; Morokuma, K.; Frisch, M. J. Combining Quantum Mechanics Methods with Molecular Mechanics Methods in ONIOM. J. Chem. Theory Comput. 2006, 2, 815-826.

13. Vreven, T.; Frisch, M. J.; Kudin, K. N.; Schlegel, H. B.; Morokuma, K. Geometry optimization with QM/MM methods II: Explicit quadratic coupling. Mol. Phys. 2006, 104, 701-714.

14. Vreven, T.; Morokuma, K.; Farkas, Ö.; Schlegel, H. B.; Frisch, M. J. Geometry optimization with $\mathrm{QM} / \mathrm{MM}, \mathrm{ONIOM}$, and other combined methods. I. Microiterations and constraints. J. Comp. Chem. 2003, 24, 760-769.

15. Vreven, T.; Morokuma, K. On the application of the IMOMO (integrated molecular orbital + molecular orbital) method. J. Comput. Chem. 2000, 21, 1419-1432.

16. Vreven, T.; Mennucci, B.; da Silva, C. O.; Morokuma, K.; Tomasi, J. The ONIOM-PCM method: Combining the hybrid molecular orbital method and the polarizable continuum model for solvation. Application to the geometry and properties of a merocyanine in solution. J. Chem. Phys. 2001, 115, 62-72.

17. Godbout, N.; Salahub, D. R.; Andzelm, J.; Wimmer, E. Optimization of Gaussian-type basis sets for local spin density functional calculations. Part I. Boron through neon, optimization technique and validation. Can. J. Chem. 1992, 70, 560-571.

18. Marenich, A. V.; Cramer, C. J.; Truhlar, D. G. Universal Solvation Model Based on Solute Electron Density and on a Continuum Model of the Solvent Defined by the Bulk Dielectric Constant and Atomic Surface Tensions. J. Phys. Chem. B 2009, 113, 6378-6396.

19. Wheeler, S. E.; Houk, K. N. Integration Grid Errors for Meta-GGA-Predicted Reaction Energies: Origin of Grid Errors for the M06 Suite of Functionals. J. Chem. Theory Comput. 2010, 6, 395-404.

20. Funes-Ardoiz, I.; Paton, R. GoodVibes, v.2.0.3. DOI: 10.5281/zenodo.1435820.

21. Boys, S. F.; and Bernardi, F. Calculation of Small Molecular Interactions by Differences of Separate Total Energies - Some Procedures with Reduced Errors. Mol. Phys. 1970, 19, 553566. 
22. Simon, S.; Duran, M.; Dannenberg, J.J. How does basis set superposition error change the potential surfaces for hydrogen bonded dimers? J. Chem. Phys. 1996, 105, 11024-11031.

23. Pettersen, E. F.; Goddard, T. D.; Huang, C. C.; Couch, G. S.; Greenblatt, D. M.; Meng, E. C.; Ferrin, T. E. UCSF Chimera-A visualization system for exploratory research and analysis. J. Comput. Chem. 2004, 25, 1605-1612.

24. Humphrey, W.; Dalke, A.; Schulten, K. VMD: Visual molecular dynamics. J. Mol. Graphics. 1996, 14, 33-38.

25. Persistence of Vision(TM) Raytracer, 3.6; Williamstown, Victoria, Australia, 2004.

26. Stone, J. An Efficient Library for Parallel Ray Tracing and Animation. University of MissouriRolla, 1998. 


\section{NMR spectra}

\section{${ }^{1} \mathrm{H}$ NMR of $\mathbf{S 1}$}

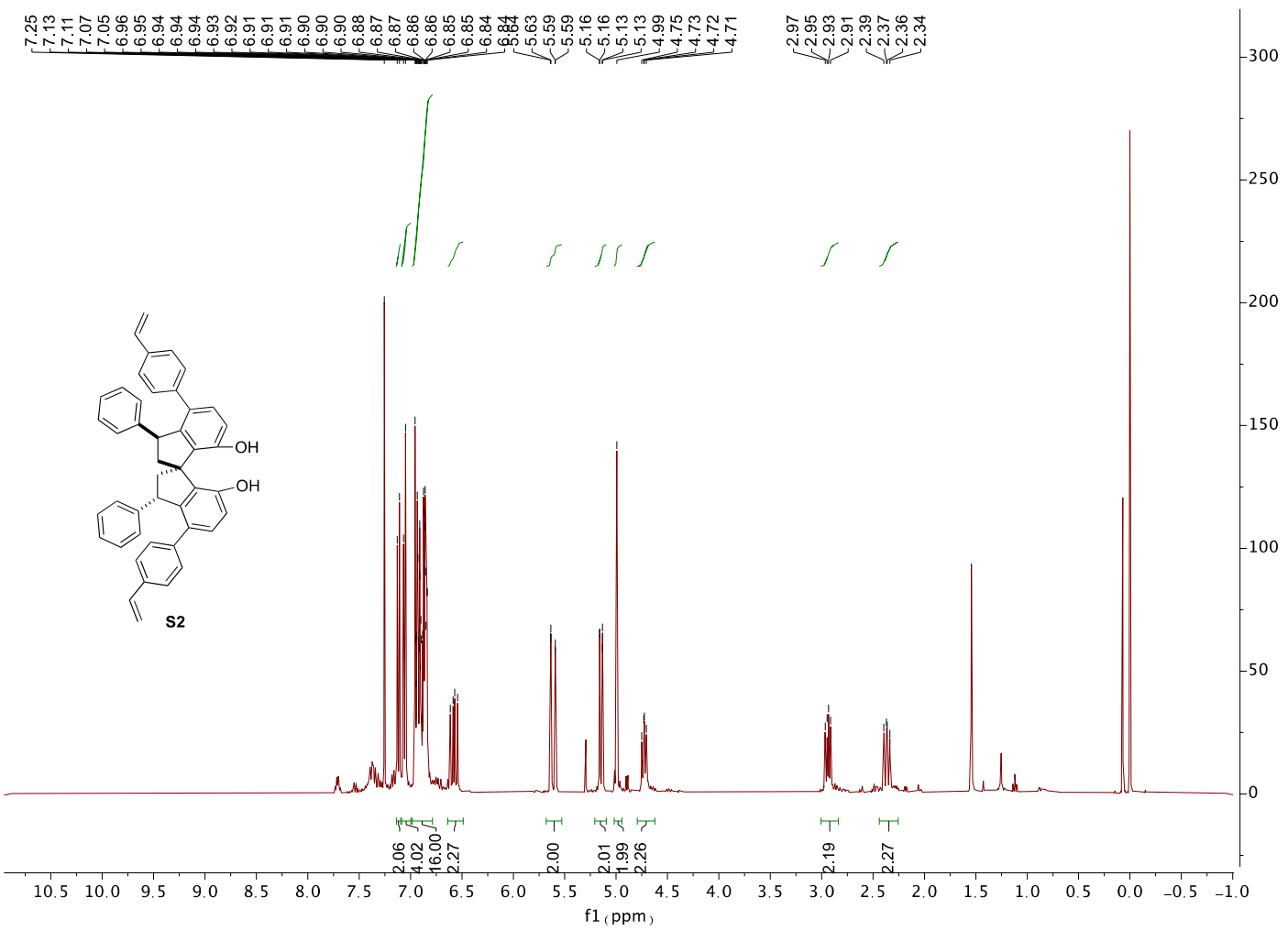

${ }^{13}$ C NMR of S1

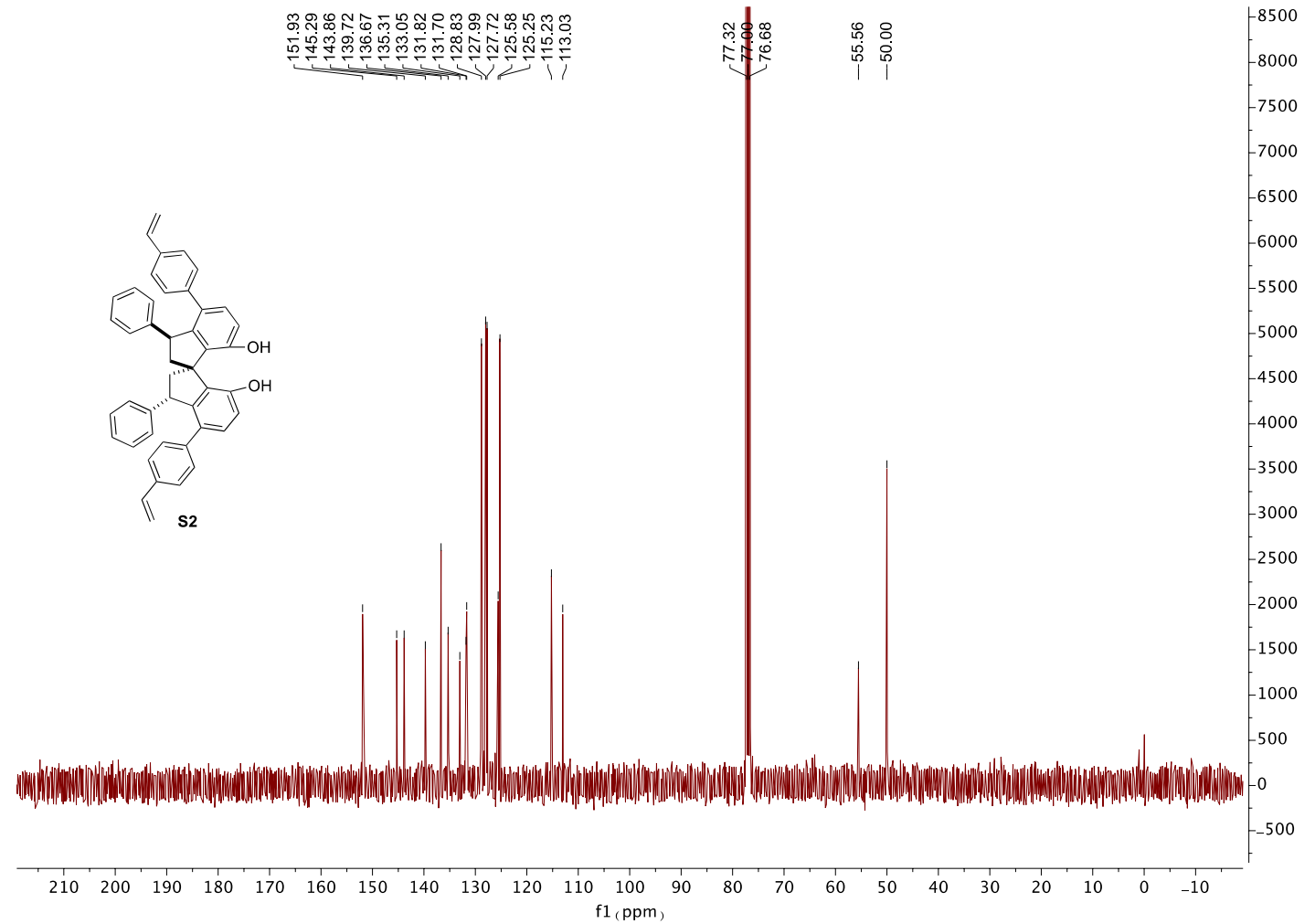


${ }^{1} \mathrm{H}$ NMR of S4

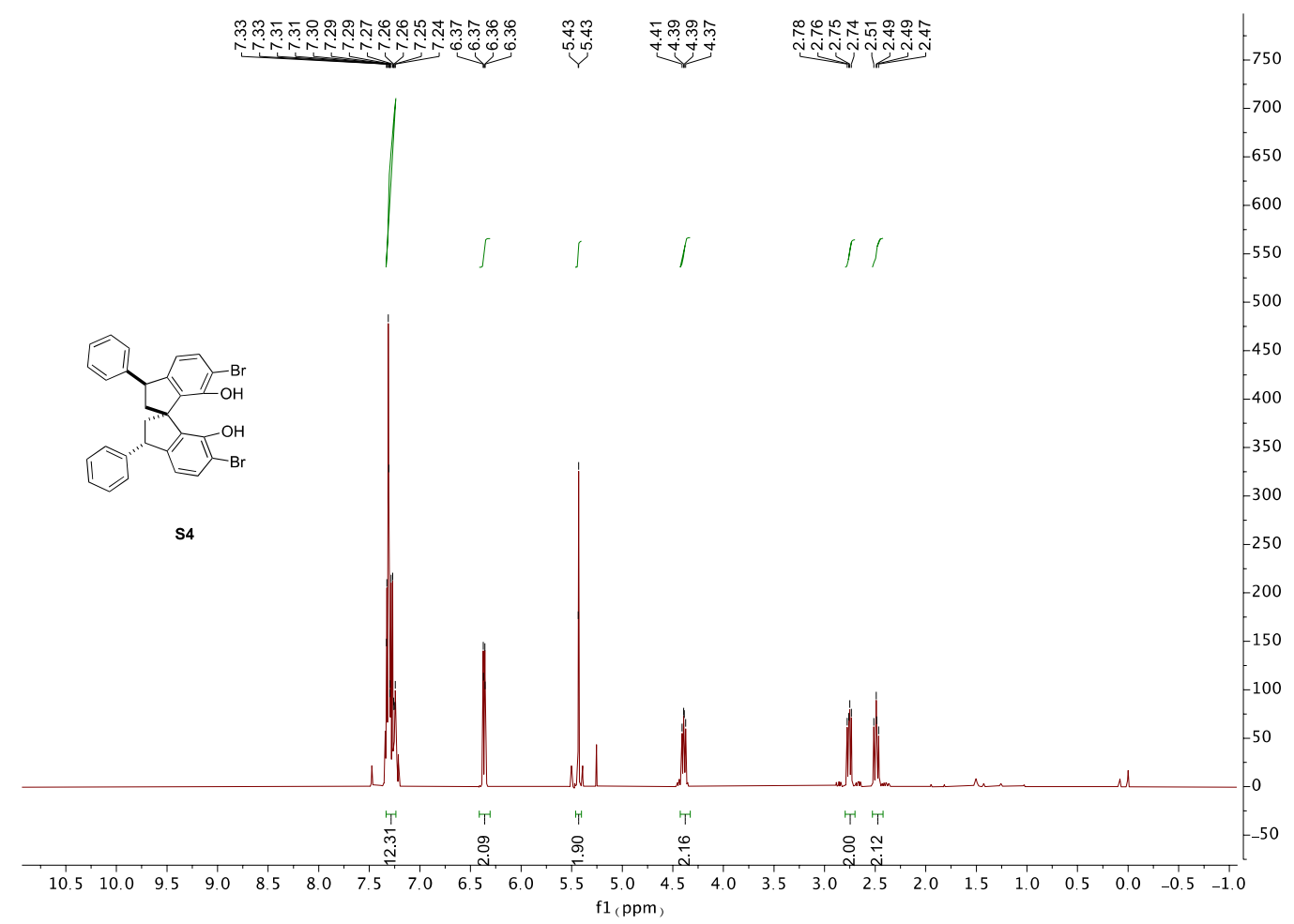

${ }^{13} \mathrm{C}$ NMR of S4

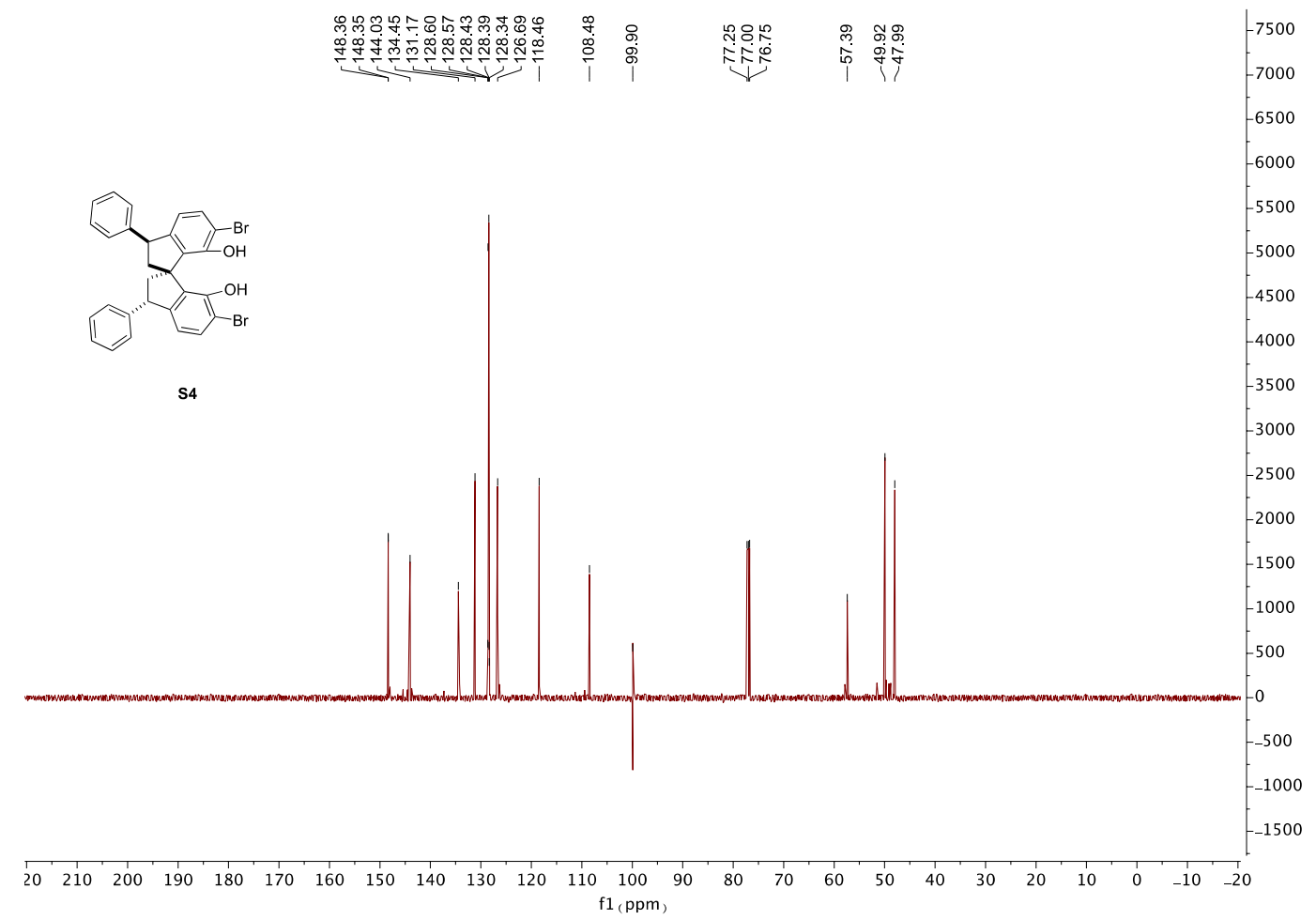




\section{${ }^{1} \mathrm{H}$ NMR of S5}

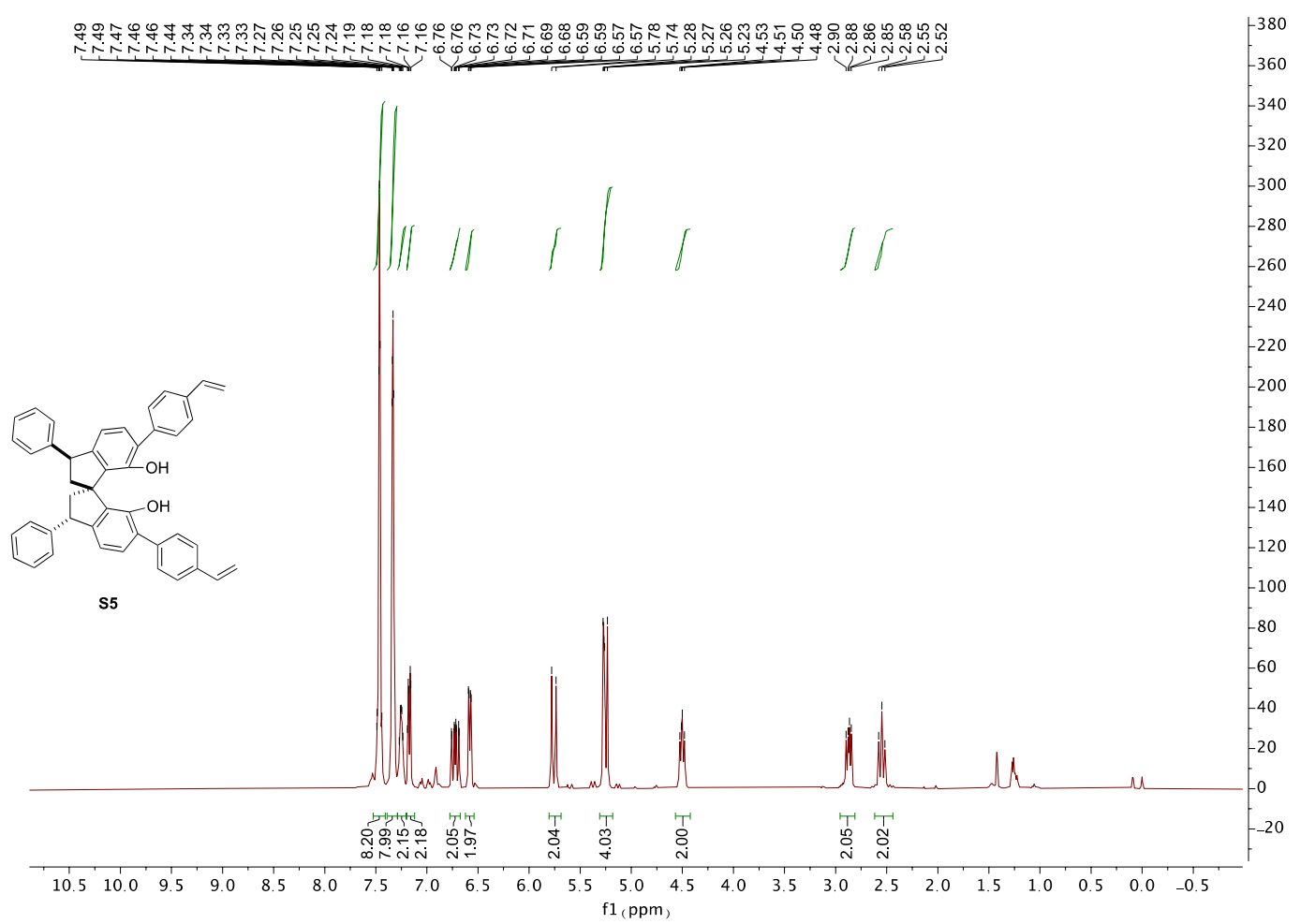

\section{${ }^{13} \mathrm{C}$ NMR of $\mathbf{S 5}$}

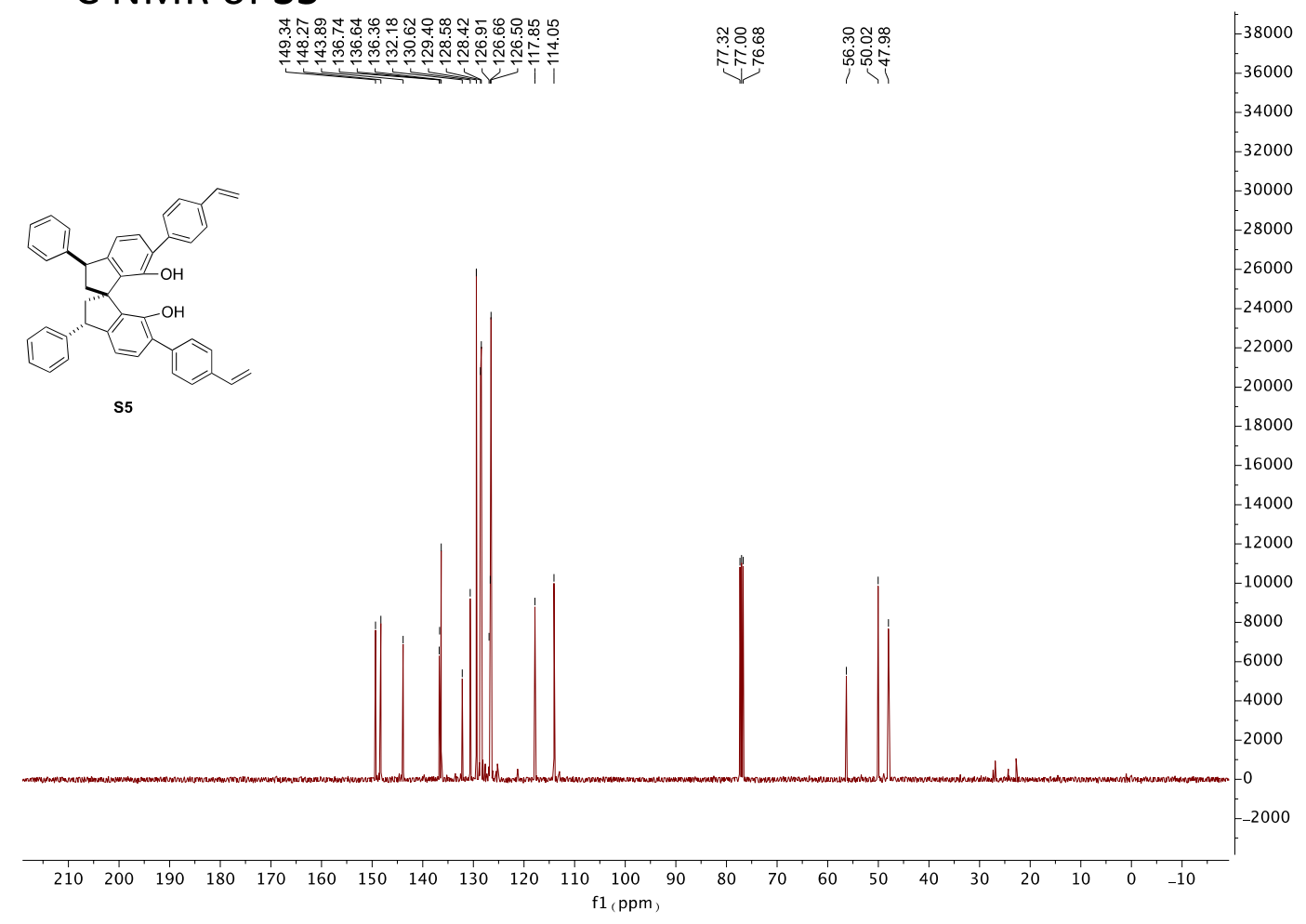




\section{${ }^{1} \mathrm{H}$ NMR of $\mathbf{S 7}$}

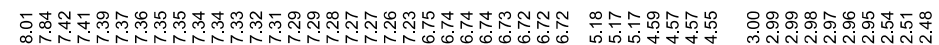

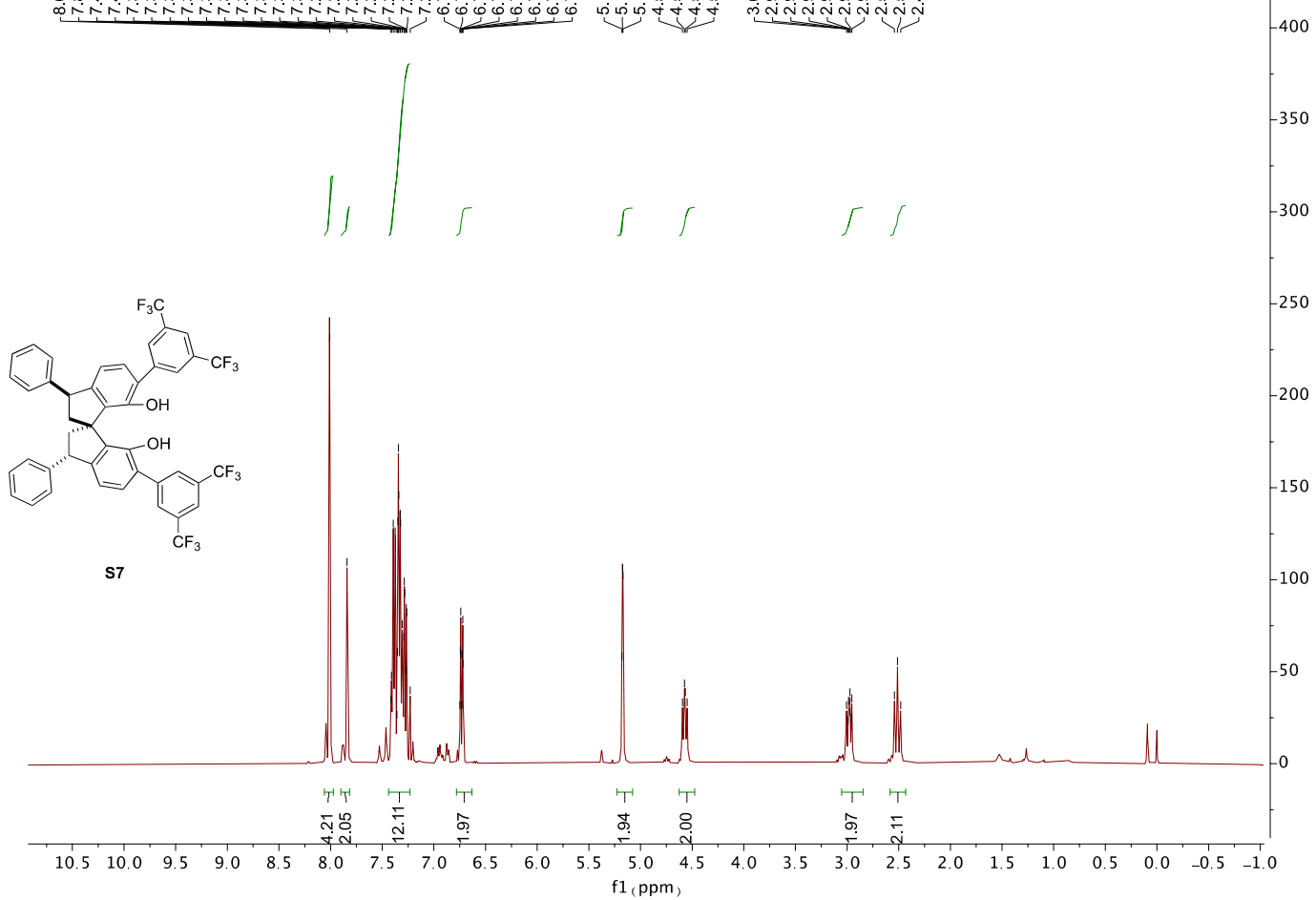

\section{${ }^{13} \mathrm{C}$ NMR of S7}

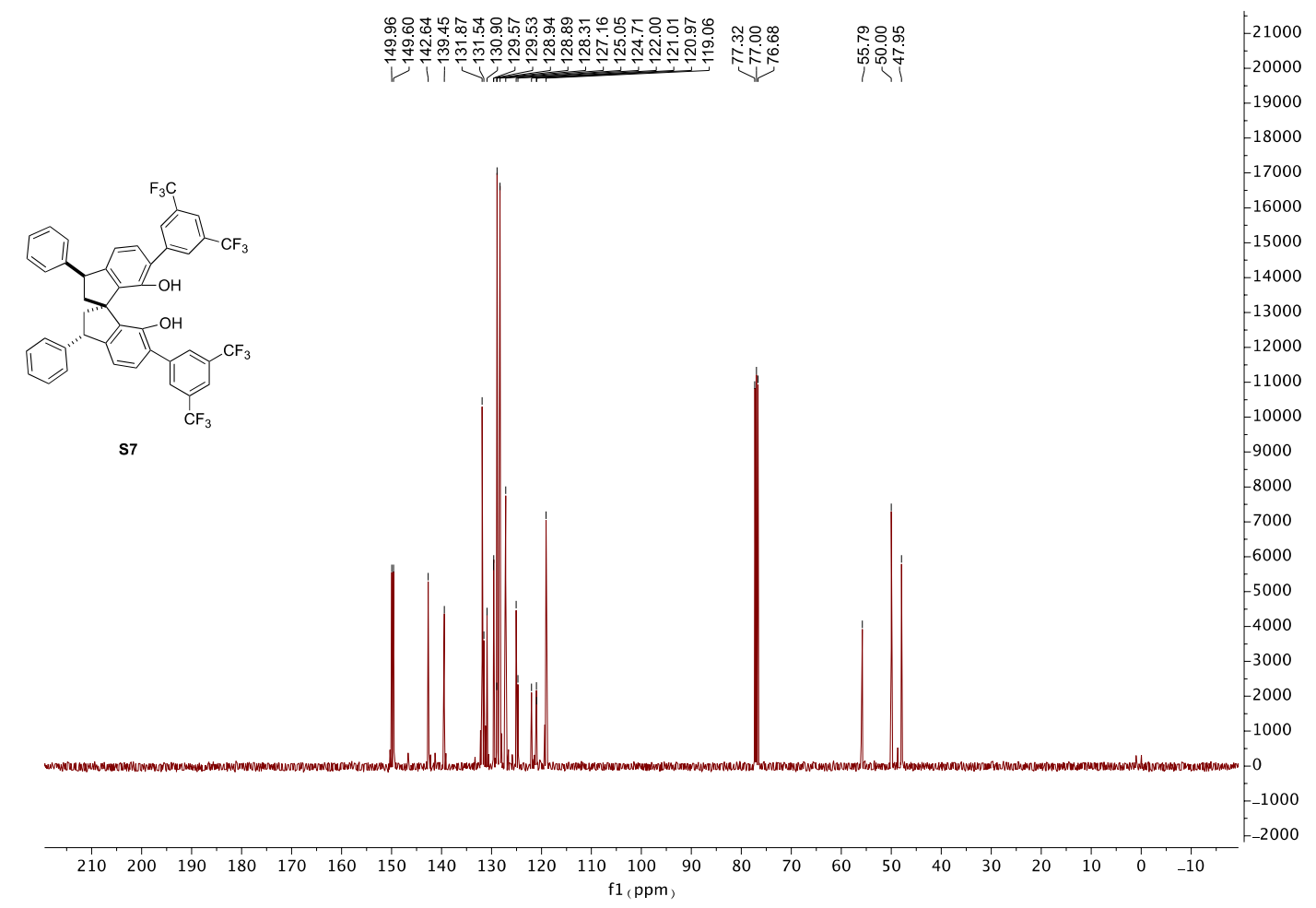


${ }^{19} \mathrm{~F}$ NMR of S7

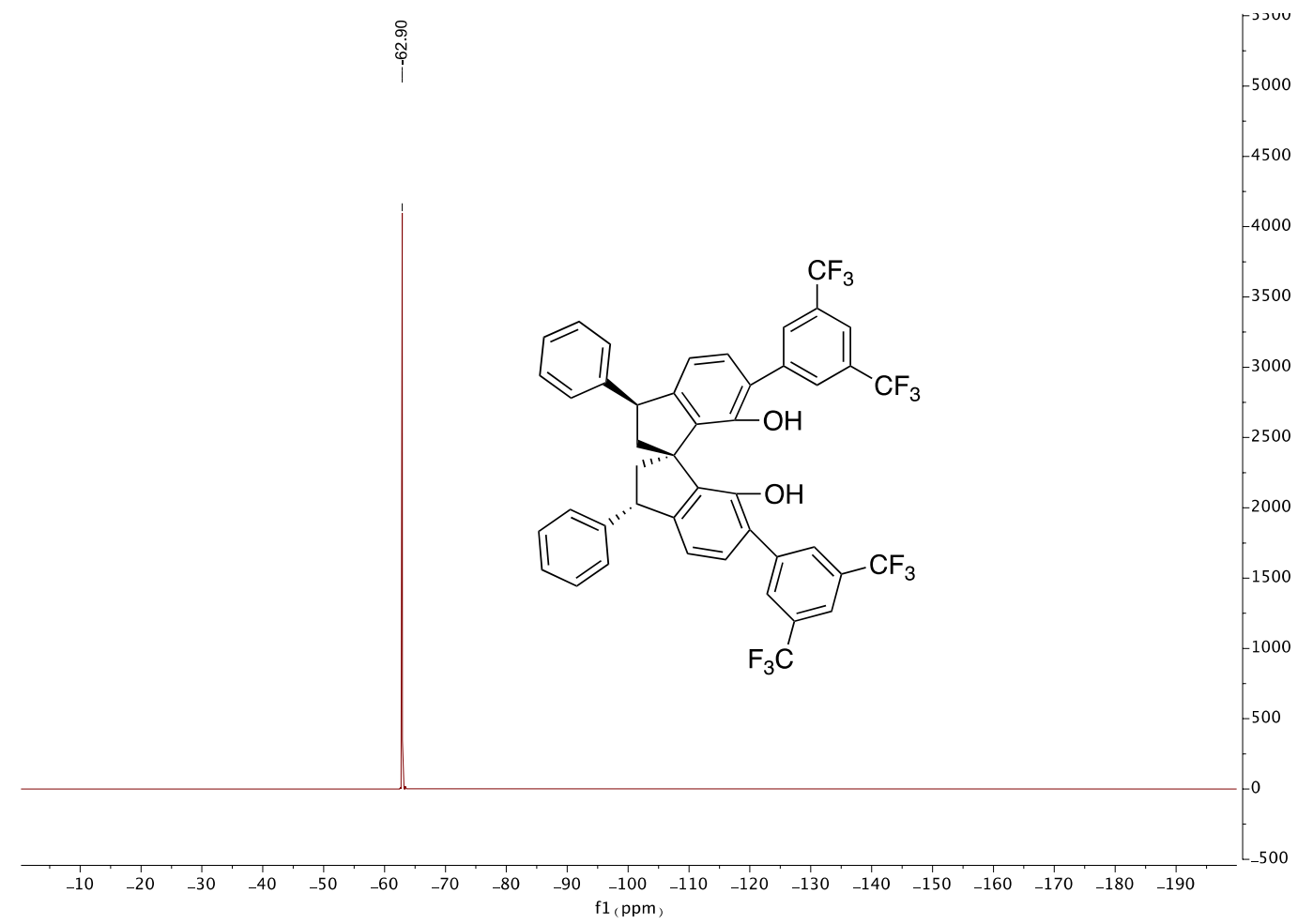


${ }^{1} \mathrm{H}$ NMR of S8

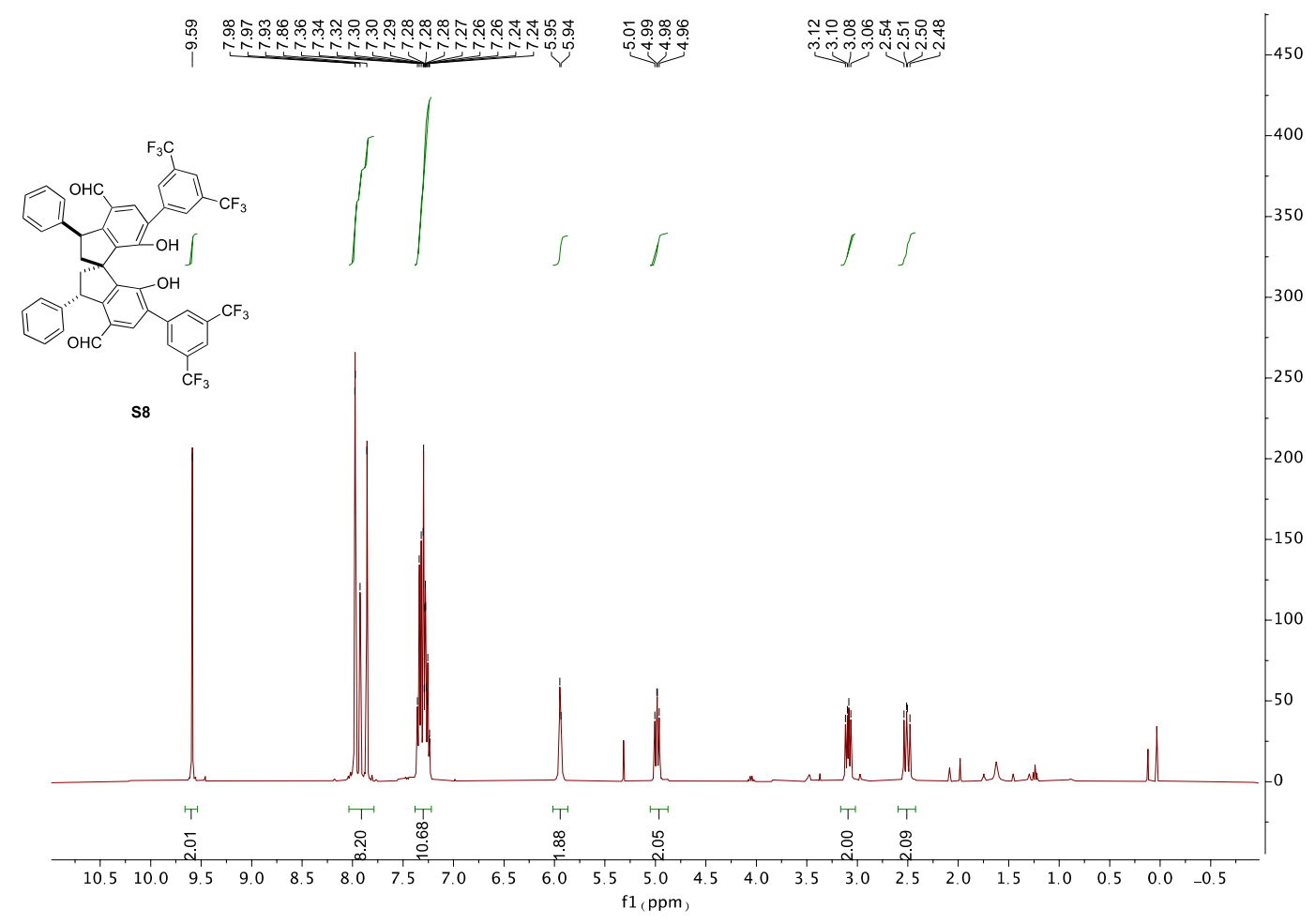

${ }^{13} \mathrm{C}$ NMR of $\mathbf{S 8}$

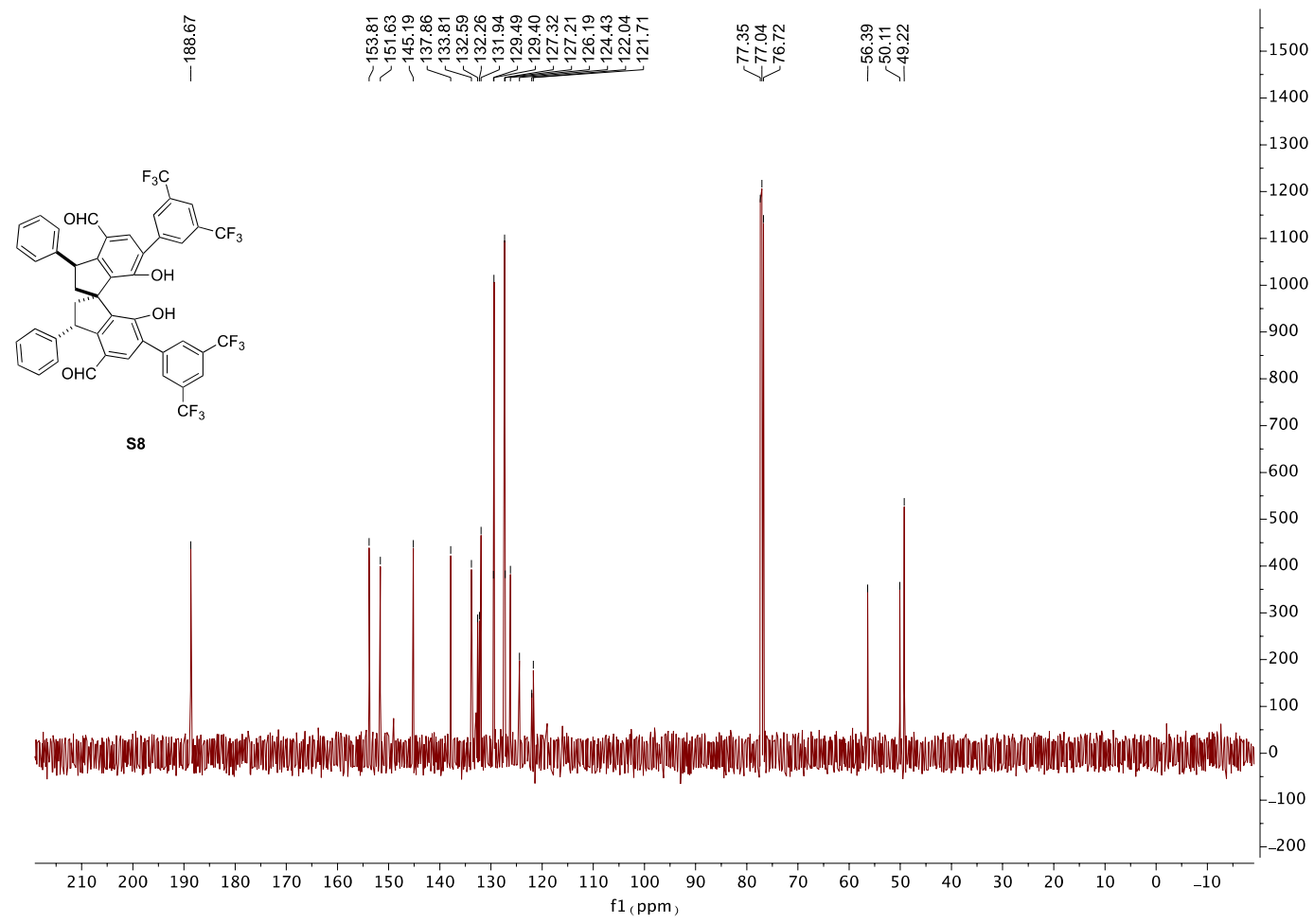


${ }^{19} \mathrm{~F}$ NMR of $\mathbf{S 8}$

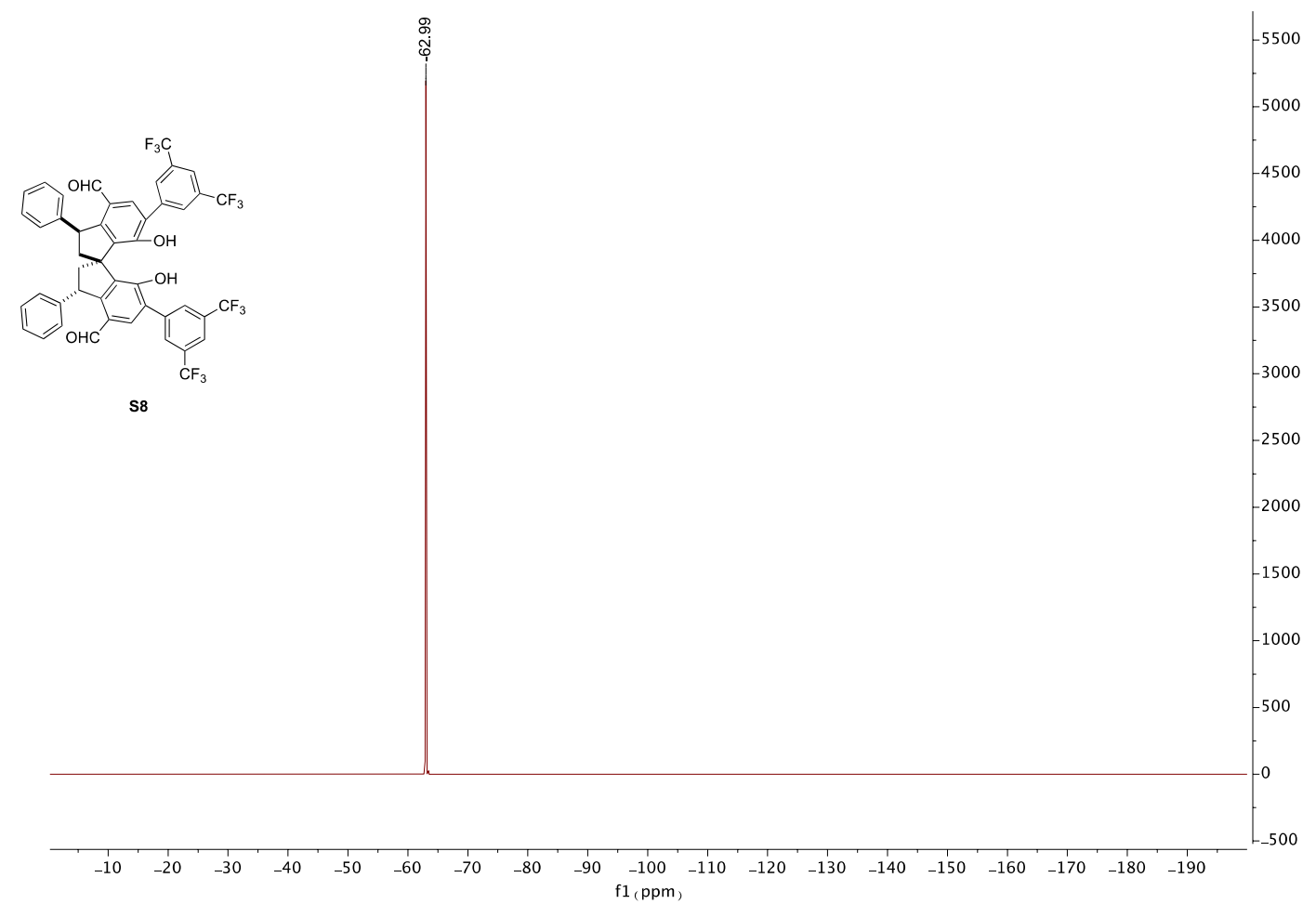

${ }^{19} \mathrm{~F}$ NMR of S9

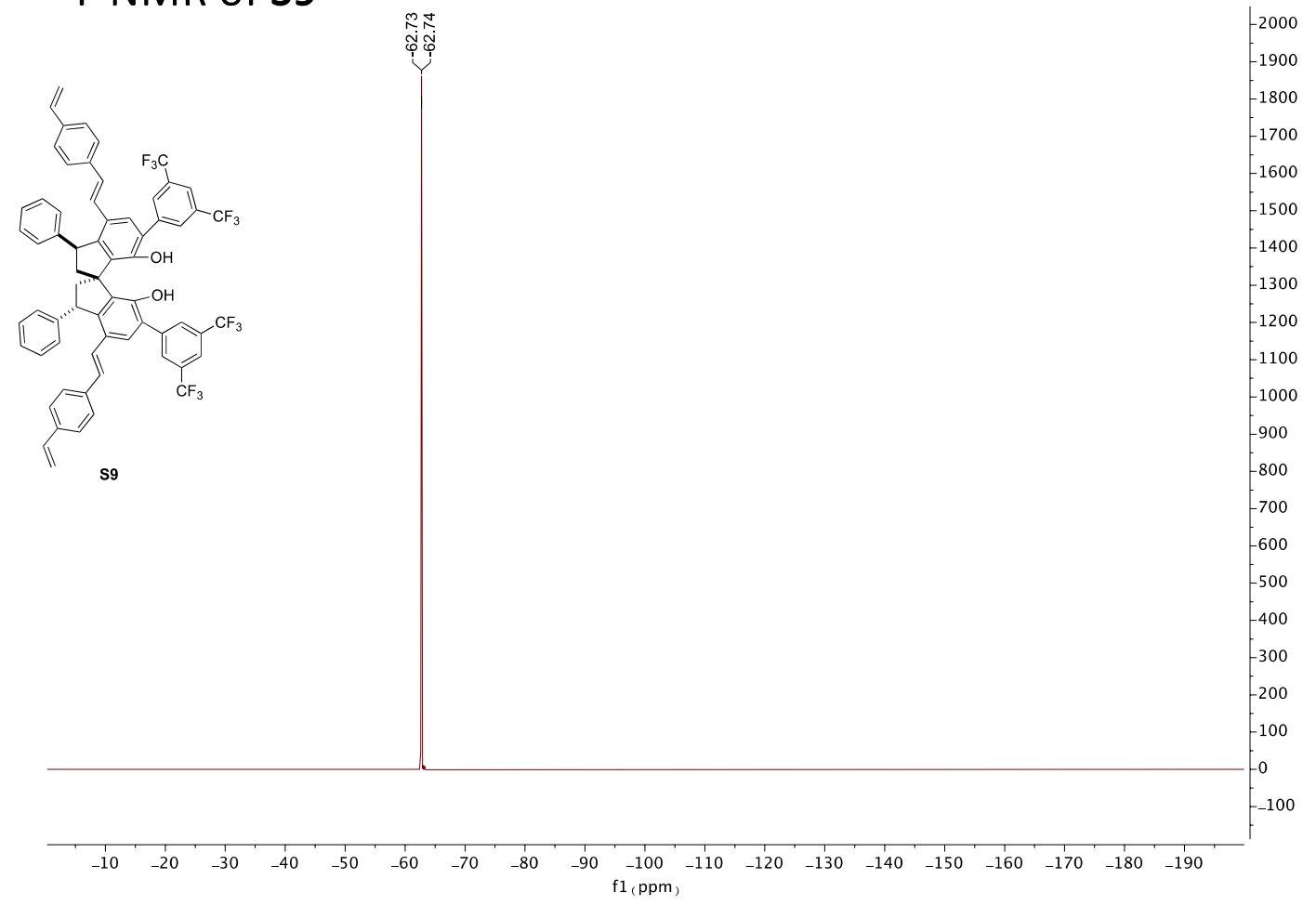




\section{${ }^{1} \mathrm{H} N M R$ of $\mathbf{S 9}$}

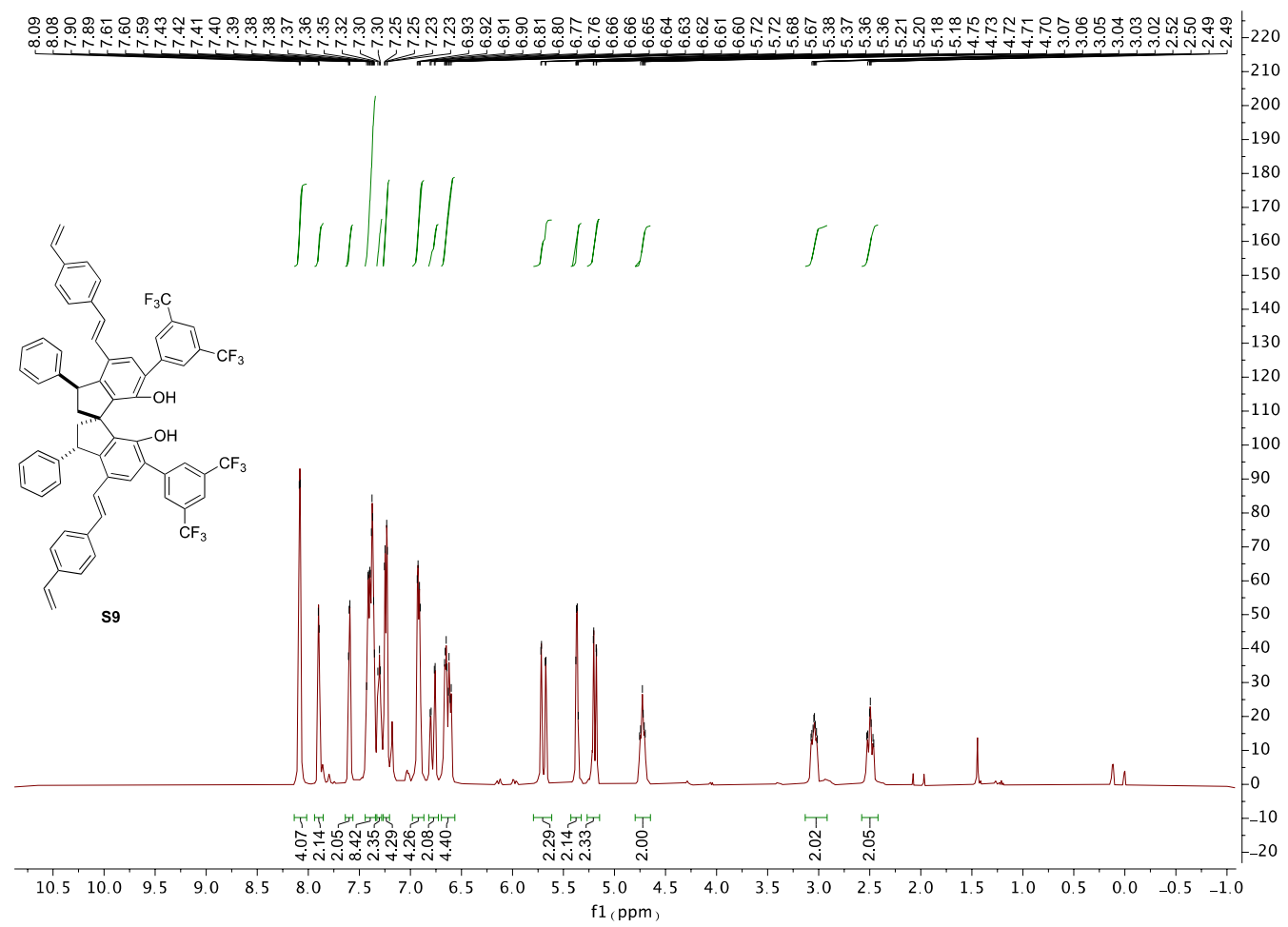

\section{${ }^{13}$ C NMR of S9}

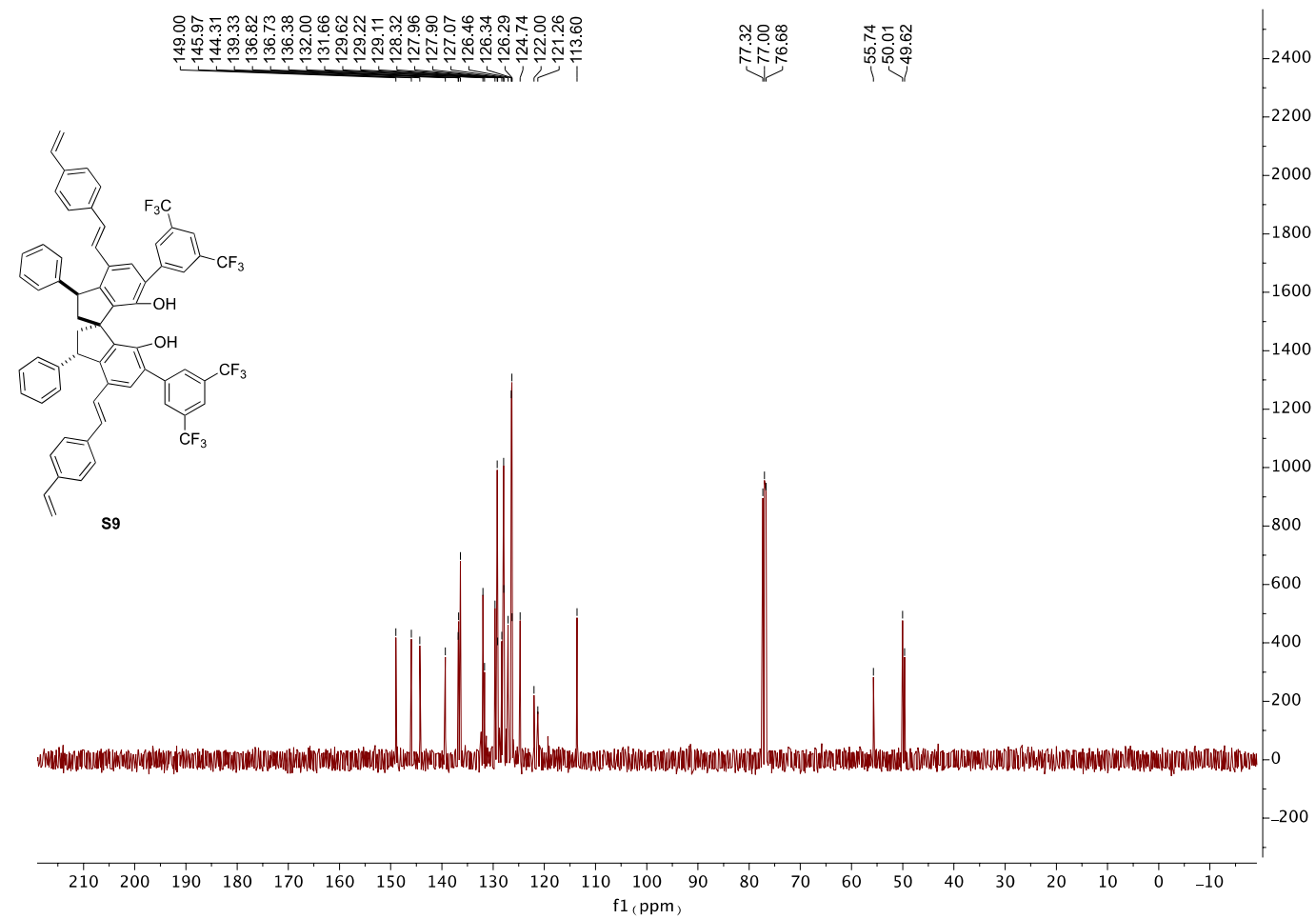




\section{${ }^{1} \mathrm{H}$ NMR of 13}

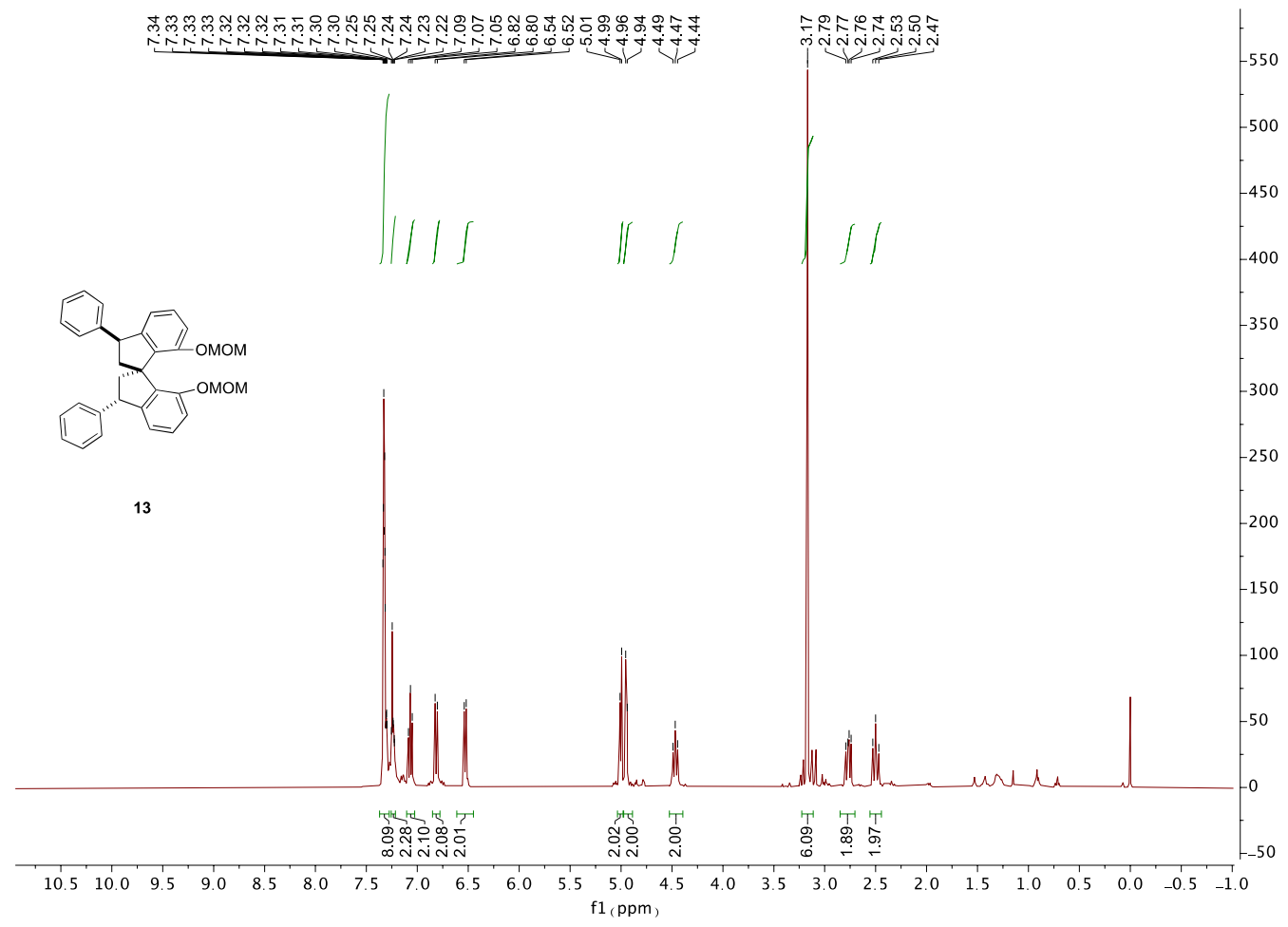

${ }^{13} \mathrm{C}$ NMR of 13

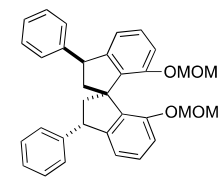

13
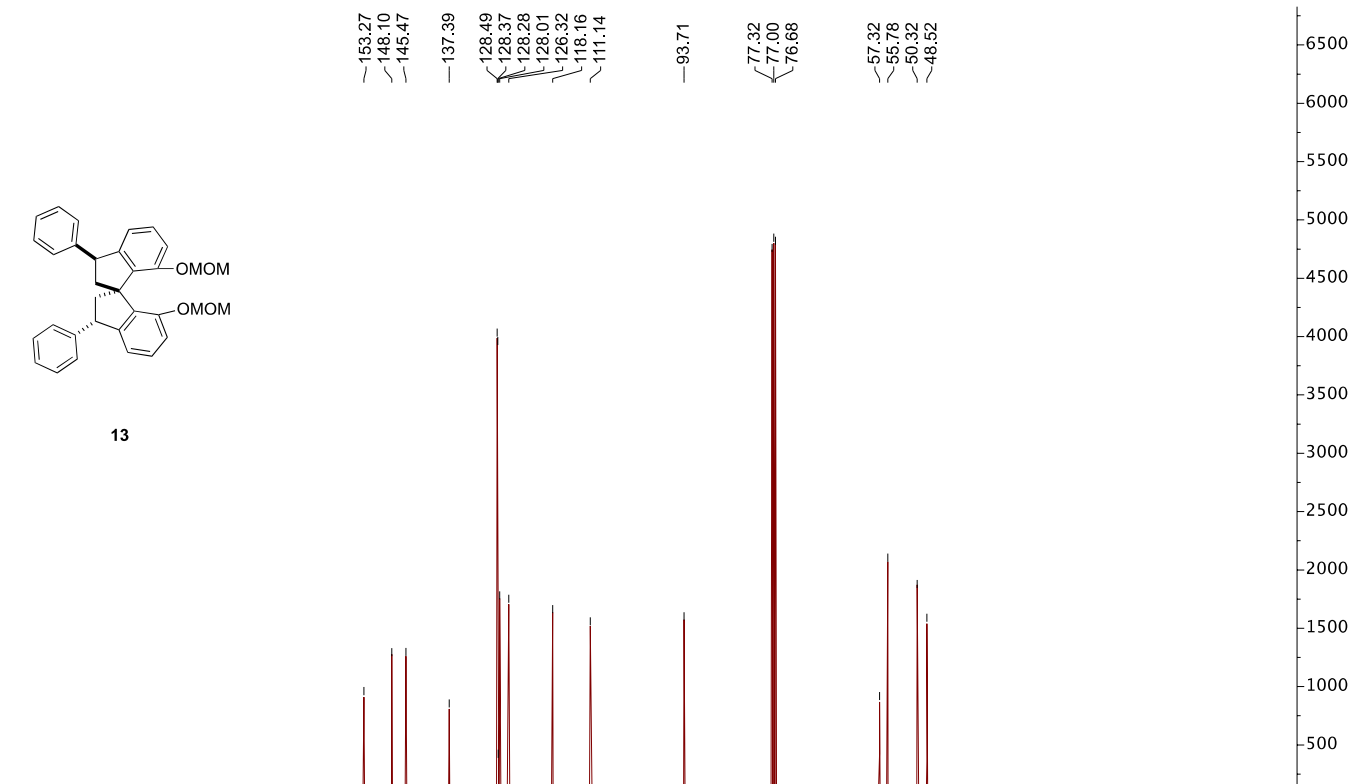

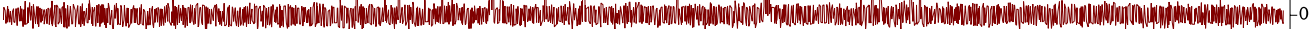

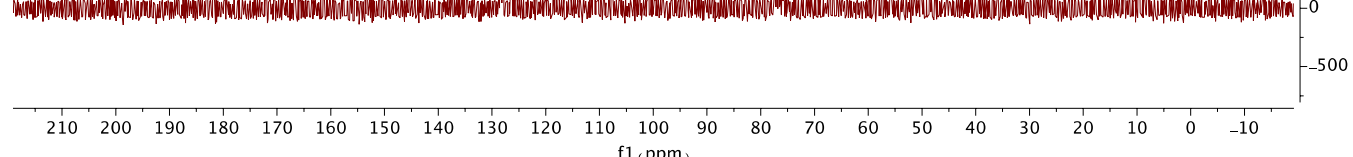




\section{${ }^{1} \mathrm{H}$ NMR of 14}

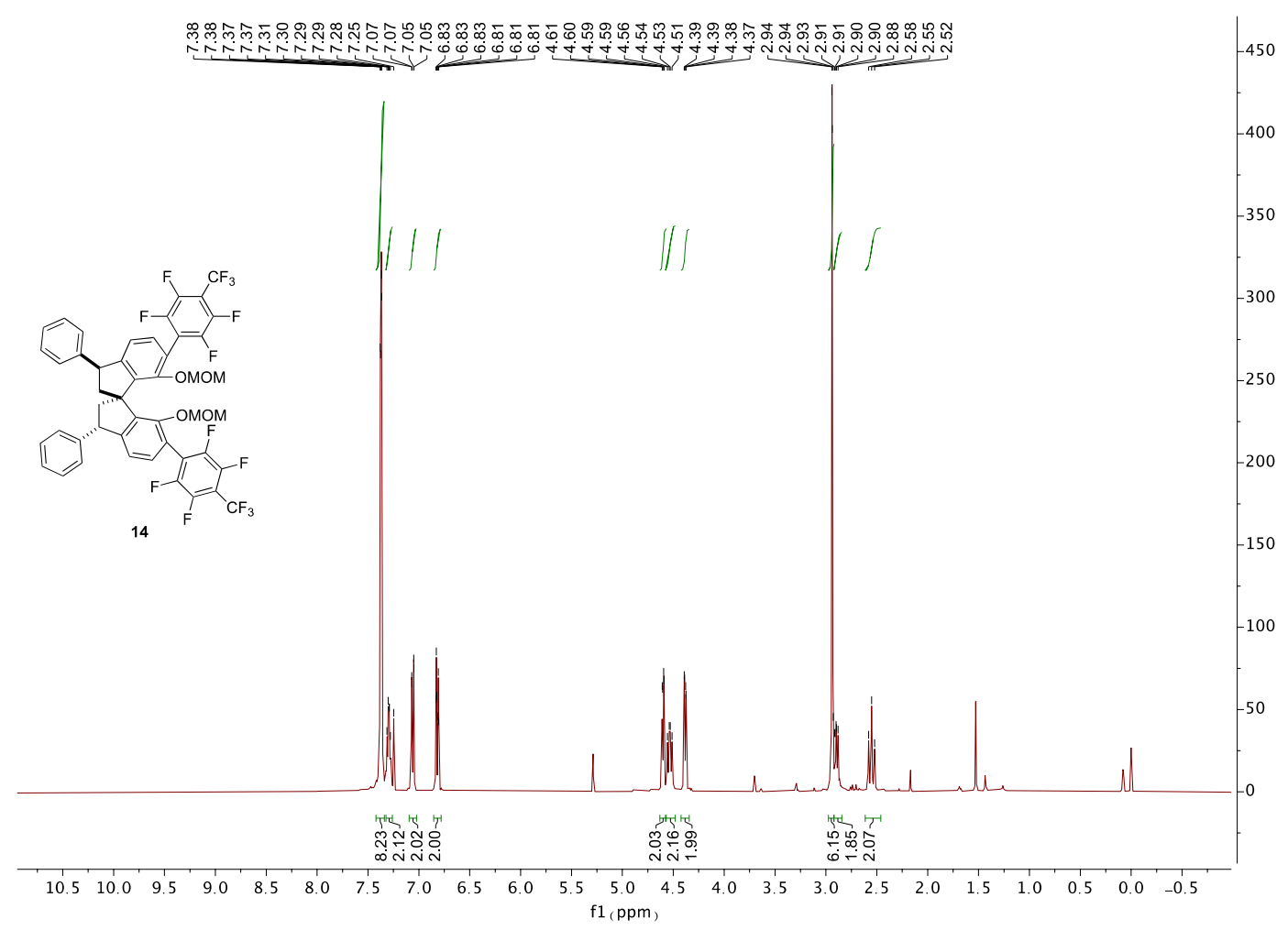

\section{${ }^{13}$ C NMR of 14}

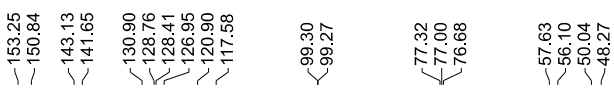
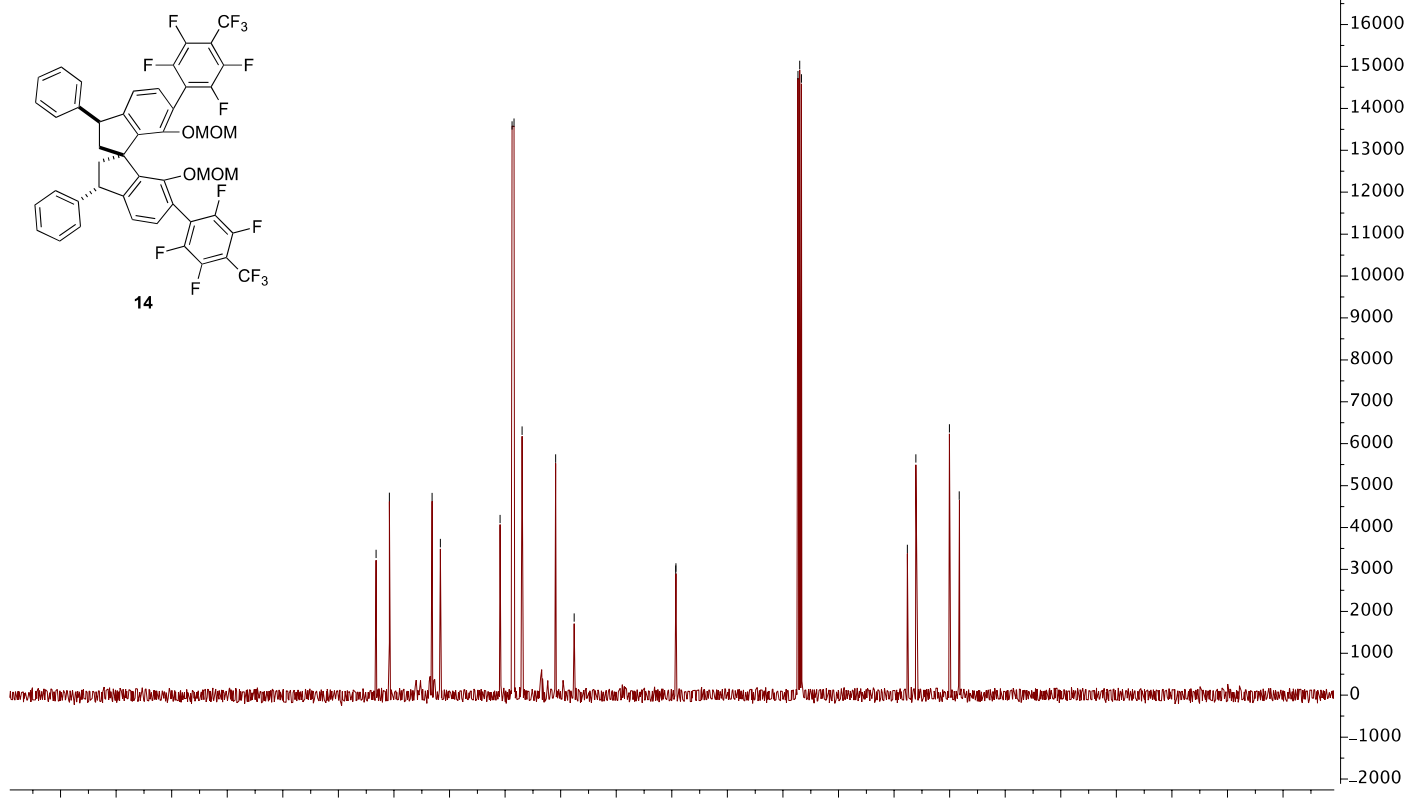

$\begin{array}{lllllllllllllllllllllllllll}210 & 200 & 190 & 180 & 170 & 160 & 150 & 140 & 130 & 120 & 110 & 100 & 90 & 80 & 70 & 60 & 50 & 40 & 30 & 20 & 10 & 0 & -10\end{array}$ 
${ }^{19} \mathrm{~F}$ NMR of 14

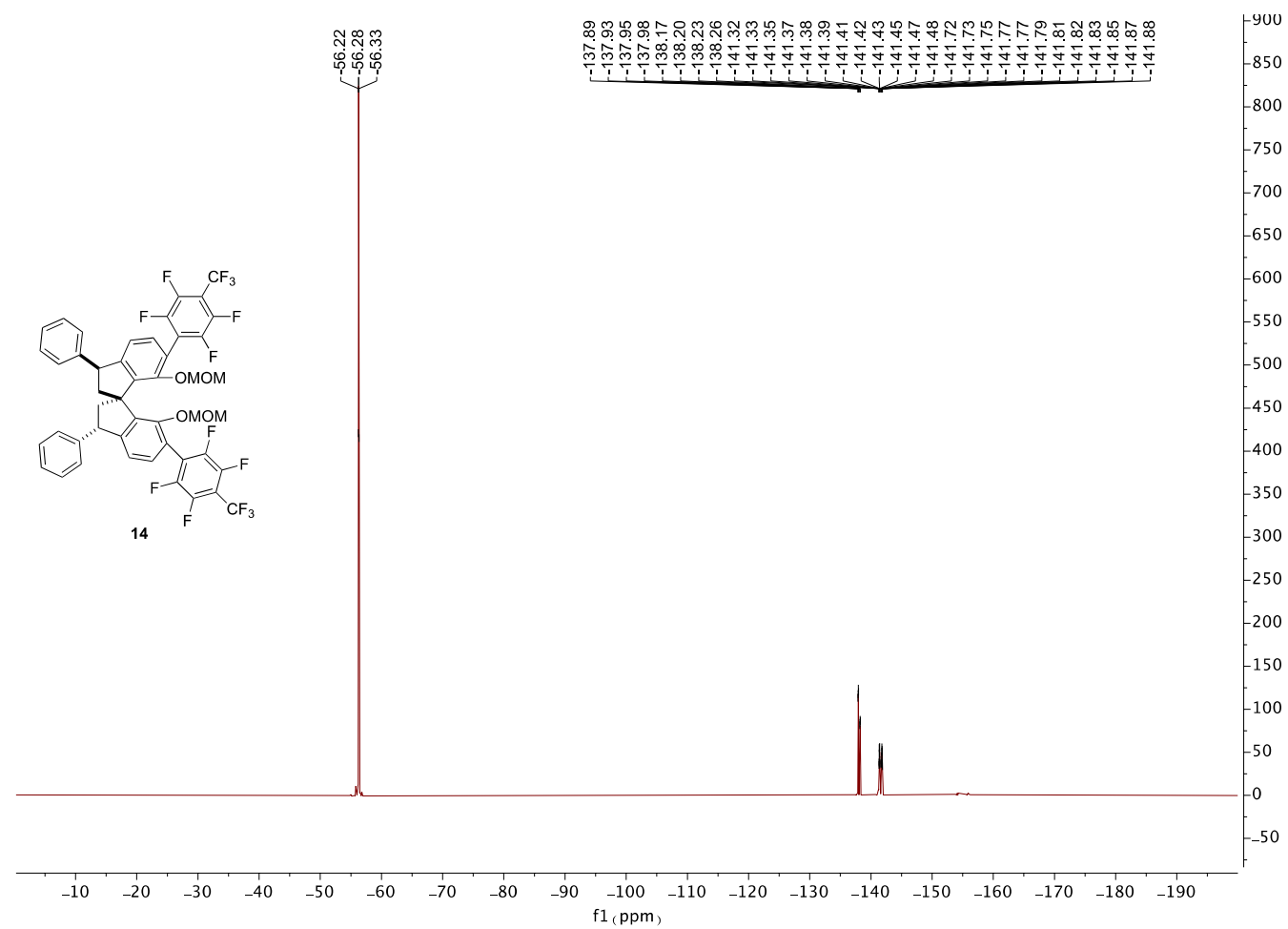

${ }^{19} \mathrm{~F}$ NMR of 15

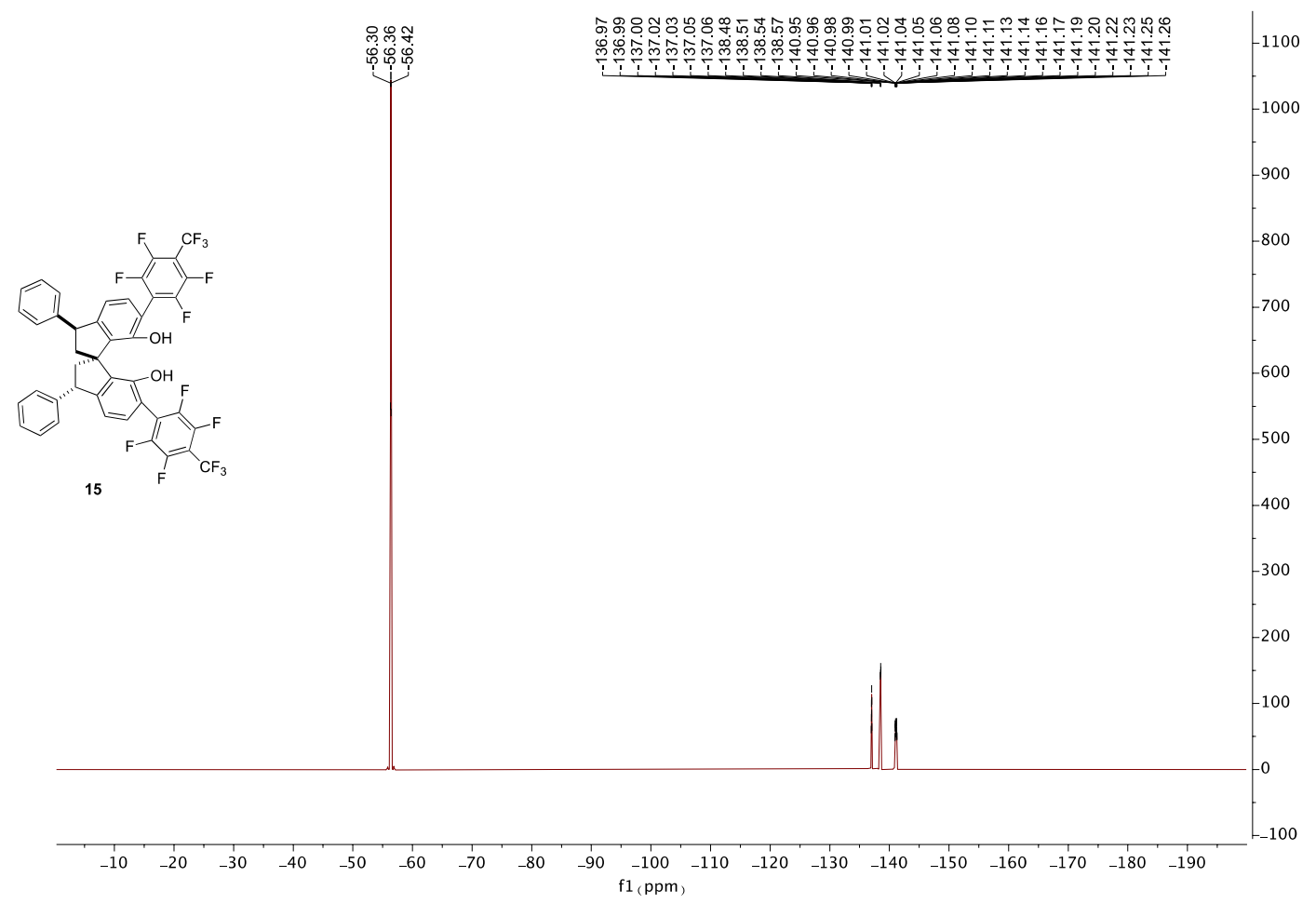




\section{${ }^{1} \mathrm{H}$ NMR of 15}

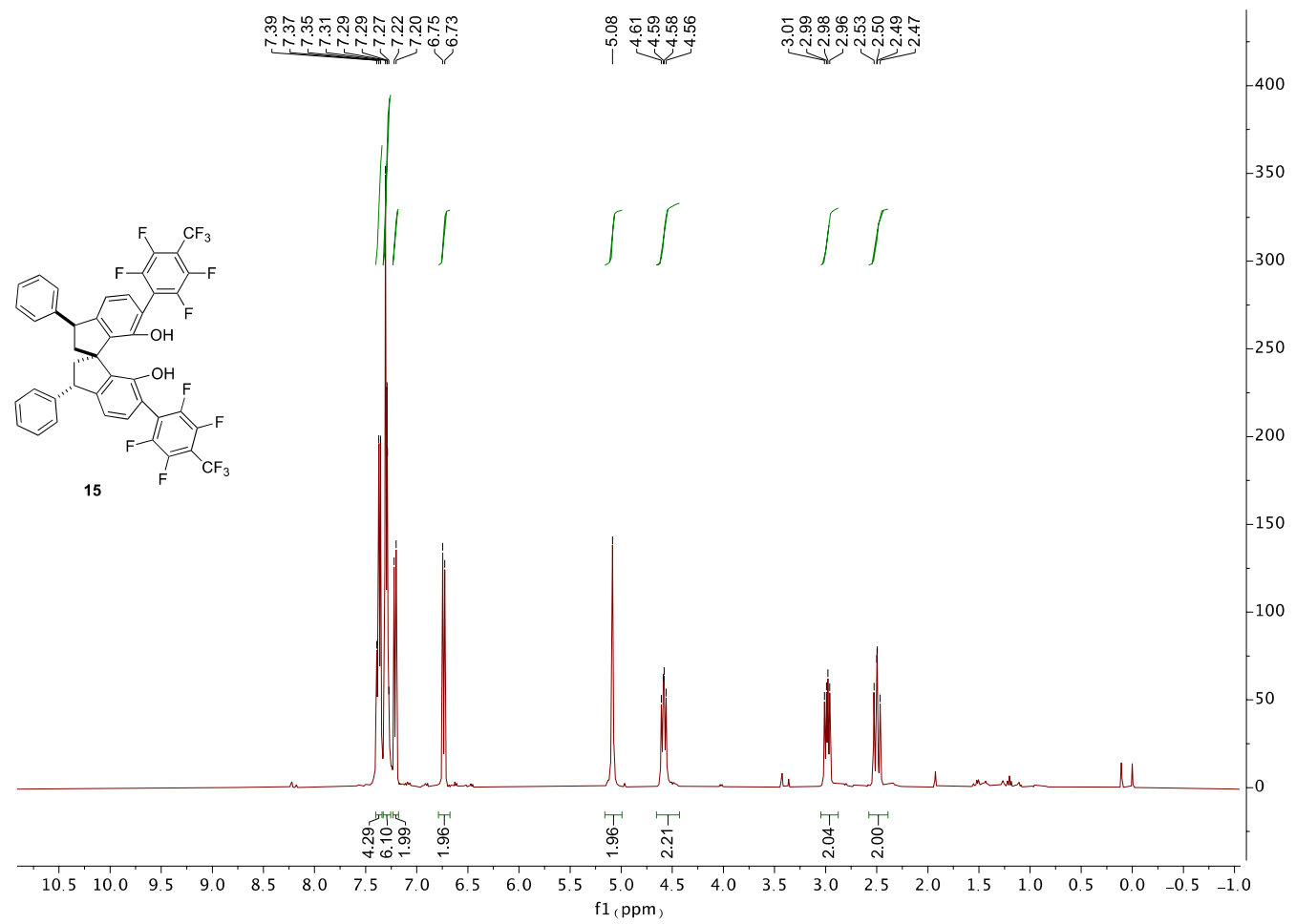

\section{${ }^{13} \mathrm{C}$ NMR of 15}

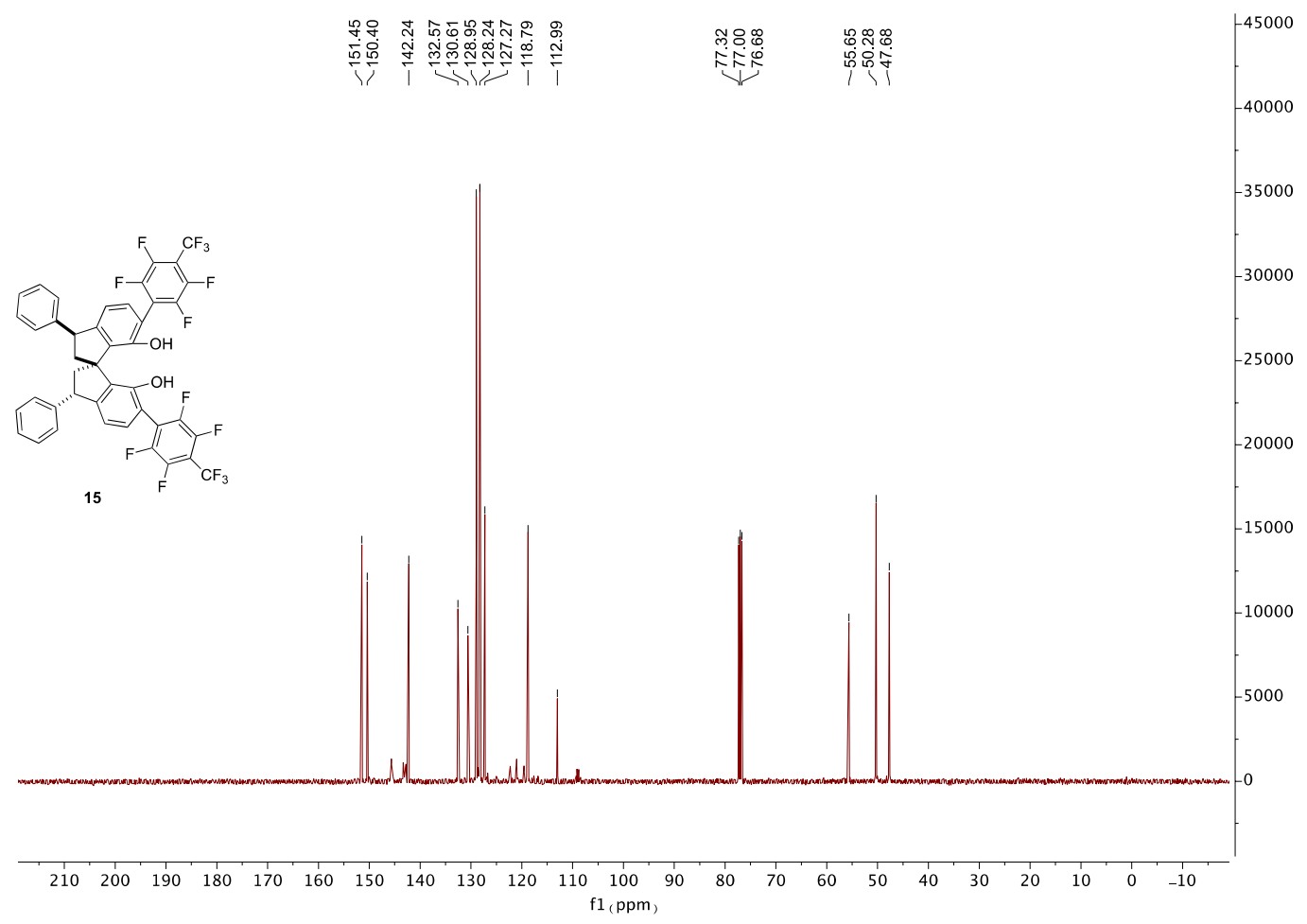


${ }^{1} \mathrm{H}$ NMR of 16

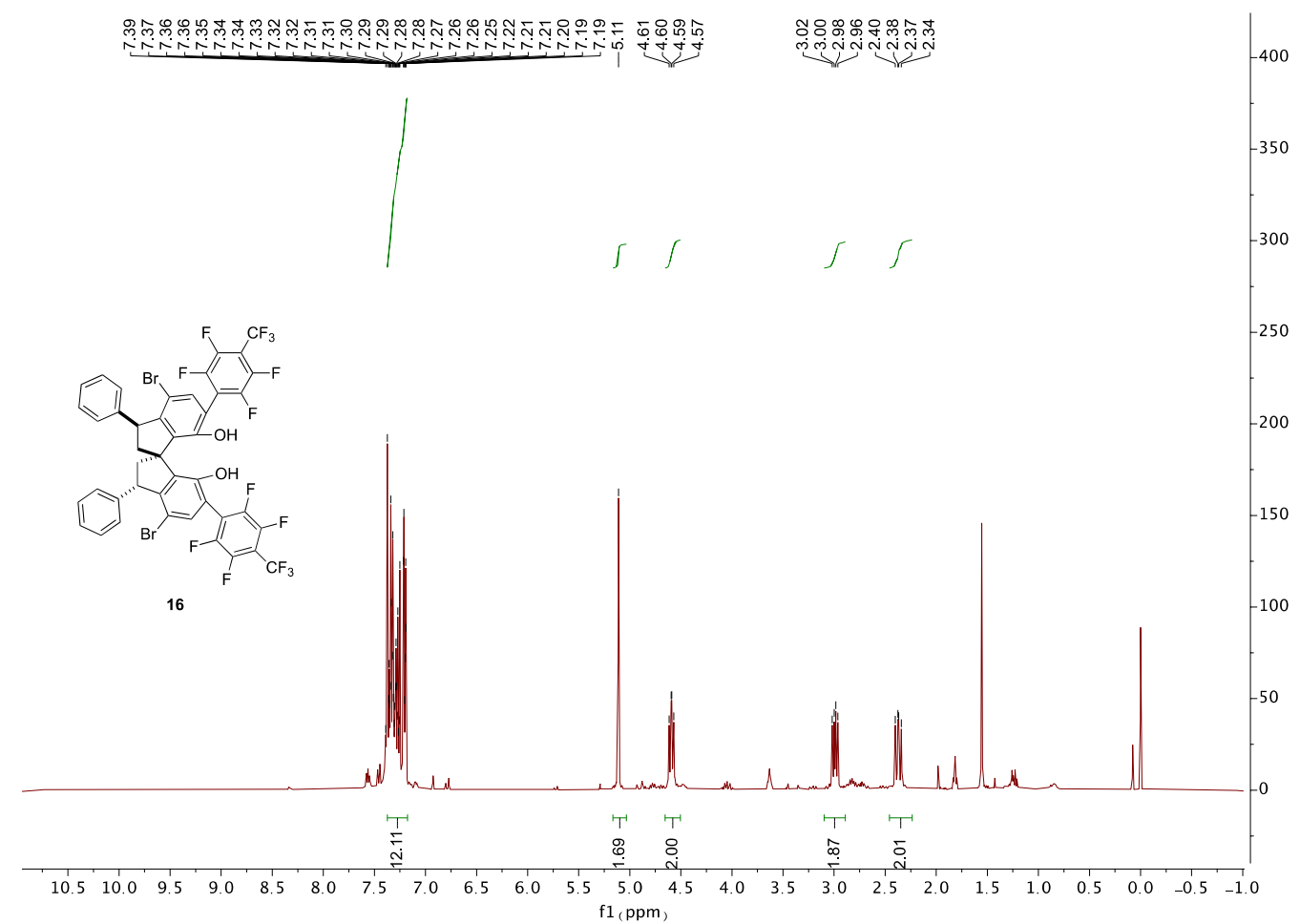

${ }^{13} \mathrm{C}$ NMR of 16

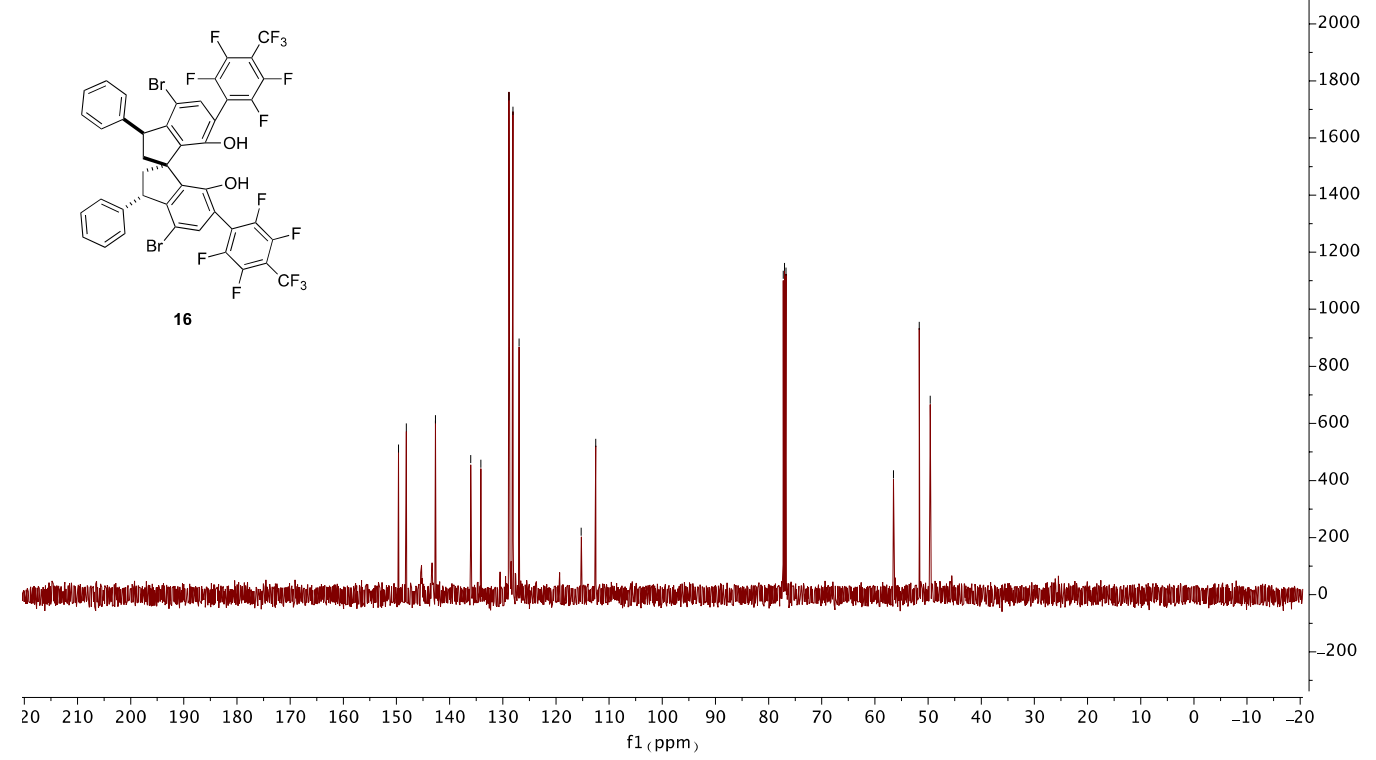


${ }^{19} \mathrm{~F}$ NMR of 16

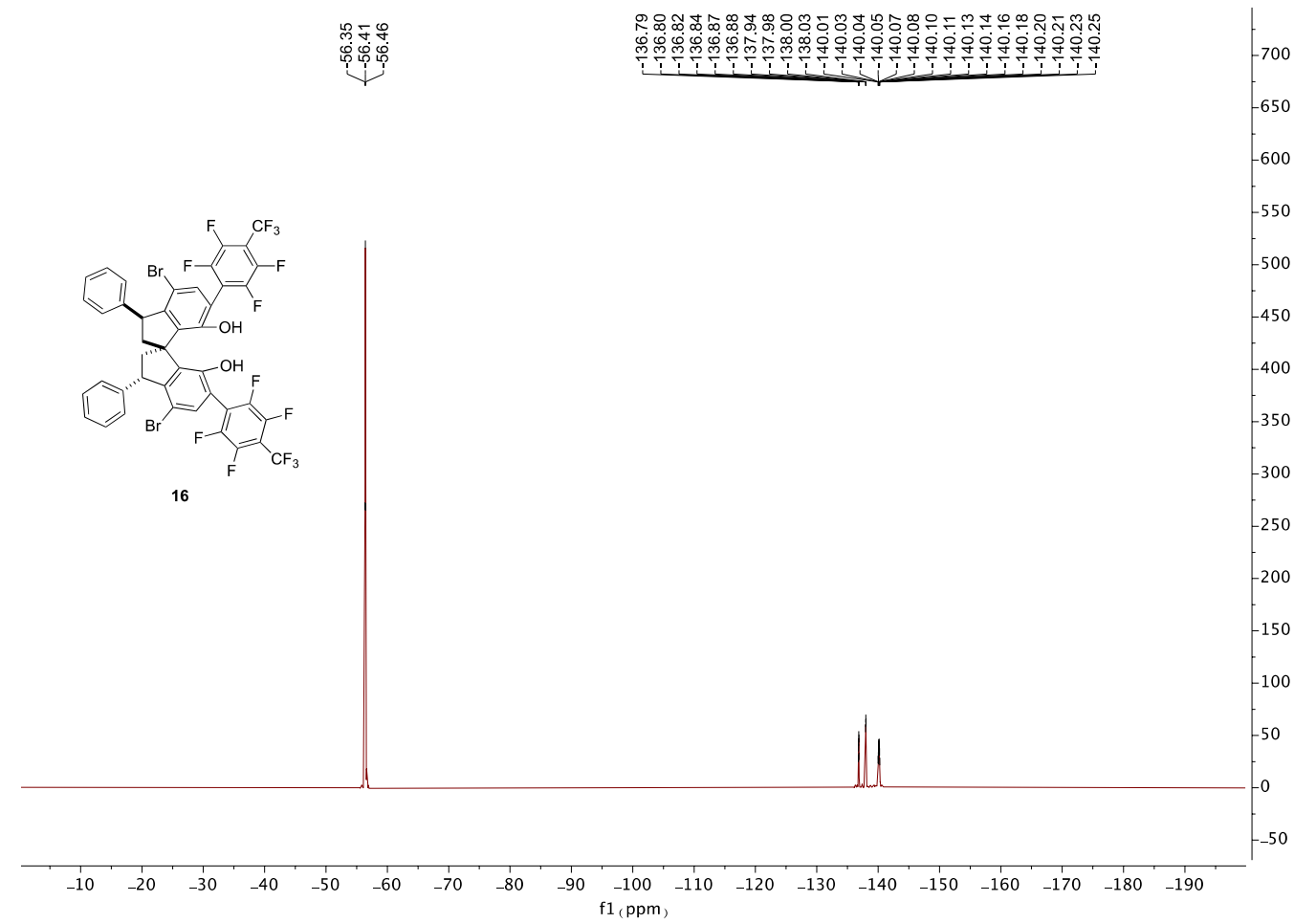

${ }^{19} \mathrm{~F}$ NMR of S11

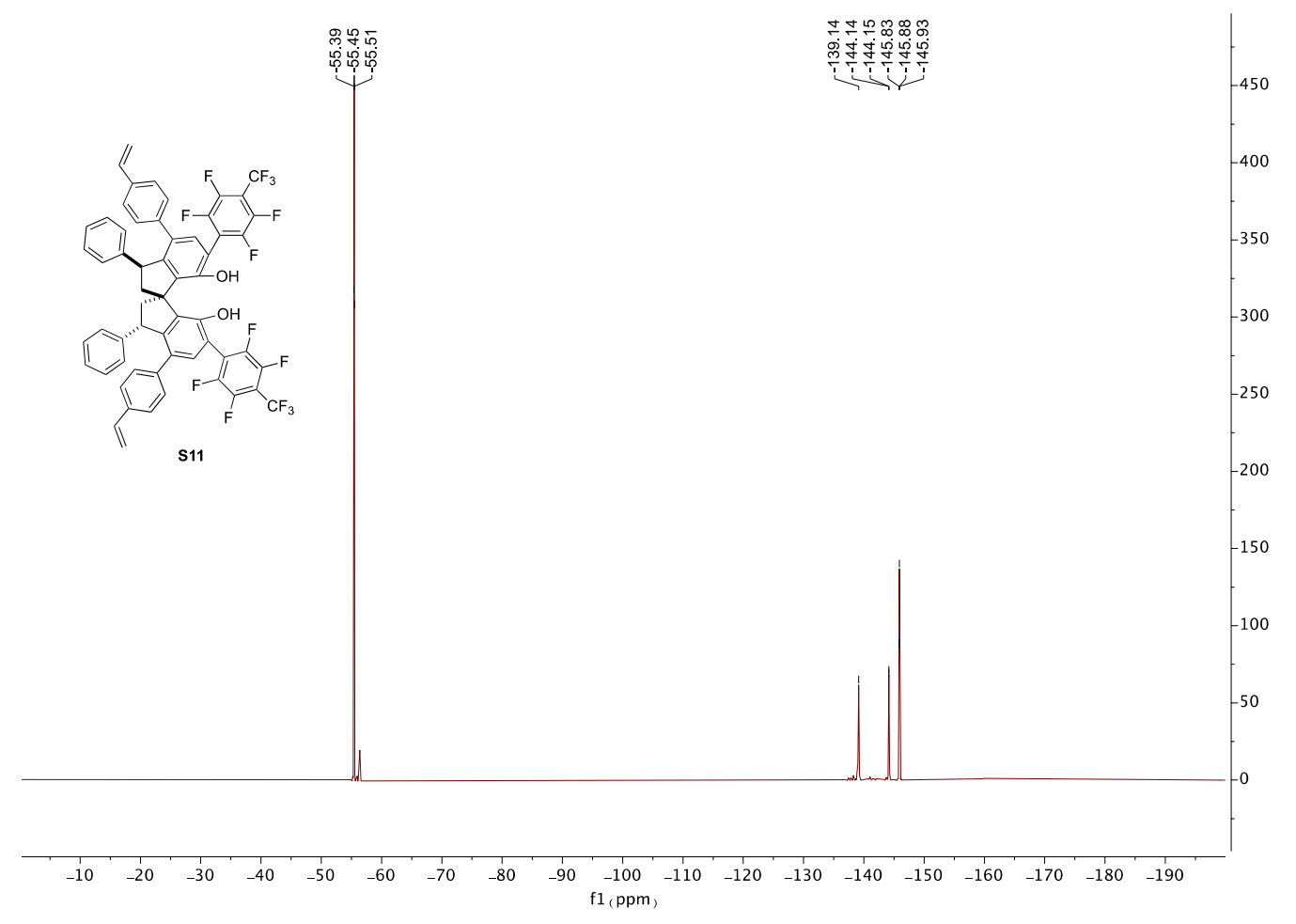




\section{${ }^{1} \mathrm{H}$ NMR of S11}

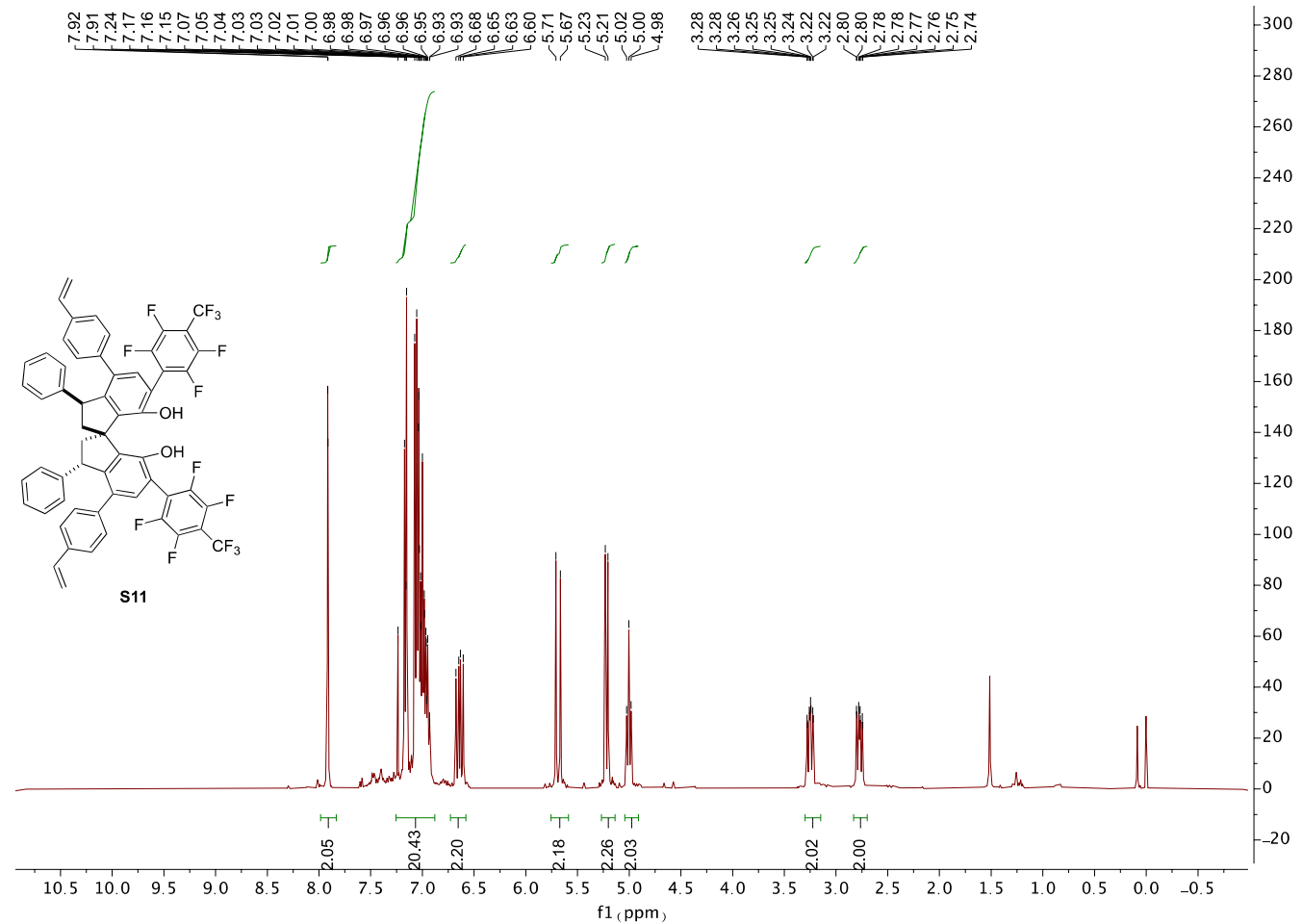

${ }^{13} \mathrm{C}$ NMR of S11

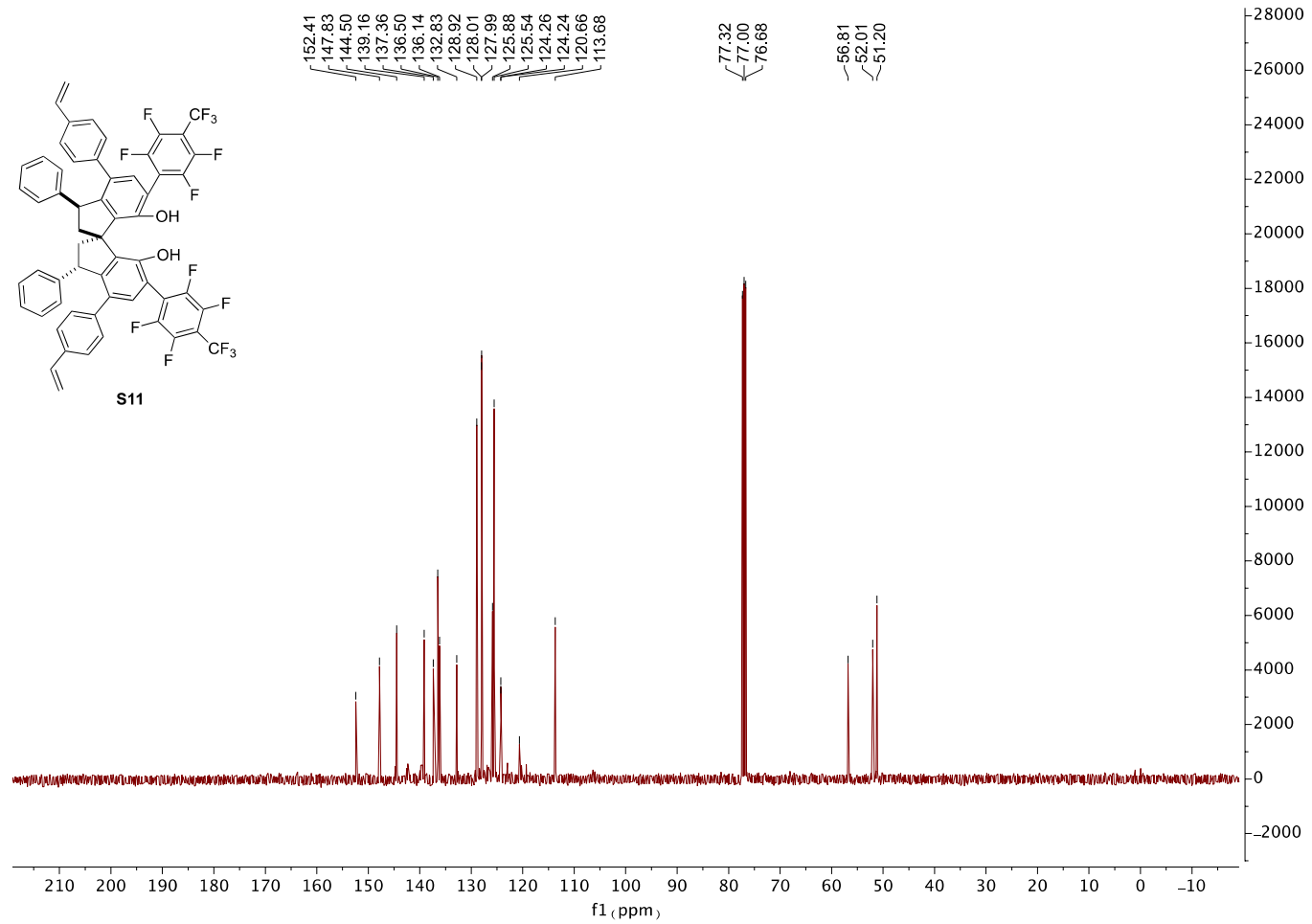




\section{${ }^{1} \mathrm{H}$ NMR of 17}

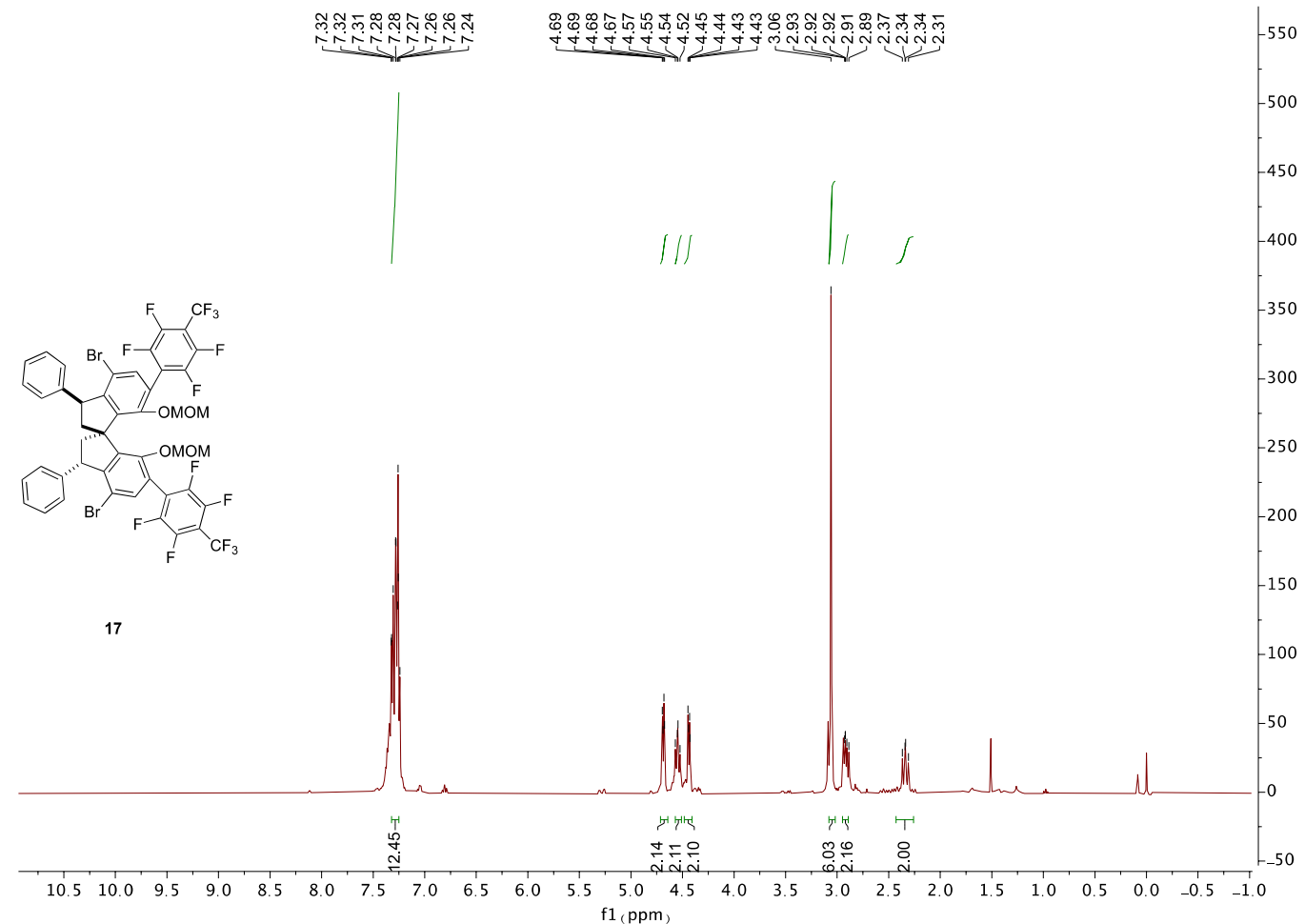

\section{${ }^{13} \mathrm{C}$ NMR of 17}

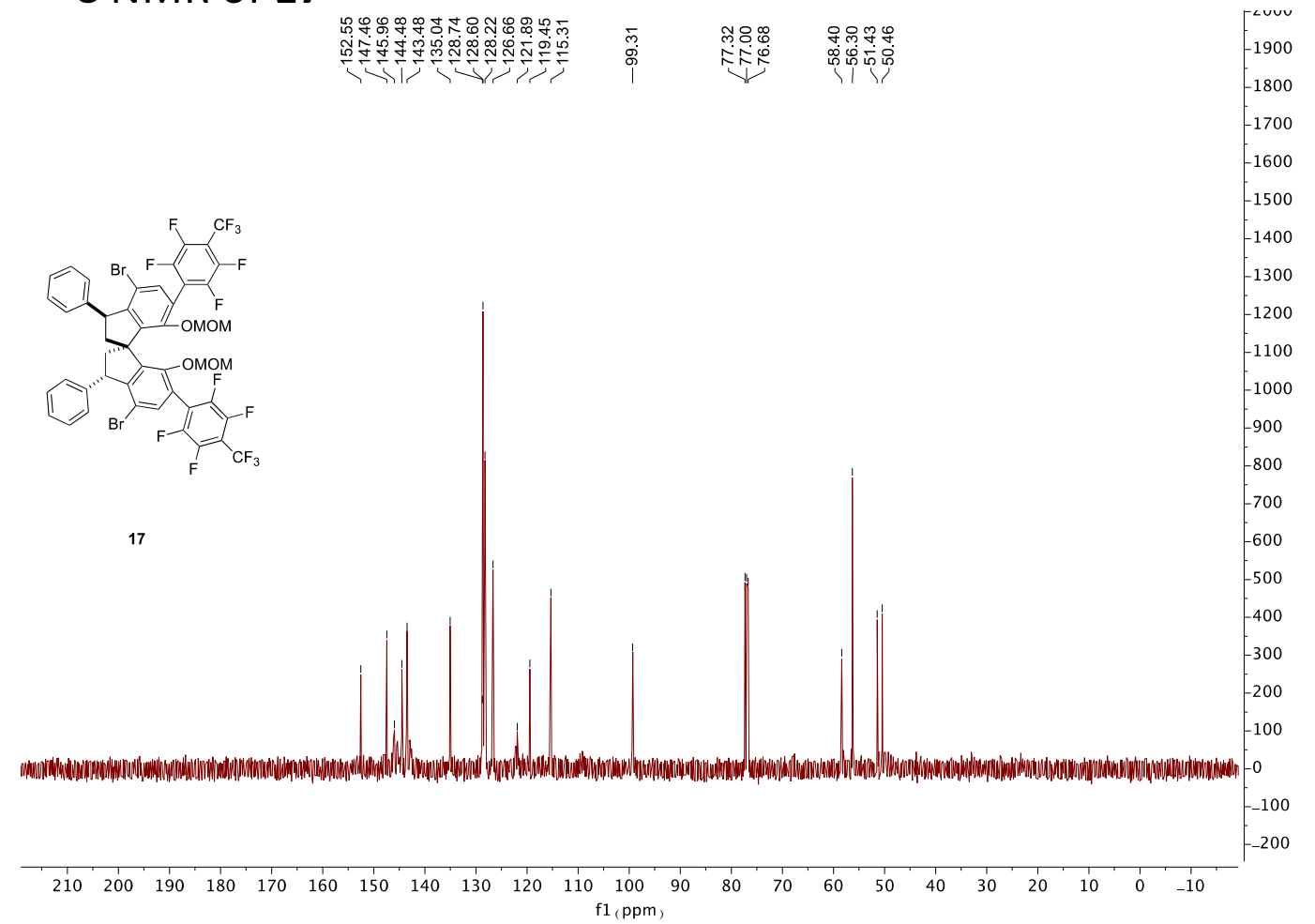




\section{${ }^{19} \mathrm{~F}$ NMR of 17}

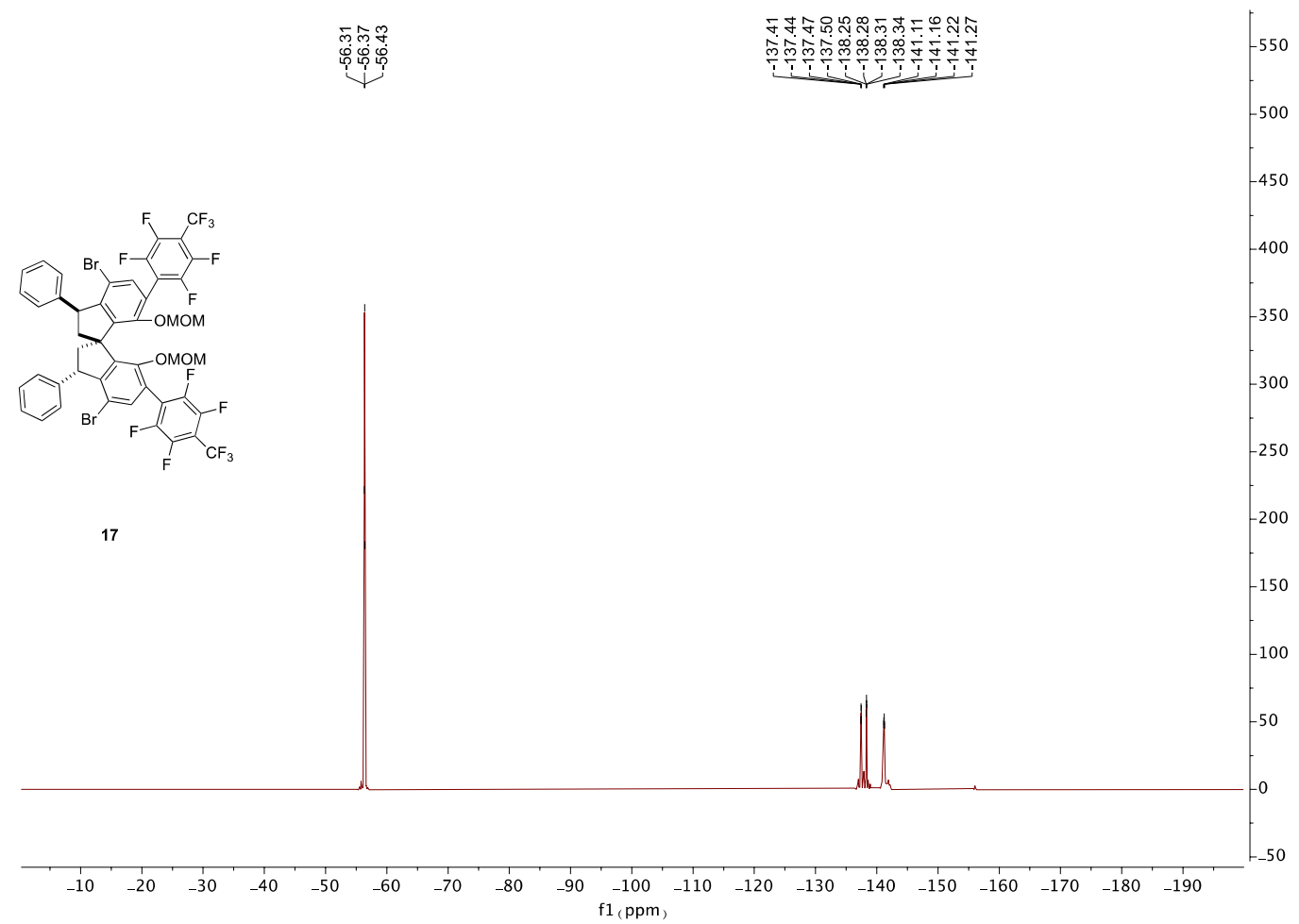

\section{${ }^{19} \mathrm{~F}$ NMR of 18}

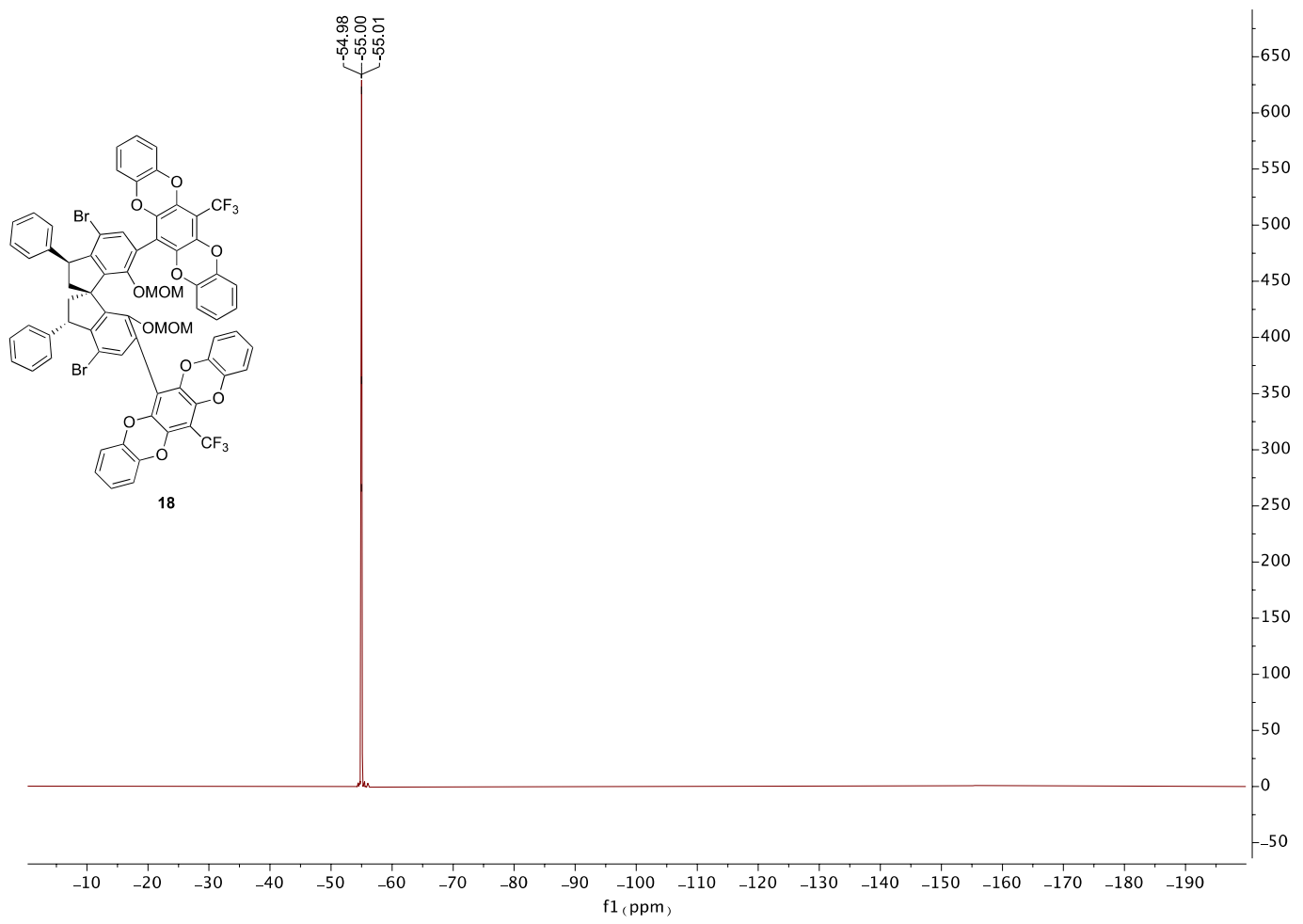




\section{${ }^{1} \mathrm{H}$ NMR of 18}

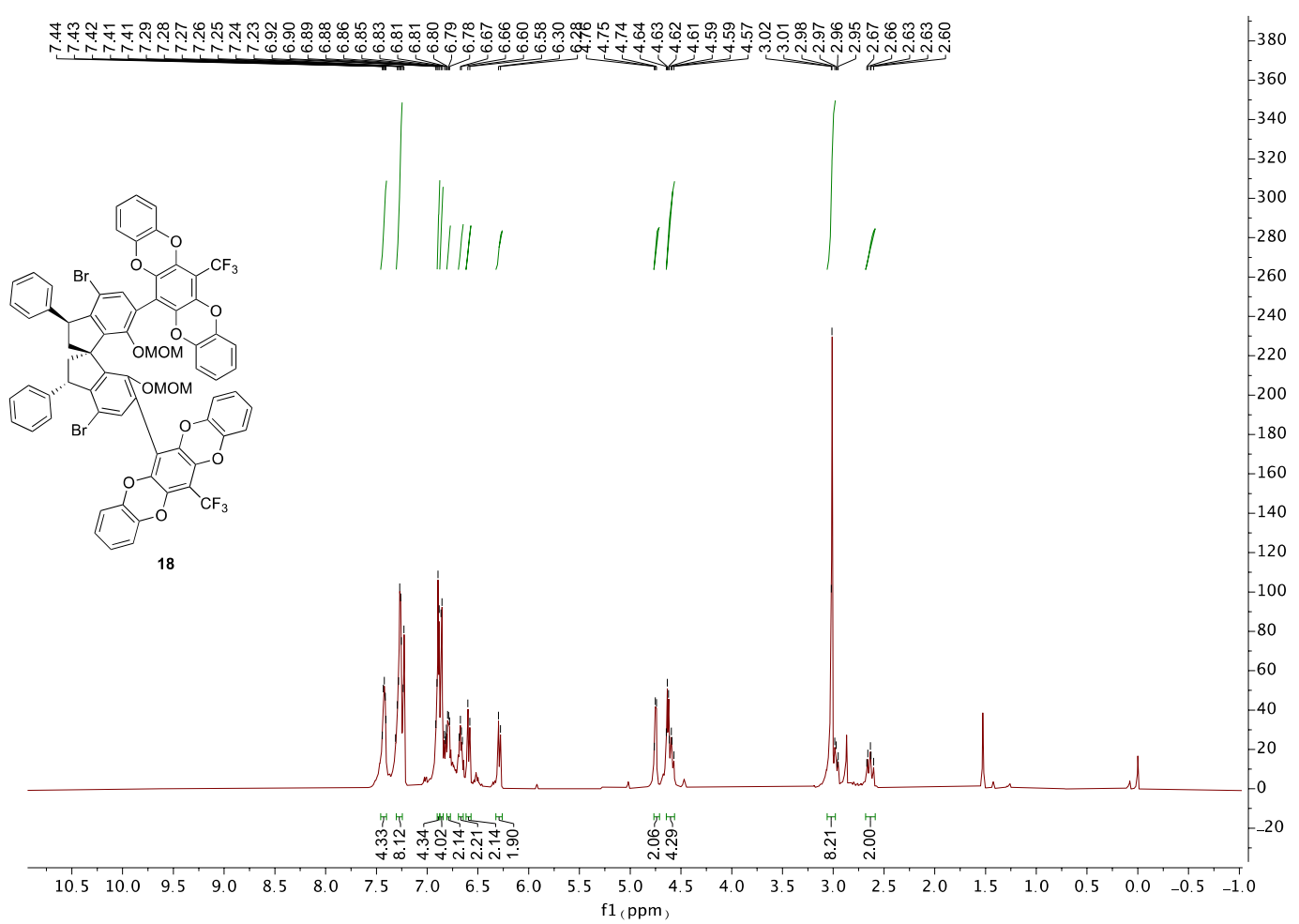

\section{${ }^{13} \mathrm{C}$ NMR of 18}

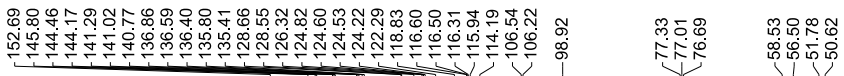

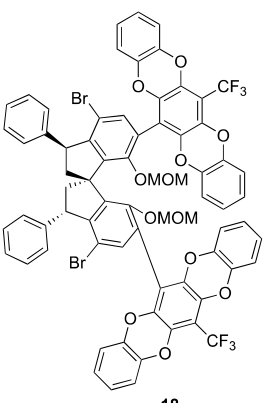

18

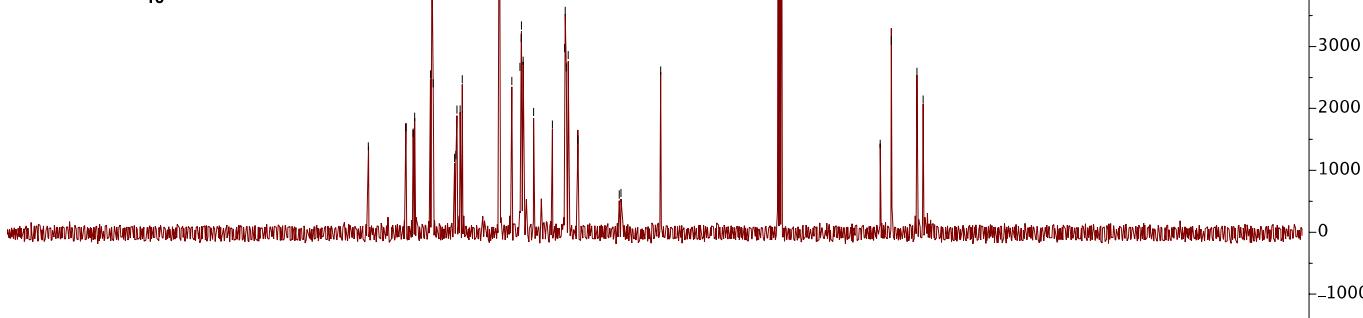

$\begin{array}{lllllllllllllllllllllllll}210 & 200 & 190 & 180 & 170 & 160 & 150 & 140 & 130 & 120 & 110 & 100 & 90 & 80 & 70 & 60 & 50 & 40 & 30 & 20 & 10 & 0 & -10 \\ \mathrm{f} 1(\mathrm{ppm}) & & \end{array}$ 


\section{${ }^{1} \mathrm{H}$ NMR of 19}

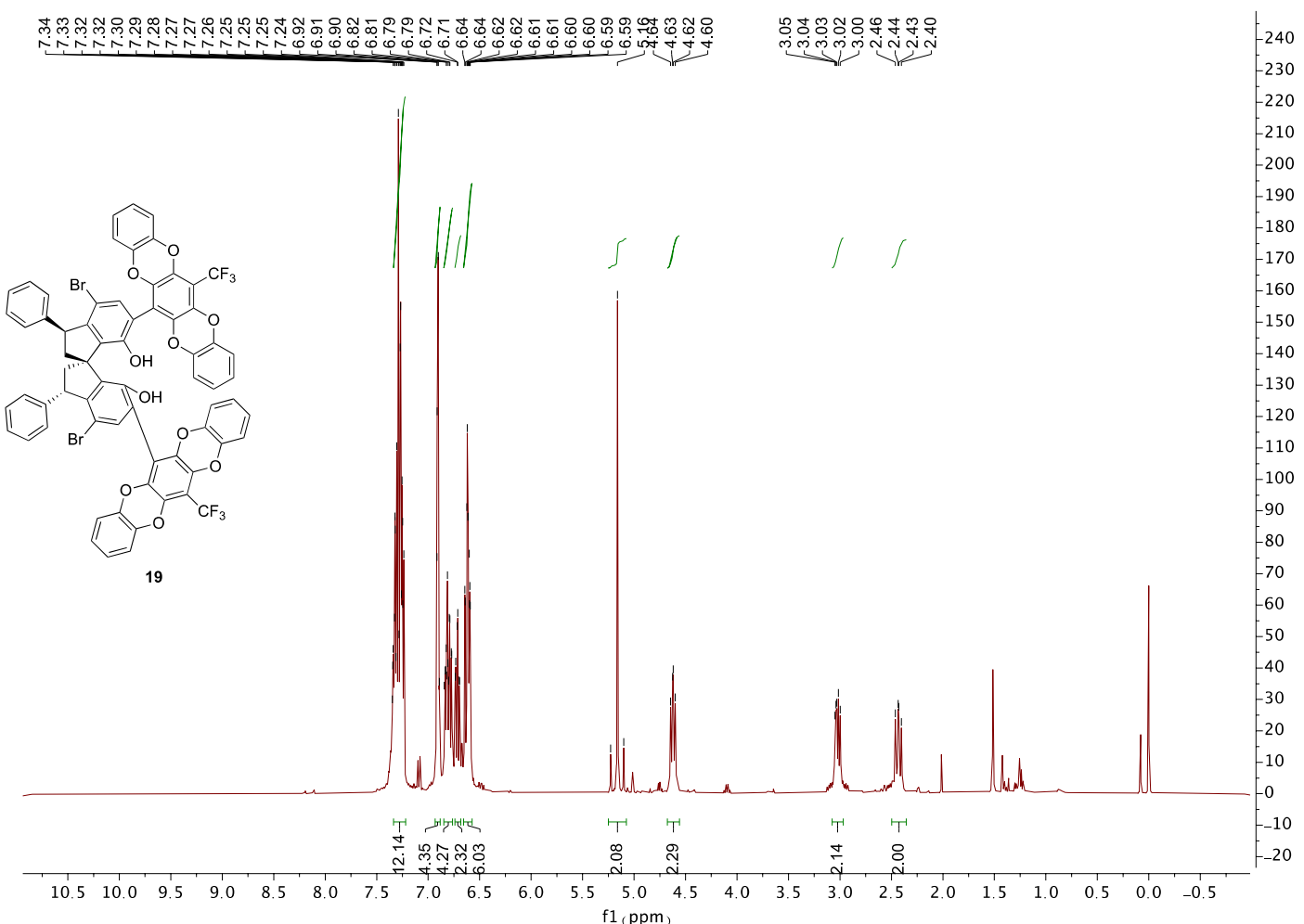

\section{${ }^{13} \mathrm{C}$ NMR of 19}

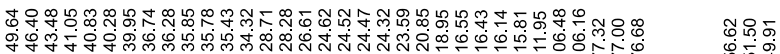

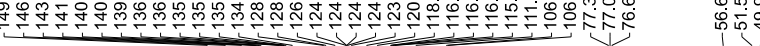
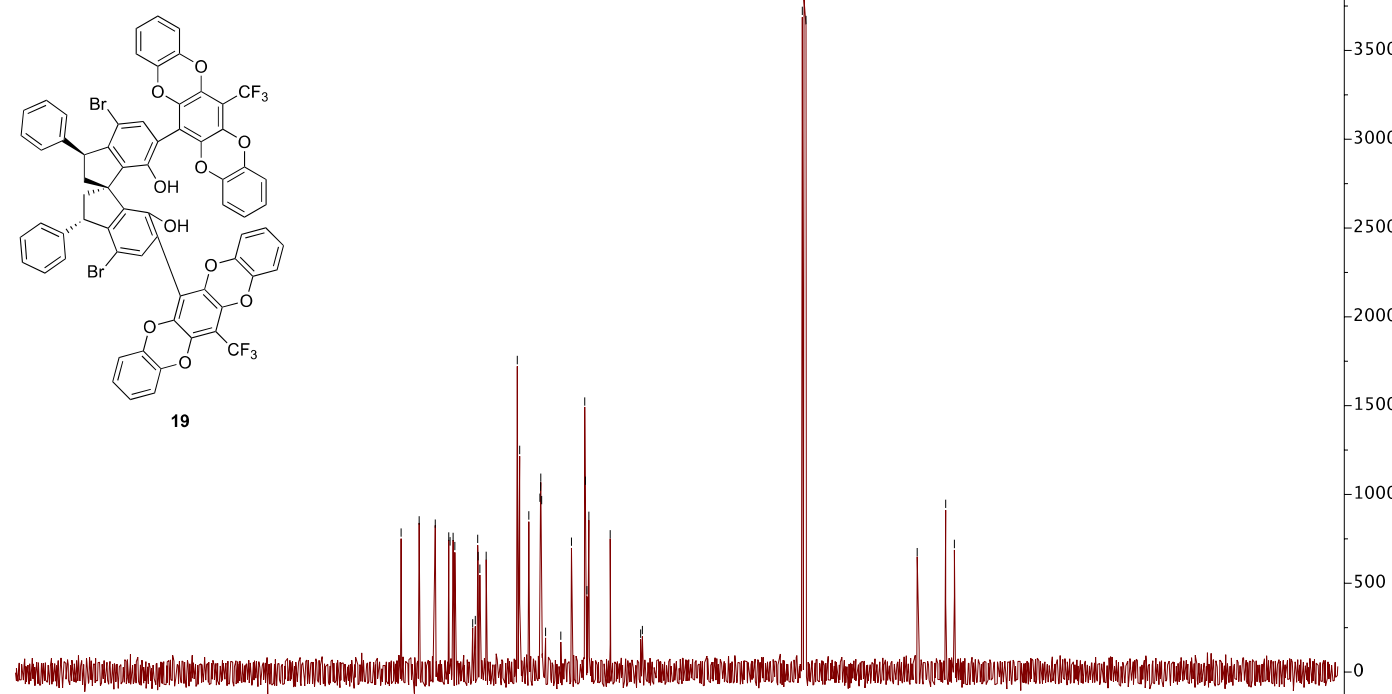
${ }^{19} \mathrm{~F}$ NMR of 19

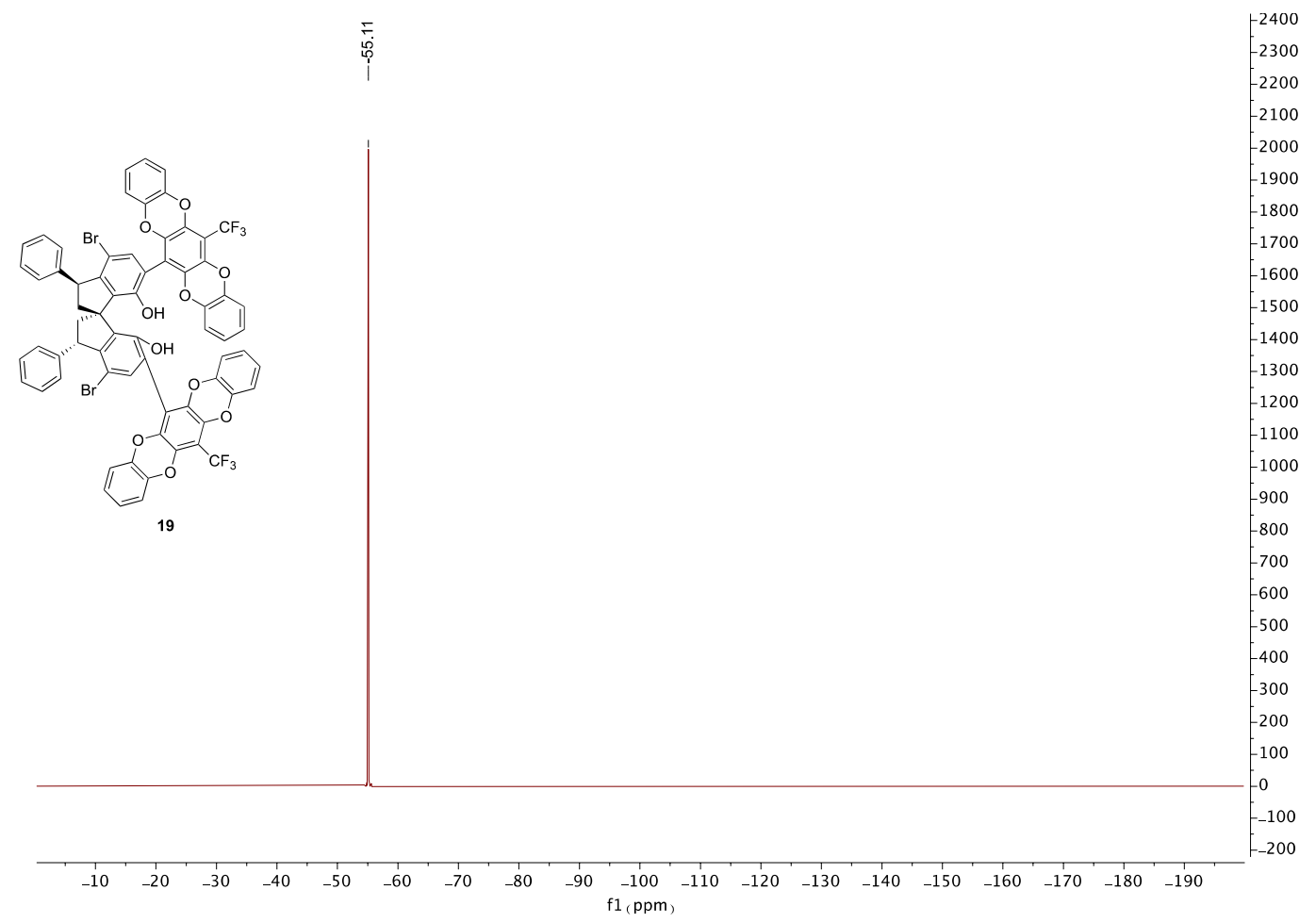

${ }^{19} \mathrm{~F}$ NMR of 20

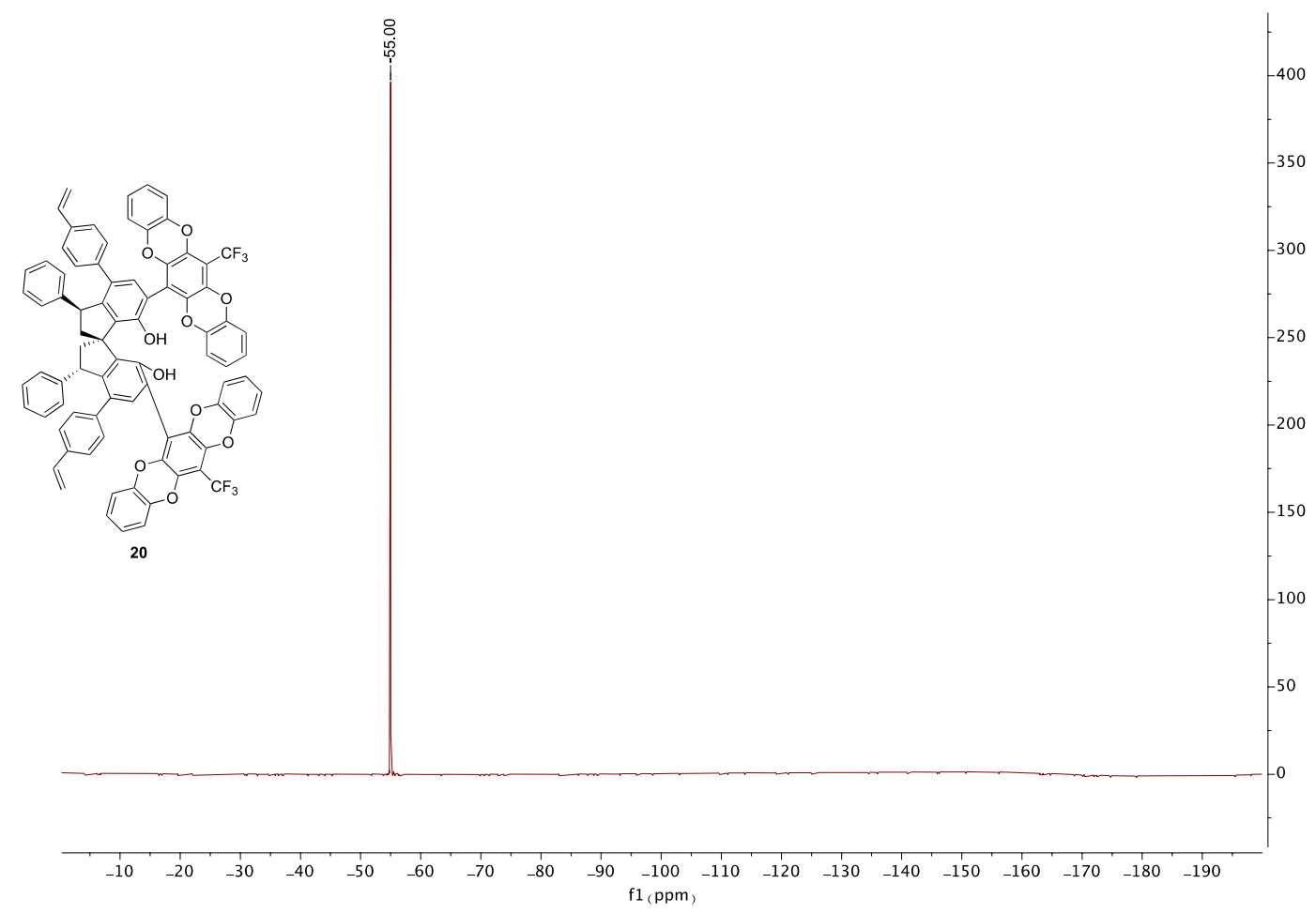




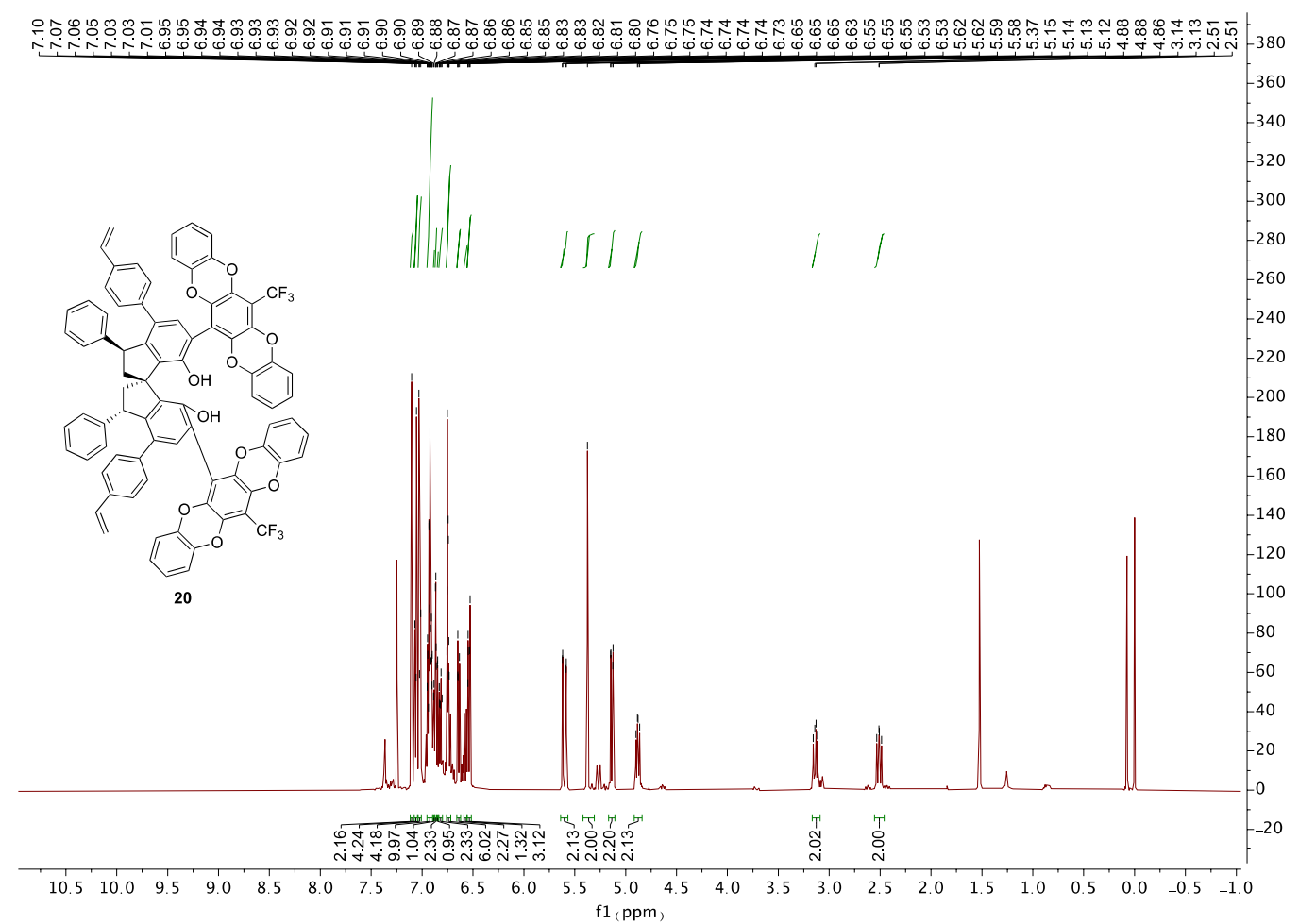

\section{${ }^{13} \mathrm{C}$ NMR of 20}

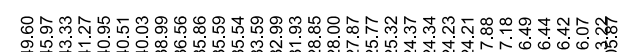

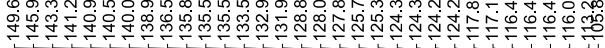

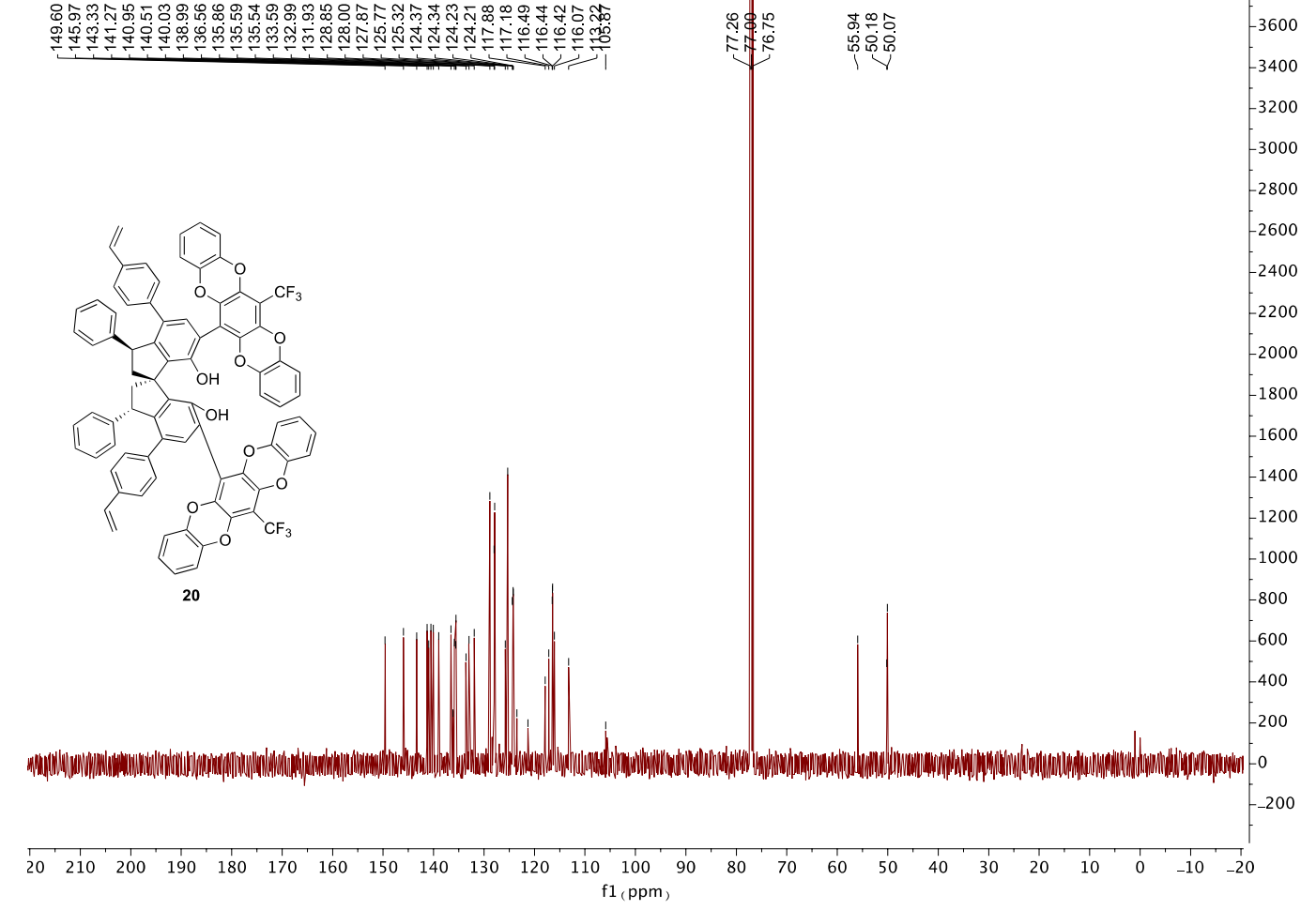

$\mathrm{f} 1$ ( $\mathrm{ppm}$ 


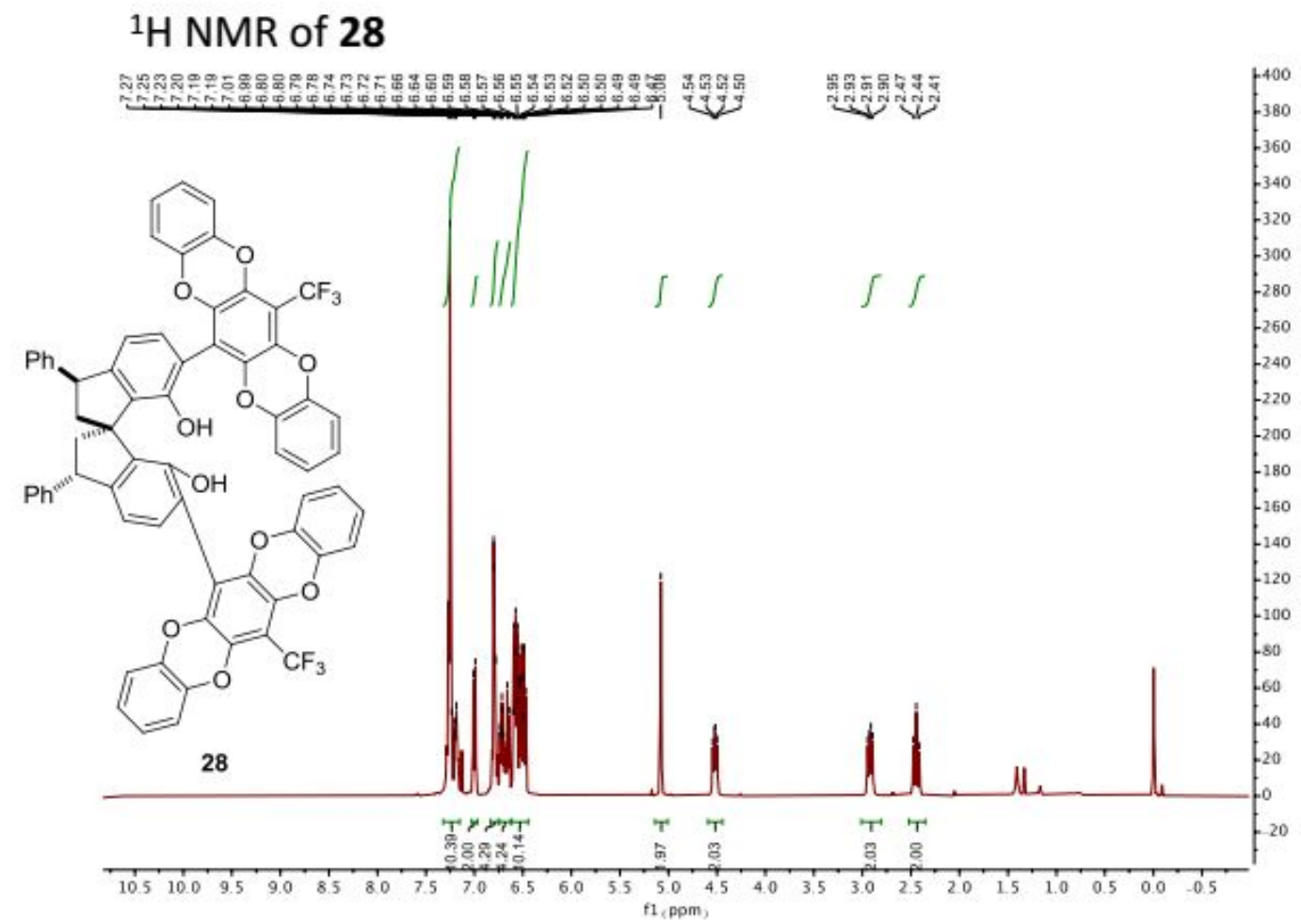

\section{${ }^{13} \mathrm{C}$ NMR of 28}

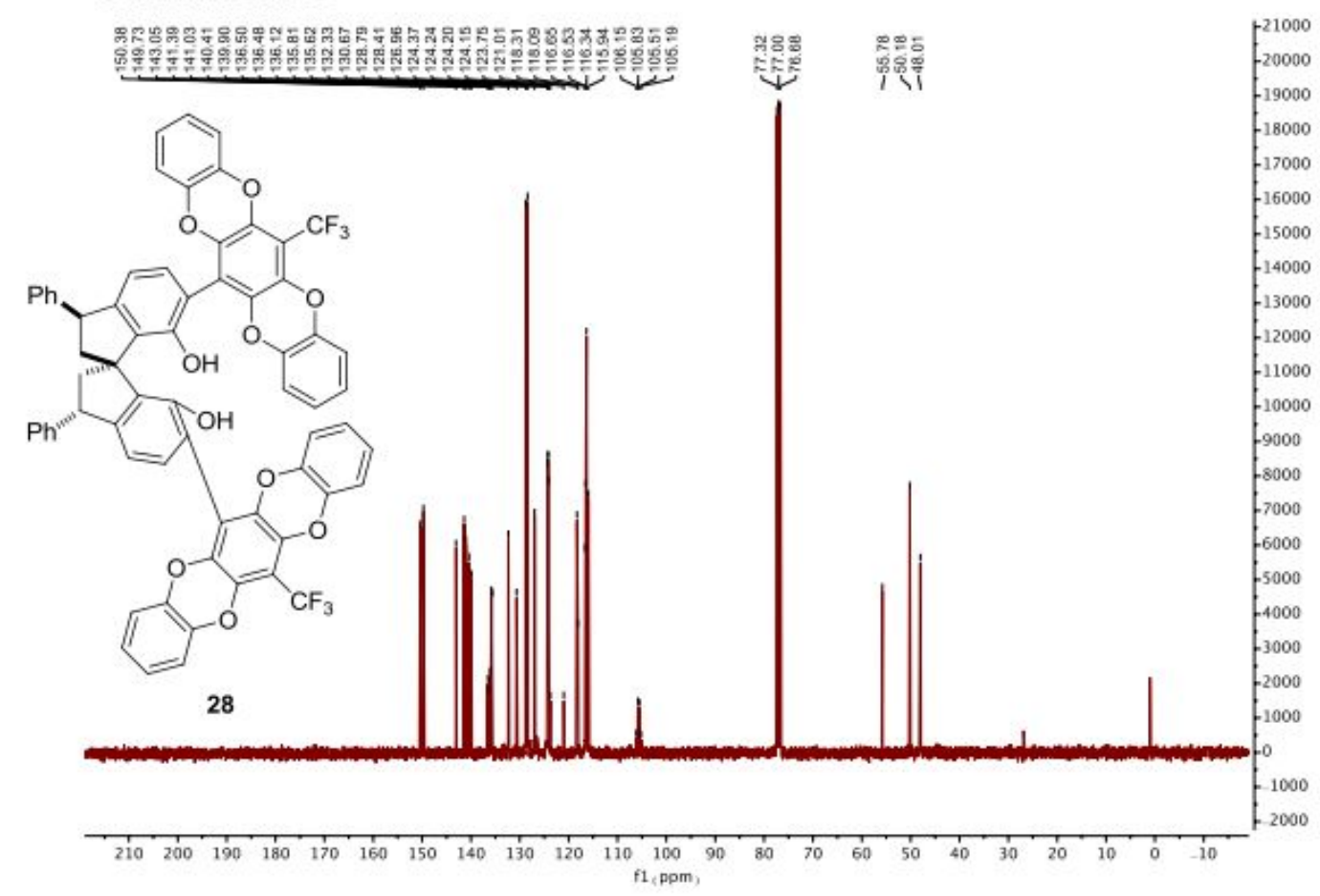


${ }^{19} \mathrm{~F}$ NMR of 28

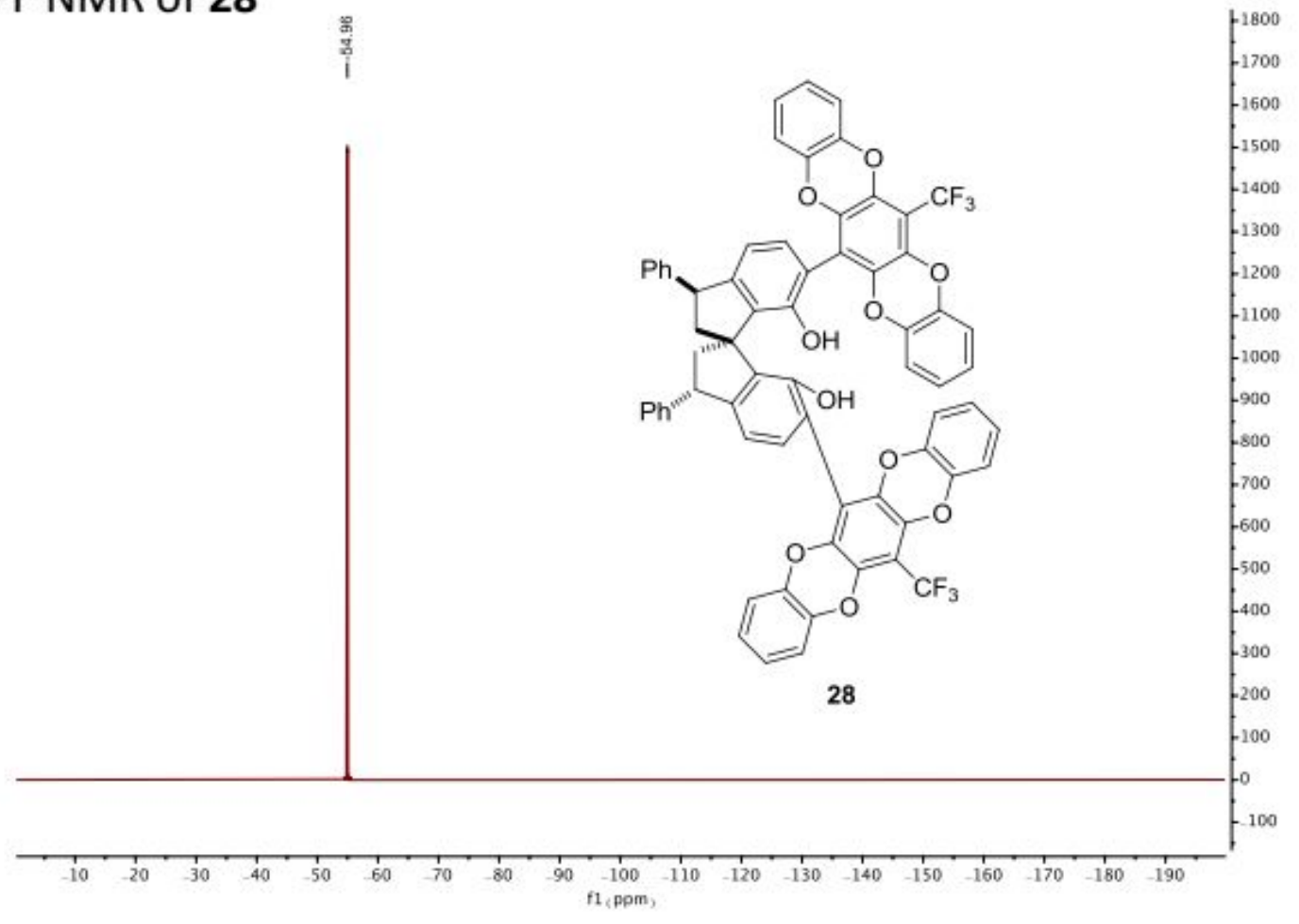




\section{${ }^{1} \mathrm{H}$ NMR of Cat $\mathbf{g}$}

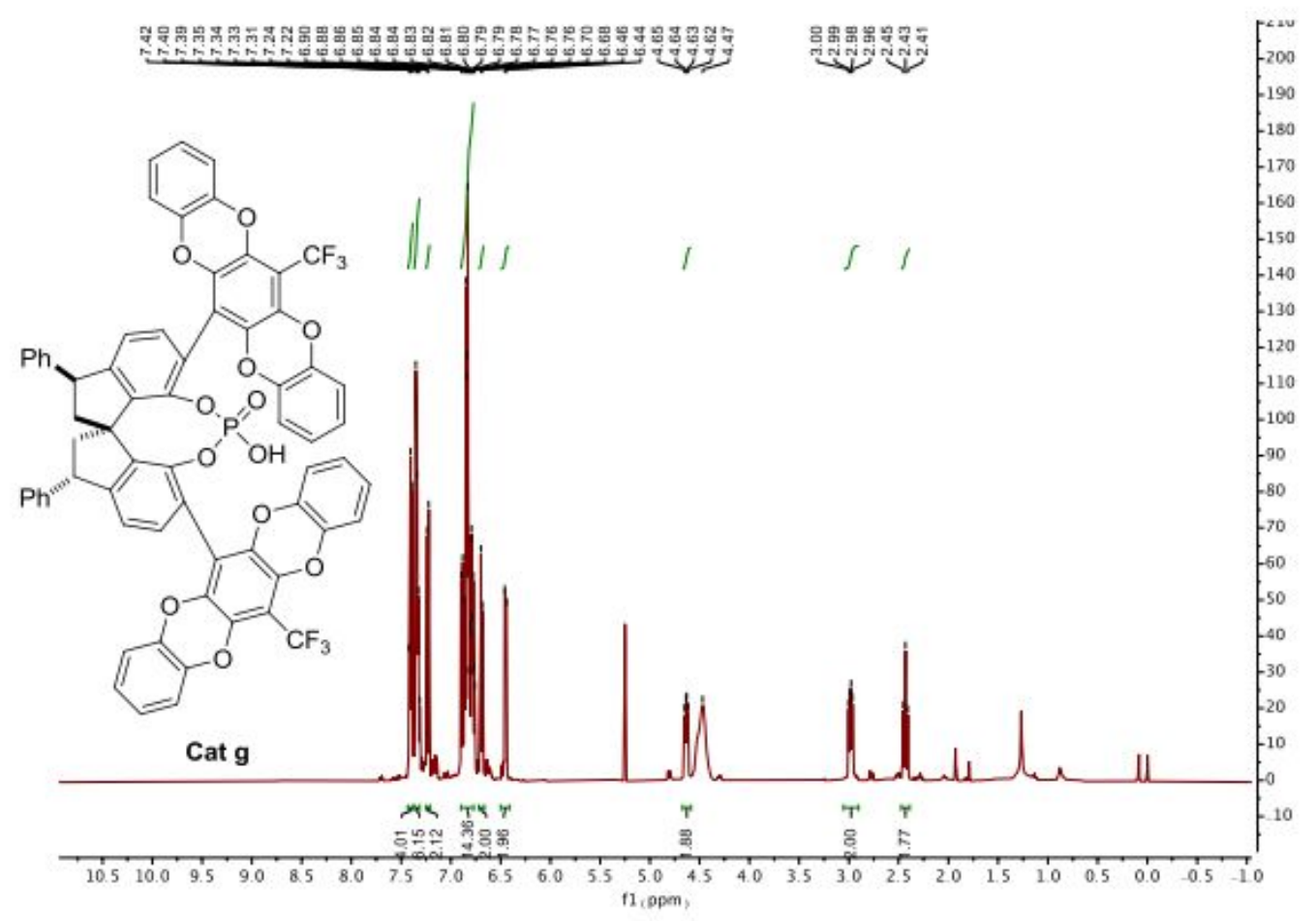

\section{${ }^{13} \mathrm{C}$ NMR of Cat $\mathbf{g}$}

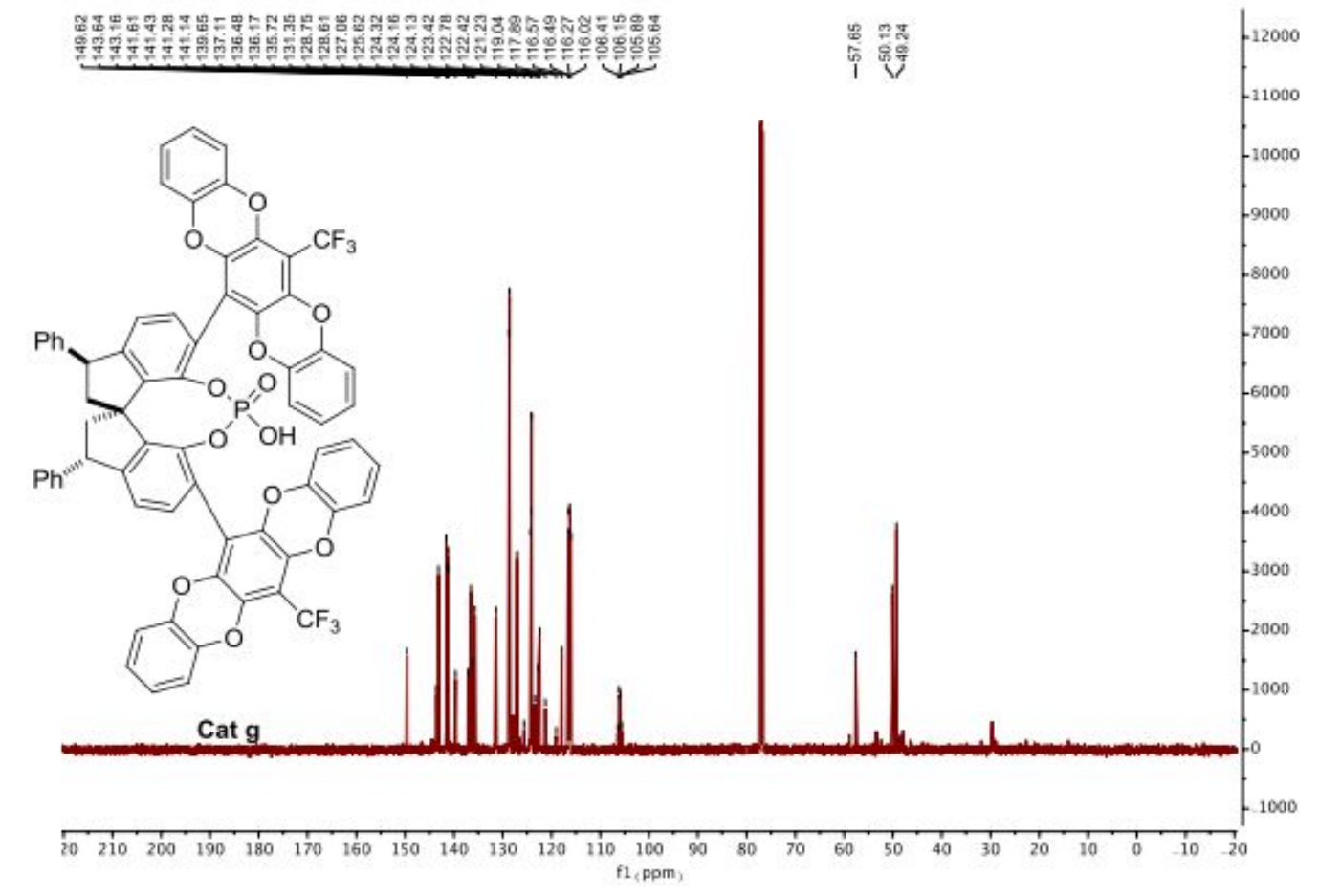




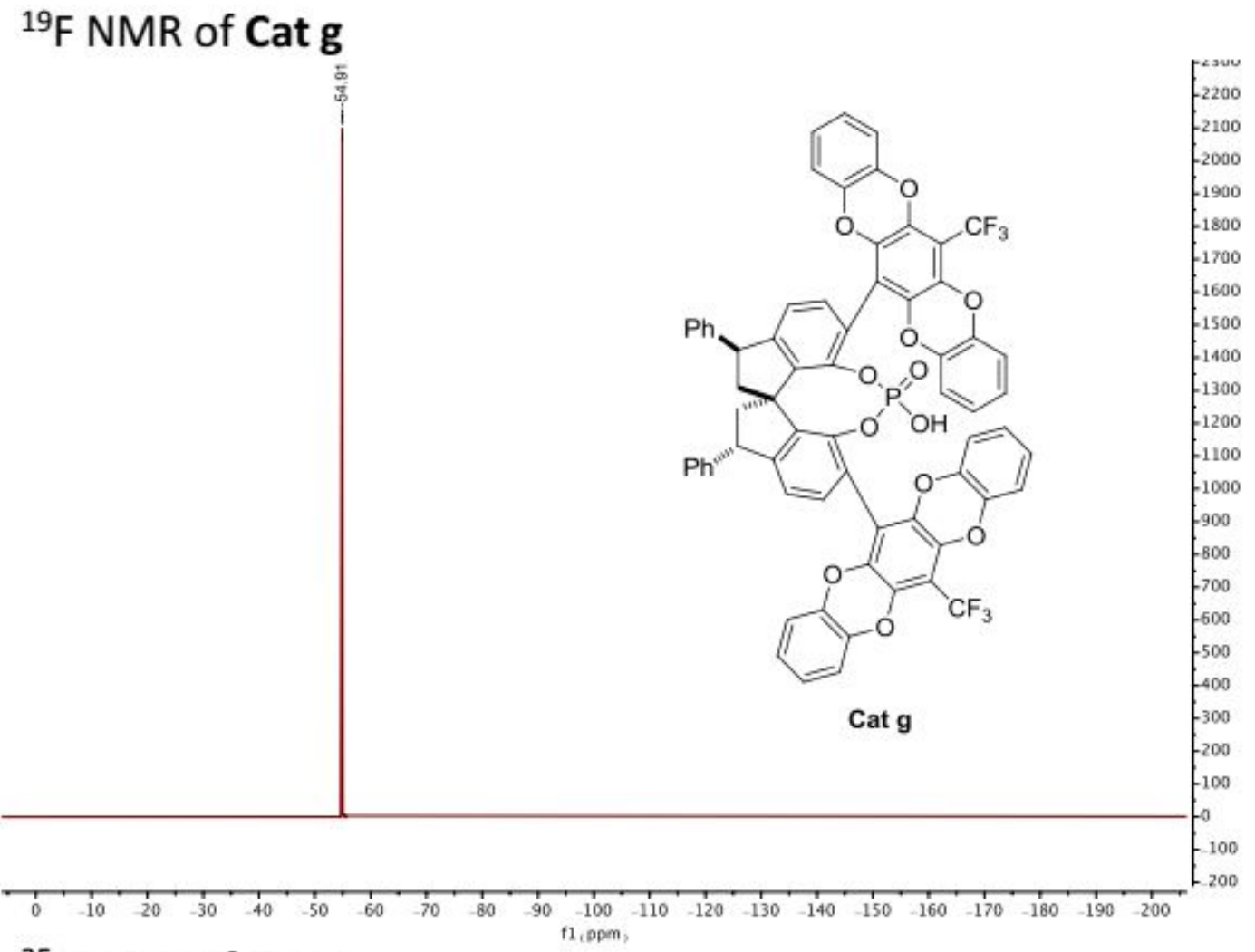

\section{${ }^{35} \mathrm{P}$ NMR of Cat $\mathbf{g}$}

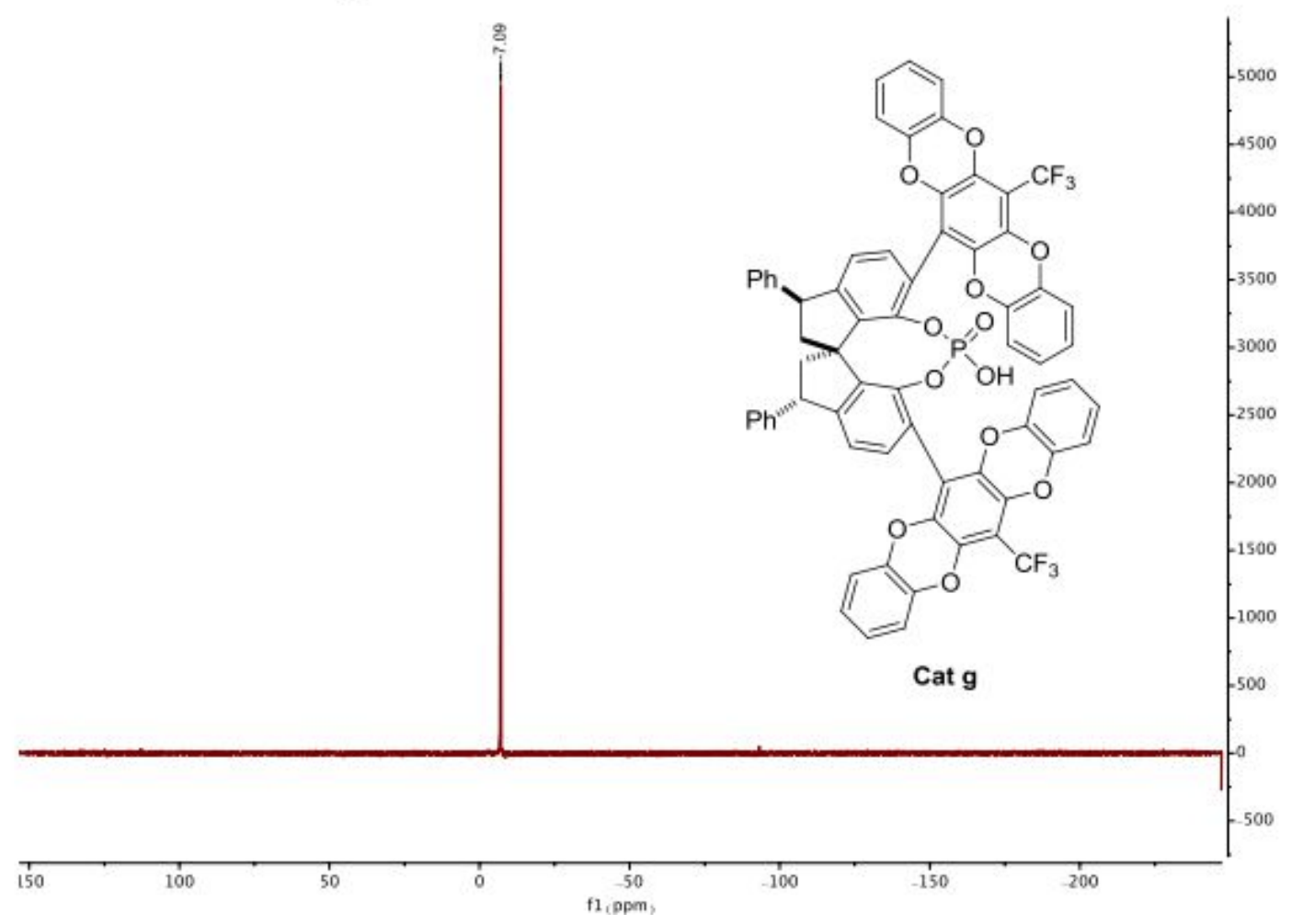




\section{${ }^{1} \mathrm{H}$ NMR of 24aa}

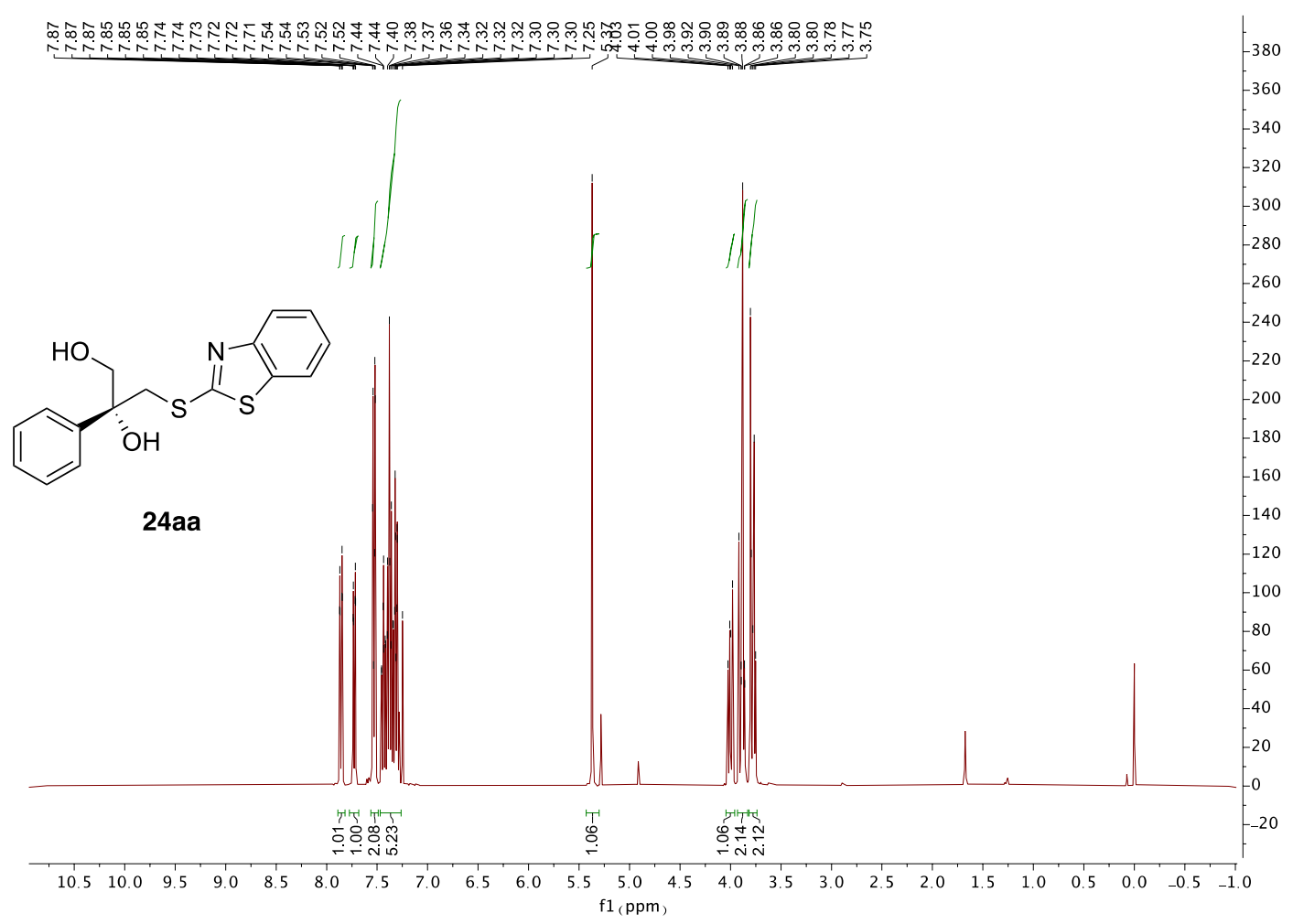

${ }^{13} \mathrm{C}$ NMR of 24aa

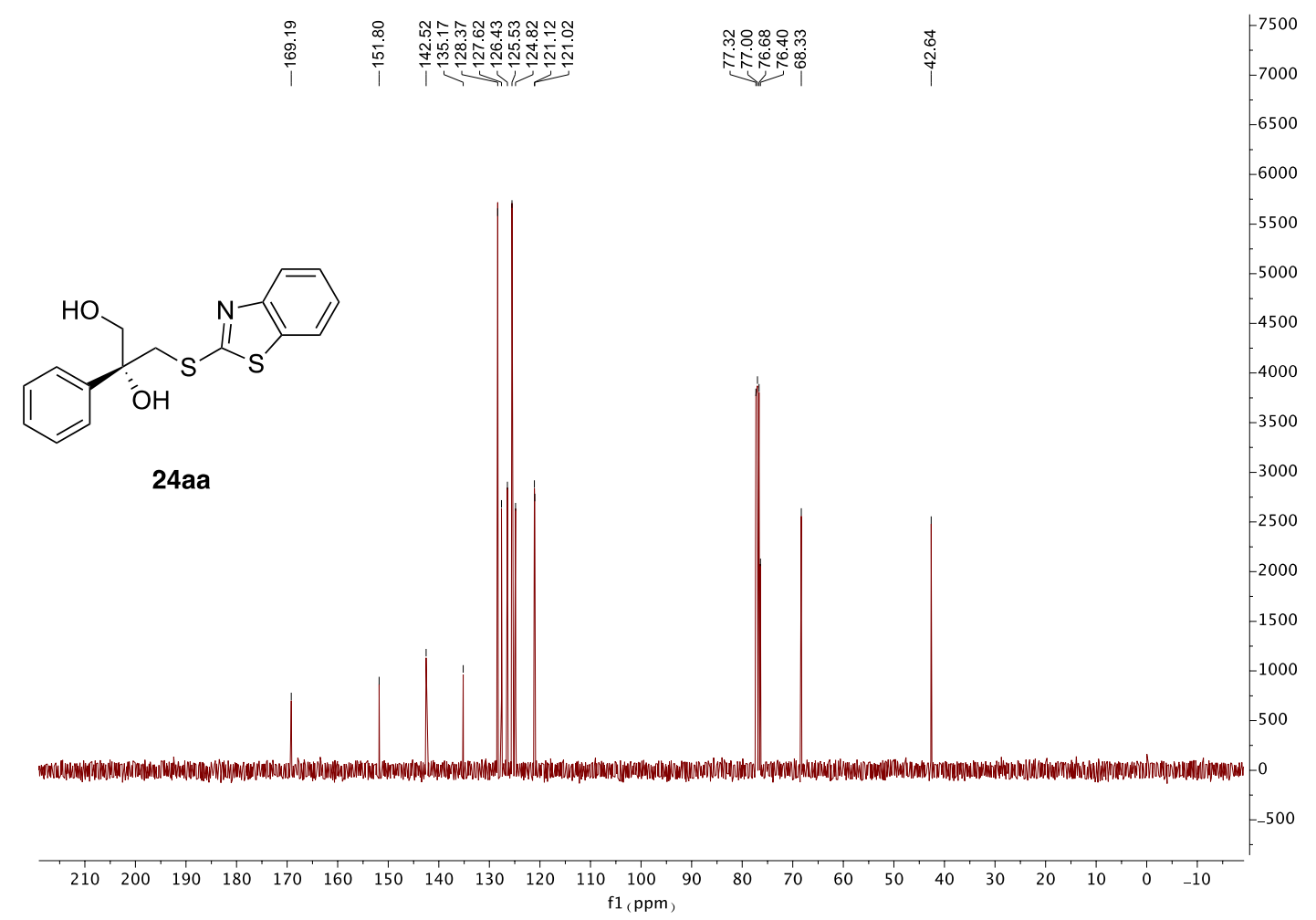




\section{${ }^{1} \mathrm{H}$ NMR of $24 \mathrm{ba}$}

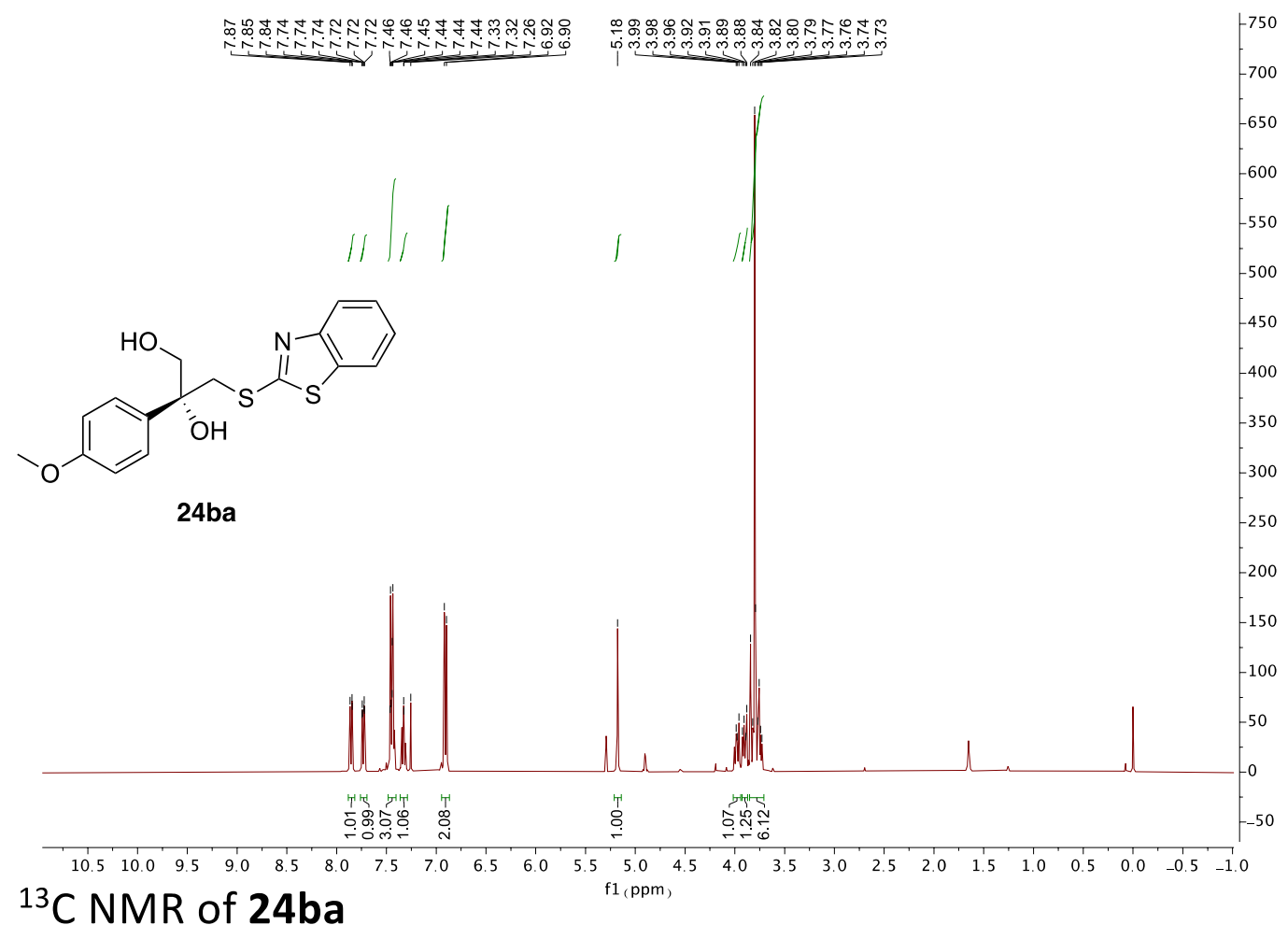

${ }^{13} \mathrm{C}$ NMR of $24 \mathrm{ba}$

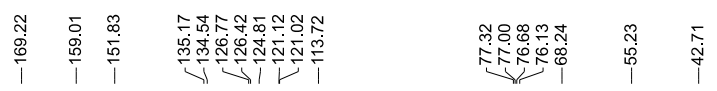

$-40000$

35000

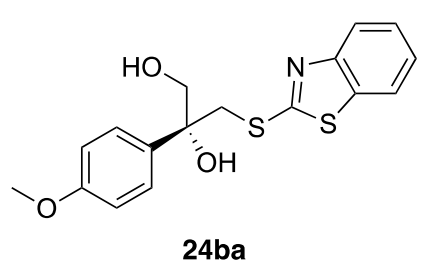

30000

5000

20000

15000

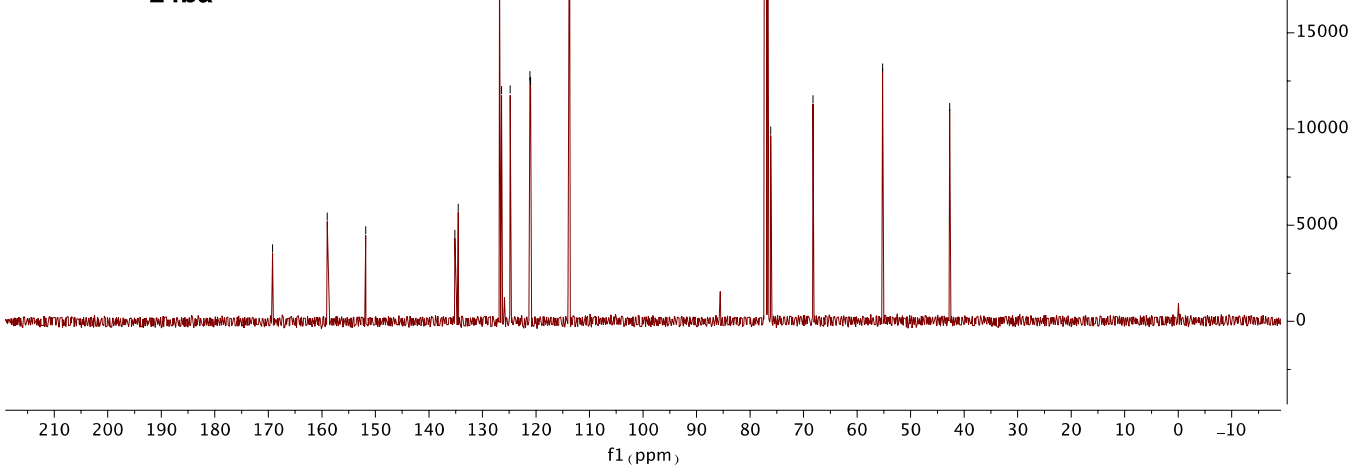




\section{${ }^{1} \mathrm{H}$ NMR of $\mathbf{2 4 c a}$}

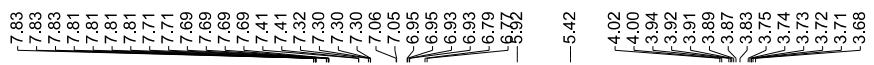

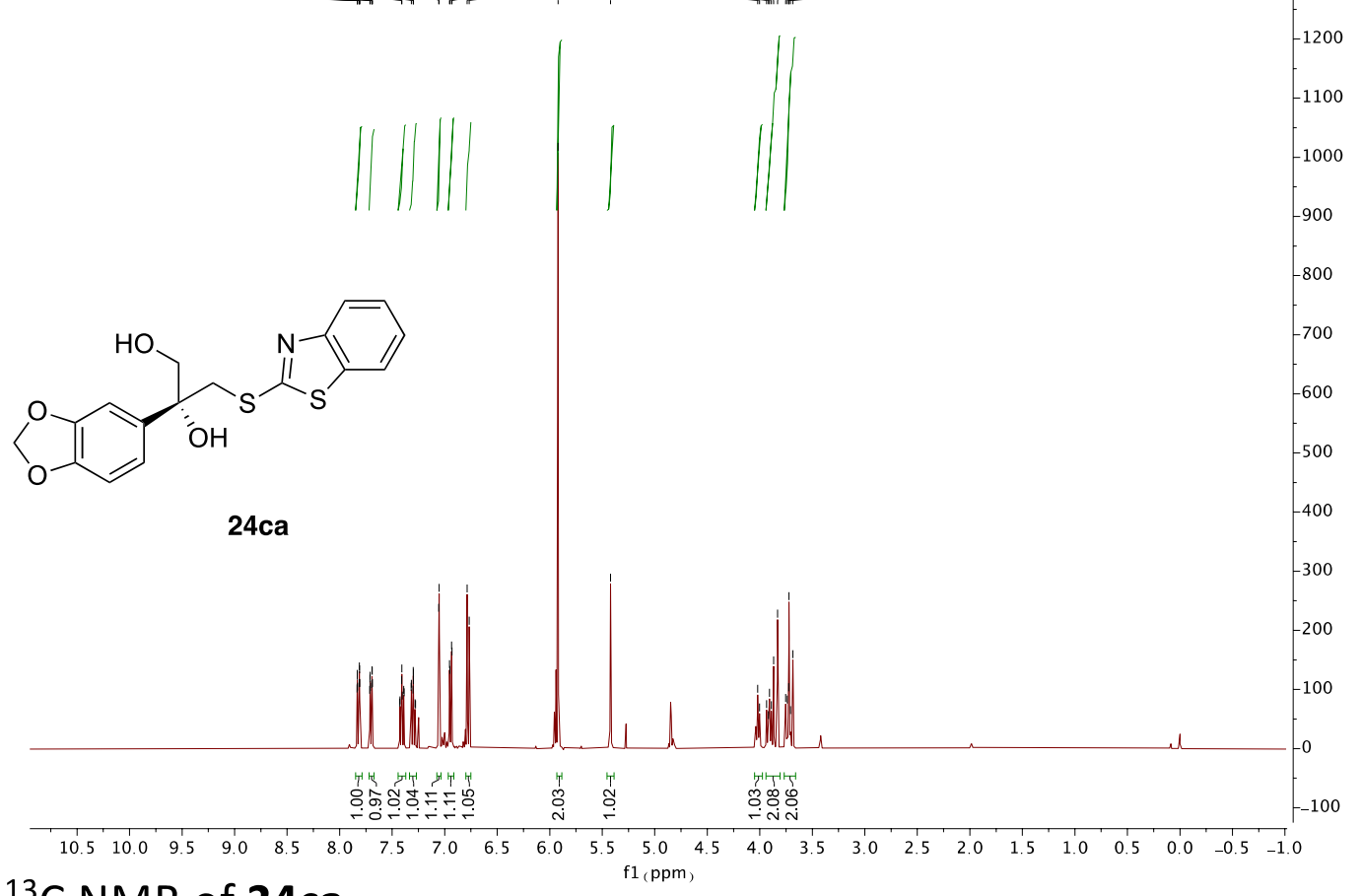

${ }^{13} \mathrm{C}$ NMR of $24 \mathrm{ca}$

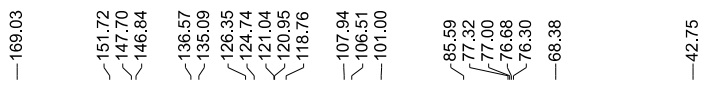

9000

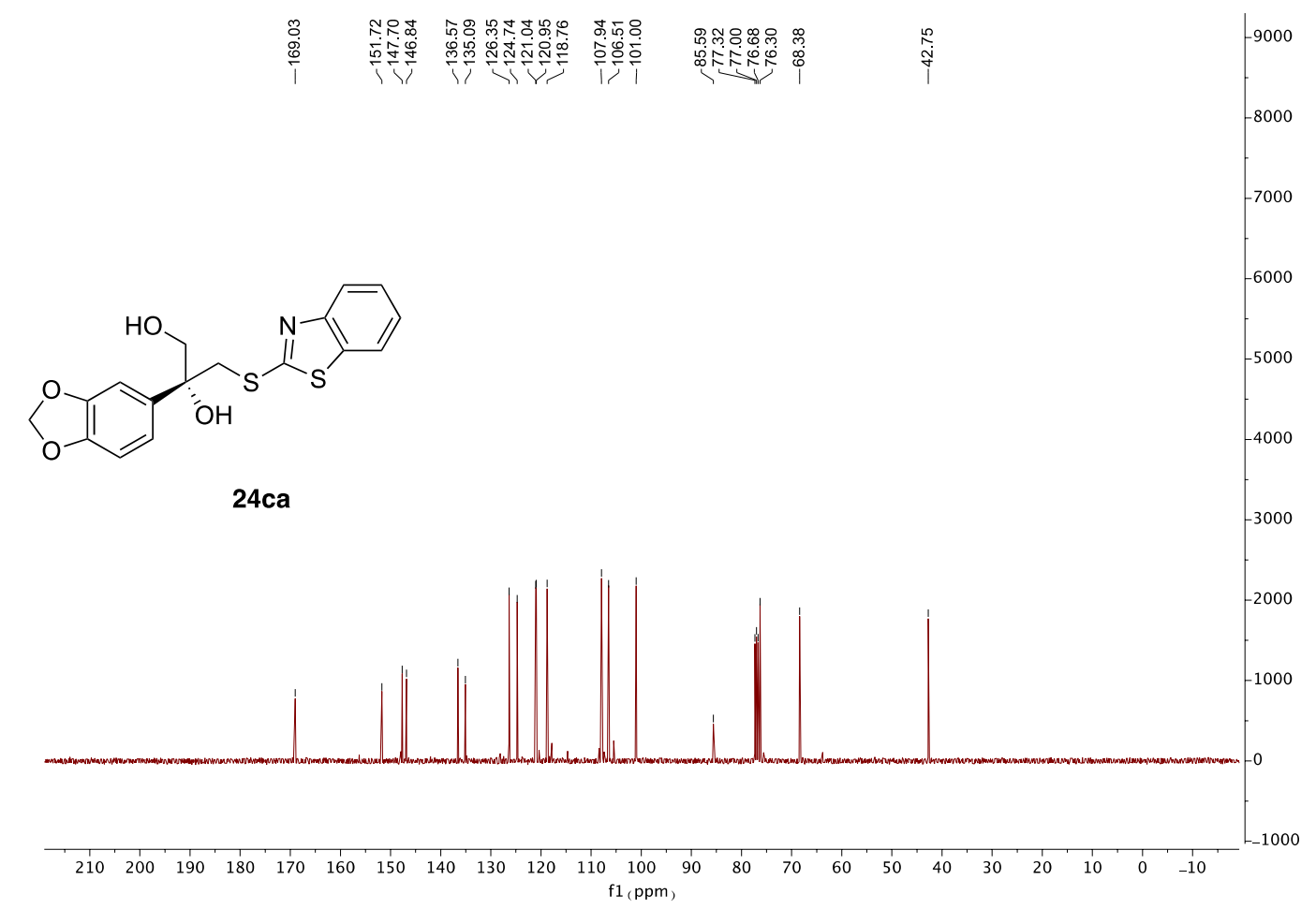




\section{${ }^{1}$ H NMR of 24da}

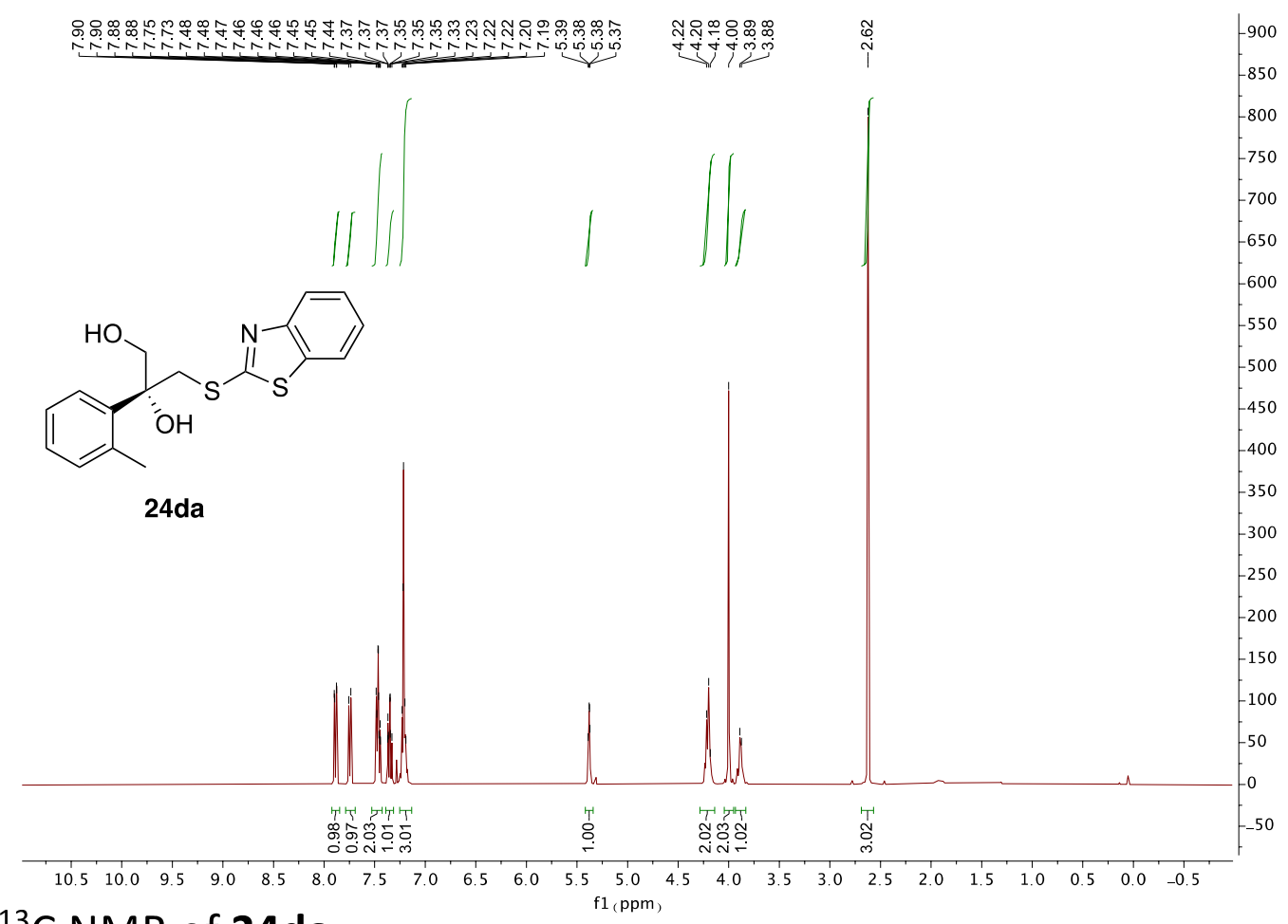

${ }^{13} \mathrm{C}$ NMR of $24 \mathrm{da}$

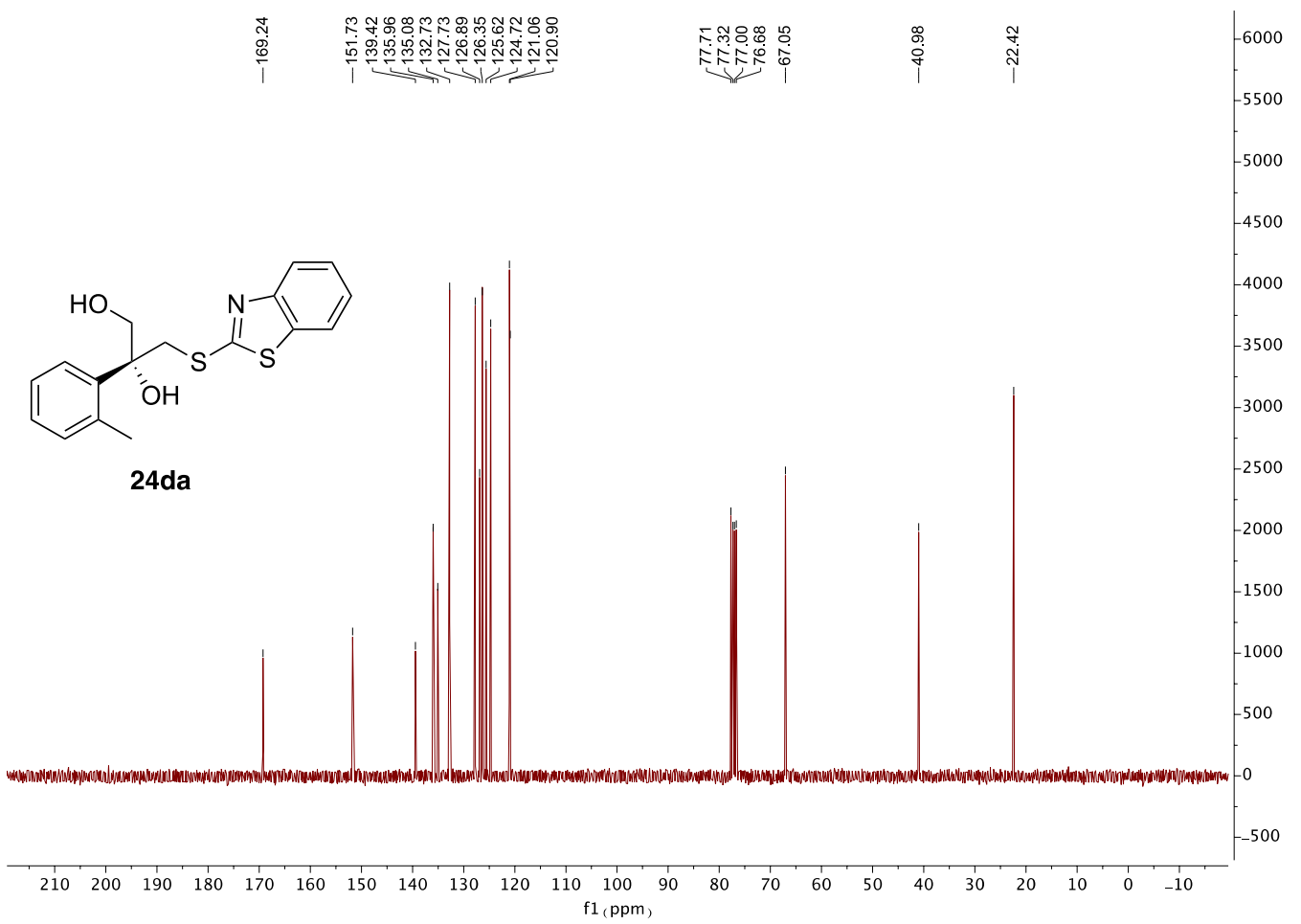




\section{${ }^{1} \mathrm{H}$ NMR of 24ea}

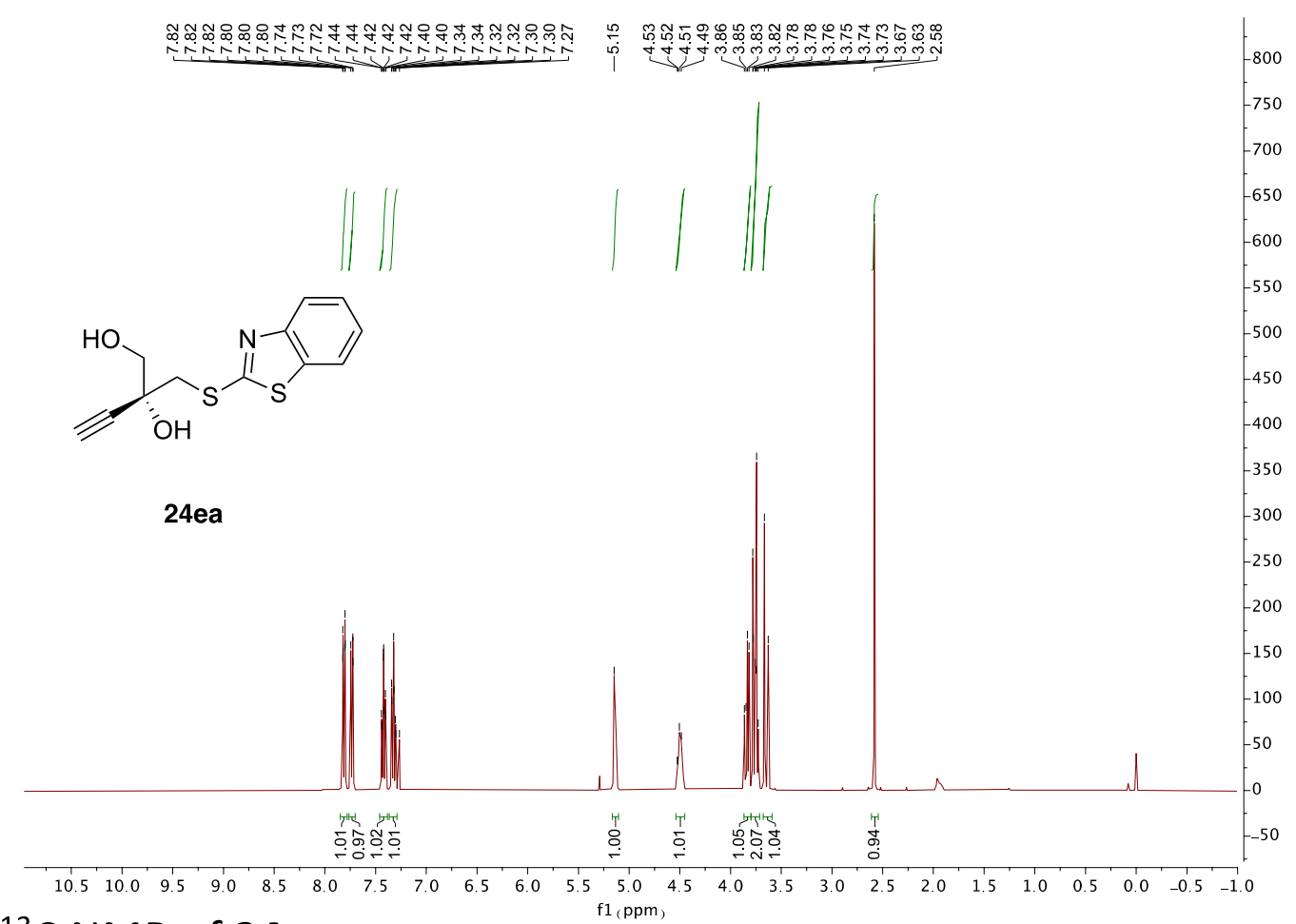

${ }^{13} \mathrm{C}$ NMR of 24 ea

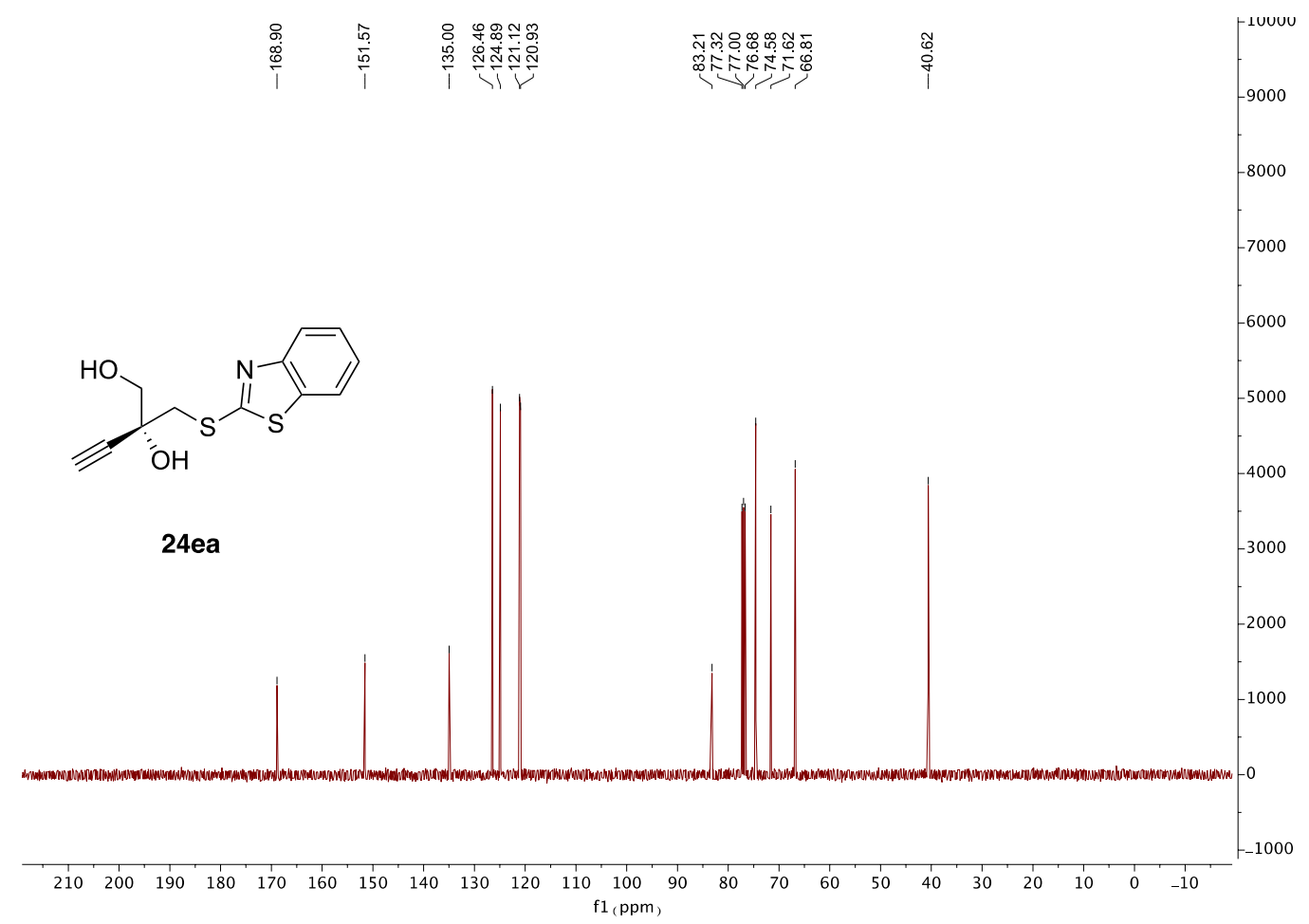




\section{${ }^{1} \mathrm{H}$ NMR of $\mathbf{2 4 f a}$}

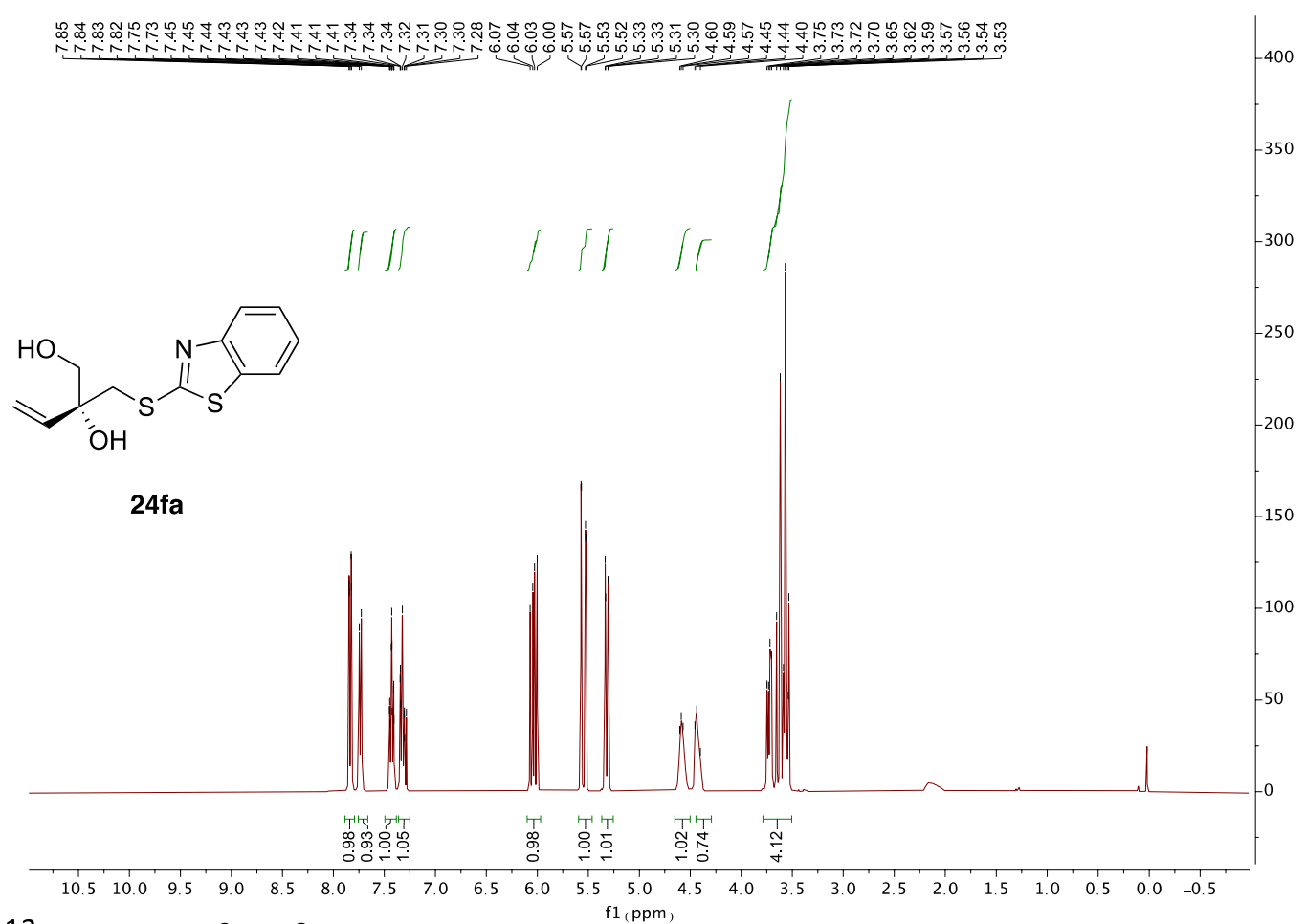

${ }^{13} \mathrm{C}$ NMR of $24 \mathrm{fa}$

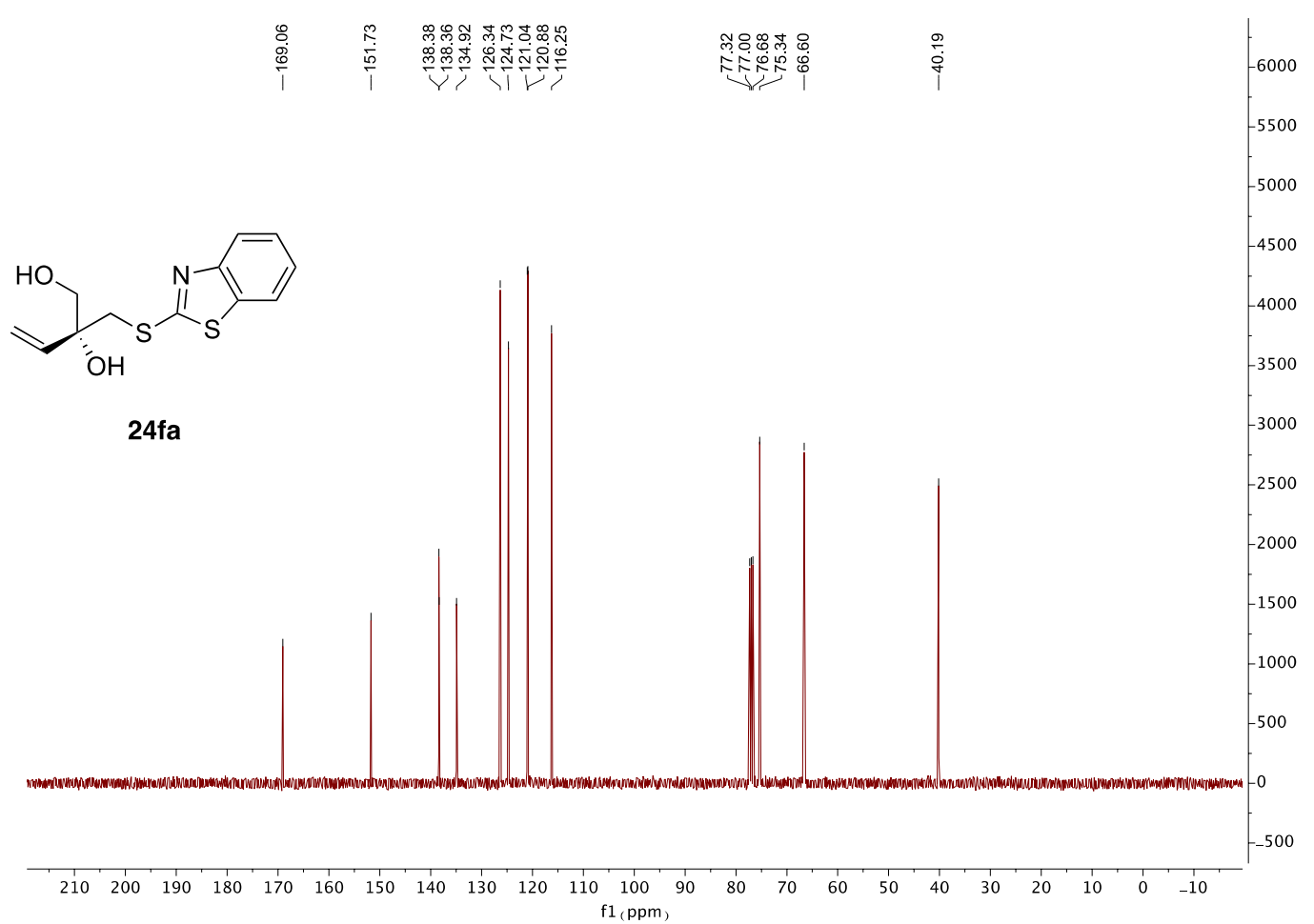




\section{${ }^{1} \mathrm{H}$ NMR of $24 \mathrm{ga}$}

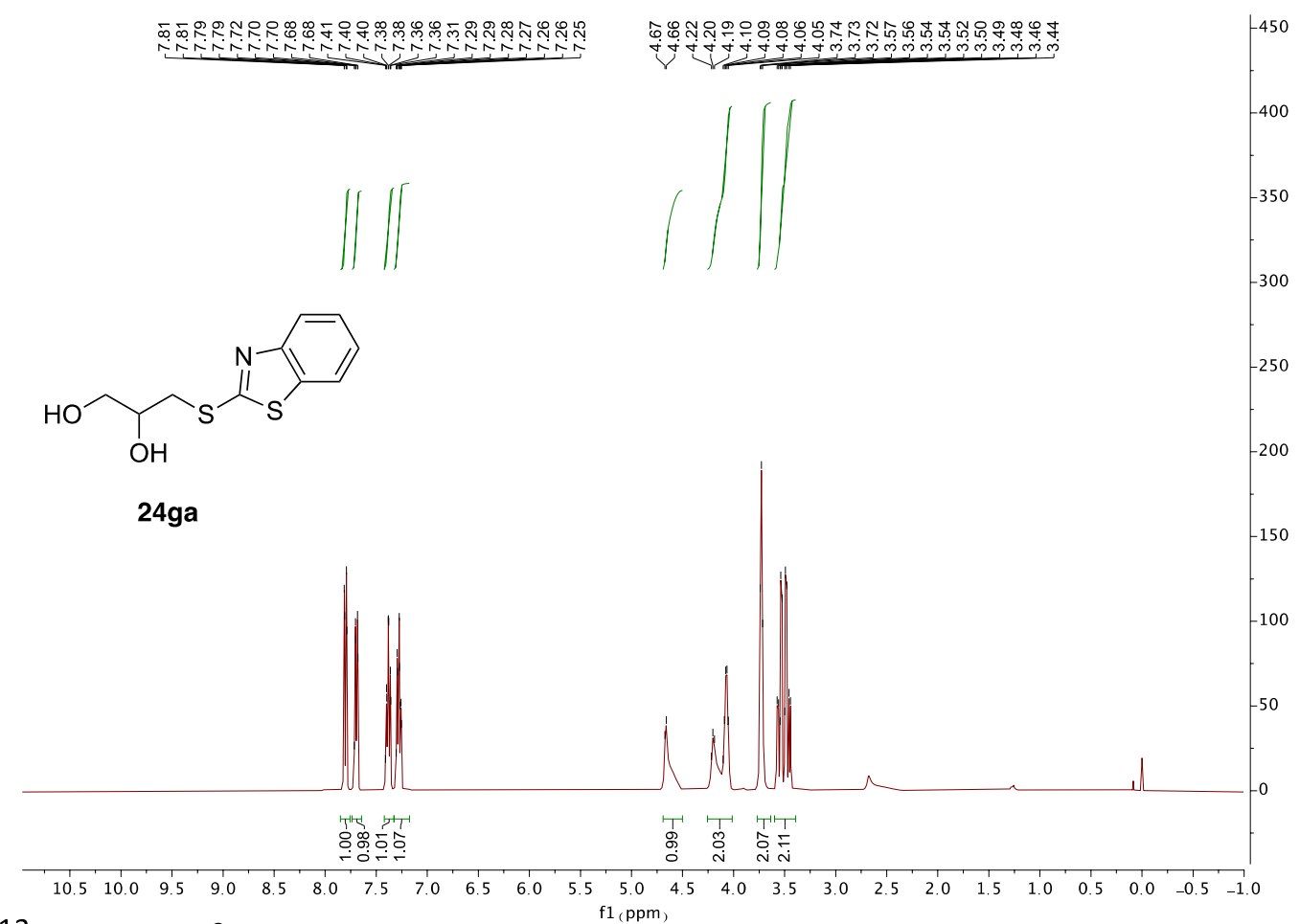

${ }^{13} \mathrm{C}$ NMR of 24ga

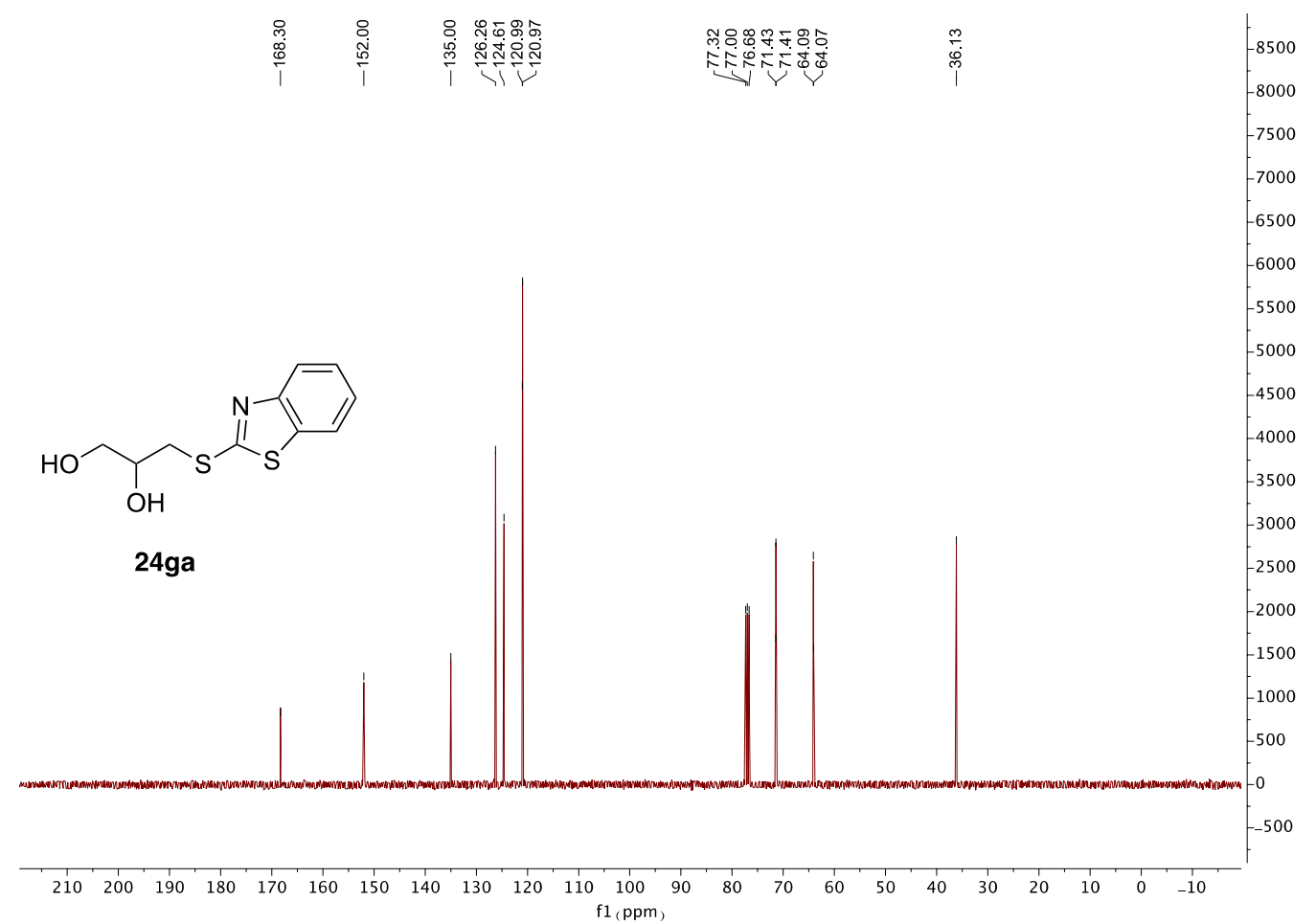




\section{${ }^{1} \mathrm{H}$ NMR of 24 ha}

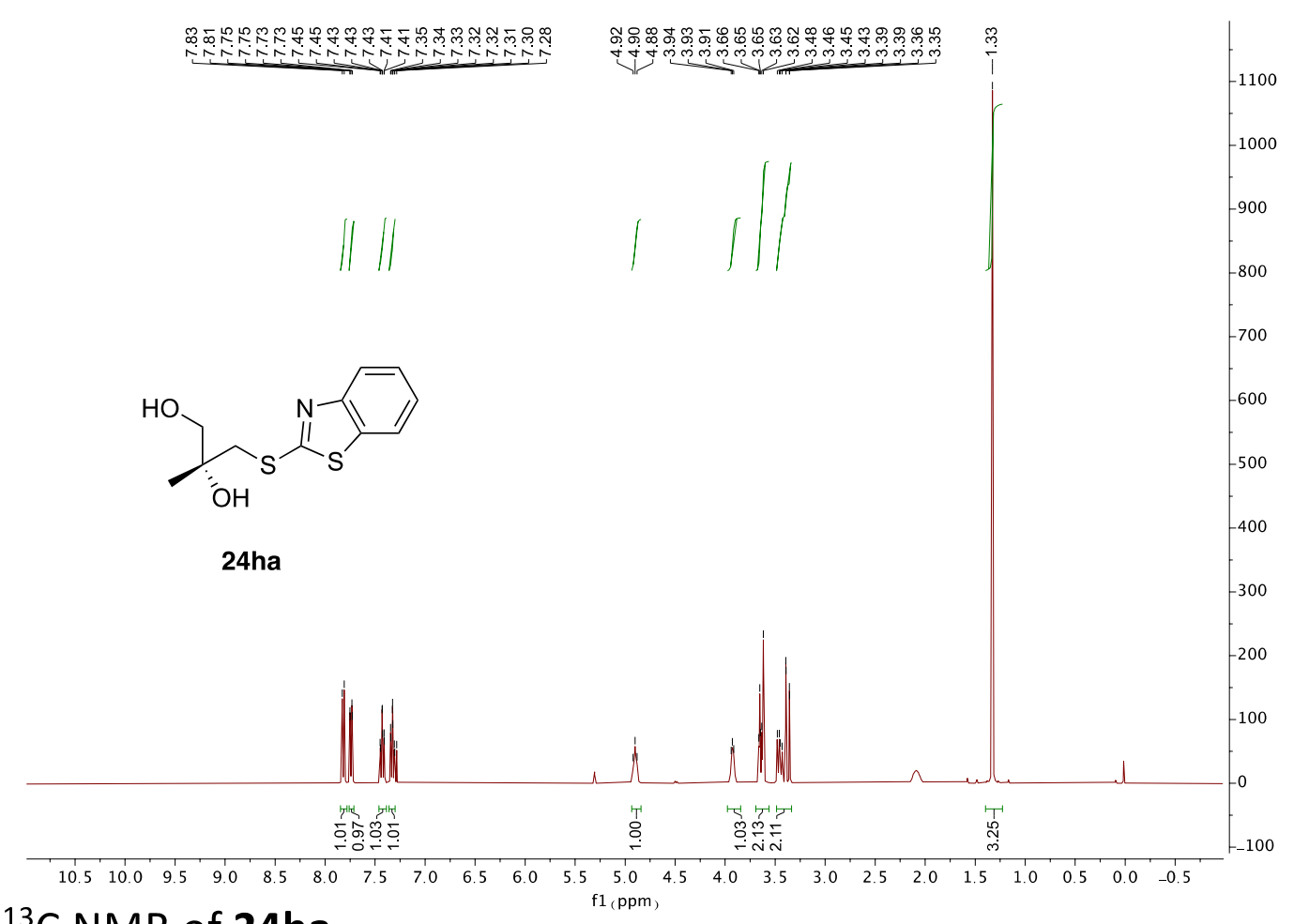

${ }^{13} \mathrm{C}$ NMR of $24 \mathrm{ha}$

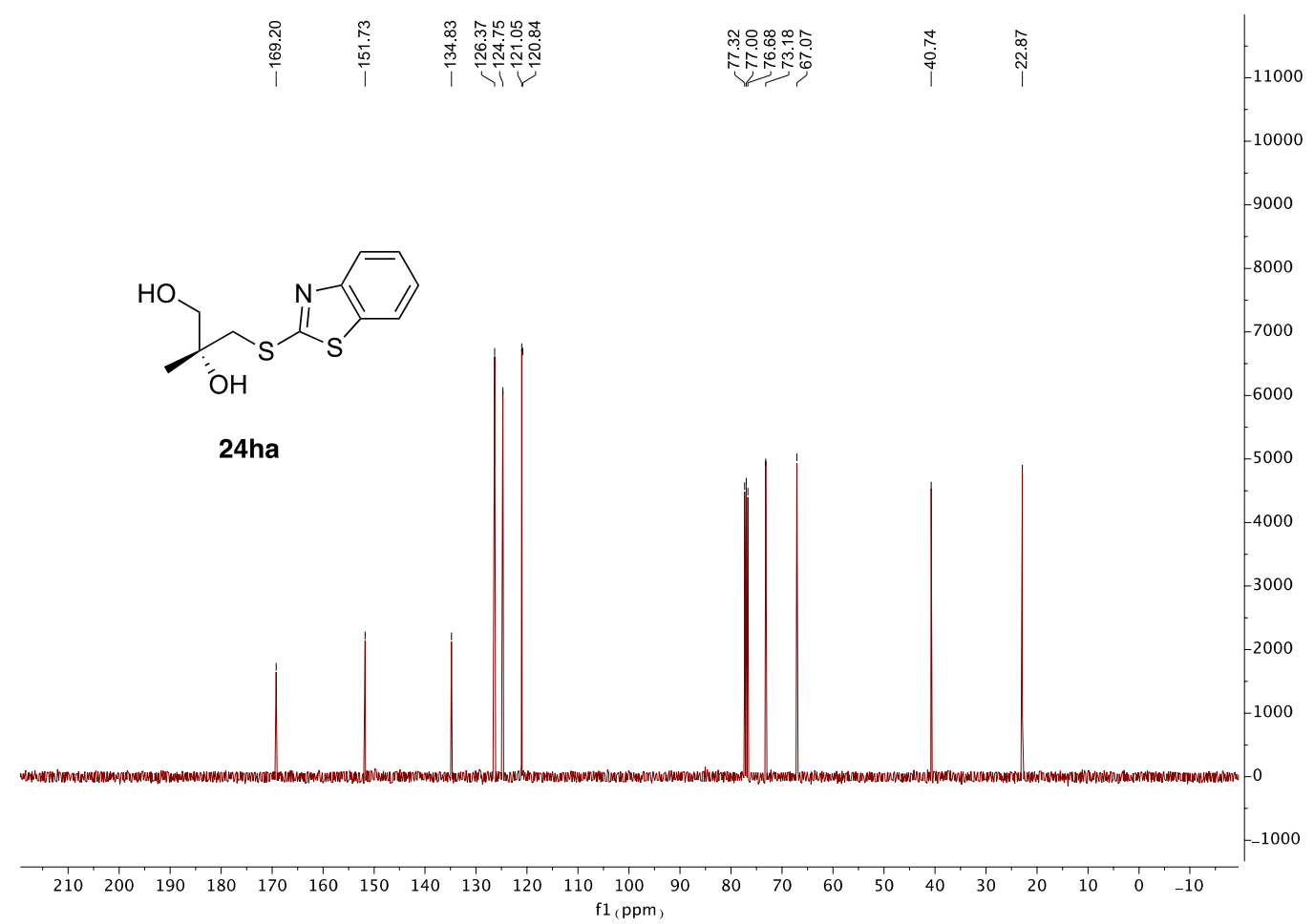




\section{${ }^{1} \mathrm{H}$ NMR of 24ia}

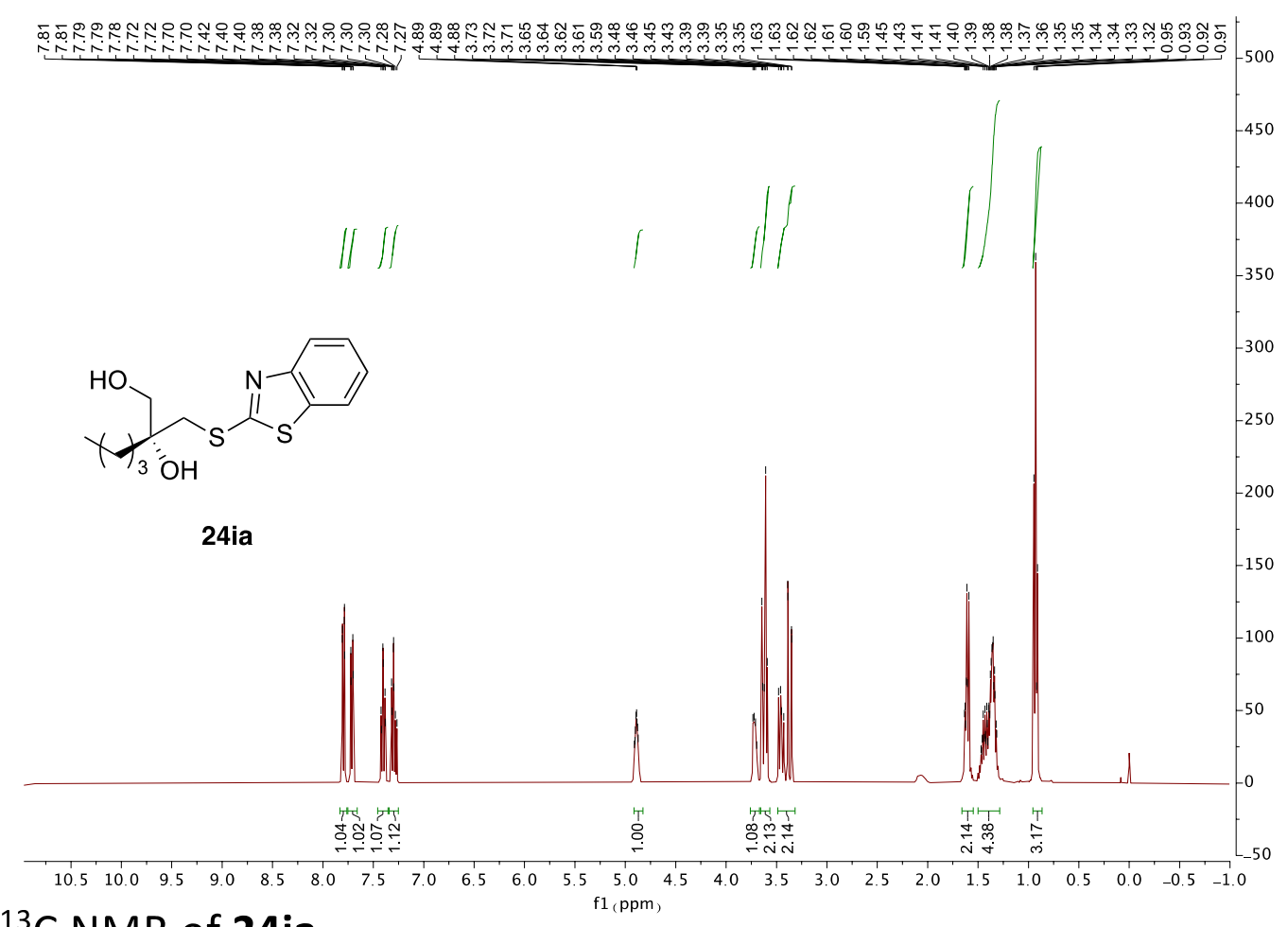

${ }^{13}$ C NMR of 24ia

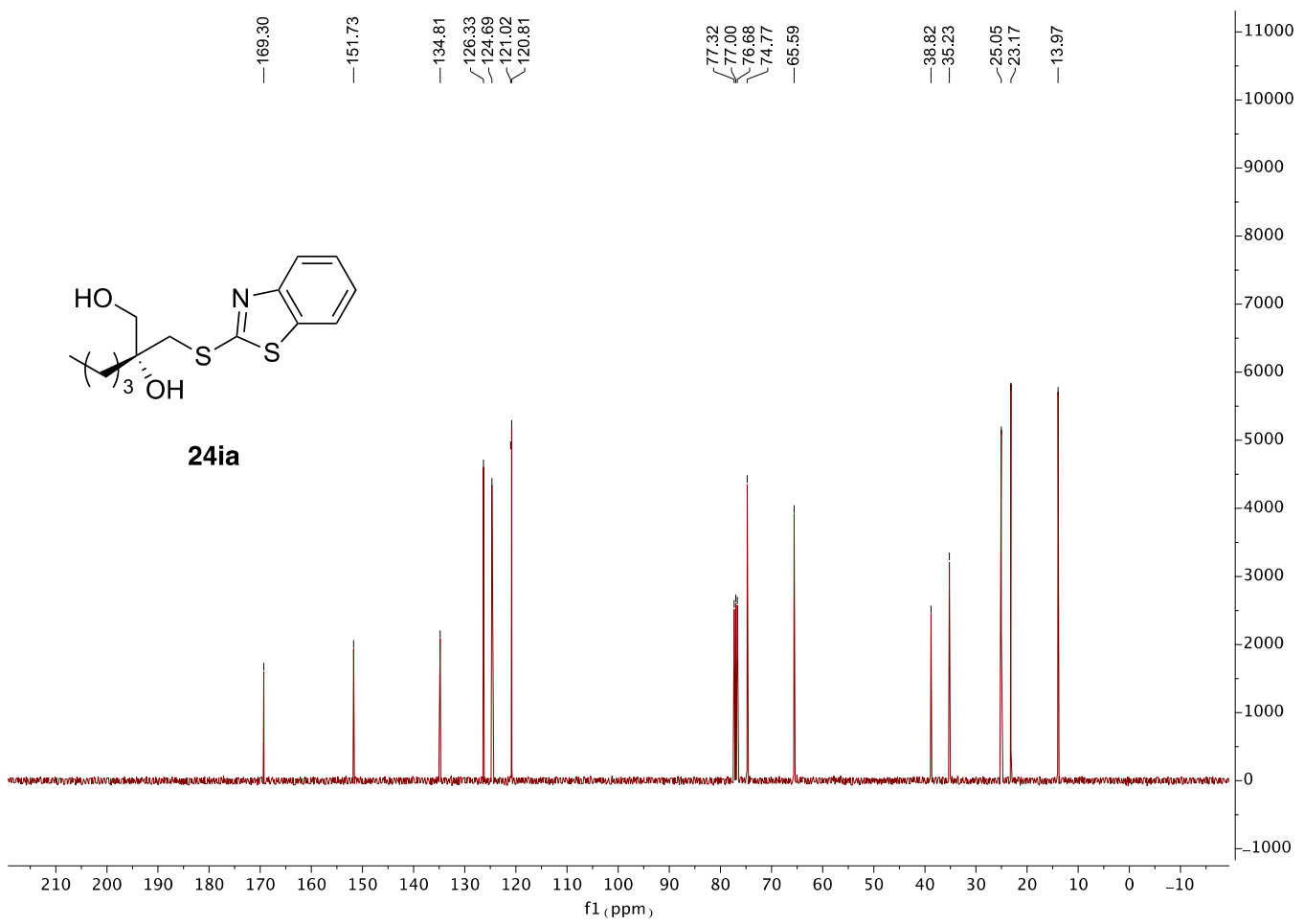




\section{${ }^{1} \mathrm{H}$ NMR of $24 \mathrm{ja}$}

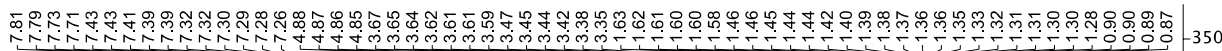

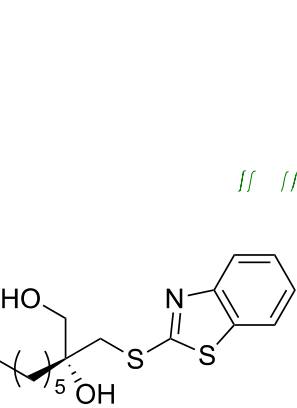

24ja

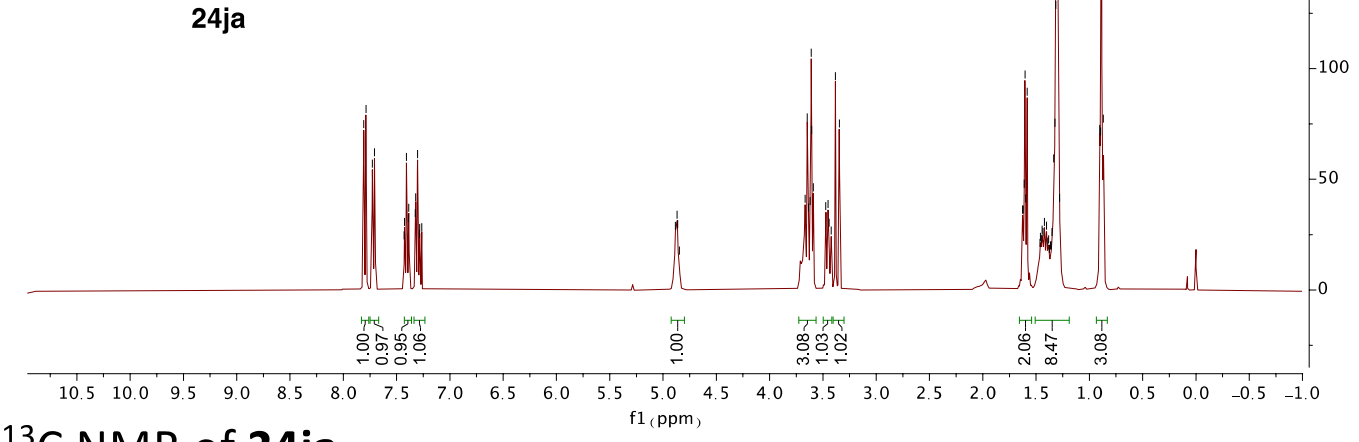

${ }^{13} \mathrm{C}$ NMR of $24 \mathrm{ja}$

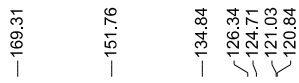

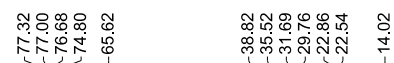

13000

12000

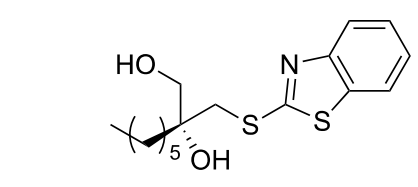

8000

24ja

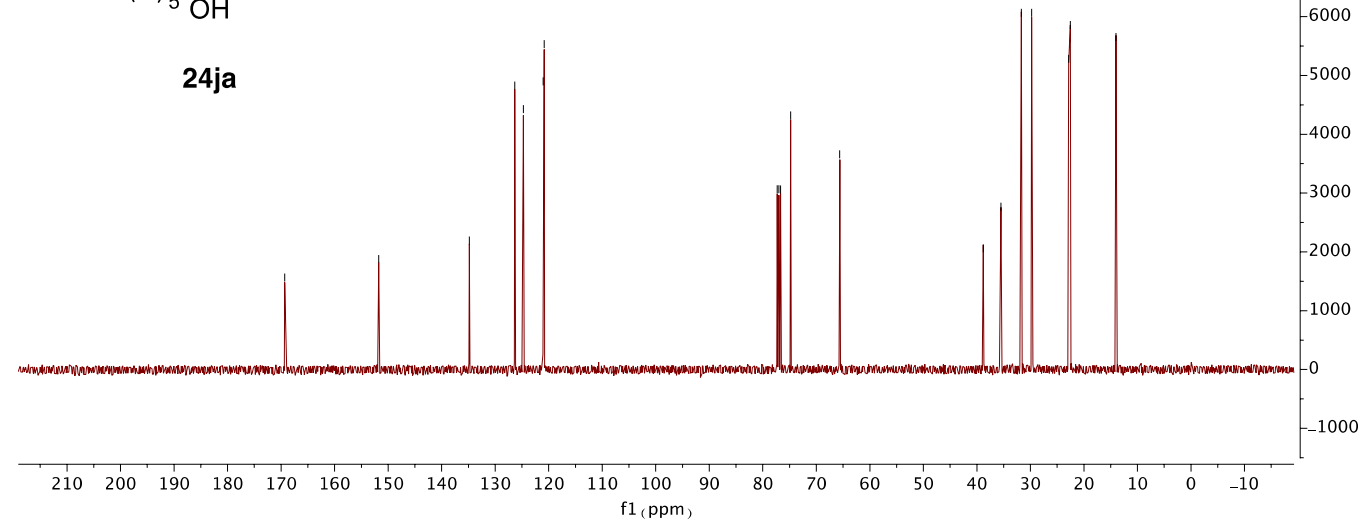




\section{${ }^{1} \mathrm{H}$ NMR of $24 \mathrm{ka}$}

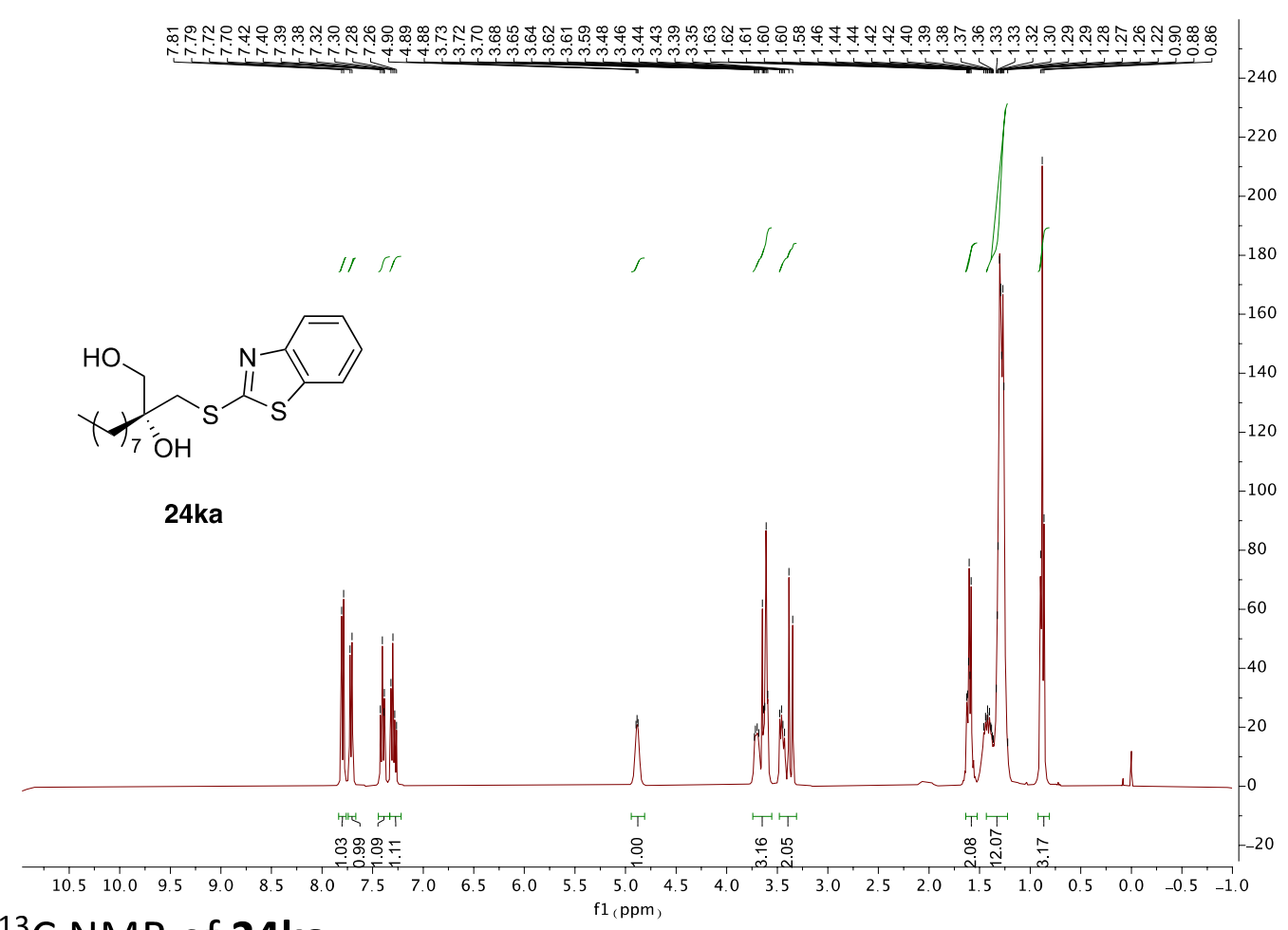

${ }^{13} \mathrm{C}$ NMR of $\mathbf{2 4 k a}$

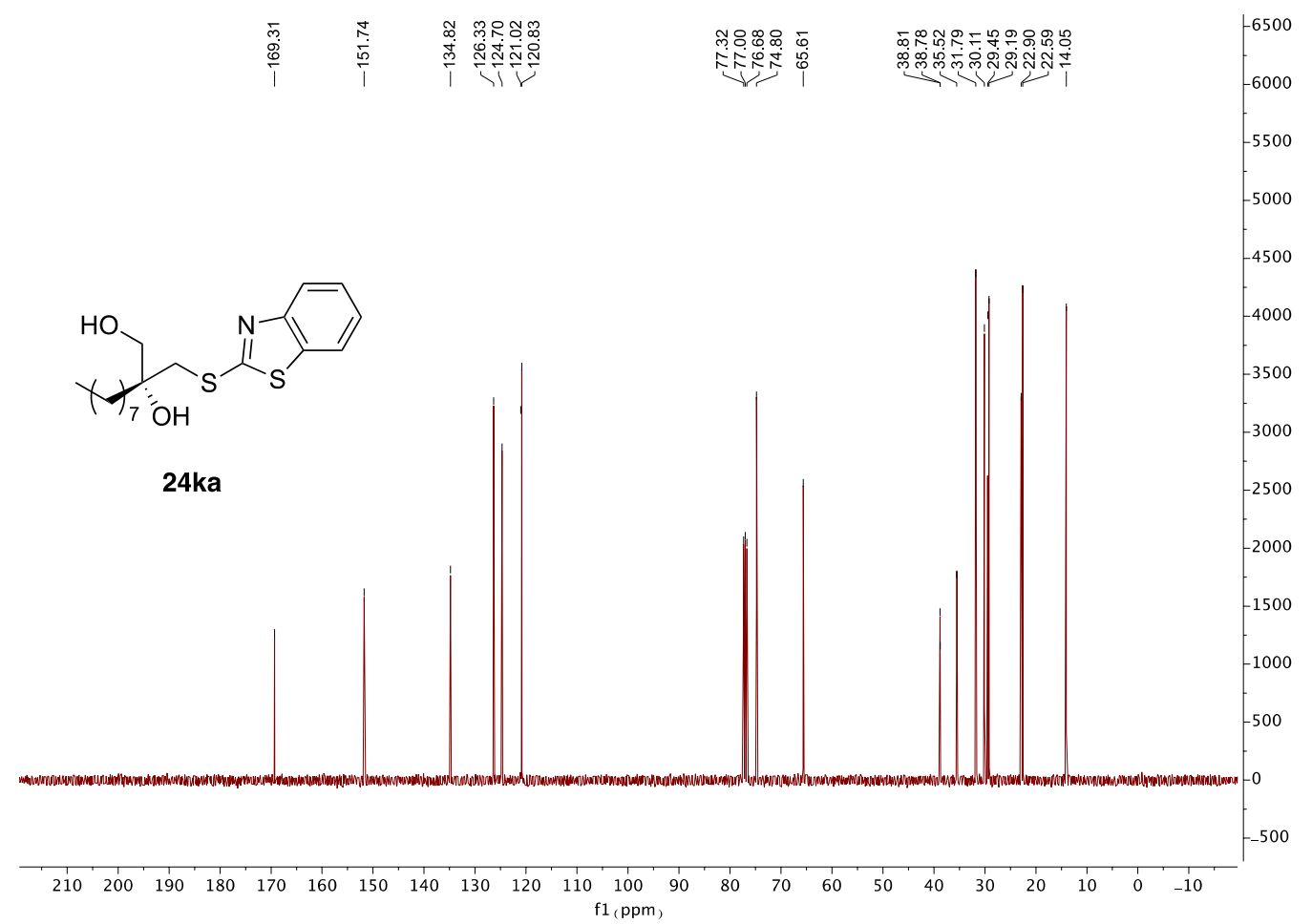




\section{${ }^{1} \mathrm{H}$ NMR of 24la}

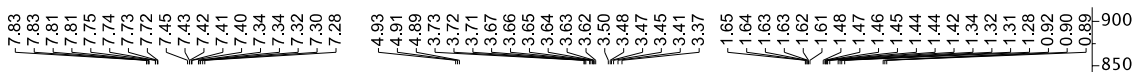

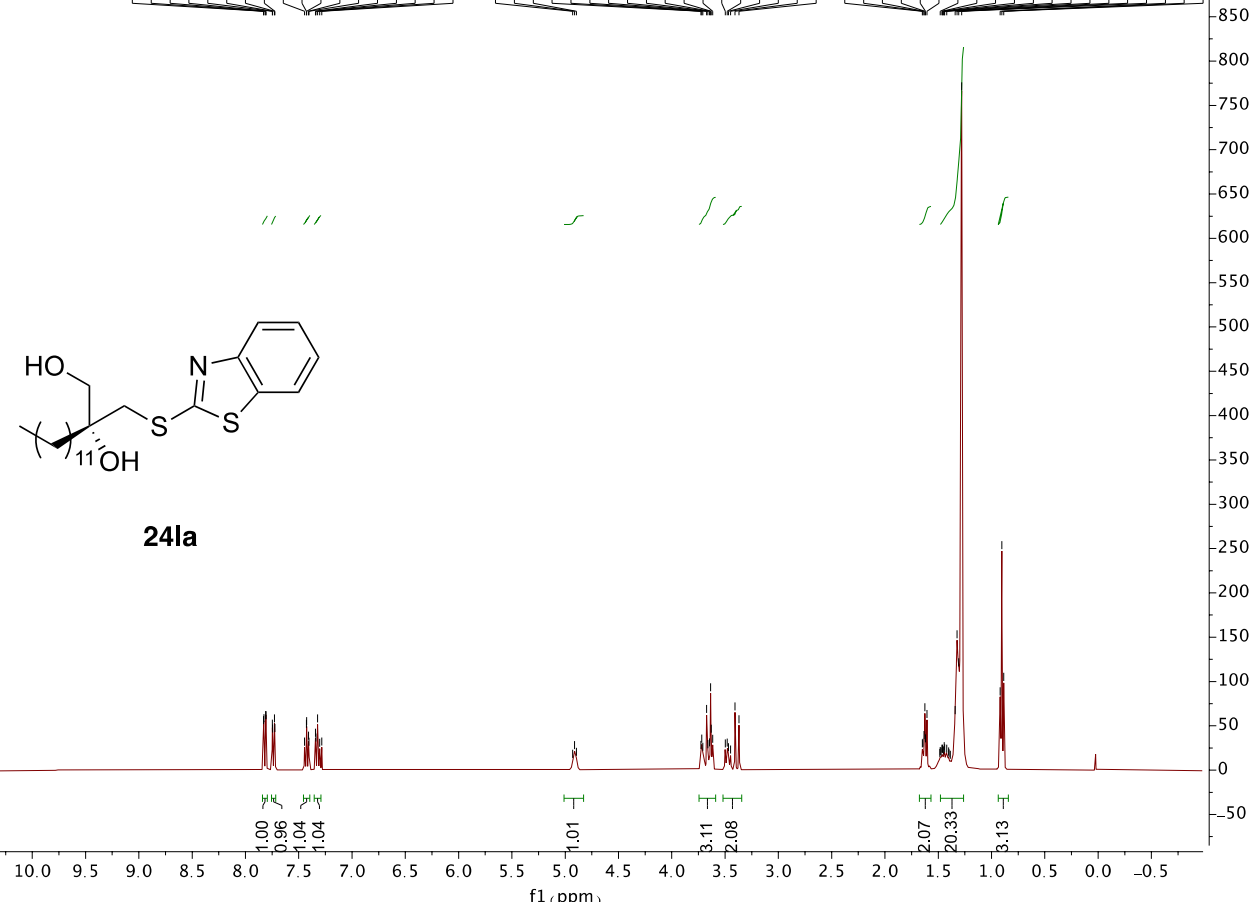

${ }^{13} \mathrm{C}$ NMR of 24la<smiles>OC[C@H](O)CSc1nc2ccccc2s1</smiles>

$241 a$

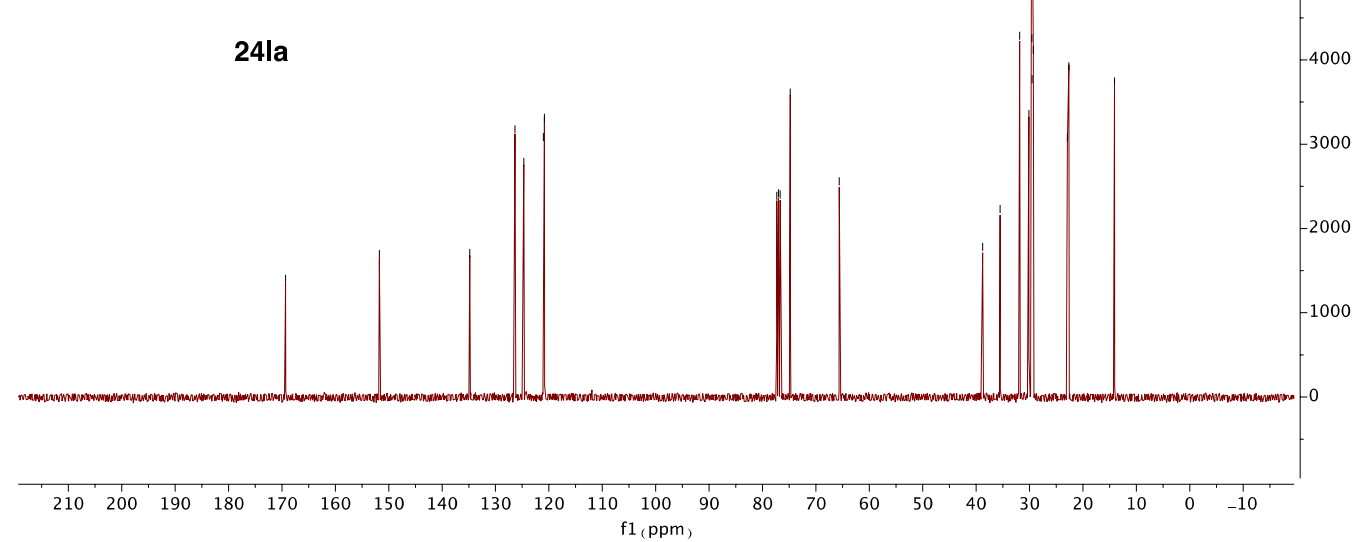




\section{${ }^{1} \mathrm{H}$ NMR of $24 \mathrm{ma}$}

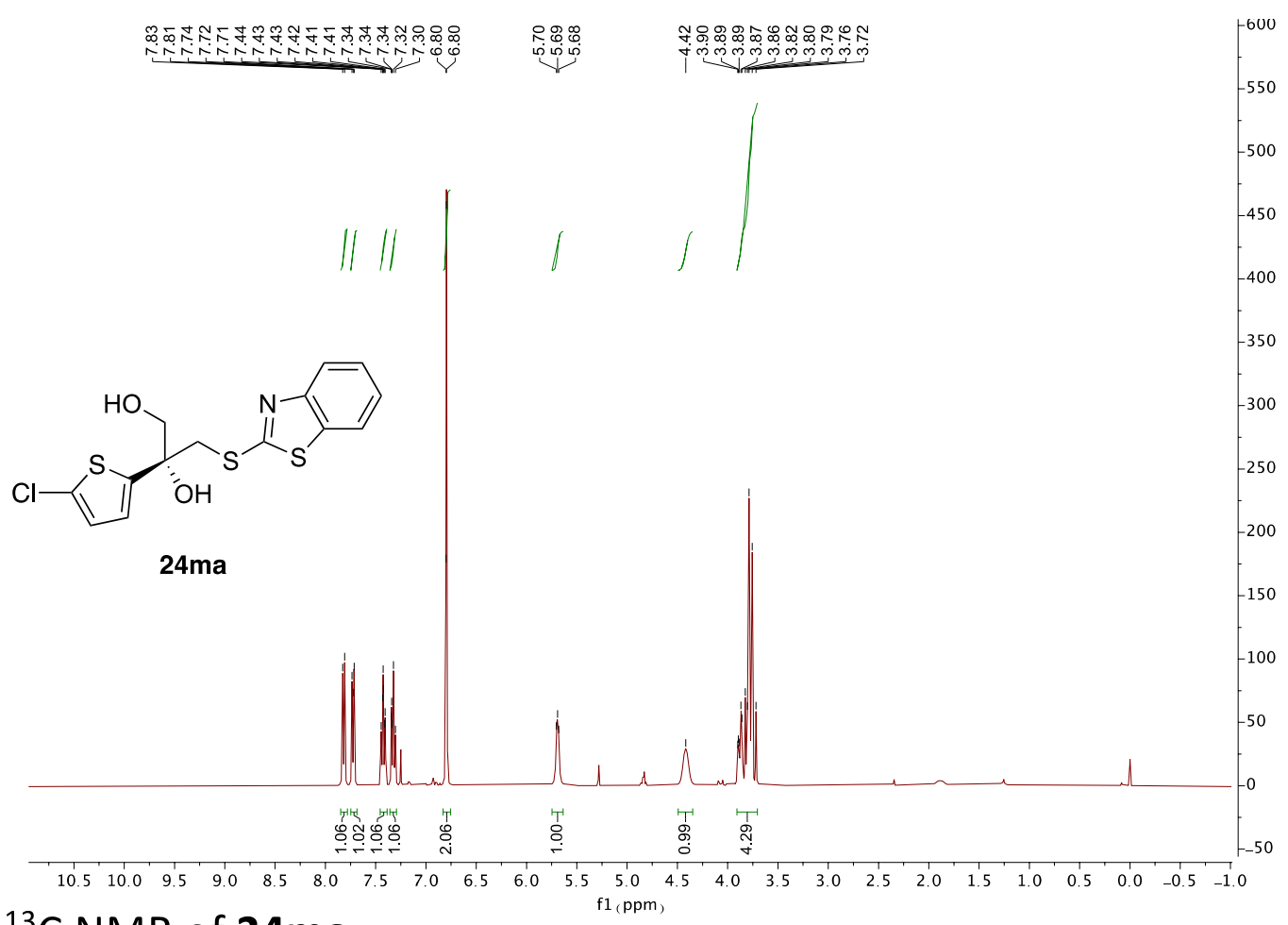

${ }^{13} \mathrm{C}$ NMR of $24 \mathrm{ma}$

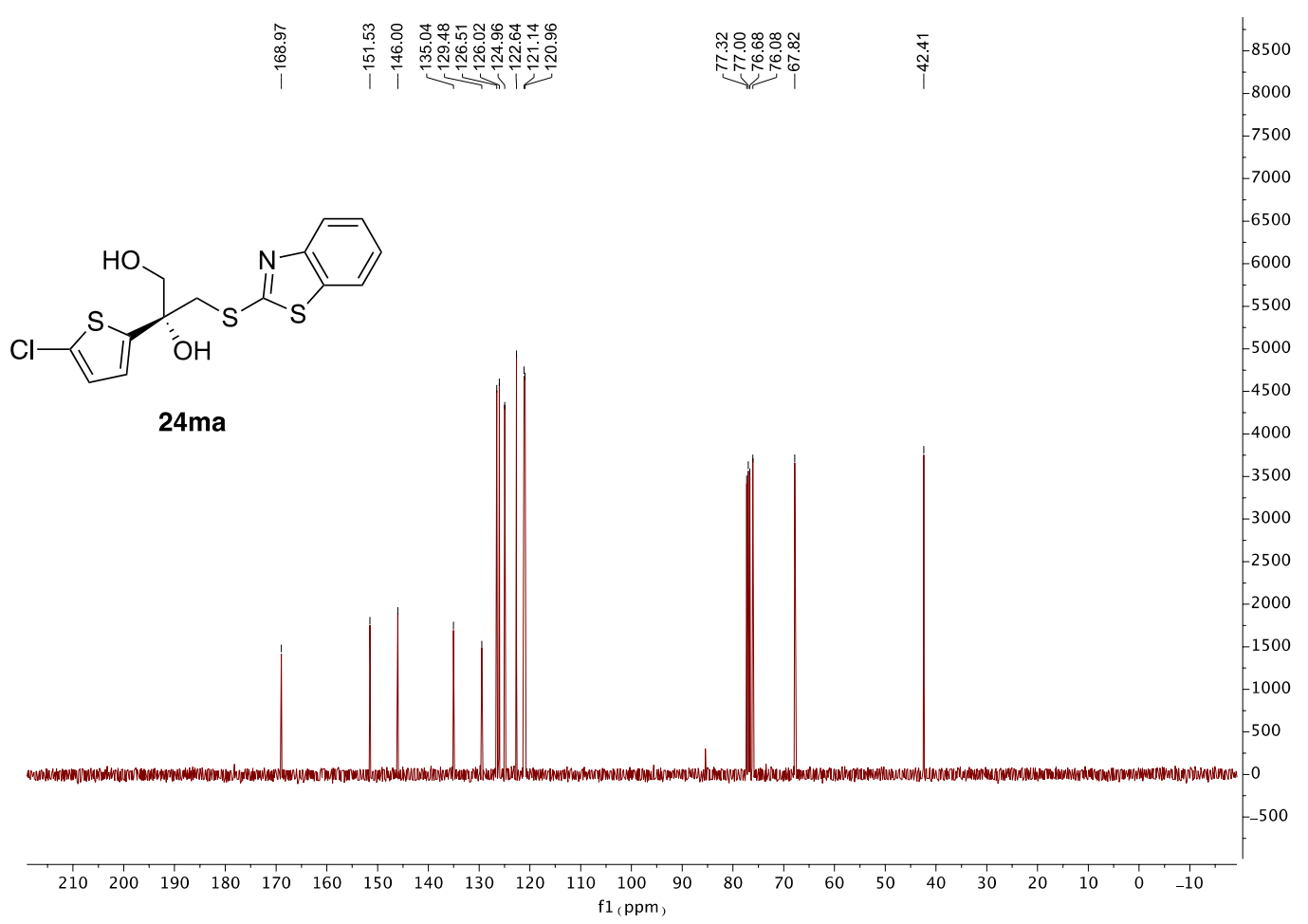




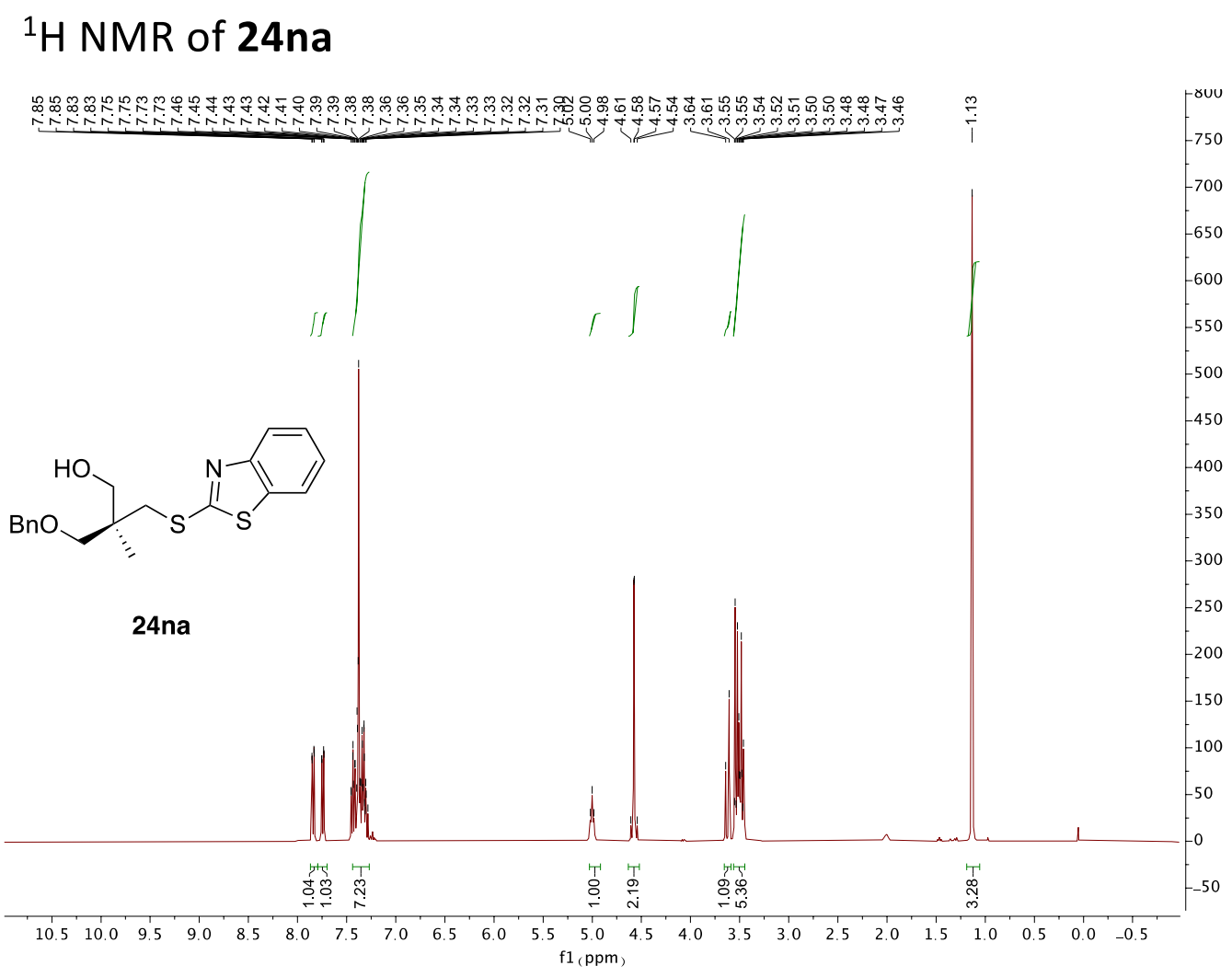

${ }^{13}$ C NMR of $24 n a$

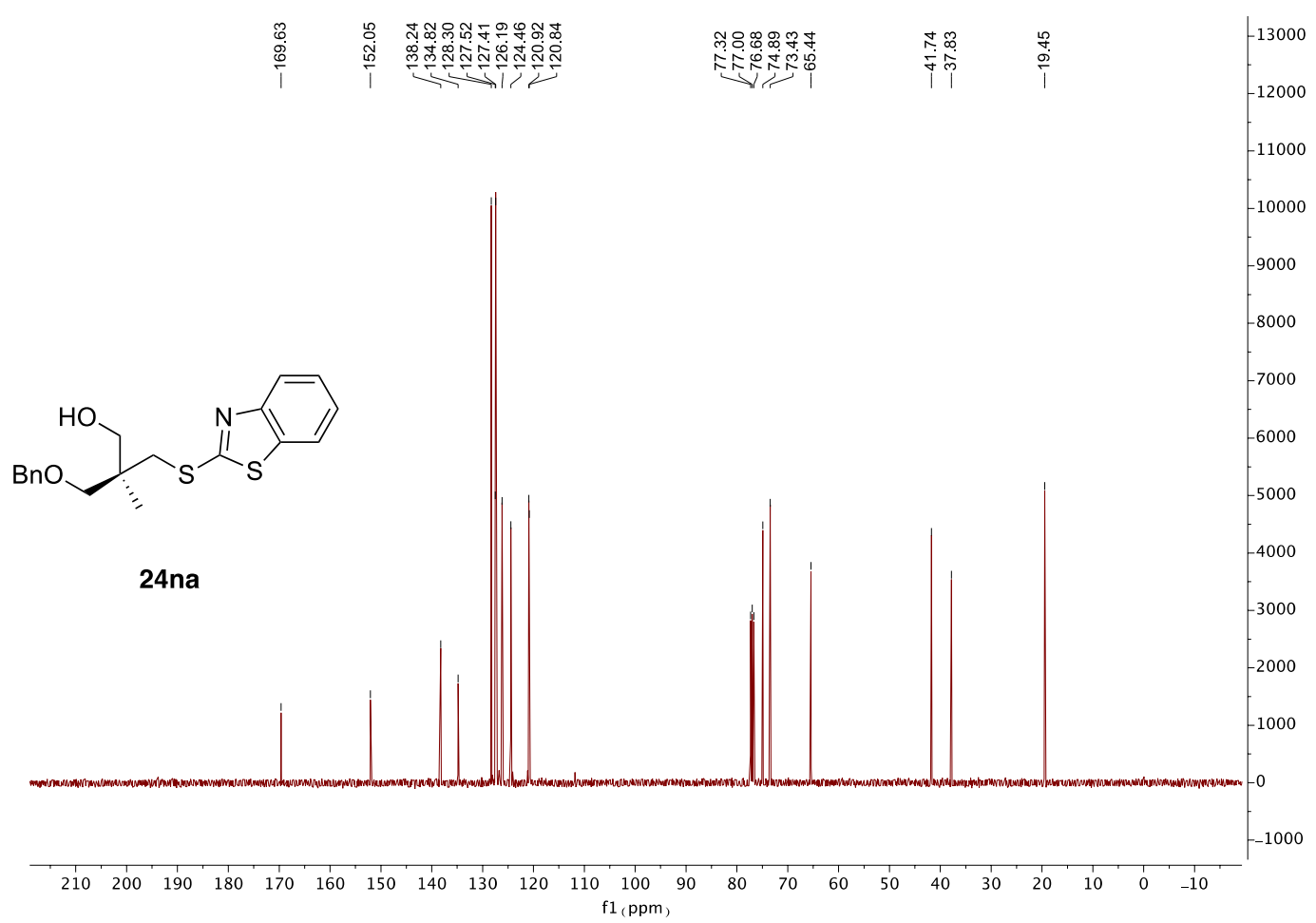




\section{${ }^{1} \mathrm{H}$ NMR of $24 a b$}

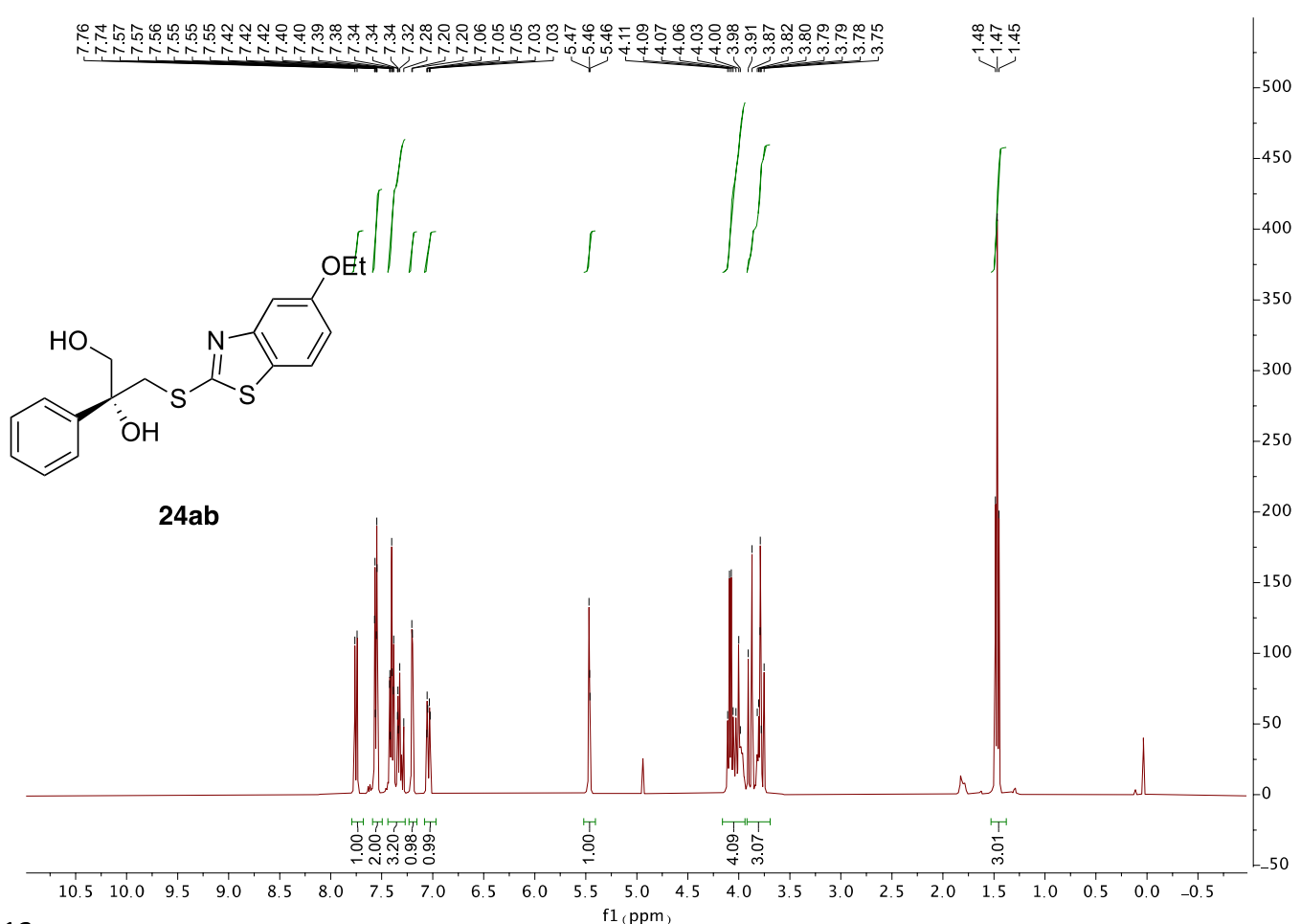

${ }^{13} \mathrm{C}$ NMR of $24 a b$

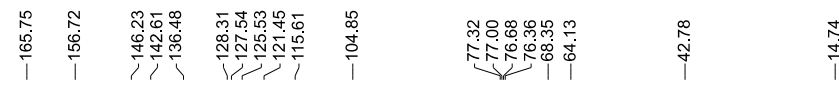

0000

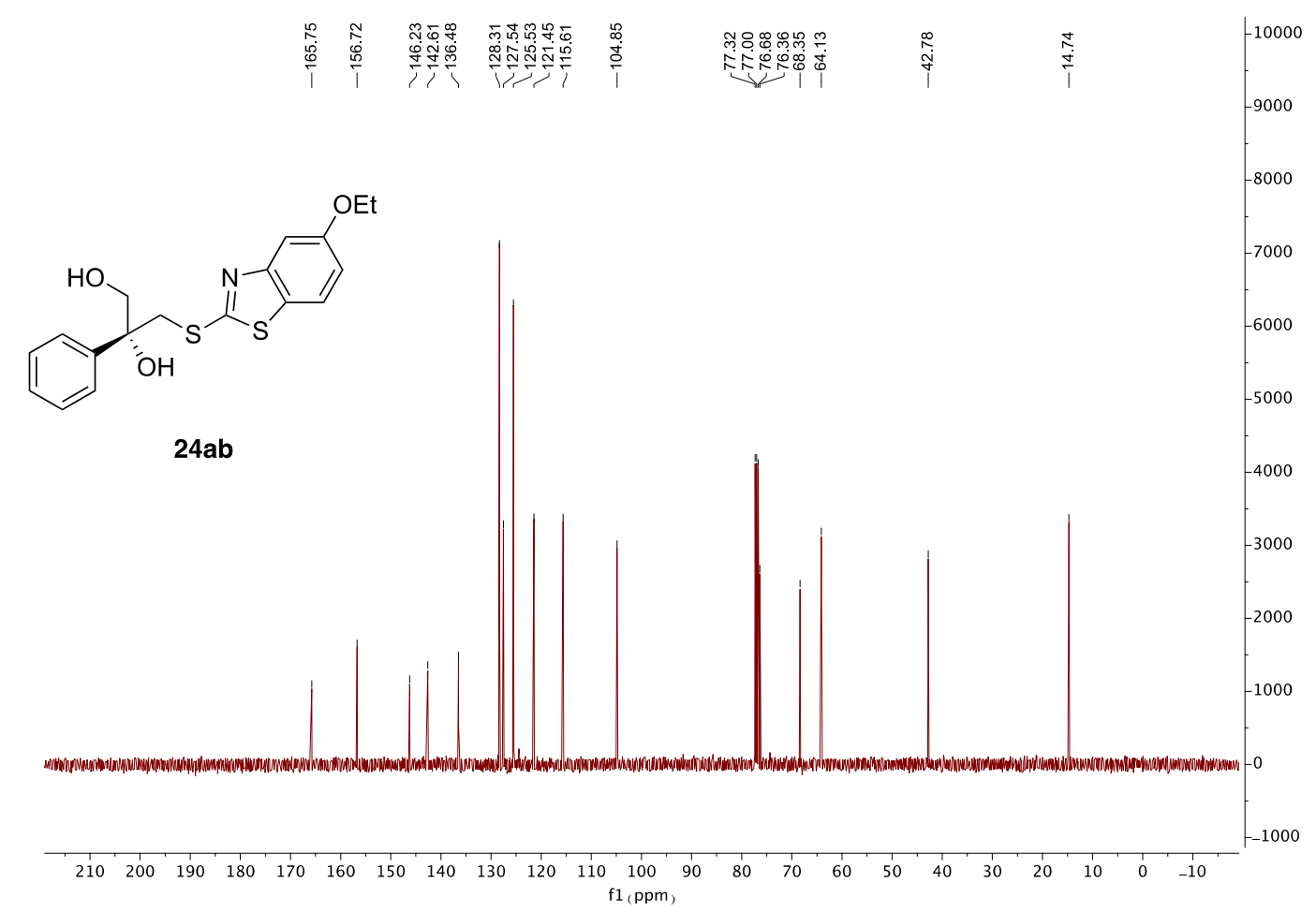




\section{${ }^{1} \mathrm{H}$ NMR of $24 \mathrm{ac}$}

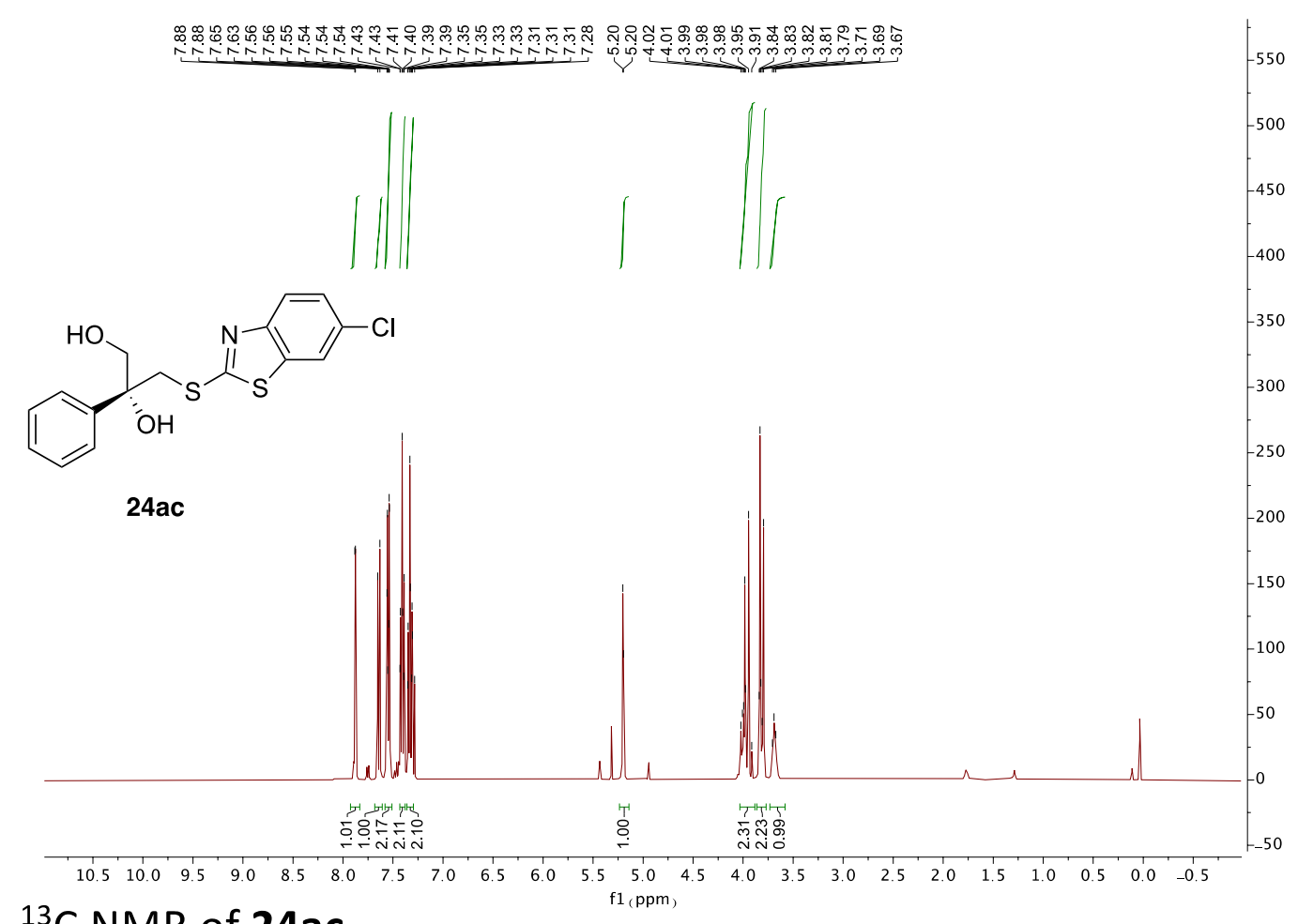

${ }^{13} \mathrm{C}$ NMR of $24 a c$

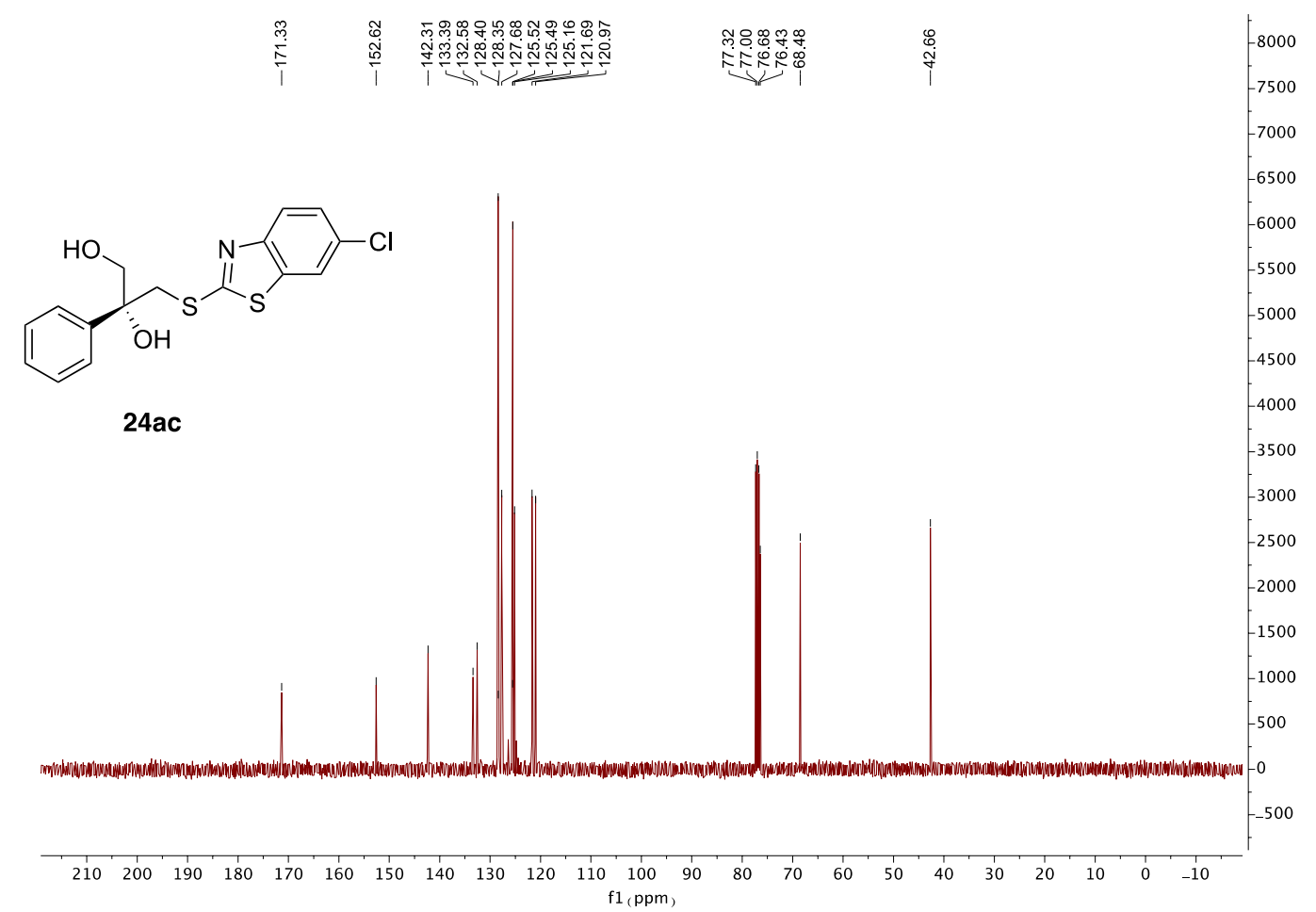




\section{${ }^{1} \mathrm{H}$ NMR of $24 \mathrm{ad}$}

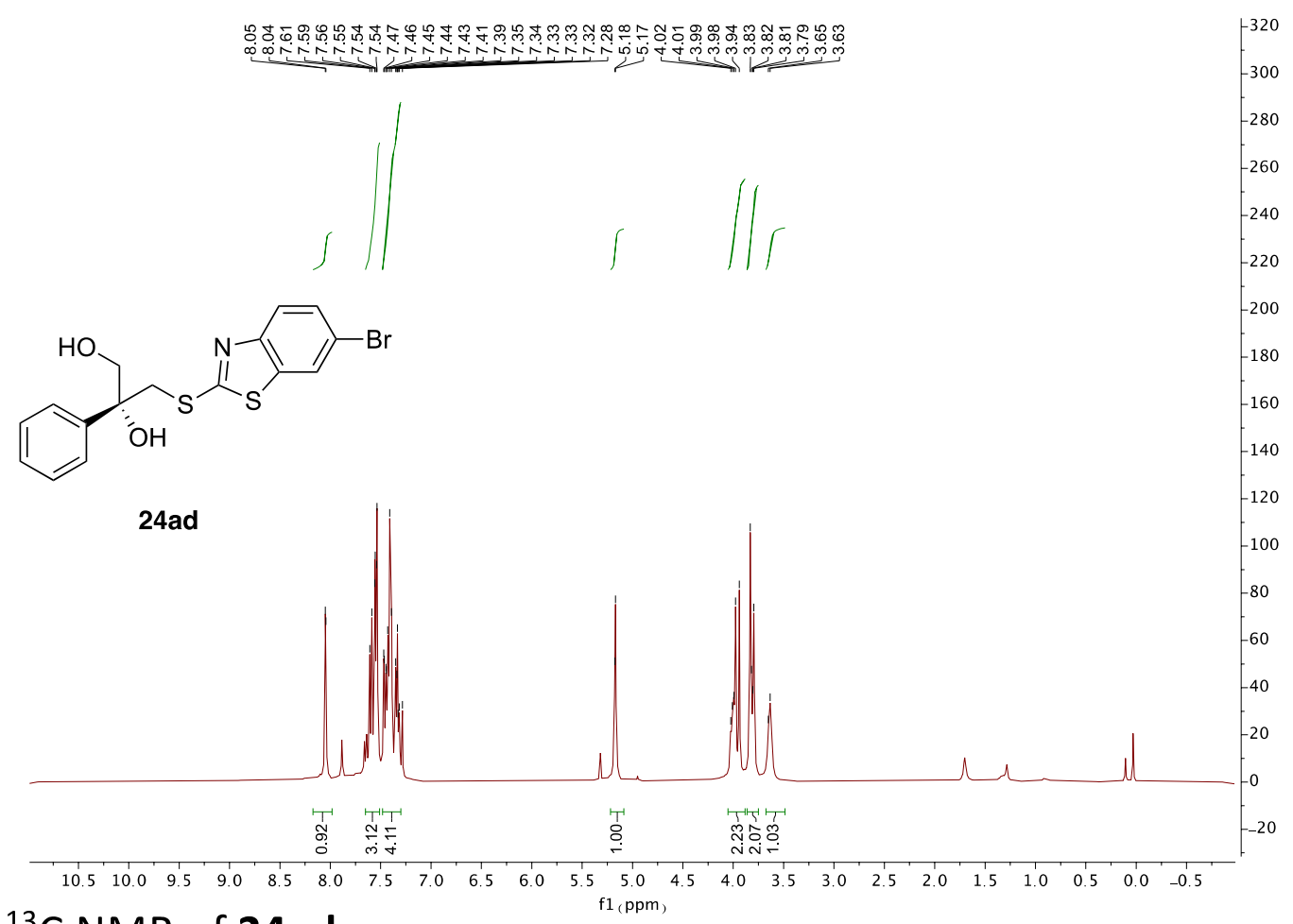

${ }^{13} \mathrm{C}$ NMR of $24 \mathrm{ad}$

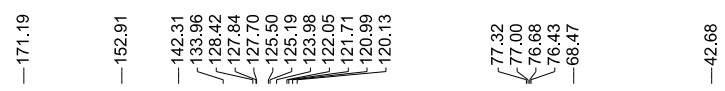

$-4000$<smiles>OCC(O)(CSc1nc2ccc(Br)cc2s1)c1ccccc1</smiles>

24ad

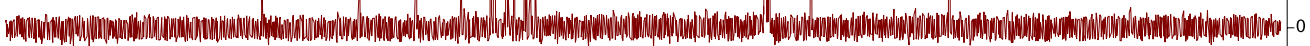

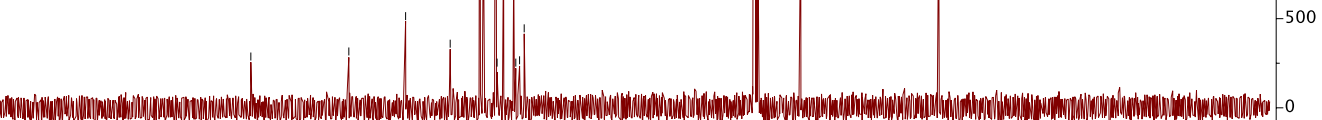

$\begin{array}{llllllllllllll}210 & 200 & 190 & 180 & 170 & 160 & 150 & 140 & 130 & 120 & 110 & 100 & 90 \\ \mathrm{f} 1(\mathrm{ppm})\end{array}$ 


\section{HPLC chromatograms}

HPLC of rac 24aa

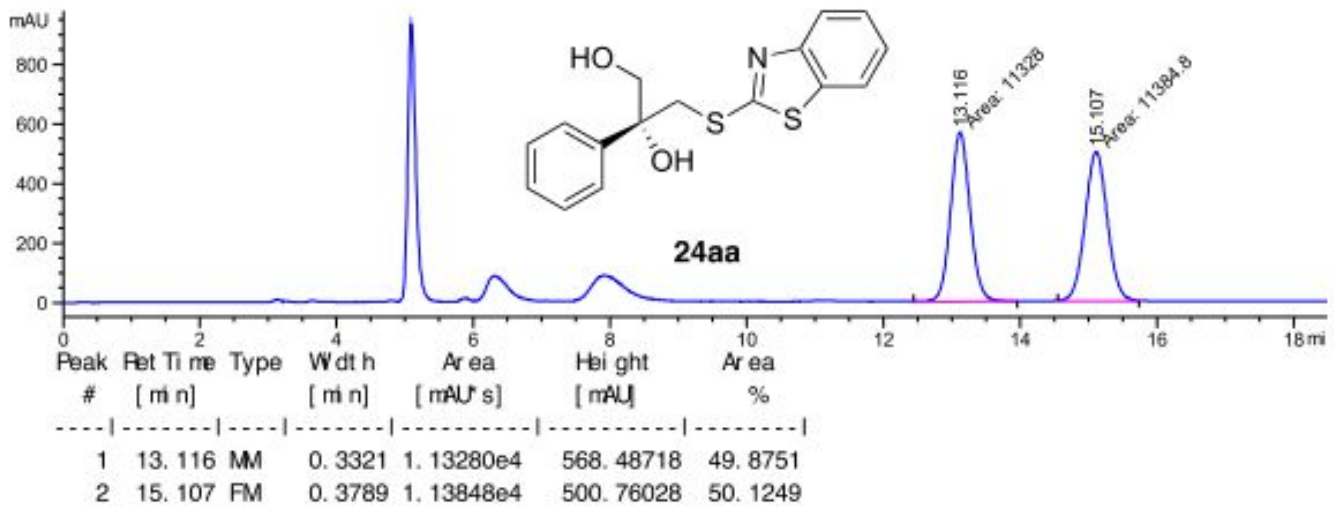

HPLC of 24aa (result for Cat f)

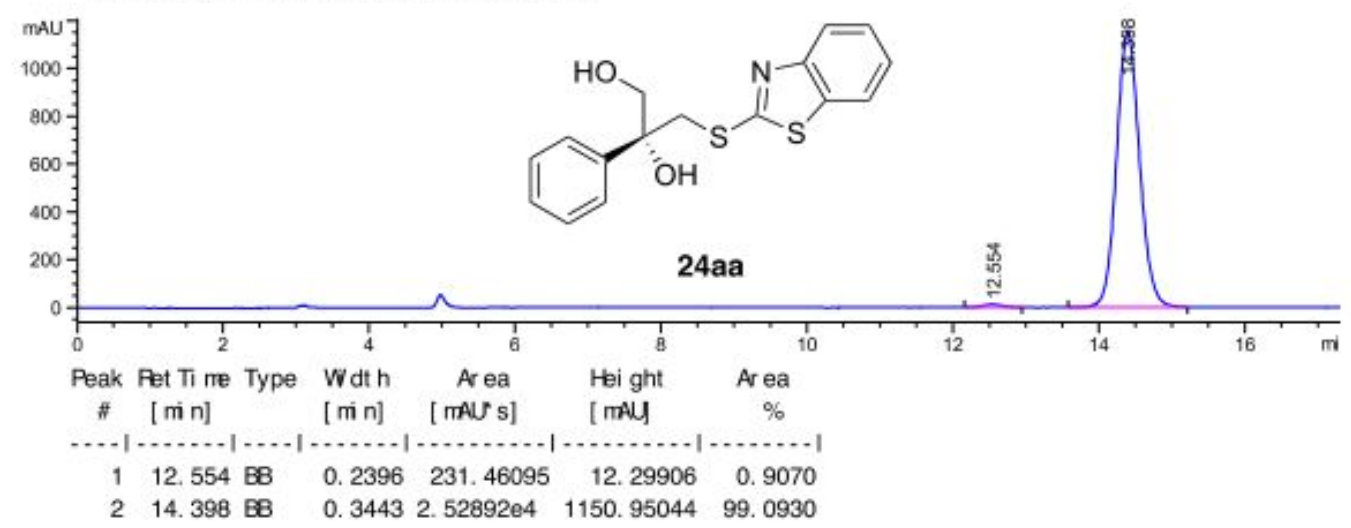

HPLC of 24aa (result for Cat g)

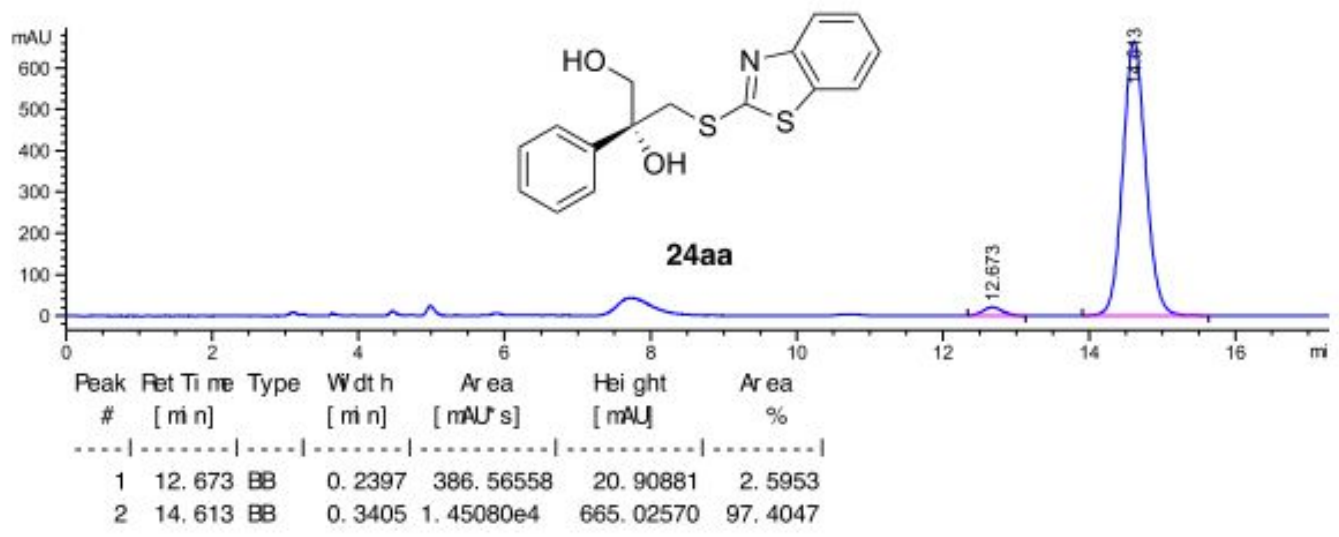




\section{HPLC of rac 24ba}

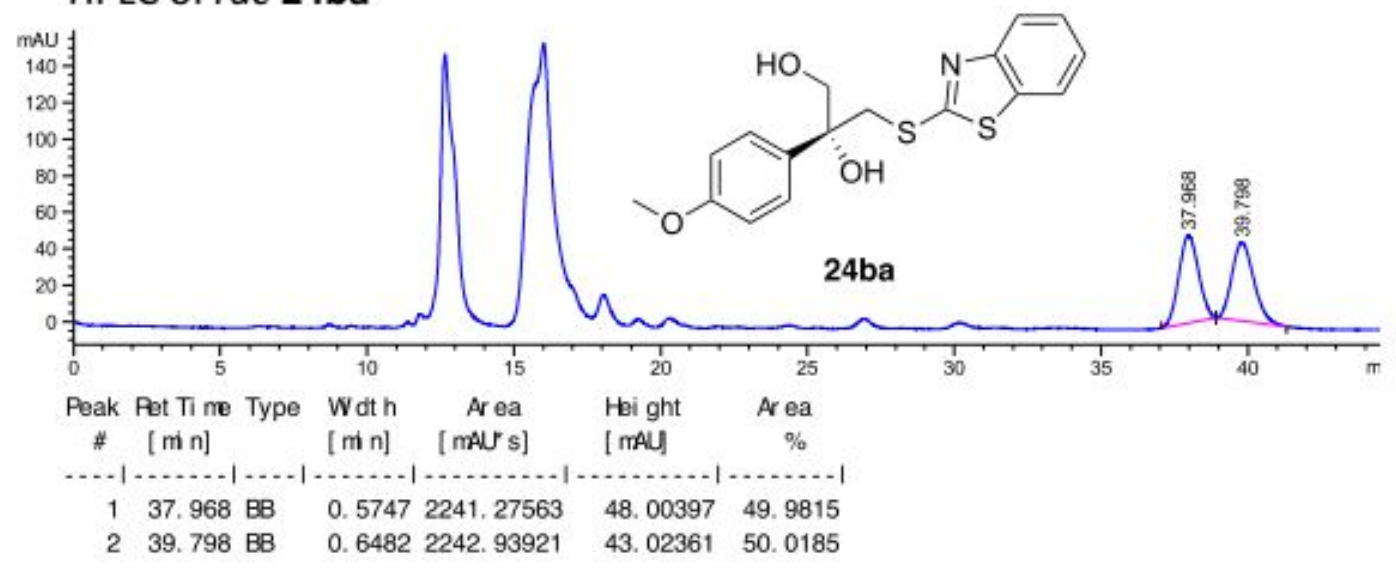

HPLC of 24ba (result for Cat f)

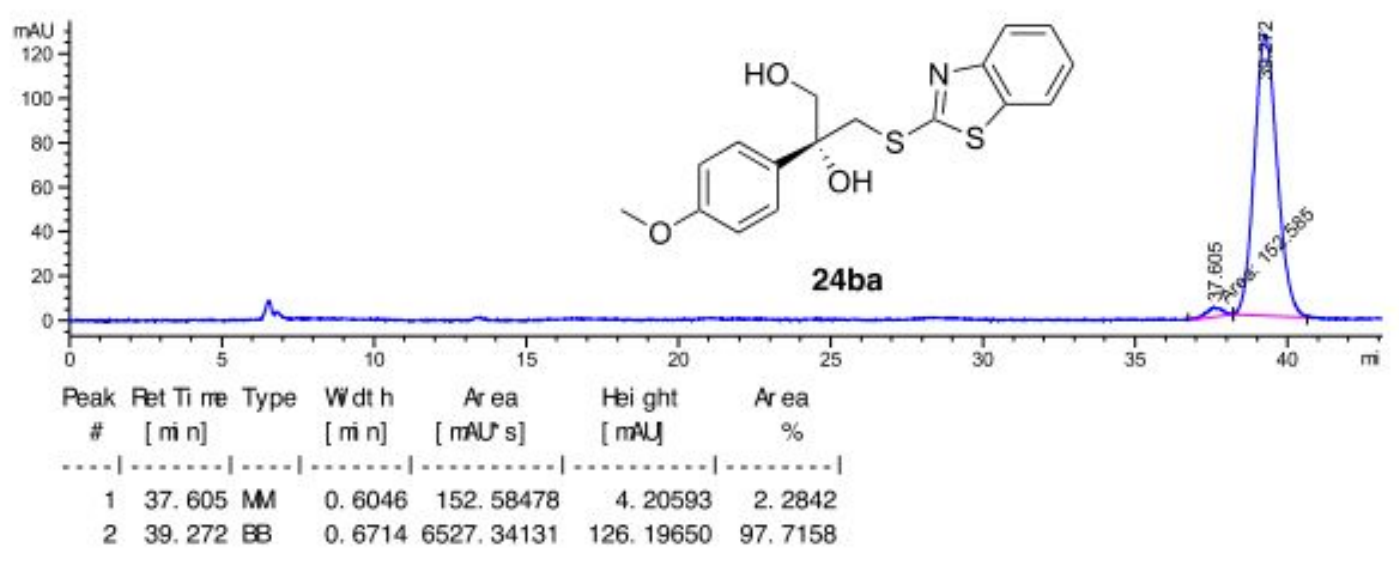

HPLC of 24ba (result for Cat g)

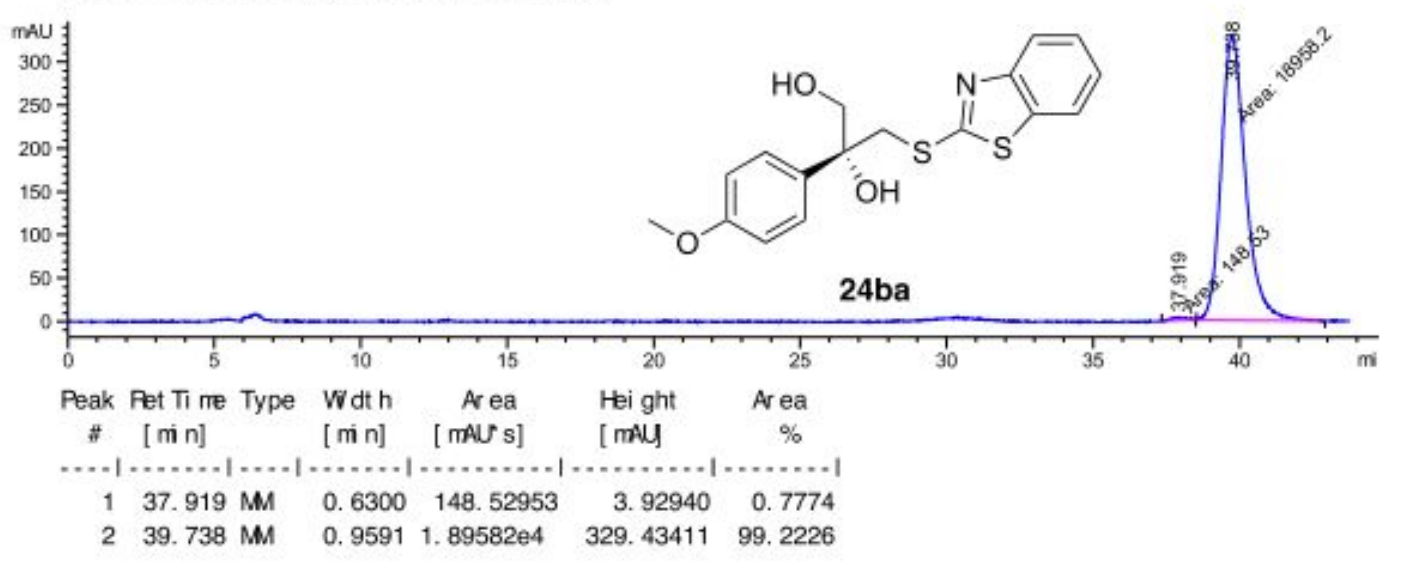




\section{HPLC of rac 24ca}

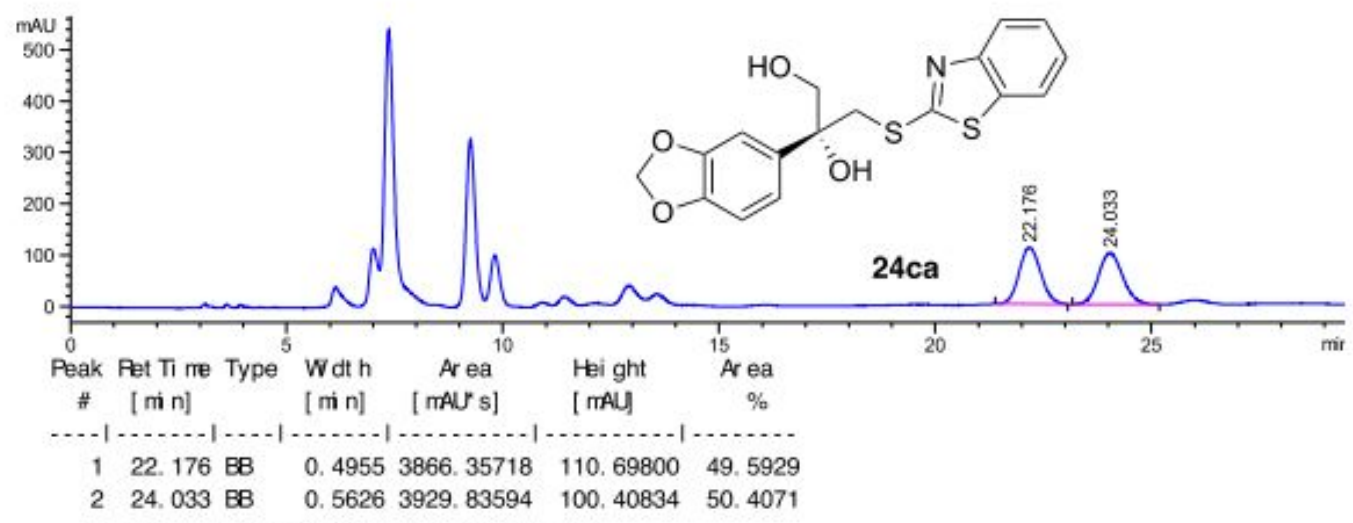

\section{HPLC of 24ca (result for Cat f)}

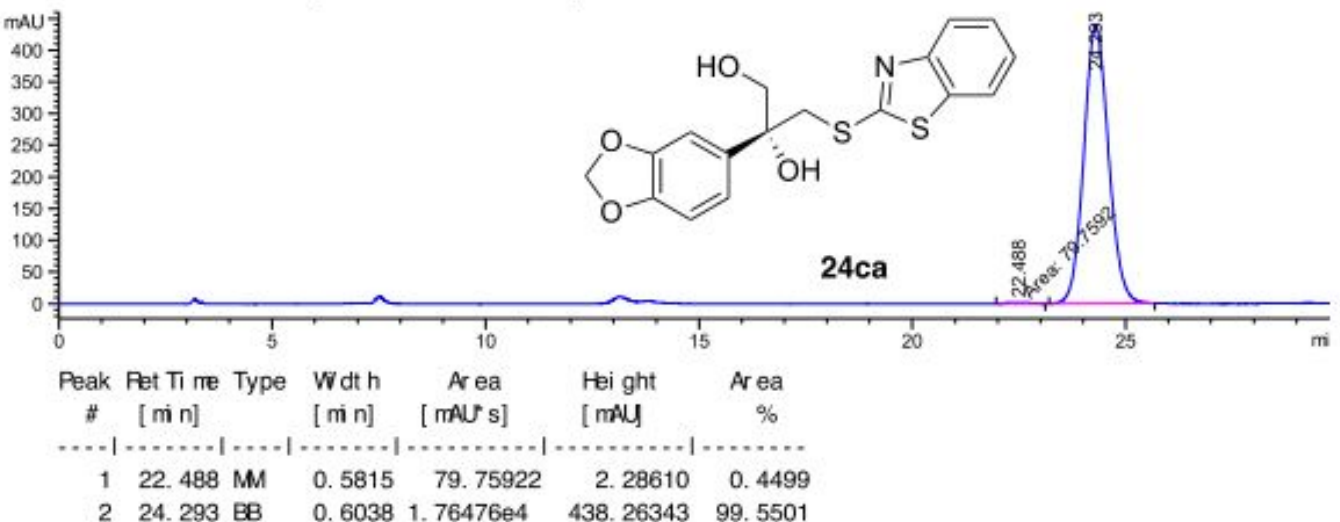

HPLC of rac 24ca

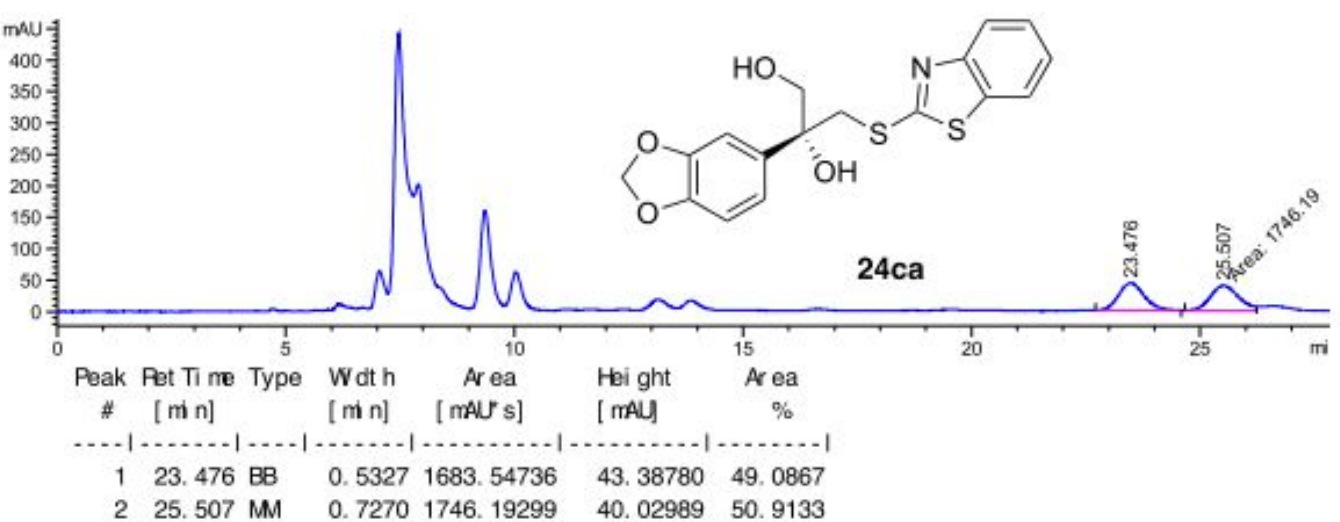

HPLC of 24ca (result for Cat g)

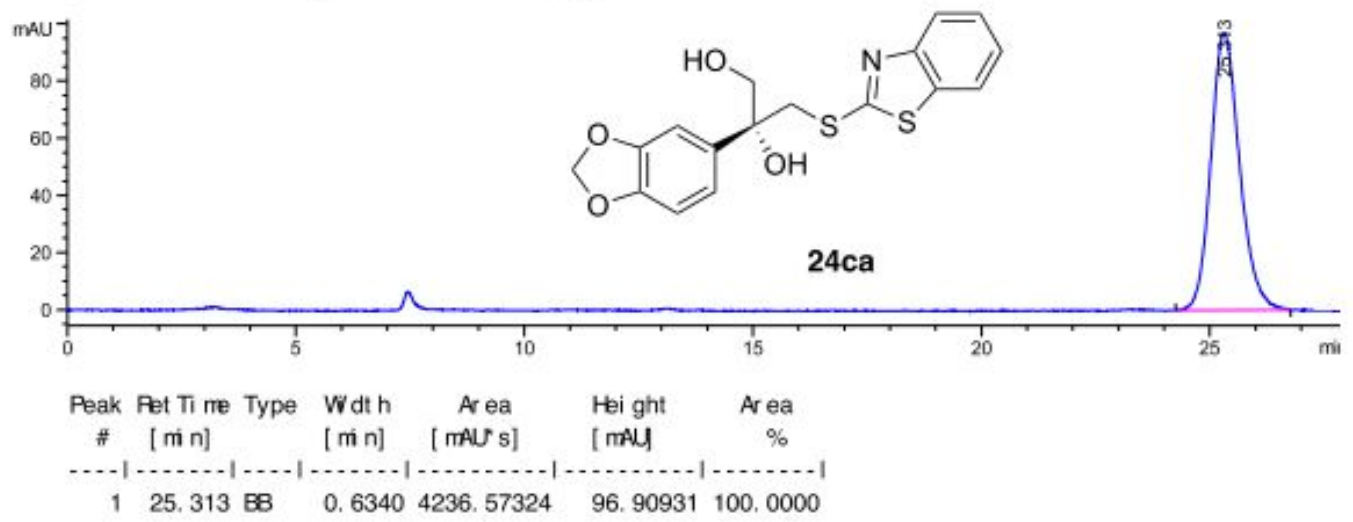




\section{HPLC of rac 24da}

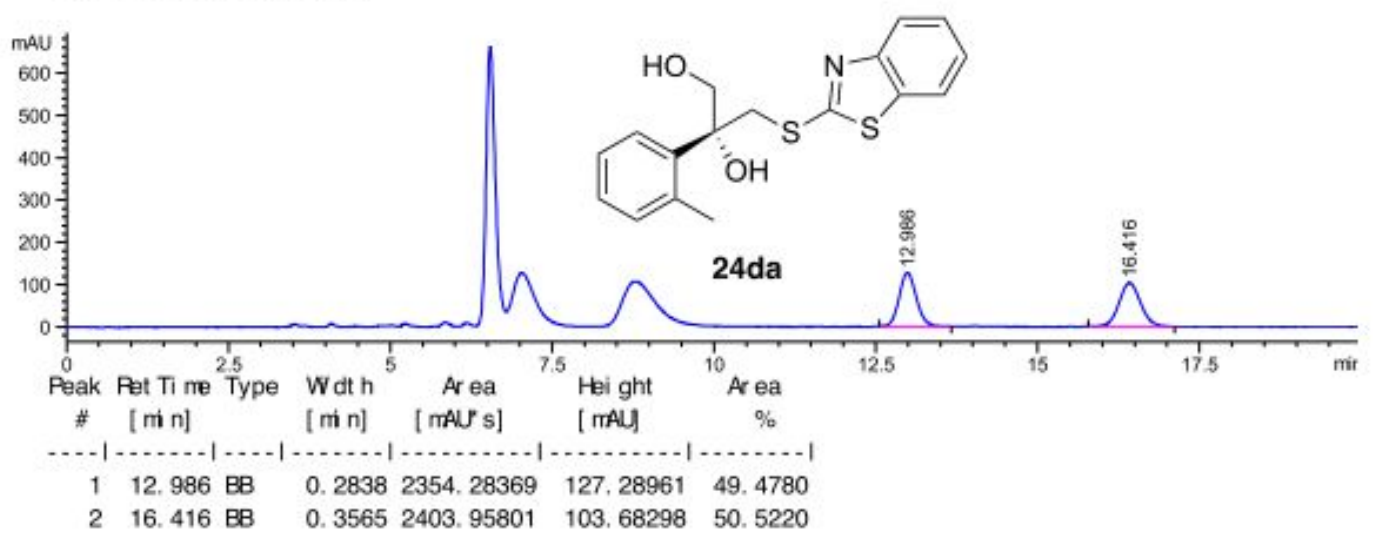

HPLC of 24da (result for Cat $f$ )

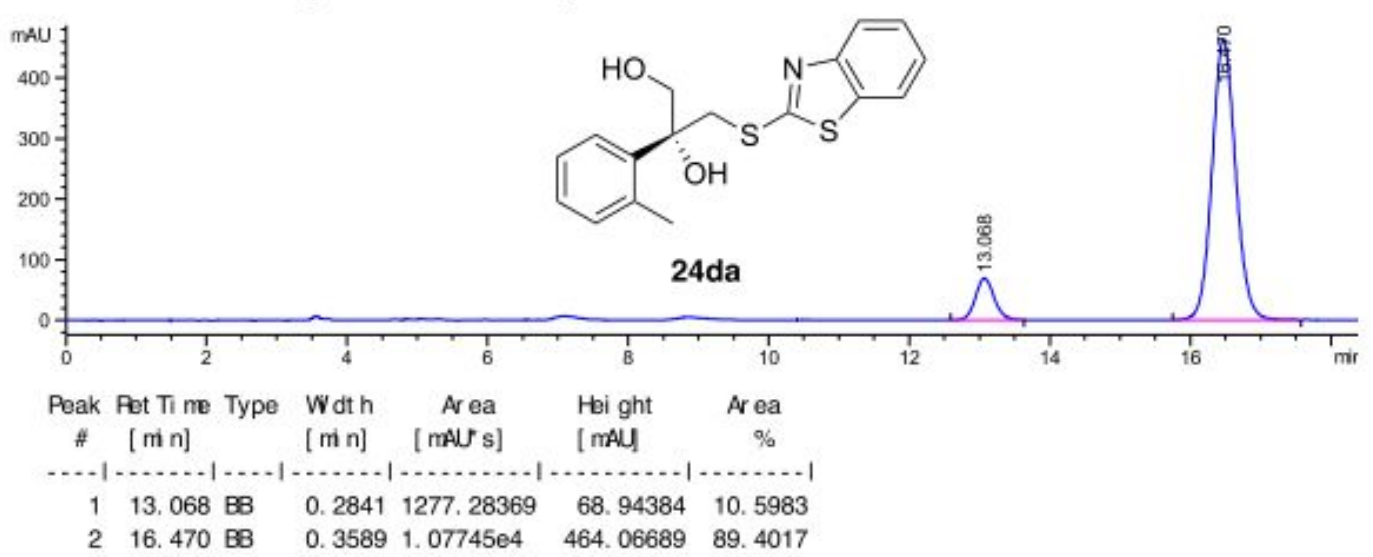

\section{HPLC of rac 24da}

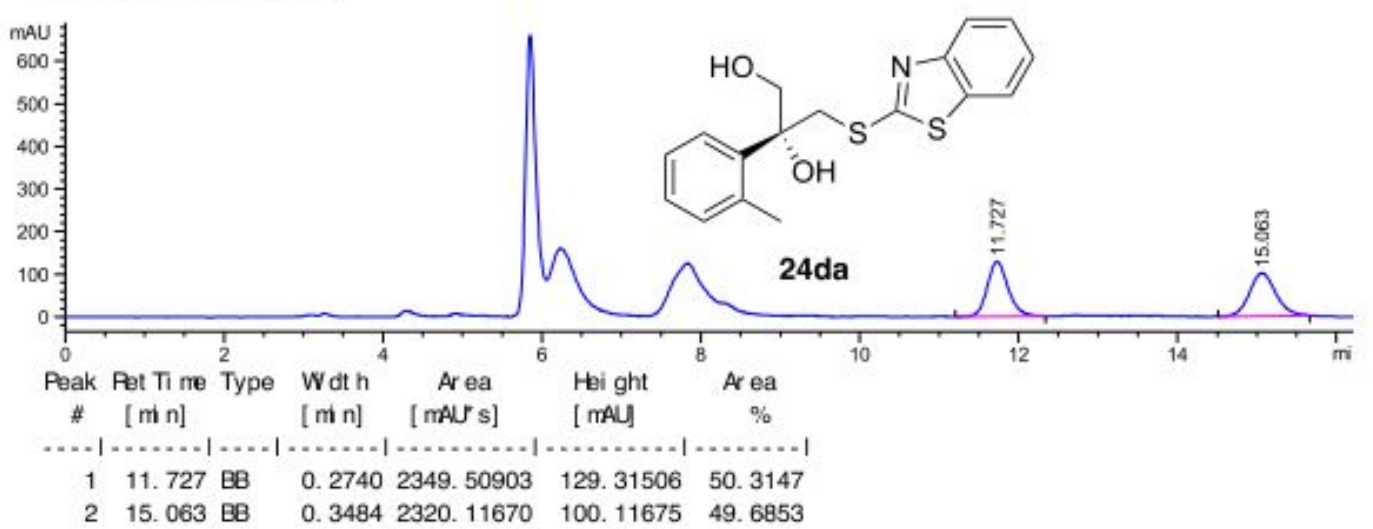

\section{HPLC of 24da (result for Cat g)}

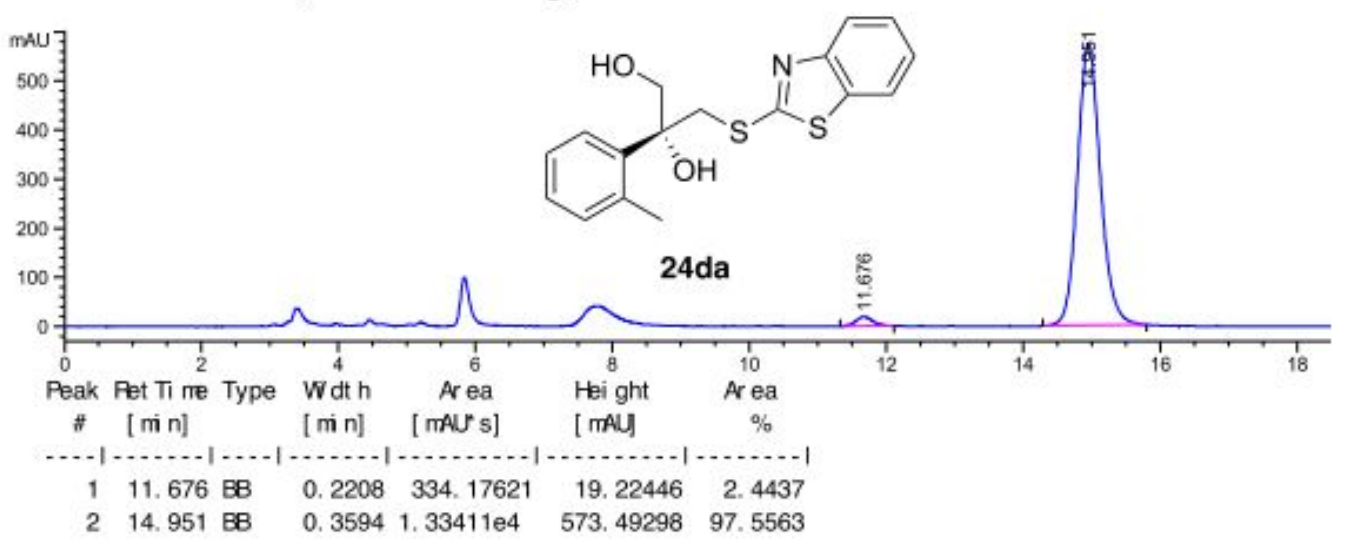




\section{HPLC of rac 24ea}

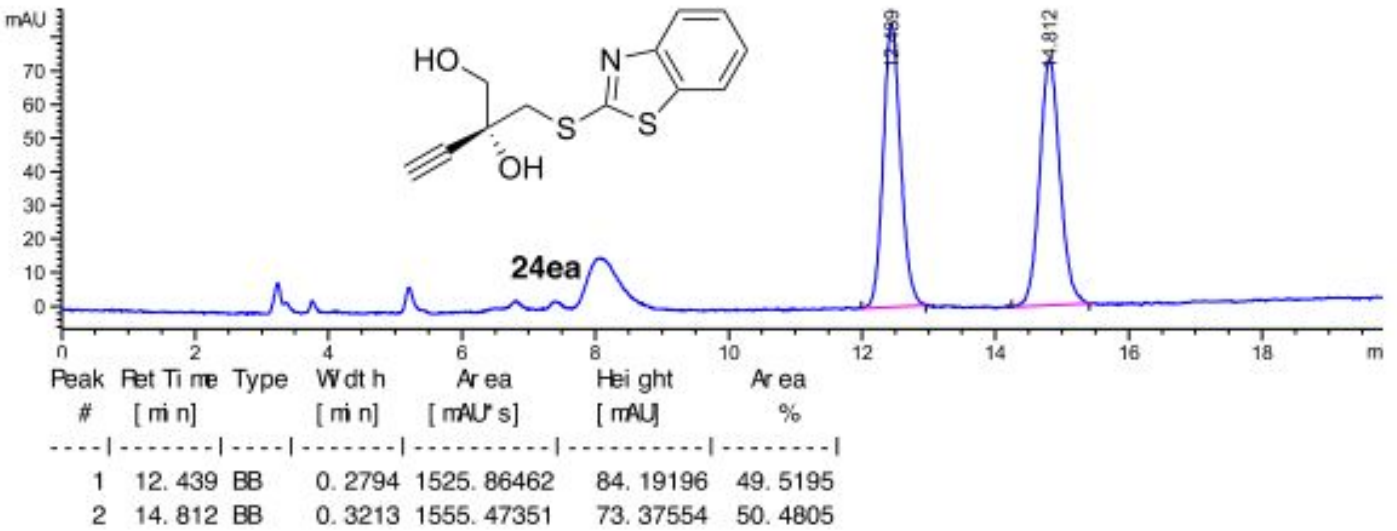

HPLC of 24ea (result for Cat f)

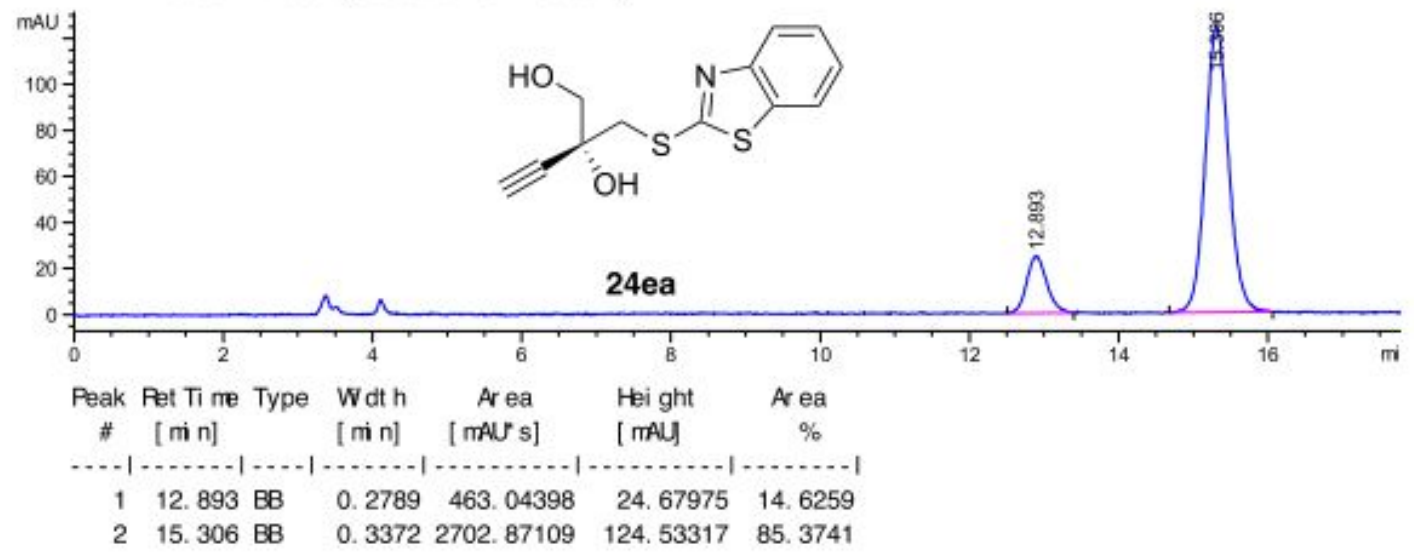

HPLC of 24ea (result for Cat g)

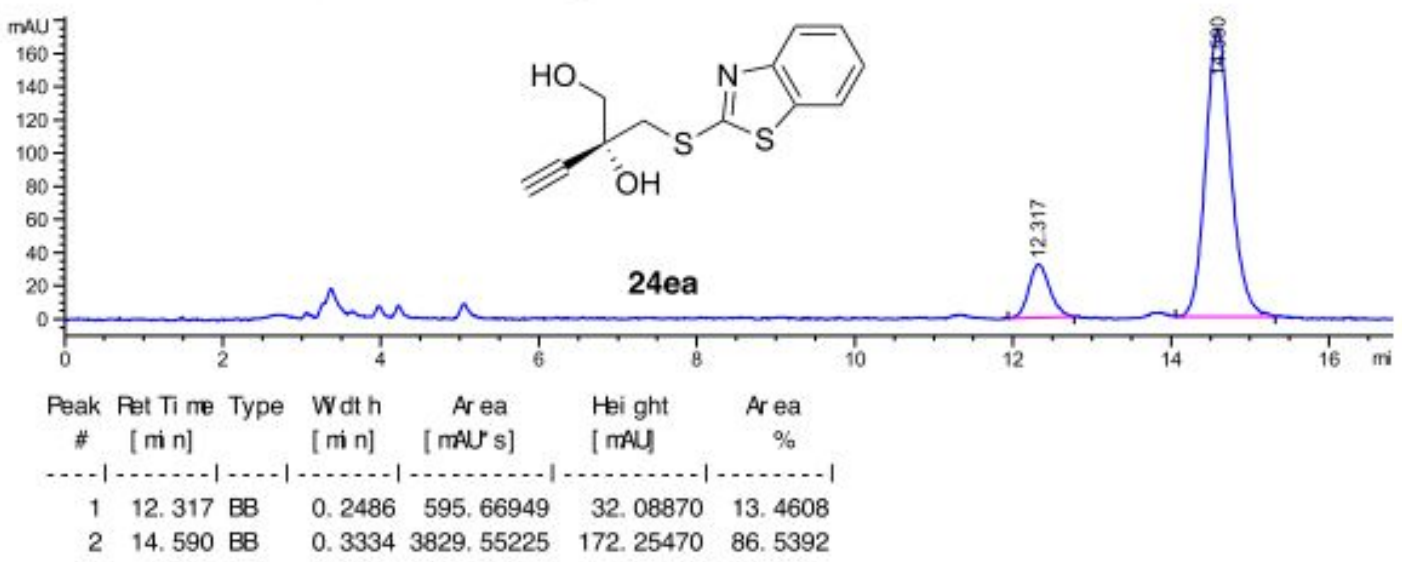




\section{HPLC of rac $\mathbf{2 4 f a}$}

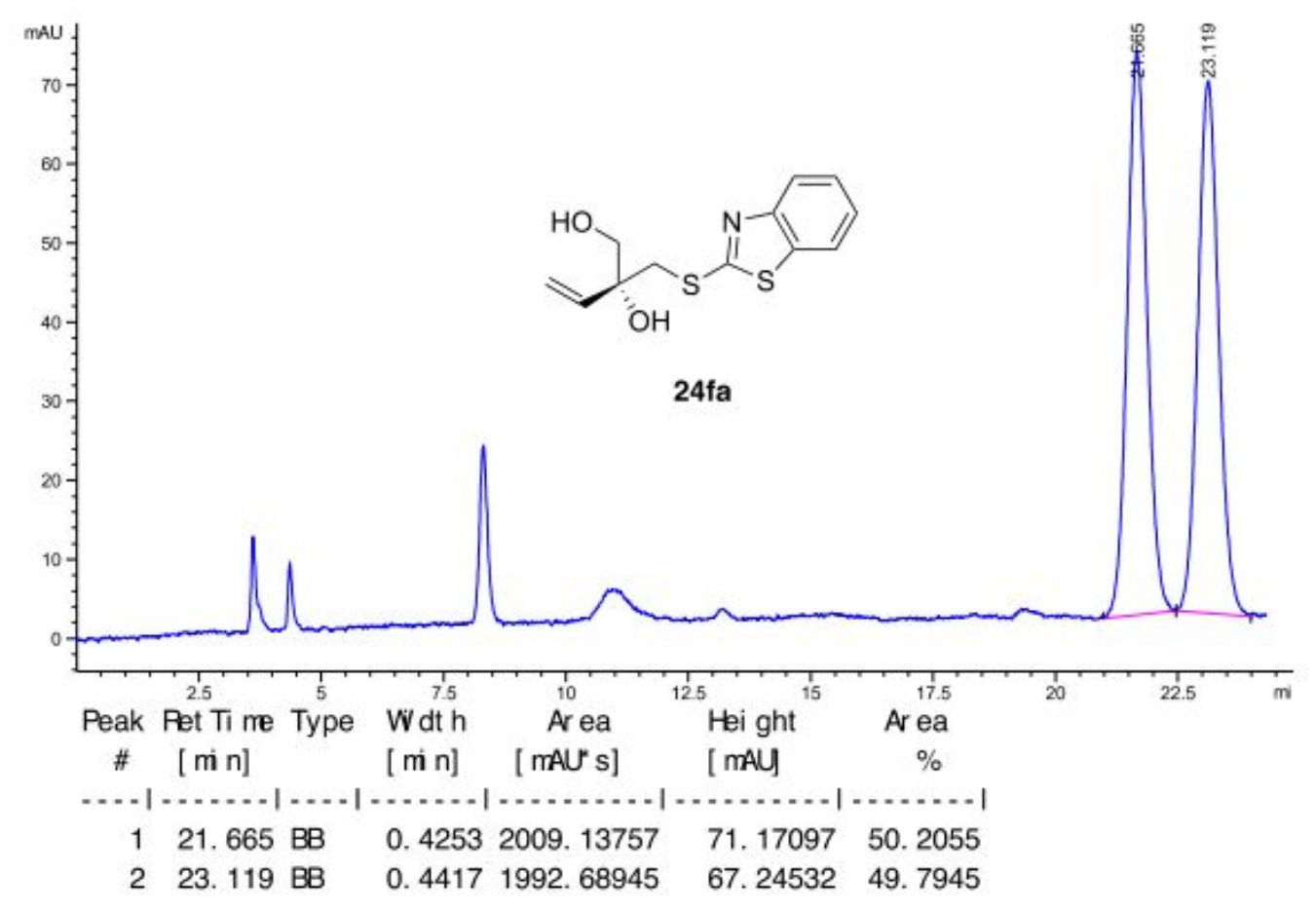

HPLC of $\mathbf{2 4 f a}$ (result for Cat f)

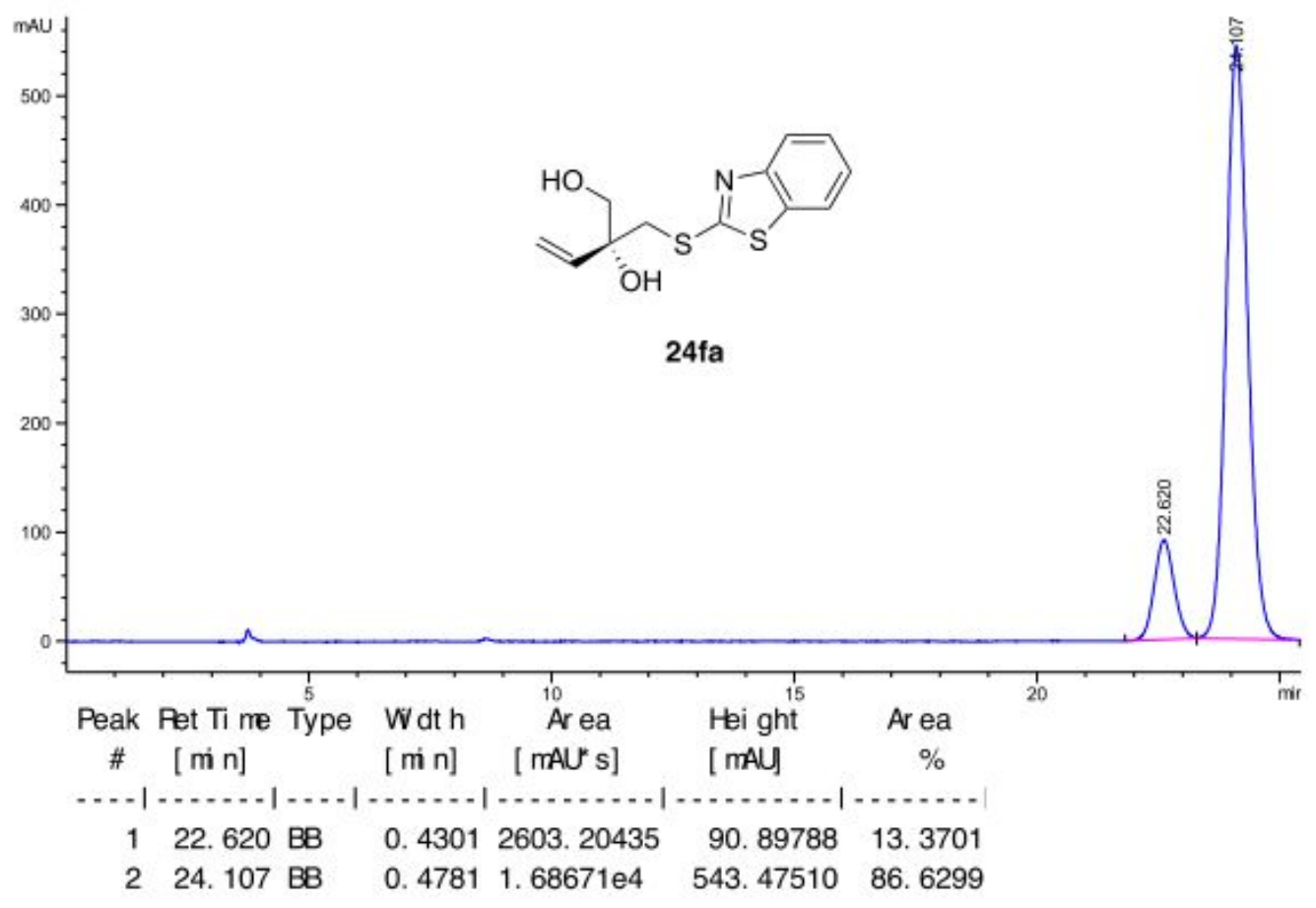


HPLC of rac 24ga

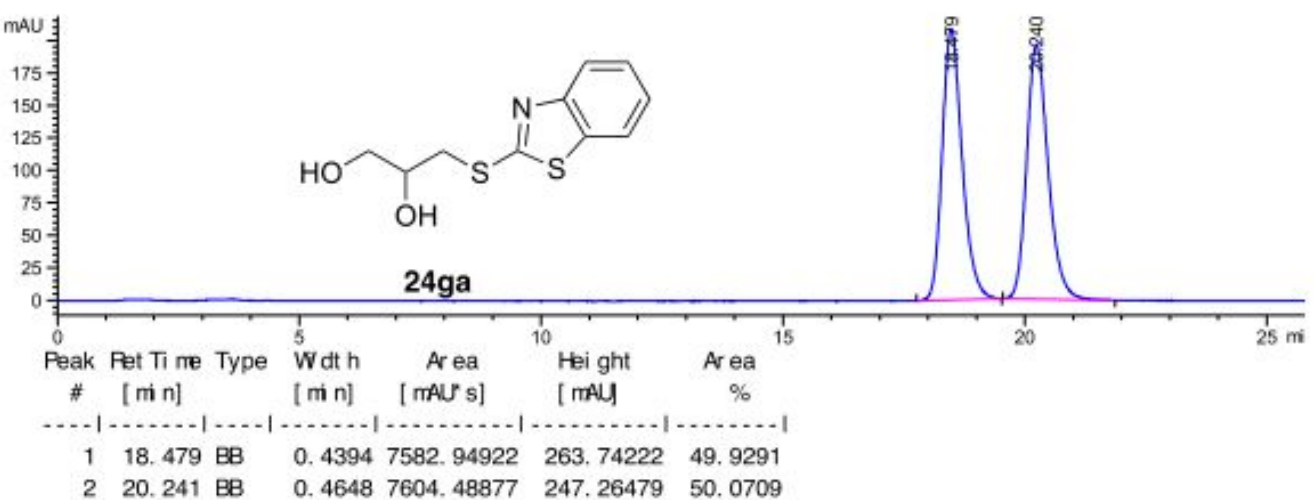

HPLC of 24ga (result for Cat f)

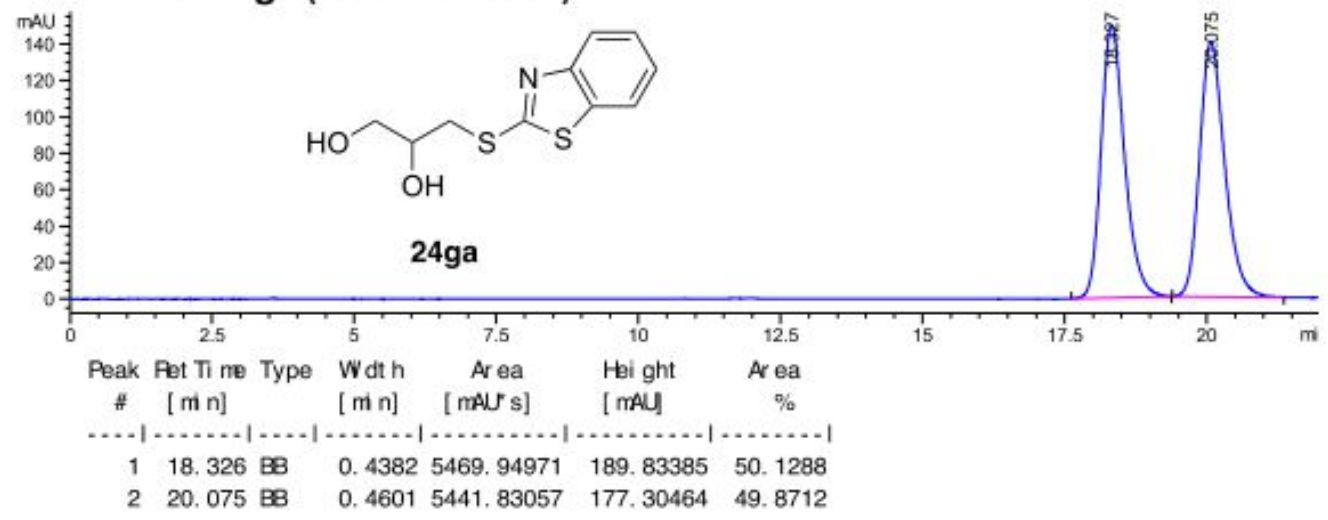

HPLC of rac 24ga

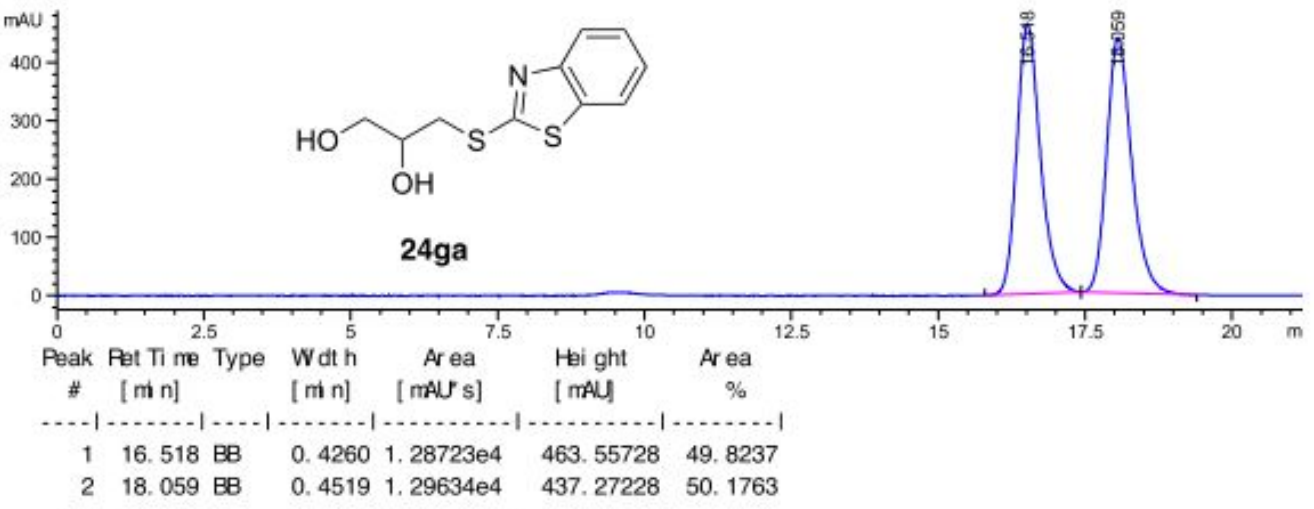

HPLC of 24ga (result for Cat g)

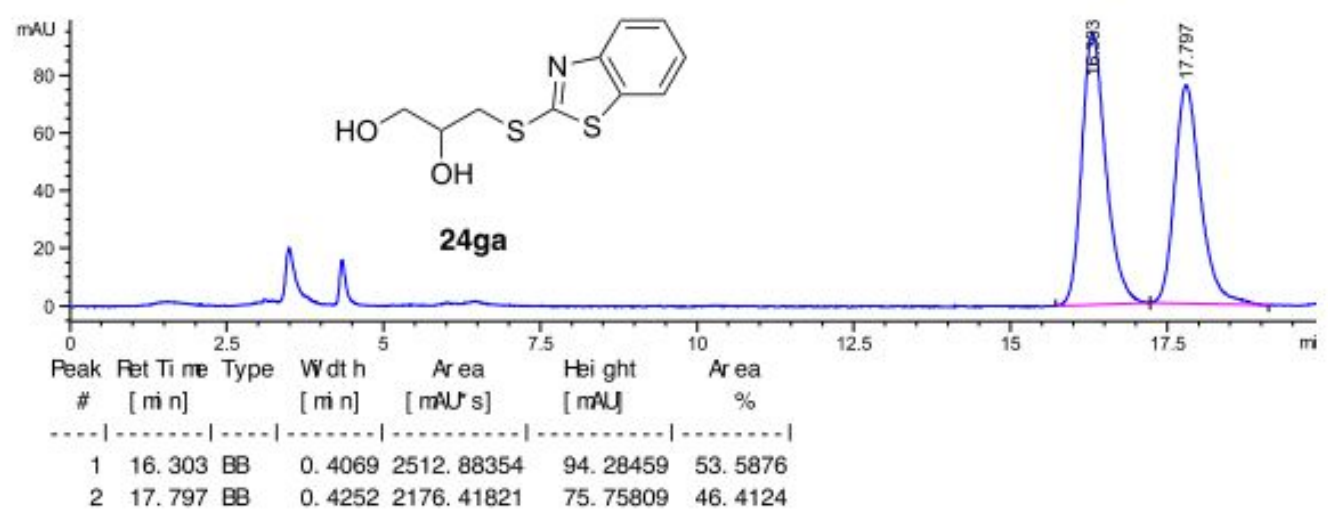


HPLC of rac 24ha

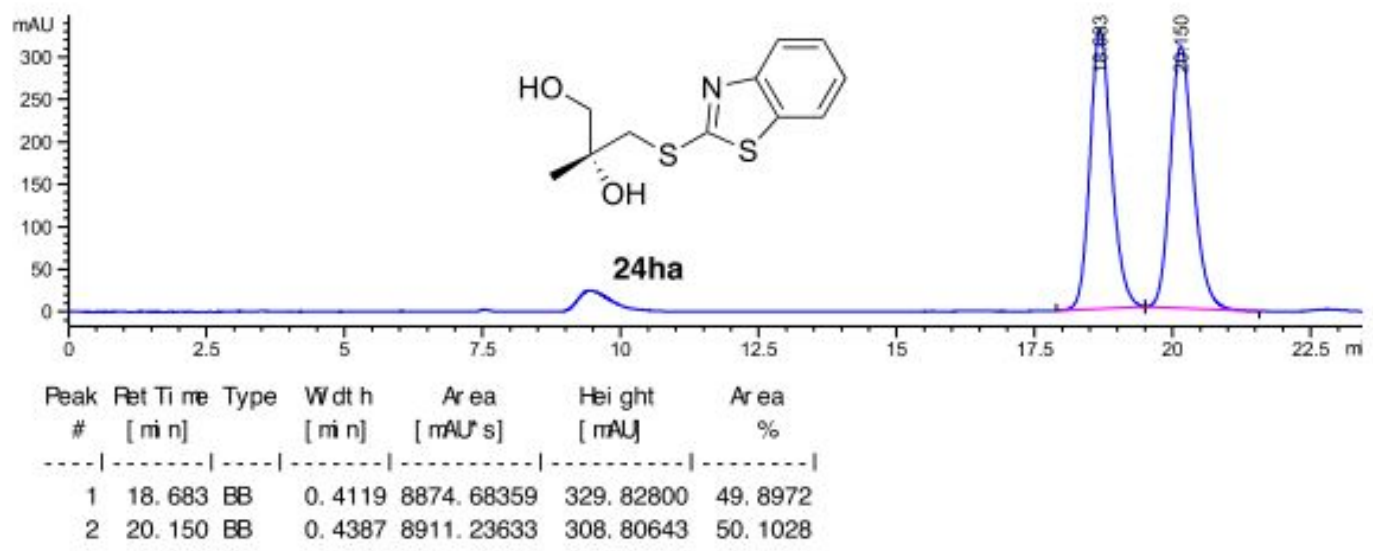

\section{HPLC of 24ha (result for Cat f)}

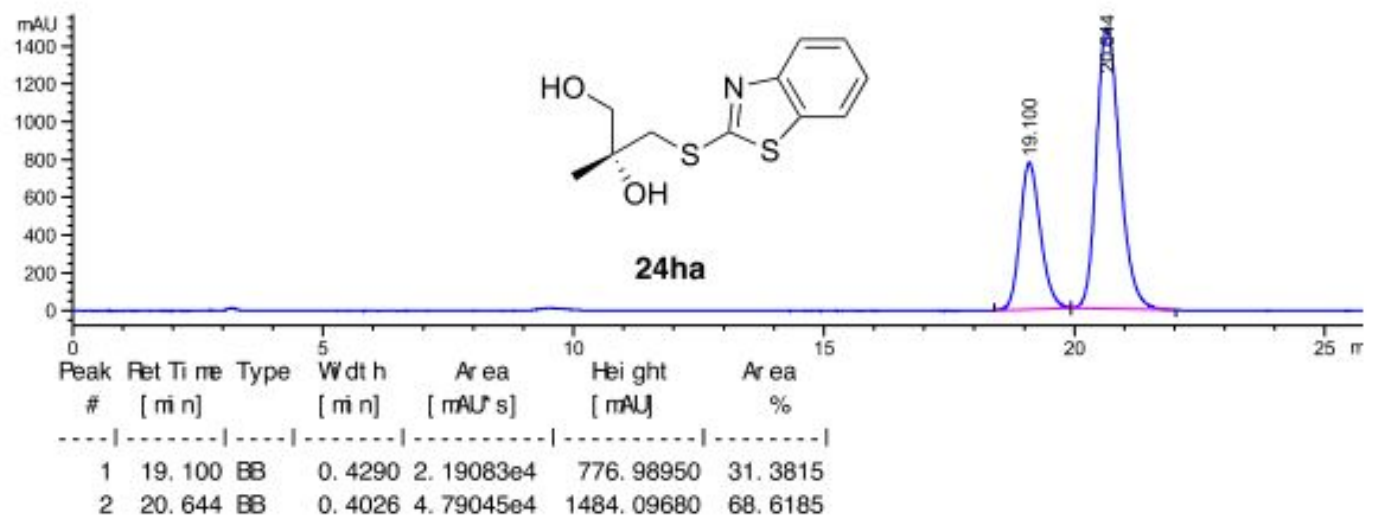

HPLC of 24ha (result for Cat g)

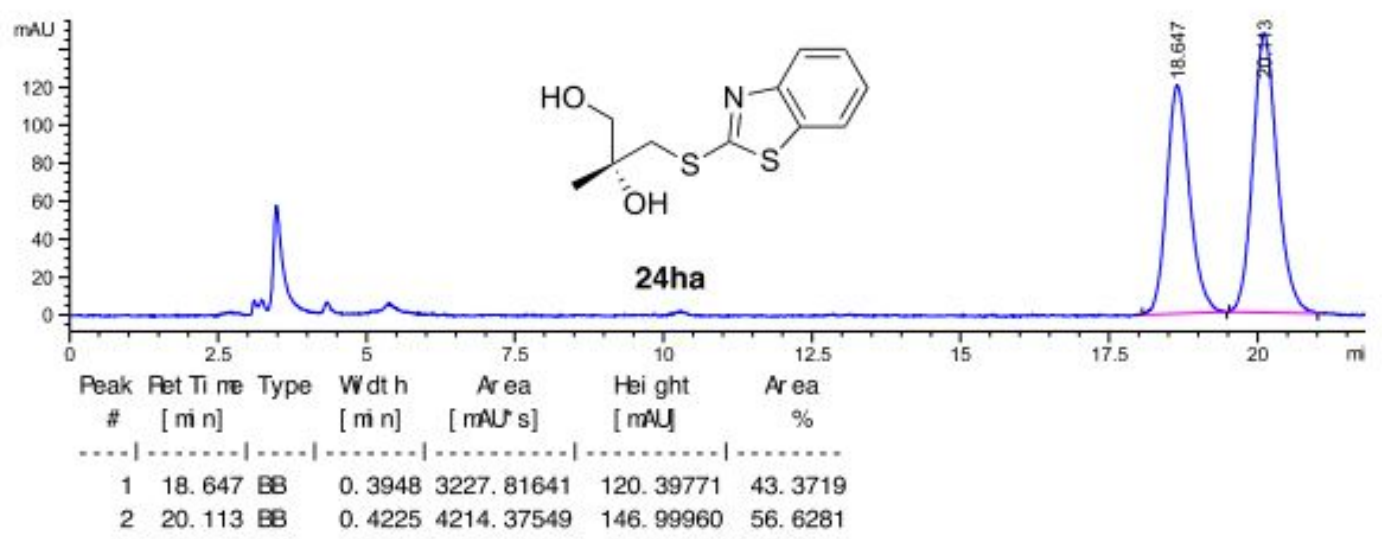


HPLC of rac 24ia

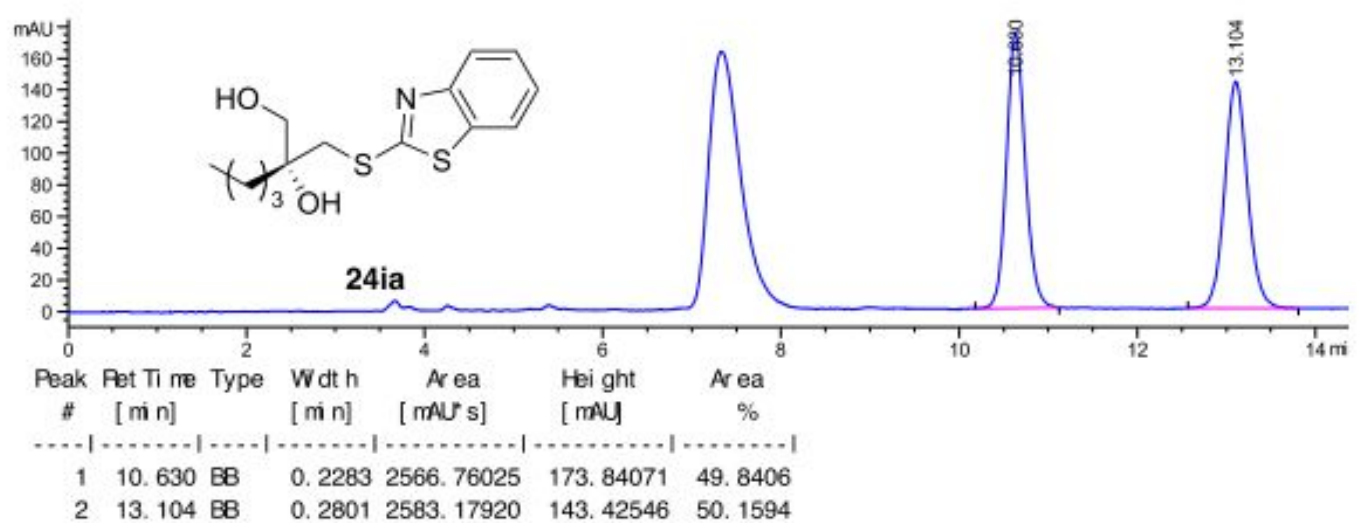

\section{HPLC of 24ia (result for Cat $f$ )}

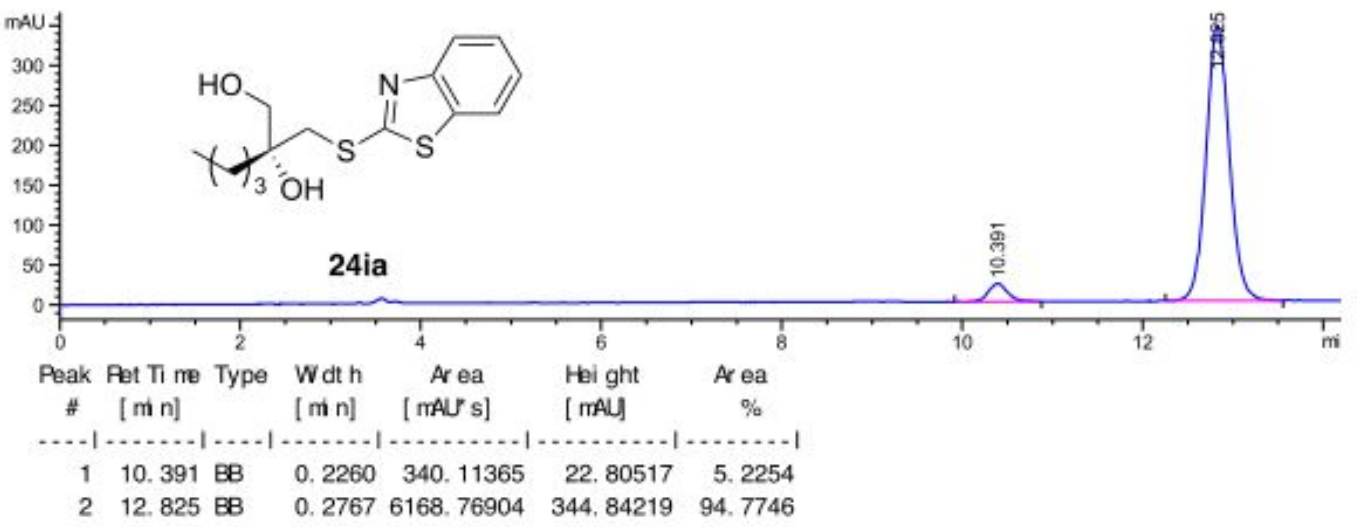

\section{HPLC of rac 24ia}

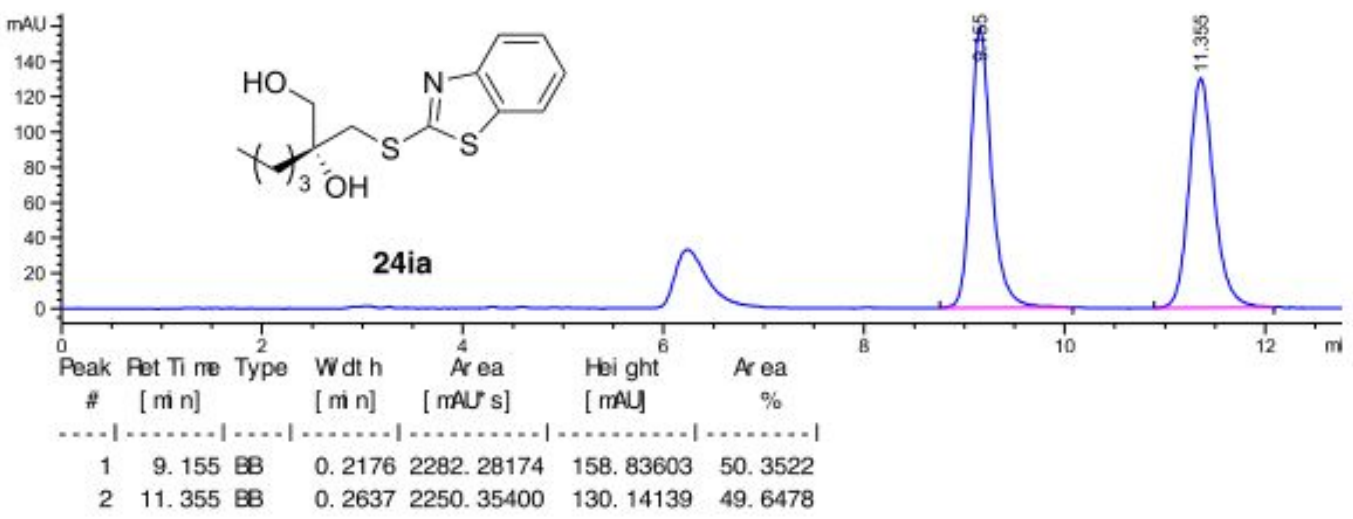

HPLC of 24ia (result for Cat g)

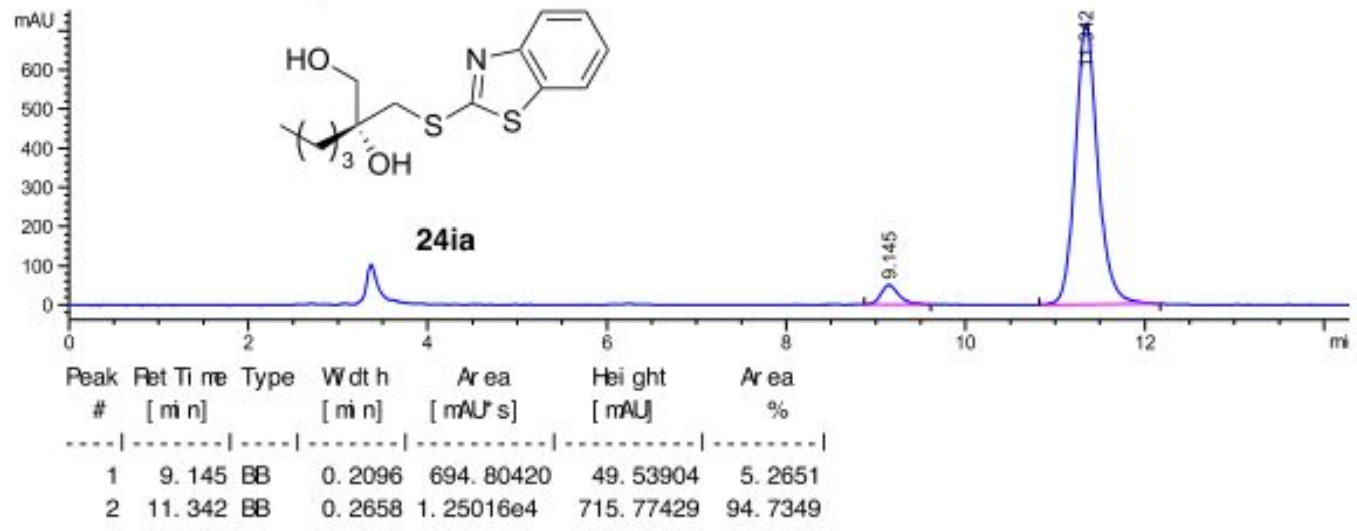


HPLC of rac 24ja

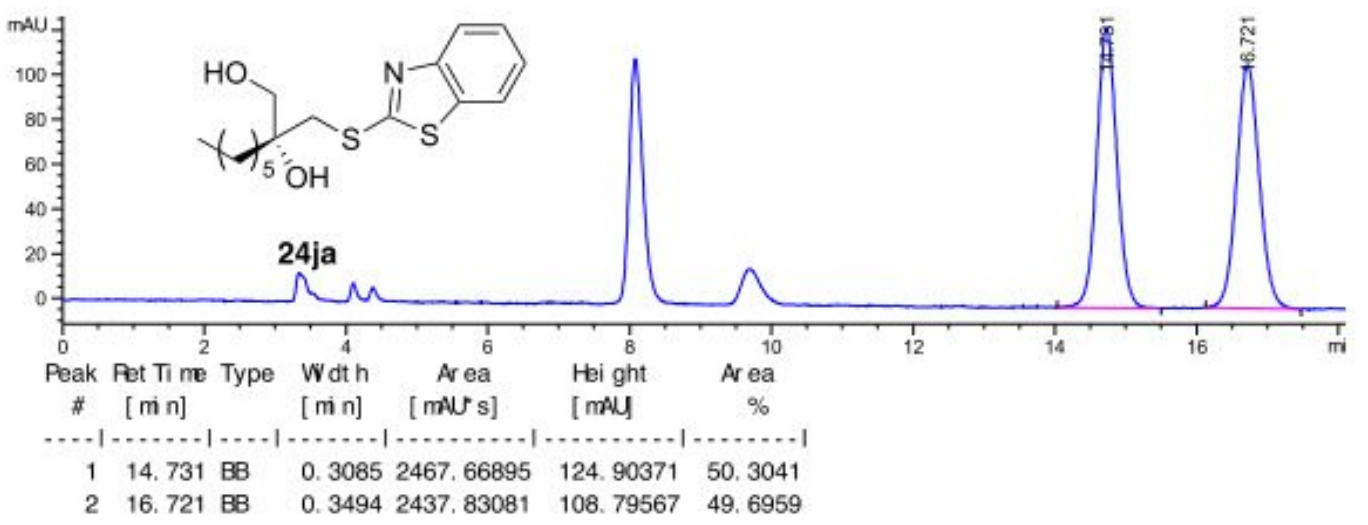

HPLC of 24ja (reșult for Cat f)

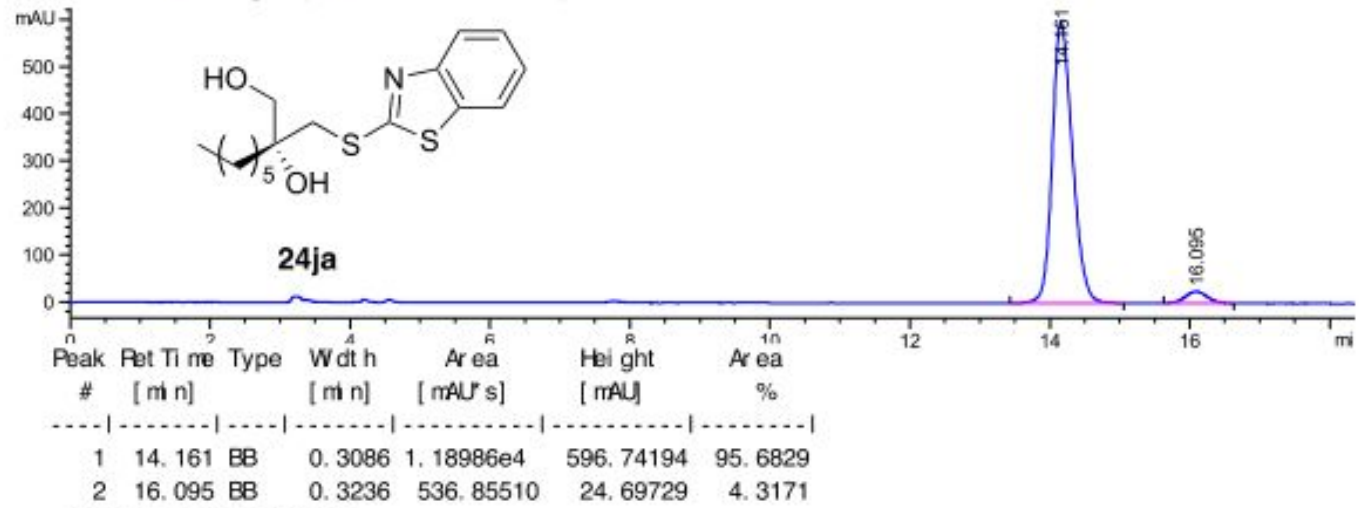

HPLC of rac 24ja

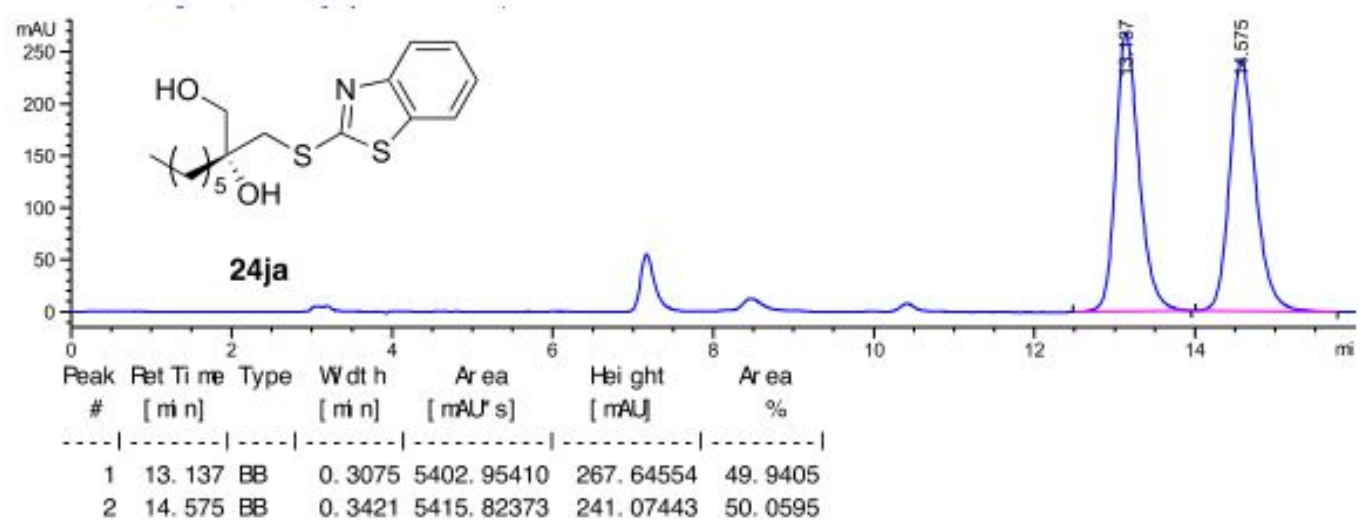

HPLC of 24ja (result for Cat g)

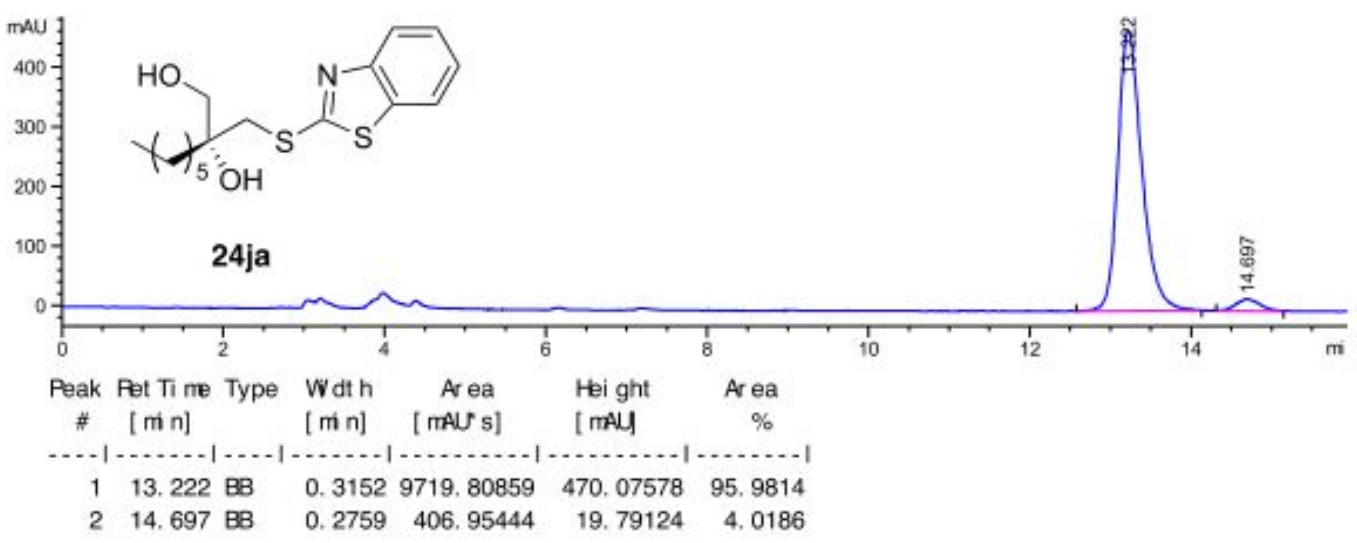




\section{HPLC of rac 24ka}

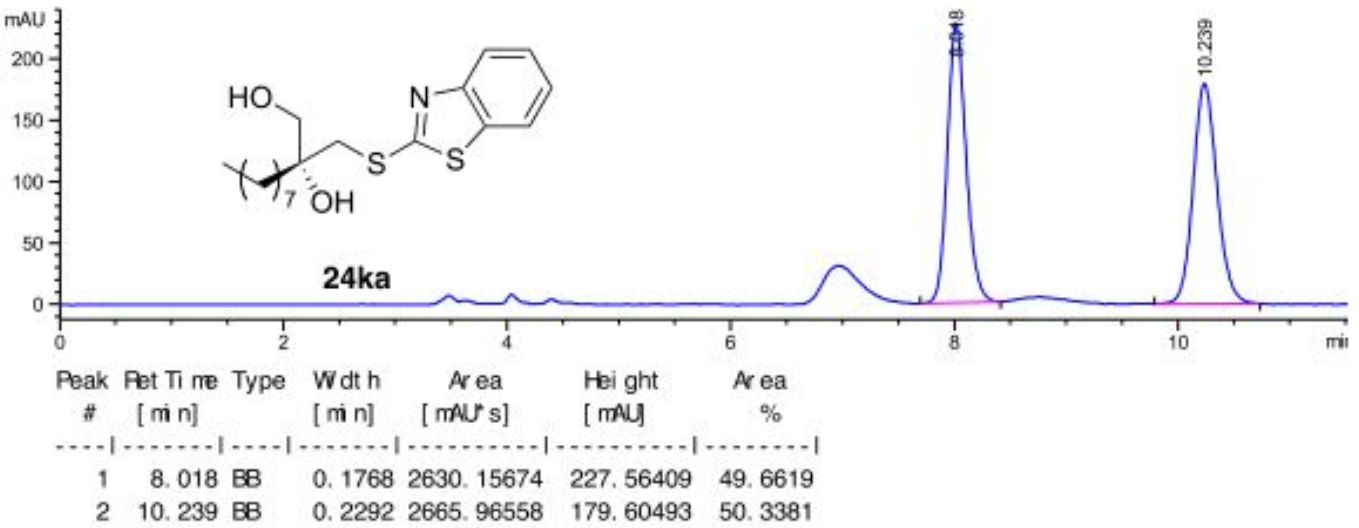

\section{HPLC of 24ka (result for Cat f)}

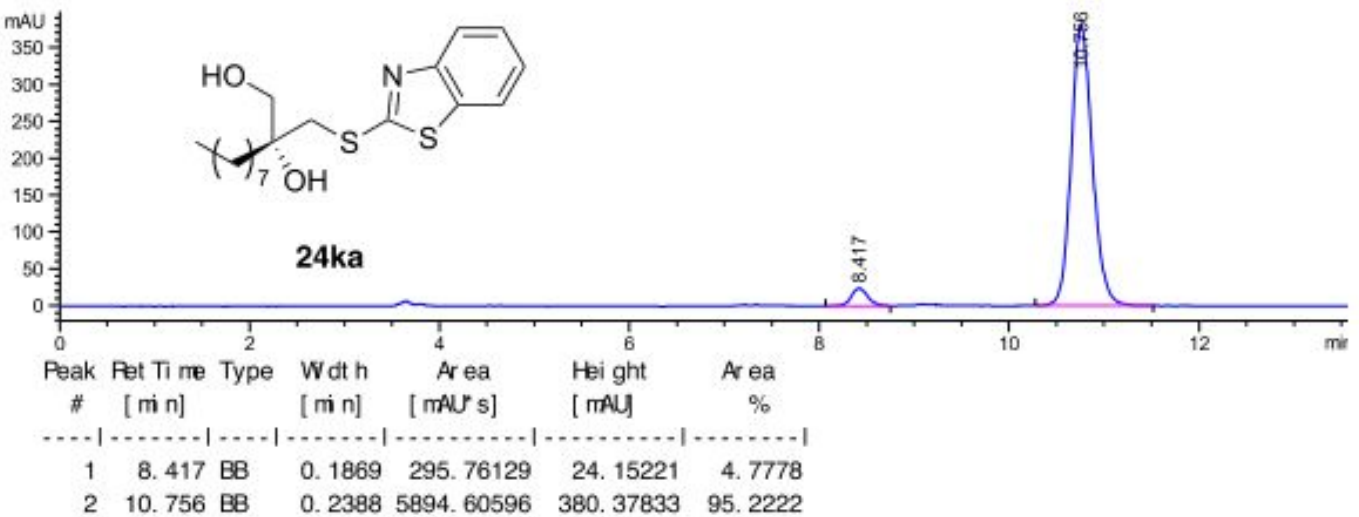

\section{HPLC of rac 24ka}

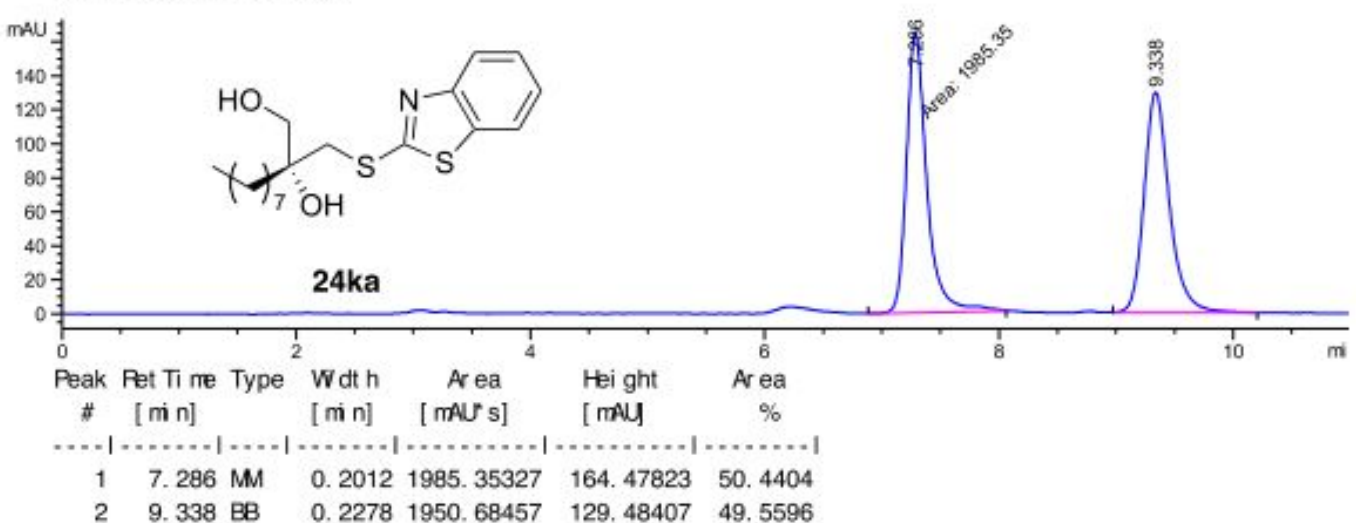

\section{HPLC of 24ka (result for Cat g)}

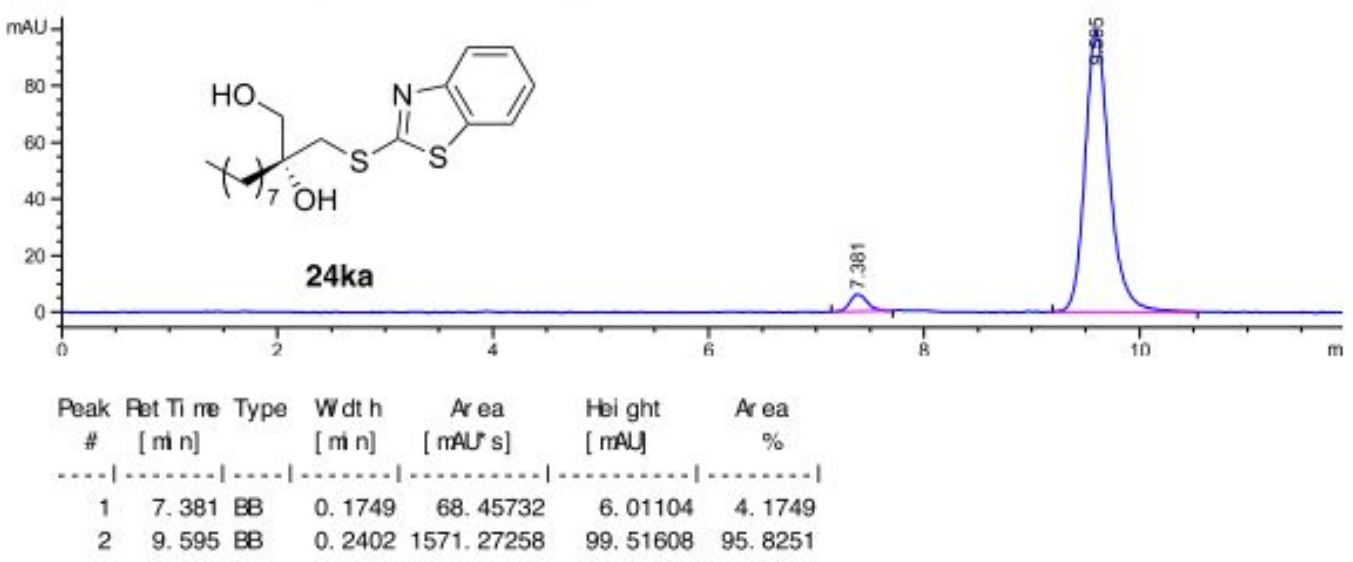




\section{HPLC of rac 24la}

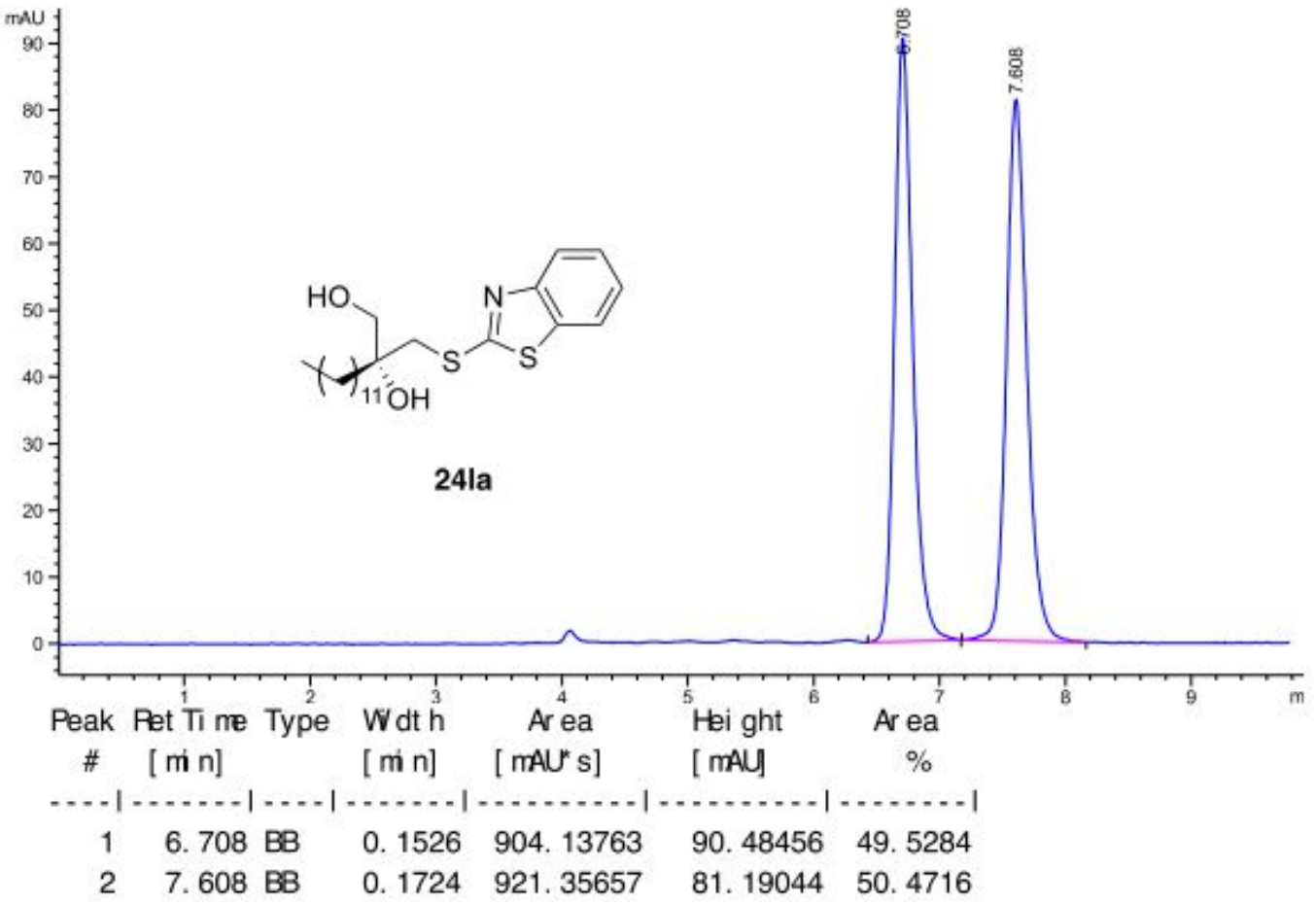

HPLC of 24la (result for Cat f)

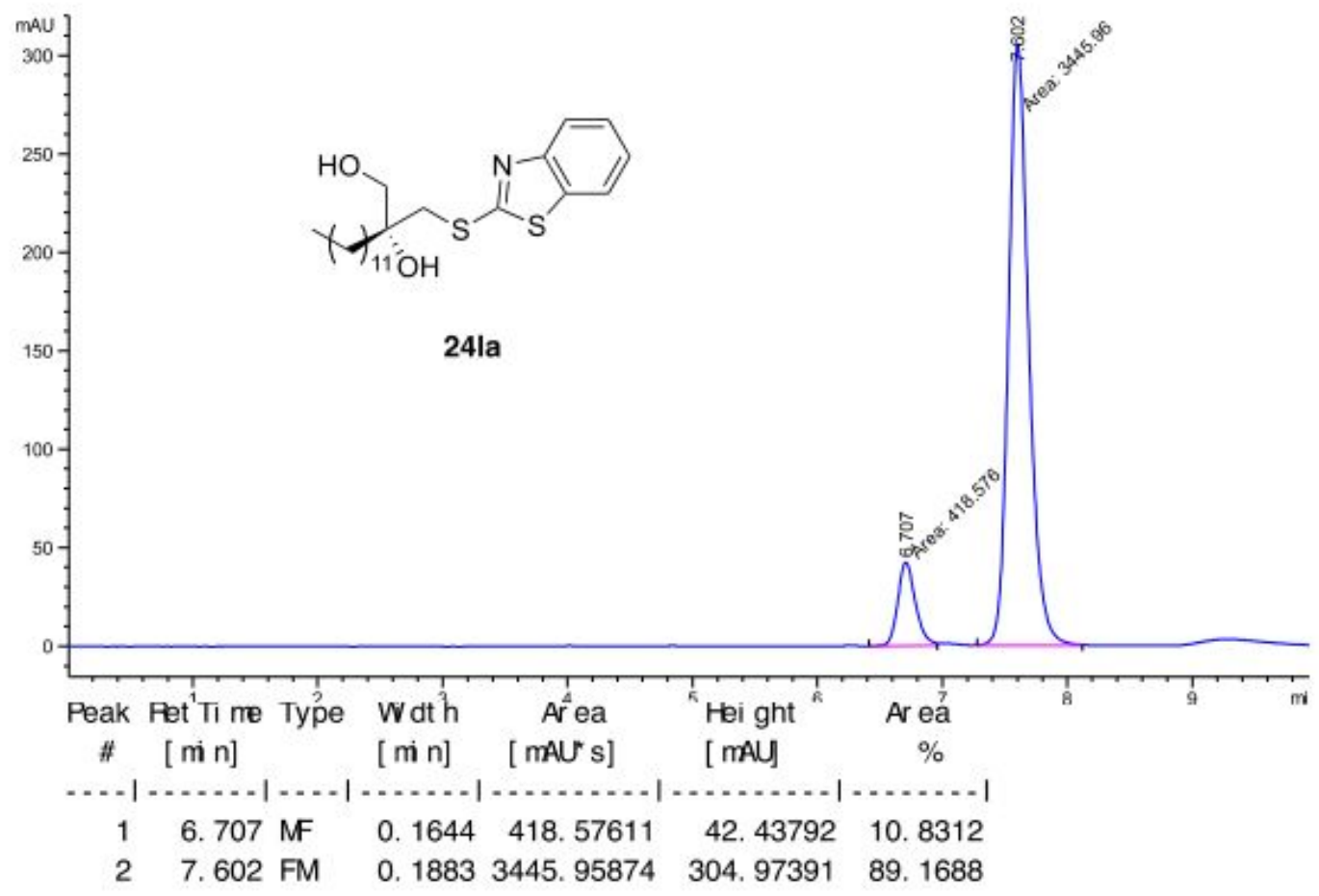




\section{HPLC of rac 24ma}

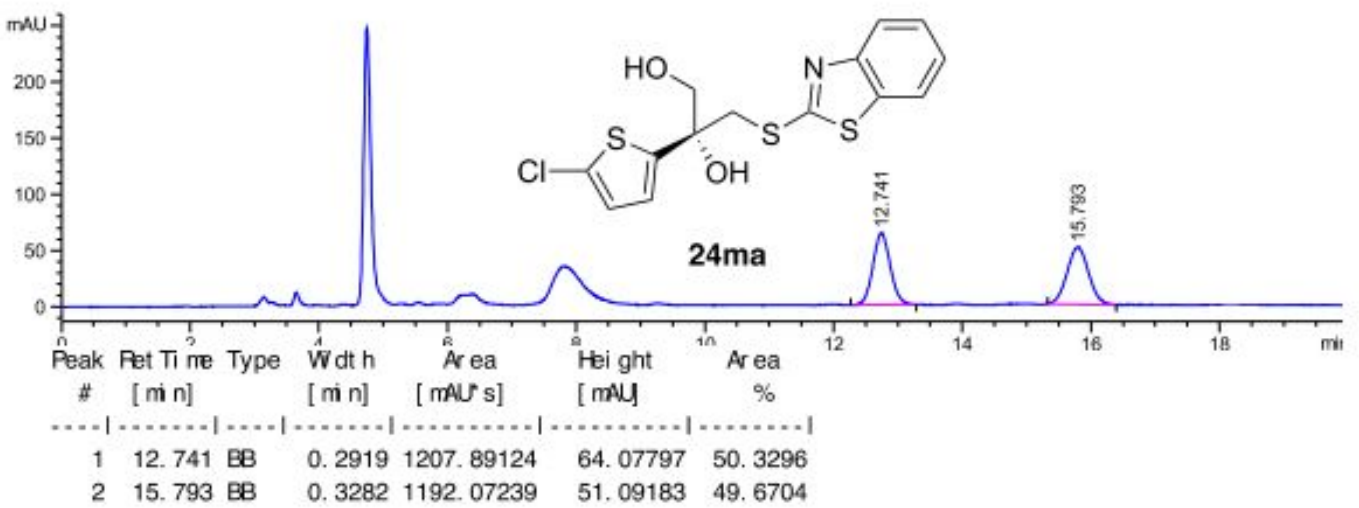

HPLC of 24ma (result for Cat $f$ )

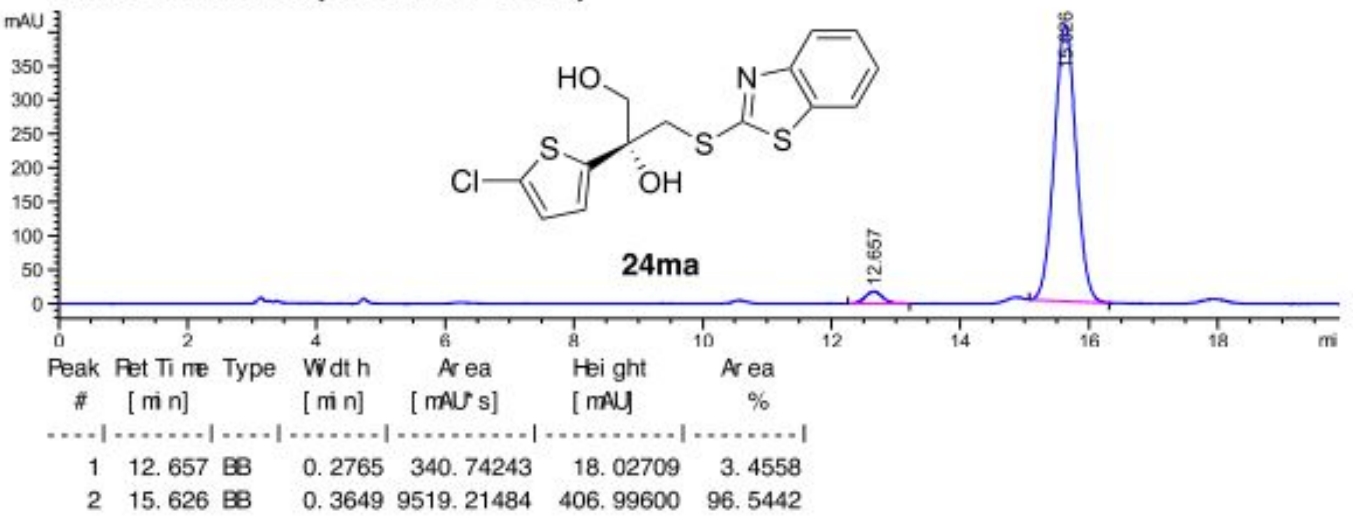

HPLC of rac 24ma

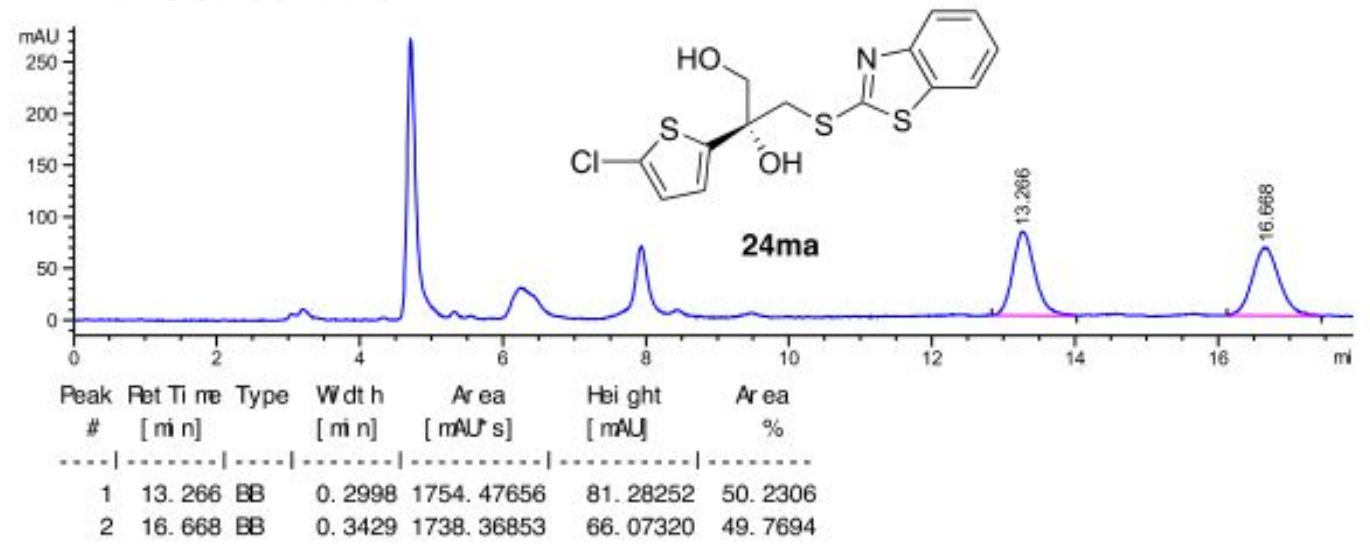

\section{HPLC of 24ma (result for Cat $\mathbf{g}$ )}

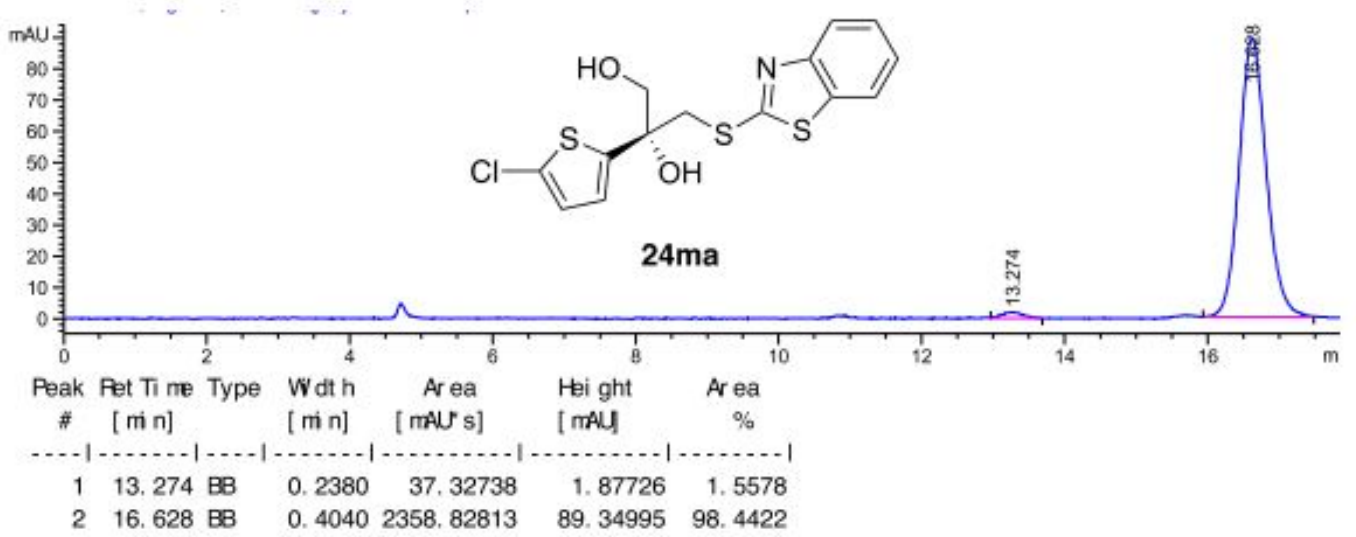


HPLC of rac 24na

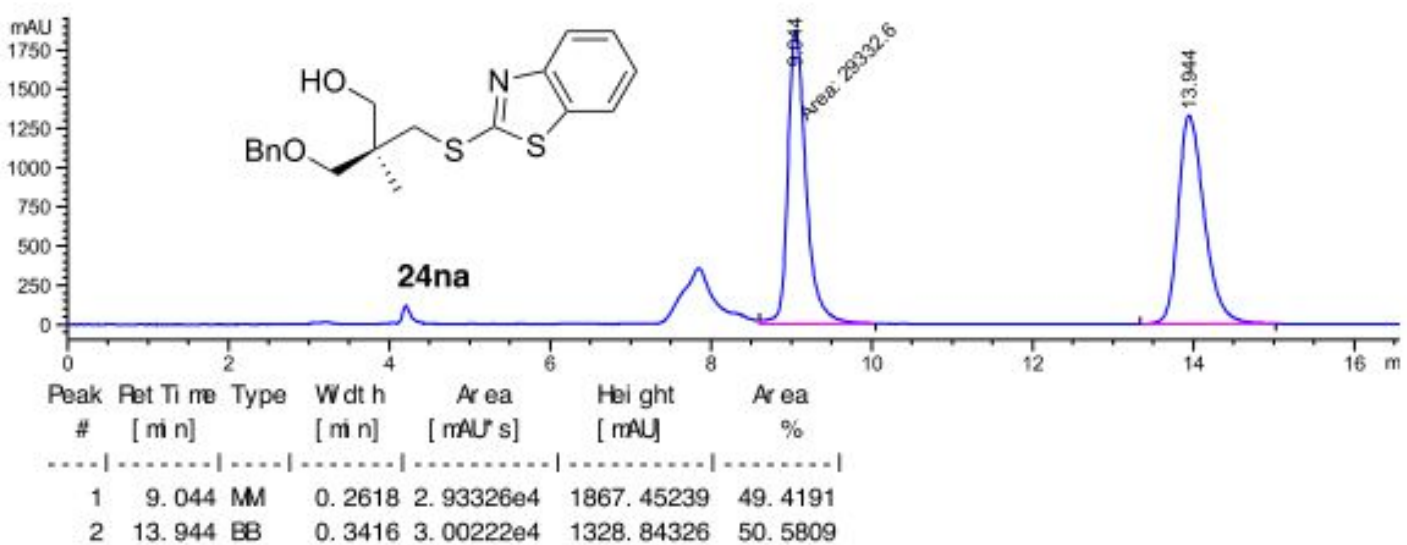

HPLC of 24na (result for Cat f)

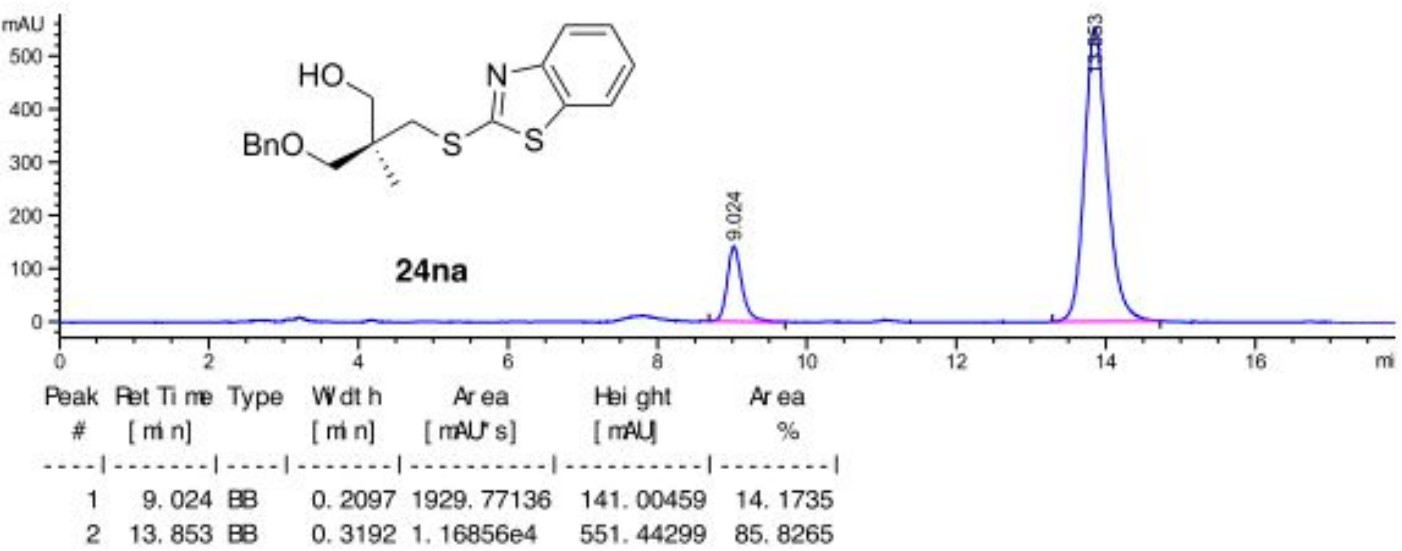

HPLC of 24na (result for Cat g)

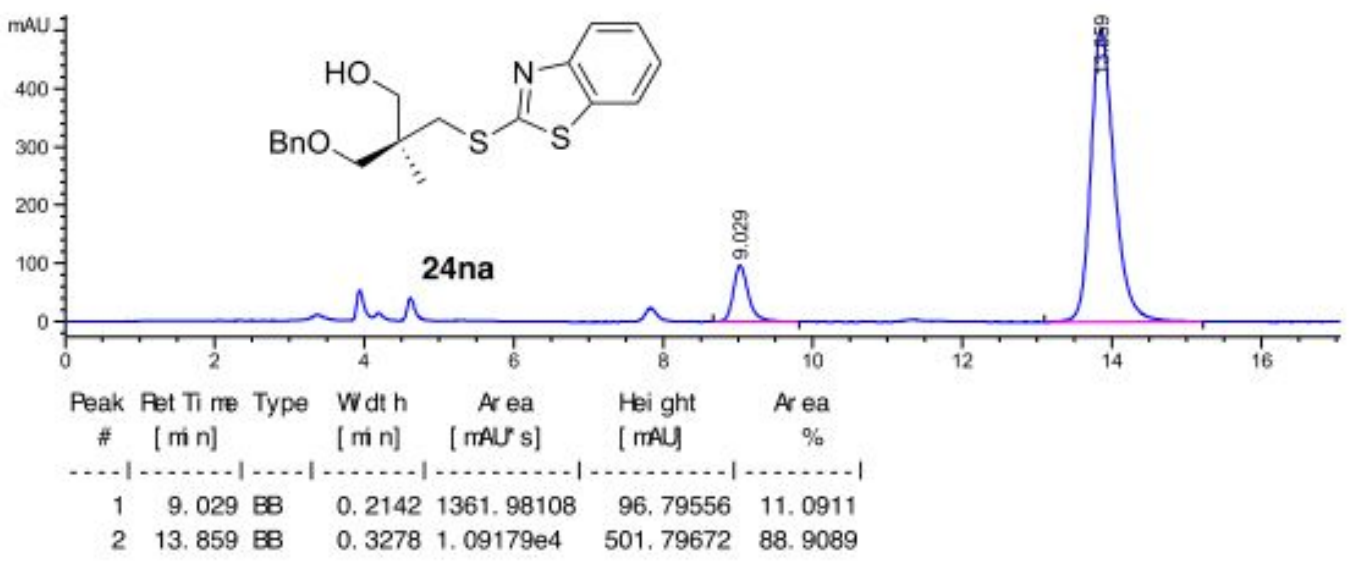




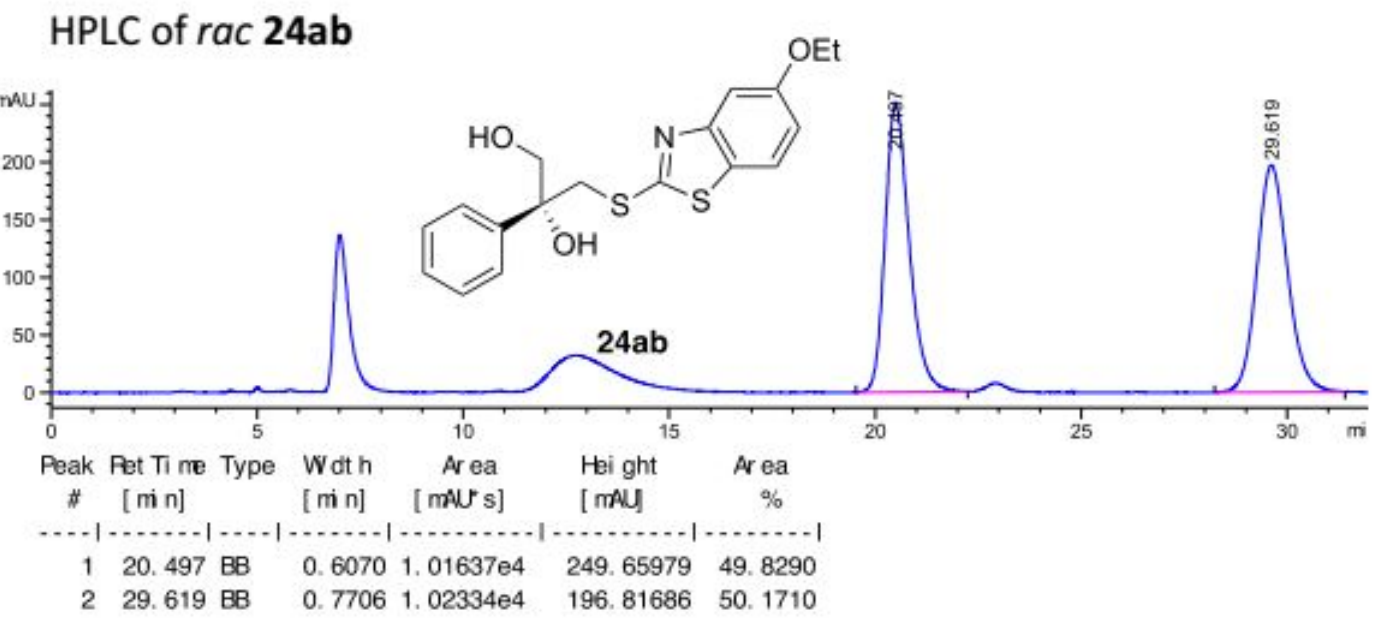

HPLC of 24ab (result for Cat $f$ )

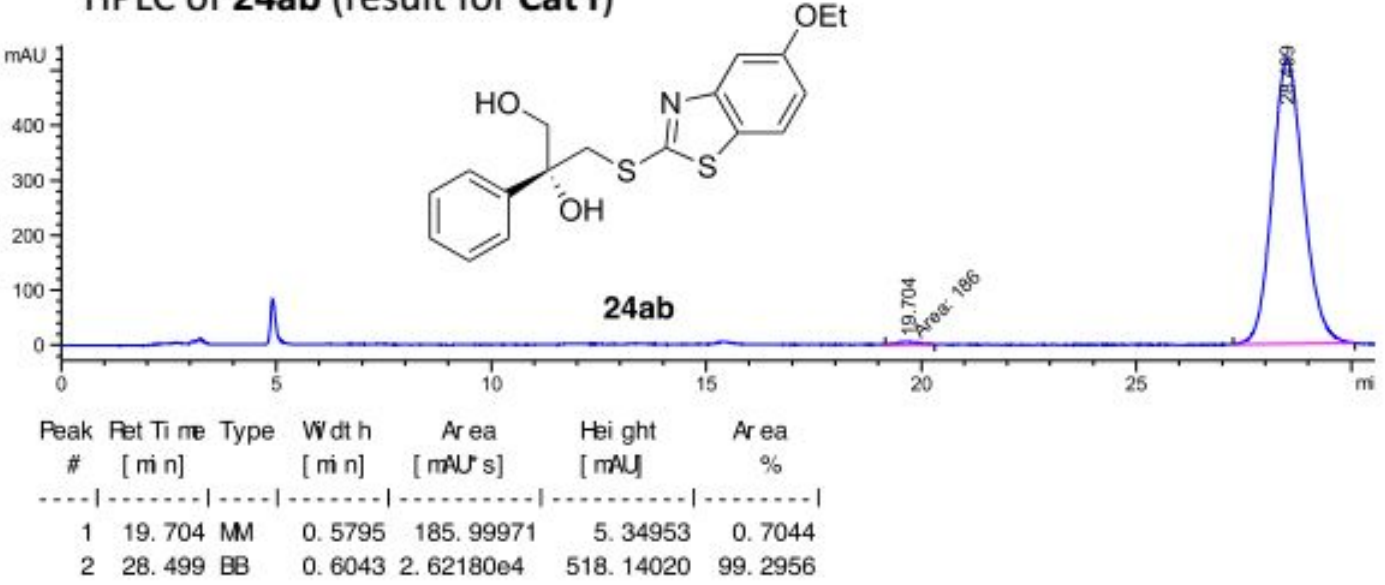

HPLC of 24ab (result for Cat g)

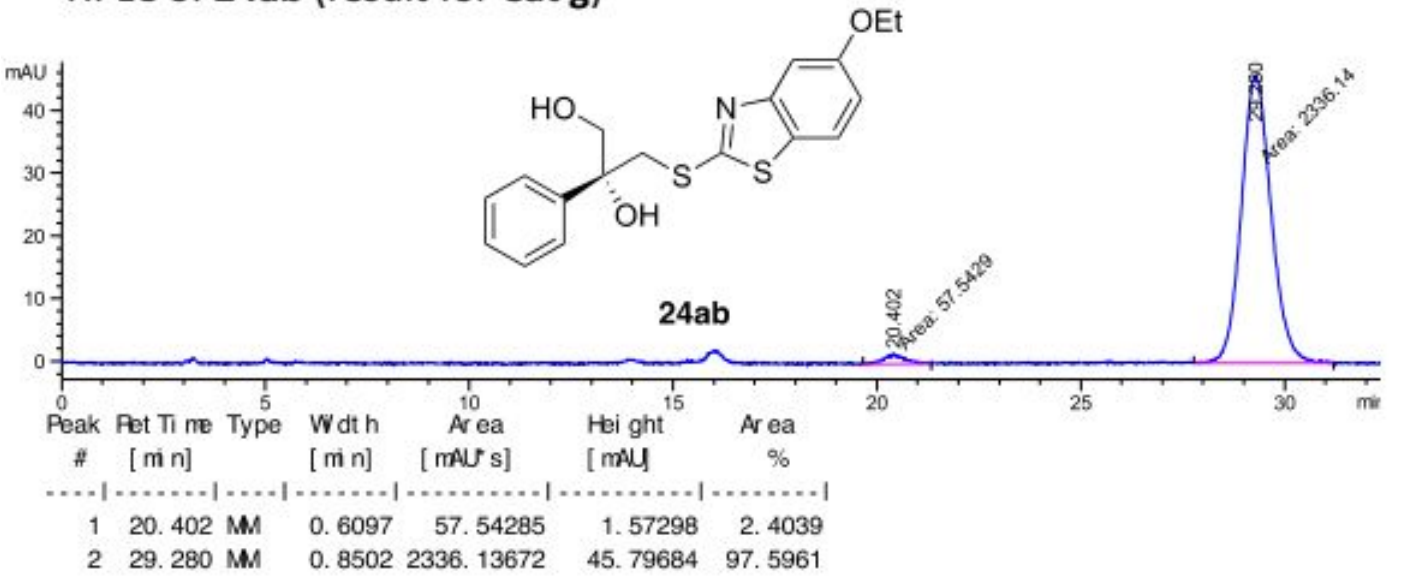


HPLC of rac 24ac

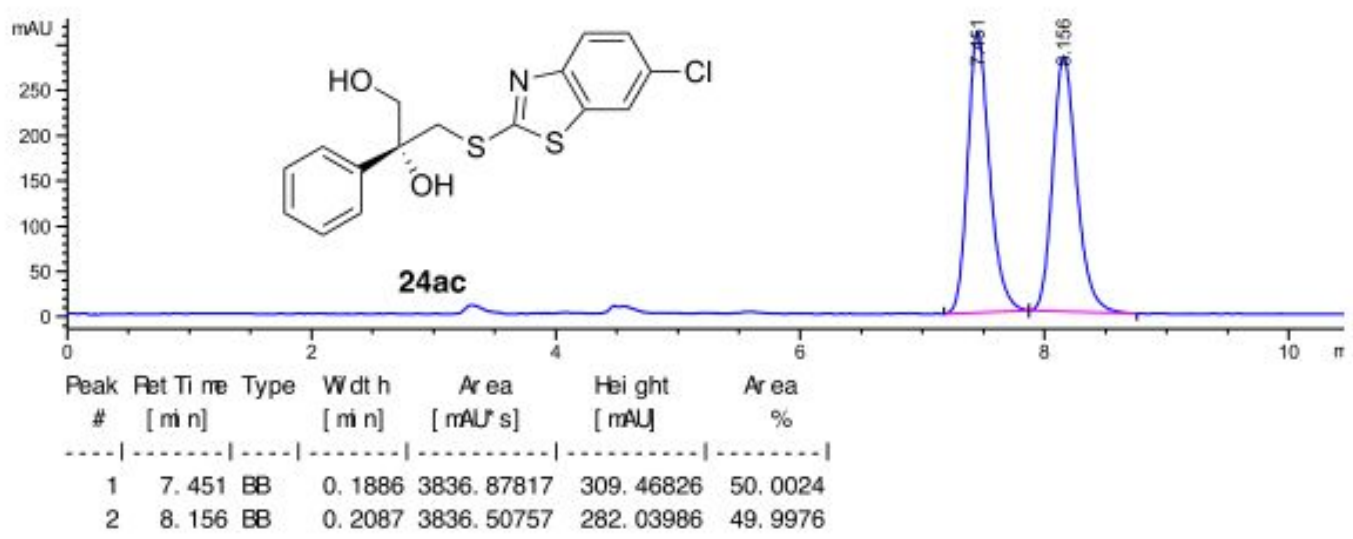

HPLC of 24ac (result for Cat $\mathbf{f}$ )

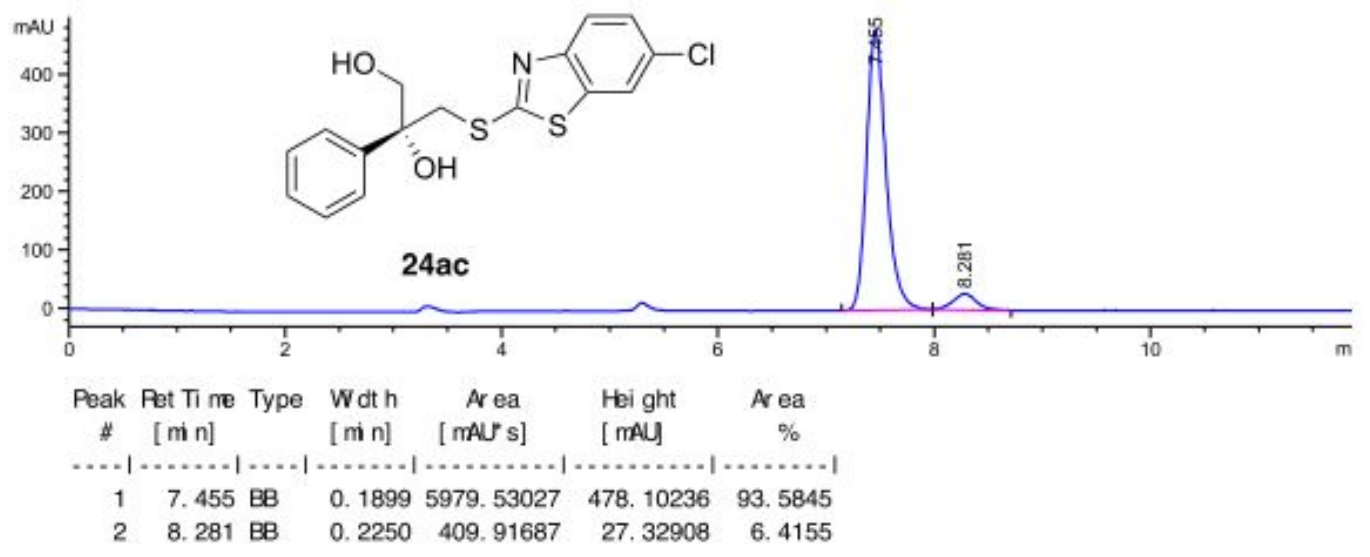

HPLC of 24ac (result for Cat g)

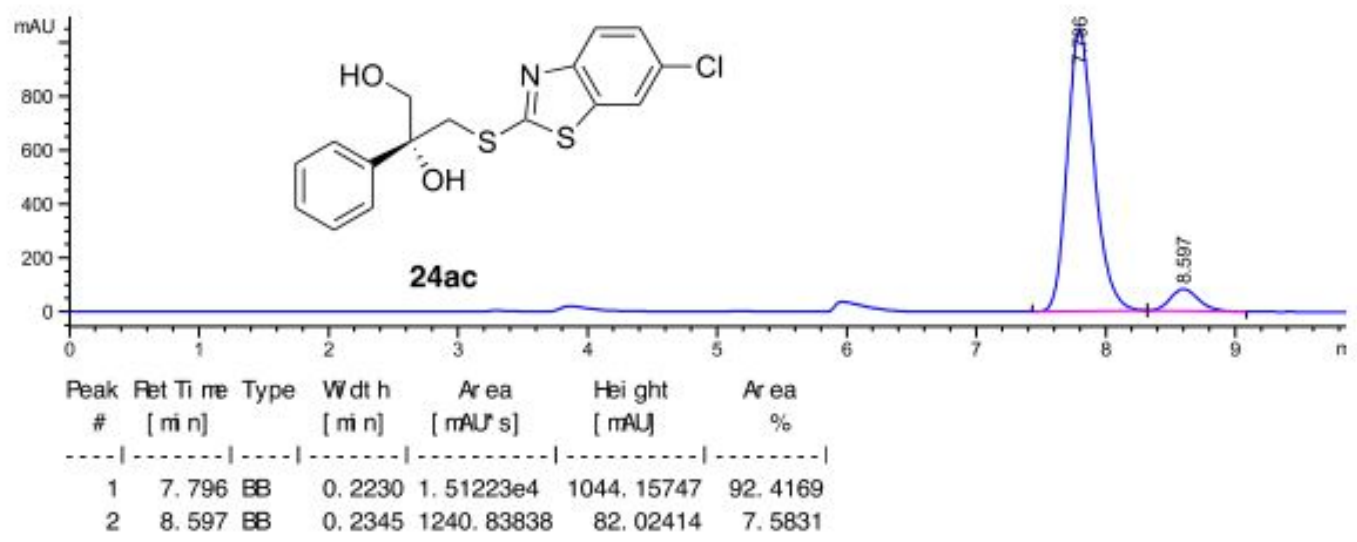


HPLC of rac 24ad

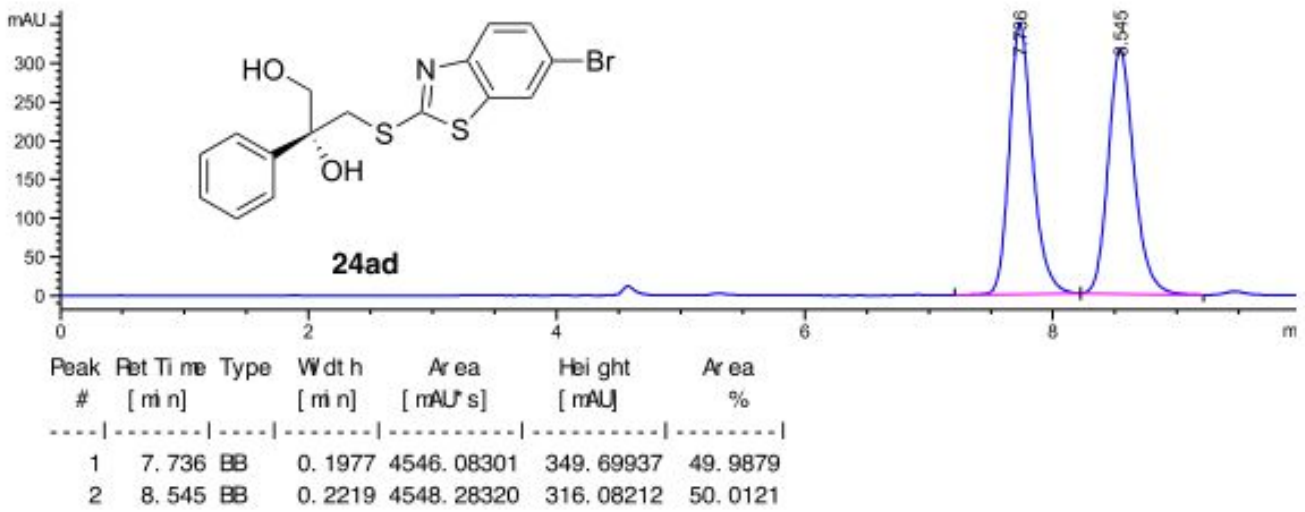

HPLC of 24ad (result for Cat f)

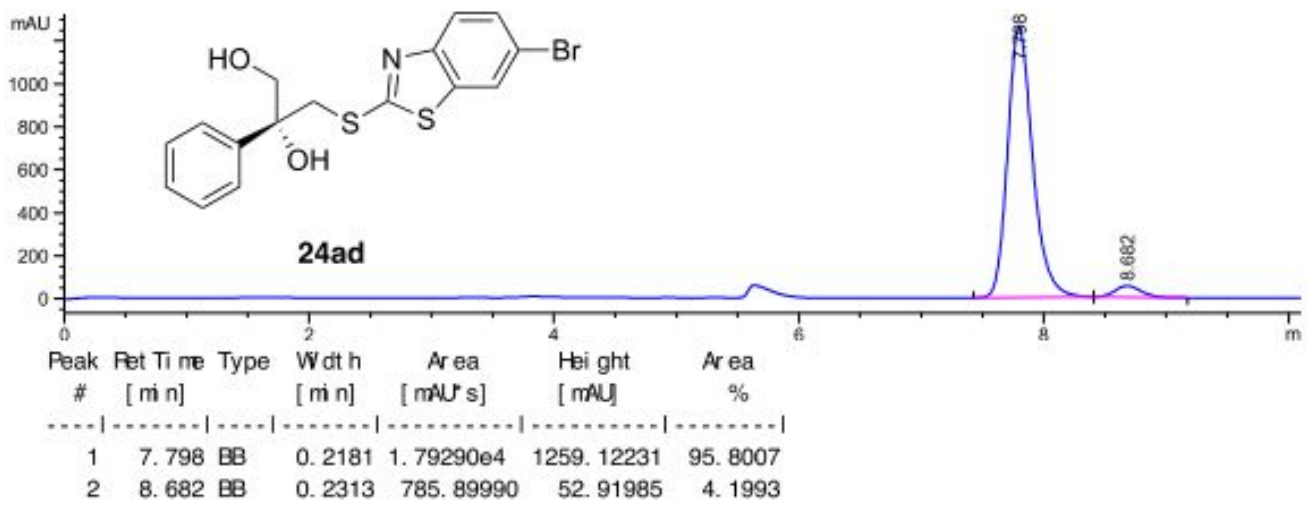

HPLC of 24ad (result for Cat g)

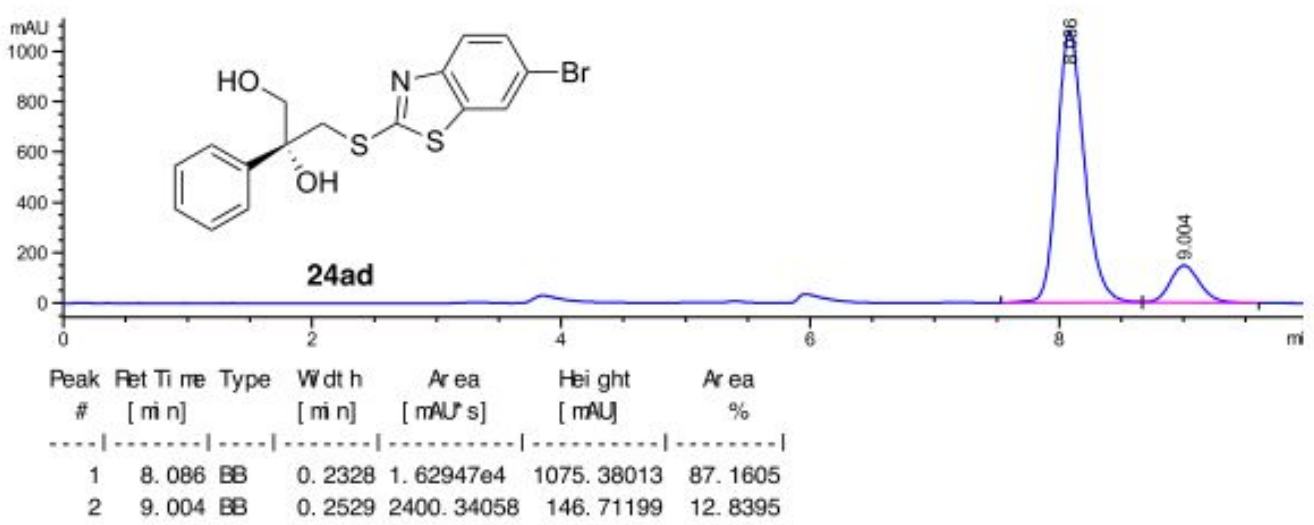

